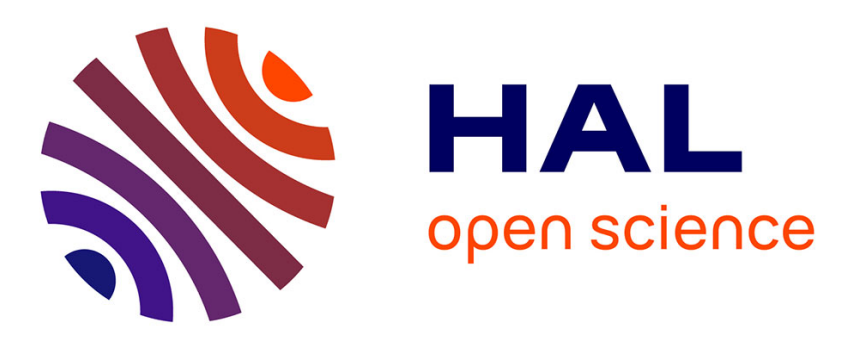

\title{
Global transcriptional profiling of neural and mesenchymal progenitors derived from human embryonic stem cells reveals alternative developmental signaling pathways.
}

Jérôme Alexandre Denis, Christelle Rochon-Beaucourt, Benoite Champon, Geneviève Pietu

\section{To cite this version:}

Jérôme Alexandre Denis, Christelle Rochon-Beaucourt, Benoite Champon, Geneviève Pietu. Global transcriptional profiling of neural and mesenchymal progenitors derived from human embryonic stem cells reveals alternative developmental signaling pathways.. Stem Cells and Development, 2011, 20 (8), pp.1395-409. 10.1089/scd.2010.0331 . hal-00547262

\author{
HAL Id: hal-00547262 \\ https://hal.science/hal-00547262
}

Submitted on 15 Dec 2010

HAL is a multi-disciplinary open access archive for the deposit and dissemination of scientific research documents, whether they are published or not. The documents may come from teaching and research institutions in France or abroad, or from public or private research centers.
L'archive ouverte pluridisciplinaire HAL, est destinée au dépôt et à la diffusion de documents scientifiques de niveau recherche, publiés ou non, émanant des établissements d'enseignement et de recherche français ou étrangers, des laboratoires publics ou privés. 

human embryonic stem cells reveals alternative developmental signaling pathways

Jérôme Alexandre Denis ${ }^{1}$, Christelle Rochon-Beaucourt ${ }^{1 £}$, Benoite Champon ${ }^{2}$, Geneviève Pietu $^{1 \S}$

1. INSERM/UEVE U-861, I-STEM, AFM, Institute for Stem cell Therapy and Exploration of Monogenic diseases, 5 rue Henri Desbruères, 91030 Evry cedex, France

2. CECS/AFM, I-STEM, Centre d'Etude des Cellules Souches, 5 rue Henri Desbruères, 91030 Evry cedex, France

$£$ Present address: Cellectis BioResearch Parc Biocitech 102, Av. Gaston Roussel 93235 ROMAINVILLE Cedex

$\S$ To whom correspondence should be addressed:

Dr Genevieve PIETU,

I-STEM Genopole Campus, 15 rue Henri Desbruères 91030 Evry Cedex France

Tel : 33(0)1 699085 24; Fax : 33(01) 69908521

e-mail : gpietu@istem.fr

Keys words : Human embryonic stem cells ; Neural precursors ; mesenchymal progenitors; cell fate decision ; microarrays

\section{ABSTRACT}


Human embryonic stem cells can be differentiated along different lineages, providing the possibility of a precise analysis of genes profiles associated with specific commitments. Subtractive gene expression profiling between differentiated and undifferentiated cells provides lists of potential actors in this commitment. This combines, however, genes that are specifically associated with development and others that are over expressed because of nonlineage-specific differentiation systems. As a way to establish gene profiles associated with the neural and/or to the mesodermal commitments of human embryonic stem cells more precisely, we have carried out a two-step analysis. We first performed a subtractive analysis of gene profiles of each of these lineages as compared to the undifferentiated stage. Then, we extended the analysis by comparing the two sets of results with each other. This strategy has allowed us to eliminate large numbers of genes that were over expressed in both sets of results and to uniquely associate different gene networks with either the neural or the mesodermal commitments.

\section{INTRODUCTION}


For one decade, human embryonic stem cells (hES) have been recognized as a valuable model for studies of early steps of human development since the acquisition of a defined phenotype in vitro follows sequential activation of gene networks and epigenetic changes that closely mimic events occurring in vivo during embryogenesis [1-5]. Identification of culture conditions that specifically direct hES toward unique phenotypes provides new ways to analyze molecular correlates of early developmental transition phases. One of the major technical advances for the study of such transitions is the development of efficient technologies such as DNA microarray that enable monitoring gene expression at the level of mRNA on a genomic scale. Nevertheless, analysis of how genes are controlled during transition toward a dedicated developmental path is restricted mainly by the ability to obtain homogeneous populations of cells because their phenotypes are continuously changing over time. Despite this, several studies clearly demonstrate that such approaches applied to hES and their progenies can provided useful informations about gene expression involved in the developmental processes [6,7]. As an example of meta-analyze see [8] and for review on human embryonic stem cell transcriptome profile see also [9].

Over the last few years, important efforts focused on the possibility of controlling the differentiation of hES particularly for neural fate. Indeed, using protocols based on the coculture of hES with defined stromal cell lines exhibiting neural inducing activity, it is possible to directly visualize the process of neural conversion by forming of columnar epithelial cells radially organized resembling a cross section of neural tube named "neural rosettes" [10,11]. These neurectodermic clusters of cells are greatly enriched in neural progenitor cells (NPC) which have the ability to differentiate into various subtypes of neurons, astrocytes and oligodendrocytes [10-14]. Although this system of culture is considered to be relevant for neural induction, neural cells differentiated in vitro from $\mathrm{hES}$ are widely heterogeneous, 
probably due to no synchronized processes [15]. Consequently, neural rosettes represent structures that contain a mixture of cells, including neuroepithelial progenitor cells (NEPC), neural stem cells (NSC), committed neurons at different stages of their development and probably neural crest derivatives at their periphery. For this reason, several groups have attempted to develop cell selection strategies including the use of fluorescence-activated cell sorting (FACS). To date, only few membrane markers have been used to enrich cultures in neural precursors and neurons [16]. Among these markers, we used Neural Cell Adhesion Molecule (NCAM/CD56) in this work [17].

The production of mesenchymal precursors (MPC) has not been as widely reported as that of neural precursors. By taking advantages of the recent development of protocols triggering the differentiation of hES toward a near-homogenous amplifiable population of mesenchymal progenitors exhibiting a phenotype of Mesenchymal Stem Cells-like (MSC) can be obtained [18-23]. In this study, we produced highly homogenous cell populations for both neural and mesenchymal precursors by engagement of the hES cells into either the neural or the mesodermal lineages. The analysis of gene expression patterns of these two populations, sharing the same genetic background, compared to the same starting population that were hES cells, using strictly identical procedures for hybridization and statistical analysis, allowed us to select genes that were modulated in opposite directions during commitment to either neural or mesenchymal fates. After this subtractive analysis, selected genes exhibiting modulations specific for either neural or mesenchymal precursors were used to build in silico global gene networks and, using a comparative strategy, to determine their implications as actors in the main signalling pathways involved in early steps of human development.

\section{MATERIALS AND METHODS}




\section{Human ES Cells Culture}

Two hES cell lines, SA01, (XY, passage 40, Cellartis, Sweden) and VUB01 (XY, passage 80, AZ-VUB, Belgium) were maintained and propagated on a feeder layer of STO (SIM mice Thioguanine and Ouabaine resistant) murine embryonic fibroblast cells inactivated by Mitomycin C (Sigma Aldrich, $2.5 \mu \mathrm{g}$ per $\mathrm{mL}$ overnight at $37^{\circ} \mathrm{C}$ ). Cells were cultured in a humidified $5 \% \mathrm{CO} 2$ incubator at $37^{\circ} \mathrm{C}$ in a serum replacement medium (Knock-out DMEM, $20 \%$ Knock-out Serum Remplacement (KSR), 1\% Glutamax 1mM, 1\% Non Essential Amino Acid (NEAA), 0.1\% Beta-Mercaptoethanol (BM) $0.1 \%$ and $1 \%$ Penicillin/streptomycin (P/S), all from GIBCO) supplemented with $8 \mathrm{ng} / \mathrm{mL}$ of bFGF (Invitrogen). The culture media was changed daily and routine passages routinely performed by mechanical cutting of hES cells on a fresh feeder layer every 4-5 days.

\section{Differentiation of hES cells toward NPC, neurons and astrocytes}

Differentiation of hES cells toward NPC

The differentiation of hES toward neurectodermal rosettes was adapted from the Stromal Differentiation Inducing Activity protocol as described elsewhere [10]. Briefly, hES cells were manually dissociated from the STO feeder layer and plated at a density of approximately $10^{3}$ cells per $\mathrm{cm}^{2}$ on a confluent layer of mitotically inactivated murine stromal feeder cells (MS5). Cells were cultured in KSR medium (Knock-out DMEM, 15 \% KSR; $1 \%$ Glutamax; $1 \%$ NEAA and $0.1 \%$ BM, all from GIBCO) for 14-16 DIV (Days $\underline{I} n$ Vitro), when the medium was replaced by Neurobasal medium, N2 (DMEM-F12+ Glutamax, 1\% N2 supplement and $1 \% \mathrm{P} / \mathrm{S})$ until DIV21.

Cell sorting

Cells were harvested at DIV21 using TrypLE Express (GIBCO) and about $5.10^{6}$ cells were suspended in PBS-2\% Fetal Calf Serum containing 1\% 7-amino-actinomycin D (7AAD) 


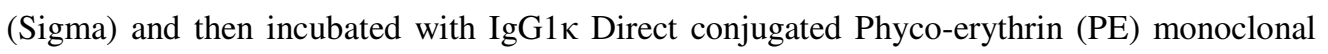
anti human Neural Cell Adhesion Molecule (hNCAM) antibody diluted 1/10 provided by BD Biosciences Pharmingen ${ }^{\mathrm{TM}}$. This antibody recognizes an extracellular immunoglobulin-like domain common to three molecular weight forms -Mw 120, 140 and 180 kilodaltons -of the NCAM protein. The cell sorting was performed by a MoFlow Cell Sorter Cytometer from Cytomation and positive and negative fractions were collected in $1 \mathrm{~mL}$ of $\mathrm{N} 2$ medium with $1 \% \mathrm{P} / \mathrm{S}$.

Differentiation of NPC toward neurons and astrocytes

After sorting, the NCAM+ cells were seeded on Poly-Ornithine $(15 \mu \mathrm{g} / \mathrm{mL}, \mathrm{Gibco}) / \mathrm{laminin}$ $\left(1 \mathrm{mg} / \mathrm{mL}\right.$, Sigma) coated dishes $\left(50 \times 10^{3} / \mathrm{cm} 2\right)$ in $\mathrm{N} 2$ medium supplemented with growth factors bFGF (20ng/ml, Invitrogen), and EGF (10ng/ml, Abcys) to allow their proliferation for 10- 15 days. The medium was changed every two days. When confluent, they were passaged (P1) after exposure to collagenase $1 \mathrm{mg} / \mathrm{ml}$ for 15 minutes at $37^{\circ} \mathrm{C}$ and plated in $\mathrm{N} 2$ medium supplemented for differentiation toward either neurons or astrocytes. For neuronal differentiation, BDNF (10 ng/mL) and AA ascorbic acid (10 ng/mL) was added in the N2 medium which was changed every 2-3 days for 2 weeks after which the cells were fixed. For differentiation toward astrocytes, medium was supplemented with EGF and CNTF (20ng/ml, R\&D) for 15 days. After that, they were passaged and maintained in N2 medium containing only CNTF until around 100 DIV and then fixed for immunochemistry.

\author{
Differentiation of hES cells toward MPC cells, osteoblasts and adipocytes \\ Differentiation of hES cells toward MPC cells
}


Mesodermal differentiation was obtained as previously described by our laboratory [22] using a modified protocol from Barberi et al [23]. Briefly, differentiation was induced by plating $2 * 10^{4}$ ES cells $/ \mathrm{cm}^{2}$ on $0.1 \%$ gelatin coated dishes in the presence of KO-DMEM medium supplemented with 20\% Fetal Bovine Serum (FBS, Invitrogen), 1mM L-glutamine, $1 \%$ NEAA, $1 \% \mathrm{P} / \mathrm{S}$ and $0.1 \mathrm{mM}$ BM. Medium was changed every 3 days. Confluent cells were passed with trypsin/EDTA $1 \mathrm{X}$ (Invitrogen) in new gelatine coated dishes. Immunophenotyping was carried out using a FACScalibur and the Cell Quest software (Becton\&Dickinson Biosciences). More than 10,000 events were acquired for each sample and analysed. Cells were harvested as previously described and incubated for 30 minutes at room temperature with one of the following anti-human antibodies: CD73-PE (SH3/NT5E), CD44-PE, CD54-PE (I-CAM-1), CD29-PE (integrin $\beta 1$ ), CD106-PE (VCAM), CD166-PE (ALCAM), CD14-PE, CD31-PE (PECAM-1), CD56-PE (NCAM), HLA-ABC-PE, HLA-DRPE, CD34-APC, CD45-FITC (all from Becton\&Dickinson Biosciences/Pharmingen); CD105PE (SH2/Endoglin; Caltag); Nestin-PE (R\&D systems) and primary monoclonal antibody vimentin and Stro1 were used with mouse IgG- or IgM-Alexa as secondary antibody. Mouse isotype antibodies served as respective controls (Becton\&Dickinson). The acquisition was performed by the FACScalibur cytometer and data were analyzed with Cell Quest Pro Software (Beckton-Dikinson).

\section{Differentiation of MPC cells toward osteoblasts and adipocytes}

To induce osteoblastic differentiation, cells were plated at a density of 30000 cells $/ \mathrm{cm}^{2}$ in a specific medium (Cambrex), containing dexamethasone, ascorbate and B-glycerophosphate (Sigma-Aldrich). After 21 days, cell phenotype was analysed by alkaline phosphatase activity (Sigma-Aldrich). Adipogenic differentiation was induced by culturing the cells in the specific medium (Cambrex) supplemented with $100 \mu \mathrm{M}$ linoleic acid. Adipogenesis was detected by the presence of neutral lipids in the cytoplasm stained with Oil Red Oil. 


\section{Immunocytochemistry}

Cells were fixed in paraformaldehyde for 20 minutes at room temperature, rinsed with PBS and blocked with $1 \%$ BSA, $5 \%$ goat serum $0,1 \%$ triton in PBS solution for 1 hour and thereafter were incubated with the appropriate primary antibodies overnight at $4^{\circ} \mathrm{C}$. Rabbit polyclonal antibodies included Nestin (dilution 1/500: Chemicon) and GFAP (dilution 1/1000; DAKO). Mouse Monoclonal antibodies ( $\operatorname{IgG}$ ) included TujI (dilution 1/500, Covance), Stro-1 (dilution 1:100) and SMA (alpha smooth muscle: 1:100, DAKO). Appropriate Alexa 488 and Alexa 555 labeled secondary antibodies were used at 1/500 and 4',6-diamino-2-phenylindole at $2 \mu \mathrm{g} / \mathrm{ml}$ (Sigma) for counterstaining.

The preparations were analyzed by epifluorescence microscopy (Zeiss Imager Z1 and Zeiss Axiovert 40CFL) and images were captured with Axiocam mRM (Zeiss).

\section{RNA Sample preparation}

mRNA samples were extracted using the RNeasy Mini kit (Qiagen) according to the manufacturing protocols for undifferentiated hES cells, NPC and MPC. RNA samples were quantified using the Nanodrop photometer and quality controls were performed on BioAnalyzer 2100 (Agilent). For RT-PCR analysis, cDNAs were prepared by reverse transcription of $500 \mathrm{ng}$ of mRNA using the SuperScript II Reverse Transcriptase kit according to the manufacturer's instructions (Invitrogen). Primers used in this study are shown in Supplementary Table S1. Quantitative RT-PCR analyses were performed with Chromo4 Analyser (Biorad) and calculations were performed using the delta-delta $\mathrm{C}(\mathrm{t})$ method.

\section{Hybridization and data analysis}

RNA samples were labeled and hybridized on the GeneChip Human Genome HG_U133_Plus 2.0 Array (Affymetrix) by the RNG platform (Réseau National des Génopoles, Paris, France) 
according to the Affymetrix procedures. Hybridization data were exploited using Array Assist 4.2 software (Stratagene). First, the software validated the quality controls. Next, the GCRMA statistical algorithm procedure was used to normalize hybridization intensity values. A one way-ANOVA test was applied on transformed logarithm base 2 data to retain values that did not changed significantly $(\alpha<0.05)$ among triplicate samples. Identification of modulated genes was performed using the Student parametric statistical test adjusted with the FDR Benjamini-Hodgberg correction. A gene was considered as modulated for a Fold Change (FC) $>2$ with a corrected p-value, $\alpha_{c}<0.05$. The final list of modulated genes was established by removing duplicate data (multiple probesets measuring the same gene) to retain the most modulated one. GO biological processes and canonical pathways analysis enriched in differentially expressed genes were identified using the Fischer exact test as implemented in the Ingenuity Pathways Analysis (Ingenuity ${ }^{\circledR}$ Systems, www.ingenuity.com) software.

\section{RESULTS}

\section{Obtention of two homogenous neural and mesodermal progenitor cells from hES}

Two hES cell lines (SA_01 and VUB_01) were induced in triplicate toward the neural lineage using the SDIA protocol (see Material and Methods). Under these conditions, neural rosettes appeared around 16-18 days in vitro (DIV) (Supplementary Figure S1A). To get a homogenous population, cell sorting was performed at DIV21 using the membrane marker Neural Cell Adhesion molecule (NCAM/CD56) known to be expressed in neural precursors but not expressed in MS5 mouse feeder cells and to a small extent in the undifferentiated hES cells (Supplementary Figure S1B). The NCAM positive cells, termed NPC, expressed a combination of markers known to be specific for the neurectodermic precursors such as NESTIN and the transcription factors SOX1,PAX6 and OTX2. Moreover, the NCAM positive cells exhibited a complete loss of NANOG expression and did not express GFAP (Glial 
Fibrillary Acidic Protein) by RT-PCR (Supplementary Figure S1C) or by immunochemistry (Supplementary Figure S1 D-F). The NCAM positive cell population was able to give rise to cells positive for TUJ1 ( $\beta$ III-tubulin) and for GFAP corresponding to neurons and astrocytes respectively (Supplementary Figure S1 G-H). Differentiation of ES cells into mesenchymal precursors (MPC) displaying a phenotype similar to that described by previous authors [1821] was readily obtained after about 30 days of culture (two to three passages). Cells displayed a homogeneous fibroblast-like morphology (Supplementary Figure S2A). At near homogeneity, they expressed CD29 ( $\beta 1$-integrin), CD44 (H-CAM), CD73 (SH-3, ecto-5'nucleotidase), CD105 (SH-2, endoglin), CD166 (ALCAM), and were negative for hematopoietic markers (CD34, CD45 and CD14), neuronal markers (NCAM/CD56 and FORSE1), and the endothelial marker CD31 (Supplementary S2B). Cells were immunoreactive for Stro1 and some of them are positive for $\alpha$-SMA (alpha smooth muscle actin) (Supplementary Figure S2C). In addition, these MPC cells were also able to differentiate into osteogenic or adipogeneic cells in appropriate conditions (Supplementary Figure S2D).

\section{Global analysis of gene expression}

To compare the expression patterns during the commitment of hES toward the neural and the mesenchymal fates, transcriptome analysis was performed in triplicate on the two undifferentiated hES cell lines SA01 and VUB01 and the two progenitor cell populations, NPC and MPC (Figure 1A). The global expression profiles of these three populations were compared by correlation plot and by the Principal Component Analysis (PCA). A high correlation coefficient was observed between the expression patterns of the three replicates for each cell line and between the two cell lines indicating a small variability between the biological samples for the same stage of differentiation (Figure 1B). Moreover, when the three 


\section{Page 11 of 257}

distinct populations were plotted by PCA, samples for the same stage were very close (Figure 1C). This allowed us to group the results obtained for the two cell lines for further statistical analysis. A total of 3167 genes were found to be differentially expressed between NPC and hES cells, 1727 up-regulated and 1440 down-regulated (Supplementary Tables S2 and S3), including genes with no annotation, putative genes and expressed sequence tags (EST) that amounted to about a quarter of all modulated genes. Parallel comparison between hES and MPC, revealed 5931 genes modulated including 2212 genes up-regulated and 3719 genes down-regulated in MPC (Supplementary Tables S4 and S5). As expected, major markers of pluripotent hES cells including NANOG; OCT4 [POU5F1]; REX1 [ZFP42]; FGF4; FOXD3; CLDN6; GDF3; DNMT3A and CD2 were down regulated in both NPC and MPC. However, the expression of the pluripotency transcription factor $S O X 2$, was maintained in neural progenitors whereas it was switched off in mesenchymal progenitors.

As quality control of genes modulated in the transcriptomic experiment, we found specific neural genes expected to be up-regulated in NPC cells compared to hES, such as NCAM, PAX6, SOX1 and OTX2. On the contrary, neither markers of mesoderm such as T/Brachyury, HAND1, IGF2, CD45, FLK1, CD31, MYOD, CALP [Calponin] nor endodermal markers SOX17, FOXA2, GATA4, AFP were found modulated. In the same way, concerning genes known to be implicated in the MSC phenotype, CD73/NT5E, CD105/ENDOGLIN, CD44, INTEGRIN $\beta 1 / I T G B 1, A L C A M / C D 166$ and VIMENTIN, were all found to be up-regulated in MPC compared to hES.

\section{Subtractive gene expression profiling}


The lists of the modulated genes in the NPC and the MPC cell populations were compared (Figure 1D). The 785 genes which are up regulated in NPC but not in MPC (Supplementary Table S6) and the subset of 306 genes which were up regulated in NPC and down-regulated in MPC were selected as potential candidate genes involved in the neuralizing process (Supplementary Table S7). Conversely, the 1479 genes which are up-regulated in MPC but not in NPC (Supplementary Table S8) and the subset of 94 genes which were up-regulated in MPC and down-regulated in NPC were selected as potential candidate genes involved in the mesenchymal differentiation (Supplementary Table S9). Between the two selections of genes specifically implicated in the neuralization process or in the mesenchymal differentiation, we focused on transcription regulators which were specifically up-regulated in each of both processes (Table S10). For the neuralization process, among the 127 up-regulated transcription regulators (Table S10, left panel), for the eleven most modulated genes with a FC> 10, eight were annotated in nervous system development (GO and IPA classifications) including genes such as LHX2, PAX6, ZIC1, FOXG1B, TFAP2B, ZBTB16 and EMX2 all implicated in the neural progenitor signature. Besides these genes, others were annotated to be involved in neural and other developmental processes, such DACH1 and LEF1 or several members of family transcription factor such as the Inhibitor of DNA binding (ID2 and ID4), the POU domain family members (POU3F2 and POU4F1) but also the homeobox family members HOX (HOXAl and HOXA9). In addition, genes found to be up-regulated in NPC and strongly down-regulated in MPC included SRY-related box protein members such as SOX3 and SOX11 or the member of the bicoïd sub-family of homeodomain-containing transcription factors such as OTX2 which encodes gene already reported to be involved in neurogenesis.

For the mesenchymal differentiation of the 118 up-regulated transcription regulators (Table S10, right panel), 25 were implicated in connective tissue development (IPA classification). 
The most modulated genes were SIXI which encodes of the homeobox gene superfamily and two genes related to TGF-beta signaling were highly up-regulated, $C D K N 2 B$ and $T G F 1 B 111$. Other modulated genes involved in development included several members of the Forkheadbox family (FOXD1, FOXF1, FOXF2, FOXJ2, FOXL1 and FOXP1), the basic helix-loophelix family (BHLHE40 and BHLHE4), the Krüppel-like factors (KLF2, KLF7 and KLF9), signal transducers and activators of transcription (STAT1, STAT2, STAT3 and STAT6), T-box members (TBX2 and TBX3) and homeobox family members (HOXB2 and HOXB7).

\section{Transcriptional networks using an in silico approach}

Global gene networks were built based on selected genes exhibiting specific overexpression in each precursor, as described above, for either the neural (Figure 2A) or the mesenchymal differentiation (Figure 2B). Starting from the core pluripotency gene network composed of the three transcription factors, NANOG, OCT4 and $S O X 2$, their potential targets were explored by selecting genes that were specifically up-regulated in each precursor cell population.

For the neural gene network, the starting point was the binding relationship linking PAX6 and LHX2 promoters with NANOG, OCT4 and SOX2 proteins encoded by the core pluripotency genes. Using this strategy, the construction of the gene network step by step revealed some nodes that included key transcription factors including downstream NOTCH1 targets such as HES1 HES5, LEF1 but also PAX3 that may support a pivotal role with its complex partner SOX10. Otherwise, NOTCHI downstream signal was also connected with the SWI/SNF DNA remodelling complex (SMARCC4 and SMARCE1).

For the mesenchymal gene network, WWTRI (also termed TAZ) implicated downstream of $T G F B 1$, acted on the level of expression of $N A N O G$ and POU5F1 and thus may control several developmental genes including GATA6. Moreover, SOX2 (whose expression was maintained in neural but greatly decreased in mesenchymal differentiation) was connected with two critical genes involved in osteogenic differentiation, JUN and TWISTI. Organisation 
of the network also included PPARG which is involved in differentiation of mesenchymal stem cells toward adipocytes and STAT3 which acts downstream of the FGF signalling pathway and is involved in differentiation process.

\section{Alternative signaling pathways controlling cell fate decisions}

Levels of gene expression were explored in three signaling pathways, Notch, Wnt, and TGFß/Activin/BMP that are known to be involved in hES cell fates determination but required different partners depending of lineage-specific differentiation.

Notch signaling pathway

Some genes encoding for ligands of Notch, such as JAGl and DLLI were found to be upregulated in the NPC but not in the MPC cells (Figure 3A and 3B). Regarding the receptors in this pathway, NOTCH1 was specifically up-regulated in the NPC cells, NOTCH2 was found up-regulated in both progenitor cell types whereas other family members of NOTCH receptors were not modulated. Genes implicated in the modulation of the activity of NOTCH signaling that were specifically up-regulated in NPC cells included : LFNG (encoded a fucose-specific glycosyltransferase), ADAM17 (encoded a metallopeptidase involved in the proteolytic release of Notch intracellular domain from the Notch1 receptor) and PSEN1 (presenilins-1) involved in the cleavage of the Notch receptor and the regulation of gamma secretase activity. On the contrary, $N U M B$ encoding for an inhibitor of the Notch pathway and playing a role in the determination of cell fates during development was specifically over expressed in MPC. At least, specific transcriptional factors HES1 and HES5, downtream targets of Notch signaling were found specifically up-regulated in NPC cells but not in MPC whereas others family members were not modulated in either progenitor cell types. On the contrary, $N U M B$, encoding for an inhibitor of the Notch pathway and playing a role in the determination of cell fates during development. Additionally, others downstream 


\section{Page 15 of 257}

transcriptional factors of the Hairy/enhancer-of split related with YRPW motif family exhibited similar expression profiles in the two progenitor cell types (ie up-regulation of HEY1 and down regulation of HEY2). Specific over expression of DLL1, NOTCH1, HES1 and HES5 were confirmed in NPC by quantitative RT-PCR (Figure 3C).

Wnt Signaling pathway

Concerning genes involved in the Wnt signaling pathway, over expression of genes encoding negative regulators of this canonical pathway was observed in the NPC whereas genes modulated in MPC cells rather reflected its activation (Figure 4A and 4B). Indeed, Wnt inhibitors including secreted antagonists such as DKK1, SFRP2 and FRZB were all over expressed specifically in NPC. In addition, genes encoding for Wnt ligands such as WNT2B, reported to be a repressor of the canonical pathway, appeared to be specifically up-regulated in NPC cells whereas $W N T 5 A$ and $W N T 5 B$, two non canonical ligands, were found to be modulated in the two types of progenitors. For the Wnt Receptors, notably Frizzled proteins, FZD3 and FZD5 were found respectively up or not regulated in neural progenitors whereas they were both switched off in mesenchymal progenitors. Concerning transcriptional regulators involved downstream of Wnt signalling pathways, genes involved in the repression of the $\beta$-catenin complex were found to be up-regulated only in NPC such as the SOX transcription factor SOX3 but also CTNNBIP1, a gene encoding a small soluble inhibitory protein also termed ICAT (Inhibitory of beta-catenin and TCF) which prevents the interaction of $\beta$-catenin with different binding partners including $L E F 1$. In addition, the gene encoding for the transcription repressor TLE4, a member of the Groucho family, was also found upregulated only in the NPC cells. Finally, when looking for the expression of genes known to be controlled directly downstream of the canonical $\beta$-catenin pathways, some genes, such as DCT, POU $3 F 2$ and NRCAM, controlled downstream of the complex containing LEF1 
appeared to be induced in NPC cells whereas no modulation of these genes was observed in the MPC cells. On the contrary, genes encoding important Wnt-induced mesenchymal markers such as FOSL1, JUN, PPAR $\delta$, CD44 were all up-regulated in the MPC whereas the expression of these genes was not modulated in the NPC. To confirm these results, expression modulations for several genes, WNT2B, FRZB, SFRP2, LEF1, involved in Wnt signaling pathway were confirmed by Quantitative PCR using new biological samples in triplicate from three independent differentiation experiment. Results showed that they were found to be up regulated in NPC and not modulated or down-regulated in MPC compared to hES (Figure 4C).

TGF/Activin/BMP signalling pathway

Among the genes involved in BMP/TGF $\beta$ signaling pathways (Figure 5A and 5B), BMP7 expression was up regulated during the differentiation of hESC toward NPC, whereas it was switched off during their differentiation toward MPC. Interestingly, we also found upregulation of FST gene in MPC encoding follistatin, an inhibitor of BMP pathway (Figure 5C). In contrast, the up regulation of INHBA (inhibin beta A, also termed activin A), which encoded a strong inducer of mesendoderm was overexpressed only in MPC (Figure 5D). Differences between others modulated genes encoding for TGF $\beta$ ligands were also found such as up- regulation of TGFB1 specifically in MPC. Concerning downstream transcriptional factors target, we found an up-regulation of SMAD3 in both NPC and MPC cells respectively whereas SMAD5 was found slightly modulated only in NPC.

At last, in order to confirm expression modulation of genes specifically involved in one of these signaling pathways either in neural or mesenchymal progenitors, quantitative PCR data were carried out from new independent biological samples (Figure S3).

\section{DISCUSSION}




\section{Page 17 of 257}

The principal result of this study is the comparison of gene expression profiles in two homogenous populations of neural and mesenchymal of progenitors by subtractive gene expression profiling during early differentiation in $\mathrm{hES}$ toward either neural or mesodermal commitment. Genes whose expressions were regulated in opposite directions might be of particular interest in molecular processes involved in the alternative cell fate decisions. Using a comparative strategy, we identified actors specific for each lineage which play an alternative role at the level of epigenetic modifications, implication of morphogens and through major developmental signalling pathways.

The integration of all these data allowed the construction of a global comprehensive developmental path between neurectoderm and mesoderm (Figure 6).

\section{Specific gene expression pattern associated with each precursor cell}

Transcriptional factors found to be specifically highly up-regulated in NPC included $L H X 2$, PAX6, SIX3, SIX6, SOX1 and FOXG1B. This gene signature indicated that our neural progenitors exhibited a pattern closed to early neural progenitors appearing first in neural plate and/or neural tube in mouse and human during normal development in vivo. Unlike late neural progenitors, these cells expressed markers representative of the anterior region of the mouse developing brain including FOXG1,EMX2 or OTX2 and markers of dorsal region such as $P A X 3$ or $P A X 6$. This "anterior" pattern was associated to a broad capacity of differentiation into various types of neurons and to glial cells in response to appropriate developmental clues. Thus, the present study presented for the first time a picture of gene expression network specifically associated to the neural developmental pathway of these progenitors.

Mesenchymal progenitors derived from hES cells appeared to be the first type of progenitors exhibiting a mesodermal phenotype that can be obtained almost homogeneously [18]. 
Identification in this study of genes specifically up-regulated in MPC highlighted potential factors that may play a role in the mesoderm formation including TWIST1. This gene is expressed in presumptive mesoderm and in invaginating cells in the ventral region of fruit fly embryos and functions in a signaling cascade to initiate mesodermal development during gastrulation in multicellular organisms ranging from Drosophila to vertebrates [24-26]. TWIST plays a central role in dorsoventral patterning, which is essential for multiple steps of mesoderm development in Drosophila [27,28]. Another interesting gene includes WWTR1/TAZ which was known to control the mechanism of self-renewal through controlling Smads nucleo-cytoplasmic shuttling [29-31] and thus may contributing to the cell fate choice by controlling mesodermal genes. Among other genes that may represent important developmental nodes, $R U N X 2$, SQSTM1 that are both involved in skeletal development were highlighted suggesting that early mesodermal progenitors acquire a developmental context that enhances osteogenesis.

\section{Epigenetic modification signatures}

The transcriptional signature also deciphers the role of another type of developmental regulation that concerns genes involved in epigenetic modifications.

Among genes that are differentially expressed during neural or mesoderm differentiation, genes encoding helicases that function to open chromatin to enhance transcription in the SWI/SNF DNA chromatin remodeling complex family including SMARCC1 and SMARCE1, were found specifically up-regulated in the neural progenitors and may interact with proteins encoded by other specific genes such as ARID2 and ARIDIB [32,33]. These proteins may play a role in enhancing differentiation by coupling gene repression with global and local changes in chromatin structure [34]. In mesenchymal precursors, specific up-regulation of SMARCA2 
was observed, that has been described to be specific of mesoderm in early post-implantation mouse embryos [35].

\section{Morphogen implications}

Another aspect of the transcriptional signatures concerns morphogens that act through signalling pathways. $B M P 7$ was found to be up-regulated in neural progenitors and therefore these cells may themselves produce morphogens that contribute to control downstream genes involved in neural development including ZICI [36] and GLI3 [37]. In MPC, the upregulation of INHBA encoding the beta A subunit that forms a homodimer named Activin-A was reported to be one of the most important mesodermal morphogens in classical developmental models including Xenopus laevi [38]. More recently, Willems and Leyns described that Activin A supported self-renewal of hES and directed the nascent mesoderm toward axial mesoderm and mesendoderm [39]. This increase also coincides with upregulation of the activin receptor type 1, ACVR1. Inversely, inhibition of Activin/Nodal signalling promotes specification of hES into neurectoderm [40]. In this context, it is interesting to notify that the follistatin, encoding a protein that antagonizes activin $[41,42]$ and BMP signalling pathway in Xenopus embryo [43] is specifically up-regulated in NPC. This might suggest that follistatin may contribute to induce neural differentiation of hES in vitro by antagonising the different pathways (BMPs, Activin and TGF $\beta$ ). Indeed, currently, the most efficient protocol allowing hES differentiation toward neurectoderm uses Noggin (an inhibitor of BMPs) and SB43542 (an artificial molecule inhibating Activin and TGF $\beta$ pathways) $[44,45]$

Altogether, these data suggest that once the mesenchymal phenotype has been acquired, these precursors may maintain a mesodermal identity by producing themselves important 
morphogens such as Activin A and inversely the neurectodermal phenotype may be maintained by producing follistatin that prevents activation of activin pathways.

\section{Alternative pathway responses}

Wnt and Notch pathway responses occurred during both neural and mesenchymal differentiation. Although discussed, different studies described a "negative" effect of WNT/ $\beta$ catenin signalling pathways onto the neural induction process in vivo [46] and in vitro in mouse ES $[47,48]$. Indeed, an up-regulation of expression of some modulators/inhibitors of the Wnt signalling was observed in NPC (but not in MPC) including SFRP2, identified elsewhere to enhance neural differentiation [47] but also $F R Z B$, another Wnt inhibitor found expressed in neural plate and neural tube in overlapping areas like SFRP2 during chick development [49]. During and just after gastrulation, the Wnt pathway was also involved as an inducer of primary mesenchymal cells but specific genes involved in this process are still unknown or elusive due to differences between species. In this study, we found that gene expression modulations of Wnt pathway members in mesenchymal cells but not in neural cells including $D K K 3$ which is both temporally and spatially regulated [50] may play a role in mesoderm formation in humans has been demonstrated in Xenopus laevis [51]. Additionally, a distinct profile of genes downstream of the Wnt signalling pathway was also observed. Neural progenitors did not express numerous genes known to be controlled by CTNNB1 whereas they were expressed in MPC. In contrast, a possible mechanism would be due to the expression of the inhibitor of the interaction of $\beta$ catenin with $L E F 1$. Thus, LEF1 may interact with other proteins and control a neural gene network downstream. Concerning the Notch signaling pathway, the central role of NOTCH1 specific pathway already described was confirmed as an important path for the maintenance of the neural progenitor state $[52,53]$ whereas the down-regulation of NOTCH1 was already reported to enhance the differentiation of mesodermal cardiogenic progeny [32]. Here, DLL1, one of the NOTCH1 ligands was 


\section{Page 21 of 257}

found highly up-regulated in NPC and may contribute to induce HES1 and HES5 transcription factors acting by their dominant effect on neurogenic transcription factors and therefore to delay the differentiation of neural precursors in mature neurons maintaining a "neural precursor state". Conversely, DLL1 was down-regulated in MPC suggesting a process that closely controls this gene during the transition toward either neural or mesoderm identity.

Some genes connected to Notch signalling were also described that may support central interactions in the global neural gene network. For example, SOX10 that interacts with PAX3 may reflect some features of neural crest development as demonstrated in cells surrounding neural rosettes in culture [54] and in the neural crest origin of the Waardenburg syndrome in humans [55]. WNT inhibition pathways associated with NOTCH1 activation may be involved in expression of $L E F 1$ specific expression contributing to an explanation of the specific regulation of downstream neural genes such as NRCAM, DCT and POU3F2. Concerning the acquisition of the mesenchymal phenotype, our data suggested that the differentiation involved a developmental process mimicking the TGF $\beta$-induced epithelial-mesenchymal transition (EMT) process. Numerous genes involved downstream of TGFbeta1 signalling pathways which have already been described as major contributors of the EMT type 1 process were found to be up-regulated [56,57]. During embryonic development, the EMT is a crucial cellular process whereby adherent cells dissolve their intercellular contacts, organize their motility apparatus, and move to new locations. As observed in vivo during the EMT, during mesenchymal differentiation of hES, we found the loss of epithelial cell adhesion markers including E-box genes including E-cadherin [CDH1] but also claudin [CLDN3/6/10/23], occludin [OCLN] and loss of some polarity genes such as Discs Large (DLG3/7) [58]. Crumbs homolog3 (Crb3) [59] and conversely the acquisition of mesenchymal markers including Fibronectin [FN1], Vimentin [VIM] and thrombospondin-1 [THBS1]. It can be postulated that TGF $\beta 1$ signalling may reflect in part the biology of the mesenchymal 
progenitors. Moreover, SIX1 a developmental gene encoding for a homeodomain transcription factor was the most up-regulated transcription regulator in MPC. It is known to cooperate with TGF $\beta$ and to increase the downstream EMT-induced TGF $\beta$ signal leading to the acquisition and/or maintenance of the stem cell-like phenotype accompanying EMT [60,61].

In conclusion, based on the production of precursor cell populations at near homogeneity, our data describing modulation of gene expression should contribute to a better comprehension of gene regulations involved in fate choices during differentiation of embryonic stem cells.

\section{ACKNOWLEDGEMENTS:}

The authors wish to thank Dr Marc Peschanski for continuous support and input during this study. This work was supported by the Association Française contre les Myopathies (AFM), MediCen (IngeCell program) and Genopole. We thank the platform of RNG, Institut Curie, Paris, France, for performing Transcriptome experiments, Phillippe Rameau for cell sorting, Lina Kassar-Duchossoy for astrocytes differentiation and Drs Alexandra Benchoua, Anselme Perrier, Laetitia Aubry and Cécile Martinat for their expertise in the field and helpful discussions.

\section{AUTHORS INFORMATIONS}

The gene expression data have been deposited in GEO Data Bank with the accession number GSE8590. 


\section{LEGENDS}

Figure 1 : Global gene expression patterns analysis for the hES cells (ES) and the two precursor cells, NPC and MPC derived from the same hES cells. (A) Schematic representation of the experimental procedure. (B) Correlation plots between all arrays of the experiment hybridized with ES, NPC and MPC mRNAs ( $\mathrm{n}=6$ per condition). Correlation was normalised in a scale from $\mathrm{r}^{2}=0.79$ (green) to $\mathrm{r}^{2}=1$ (red). (C) Principal component analysis (PCA) of the three cell populations representative of intra-samples variability across whole chip for each developemental stage. Each array is represented as a colored square in a twodimensional reference. ES are represented as red squares; MPC as green squares and NPC as blue squares. (D) Genes were selected as differentially expressed either between NPC vs hES or MPC compared to hES for a thereshold of FC (Fold change) $>2$ and a corrected p-value $\alpha<0.05$. The lists of modulated genes in all the conditions were crossed by Venn diagrams.

Figure 2: Global gene networks. Networks were constructed using the Ingenuity software based on expression relationships described in the literature. Modulated genes are represented as a box with its gene symbol inside. (A) Global neural gene network and (B) global mesenchymal gene network. Genes in red were up-regulated and genes in green were downregulated in NPC or MPC compared to hES. Colour intensities of genes were correlated to fold change intensities.

Figure 3: Comparison of gene expression modulations within Notch signalling pathway either in NPC or in MPC. Modulated genes are represented as a box with its gene symbol inside. Genes up-regulated by comparison with hES are in red and those down-regulated in green. Colour intensities of genes were correlated to fold change intensities. The following 
symbol $(Q)$ indicated a relation of "inhibition" and ( $९$ ) a relation of "activation" between genes in the path. (A) Genes modulated between NPC and hES (B) Genes modulated between MPC and hES. (C) Differential gene expression measured by quantitative RT-PCR in hES (ES), MPC, NPC. Results are presented as relative expression level compared to hES cells considered arbitrarily as 1 from 3 independent samples. (t-student, $* \mathrm{p}<0,05)$.

Figure 4 : Comparison of gene expression modulations within canonical Wnt/ $\beta$ catenin pathway either in NPC or in MPC. Same legend as described in Figure 3. (A) Genes specifically modulated between NPC and hES (B) Genes modulated between MPC and hES. (C) Differential gene expression measured by quantitative RT-PCR in hES (ES), MPC, NPC. Results are presented as relative expression level compared to hES cells considered arbitrarily as 1 from 3 independent samples. (t-student, *p<0,05; **p<0,01;***p<0,001; ns statistically not significant).

Figure 5: Comparison of gene expression modulations within TGF Beta/Activin/BMP signalling pathway either in NPC or in MPC. Same legend as described in Figure 3. (A) Genes specifically modulated between NPC and hES (B) Genes modulated between MPC and hES. Differential expression measured by quantitative RT-PCR for (C) Inhibin BetaA, INHBA (Activin A) and (D) FST, Follistatin. (t-student, * $\mathrm{p}<0,05$; ns statistically not significant).

Figure 6 : Comprehensive developmental path model based on our gene expression data which may be controlled the hES cell fate decision toward either neural or mesodermal fates. 


\section{REFERENCES}

1. Thomson JA, J Itskovitz-Eldor, SS Shapiro, MA Waknitz, JJ Swiergiel, VS Marshall and JM Jones. (1998). Embryonic stem cell lines derived from human blastocysts. Science 282:1145-7.

2. Murry CE and G Keller. (2008). Differentiation of embryonic stem cells to clinically relevant populations: lessons from embryonic development. Cell 132:661-80.

3. Aberdam D, K Gambaro, A Medawar, E Aberdam, P Rostagno, S de la Forest Divonne and M Rouleau. (2007). Embryonic stem cells as a cellular model for neuroectodermal commitment and skin formation. C R Biol 330:479-84.

4. Abranches E, M Silva, L Pradier, H Schulz, O Hummel, D Henrique and E Bekman. (2009). Neural differentiation of embryonic stem cells in vitro: a road map to neurogenesis in the embryo. PLoS One 4:e6286.

5. Vallier L and RA Pedersen. (2005). Human embryonic stem cells: an in vitro model to study mechanisms controlling pluripotency in early mammalian development. Stem Cell Rev 1:119-30.

6. Brandenberger R, H Wei, S Zhang, S Lei, J Murage, GJ Fisk, Y Li, C Xu, R Fang, K Guegler, MS Rao, R Mandalam, J Lebkowski and LW Stanton. (2004). Transcriptome characterization elucidates signaling networks that control human ES cell growth and differentiation. Nat Biotechnol 22:707-16.

7. Calhoun JD, RR Rao, S Warrenfeltz, R Rekaya, S Dalton, J McDonald and SL Stice. (2004). Transcriptional profiling of initial differentiation events in human embryonic stem cells. Biochem Biophys Res Commun 323:453-64.

8. Assou S, T Le Carrour, S Tondeur, S Strom, A Gabelle, S Marty, L Nadal, V Pantesco, T Reme, JP Hugnot, S Gasca, O Hovatta, S Hamamah, B Klein and J De Vos. (2007). A meta-analysis of human embryonic stem cells transcriptome integrated into a web-based expression atlas. Stem Cells 25:961-73.

9. Robson P. (2004). The maturing of the human embryonic stem cell transcriptome profile. Trends Biotechnol 22:609-12.

10. Perrier AL, V Tabar, T Barberi, ME Rubio, J Bruses, N Topf, NL Harrison and L Studer. (2004). Derivation of midbrain dopamine neurons from human embryonic stem cells. Proc Natl Acad Sci U S A 101:12543-8.

11. Zhang SC, M Wernig, ID Duncan, O Brustle and JA Thomson. (2001). In vitro differentiation of transplantable neural precursors from human embryonic stem cells. Nat Biotechnol 19:1129-33.

12. Li XJ, ZW Du, ED Zarnowska, M Pankratz, LO Hansen, RA Pearce and SC Zhang. (2005). Specification of motoneurons from human embryonic stem cells. Nat Biotechnol 23:215-21.

13. Elkabetz Y, G Panagiotakos, G Al Shamy, ND Socci, V Tabar and L Studer. (2008). Human ES cell-derived neural rosettes reveal a functionally distinct early neural stem cell stage. Genes Dev 22:152-65.

14. Wilson PG and SS Stice. (2006). Development and differentiation of neural rosettes derived from human embryonic stem cells. Stem Cell Rev 2:67-77.

15. Stewart MH, M Bosse, K Chadwick, P Menendez, SC Bendall and M Bhatia. (2006). Clonal isolation of hESCs reveals heterogeneity within the pluripotent stem cell compartment. Nat Methods 3:807-15.

16. Pruszak J, KC Sonntag, MH Aung, R Sanchez-Pernaute and O Isacson. (2007). Markers and methods for cell sorting of human embryonic stem cell-derived neural cell populations. Stem Cells 25:2257-68. 
17. Carpenter MK, MS Inokuma, J Denham, T Mujtaba, CP Chiu and MS Rao. (2001). Enrichment of neurons and neural precursors from human embryonic stem cells. Exp Neurol 172:383-97.

18. de Peppo GM, S Svensson, M Lenneras, J Synnergren, J Stenberg, R Strehl, J Hyllner, $\mathrm{P}$ Thomsen and C Karlsson. Human Embryonic Mesodermal Progenitors Highly Resemble Human Mesenchymal Stem Cells and Display High Potential for Tissue Engineering Applications. Tissue Eng Part A.

19. Mahmood A, L Harkness, HD Schroder, BM Abdallah and M Kassem. Enhanced differentiation of human embryonic stem cells to mesenchymal progenitors by inhibition of TGF-beta/activin/nodal signaling using SB-431542. J Bone Miner Res 25:1216-33.

20. Mateizel I, A De Becker, H Van de Velde, M De Rycke, A Van Steirteghem, R Cornelissen, J Van der Elst, I Liebaers, I Van Riet and K Sermon. (2008). Efficient differentiation of human embryonic stem cells into a homogeneous population of osteoprogenitor-like cells. Reprod Biomed Online 16:741-53.

21. Olivier EN, AC Rybicki and EE Bouhassira. (2006). Differentiation of human embryonic stem cells into bipotent mesenchymal stem cells. Stem Cells 24:1914-22.

22. Giraud-Triboult K, C Rochon-Beaucourt, X Nissan, B Champon, S Aubert and G Pietu. Combined mRNA and microRNA profiling reveals that miR-148a and miR-20b control human mesenchymal stem cell phenotype via EPAS1. Physiol Genomics.

23. Barberi T, LM Willis, ND Socci and L Studer. (2005). Derivation of multipotent mesenchymal precursors from human embryonic stem cells. PLoS Med 2:e161.

24. Karreth F and DA Tuveson. (2004). Twist induces an epithelial-mesenchymal transition to facilitate tumor metastasis. Cancer Biol Ther 3:1058-9.

25. Yang J, SA Mani, JL Donaher, S Ramaswamy, RA Itzykson, C Come, P Savagner, I Gitelman, A Richardson and RA Weinberg. (2004). Twist, a master regulator of morphogenesis, plays an essential role in tumor metastasis. Cell 117:927-39.

26. Yang MH and KJ Wu. (2008). TWIST activation by hypoxia inducible factor-1 (HIF1): implications in metastasis and development. Cell Cycle 7:2090-6.

27. Castanon I and MK Baylies. (2002). A Twist in fate: evolutionary comparison of Twist structure and function. Gene 287:11-22.

28. Furlong EE, EC Andersen, B Null, KP White and MP Scott. (2001). Patterns of gene expression during Drosophila mesoderm development. Science 293:1629-33.

29. Hong JH and MB Yaffe. (2006). TAZ: a beta-catenin-like molecule that regulates mesenchymal stem cell differentiation. Cell Cycle 5:176-9.

30. Hong JH, ES Hwang, MT McManus, A Amsterdam, Y Tian, R Kalmukova, E Mueller, T Benjamin, BM Spiegelman, PA Sharp, N Hopkins and MB Yaffe. (2005). TAZ, a transcriptional modulator of mesenchymal stem cell differentiation. Science 309:1074-8.

31. Varelas X, R Sakuma, P Samavarchi-Tehrani, R Peerani, BM Rao, J Dembowy, MB Yaffe, PW Zandstra and JL Wrana. (2008). TAZ controls Smad nucleocytoplasmic shuttling and regulates human embryonic stem-cell self-renewal. Nat Cell Biol $10: 837-48$

32. Nemir M, A Croquelois, $T$ Pedrazzini and F Radtke. (2006). Induction of cardiogenesis in embryonic stem cells via downregulation of Notch1 signaling. Circ Res 98:1471-8.

33. Wang X, NG Nagl, D Wilsker, M Van Scoy, S Pacchione, P Yaciuk, PB Dallas and E Moran. (2004). Two related ARID family proteins are alternative subunits of human SWI/SNF complexes. Biochem J 383:319-25. 
34. Schaniel C, YS Ang, K Ratnakumar, C Cormier, T James, E Bernstein, IR Lemischka and PJ Paddison. (2009). Smarcc1/Baf155 couples self-renewal gene repression with changes in chromatin structure in mouse embryonic stem cells. Stem Cells 27:297991.

35. Dauvillier S, MO Ott, JP Renard and E Legouy. (2001). BRM (SNF2alpha) expression is concomitant to the onset of vasculogenesis in early mouse postimplantation development. Mech Dev 101:221-5.

36. Aruga J, T Tohmonda, S Homma and K Mikoshiba. (2002). Zic1 promotes the expansion of dorsal neural progenitors in spinal cord by inhibiting neuronal differentiation. Dev Biol 244:329-41.

37. Aruga J, K Mizugishi, H Koseki, K Imai, R Balling, T Noda and K Mikoshiba. (1999). Zic1 regulates the patterning of vertebral arches in cooperation with Gli3. Mech Dev 89:141-50.

38. Ninomiya H, S Takahashi, K Tanegashima, C Yokota and M Asashima. (1999). Endoderm differentiation and inductive effect of activin-treated ectoderm in Xenopus. Dev Growth Differ 41:391-400.

39. Willems E and L Leyns. (2008). Patterning of mouse embryonic stem cell-derived pan-mesoderm by Activin A/Nodal and Bmp4 signaling requires Fibroblast Growth Factor activity. Differentiation 76:745-59.

40. Smith JR, L Vallier, G Lupo, M Alexander, WA Harris and RA Pedersen. (2008). Inhibition of Activin/Nodal signaling promotes specification of human embryonic stem cells into neuroectoderm. Dev Biol 313:107-17.

41. Thompson TB, TF Lerch, RW Cook, TK Woodruff and TS Jardetzky. (2005). The structure of the follistatin:activin complex reveals antagonism of both type I and type II receptor binding. Dev Cell 9:535-43.

42. Harrington AE, SA Morris-Triggs, BT Ruotolo, CV Robinson, S Ohnuma and M Hyvonen. (2006). Structural basis for the inhibition of activin signalling by follistatin. Embo J 25:1035-45.

43. Iemura S, TS Yamamoto, C Takagi, H Uchiyama, T Natsume, S Shimasaki, H Sugino and N Ueno. (1998). Direct binding of follistatin to a complex of bone-morphogenetic protein and its receptor inhibits ventral and epidermal cell fates in early Xenopus embryo. Proc Natl Acad Sci U S A 95:9337-42.

44. Chambers SM, CA Fasano, EP Papapetrou, M Tomishima, M Sadelain and L Studer. (2009). Highly efficient neural conversion of human ES and iPS cells by dual inhibition of SMAD signaling. Nat Biotechnol 27:275-80.

45. Patani R, A Compston, CA Puddifoot, DJ Wyllie, GE Hardingham, ND Allen and S Chandran. (2009). Activin/Nodal inhibition alone accelerates highly efficient neural conversion from human embryonic stem cells and imposes a caudal positional identity. PLoS One 4:e7327.

46. Heeg-Truesdell E and C LaBonne. (2006). Neural induction in Xenopus requires inhibition of Wnt-beta-catenin signaling. Dev Biol 298:71-86.

47. Aubert J, H Dunstan, I Chambers and A Smith. (2002). Functional gene screening in embryonic stem cells implicates Wnt antagonism in neural differentiation. Nat Biotechnol 20:1240-5.

48. Kielman MF, M Rindapaa, C Gaspar, N van Poppel, C Breukel, S van Leeuwen, MM Taketo, S Roberts, R Smits and R Fodde. (2002). Apc modulates embryonic stem-cell differentiation by controlling the dosage of beta-catenin signaling. Nat Genet 32:594605 . 
49. Ladher RK, VL Church, S Allen, L Robson, A Abdelfattah, NA Brown, G Hattersley, V Rosen, FP Luyten, L Dale and PH Francis-West. (2000). Cloning and expression of the Wnt antagonists Sfrp-2 and Frzb during chick development. Dev Biol 218:183-98.

50. Monaghan AP, P Kioschis, W Wu, A Zuniga, D Bock, A Poustka, H Delius and C Niehrs. (1999). Dickkopf genes are co-ordinately expressed in mesodermal lineages. Mech Dev 87:45-56.

51. Pinho S and C Niehrs. (2007). Dkk3 is required for TGF-beta signaling during Xenopus mesoderm induction. Differentiation 75:957-67.

52. Hitoshi S, RM Seaberg, C Koscik, T Alexson, S Kusunoki, I Kanazawa, S Tsuji and D van der Kooy. (2004). Primitive neural stem cells from the mammalian epiblast differentiate to definitive neural stem cells under the control of Notch signaling. Genes Dev 18:1806-11.

53. Lowell S, A Benchoua, B Heavey and AG Smith. (2006). Notch promotes neural lineage entry by pluripotent embryonic stem cells. PLoS Biol 4:e121.

54. Lee G, H Kim, Y Elkabetz, G Al Shamy, G Panagiotakos, T Barberi, V Tabar and L Studer. (2007). Isolation and directed differentiation of neural crest stem cells derived from human embryonic stem cells. Nat Biotechnol 25:1468-75.

55. Potterf SB, M Furumura, KJ Dunn, H Arnheiter and WJ Pavan. (2000). Transcription factor hierarchy in Waardenburg syndrome: regulation of MITF expression by SOX10 and PAX3. Hum Genet 107:1-6.

56. Lee JM, S Dedhar, R Kalluri and EW Thompson. (2006). The epithelial-mesenchymal transition: new insights in signaling, development, and disease. J Cell Biol 172:97381.

57. Kalluri R and RA Weinberg. (2009). The basics of epithelial-mesenchymal transition. J Clin Invest 119:1420-8.

58. Cavatorta AL, AA Giri, L Banks and D Gardiol. (2008). Cloning and functional analysis of the promoter region of the human Disc large gene. Gene 424:87-95.

59. Whiteman EL, CJ Liu, ER Fearon and B Margolis. (2008). The transcription factor snail represses Crumbs3 expression and disrupts apico-basal polarity complexes. Oncogene 27:3875-9.

60. McCoy EL, R Iwanaga, P Jedlicka, NS Abbey, LA Chodosh, KA Heichman, AL Welm and HL Ford. (2009). Six1 expands the mouse mammary epithelial stem/progenitor cell pool and induces mammary tumors that undergo epithelialmesenchymal transition. J Clin Invest 119:2663-77.

61. Micalizzi DS, KL Christensen, P Jedlicka, RD Coletta, AE Baron, JC Harrell, KB Horwitz, D Billheimer, KA Heichman, AL Welm, WP Schiemann and HL Ford. (2009). The Six1 homeoprotein induces human mammary carcinoma cells to undergo epithelial-mesenchymal transition and metastasis in mice through increasing TGF-beta signaling. J Clin Invest 119:2678-90. 

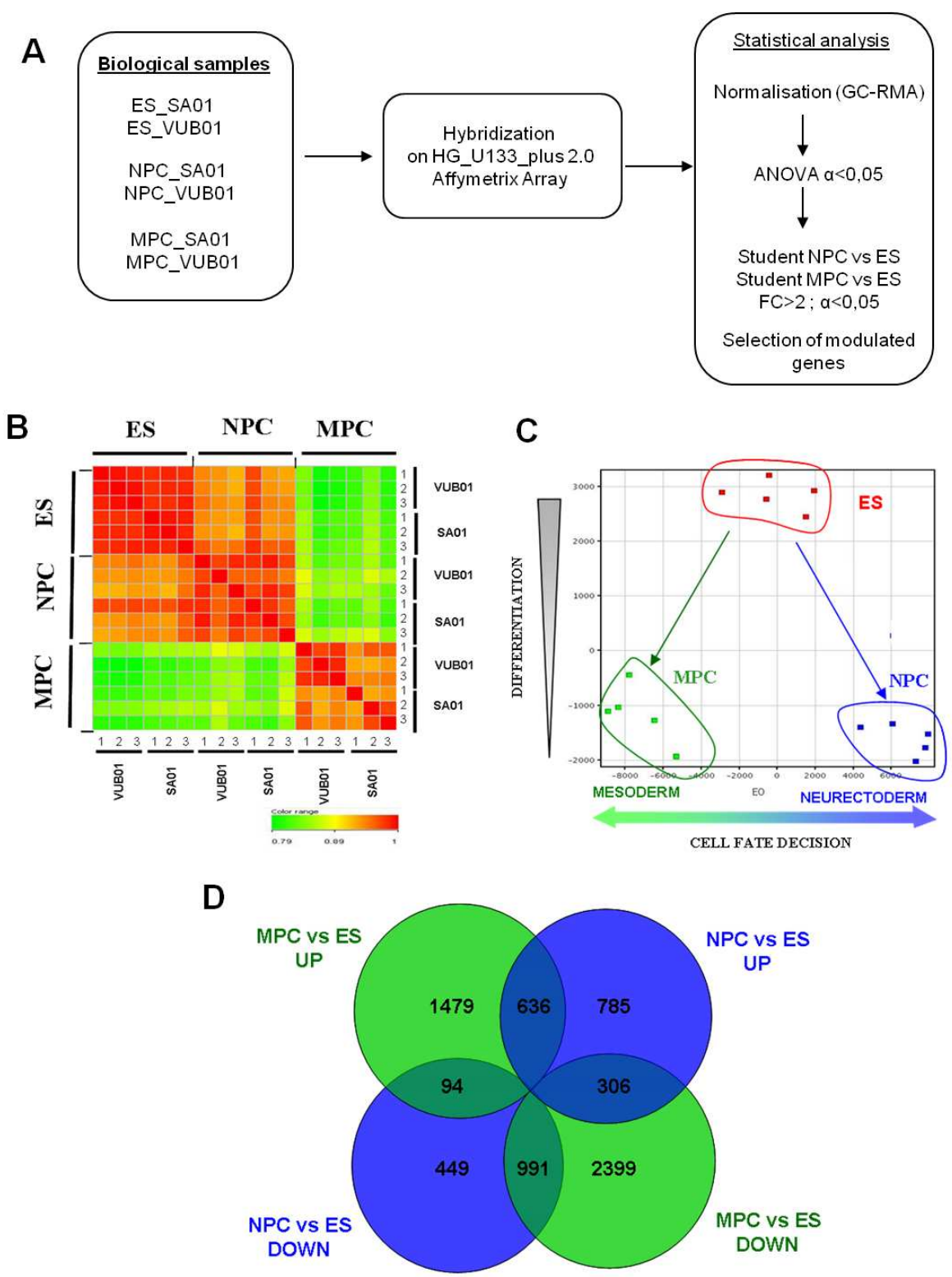

Figure 1: Global gene expression patterns analysis for the hES cells (ES) and the two precursor cells, NPC and MPC derived from the same hES cells. Schematic representation of the experimental procedure (A). Correlation plots between all arrays of the experiment hybridized with ES, NPC and MPC mRNAs ( $n=6$ per condition). (B) Correlation was normalised in a scale from $r^{2}=0.79$ (green)

to $r^{2}=1$ (red). Principal component analysis of the three cell populations (C). Comparison of the expression profiles (D). Genes were selected as differentially expressed either between NPC vs hES or MPC vs hES for a thereshold of FC (Fold change) $>2$ and a corrected $p$-value $a<0.05$. The lists of modulated genes in all the conditions were crossed by Venn diagrams. $183 \times 239 \mathrm{~mm}(150 \times 150 \mathrm{DPI})$ 


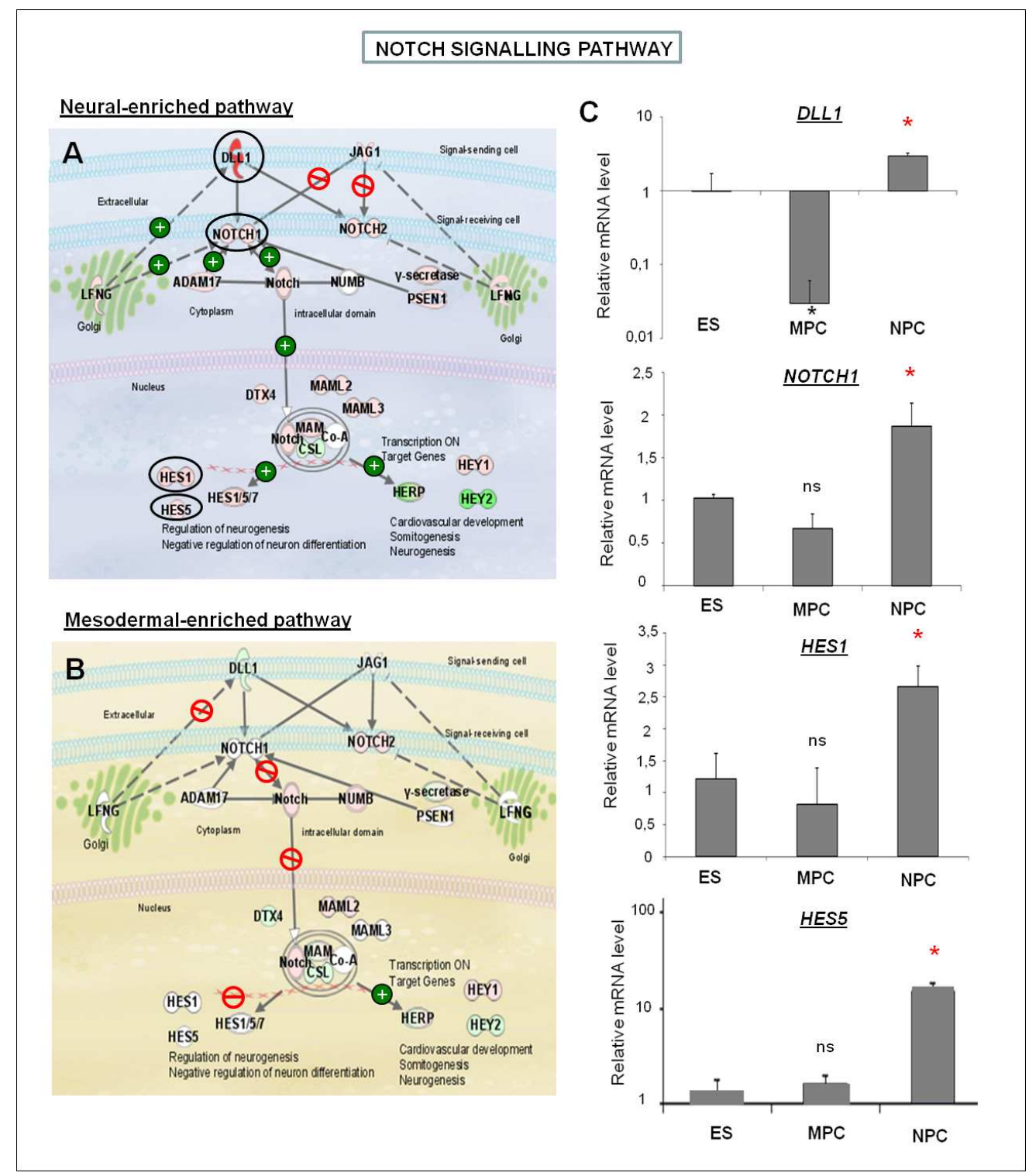

Figure 3: Comparison of gene expression modulations within Notch signalling pathway either in NPC or in MPC. Modulated genes are represented as a box with its gene symbol inside. Genes upregulated by comparison with hES are in red and those down-regulated in green. Colour intensities of genes were correlated to fold change intensities. The following symbol ( ) indicated a relation of "inhibition" and ( ) a relation of "activation" between genes in the path. (A) Genes modulated between NPC and hES (B) Genes modulated between MPC and hES. (C) Differential expression measured by quantitative RT-PCR for some genes in hES (ES), MPC, NPC. Results are presented as relative expression level compared to hES cells considered arbitrarily as 1 from 3 independent samples. (t-student, $* \mathrm{p}<0,05)$. $216 \times 249 \mathrm{~mm}(150 \times 150 \mathrm{DPI})$ 


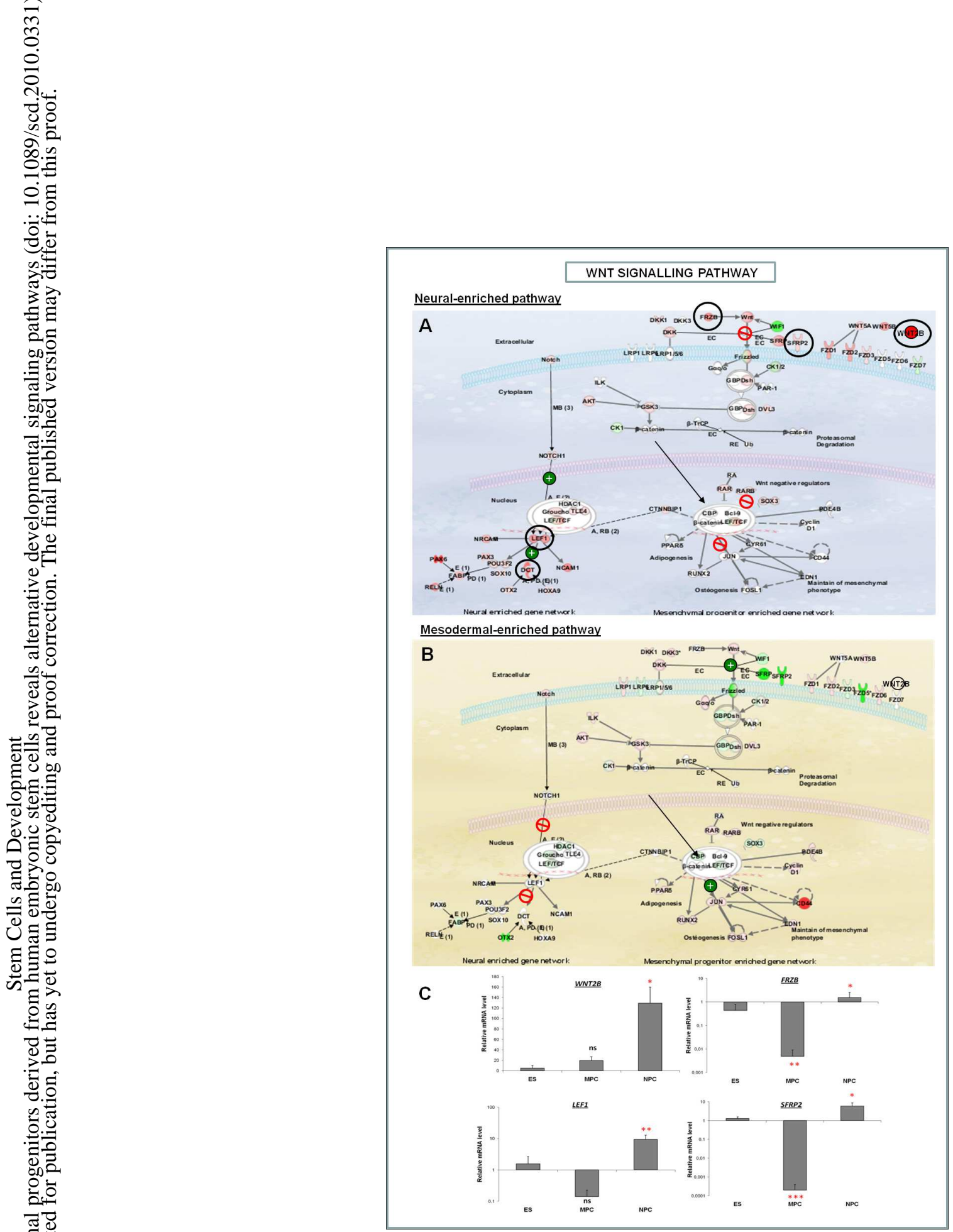

Figure 4 : Comparison of gene expression modulations within canonical Wnt/ $\beta$ catenin pathway either in NPC or in MPC. Same legend as described in Figure 3. (A) Genes specifically modulated between NPC and hES (B) Genes modulated between MPC and hES. (C) Differential expression measured by quantitative RT-PCR for some genes in hES (ES), MPC, NPC. Results are presented as relative expression level compared to hES cells considered arbitrarily as 1 from 3 independent samples. (t-student, $* \mathrm{p}<0,05 ; * * \mathrm{p}<0,01 ; * * * \mathrm{p}<0,001$; ns statistically not significant). $191 \times 334 \mathrm{~mm}(150 \times 150 \mathrm{DPI})$ 


\section{Supplementary Figure legends}

Figure S1 : Differentiation and characterization of NPC. (A) hES cells were cultured under appropriate conditions until DIV21 to obtain neural rosettes. (B) NCAM-positive cells were isolated by cell sorting. (C) Gene expression patterns of the NCAM positive cells were explored by RT-PCR. Cells do not express undifferentiated markers such as NANOG or glial makers such as GFAP whereas the induction of some expected neurectodermic markers was observed including NCAM-1; PAX6; SOX1; OTX2. No change in the expression of NES [Nestin] was observed. Immunostaining revealed that NCAM-positive cells widely expressed NESTIN (D), NCAM, SOX1 (E) and PAX6 (F). Functional characterization of these cells demonstrated their ability to generate both neurons and astrocytes under classical differentiating conditions as highlighted by positive $\beta$ III-tubulin (G) and GFAP positive staining $(\mathrm{H})$ respectively.

Figure S2 : Differentiation and characterization of MPC cells. (A) hES cells were cultured on gelatin in appropriate conditions until DIV28 to obtain cells with a fibroblast-like morphology. (B) Phenotyping was performed by FACS as described on material and methods. (C) Immunocytochemistry of two mesodermal markers Stro-1 (left, red) and aSMA (right, green) counterstained with DAPI (nuclei in blue). (D) Capacity of MPC to differentiate $\underline{\text { toward either osteoblasts or adipocytes as described on material and methods. }}$

Figure S3 : Validation by Q-PCR of key genes identified to be modulated in the transcriptome experiment. (A) Genes specifically modulated between NPC and hES (B) Genes specifically modulated between MPC and hES. Results are presented as relative expression level compared to hES cells considered arbitrarily as 1 from 3 independent samples. (t-student, $* \mathrm{p}<0,05 ; * * \mathrm{p}<0,01 ; * * * \mathrm{p}<0,001 ;$ ns statistically not significant). 


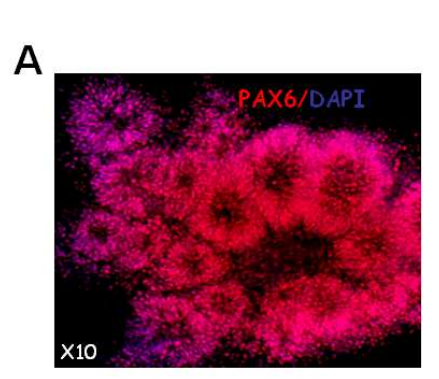

B
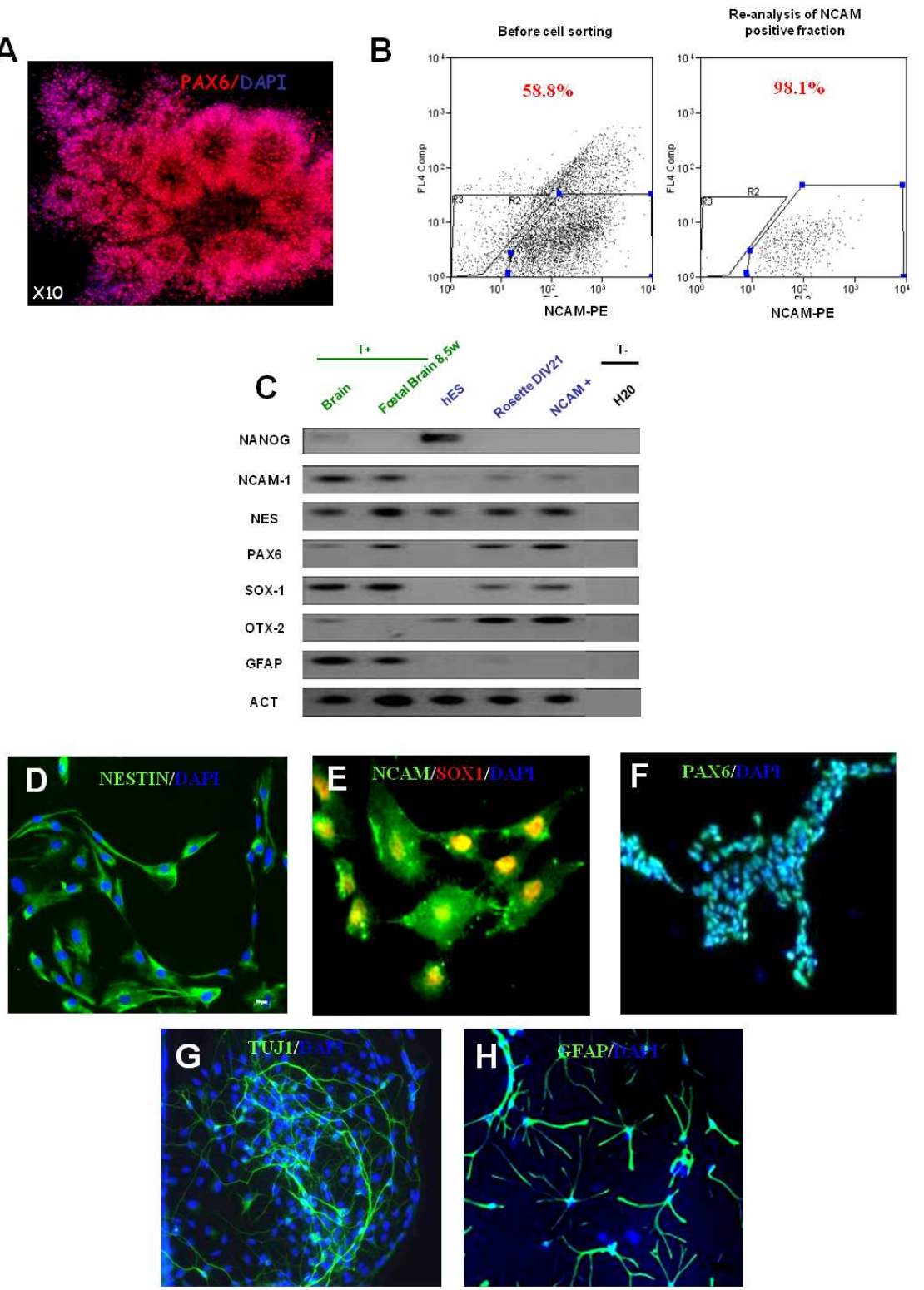

Figure S1 : Differentiation and characterization of NPC. (A) hES cells were cultured under appropriate conditions until DIV21 to obtain neural rosettes. (B) NCAM-positive cells were isolated by cell sorting. (C) Gene expression patterns of the NCAM positive cells were explored by RT-PCR. Cells do not express undifferentiated markers such as NANOG or glial makers such as GFAP whereas the induction of some expected neurectodermic markers was observed including NCAM-1; PAX6; SOX1; OTX2. No change in the expression of NES [Nestin] was observed. Immunostaining revealed that NCAM-positive cells widely expressed NESTIN (D), NCAM, SOX1 (E) and PAX6 (F). Functional characterization of these cells demonstrated their ability to generate both neurons and astrocytes under classical differentiating conditions as highlighted by positive $\beta I I I-t u b u l i n(G)$ and GFAP positive staining $(\mathrm{H})$ respectively. $181 \times 246 \mathrm{~mm}(150 \times 150 \mathrm{DPI})$ 


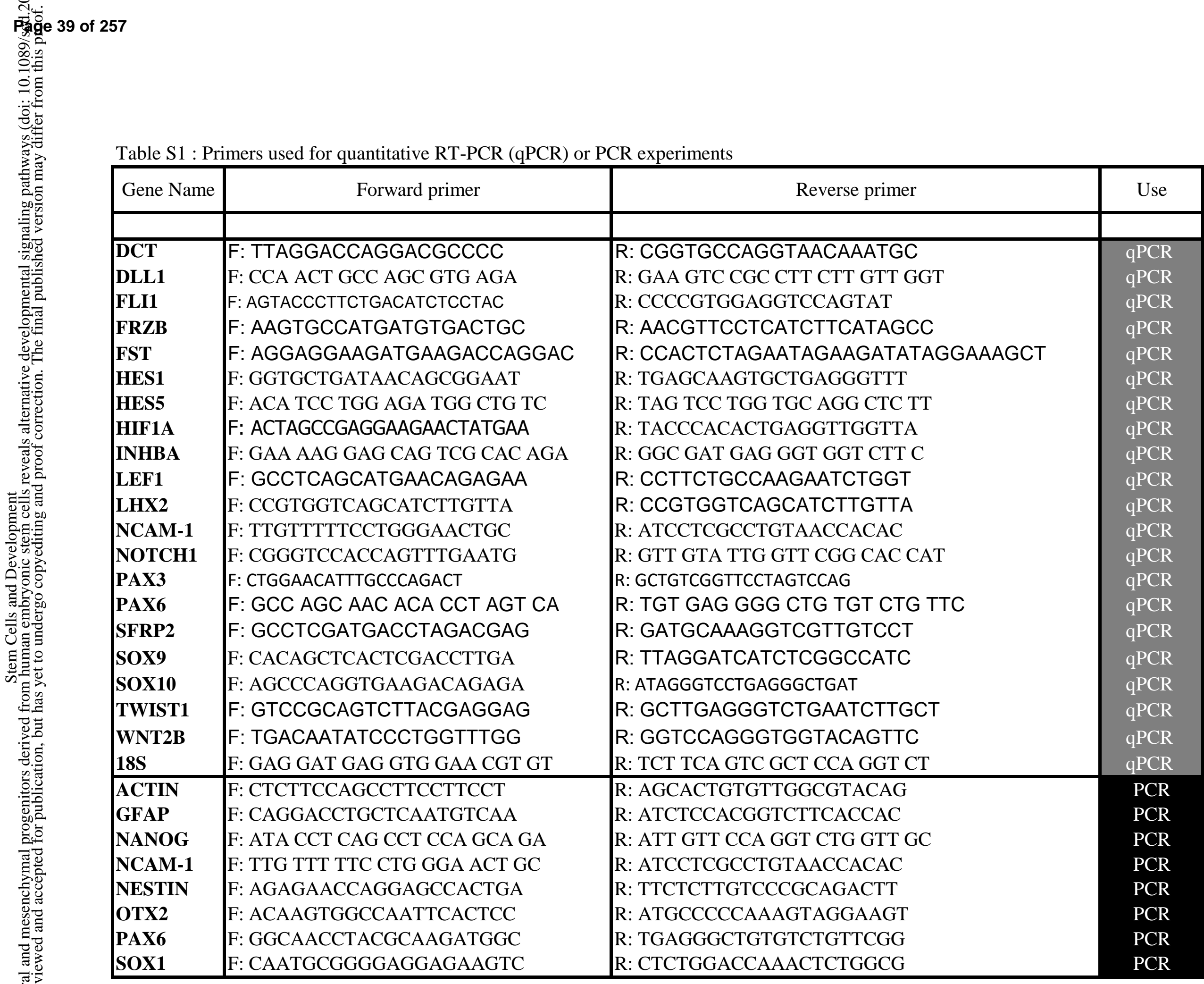




\begin{tabular}{|c|c|c|c|c|}
\hline Gene Symbol & Entrez Gene & Gene Title & NPC_up & Chromosome Number(Avadis) \\
\hline 37500 & 4735 & septin 2 & 2,38 & chr2 \\
\hline 39692 & 23176 & septin 8 & 2,05 & chr5 \\
\hline (vide) & (vide) & CDNA clone IMAGE:4152983 & 34,42 & chr12 \\
\hline $15 \mathrm{E} 1.2$ & 283459 & Hypothetical protein LOC283459 & 2,71 & chr12 \\
\hline $76 \mathrm{P}$ & 27229 & Gamma tubulin ring complex protein (76p gene) & 3,37 & chr15 \\
\hline ABHD6 & 57406 & abhydrolase domain containing 6 & 2,79 & chr3 \\
\hline $\mathrm{ABI} 2$ & 10152 & Abl interactor 2 & 2,23 & chr2 \\
\hline ABL2 & 27 & V-abl Abelson murine leukemia viral oncogene homolog 2 (arg, Abelson-related gene) & 2,02 & chr1 \\
\hline ABR & 29 & active BCR-related gene & 2,41 & chr17 \\
\hline ACOX3 & 8310 & acyl-Coenzyme A oxidase 3, pristanoyl & 3,38 & chr4 \\
\hline ACSBG2 & 81616 & Acyl-CoA synthetase bubblegum family member 2 & 2,68 & chr19 \\
\hline ACSL3 & 2181 & Acyl-CoA synthetase long-chain family member 3 & 2,04 & chr2 \\
\hline ACTA2 & 59 & actin, alpha 2, smooth muscle, aorta & 8,75 & chr10 \\
\hline ACY1L2 & 135293 & aminoacylase 1-like 2 & 2,50 & chr6 \\
\hline ADAM12 & 8038 & ADAM metallopeptidase domain 12 (meltrin alpha) & 4,25 & chr10 \\
\hline ADAM17 & 6868 & ADAM metallopeptidase domain 17 (tumor necrosis factor, alpha, converting enzyme) & 2,02 & chr2 \\
\hline ADAM19 & 8728 & ADAM metallopeptidase domain 19 (meltrin beta) & 2,15 & chr5 \\
\hline ADAM23 & 8745 & ADAM metallopeptidase domain 23 & 2,30 & chr2 \\
\hline ADAMTS1 & 9510 & ADAM metallopeptidase with thrombospondin type 1 motif, 1 & 2,62 & chr21 \\
\hline ADAMTS18 & 170692 & ADAM metallopeptidase with thrombospondin type 1 motif, 18 & 7,82 & chr16 \\
\hline ADAMTS6 & 11174 & ADAM metallopeptidase with thrombospondin type 1 motif, 6 & 5,92 & chr5 \\
\hline ADAMTS9 & 56999 & ADAM metallopeptidase with thrombospondin type 1 motif, 9 & 19,15 & chr3 \\
\hline ADARB1 & 104 & adenosine deaminase, RNA-specific, B1 (RED1 homolog rat) & 2,79 & chr21 \\
\hline ADCY6 & 112 & adenylate cyclase 6 & 2,06 & chr12 \\
\hline ADSSL1 & 122622 & adenylosuccinate synthase like 1 & 3,26 & chr14 \\
\hline AFF3 & 3899 & AF4/FMR2 family, member 3 & 2,51 & chr2 \\
\hline AFF4 & 27125 & AF4/FMR2 family, member 4 & 3,76 & chr5 \\
\hline AFG3L1 & 172 & AFG3 ATPase family gene 3-like 1 (yeast) & 2,26 & $\operatorname{chr} 16$ \\
\hline AG1 & 440673 & AG1 protein & 3,17 & chr1 \\
\hline AGPAT3 & 56894 & 1-acylglycerol-3-phosphate O-acyltransferase 3 & 2,02 & chr21 \\
\hline AGPAT4 & 56895 & 1-acylglycerol-3-phosphate O-acyltransferase 4 (lysophosphatidic acid acyltransferase, & 3,89 & chr6 \\
\hline AGTPBP1 & 23287 & ATP/GTP binding protein 1 & 3,23 & chr9 \\
\hline $\mathrm{AHI} 1$ & 54806 & Abelson helper integration site & 4,59 & chr6 \\
\hline AHNAK & 79026 & AHNAK nucleoprotein (desmoyokin) & 7,26 & chr11 \\
\hline AHSA2 & 130872 & AHA1, activator of heat shock $90 \mathrm{kDa}$ protein ATPase homolog 2 (yeast) & 3,01 & chr2 \\
\hline AKAP13 & 11214 & A kinase (PRKA) anchor protein 13 & 2,53 & chr15 \\
\hline AKAP9 & 10142 & A kinase (PRKA) anchor protein (yotiao) 9 & 2,23 & chr7 \\
\hline AKR1C1 & 1645 & aldo-keto reductase family 1 , member $\mathrm{C} 1$ (dihydrodiol dehydrogenase $1 ; 20$-alpha (3-ald & 2,43 & $\operatorname{chr} 10$ \\
\hline AKR1C2 & 1646 & aldo-keto reductase family 1 , member $\mathrm{C} 2$ (dihydrodiol dehydrogenase 2; bile acid bindin & 2,32 & chr10 \\
\hline AKT3 & 10000 & V-akt murine thymoma viral oncogene homolog 3 (protein kinase $\mathrm{B}$, gamma) & 2,78 & chr1 \\
\hline ALCAM & 214 & activated leukocyte cell adhesion molecule & 2,30 & chr3 \\
\hline ALDH1A1 & 216 & aldehyde dehydrogenase 1 family, member $\mathrm{A} 1$ & 8,67 & chr9 \\
\hline ALPK2 & 115701 & alpha-kinase 2 & 4,74 & chr18 \\
\hline ALS2CR4 & 65062 & amyotrophic lateral sclerosis 2 (juvenile) chromosome region, candidate 4 & 2,93 & chr2 \\
\hline AMIGO2 & 347902 & adhesion molecule with Ig-like domain 2 & 4,13 & chr12 \\
\hline AMOTL1 & 154810 & angiomotin like 1 & 3,05 & chr11 \\
\hline AMOTL2 & 51421 & angiomotin like 2 & 3,72 & chr3 \\
\hline AMPH & 273 & amphiphysin (Stiff-Man syndrome with breast cancer 128kDa autoantigen) & 2,77 & chr7 \\
\hline AMY1A & $77 / / / 278 / / / 27$ & amylase, alpha $1 \mathrm{~A}$; salivary /// amylase, alpha $1 \mathrm{~B}$; salivary /// amylase, alpha $1 \mathrm{C}$; salivar & 2,34 & chr1 \\
\hline ANAPC5 & 51433 & Anaphase promoting complex subunit 5 & 2,42 & chr12 \\
\hline ANAPC7 & 51434 & anaphase promoting complex subunit 7 & 2,70 & chr12 \\
\hline ANGPTL1 & 9068 & Angiopoietin-like 1 & 2,45 & chr1 \\
\hline ANGPTL2 & 23452 & angiopoietin-like 2 & 2,97 & chr9 \\
\hline ANK2 & 287 & ankyrin 2, neuronal & 4,55 & chr4 \\
\hline ANKHD1 & $04734 / / / 5488$ & ankyrin repeat and $\mathrm{KH}$ domain containing $1 / / / \mathrm{MASK}-4 \mathrm{E}-\mathrm{BP} 3$ alternate reading frame g & 2,18 & chr5 \\
\hline ANKMY2 & 57037 & ankyrin repeat and MYND domain containing 2 & 3,49 & chr7 \\
\hline ANKRD10 & 55608 & Ankyrin repeat domain 10 & 2,05 & $\operatorname{chr} 13$ \\
\hline ANKRD13 & 88455 & ankyrin repeat domain 13 & 2,00 & chr12 \\
\hline ANKRD38 & 163782 & ankyrin repeat domain 38 & 21,36 & chr1 \\
\hline ANKRD6 & 22881 & ankyrin repeat domain 6 & 6,12 & chr6 \\
\hline ANTXR2 & 118429 & Anthrax toxin receptor 2 & 2,23 & chr4 \\
\hline ANXA13 & 312 & Annexin A13 & 2,90 & chr1 \\
\hline AP3M1 & 26985 & adaptor-related protein complex 3 , mu 1 subunit & 2,12 & chr10 \\
\hline APBA2 & 321 & amyloid beta (A4) precursor protein-binding, family A, member 2 (X11-like) & 6,89 & chr15 \\
\hline APCDD1 & 147495 & adenomatosis polyposis coli down-regulated 1 & 2,95 & chr18 \\
\hline API5 & 8539 & Apoptosis inhibitor 5 & 2,93 & chr11 \\
\hline APPBP2 & 10513 & amyloid beta precursor protein (cytoplasmic tail) binding protein 2 & 3,26 & chr17 \\
\hline AQP3 & 360 & aquaporin 3 & 2,73 & chr9 \\
\hline ARHGAP2 & 83478 & Rho GTPase activating protein 24 & 2,62 & chr4 \\
\hline
\end{tabular}




\begin{tabular}{|c|c|c|c|c|}
\hline Gene Symbol & Entrez Gene & Gene Title & NPC_up & Chromosome Number(Avadis) \\
\hline ARHGAP28 & 79822 & Rho GTPase activating protein 28 & 2,76 & chr18 \\
\hline ARHGAP29 & 9411 & Rho GTPase activating protein 29 & 5,06 & chr1 \\
\hline ARHGAP5 & 394 & Rho GTPase activating protein 5 & 2,87 & chr14 \\
\hline ARHGAP6 & 395 & Rho GTPase activating protein 6 & 3,44 & $\operatorname{chrX}$ \\
\hline ARHGDIB & 397 & Rho GDP dissociation inhibitor (GDI) beta & 4,10 & chr12 \\
\hline ARHGEF17 & 9828 & Rho guanine nucleotide exchange factor (GEF) 17 & 3,07 & chr11 \\
\hline ARHGEF4 & 50649 & Rho guanine nucleotide exchange factor (GEF) 4 & 2,38 & chr2 \\
\hline ARHGEF7 & 8874 & Rho guanine nucleotide exchange factor (GEF) 7 & 2,59 & chr13 \\
\hline ARID1B & 57492 & AT rich interactive domain 1B (SWI1-like) & 2,02 & chr6 \\
\hline ARID2 & 196528 & AT rich interactive domain 2 (ARID, RFX-like) & 2,53 & chr12 \\
\hline ARID5B & 84159 & AT rich interactive domain 5B (MRF1-like) & 10,53 & $\operatorname{chr} 10$ \\
\hline ARL6IP5 & 10550 & ADP-ribosylation-like factor 6 interacting protein 5 & 2,12 & chr3 \\
\hline ARL7 & 10123 & ADP-ribosylation factor-like 7 & 13,85 & chr2 \\
\hline ARMC8 & 25852 & armadillo repeat containing 8 & 2,52 & chr3 \\
\hline ARMCX3 & 51566 & armadillo repeat containing, $X$-linked 3 & 2,58 & $\operatorname{chr} X$ \\
\hline ARNT2 & 9915 & aryl-hydrocarbon receptor nuclear translocator 2 & 2,67 & chr15 \\
\hline ARRDC4 & 91947 & arrestin domain containing 4 & 2,45 & chr15 \\
\hline ARSI & 340075 & arylsulfatase I & 2,07 & chr5 \\
\hline ARX & 170302 & aristaless related homeobox & 2,74 & $\operatorname{chr} X$ \\
\hline ASCC3L1 & 23020 & Activating signal cointegrator 1 complex subunit 3 -like 1 & 2,88 & chr17 \\
\hline ASH1L & 55870 & POU domain, class 6 , transcription factor 1 & 8,38 & (vide) \\
\hline ASPH & 444 & Aspartate beta-hydroxylase & 4,17 & chr8 \\
\hline ASTN & 460 & astrotactin & 4,43 & chr1 \\
\hline ASXL1 & 171023 & additional sex combs like 1 (Drosophila) & 7,40 & chr20 \\
\hline ATAD1 & 84896 & ATPase family, AAA domain containing 1 & 2,19 & chr10 \\
\hline ATBF1 & 463 & AT-binding transcription factor 1 & 3,03 & chr16 \\
\hline ATF6 & 22926 & Activating transcription factor 6 & 2,82 & chr1 \\
\hline ATP10D & 57205 & ATPase, Class V, type 10D & 2,81 & chr4 \\
\hline ATP11A & 23250 & ATPase, Class VI, type 11A & 2,28 & chr13 \\
\hline ATP1A2 & 477 & ATPase, $\mathrm{Na}+/ \mathrm{K}+$ transporting, alpha $2(+)$ polypeptide & 2,48 & chr1 \\
\hline ATP2B1 & 490 & ATPase, Ca++ transporting, plasma membrane 1 & 3,56 & chr12 \\
\hline ATP2B4 & 493 & ATPase, Ca++ transporting, plasma membrane 4 & 2,75 & chr1 \\
\hline ATP2C1 & 27032 & ATPase, $\mathrm{Ca}++$ transporting, type 2C, member 1 & 2,89 & chr3 \\
\hline ATP7A & 538 & ATPase, $\mathrm{Cu}++$ transporting, alpha polypeptide (Menkes syndrome) & 3,10 & $\operatorname{chrX}$ \\
\hline ATP7B & 540 & ATPase, Cu++ transporting, beta polypeptide (Wilson disease) & 3,42 & chr13 \\
\hline ATP9A & 10079 & ATPase, Class II, type 9A & 3,03 & chr20 \\
\hline ATRX & 546 & Alpha thalassemia/mental retardation syndrome X-linked (RAD54 homolog, S. cerevisia & 2,13 & $\operatorname{chr} X$ \\
\hline AXL & 558 & AXL receptor tyrosine kinase & 2,71 & chr19 \\
\hline B3GALT6 & 126792 & UDP-Gal:betaGal beta 1,3-galactosyltransferase polypeptide 6 & 2,66 & chr1 \\
\hline B3GNT5 & 84002 & UDP-GIcNAc:betaGal beta-1,3-N-acetylglucosaminyltransferase 5 & 3,05 & chr3 \\
\hline B3GTL & 145173 & beta 3-glycosyltransferase-like & 2,45 & chr13 \\
\hline BACE1 & 23621 & beta-site APP-cleaving enzyme 1 & 3,31 & chr11 \\
\hline BACH2 & 60468 & BTB and CNC homology 1, basic leucine zipper transcription factor $2 / / / \mathrm{BTB}$ and $\mathrm{CNC} \mid$ & 4,16 & chr6 \\
\hline BAT1 & 7919 & HLA-B associated transcript 1 & 2,16 & chr6 \\
\hline BBS1 & 582 & Bardet-Biedl syndrome 1 & 2,61 & chr11 \\
\hline BBS2 & 583 & Bardet-Biedl syndrome 2 & 2,27 & chr16 \\
\hline BCAR3 & 8412 & Breast cancer anti-estrogen resistance 3 & 2,17 & chr1 \\
\hline BCHE & 590 & butyrylcholinesterase & 7,49 & chr3 \\
\hline BCL2 & 596 & B-cell CLL/ymphoma 2 & 2,47 & chr18 \\
\hline BCL2L11 & 10018 & BCL2-like 11 (apoptosis facilitator) & 3,19 & chr2 \\
\hline BCL7A & 605 & B-cell CLL/lymphoma 7A & 2,02 & chr12 \\
\hline BDH1 & 622 & 3-hydroxybutyrate dehydrogenase, type 1 & 2,97 & chr3 \\
\hline BID & 637 & BH3 interacting domain death agonist & 2,08 & chr22 \\
\hline BIN1 & 274 & bridging integrator 1 & 2,08 & chr2 \\
\hline BIRC1 & 4671 & baculoviral IAP repeat-containing 1 & 3,46 & chr5 \\
\hline BIRC6 & 57448 & Splicing factor, arginine/serine-rich 12 & 2,11 & chr2 \\
\hline BMP7 & 655 & Bone morphogenetic protein 7 (osteogenic protein 1) & 2,09 & chr20 \\
\hline BMPR2 & 659 & bone morphogenetic protein receptor, type II (serine/threonine kinase) & 2,29 & chr2 \\
\hline BNC1 & 646 & basonuclin 1 & 2,63 & chr15 \\
\hline BNIP3L & 665 & BCL2/adenovirus E1B 19kDa interacting protein 3-like /// BCL2/adenovirus E1B 19kDa & 2,63 & chr8 \\
\hline BOC & 91653 & brother of CDO & 11,85 & chr3 \\
\hline BRUNOL5 & 60680 & bruno-like 5, RNA binding protein (Drosophila) & 3,57 & chr19 \\
\hline BRWD2 & 55717 & bromodomain and WD repeat domain containing 2 & 3,05 & chr10 \\
\hline BST2 & 684 & bone marrow stromal cell antigen 2 & 2,18 & chr19 \\
\hline |BTAF1 & 9044 & BTAF1 RNA polymerase II, B-TFIID transcription factor-associated, 170kDa (Mot1 hom & 2,00 & chr10 \\
\hline |BTBD3 & 22903 & BTB (POZ) domain containing 3 & 2,86 & chr20 \\
\hline BTBD5 & 54813 & BTB (POZ) domain containing 5 & 2,46 & chr14 \\
\hline D7 & 727 & $\mathrm{~B}(\mathrm{POZ})$ domain containing 7 & 3 & chr14 \\
\hline
\end{tabular}




\begin{tabular}{|c|c|c|c|c|}
\hline Gene Symbol & Entrez Gene & Gene Title & NPC_up & Chromosome Number(Avadis) \\
\hline BTG1 & 694 & B-cell translocation gene 1 , anti-proliferative & 2,19 & chr12 \\
\hline BTG2 & 7832 & BTG family, member 2 & 3,78 & chr1 \\
\hline BZRAP1 & 9256 & benzodiazapine receptor (peripheral) associated protein 1 & 2,39 & chr17 \\
\hline C10orf104 & 119504 & chromosome 10 open reading frame 104 & 2,81 & chr10 \\
\hline C10orf18 & 54906 & Chromosome 10 open reading frame 18 & 2,18 & chr10 \\
\hline C10orf30 & 222389 & Chromosome 10 open reading frame 30 & 2,34 & chr10 \\
\hline C10orf32 & 119032 & chromosome 10 open reading frame 32 & 3,17 & chr10 \\
\hline C10orf33 & 84795 & chromosome 10 open reading frame 33 & 2,13 & chr10 \\
\hline C10orf38 & 221061 & chromosome 10 open reading frame 38 & 2,17 & chr10 \\
\hline C10orf45 & 83641 & chromosome 10 open reading frame 45 & 2,47 & chr10 \\
\hline C10orf46 & 143384 & Chromosome 10 open reading frame 46 & 2,40 & chr1 \\
\hline C10orf56 & 219654 & chromosome 10 open reading frame 56 & 2,65 & chr10 \\
\hline C10orf58 & 84293 & Chromosome 10 open reading frame 58 & 3,91 & chr10 \\
\hline C11orf17 & $56672 / / / / 8178 \varepsilon$ & chromosome 11 open reading frame $17 / / /$ chromosome 11 open reading frame $17 / / / \mathrm{N}$ & 5,37 & chr1 \\
\hline C11orf9 & 745 & chromosome 11 open reading frame 9 & 3,92 & chr11 \\
\hline C12orf22 & 81566 & chromosome 12 open reading frame 22 /// chromosome 12 open reading frame 22 & 2,07 & chr12 \\
\hline C14orf101 & 54916 & chromosome 14 open reading frame 101 & 2,39 & chr14 \\
\hline C14orf162 & 56936 & chromosome 14 open reading frame 162 & 4,47 & chr14 \\
\hline C14orf32 & 93487 & Chromosome 14 open reading frame 32 & 2,64 & chr14 \\
\hline C14orf39 & 317761 & chromosome 14 open reading frame 39 & 3,15 & chr14 \\
\hline C14orf65 & 317762 & chromosome 14 open reading frame 65 & 2,02 & chr14 \\
\hline C15orf17 & 57184 & chromosome 15 open reading frame 17 & 2,25 & chr15 \\
\hline C15orf21 & 283651 & Chromosome 15 open reading frame 21 & 2,54 & chr15 \\
\hline C15orf29 & 79768 & chromosome 15 open reading frame 29 & 2,96 & chr15 \\
\hline C15orf38 & 348110 & Chromosome 15 open reading frame 38 & 2,46 & chr15 \\
\hline C16orf46 & 123775 & Chromosome 16 open reading frame 46 & 2,60 & (vide) \\
\hline C16orf48 & 84080 & chromosome 16 open reading frame 48 & 2,06 & chr16 \\
\hline C16orf55 & 124045 & chromosome 16 open reading frame 55 & 2,59 & chr16 \\
\hline C17orf42 & 79736 & Chromosome 17 open reading frame 42 & 2,99 & chr17 \\
\hline C17orf45 & 125144 & Chromosome 17 open reading frame 45 & 4,23 & chr17 \\
\hline C18orf51 & 125704 & chromosome 18 open reading frame 51 & 2,70 & chr18 \\
\hline C1GALT1C1 & 29071 & C1GALT1-specific chaperone 1 & 3,05 & $\operatorname{chr} X$ \\
\hline C1orf104 & 284618 & Chromosome 1 open reading frame 104 & 2,31 & chr1 \\
\hline C1orf112 & 55732 & Chromosome 1 open reading frame 112 & 2,03 & chr1 \\
\hline C1orf139 & 79971 & chromosome 1 open reading frame 139 & 20,12 & chr1 \\
\hline C1orf168 & 199920 & chromosome 1 open reading frame 168 & 2,86 & chr1 \\
\hline C1orf21 & 81563 & chromosome 1 open reading frame $21 / / /$ chromosome 1 open reading frame 21 & 2,25 & chr1 \\
\hline C1orf54 & 79630 & chromosome 1 open reading frame 54 & 2,08 & chr1 \\
\hline C1orf63 & 57035 & chromosome 1 open reading frame 63 & 2,16 & chr1 \\
\hline C1orf85 & 112770 & Chromosome 1 open reading frame 85 & 2,19 & chr1 \\
\hline C1orf9 & 51430 & chromosome 1 open reading frame 9 & 2,08 & chr1 \\
\hline C1QBP & 708 & Complement component 1 , q subcomponent binding protein & 2,30 & chr17 \\
\hline C1RL & 51279 & complement component 1 , $r$ subcomponent-like & 2,35 & chr12 \\
\hline C20orf108 & 116151 & chromosome 20 open reading frame 108 & 2,36 & chr20 \\
\hline C20orf112 & 140688 & chromosome 20 open reading frame 112 & 2,82 & chr20 \\
\hline C20orf12 & 55184 & Chromosome 20 open reading frame 12 & 2,05 & chr13 \\
\hline C20orf133 & 140733 & chromosome 20 open reading frame 133 & 3,27 & chr20 \\
\hline C20orf142 & 128486 & chromosome 20 open reading frame 142 & 2,24 & chr20 \\
\hline C20orf194 & 25943 & chromosome 20 open reading frame 194 & 2,38 & chr20 \\
\hline C20orf58 & 128414 & chromosome 20 open reading frame 58 & 2,37 & chr20 \\
\hline C20orf81 & 64773 & chromosome 20 open reading frame 81 & 2,76 & chr20 \\
\hline C21 orf25 & 25966 & chromosome 21 open reading frame 25 & 2,14 & chr21 \\
\hline C21 orf51 & 54065 & chromosome 21 open reading frame 51 & 2,85 & chr21 \\
\hline C21 orf66 & 94104 & Chromosome 21 open reading frame 66 & 4,60 & chr21 \\
\hline C2orf23 & 65055 & chromosome 2 open reading frame 23 & 2,73 & chr2 \\
\hline C2orf4 & 51072 & Chromosome 2 open reading frame 4 & 2,57 & chr2 \\
\hline C3orf15 & 89876 & chromosome 3 open reading frame 15 & 7,16 & chr3 \\
\hline C3orf6 & 152137 & chromosome 3 open reading frame 6 & 8,53 & chr3 \\
\hline $\mathrm{C} 5$ & 727 & complement component 5 & 2,52 & chr9 \\
\hline C5orf13 & 9315 & chromosome 5 open reading frame 13 & 3,04 & chr5 \\
\hline C5orf5 & 51306 & chromosome 5 open reading frame 5 & 2,41 & chr5 \\
\hline C6orf111 & 25957 & chromosome 6 open reading frame 111 & 3,14 & chr6 \\
\hline C6orf129 & 154467 & chromosome 6 open reading frame 129 & 2,24 & chr6 \\
\hline C6orf141 & 135398 & chromosome 6 open reading frame 141 & 4,10 & chr6 \\
\hline C6orf145 & 221749 & chromosome 6 open reading frame 145 & 3,27 & chr6 \\
\hline C6orf155 & 79940 & Chromosome 6 open reading frame 155 & 5,74 & chr6 \\
\hline C6orf166 & 55122 & Chromosome 6 open reading frame 166 & 2,56 & chr6 \\
\hline orf49 & 29964 & Chromosome 6 open reading frame 49 & 3 , & chr6 \\
\hline
\end{tabular}




\begin{tabular}{|c|c|c|c|c|}
\hline Gene Symbol & Entrez Gene & Gene Title & NPC_up & Chromosome Number(Avadis) \\
\hline C6orf59 & 79992 & chromosome 6 open reading frame 59 & 2,63 & chr6 \\
\hline C6orf69 & 222658 & chromosome 6 open reading frame 69 & 2,01 & chr6 \\
\hline C6orf84 & 22832 & chromosome 6 open reading frame 84 & 2,27 & chr6 \\
\hline C6orf89 & 221477 & Chromosome 6 open reading frame 89 & 2,71 & chr6 \\
\hline C7orf19 & 80228 & chromosome 7 open reading frame 19 & 2,46 & chr7 \\
\hline C8orf13 & 83648 & chromosome 8 open reading frame 13 & 2,22 & chr8 \\
\hline C8orf32 & 55093 & chromosome 8 open reading frame 32 & 2,19 & chr8 \\
\hline C8orf70 & 51101 & Chromosome 8 open reading frame 70 & 2,13 & chr8 \\
\hline C8orf72 & 90362 & chromosome 8 open reading frame 72 & 5,84 & chr8 \\
\hline C9orf28 & 89853 & chromosome 9 open reading frame 28 & 2,56 & chr9 \\
\hline c9orf5 & 23731 & chromosome 9 open reading frame 5 & 2,17 & chr9 \\
\hline C9orf7 & 11094 & chromosome 9 open reading frame 7 & 2,46 & chr9 \\
\hline C9orf72 & 203228 & chromosome 9 open reading frame 72 & 2,90 & chr9 \\
\hline C9orf80 & 58493 & Chromosome 9 open reading frame 80 & 2,73 & chr9 \\
\hline CA2 & 760 & carbonic anhydrase II & 2,38 & chr8 \\
\hline CAB39L & 81617 & calcium binding protein 39-like & 2,55 & chr13 \\
\hline CABLES2 & 81928 & Cdk5 and Abl enzyme substrate 2 & 2,03 & chr20 \\
\hline CACNA1A & 773 & calcium channel, voltage-dependent, $\mathrm{P} / \mathrm{Q}$ type, alpha $1 \mathrm{~A}$ subunit & 3,30 & chr19 \\
\hline CACNB3 & 784 & calcium channel, voltage-dependent, beta 3 subunit & 3,47 & chr12 \\
\hline CALD1 & 800 & caldesmon 1 & 2,11 & chr7 \\
\hline CALM2 & 805 & Calmodulin 2 (phosphorylase kinase, delta) & 2,30 & chr2 \\
\hline CALML4 & 91860 & calmodulin-like 4 & 2,34 & chr15 \\
\hline CAMK2D & 817 & Calcium/calmodulin-dependent protein kinase (CaM kinase) II delta & 3,07 & chr4 \\
\hline CAMK2N1 & 55450 & calcium/calmodulin-dependent protein kinase II inhibitor 1 & 7,69 & chr1 \\
\hline CAMTA1 & 23261 & calmodulin binding transcription activator 1 & 3,03 & chr1 \\
\hline CAND1 & 55832 & cullin-associated and neddylation-dissociated 1 & 2,44 & chr12 \\
\hline CAP2 & 10486 & CAP, adenylate cyclase-associated protein, 2 (yeast) & 2,87 & chr6 \\
\hline CAP350 & 9857 & centrosome-associated protein 350 & 2,11 & chr1 \\
\hline CAPN1 & 823 & calpain $1,(\mathrm{mu} / \mathrm{l})$ large subunit & 3,99 & chr11 \\
\hline CAPN2 & 824 & calpain 2, (m/II) large subunit & 4,13 & chr1 \\
\hline CAPN6 & 827 & calpain 6 & 6,34 & $\operatorname{chr} X$ \\
\hline CARD8 & 22900 & caspase recruitment domain family, member 8 & 2,80 & chr19 \\
\hline CART1 & 8092 & cartilage paired-class homeoprotein 1 & 3,09 & chr12 \\
\hline CASKIN1 & 57524 & CASK interacting protein 1 & 2,06 & chr16 \\
\hline CAST & 831 & calpastatin & 2,51 & chr5 \\
\hline CAST1 & 26059 & CAZ-associated structural protein & 2,17 & chr3 \\
\hline CBFB & 865 & core-binding factor, beta subunit & 2,57 & chr16 \\
\hline CBR4 & 84869 & carbonic reductase 4 & 2,28 & chr4 \\
\hline СBX5 & 23468 & Chromobox homolog 5 (HP1 alpha homolog, Drosophila) & 2,23 & chr12 \\
\hline CCAR1 & 55749 & Cell division cycle and apoptosis regulator 1 & 2,07 & chr10 \\
\hline CCDC14 & 64770 & coiled-coil domain containing 14 & 2,04 & chr3 \\
\hline CCDC3 & 83643 & coiled-coil domain containing 3 & 3,17 & chr10 \\
\hline CCDC52 & 152185 & Coiled-coil domain containing 52 & 2,39 & chr3 \\
\hline CCNG2 & 901 & Cyclin G2 & 3,33 & chr4 \\
\hline CCNL2 & 81669 & cyclin L2 & 2,64 & chr1_random \\
\hline ССТ6В & 10693 & chaperonin containing TCP1, subunit 6B (zeta 2) & 2,07 & chr17 \\
\hline CD24 & 934 & CD24 antigen (small cell lung carcinoma cluster 4 antigen) & 2,92 & chr5 \\
\hline CD47 & 961 & CD47 antigen (Rh-related antigen, integrin-associated signal transducer) & 9,00 & chr3 \\
\hline CD99 & 4267 & CD99 antigen & 3,43 & $\operatorname{chr} X$ \\
\hline CD99L2 & 83692 & CD99 antigen-like 2 & 2,41 & $\operatorname{chrX}$ \\
\hline CDC2L6 & 23097 & cell division cycle 2-like 6 (CDK8-like) & 3,25 & chr6 \\
\hline CDC42BPB & 9578 & CDC42 binding protein kinase beta (DMPK-like) & 2,84 & chr14 \\
\hline CDC42EP3 & 10602 & CDC42 effector protein (Rho GTPase binding) 3 & 2,66 & chr2 \\
\hline $\mathrm{CDH} 10$ & 1008 & cadherin 10, type 2 (T2-cadherin) & 4,90 & chr5 \\
\hline $\mathrm{CDH} 11$ & 1009 & cadherin 11 , type 2 , OB-cadherin (osteoblast) & 8,41 & chr16 \\
\hline $\mathrm{CDH} 2$ & 1000 & cadherin 2, type 1, $\mathrm{N}$-cadherin (neuronal) & 6,38 & chr18 \\
\hline $\mathrm{CDH} 6$ & 1004 & cadherin 6, type 2, K-cadherin (fetal kidney) & 16,58 & chr5 \\
\hline CDK5RAP3 & 80279 & CDK5 regulatory subunit associated protein 3 & 2,15 & chr17 \\
\hline CDK6 & 1021 & cyclin-dependent kinase 6 & 3,71 & chr7 \\
\hline CDKL3 & 51265 & cyclin-dependent kinase-like 3 & 2,12 & chr5 \\
\hline CDKN1C & 1028 & Cyclin-dependent kinase inhibitor 1C (p57, Kip2) & 3,14 & chr11 \\
\hline CDON & 50937 & Cdon homolog (mouse) & 3,91 & chr11 \\
\hline CDV1 & 28981 & carnitine deficiency-associated, expressed in ventricle 1 & 3,30 & chr12 \\
\hline CENTB5 & 116983 & centaurin, beta 5 & 2,05 & chr1 \\
\hline CENTG2 & 116987 & Centaurin, gamma 2 & 2,01 & chr2 \\
\hline CEP1 & 11064 & centrosomal protein 1 & 2,32 & chr9 \\
\hline Cep152 & 22995 & |KIAA0912 protein & 2,03 & chr15 \\
\hline p164 & 22897 & AA1052 protein & 2,54 & $\operatorname{chr} 11$ \\
\hline
\end{tabular}




\begin{tabular}{|c|c|c|c|c|}
\hline Gene Symbol & Entrez Gene & Gene Title & NPC_up & Chromosome Number(Avadis) \\
\hline CEP68 & 23177 & Centrosomal protein $68 \mathrm{kDa}$ & 2,54 & chr2 \\
\hline CHD1L & 9557 & Chromodomain helicase DNA binding protein 1-like & 2,83 & chr1 \\
\hline CHD2 & 1106 & chromodomain helicase DNA binding protein 2 & 2,21 & chr15 \\
\hline CHD7 & 55636 & chromodomain helicase DNA binding protein 7 & 5,92 & chr8 \\
\hline CHD9 & 80205 & Chromodomain helicase DNA binding protein 9 & 3,66 & chr16 \\
\hline $\mathrm{CHKB}$ & $1120 / / / 1375$ & choline kinase beta /// carnitine palmitoyltransferase 1B (muscle) & 3,33 & chr22 \\
\hline $\mathrm{CHN} 2$ & 1124 & Chimerin (chimaerin) 2 & 3,09 & chr7 \\
\hline CHST5 & 23563 & carbohydrate (N-acetylglucosamine 6-O) sulfotransferase 5 & 2,87 & chr16 \\
\hline CHST5 & $23563 / / / 84836$ & carbohydrate ( $\mathrm{N}$-acetylglucosamine 6-O) sulfotransferase 5 /// hypothetical protein MGd & 2,15 & chr16 \\
\hline CIRBP & 1153 & cold inducible RNA binding protein & 3,04 & chr19 \\
\hline CKLFSF3 & 123920 & chemokine-like factor superfamily 3 & 2,39 & chr16 \\
\hline CLCN4 & 1183 & Chloride channel 4 & 2,43 & $\operatorname{chr} X$ \\
\hline CLGN & 1047 & calmegin & 6,29 & chr4 \\
\hline CLIPR-59 & 25999 & CLIP-170-related protein & 3,37 & chr19 \\
\hline CLK1 & 1195 & CDC-like kinase 1 & 2,62 & chr2 \\
\hline CMKOR1 & 57007 & chemokine orphan receptor 1 & 4,25 & chr2 \\
\hline CMPK & 51727 & cytidylate kinase & 2,09 & chr1 \\
\hline $\mathrm{CNIH}$ & 10175 & Cornichon homolog (Drosophila) & 2,08 & chr14 \\
\hline CNKSR3 & 154043 & CNKSR family member 3 & 2,86 & chr6 \\
\hline CNNM3 & 26505 & Cyclin M3 & 2,43 & chr2 \\
\hline CNOT7 & 29883 & CCR4-NOT transcription complex, subunit 7 & 2,28 & chr1 \\
\hline CNTN2 & 6900 & contactin 2 (axonal) & 3,66 & chr1 \\
\hline CNTN4 & 152330 & contactin 4 & 4,47 & chr3 \\
\hline COL11A1 & 1301 & collagen, type XI, alpha 1 & 13,78 & chr1 \\
\hline COL12A1 & 1303 & collagen, type XII, alpha 1 & 5,97 & chr6 \\
\hline COL13A1 & 1305 & collagen, type XIII, alpha 1 & 2,86 & chr10 \\
\hline COL1A1 & 1277 & collagen, type I, alpha 1 & 3,89 & chr17 \\
\hline COL1A2 & 1278 & Collagen, type I, alpha 2 & 8,89 & chr7 \\
\hline COL22A1 & 169044 & collagen, type XXII, alpha 1 & 2,71 & chr8 \\
\hline COL27A1 & 85301 & Collagen, type XXVII, alpha 1 & 2,69 & chr9 \\
\hline COL2A1 & 1280 & collagen, type II, alpha 1 (primary osteoarthritis, spondyloepiphyseal dysplasia, congenit & 21,25 & chr12 \\
\hline COL3A1 & 1281 & collagen, type III, alpha 1 (Ehlers-Danlos syndrome type IV, autosomal dominant) & 64,06 & (vide) \\
\hline COL4A2 & 1284 & collagen, type IV, alpha 2 & 2,39 & chr13 \\
\hline COL4A5 & 1287 & collagen, type IV, alpha 5 (Alport syndrome) & 3,80 & $\operatorname{chrX}$ \\
\hline COL4A6 & 1288 & collagen, type IV, alpha 6 & 8,80 & $\operatorname{chr} X$ \\
\hline COL5A1 & 1289 & collagen, type $\mathrm{V}$, alpha 1 & 11,41 & chr9 \\
\hline COL5A2 & 1290 & collagen, type $\mathrm{V}$, alpha 2 & 8,05 & chr2 \\
\hline COL6A3 & 1293 & collagen, type $\mathrm{VI}$, alpha 3 & 27,77 & chr2 \\
\hline COL9A1 & 1297 & collagen, type IX, alpha 1 & 3,10 & chr6 \\
\hline COLEC12 & 81035 & collectin sub-family member 12 /// collectin sub-family member 12 & 8,88 & chr18 \\
\hline COMMD3 & 23412 & COMM domain containing 3 & 2,43 & chr10 \\
\hline COPG2 & 26958 & Coatomer protein complex, subunit gamma 2 & 6,24 & chr7 \\
\hline CORO1C & 23603 & coronin, actin binding protein, $1 \mathrm{C}$ & 2,55 & chr12 \\
\hline $\operatorname{cox} 411$ & 1327 & cytochrome c oxidase subunit IV isoform 1 & 2,15 & chr16 \\
\hline CPAMD8 & 27151 & C3 and PZP-like, alpha-2-macroglobulin domain containing 8 & 2,63 & chr19 \\
\hline CPE & 1363 & carboxypeptidase E & 3,56 & chr4 \\
\hline CPS1 & 1373 & carbamoyl-phosphate synthetase 1 , mitochondrial & 4,28 & chr2 \\
\hline CPXM & 56265 & carboxypeptidase X (M14 family) & 2,40 & chr20 \\
\hline CREB5 & 9586 & cAMP responsive element binding protein 5 & 2,73 & chr7 \\
\hline CRELD1 & 78987 & cysteine-rich with EGF-like domains 1 & 2,58 & chr3 \\
\hline CREM & 1390 & CAMP responsive element modulator & 2,01 & chr10 \\
\hline CRLF3 & 51379 & Cytokine receptor-like factor 3 & 5,71 & chr17 \\
\hline CROCC & 9696 & ciliary rootlet coiled-coil, rootletin & 2,42 & chr1 \\
\hline CROT & 54677 & carnitine O-octanoyltransferase & 4,57 & chr7 \\
\hline CRYZL1 & 9946 & crystallin, zeta (quinone reductase)-like 1 & 3,27 & chr21 \\
\hline CSAD & 51380 & cysteine sulfinic acid decarboxylase & 3,08 & chr12 \\
\hline CSK & 1445 & c-src tyrosine kinase & 2,02 & chr15 \\
\hline CSPG3 & 1463 & chondroitin sulfate proteoglycan 3 (neurocan) & 3,86 & chr19 \\
\hline CTDSPL & 10217 & CTD (carboxy-terminal domain, RNA polymerase II, polypeptide A) small phosphatase-I & 2,40 & chr3 \\
\hline CTGLF1 & 99753 /// 3997 & centaurin, gamma-like family, member 1 /// hypothetical gene supported by AK093334; & 2,44 & chr10 \\
\hline CTGLF1 /// LOC3\$ & $\oint 9753$ /// 3997 & centaurin, gamma-like family, member 1 /// hypothetical gene supported by AK093334; & 2,86 & chr10 \\
\hline CTNNA1 & 1495 & Catenin (cadherin-associated protein), alpha $1,102 \mathrm{kDa}$ & 2,08 & chr5 \\
\hline CTNNA2 & 1496 & catenin (cadherin-associated protein), alpha 2 & 7,17 & chr2 \\
\hline CTNNBIP1 & 56998 & catenin, beta interacting protein 1 & 3,94 & chr1 \\
\hline CTNND2 & 1501 & catenin (cadherin-associated protein), delta 2 (neural plakophilin-related arm-repeat pro & 3,29 & chr5 \\
\hline CTTNBP2 & 83992 & Cortactin binding protein 2 & 2,30 & chr7 \\
\hline CTTNBP2NL & 55917 & CTTNBP2 N-terminal like & 2,50 & chr1 \\
\hline CUEDC1 & 404093 & CUE domain containing 1 & 2,03 & $\operatorname{chr} 17$ \\
\hline
\end{tabular}




\begin{tabular}{|c|c|c|c|c|}
\hline \multicolumn{5}{|c|}{ Table S2 : Genes overexpressed in NPC compared to hES (Fold Change $>2 ; \alpha<0.05$ ) } \\
\hline Gene Symbol & Entrez Gene & Gene Title & NPC_up & Chromosome Number(Avadis) \\
\hline CUGBP2 & 10659 & CUG triplet repeat, RNA binding protein 2 & 3,44 & chr10 \\
\hline CUL1 & 8454 & Cullin 1 & 2,13 & chr7 \\
\hline CUL3 & 8452 & Cullin 3 & 3,04 & chr2 \\
\hline CUL7 & 9820 & cullin 7 & 2,40 & chr6 \\
\hline CUTL1 & 1523 & Cut-like 1, CCAAT displacement protein (Drosophila) & 2,31 & chr7 \\
\hline CXCL14 & 9547 & chemokine (C-X-C motif) ligand 14 & 16,10 & chr5 \\
\hline CXCR4 & 7852 & chemokine (C-X-C motif) receptor 4 & 4,95 & chr2 \\
\hline CXorf33 & 139322 & chromosome $\mathrm{X}$ open reading frame 33 & 2,21 & $\operatorname{chr} \mathrm{x}$ \\
\hline CXorf39 & 139231 & Chromosome $\mathrm{X}$ open reading frame 39 & 2,21 & $\operatorname{chr} X$ \\
\hline $\mathrm{CXXC4}$ & 80319 & CXXC finger 4 & 3,00 & chr4 \\
\hline CYFIP2 & 26999 & cytoplasmic FMR1 interacting protein 2 /// cytoplasmic FMR1 interacting protein 2 & 2,34 & chr5 \\
\hline D2LIC & 51626 & dynein 2 light intermediate chain & 2,17 & chr2 \\
\hline DAB2 & 1601 & Disabled homolog 2, mitogen-responsive phosphoprotein (Drosophila) & 3,84 & chr5 \\
\hline DACH1 & 1602 & dachshund homolog 1 (Drosophila) & 27,40 & chr13 \\
\hline DCBLD2 & 131566 & discoidin, CUB and LCCL domain containing 2 & 2,47 & chr3 \\
\hline DCHS1 & 8642 & dachsous 1 (Drosophila) & 2,11 & chr11 \\
\hline DCT & 1638 & dopachrome tautomerase (dopachrome delta-isomerase, tyrosine-related protein 2) & 17,25 & $\operatorname{chr} 13$ \\
\hline DCTN3 & 11258 & dynactin 3 (p22) & 2,42 & chr9 \\
\hline DCUN1D4 & 23142 & DCN1, defective in cullin neddylation 1 , domain containing 4 (S. cerevisiae) & 3,75 & chr4 \\
\hline $\mathrm{DCX}$ & 1641 & doublecortex; lissencephaly, X-linked (doublecortin) & 3,86 & $\operatorname{chr} X$ \\
\hline DDEF1 & 50807 & development and differentiation enhancing factor 1 & 2,69 & chr8 \\
\hline DDEF2 & 8853 & development and differentiation enhancing factor 2 & 4,81 & chr2 \\
\hline DDHD2 & 23259 & DDHD domain containing 2 & 5,47 & chr8 \\
\hline DDR2 & 4921 & Discoidin domain receptor family, member 2 & 4,77 & chr1 \\
\hline DDX17 & 10521 & DEAD (Asp-Glu-Ala-Asp) box polypeptide 17 & 2,13 & chr22 \\
\hline DDX50 & 79009 & DEAD (Asp-Glu-Ala-Asp) box polypeptide 50 & 2,13 & chr10 \\
\hline DENND2A & 27147 & DENN/MADD domain containing 2A & 3,98 & chr7 \\
\hline DERA & 51071 & 2-deoxyribose-5-phosphate aldolase homolog (C. elegans) & 2,83 & chr12 \\
\hline DFNA5 & 1687 & deafness, autosomal dominant 5 & 2,39 & chr7 \\
\hline DGCR8 & 54487 & DiGeorge syndrome critical region gene 8 & 2,06 & chr22 \\
\hline DGKD & 8527 & diacylglycerol kinase, delta $130 \mathrm{kDa}$ & 2,09 & chr2 \\
\hline DHX32 & 55760 & DEAH (Asp-Glu-Ala-His) box polypeptide 32 & 2,52 & chr10 \\
\hline DHX57 & 90957 & DEAH (Asp-Glu-Ala-Asp/His) box polypeptide 57 & 2,16 & chr2 \\
\hline DICER1 & 23405 & Dicer1, Dcr-1 homolog (Drosophila) & 2,11 & chr14 \\
\hline DIO3 & 1735 & deiodinase, iodothyronine, type III & 2,97 & chr14 \\
\hline DIP13B & 55198 & DIP13 beta & 2,42 & chr12 \\
\hline DIP2A & 23181 & DIP2 disco-interacting protein 2 homolog A (Drosophila) & 2,66 & chr21 \\
\hline DJ328E19.C1.1// & | /// $440670 / / /$ & hypothetical protein DJ328E19.C1.1 /// hypothetical protein FLJ20719 /// hypothetical prd & 3,04 & chr1_random \\
\hline DJ328E19.C1.1/ & |1967 /// 4406 & hypothetical protein DJ328E19.C1.1 /// hypothetical protein FLJ20719 /// hypothetical pr & 2,46 & chr1_random \\
\hline DKFZp313A2432 & 258010 & hypothetical protein DKFZp313A2432 & 2,35 & chr11 \\
\hline DKFZP434B0335 & 25851 & DKFZP434B0335 protein & 2,74 & chr7 \\
\hline DKFZP434F0318 & 81575 & hypothetical protein DKFZp434F0318 /// hypothetical protein DKFZp434F0318 & 2,14 & chr12 \\
\hline DKFZP434H0115 & 83538 & hypothetical protein DKFZp434H0115 & 2,36 & chr17 \\
\hline DKFZp434P055 & 91531 & hypothetical protein DKFZp434P055 & 2,42 & chr2 \\
\hline DKFZp451M2119 & 285023 & Hypothetical protein DKFZp451M2119 & 3,08 & chr2 \\
\hline DKFZP564D172 & 83989 & hypothetical protein DKFZp564D172 & 2,09 & chr5 \\
\hline DKFZP566N034 & 81615 & hypothetical protein DKFZp566N034 & 2,62 & chr2 \\
\hline DKFZP586H2123 & 25891 & regeneration associated muscle protease & 13,26 & chr11 \\
\hline DKFZP761M1511 & 54492 & hypothetical protein DKFZP761M1511 & 2,22 & chr5 \\
\hline DKFZP761N0912 & 57183 & hypothetical protein DKFZp761N09121 & 10,12 & (vide) \\
\hline DKFZp761O2018 & 92293 & hypothetical protein DKFZp76102018 & 4,07 & chr12 \\
\hline DKFZp762A217 & 160335 & hypothetical protein DKFZp762A217 & 3,19 & chr12 \\
\hline DKK1 & 22943 & dickkopf homolog 1 (Xenopus laevis) & 4,99 & $\operatorname{chr} 10$ \\
\hline DLC1 & 10395 & deleted in liver cancer 1 & 8,48 & chr8 \\
\hline DLK1 & 8788 & delta-like 1 homolog (Drosophila) & 39,62 & chr14 \\
\hline DLL1 & 28514 & delta-like 1 (Drosophila) & 24,34 & chr6 \\
\hline DLX1 & 1745 & distal-less homeo box 1 & 2,82 & chr2 \\
\hline DLX2 & 1746 & distal-less homeo box 2 & 4,26 & chr2 \\
\hline DMD & 1756 & dystrophin (muscular dystrophy, Duchenne and Becker types) & 3,45 & $\operatorname{chr} X$ \\
\hline DMRT3 & 58524 & doublesex and mab-3 related transcription factor 3 & 3,27 & chr9 \\
\hline DMXL2 & 23312 & Dmx-like 2 & 5,22 & chr15 \\
\hline DNAJC1 & 64215 & DnaJ (Hsp40) homolog, subfamily C, member 1 & 3,02 & chr10 \\
\hline DNAJC12 & 56521 & DnaJ (Hsp40) homolog, subfamily C, member 12 & 3,13 & chr10 \\
\hline DNAJC6 & 9829 & DnaJ (Hsp40) homolog, subfamily C, member 6 & 3,57 & chr1 \\
\hline DNALI1 & 7802 & dynein, axonemal, light intermediate polypeptide 1 & 4,36 & chr1 \\
\hline DNCH2 & 79659 & dynein, cytoplasmic, heavy polypeptide 2 & 2,28 & chr11 \\
\hline DNM1 & 1759 & dynamin 1 & 4,23 & chr9 \\
\hline DOC1 & 11259 & downregulated in ovarian cancer 1 & 3,17 & chr3 \\
\hline
\end{tabular}




\begin{tabular}{|c|c|c|c|c|}
\hline Gene Symbol & Entrez Gene & Gene Title & NPC_up & Chromosome Number(Avadis) \\
\hline DOCK1 & 1793 & dedicator of cytokinesis 1 & 2,41 & chr10 \\
\hline DOCK3 & 1795 & dedicator of cytokinesis 3 & 2,46 & chr3 \\
\hline DOCK7 & 85440 & Dedicator of cytokinesis 7 & 2,75 & chr1 \\
\hline DOT1L & 84444 & DOT1-like, histone H3 methyltransferase (S. cerevisiae) & 2,79 & chr19 \\
\hline DPY19L1 & 23333 & dpy-19-like 1 (C. elegans) & 2,45 & chr7 \\
\hline DPY19L2 & 283417 & dpy-19-like 2 (C. elegans) & 2,11 & chr7 \\
\hline DPYD & 1806 & dihydropyrimidine dehydrogenase & 2,27 & chr1 \\
\hline DPYSL5 & 56896 & dihydropyrimidinase-like 5 & 3,84 & chr2 \\
\hline DREV1 & 51108 & DORA reverse strand protein 1 & 2,32 & chr16 \\
\hline DSC2 & 1824 & desmocollin 2 & 4,07 & chr18 \\
\hline DSC3 & 1825 & desmocollin 3 & 3,96 & chr18 \\
\hline DSCR1 & 1827 & Down syndrome critical region gene 1 & 2,04 & chr21 \\
\hline DSCR6 & 53820 & Down syndrome critical region gene 6 & 2,65 & chr21 \\
\hline DST & 667 & dystonin & 4,69 & chr6 \\
\hline DTX4 & 23220 & deltex 4 homolog (Drosophila) & 4,18 & chr11 \\
\hline DUSP4 & 1846 & dual specificity phosphatase 4 & 7,87 & chr8 \\
\hline DVL3 & 1857 & dishevelled, dsh homolog 3 (Drosophila) & 2,40 & chr3 \\
\hline DZIP1 & 22873 & DAZ interacting protein 1 & 2,07 & chr13 \\
\hline EBF & 1879 & Early B-cell factor & 2,92 & chr5 \\
\hline ECHDC1 & 55862 & Enoyl Coenzyme A hydratase domain containing 1 & 3,52 & chr6 \\
\hline EDG2 & 1902 & endothelial differentiation, lysophosphatidic acid G-protein-coupled receptor, 2 & 4,01 & chr9 \\
\hline EDG3 & 1903 & endothelial differentiation, sphingolipid G-protein-coupled receptor, 3 & 7,18 & chr9 \\
\hline EDNRA & 1909 & endothelin receptor type $A$ & 6,36 & chr4 \\
\hline EFEMP1 & 2202 & EGF-containing fibulin-like extracellular matrix protein 1 & 3,92 & chr2 \\
\hline EFEMP2 & 30008 & EGF-containing fibulin-like extracellular matrix protein 2 & 2,51 & chr11 \\
\hline EFHC1 & 114327 & EF-hand domain (C-terminal) containing 1 & 3,83 & chr6 \\
\hline EFHD1 & 80303 & EF-hand domain family, member D1 & 3,22 & chr2 \\
\hline EFNA1 & 1942 & ephrin-A1 & 2,08 & chr1 \\
\hline EFNA5 & 1946 & Ephrin-A5 & 10,87 & chr5 \\
\hline EFNB2 & 1948 & ephrin-B2 & 5,61 & chr13 \\
\hline EFNB3 & 1949 & ephrin-B3 & 3,08 & chr17 \\
\hline EFS & 10278 & embryonal Fyn-associated substrate & 3,80 & chr14 \\
\hline EGFL5 & 1955 & EGF-like-domain, multiple 5 & 2,94 & chr9 \\
\hline EGFR & 1956 & epidermal growth factor receptor (erythroblastic leukemia viral (v-erb-b) oncogene hom & 2,62 & chr7 \\
\hline EGR1 & 1958 & Early growth response 1 & 6,71 & chr5 \\
\hline EIF1 & 10209 & Eukaryotic translation initiation factor 1 & 2,08 & chr17 \\
\hline EIF2C3 & 192669 & eukaryotic translation initiation factor $2 \mathrm{C}, 3$ & 2,38 & chr1 \\
\hline EIF2C4 & 192670 & Eukaryotic translation initiation factor $2 \mathrm{C}, 4$ & 2,87 & chr1 \\
\hline EIF2S1 & 1965 & eukaryotic translation initiation factor 2 , subunit 1 alpha, $35 \mathrm{kDa}$ & 2,63 & chr14 \\
\hline EIF4G3 & 8672 & Eukaryotic translation initiation factor 4 gamma, 3 & 2,64 & chr1 \\
\hline EIF5 & 1983 & eukaryotic translation initiation factor 5 & 2,08 & chr14 \\
\hline ELAC1 & 55520 & elaC homolog 1 (E. coli) & 2,01 & chr18 \\
\hline ELAVL1 & 1994 & ELAV (embryonic lethal, abnormal vision, Drosophila)-like 1 (Hu antigen R) & 3,31 & chr19 \\
\hline ELAVL4 & 1996 & ELAV (embryonic lethal, abnormal vision, Drosophila)-like 4 (Hu antigen D) & 4,59 & chr1 \\
\hline ELOVL2 & 54898 & elongation of very long chain fatty acids (FEN1/Elo2, SUR4/Elo3, yeast)-like 2 & 5,49 & chr6 \\
\hline ELOVL5 & 60481 & ELOVL family member 5 , elongation of long chain fatty acids (FEN1/Elo2, SUR4/Elo3-lil & 2,05 & chr6 \\
\hline EMID2 & 136227 & EMI domain containing 2 & 2,55 & chr7 \\
\hline EMILIN2 & 84034 & elastin microfibril interfacer 2 /// elastin microfibril interfacer 2 & 2,47 & chr18 \\
\hline EML1 & 2009 & echinoderm microtubule associated protein like 1 & 2,27 & chr14 \\
\hline EMP1 & 2012 & epithelial membrane protein 1 & 5,85 & chr12 \\
\hline EMP2 & 2013 & epithelial membrane protein 2 & 3,91 & chr16 \\
\hline EMX2 & 2018 & empty spiracles homolog 2 (Drosophila) & 10,30 & chr10 \\
\hline EMX2OS & 196047 & empty spiracles homolog 2 (Drosophila) opposite strand & 2,81 & chr10 \\
\hline ENAH & 55740 & enabled homolog (Drosophila) & 2,55 & chr1 \\
\hline ENC1 & 8507 & ectodermal-neural cortex (with BTB-like domain) & 8,44 & chr5 \\
\hline ENO3 & 2027 & enolase 3 (beta, muscle) & 3,30 & chr17 \\
\hline ENOSF1 & 55556 & enolase superfamily member 1 & 2,13 & chr18 \\
\hline ENPP2 & 5168 & ectonucleotide pyrophosphatase/phosphodiesterase 2 (autotaxin) & 2,43 & chr8 \\
\hline ENTPD4 & 9583 & ectonucleoside triphosphate diphosphohydrolase 4 & 3,54 & chr8 \\
\hline EP400 & 57634 & E1A binding protein $\mathrm{p} 400$ & 2,38 & chr12 \\
\hline EPAS1 & 2034 & endothelial PAS domain protein 1 & 8,51 & chr2 \\
\hline EPB41 & 2035 & Erythrocyte membrane protein band 4.1 (elliptocytosis 1, RH-linked) & 2,55 & chr1 \\
\hline EPB41L5 & 57669 & erythrocyte membrane protein band 4.1 like 5 & 4,25 & chr2 \\
\hline EPC1 & 80314 & Enhancer of polycomb homolog 1 (Drosophila) & 2,24 & chr10 \\
\hline EPHA4 & 2043 & EPH receptor A4 & 12,56 & chr2 \\
\hline EPHA7 & 2045 & EPH receptor A7 & 7,42 & chr6 \\
\hline EPHB2 & 2048 & EPH receptor B2 & 2,96 & chr1 \\
\hline EPHB3 & 2049 & EPH receptor B3 & 2,56 & chr3 \\
\hline
\end{tabular}




\begin{tabular}{|c|c|c|c|c|}
\hline Gene Symbol & Entrez Gene & Gene Title & NPC_up & Chromosome Number(Avadis) \\
\hline EPM2AIP1 & 9852 & EPM2A (laforin) interacting protein 1 & 6,40 & chr3 \\
\hline EPS8 & 2059 & epidermal growth factor receptor pathway substrate 8 & 3,04 & chr12 \\
\hline ESRRG & 2104 & estrogen-related receptor gamma & 2,58 & chr1 \\
\hline EVI1 & 2122 & ecotropic viral integration site 1 & 9,29 & chr3 \\
\hline EVL & 51466 & Enah/Vasp-like & 3,39 & chr14 \\
\hline EWSR1 & 2130 & Ewing sarcoma breakpoint region 1 & 3,64 & chr22 \\
\hline EXT1 & 2131 & Exostoses (multiple) 1 & 2,75 & chr8 \\
\hline EYA1 & 2138 & eyes absent homolog 1 (Drosophila) & 3,91 & chr8 \\
\hline EYA2 & 2139 & eyes absent homolog 2 (Drosophila) & 4,33 & chr20 \\
\hline EYA4 & 2070 & Eyes absent homolog 4 (Drosophila) & 4,11 & chr6 \\
\hline F2RL2 & 2151 & coagulation factor II (thrombin) receptor-like 2 & 7,64 & chr5 \\
\hline FABP7 & 2173 & fatty acid binding protein 7 , brain & 7,66 & chr6 \\
\hline FAM13C1 & 220965 & family with sequence similarity 13 , member $\mathrm{C} 1$ & 2,78 & chr10 \\
\hline FAM19A5 & 25817 & family with sequence similarity 19 (chemokine (C-C motif)-like), member A5 & 2,06 & chr22 \\
\hline FAM46A & 55603 & family with sequence similarity 46 , member $A$ & 2,92 & chr6 \\
\hline FAM51A1 & 54960 & Family with sequence similarity 51 , member A1 & 2,03 & $\operatorname{chr} X$ \\
\hline FAM64A & 54478 & Family with sequence similarity 64 , member $\mathrm{A}$ & 2,03 & chr17 \\
\hline FAM77D & 286183 & Family with sequence similarity 77 , member $\mathrm{D}$ & 5,59 & chr8 \\
\hline FAM7A2 & 89839 & Family with sequence similarity 7 , member A2 & 2,31 & (vide) \\
\hline FAM89A & 375061 & family with sequence similarity 89 , member $\mathrm{A}$ & 4,01 & chr1 \\
\hline FAM89B & 23625 & family with sequence similarity 89 , member $B$ & 2,11 & chr11 \\
\hline FANCA & 2175 & Fanconi anemia, complementation group A & 2,35 & chr16 \\
\hline FANK1 & 92565 & fibronectin type III and ankyrin repeat domains 1 & 3,56 & $\operatorname{chr} 10$ \\
\hline FARP1 & 10160 & FERM, RhoGEF (ARHGEF) and pleckstrin domain protein 1 (chondrocyte-derived) & 2,75 & chr13 \\
\hline FBLN1 & 2192 & fibulin 1 & 2,75 & chr22 \\
\hline FBN1 & 2200 & fibrillin 1 (Marfan syndrome) & 10,66 & chr15 \\
\hline FBN2 & 2201 & fibrillin 2 (congenital contractural arachnodactyly) & 11,24 & chr5 \\
\hline FBXL14 & 144699 & F-box and leucine-rich repeat protein 14 & 6,25 & chr12 \\
\hline FBXL17 & 64839 & F-box and leucine-rich repeat protein 17 & 2,15 & chr5 \\
\hline FBXO33 & 254170 & F-box protein 33 & 2,04 & chr14 \\
\hline FBXW8 & 26259 & F-box and WD-40 domain protein 8 & 2,27 & chr12 \\
\hline FCHSD2 & 9873 & $\mathrm{FCH}$ and double $\mathrm{SH} 3$ domains 2 & 2,45 & chr11 \\
\hline FCMD & 2218 & Fukuyama type congenital muscular dystrophy (fukutin) & 2,04 & chr9 \\
\hline FGF7 & 2252 & Fibroblast growth factor 7 (keratinocyte growth factor) & 2,25 & (vide) \\
\hline FGF9 & 2254 & fibroblast growth factor 9 (glia-activating factor) & 21,83 & chr13 \\
\hline FHOD3 & 80206 & formin homology 2 domain containing 3 & 7,10 & chr18 \\
\hline FIGN & 55137 & Fidgetin & 4,91 & chr2 \\
\hline FILIP1 & 27145 & filamin A interacting protein 1 & 3,28 & chr6 \\
\hline FJX1 & 24147 & four jointed box 1 (Drosophila) & 6,85 & chr11 \\
\hline FKBP7 & 51661 & FK506 binding protein 7 & 3,51 & chr2 \\
\hline FKBP9 & 11328 & FK506 binding protein 9, $63 \mathrm{kDa}$ & 3,15 & chr7 \\
\hline FLJ10081 & 55683 & hypothetical protein FLJ10081 & 2,26 & chr2 \\
\hline FLJ10099 & 55069 & Hypothetical protein FLJ10099 & 2,31 & chr7 \\
\hline FLJ10154 & 55082 & Hypothetical protein FLJ10154 & 4,51 & $\operatorname{chr} 13$ \\
\hline FLJ10159 & 55084 & hypothetical protein FLJ10159 & 3,28 & chr6 \\
\hline FLJ10178 & 55086 & hypothetical protein FLJ10178 & 3,09 & $\operatorname{chrX}$ \\
\hline FLJ10213 & 55096 & hypothetical protein FLJ10213 & 2,20 & chr3 \\
\hline FLJ10287 & 54482 & hypothetical protein FLJ10287 & 3,29 & chr1 \\
\hline FLJ10357 & 55701 & hypothetical protein FLJ10357 & 4,05 & chr14 \\
\hline FLJ10996 & 54520 & hypothetical protein FLJ10996 & 2,19 & chr2 \\
\hline FLJ12700 & 79970 & hypothetical protein FLJ12700 & 2,18 & $\operatorname{chr} 7$ \\
\hline FLJ13089 & 80018 & hypothetical protein FLJ13089 & 2,41 & chr12 \\
\hline FLJ13197 & 79667 & hypothetical protein FLJ13197 & 2,52 & $\operatorname{chr} 4$ \\
\hline FLJ14054 & 79614 & hypothetical protein FLJ14054 & 9,16 & chr5 \\
\hline FLJ14525 & 84886 & hypothetical protein FLJ14525 & 2,08 & chr1 \\
\hline FLJ14640 & 84902 & hypothetical protein FLJ14640 & 2,08 & chr19 \\
\hline FLJ16008 & 339761 & FLJ16008 protein & 3,85 & chr2 \\
\hline FLJ20054 & 54530 & hypothetical protein FLJ20054 & 3,69 & chr1 \\
\hline FLJ20719 & 55672 & hypothetical protein FLJ20719 & 4,93 & chr1 \\
\hline FLJ20719 & $40670 / / / 4406$ & hypothetical protein FLJ20719 /// hypothetical protein LOC200030 /// hypothetical proteir & 2,59 & chr1 \\
\hline FLJ20719 & $40673 / / / 5567$ & hypothetical protein FLJ20719 /// AG1 protein & 2,41 & chr1_random \\
\hline FLJ20719 & | /// $440673 / / /$ & |hypothetical protein FLJ20719 /// hypothetical protein MGC8902 /// AE01 mRNA /// AG1 & 2,35 & chr1 \\
\hline FLJ21125 & 79680 & hypothetical protein FLJ21125 & 2,04 & chr22 \\
\hline FLJ21127 & 79600 & hypothetical protein FLJ21127 & 2,77 & chr12 \\
\hline FLJ21616 & 79618 & Hypothetical protein FLJ21616 & 2,28 & chr8 \\
\hline FLJ21827 & 56912 & hypothetical protein FLJ21827 & 2,19 & chr11 \\
\hline FLJ21865 & 64772 & endo-beta- $\mathrm{N}$-acetylglucosaminidase & 2,32 & chr17 \\
\hline J21986 & 974 & ypothetical protein FLJ21986 & 2 & chr7 \\
\hline
\end{tabular}


Table S2 : Genes overexpressed in NPC compared to hES (Fold Change $>2 ; \alpha<0.05$ )

\begin{tabular}{|c|c|c|c|c|}
\hline Gene Symbol & Entrez Gene & Gene Title & NPC_up & Chromosome Number(Avadis) \\
\hline FLJ22313 & 64224 & hypothetical protein FLJ22313 & 2,34 & chr7 \\
\hline FLJ22471 & 80212 & limkain beta 2 & 6,73 & $\operatorname{chr} 12$ \\
\hline FLJ23191 & 79625 & hypothetical protein FLJ23191 & 4,87 & chr4 \\
\hline FLJ23342 & 79684 & Hypothetical protein FLJ23342 & 2,60 & chr11 \\
\hline FLJ23518 & 79780 & hypothetical protein FLJ23518 & 2,21 & chr11 \\
\hline FLJ25076 & 134111 & similar to CG4502-PA & 2,40 & chr5 \\
\hline FLJ25476 & 149076 & FLJ25476 protein & 2,24 & chr1 \\
\hline FLJ25694 & 283492 & hypothetical protein FLJ25694 & 5,59 & chr13 \\
\hline FLJ25715 & 284241 & Hypothetical protein FLJ25715 & 2,12 & chr18 \\
\hline FLJ25967 & 440823 & hypothetical gene supported by AK098833 & 2,71 & chr22 \\
\hline FLJ30092 & 196515 & AF-1 specific protein phosphatase /// AF-1 specific protein phosphatase & 2,01 & chr12 \\
\hline FLJ30851 & 375190 & FLJ30851 protein & 2,71 & chr2 \\
\hline FLJ30901 & 150378 & hypothetical protein FLJ30901 & 7,03 & chr22 \\
\hline FLJ31438 & 130162 & hypothetical protein FLJ31438 & 2,95 & chr2 \\
\hline FLJ31818 & 154743 & hypothetical protein FLJ31818 & 2,18 & chr7 \\
\hline FLJ31951 & 153830 & Hypothetical protein FLJ31951 & 3,92 & chr5 \\
\hline FLJ34208 & 401106 & Hypothetical gene supported by AK091527 & 2,15 & chr3 \\
\hline FLJ34443 & 285464 & hypothetical protein FLJ34443 & 2,90 & chr4 \\
\hline FLJ36031 & 168455 & Hypothetical protein FLJ36031 & 3,36 & chr7 \\
\hline FLJ36166 & 349152 & Hypothetical protein FLJ36166 & 2,36 & chr7 \\
\hline FLJ37440 & 129804 & hypothetical protein FLJ37440 & 2,05 & chr2 \\
\hline FLJ37562 & 134553 & hypothetical protein FLJ37562 & 2,50 & chr5 \\
\hline FLJ38379 & 285097 & hypothetical protein FLJ38379 & 2,87 & chr2 \\
\hline FLJ39155 & 133584 & hypothetical protein FLJ39155 & 3,06 & chr5 \\
\hline FLJ39378 & 353116 & hypothetical protein FLJ39378 & 2,38 & chr12 \\
\hline FLJ39653 & 202020 & hypothetical protein FLJ39653 & 2,30 & chr4 \\
\hline FLJ42393 & 401105 & FLJ42393 protein & 2,27 & chr3 \\
\hline FLJ42709 & 441094 & hypothetical gene supported by AK124699 & 3,93 & chr5 \\
\hline FLJ42957 & 400077 & FLJ42957 protein & 2,28 & chr12 \\
\hline FLJ44216 & 375484 & FLJ44216 protein & 2,75 & chr5 \\
\hline FLJ45187 & 387640 & FLJ45187 protein & 13,87 & chr10 \\
\hline FLJ45831 & 400576 & FLJ45831 protein /// MRNA; cDNA DKFZp564A222 (from clone DKFZp564A222) & 2,06 & chr2 \\
\hline FLJ90757 & 440465 & FLJ90757 protein & 4,70 & chr17 \\
\hline FLRT2 & 23768 & fibronectin leucine rich transmembrane protein 2 & 6,72 & chr14 \\
\hline FLRT3 & 23767 & fibronectin leucine rich transmembrane protein 3 & 20,56 & chr20 \\
\hline FNBP1 & 23048 & Formin binding protein 1 & 3,16 & chr9 \\
\hline FNBP1L & 54874 & Formin binding protein 1 -like & 2,21 & chr1 \\
\hline FNBP4 & 23360 & formin binding protein 4 & 2,24 & chr11 \\
\hline FNDC5 & 252995 & fibronectin type III domain containing 5 & 3,30 & chr1 \\
\hline FOSL2 & 2355 & FOS-like antigen 2 & 2,36 & chr2 \\
\hline FOXC1 & 2296 & forkhead box $\mathrm{C} 1$ & 5,17 & chr6 \\
\hline FOXG1B & 2290 & forkhead box G1B & 14,70 & chr14 \\
\hline FOXK1 & 221937 & Forkhead box K1 & 2,38 & chr7 \\
\hline FOXOЗA & 2309 & forkhead box O3A & 2,14 & chr6 \\
\hline FOXP1 & 27086 & Forkhead box P1 & 3,05 & chr3 \\
\hline FRMD4A & 55691 & FERM domain containing $4 \mathrm{~A}$ & 3,61 & chr10 \\
\hline FRMD4A & $1810 / / / 55691$ & FERM domain containing 4A /// Down-regulator of transcription 1, TBP-binding (negative & 6,08 & chr1 \\
\hline FRMD4B & 23150 & FERM domain containing 4B & 2,36 & chr3 \\
\hline FRMD6 & 122786 & FERM domain containing 6 & 3,92 & chr14 \\
\hline FRZB & 2487 & frizzled-related protein & 13,30 & chr2 \\
\hline FSD1CL & 405752 & GTPase activating Rap/RanGAP domain-like 1 & 3,17 & chr9 \\
\hline FSD1L & 405752 & FSD1-like & 2,01 & $\operatorname{chr} 9$ \\
\hline FST & 10468 & follistatin & 3,11 & chr5 \\
\hline FUBP1 & 8880 & Far upstream element (FUSE) binding protein 1 & 2,47 & chr1 \\
\hline FXYD6 & 53826 & FXYD domain containing ion transport regulator 6 & 2,91 & chr11 \\
\hline FYTTD1 & 84248 & forty-two-three domain containing 1 & 2,72 & chr3 \\
\hline FZD1 & 8321 & frizzled homolog 1 (Drosophila) & 10,39 & chr7 \\
\hline FZD2 & 2535 & frizzled homolog 2 (Drosophila) & 10,50 & chr17 \\
\hline FZD3 & 7976 & frizzled homolog 3 (Drosophila) & 3,20 & chr8 \\
\hline GAB2 & 9846 & GRB2-associated binding protein 2 & 2,33 & chr11 \\
\hline GABRP & 2568 & gamma-aminobutyric acid (GABA) A receptor, pi & 15,76 & chr5 \\
\hline GAD1 & 2571 & glutamate decarboxylase 1 (brain, 67kDa) & 3,77 & chr2 \\
\hline GALNT10 & 55568 & UDP-N-acetyl-alpha-D-galactosamine:polypeptide $\mathrm{N}$-acetylgalactosaminyltransferase 10 & 2,68 & chr5 \\
\hline GALNTL1 & 57452 & UDP-N-acetyl-alpha-D-galactosamine:polypeptide N-acetylgalactosaminyltransferase-lih & 3,56 & chr14 \\
\hline GAPVD1 & 26130 & GTPase activating protein and VPS9 domains 1 & 2,15 & (vide) \\
\hline GAS1 & 2619 & growth arrest-specific 1 & 29,68 & chr9 \\
\hline GAS2L3 & 283431 & growth arrest-specific 2 like 3 & 2,33 & chr12 \\
\hline GAS6 & 2621 & growth arrest-specific 6 & 2,91 & chr13 \\
\hline
\end{tabular}




\begin{tabular}{|c|c|c|c|c|}
\hline Gene Symbol & Entrez Gene & Gene Title & NPC_up & Chromosome Number(Avadis) \\
\hline GAS7 & 8522 & growth arrest-specific 7 & 2,85 & chr17 \\
\hline GATA3 & 2625 & GATA binding protein 3 & 5,80 & $\operatorname{chr} 10$ \\
\hline GDPD1 & 284161 & Glycerophosphodiester phosphodiesterase domain containing 1 & 2,17 & chr17 \\
\hline GGA2 & 23062 & golgi associated, gamma adaptin ear containing, ARF binding protein 2 & 2,26 & chr16 \\
\hline GGH & 8836 & gamma-glutamyl hydrolase (conjugase, folylpolygammaglutamyl hydrolase) & 2,88 & chr8 \\
\hline GKAP1 & 80318 & G kinase anchoring protein 1 & 3,06 & chr9 \\
\hline GLCCl1 & 113263 & glucocorticoid induced transcript 1 & 2,40 & $\operatorname{chr} 7$ \\
\hline GLI3 & 2737 & GLI-Kruppel family member GLI3 (Greig cephalopolysyndactyly syndrome) & 4,08 & $\operatorname{chr} 7$ \\
\hline GLIS3 & 169792 & GLIS family zinc finger 3 & 9,00 & chr9 \\
\hline GLRB & 2743 & glycine receptor, beta & 2,22 & chr4 \\
\hline GLT25D2 & 23127 & glycosyltransferase 25 domain containing 2 & 11,19 & chr1 \\
\hline GLUD1 & 2746 & glutamate dehydrogenase 1 & 2,37 & $\operatorname{chr} X$ \\
\hline GNAS & 2778 & GNAS complex locus & 2,01 & chr20 \\
\hline GNAZ & 2781 & guanine nucleotide binding protein ( $G$ protein), alpha z polypeptide & 4,73 & chr22 \\
\hline GNB1 & 2782 & guanine nucleotide binding protein ( $G$ protein), beta polypeptide 1 & 2,25 & chr1 \\
\hline GNE & 10020 & glucosamine (UDP-N-acetyl)-2-epimerase/N-acetylmannosamine kinase & 2,12 & chr9 \\
\hline GNG12 & 55970 & guanine nucleotide binding protein ( $G$ protein), gamma 12 & 3,30 & chr1 \\
\hline GNG2 & 54331 & Guanine nucleotide binding protein ( $G$ protein), gamma 2 & 2,66 & chr14 \\
\hline GOLGA8A & 23015 & golgi autoantigen, golgin subfamily a, $8 \mathrm{~A}$ & 6,17 & chr15 \\
\hline GOLGA8A & $3015 / / / 44027$ & golgi autoantigen, golgin subfamily a, 8A /// golgi autoantigen, golgin subfamily a, 8B & 4,71 & chr15 \\
\hline GOLGA8B & 440270 & golgi autoantigen, golgin subfamily a, 8B & 5,30 & chr15 \\
\hline GOPC & 57120 & Golgi associated PDZ and coiled-coil motif containing & 3,70 & chr6 \\
\hline GOSR2 & 9570 & Golgi SNAP receptor complex member 2 & 2,63 & chr17 \\
\hline GPC1 & 2817 & glypican 1 & 3,13 & chr2 \\
\hline GPC3 & 2719 & glypican 3 & 9,11 & $\operatorname{chrX}$ \\
\hline GPC6 & 10082 & Glypican 6 & 3,22 & chr13 \\
\hline GPM6A & 2823 & glycoprotein M6A & 17,26 & chr4 \\
\hline GPR153 & 387509 & G protein-coupled receptor 153 & 2,69 & chr1 \\
\hline GPR161 & 23432 & G protein-coupled receptor 161 & 6,56 & chr1 \\
\hline GPR23 & 2846 & G protein-coupled receptor 23 & 2,36 & $\operatorname{chrX}$ \\
\hline GPR24 & 2847 & G protein-coupled receptor 24 & 2,29 & chr22 \\
\hline GPR56 & 9289 & G protein-coupled receptor 56 & 10,06 & chr16 \\
\hline GPRASP1 & 9737 & G protein-coupled receptor associated sorting protein 1 & 3,08 & $\operatorname{chr} X$ \\
\hline GPSM1 & 26086 & G-protein signalling modulator 1 (AGS3-like, C. elegans) & 4,14 & chr9 \\
\hline GRAMD1B & 57476 & GRAM domain containing 1B & 2,28 & chr11 \\
\hline GREB1 & 9687 & GREB1 protein & 4,39 & chr2 \\
\hline GREM1 & 26585 & gremlin 1, cysteine knot superfamily, homolog (Xenopus laevis) & 6,32 & chr15 \\
\hline GRHL1 & 29841 & grainyhead-like 1 (Drosophila) & 2,15 & chr2 \\
\hline GRIA1 & 2890 & glutamate receptor, ionotropic, AMPA 1 & 4,04 & chr5 \\
\hline GRK5 & 2869 & G protein-coupled receptor kinase 5 & 2,95 & chr10 \\
\hline GRM3 & 2913 & glutamate receptor, metabotropic 3 & 3,02 & chr7 \\
\hline GSDML & 55876 & gasdermin-like & 2,64 & chr17 \\
\hline GSK3B & 2932 & Glycogen synthase kinase 3 beta & 3,45 & chr3 \\
\hline GSTA4 & 2941 & glutathione S-transferase A4 & 3,57 & chr6 \\
\hline GSTM3 & 2947 & glutathione S-transferase M3 (brain) & 3,47 & chr1 \\
\hline GUCY1A3 & 2982 & guanylate cyclase 1 , soluble, alpha 3 & 8,68 & $\operatorname{chr} 4$ \\
\hline $\mathrm{H} 19$ & 283120 & H19, imprinted maternally expressed untranslated mRNA & 28,54 & chr11 \\
\hline H1FO & 3005 & $\mathrm{H} 1$ histone family, member 0 & 3,22 & chr22 \\
\hline $\mathrm{H} 1 \mathrm{FX}$ & 8971 & $\mathrm{H} 1$ histone family, member $\mathrm{X}$ & 2,56 & chr3 \\
\hline H2AFV & 94239 & H2A histone family, member $V$ & 2,99 & chr7 \\
\hline H2AFY & 9555 & $\mathrm{H} 2 \mathrm{~A}$ histone family, member $\mathrm{Y}$ & 4,70 & chr5 \\
\hline H2AFY2 & 55506 & H2A histone family, member Y2 & 3,19 & chr10 \\
\hline H3F3B & 3021 & $\mathrm{H} 3$ histone, family $3 \mathrm{~B}(\mathrm{H} 3.3 \mathrm{~B})$ & 2,65 & chr12 \\
\hline HABP4 & 22927 & hyaluronan binding protein 4 & 2,37 & chr9 \\
\hline HAPLN1 & 1404 & Hyaluronan and proteoglycan link protein 1 & 12,40 & chr5 \\
\hline HDAC6 & 10013 & histone deacetylase 6 & 2,43 & $\operatorname{chrX}$ \\
\hline HECA & 51696 & headcase homolog (Drosophila) & 2,85 & chr6 \\
\hline |HECW2 & 57520 & HECT, C2 and WW domain containing E3 ubiquitin protein ligase 2 & 2,85 & chr2 \\
\hline HEG1 & 57493 & HEG homolog 1 (zebrafish) & 4,43 & chr3 \\
\hline |HEL308 & 113510 & DNA helicase HEL308 & 2,25 & chr4 \\
\hline HES1 & 3280 & hairy and enhancer of split 1, (Drosophila) & 5,20 & chr3 \\
\hline HES5 & 388585 & hairy and enhancer of split 5 (Drosophila) & 3,79 & chr1 \\
\hline |HEXIM1 & 10614 & hexamethylene bis-acetamide inducible 1 & 2,00 & chr17 \\
\hline HEY1 & 23462 & hairy/enhancer-of-split related with YRPW motif 1 & 4,70 & chr8 \\
\hline HHAT & 55733 & hedgehog acyltransferase & 2,02 & chr1 \\
\hline HIC2 & 23119 & hypermethylated in cancer 2 & 2,01 & chr22 \\
\hline HIPK2 & 28996 & Homeodomain interacting protein kinase 2 & 4,15 & chr7 \\
\hline |HIST1H2BD & 3017 & Histone 1, H2bd & 2,88 & chr6 \\
\hline
\end{tabular}


Table S2 : Genes overexpressed in NPC compared to hES (Fold Change $>2 ; \alpha<0.05$ )

\begin{tabular}{|c|c|c|c|c|}
\hline Gene Symbol & Entrez Gene & Gene Title & NPC_up & Chromosome Number(Avadis) \\
\hline HMCN1 & 83872 & hemicentin 1 & 3,88 & chr1 \\
\hline HNRPAO & 10949 & Heterogeneous nuclear ribonucleoprotein A0 & 2,32 & chr5 \\
\hline HNRPA1 & 3178 & heterogeneous nuclear ribonucleoprotein A1 & 2,02 & chr12 \\
\hline HNRPA3 & 220988 & heterogeneous nuclear ribonucleoprotein A3 & 2,37 & chr2 \\
\hline HNRPC & 3183 & heterogeneous nuclear ribonucleoprotein $\mathrm{C}(\mathrm{C} 1 / \mathrm{C} 2)$ & 2,87 & chr14 \\
\hline HNRPD & 3184 & Heterogeneous nuclear ribonucleoprotein D (AU-rich element RNA binding protein 1, 37 & 2,64 & chr4 \\
\hline HNRPR & 10236 & heterogeneous nuclear ribonucleoprotein $\mathrm{R}$ & 3,46 & chr1 \\
\hline HNT & 50863 & neurotrimin & 2,01 & chr11 \\
\hline HOMER2 & 9455 & Homer homolog 2 (Drosophila) & 2,13 & chr15 \\
\hline HOMER3 & 9454 & homer homolog 3 (Drosophila) & 2,99 & $\operatorname{chr} 19$ \\
\hline HOXA1 & 3198 & homeo box A1 & 3,61 & $\operatorname{chr} 7$ \\
\hline HOXA9 & 3205 & homeo box A9 & 3,29 & chr7 \\
\hline HOXB2 & 3212 & homeo box B2 & 4,48 & $\operatorname{chr} 17$ \\
\hline HP1BP3 & 50809 & heterochromatin protein 1 , binding protein 3 & 2,63 & chr1 \\
\hline HRB2 & 11103 & HIV-1 rev binding protein 2 & 2,13 & chr12 \\
\hline HRMT1L1 & 3275 & HMT1 hnRNP methyltransferase-like 1 (S. cerevisiae) & 2,44 & chr21 \\
\hline HS6ST2 & 90161 & heparan sulfate 6-O-sulfotransferase 2 & 3,89 & $\operatorname{chr} X$ \\
\hline HSA277841 & 55421 & ELG protein & 2,31 & chr17 \\
\hline HSF2 & 3298 & heat shock transcription factor 2 & 2,95 & chr6 \\
\hline HSPA5 & 3309 & heat shock $70 \mathrm{kDa}$ protein 5 (glucose-regulated protein, $78 \mathrm{kDa}$ ) & 2,49 & chr9 \\
\hline HSPA5BP1 & 54972 & heat shock $70 \mathrm{kDa}$ protein 5 (glucose-regulated protein, $78 \mathrm{kDa}$ ) binding protein 1 & 2,91 & chr11 \\
\hline HSPC065 & 29070 & HSPC065 protein & 2,05 & chr16 \\
\hline HTR1E & 3354 & 5-hydroxytryptamine (serotonin) receptor $1 \mathrm{E}$ & 2,01 & chr6 \\
\hline HUNK & 30811 & hormonally upregulated Neu-associated kinase & 2,65 & chr21 \\
\hline ID2 & 3398 & inhibitor of DNA binding 2, dominant negative helix-loop-helix protein & 6,35 & chr2 \\
\hline ID2 /// ID2B & $3398 / / / 84099$ & inhibitor of DNA binding 2, dominant negative helix-loop-helix protein /// inhibitor of DNA & 5,95 & chr2 \\
\hline ID3 & 3399 & inhibitor of DNA binding 3, dominant negative helix-loop-helix protein & 2,55 & chr1 \\
\hline ID4 & 3400 & Inhibitor of DNA binding 4, dominant negative helix-loop-helix protein & 6,66 & chr6 \\
\hline IDH2 & 3418 & isocitrate dehydrogenase $2(\mathrm{NADP}+)$, mitochondrial & 2,16 & chr15 \\
\hline IER5L & 389792 & immediate early response 5 -like & 7,56 & chr9 \\
\hline IFI16 & 3428 & interferon, gamma-inducible protein 16 & 3,56 & chr1 \\
\hline IFNGR1 & 3459 & Interferon gamma receptor 1 & 2,05 & chr6 \\
\hline IGFBP3 & 3486 & insulin-like growth factor binding protein 3 & 11,27 & chr7 \\
\hline IGFBP5 & 3488 & insulin-like growth factor binding protein 5 & 25,08 & chr2 \\
\hline IGFBP7 & 3490 & insulin-like growth factor binding protein 7 & 8,27 & chr4 \\
\hline IGSF11 & 152404 & immunoglobulin superfamily, member 11 & 2,05 & chr3 \\
\hline IGSF4 & 23705 & Immunoglobulin superfamily, member 4 & 8,38 & chr11 \\
\hline IKIP & 121457 & IKK interacting protein & 2,81 & chr12 \\
\hline IL11RA & 3590 & interleukin 11 receptor, alpha & 2,53 & chr9 \\
\hline IL17D & 53342 & Interleukin 17D & 2,19 & chr13 \\
\hline IL1R1 & 3554 & interleukin 1 receptor, type I & 2,39 & chr2 \\
\hline IL6ST & 3572 & interleukin 6 signal transducer (gp130, oncostatin M receptor) & 2,88 & chr17 \\
\hline IMMP1L & 196294 & IMP1 inner mitochondrial membrane peptidase-like (S. cerevisiae) & 2,15 & chr11 \\
\hline ING3 & 54556 & inhibitor of growth family, member 3 & 2,11 & chr7 \\
\hline ING4 & 51147 & inhibitor of growth family, member 4 & 2,29 & chr12 \\
\hline INPP1 & 3628 & inositol polyphosphate-1-phosphatase & 2,42 & chr2 \\
\hline INPP5E & 56623 & inositol polyphosphate-5-phosphatase, $72 \mathrm{kDa}$ & 2,21 & chr9 \\
\hline INSM1 & 3642 & insulinoma-associated 1 & 2,84 & chr20 \\
\hline IPO9 & 55705 & Importin 9 & 2,55 & chr1 \\
\hline IPP & 3652 & intracisternal A particle-promoted polypeptide & 2,12 & chr1 \\
\hline IQCE & 23288 & IQ motif containing $\mathrm{E}$ & 2,16 & chr7 \\
\hline IRS4 & 8471 & Insulin receptor substrate 4 & 7,48 & $\operatorname{chr} X$ \\
\hline ISL1 & 3670 & ISL1 transcription factor, LIM/homeodomain, (islet-1) & 4,91 & chr5 \\
\hline ITGA4 & 3676 & integrin, alpha 4 (antigen CD49D, alpha 4 subunit of VLA-4 receptor) & 4,20 & chr2 \\
\hline ITGAV & 3685 & Integrin, alpha $\mathrm{V}$ (vitronectin receptor, alpha polypeptide, antigen CD51) & 6,90 & chr2 \\
\hline ITGB8 & 3696 & Integrin, beta 8 & 2,37 & chr7 \\
\hline IVNS1ABP & 10625 & influenza virus NS1A binding protein & 2,06 & chr1 \\
\hline JAG1 & 182 & Jagged 1 (Alagille syndrome) & 3,09 & chr20 \\
\hline JRK & 8629 & jerky homolog (mouse) & 2,16 & chr8 \\
\hline JUB & 84962 & jub, ajuba homolog (Xenopus laevis) & 4,72 & chr14 \\
\hline KALRN & 8997 & kalirin, RhoGEF kinase & 2,89 & chr3 \\
\hline KBTBD11 & 9920 & kelch repeat and BTB (POZ) domain containing 11 & 2,36 & chr8 \\
\hline KBTBD9 & 114818 & kelch repeat and BTB (POZ) domain containing 9 & 3,93 & chr2 \\
\hline KCNJ13 & 3769 & potassium inwardly-rectifying channel, subfamily J, member 13 & 4,61 & chr2 \\
\hline KCNJ2 & 3759 & potassium inwardly-rectifying channel, subfamily J, member 2 & 2,38 & chr17 \\
\hline KCNJ4 & 3761 & potassium inwardly-rectifying channel, subfamily J, member 4 & 3,63 & chr22 \\
\hline KCNK10 & 54207 & potassium channel, subfamily $\mathrm{K}$, member 10 & 6,54 & chr14 \\
\hline KCNT2 & 343450 & potassium channel, subfamily $\mathrm{T}$, member 2 & 4,19 & chr1 \\
\hline
\end{tabular}




\begin{tabular}{|c|c|c|c|c|}
\hline Gene Symbol & Entrez Gene & Gene Title & NPC_up & Chromosome Number(Avadis) \\
\hline KCTD1 & 284252 & potassium channel tetramerisation domain containing 1 & 2,13 & chr18 \\
\hline |KIAA0101 & 9768 & |KIAA0101 /// KIAA0101 & 2,65 & chr15 \\
\hline |KIAA0247 & 9766 & |KIAA0247 & 2,68 & chr14 \\
\hline |KIAA0265 & 23008 & KIAA0265 protein & 2,79 & chr7 \\
\hline |KIAA0323 & 23351 & |KIAA0323 & 2,30 & chr14 \\
\hline |KIAA0500 & 57237 & KIAA0500 protein & 3,13 & chr14 \\
\hline |KIAA0515 & 84726 & KIAA0515 & 2,11 & chr9 \\
\hline |KIAA0556 & 23247 & KIAA0556 protein & 2,03 & chr16 \\
\hline |KIAA0582 & 23177 & KIAA0582 & 2,21 & chr2 \\
\hline |KIAA0690 & 23223 & |KIAA0690 & 2,10 & $\operatorname{chr} 10$ \\
\hline |KIAA0738 & 9747 & KIAA0738 gene product & 2,17 & chr7 \\
\hline |KIAA0826 & 23045 & KIAA0826 & 2,05 & chr4 \\
\hline |KIAA0830 & 23052 & KIAA0830 protein & 3,25 & chr11 \\
\hline |KIAA0841 & 23354 & KIAA0841 & 3,12 & chr19 \\
\hline |KIAA0882 & 23158 & KIAA0882 protein & 15,64 & chr4 \\
\hline |KIAA0889 & 25781 & KIAA0889 protein & 3,19 & chr20 \\
\hline |KIAA0895 & 23366 & KIAA0895 protein & 2,17 & $\operatorname{chr} 7$ \\
\hline |KIAA0934 & 22982 & KIAA0934 & 3,36 & chr10 \\
\hline |KIAA0960 & 23249 & |KIAA0960 protein & 2,17 & chr7 \\
\hline |KIAA0992 & 23022 & palladin & 8,10 & chr4 \\
\hline |KIAA1043 & 23331 & KIAA1043 protein & 4,72 & chr22 \\
\hline |KIAA1102 & 22998 & KIAA1102 protein & 4,18 & chr4 \\
\hline |KIAA1128 & 54462 & KIAA1128 & 2,94 & chr10 \\
\hline |KIAA1217 & 56243 & |KIAA1217 & 3,05 & chr10 \\
\hline |KIAA1276 & 27146 & KIAA1276 protein & 2,63 & chr4 \\
\hline |KIAA1434 & 56261 & hypothetical protein KIAA1434 & 2,54 & chr20 \\
\hline |KIAA1447 & 57597 & KIAA1447 protein & 4,03 & chr17 \\
\hline |KIAA1524 & 57650 & KIAA1524 & 2,62 & chr3 \\
\hline |KIAA1530 & 57654 & KIAA1530 protein & 3,20 & chr4 \\
\hline |KIAA1545 & 57666 & KIAA1545 protein & 2,26 & chr12 \\
\hline |KIAA1546 & 57667 & KIAA1546 protein & 3,62 & chr4 \\
\hline |KIAA1641 & 57730 & |KIAA1641 & 5,13 & (vide) \\
\hline |KIAA1704 & 55425 & |KIAA1704 & 2,99 & chr13 \\
\hline |KIAA1713 & 80816 & KIAA1713 & 6,29 & chr18 \\
\hline |KIAA1729 & 85460 & KIAA1729 protein & 10,42 & chr4 \\
\hline |KIAA1772 & 80000 & |KIAA1772 & 7,10 & $\operatorname{chr} 18$ \\
\hline |KIAA1838 & 84498 & KIAA1838 & 3,14 & chr6 \\
\hline |KIAA1841 & 84542 & |KIAA1841 protein & 2,92 & chr2 \\
\hline |KIAA1856 & 84629 & KIAA1856 protein & 2,07 & chr7 \\
\hline |KIAA1909 & 153478 & |KIAA1909 protein & 2,27 & chr5 \\
\hline |KIAA1919 & 91749 & |KIAA1919 & 2,27 & chr6 \\
\hline |KIAA2022 & 340533 & |KIAA2022 protein & 2,43 & $\operatorname{chr} X$ \\
\hline KIDINS220 & 57498 & Kinase D-interacting substance of $220 \mathrm{kDa}$ & 2,05 & chr2 \\
\hline KIF3A & 11127 & kinesin family member $3 \mathrm{~A}$ & 2,04 & chr5 \\
\hline KIF5C & 3800 & Kinesin family member $5 \mathrm{C}$ & 2,34 & chr2 \\
\hline |KIFAP3 & 22920 & kinesin-associated protein 3 & 2,78 & chr1 \\
\hline KIRREL & 55243 & kin of IRRE like (Drosophila) & 3,89 & chr1 \\
\hline |KLF11 & 8462 & Kruppel-like factor 11 & 2,85 & chr2 \\
\hline KLF12 & 11278 & Kruppel-like factor 12 & 2,12 & chr13 \\
\hline KLF3 & 51274 & Kruppel-like factor 3 (basic) & 3,38 & chr4 \\
\hline KLF6 & 1316 & Kruppel-like factor 6 & 3,24 & chr10 \\
\hline KLHDC5 & 57542 & kelch domain containing 5 & 2,70 & chr12 \\
\hline KLHDC8A & 55220 & Kelch domain containing $8 \mathrm{~A}$ & 9,89 & chr1 \\
\hline KLHL14 & 57565 & kelch-like 14 (Drosophila) & 7,04 & chr18 \\
\hline KLHL22 & 84861 & kelch-like 22 (Drosophila) & 2,78 & chr22 \\
\hline |KLHL24 & 54800 & |kelch-like 24 (Drosophila) & 2,13 & chr3 \\
\hline KLHL5 & 51088 & Kelch-like 5 (Drosophila) & 2,82 & chr4 \\
\hline KLHL8 & 57563 & kelch-like 8 (Drosophila) & 3,15 & chr4 \\
\hline |KPNA1 & 3836 & karyopherin alpha 1 (importin alpha 5) & 2,19 & chr3 \\
\hline KPNB1 & 3837 & Karyopherin (importin) beta 1 & 2,02 & chr17 \\
\hline |L3MBTL & 26013 & |(3)mbt-like (Drosophila) & 2,49 & chr20 \\
\hline L3MBTL3 & 84456 & |(3)mbt-like 3 (Drosophila) & 2,09 & chr6 \\
\hline LAMA1 & 284217 & laminin, alpha 1 & 2,36 & chr18 \\
\hline LAMB2 & 3913 & laminin, beta 2 (laminin S) & 2,96 & chr3 \\
\hline |LASS5 & 91012 & LAG1 longevity assurance homolog 5 (S. cerevisiae) & 2,08 & chr12 \\
\hline |LASS6 & 253782 & LAG1 longevity assurance homolog 6 (S. cerevisiae) & 2,60 & chr2 \\
\hline LDOC1L & 84247 & leucine zipper, down-regulated in cancer 1 -like & 2,23 & chr22 \\
\hline AP-2 & 6842 & liver-expressed antimicrobial peptide 2 & 3 & chr5 \\
\hline
\end{tabular}


Table S2 : Genes overexpressed in NPC compared to hES (Fold Change $>2 ; \alpha<0.05$ )

\begin{tabular}{|c|c|c|c|c|}
\hline Gene Symbol & Entrez Gene & Gene Title & NPC_up & Chromosome Number(Avadis) \\
\hline LEF1 & 51176 & lymphoid enhancer-binding factor 1 & 12,44 & chr4 \\
\hline LEMD1 & 93273 & LEM domain containing 1 & 3,95 & chr1 \\
\hline LEPR & 3953 & Leptin receptor & 4,22 & chr1 \\
\hline LEPROT & 54741 & leptin receptor overlapping transcript & 2,90 & chr1 \\
\hline LFNG & 3955 & lunatic fringe homolog (Drosophila) & 2,74 & chr7 \\
\hline LGALS8 & 3964 & lectin, galactoside-binding, soluble, 8 (galectin 8) & 3,87 & chr1 \\
\hline LGl1 & 9211 & leucine-rich, glioma inactivated 1 & 8,42 & chr10 \\
\hline LGR5 & 8549 & leucine-rich repeat-containing G protein-coupled receptor 5 & 6,45 & chr12 \\
\hline LHCGR & 3973 & Luteinizing hormone/choriogonadotropin receptor & 4,91 & chr2 \\
\hline LHX2 & 9355 & LIM homeobox 2 & 135,71 & chr9 \\
\hline LHX9 & 56956 & LIM homeobox 9 & 3,81 & chr1 \\
\hline LIX1 & 167410 & Lix1 homolog (mouse) & 24,29 & chr5 \\
\hline LIX1L & 128077 & Lix1 homolog (mouse) like & 6,85 & chr1 \\
\hline LKAP & 9665 & limkain b1 & 2,03 & chr16 \\
\hline LMO2 & 4005 & LIM domain only 2 (rhombotin-like 1) & 2,35 & chr11 \\
\hline LMO3 & 55885 & LIM domain only 3 (rhombotin-like 2) & 26,62 & chr12 \\
\hline LNPEP & 4012 & leucyl/cystinyl aminopeptidase & 2,30 & chr5 \\
\hline LOC112476 & 112476 & similar to lymphocyte antigen 6 complex, locus G5B; G5b protein; open reading frame 3 & 4,16 & chr16 \\
\hline LOC113386 & 113386 & similar to envelope protein & 2,25 & $\operatorname{chr} 19$ \\
\hline LOC115648 & 115648 & similar to hypothetical protein FLJ13659 & 3,51 & chr19 \\
\hline LOC115648 & $48203 / / / 2844$ & similar to hypothetical protein FLJ13659 /// hypothetical protein LOC148203 /// zinc finge & 2,01 & chr19 \\
\hline LOC132241 & 132241 & hypothetical protein LOC132241 & 2,86 & chr3 \\
\hline LOC143381 & 143381 & hypothetical protein LOC143381 & 16,72 & chr10 \\
\hline LOC144997 & 144997 & hypothetical protein LOC144997 & 7,95 & $\operatorname{chr} 13$ \\
\hline LOC145786 & 145786 & hypothetical protein LOC145786 & 72,07 & chr15 \\
\hline LOC147670 & 147670 & hypothetical protein LOC147670 & 2,25 & (vide) \\
\hline LOC148898 & 148898 & hypothetical protein BC007899 & 6,09 & chr1 \\
\hline LOC150759 & 150759 & hypothetical protein LOC150759 & 4,35 & chr2 \\
\hline LOC151194 & 151194 & similar to hepatocellular carcinoma-associated antigen HCA557b & 2,43 & chr2 \\
\hline LOC153222 & 153222 & adult retina protein & 2,68 & chr5 \\
\hline LOC153561 & 153561 & Hypothetical protein LOC153561 & 4,43 & chr5 \\
\hline LOC153682 & 153682 & Hypothetical protein LOC153682 & 2,28 & chr5 \\
\hline LOC158563 & 158563 & hypothetical protein LOC158563 & 3,15 & $\operatorname{chrX}$ \\
\hline LOC200030 & p0030 /// 40078 & hypothetical protein LOC200030 /// hypothetical LOC400781 & 4,14 & chr1 \\
\hline LOC200030 & 200030 & hypothetical protein LOC200030 & 2,48 & chr1 \\
\hline LOC220930 & 220930 & hypothetical protein LOC220930 & 3,37 & chr10 \\
\hline LOC221362 & 221362 & hypothetical protein LOC221362 & 2,68 & chr6 \\
\hline LOC221981 & 221981 & hypothetical protein LOC221981 & 2,99 & chr7 \\
\hline LOC255326 & 255326 & hypothetical protein LOC255326 & 2,64 & chr10 \\
\hline LOC283130 & 283130 & Hypothetical protein LOC283130 & 4,59 & chr11 \\
\hline LOC283464 & 283464 & hypothetical protein LOC283464 & 2,56 & chr12 \\
\hline LOC283481 & 283481 & hypothetical protein LOC283481 & 2,87 & chr13 \\
\hline LOC283508 & 283508 & hypothetical protein LOC283508 & 3,97 & chr13 \\
\hline LOC283677 & 283677 & hypothetical LOC283677 & 2,56 & chr4 \\
\hline LOC284244 & 284244 & hypothetical protein LOC284244 & 2,93 & chr18 \\
\hline LOC284262 & 284262 & hypothetical protein LOC284262 & 3,78 & chr18 \\
\hline LOC284356 & 284356 & hypothetical protein LOC284356 & 3,97 & chr19 \\
\hline LOC284409 & 284409 & hypothetical LOC284409 & 2,65 & chr19 \\
\hline LOC285382 & 285382 & hypothetical gene supported by AK091454 & 2,98 & chr3 \\
\hline LOC285431 & 285431 & hypothetical protein LOC285431 & 2,27 & chr4 \\
\hline LOC285535 & 285535 & hypothetical protein LOC285535 & 3,18 & chr4 \\
\hline LOC285831 & 285831 & hypothetical protein LOC285831 & 3,15 & chr6 \\
\hline LOC285989 & 285989 & hypothetical protein LOC285989 & 2,26 & chr7 \\
\hline LOC286052 & 286052 & hypothetical protein LOC286052 & 2,19 & chr8 \\
\hline LOC286170 & 286170 & hypothetical protein LOC286170 & 2,11 & chr8 \\
\hline LOC286334 & 286334 & Hypothetical protein LOC286334 & 2,61 & chr9 \\
\hline LOC286382 & 286382 & hypothetical protein LOC286382 & 2,95 & chr9 \\
\hline LOC338758 & 338758 & hypothetical protein LOC338758 & 3,78 & chr12 \\
\hline LOC339025 & 339025 & Hypothetical LOC339025 & 2,12 & chr15 \\
\hline LOC339287 & 339287 & hypothetical protein LOC339287 & 2,25 & chr17 \\
\hline LOC340281 & 340281 & hypothetical protein LOC340281 & 2,55 & chr7 \\
\hline LOC346355 & 46355 /// 3926 & similar to RIKEN cDNA A930017N06 gene /// similar to RIKEN cDNA A930017N06 gen & 2,25 & chr7 \\
\hline LOC347475 & 347475 & hypothetical gene supported by BC017958 & 2,57 & $\operatorname{chrX}$ \\
\hline LOC348094 & 348094 & hypothetical protein LOC348094 & 2,40 & chr15 \\
\hline LOC387758 & 387758 & similar to RIKEN cDNA 1110018M03 & 6,45 & chr11 \\
\hline LOC387790 & 387790 & Hypothetical LOC387790 & 3,05 & chr11 \\
\hline LOC387978 & 387978 & hypothetical gene supported by BX248251 & 2,08 & chr14 \\
\hline LOC388279 & 388279 & Hypothetical gene supported by AF275804 & 2,54 & chr16 \\
\hline
\end{tabular}




\begin{tabular}{|c|c|c|c|c|}
\hline Gene Symbol & Entrez Gene & Gene Title & NPC_up & Chromosome Number(Avadis) \\
\hline LOC388620 & 388620 & Similar to implantation-associated protein & 2,64 & chr1 \\
\hline LOC388889 & B8889 /// 4408 & Hypothetical LOC388889 /// Hypothetical protein LOC150271 & 3,20 & chr22 \\
\hline LOC388969 & 388969 & Hypothetical LOC388969 & 2,57 & chr2 \\
\hline LOC389295 & 389295 & Hypothetical protein LOC153561 & 8,12 & chr5 \\
\hline LOC389432 & 389432 & SAM domain containing 1 & 3,76 & chr6 \\
\hline LOC389765 & 389765 & similar to KIF27C & 2,91 & chr9 \\
\hline LOC390299 & 390299 & Tetraspanin 11 & 3,03 & chr12 \\
\hline LOC390551 & p0551/// 44023 & similar to hect domain and RLD 2 /// similar to hect domain and RLD 2 & 2,49 & chr15_random \\
\hline LOC391269 & 391269 & Similar to ankyrin repeat domain $20 \mathrm{~A}$ & 2,23 & chr21 \\
\hline LOC391491 & 391491 & Similar to guanidinoacetate methyltransferase; GAMT & 2,74 & chr2 \\
\hline LOC399763 & 399763 & similar to LINE-1 reverse transcriptase homolog & 3,22 & chr10 \\
\hline LOC399959 & 399959 & Hypothetical gene supported by BX647608 & 5,79 & chr11 \\
\hline LOC400043 & 400043 & hypothetical gene supported by BC009385 & 6,24 & chr12 \\
\hline LOC400642 & 400642 & hypothetical gene supported by BC041875; BX648984 & 2,53 & chr18 \\
\hline LOC400685 & 400685 & Hypothetical gene supported by BC045806 & 2,59 & chr19 \\
\hline LOC400960 & 400960 & hypothetical gene supported by BC040598 & 2,23 & chr2 \\
\hline LOC401394 & p1394 /// 4025 & hypothetical LOC401394 /// hypothetical LOC402578 & 3,67 & chr7 \\
\hline LOC401528 & $\beta / / / 401530 / / /$ & hypothetical gene supported by BC032955 /// hypothetical gene supported by BC03295 & 5,57 & chr9 \\
\hline LOC402485 & 402485 & Hypothetical LOC401328 & 6,86 & chr7 \\
\hline LOC402530 & p1363 /// 40253 & Hypothetical protein FLJ25037 /// Hypothetical LOC441242 & 2,48 & chr11 \\
\hline LOC402560 & 402560 & Hypothetical LOC401384 & 4,04 & chr7 \\
\hline LOC439994 & 439994 & hypothetical gene supported by AF064843; AK025716 & 2,22 & chr2 \\
\hline LOC440135 & 440135 & LOC440135 & 2,22 & chr13 \\
\hline LOC440282 & 440282 & Hypothetical protein LOC145783 & 3,62 & chr15 \\
\hline LOC440460 & 440460 & SH3-domain GRB2-like pseudogene 3 & 2,03 & chr17 \\
\hline LOC440526 & 440526 & LOC440526 & 2,16 & chr19 \\
\hline LOC440934 & 440934 & Hypothetical gene supported by BC008048 & 7,62 & chr2 \\
\hline LOC440944 & 440944 & Hypothetical gene supported by AK128398 & 2,50 & chr3 \\
\hline LOC440995 & 440995 & hypothetical gene supported by BC034933; BC068085 & 2,09 & chr3 \\
\hline LOC440996 & 440996 & Hypothetical gene supported by BC053580 & 3,68 & chr5 \\
\hline LOC441022 & 441022 & similar to RUN and FYVE domain-containing 2; Run- and FYVE-domain containing prote & 3,04 & chr4 \\
\hline LOC441241 & $41241 / / / 4412$ & chaperonin containing TCP1, subunit $6 \mathrm{~A}$ (zeta 1 )-like /// chaperonin containing TCP1, su & 2,57 & chr7 \\
\hline LOC441300 & 441300 & LOC441300 & 2,83 & chr7 \\
\hline LOC441351 & 441351 & Hypothetical gene supported by BX537900 & 4,46 & chr8 \\
\hline LOC441428 & 441428 & Hypothetical gene supported by BX641014 & 2,38 & chr9 \\
\hline LOC492304 & 492304 & putative insulin-like growth factor II associated protein & 16,83 & chr11 \\
\hline LOC494141 & 494141 & Similar to mitochondrial carrier triple repeat 1 & 2,36 & chr5 \\
\hline LOC56757 & 56757 & hypothetical protein LOC56757 & 3,05 & chr5 \\
\hline LOC641522 & 641522 & ADP-ribosylation factor-like 17 pseudogene 1 & 2,01 & chr17 \\
\hline LOC90110 & 90110 & hypothetical protein LOC90110 & 2,99 & chr17 \\
\hline LOC90693 & 90693 & LOC90693 protein & 2,51 & chr7 \\
\hline LOC91137 & 91137 & Hypothetical protein BC017169 & 3,24 & chr5 \\
\hline LOC91316 & 91316 & Similar to bK246H3.1 (immunoglobulin lambda-like polypeptide 1, pre-B-cell specific) & 7,34 & chr5 \\
\hline LOC91461 & 91461 & hypothetical protein BC007901 & 5,60 & chr2 \\
\hline LOC92162 & 92162 & similar to RIKEN cDNA $2600017 \mathrm{H} 02$ & 6,69 & chr17 \\
\hline LOC92312 & 92312 & Hypothetical protein LOC92312 & 2,58 & chr1 \\
\hline LOC92691 & 92691 & hypothetical protein BC008604 & 7,23 & chr2 \\
\hline LOC94431 & 94431 & similar to RNA polymerase I transcription factor RRN3 & 2,77 & chr16 \\
\hline LONPL & 83752 & Peroxisomal LON protease like & 4,46 & chr16 \\
\hline LOXL1 & 4016 & lysyl oxidase-like 1 & 2,84 & chr15 \\
\hline LOXL2 & 4017 & lysyl oxidase-like 2 & 3,76 & chr8 \\
\hline LPGAT1 & 9926 & Lysophosphatidylglycerol acyltransferase 1 & 2,33 & chr1 \\
\hline LPHN3 & 23284 & latrophilin 3 & 6,67 & chr4 \\
\hline LPIN1 & 23175 & lipin 1 & 5,55 & chr2 \\
\hline LPIN2 & 9663 & lipin 2 & 2,31 & chr18 \\
\hline LPL & 4023 & lipoprotein lipase & 3,81 & chr8 \\
\hline $\mathrm{LRCH} 2$ & 57631 & leucine-rich repeats and calponin homology $(\mathrm{CH})$ domain containing 2 & 2,07 & $\operatorname{chr} X$ \\
\hline $\mathrm{LRCH} 3$ & 84859 & leucine-rich repeats and calponin homology $(\mathrm{CH})$ domain containing 3 & 3,56 & chr3 \\
\hline LRP12 & 29967 & low density lipoprotein-related protein 12 & 2,62 & chr8 \\
\hline LRP2 & 4036 & Low density lipoprotein-related protein 2 & 21,79 & chr2 \\
\hline LRP4 & 4038 & low density lipoprotein receptor-related protein 4 & 3,17 & chr11 \\
\hline LRPAP1 & 4043 & Low density lipoprotein receptor-related protein associated protein 1 & 3,71 & chr4 \\
\hline LRRC17 & 10234 & leucine rich repeat containing 17 & 5,05 & chr7 \\
\hline LRRC3B & 116135 & leucine rich repeat containing $3 \mathrm{~B}$ & 2,35 & chr3 \\
\hline LRRC49 & 54839 & leucine rich repeat containing 49 & 2,20 & chr15 \\
\hline LRRC4C & 57689 & leucine rich repeat containing $4 \mathrm{C}$ & 3,58 & chr11 \\
\hline LRRN3 & 54674 & leucine rich repeat neuronal 3 & 6,49 & chr7 \\
\hline LSAMP & 4045 & limbic system-associated membrane protein & 2,27 & chr3 \\
\hline
\end{tabular}




\begin{tabular}{|c|c|c|c|c|}
\hline Gene Symbol & Entrez Gene & Gene Title & NPC_up & Chromosome Number(Avadis) \\
\hline LSM8 & 51691 & LSM8 homolog, U6 small nuclear RNA associated (S. cerevisiae) & 2,69 & chr7 \\
\hline LTBP1 & 4052 & latent transforming growth factor beta binding protein 1 & 3,84 & chr2 \\
\hline LUM & 4060 & lumican & 16,09 & chr12 \\
\hline LZIC & 84328 & Leucine zipper and CTNNBIP1 domain containing & 2,15 & chr1 \\
\hline LZTFL1 & 54585 & leucine zipper transcription factor-like 1 & 2,74 & chr3 \\
\hline MAB21L1 & 4081 & mab-21-like 1 (C. elegans) & 5,33 & chr13 \\
\hline MAB21L2 & 10586 & mab-21-like 2 (C. elegans) & 11,53 & chr4 \\
\hline MAF & 4094 & v-maf musculoaponeurotic fibrosarcoma oncogene homolog (avian) & 6,36 & chr16 \\
\hline MAGEH1 & 28986 & melanoma antigen family $\mathrm{H}, 1$ & 2,63 & $\operatorname{chr} X$ \\
\hline MAGI3 & 260425 & membrane associated guanylate kinase, WW and PDZ domain containing 3 & 2,96 & chr1 \\
\hline MALAT1 & 378938 & metastasis associated lung adenocarcinoma transcript 1 (non-coding RNA) & 7,76 & chr11 \\
\hline MAML2 & 84441 & Mastermind-like 2 (Drosophila) & 2,20 & chr11 \\
\hline MAML3 & 55534 & Mastermind-like 3 (Drosophila) & 2,68 & chr4 \\
\hline MAN1A1 & 4121 & Mannosidase, alpha, class $1 \mathrm{~A}$, member 1 & 4,33 & chr6 \\
\hline MAN1A2 & 10905 & Mannosidase, alpha, class $1 \mathrm{~A}$, member 2 & 2,49 & chr1 \\
\hline MAN2A2 & 4122 & mannosidase, alpha, class 2A, member 2 & 2,72 & chr15 \\
\hline MAP2 & 4133 & Microtubule-associated protein 2 & 25,86 & chr2 \\
\hline MAP2K1IP1 & 8649 & Mitogen-activated protein kinase kinase 1 interacting protein 1 & 2,40 & chr4 \\
\hline MAP3K1 & 4214 & Mitogen-activated protein kinase kinase kinase 1 & 2,42 & chr5 \\
\hline MAP6 & 4135 & microtubule-associated protein 6 & 4,48 & chr11 \\
\hline MAPK10 & 5602 & mitogen-activated protein kinase 10 & 9,24 & chr4 \\
\hline MARCH6 & 10299 & membrane-associated ring finger (C3HC4) 6 & 2,16 & chr5 \\
\hline MARCKS & 4082 & Myristoylated alanine-rich protein kinase $\mathrm{C}$ substrate & 2,91 & chr6 \\
\hline MASP2 & 10747 & Mannan-binding lectin serine peptidase 2 & 6,08 & chr1 \\
\hline MAST4 & 23227 & microtubule associated serine/threonine kinase family member 4 & 2,90 & chr5 \\
\hline MBD2 & 8932 & methyl-CpG binding domain protein 2 & 3,34 & chr18 \\
\hline MBNL2 & 10150 & Muscleblind-like 2 (Drosophila) & 9,49 & chr13 \\
\hline MCART6 & 401612 & Mitochondrial carrier triple repeat 6 & 3,04 & $\operatorname{chr} X$ \\
\hline MCF2L & 23263 & MCF.2 cell line derived transforming sequence-like & 3,95 & chr13 \\
\hline MDC1 & 9656 & mediator of DNA damage checkpoint 1 & 2,30 & chr6 \\
\hline MDFIC & 29969 & MyoD family inhibitor domain containing /// MyoD family inhibitor domain containing & 3,14 & chr7 \\
\hline MDM4 & 4194 & Mdm4, transformed 3T3 cell double minute 4, p53 binding protein (mouse) & 2,66 & chr1 \\
\hline ME3 & 10873 & malic enzyme 3, NADP(+)-dependent, mitochondrial & 8,66 & chr11 \\
\hline MECP2 & 4204 & methyl $\mathrm{CpG}$ binding protein 2 (Rett syndrome) & 2,09 & $\operatorname{chr} \mathrm{X}$ \\
\hline MED6 & 10001 & mediator of RNA polymerase II transcription, subunit 6 homolog (yeast) & 2,15 & chr14 \\
\hline MEF2C & 4208 & MADS box transcription enhancer factor 2, polypeptide $\mathrm{C}$ (myocyte enhancer factor $2 \mathrm{C}$ ) & 5,04 & chr5 \\
\hline MEIS1 & 4211 & Meis1, myeloid ecotropic viral integration site 1 homolog (mouse) & 8,07 & chr2 \\
\hline MEIS2 & 4212 & Meis1, myeloid ecotropic viral integration site 1 homolog 2 (mouse) & 31,73 & chr15 \\
\hline METAP2 & 10988 & Methionyl aminopeptidase 2 & 2,94 & chr12 \\
\hline MGAT3 & 4248 & Mannosyl (beta-1,4-)-glycoprotein beta-1,4-N-acetylglucosaminyltransferase & 2,44 & chr22 \\
\hline MGC10850 & 84736 & hypothetical protein MGC10850 & 2,25 & chr11 \\
\hline MGC10854 & 84260 & trichoplein & 3,82 & chr12 \\
\hline MGC12760 & 84809 & hypothetical protein MGC12760 /// hypothetical protein MGC12760 & 3,31 & chr1 \\
\hline MGC13057 & 84281 & Hypothetical protein MGC13057 & 2,58 & chr2 \\
\hline MGC15407 & 112942 & Similar to RIKEN cDNA 4931428D14 gene & 2,54 & chr2 \\
\hline MGC15875 & 85007 & hypothetical protein MGC15875 & 2,82 & chr5 \\
\hline MGC17839 & 219902 & hypothetical protein MGC17839 & 2,07 & chr11 \\
\hline MGC19764 & 162394 & likely ortholog of mouse schlafen 5 & 2,22 & chr17 \\
\hline MGC21644 & 153768 & Hypothetical protein MGC21644 & 2,08 & chr5 \\
\hline MGC22265 & 349035 & (clone CB1) mRNA fragment /// Hypothetical protein MGC22265 & 2,37 & chr5 \\
\hline MGC24039 & 160518 & hypothetical protein MGC24039 & 3,90 & chr12 \\
\hline MGC25181 & 257054 & hypothetical protein MGC25181 & 2,26 & chr2_random \\
\hline MGC2752 & 65996 & hypothetical protein MGC2752 & 2,12 & chr19 \\
\hline MGC2803 & 79002 & hypothetical protein MGC2803 & 2,14 & chr19 \\
\hline MGC3032 & 65998 & hypothetical protein MGC3032 & 2,54 & chr11 \\
\hline MGC3121 & 78994 & hypothetical protein MGC3121 & 2,11 & chr16 \\
\hline MGC33212 & 255758 & hypothetical protein MGC33212 & 2,44 & chr3 \\
\hline MGC33302 & 256471 & Hypothetical protein MGC33302 & 2,33 & chr4 \\
\hline MGC33926 & 130733 & hypothetical protein MGC33926 & 3,27 & chr2 \\
\hline MGC35048 & 124152 & hypothetical protein MGC35048 & 2,96 & chr16 \\
\hline MGC35097 & 200942 & hypothetical protein MGC35097 & 3,06 & chr3 \\
\hline MGC35366 & 144193 & hypothetical protein MGC35366 & 2,52 & chr12 \\
\hline MGC39900 & 286527 & hypothetical protein MGC39900 & 7,63 & $\operatorname{chrX}$ \\
\hline MGC40499 & 245812 & hypothetical protein MGC40499 & 2,01 & chr7 \\
\hline MGC4707 & 79096 & MGC4707 protein & 2,40 & chr11 \\
\hline MGC52110 & 493753 & hypothetical protein MGC52110 & 2,02 & chr2 \\
\hline MGC5509 & 79074 & Hypothetical protein MGC5509 & 3,05 & chr2 \\
\hline MGC5576 & 79022 & hypothetical protein MGC5576 & 2,35 & chr12 \\
\hline
\end{tabular}




\begin{tabular}{|c|c|c|c|c|}
\hline Gene Symbol & Entrez Gene & Gene Title & NPC_up & Chromosome Number(Avadis) \\
\hline MGEA5 & 10724 & meningioma expressed antigen 5 (hyaluronidase) & 3,74 & chr10 \\
\hline MID1 & 4281 & Midline 1 (Opitz/BBB syndrome) & 2,52 & $\operatorname{chr} \mathrm{X}$ \\
\hline MITF & 4286 & microphthalmia-associated transcription factor & 6,58 & chr3 \\
\hline MKL2 & 57496 & MKL/myocardin-like 2 & 2,04 & chr16 \\
\hline MLL3 & 58508 & myeloid/lymphoid or mixed-lineage leukemia 3 & 2,24 & chr7 \\
\hline MLLT10 & 8028 & myeloid/lymphoid or mixed-lineage leukemia (trithorax homolog, Drosophila); translocate & 2,59 & chr10 \\
\hline MLLT3 & 4300 & Myeloid/lymphoid or mixed-lineage leukemia (trithorax homolog, Drosophila); translocate & 2,02 & chr9 \\
\hline MLLT4 & 4301 & myeloid/lymphoid or mixed-lineage leukemia (trithorax homolog, Drosophila); translocate & 4,22 & chr6 \\
\hline |MLLT6 & 4302 & myeloid/lymphoid or mixed-lineage leukemia (trithorax homolog, Drosophila); translocatf & 2,45 & chr17 \\
\hline MLR1 & 254251 & transcription factor MLR1 & 2,49 & chr4 \\
\hline MLR2 & 84458 & ligand-dependent corepressor & 2,87 & chr10 \\
\hline MMRN1 & 22915 & multimerin 1 & 2,24 & chr4 \\
\hline MN1 & 4330 & meningioma (disrupted in balanced translocation) 1 & 3,06 & chr22 \\
\hline MOBKL2B & 79817 & MOB1, Mps One Binder kinase activator-like 2B (yeast) & 2,08 & chr9 \\
\hline MON2 & 23041 & MON2 homolog (yeast) & 2,64 & chr12 \\
\hline MORC4 & 79710 & MORC family $\mathrm{CW}$-type zinc finger 4 & 4,44 & $\operatorname{chrX}$ \\
\hline MORF4L2 & 9643 & Mortality factor 4 like 2 & 2,04 & $\operatorname{chr} X$ \\
\hline MOXD1 & 26002 & monooxygenase, DBH-like 1 & 4,91 & chr6 \\
\hline MPHOSPH9 & 10198 & M-phase phosphoprotein 9 & 2,36 & chr12 \\
\hline MPZL1 & 9019 & myelin protein zero-like 1 & 2,71 & chr1 \\
\hline MRC2 & 9902 & mannose receptor, C type 2 & 3,84 & chr17 \\
\hline MRPS22 & 56945 & Mitochondrial ribosomal protein S22 & 2,80 & chr3 \\
\hline MRPS6 & 64968 & Mitochondrial ribosomal protein S6 & 2,84 & chr21 \\
\hline MSH5 & 4439 & mutS homolog 5 (E. coli) & 2,77 & chr6 \\
\hline MSRB2 & 22921 & methionine sulfoxide reductase B2 & 2,21 & $\operatorname{chr} 10$ \\
\hline MSRB3 & 253827 & methionine sulfoxide reductase B3 & 9,04 & chr12 \\
\hline MST1 & 4485 & macrophage stimulating 1 (hepatocyte growth factor-like) & 2,15 & chr1 \\
\hline MSX1 & 4487 & msh homeo box homolog 1 (Drosophila) & 7,61 & chr4 \\
\hline MSX2 & 4488 & msh homeo box homolog 2 (Drosophila) & 3,02 & chr5 \\
\hline MTCH2 & 23788 & mitochondrial carrier homolog 2 (C. elegans) & 2,17 & chr11 \\
\hline MTDH & 92140 & Metadherin & 2,02 & chr8 \\
\hline MTERFD2 & 130916 & MTERF domain containing 2 & 2,25 & chr2 \\
\hline MTERFD3 & 80298 & MTERF domain containing 3 & 2,20 & chr12 \\
\hline MUM1L1 & 139221 & melanoma associated antigen (mutated) 1-like 1 & 3,66 & $\operatorname{chrX}$ \\
\hline MUSTN1 & 389125 & musculoskeletal, embryonic nuclear protein 1 & 2,37 & chr3 \\
\hline MXD1 & 4084 & MAX dimerization protein 1 & 2,79 & chr2 \\
\hline MXD4 & 10608 & MAX dimerization protein 4 & 3,11 & chr4 \\
\hline MXI1 & 4601 & MAX interactor 1 /// MAX interactor 1 & 2,39 & chr10 \\
\hline MXRA5 & 25878 & matrix-remodelling associated 5 & 4,01 & $\operatorname{chr} X$ \\
\hline MXRA7 & 439921 & matrix-remodelling associated 7 & 2,38 & chr17 \\
\hline MXRA8 & 54587 & matrix-remodelling associated 8 & 3,26 & chr1_random \\
\hline MYADM & 91663 & myeloid-associated differentiation marker & 2,11 & chr19 \\
\hline MYBL1 & 4603 & v-myb myeloblastosis viral oncogene homolog (avian)-like 1 & 2,23 & chr8 \\
\hline MYEF2 & 50804 & myelin expression factor 2 & 2,04 & chr15 \\
\hline MYLK & 4638 & myosin, light polypeptide kinase /// myosin, light polypeptide kinase & 4,39 & chr6 \\
\hline MYST3 & 7994 & MYST histone acetyltransferase (monocytic leukemia) 3 & 2,94 & chr8 \\
\hline NAALAD2 & 10003 & $\mathrm{~N}$-acetylated alpha-linked acidic dipeptidase 2 & 2,66 & chr11 \\
\hline NAB1 & 4664 & NGFI-A binding protein 1 (EGR1 binding protein 1) & 2,65 & chr2 \\
\hline NAB2 & 4665 & NGFI-A binding protein 2 (EGR1 binding protein 2) & 2,01 & chr12 \\
\hline NAG6 & 64753 & hypothetical protein DKFZp434G156 & 2,52 & chr7 \\
\hline NALP1 & 22861 & $\mathrm{NACHT}$, leucine rich repeat and PYD (pyrin domain) containing 1 & 5,90 & chr17 \\
\hline NAPE-PLD & 222236 & N-acyl-phosphatidylethanolamine-hydrolyzing phospholipase D & 2,16 & chr7 \\
\hline NASP & 4678 & Nuclear autoantigenic sperm protein (histone-binding) & 2,41 & chr1 \\
\hline NAV1 & 89796 & neuron navigator 1 & 12,31 & chr1 \\
\hline NAV3 & 89795 & neuron navigator 3 & 5,50 & chr12 \\
\hline NBEA & 26960 & neurobeachin & 2,44 & chr13 \\
\hline NBLA04196 & 64921 & Putative protein product of Nbla04196 & 2,86 & chr7 \\
\hline NBPF8 & 641559 & Neuroblastoma breakpoint family, member 20 & 4,08 & chr1 \\
\hline NCALD & 83988 & neurocalcin delta /// neurocalcin delta & 10,89 & chr8 \\
\hline NCAM1 & 4684 & neural cell adhesion molecule 1 & 13,84 & chr11 \\
\hline NCOA5 & 57727 & Nuclear receptor coactivator 5 & 2,49 & chr20 \\
\hline NCOA6 & 23054 & nuclear receptor coactivator 6 & 2,51 & chr20 \\
\hline NCOR1 & 9611 & Nuclear receptor co-repressor 1 & 2,05 & chr17 \\
\hline NDN & 4692 & necdin homolog (mouse) & 2,01 & chr15 \\
\hline NDRG1 & 10397 & $\mathrm{~N}$-myc downstream regulated gene 1 & 2,51 & chr8 \\
\hline NEBL & 10529 & nebulette & 4,19 & chr10 \\
\hline NEDD9 & 4739 & neural precursor cell expressed, developmentally down-regulated 9 & 12,90 & chr6 \\
\hline K3 & 4752 & MA (never in mitosis gene a)-rel & 5 & $\operatorname{chr} 13$ \\
\hline
\end{tabular}




\begin{tabular}{|c|c|c|c|c|}
\hline Gene Symbol & Entrez Gene & Gene Title & NPC_up & Chromosome Number(Avadis) \\
\hline NEK9 & 91754 & NIMA (never in mitosis gene a)- related kinase 9 & 4,50 & chr14 \\
\hline NELL2 & 4753 & NEL-like 2 (chicken) /// NEL-like 2 (chicken) & 3,91 & chr12 \\
\hline NEO1 & 4756 & neogenin homolog 1 (chicken) & 2,57 & chr15 \\
\hline NEUROD1 & 4760 & neurogenic differentiation 1 & 2,50 & chr2 \\
\hline NEXN & 91624 & nexilin ( $\mathrm{F}$ actin binding protein) & 2,29 & chr1 \\
\hline NFAT5 & 10725 & nuclear factor of activated T-cells 5 , tonicity-responsive & 2,88 & chr16 \\
\hline NFATC1 & 4772 & nuclear factor of activated T-cells, cytoplasmic, calcineurin-dependent 1 & 2,57 & chr18 \\
\hline NFATC4 & 4776 & nuclear factor of activated T-cells, cytoplasmic, calcineurin-dependent 4 & 3,45 & chr14 \\
\hline NHLH2 & 4808 & nescient helix loop helix 2 & 2,79 & chr1 \\
\hline NHLRC2 & 374354 & NHL repeat containing 2 & 2,24 & chr10 \\
\hline NID1 & 4811 & nidogen 1 & 3,25 & chr1 \\
\hline NID2 & 22795 & nidogen 2 (osteonidogen) & 11,71 & chr14 \\
\hline NIN & 51199 & ninein (GSK3B interacting protein) & 6,35 & chr14 \\
\hline $\mathrm{NISCH}$ & 11188 & nischarin & 2,21 & chr3 \\
\hline NKTR & 4820 & natural killer-tumor recognition sequence & 2,73 & chr3 \\
\hline NLGN1 & 22871 & neuroligin 1 & 3,38 & chr3 \\
\hline NME5 & 8382 & non-metastatic cells 5, protein expressed in (nucleoside-diphosphate kinase) & 2,77 & chr5 \\
\hline NMT2 & 9397 & $\mathrm{~N}$-myristoyltransferase 2 & 2,22 & chr10 \\
\hline NOL7 & 51406 & Nucleolar protein $7,27 \mathrm{kDa}$ & 2,36 & chr6 \\
\hline NOPE & 57722 & likely ortholog of mouse neighbor of Punc E11 & 5,49 & chr15 \\
\hline $\mathrm{NOTCH} 1$ & 4851 & Notch homolog 1, translocation-associated (Drosophila) & 2,73 & chr9 \\
\hline NOTCH2 & 4853 & Notch homolog 2 (Drosophila) & 2,41 & chr1 \\
\hline $\mathrm{NOTCH} 2 \mathrm{NL}$ & 388677 & Notch homolog 2 (Drosophila) N-terminal like & 4,78 & chr1 \\
\hline NOVA1 & 4857 & neuro-oncological ventral antigen 1 & 2,89 & chr14 \\
\hline NOX4 & 50507 & NADPH oxidase 4 & 2,72 & chr11 \\
\hline NPHP3 & 27031 & nephronophthisis 3 (adolescent) & 2,91 & chr3 \\
\hline NPR3 & 4883 & natriuretic peptide receptor $\mathrm{C} /$ guanylate cyclase $\mathrm{C}$ (atrionatriuretic peptide receptor $\mathrm{C}$ ) & 3,70 & chr5 \\
\hline NPY2R & 4887 & neuropeptide $\mathrm{Y}$ receptor Y2 & 3,47 & chr4 \\
\hline NR2C2 & 7182 & Nuclear receptor subfamily 2 , group $\mathrm{C}$, member 2 & 2,19 & chr3 \\
\hline NR2E1 & 7101 & nuclear receptor subfamily 2 , group $E$, member 1 & 3,16 & chr6 \\
\hline $\mathrm{NR} 2 \mathrm{~F} 1$ & 7025 & Nuclear receptor subfamily 2 , group $F$, member 1 & 8,52 & chr5 \\
\hline NR2F2 & 7026 & nuclear receptor subfamily 2 , group $F$, member 2 & 11,84 & chr15 \\
\hline NRBP2 & 340371 & nuclear receptor binding protein 2 & 2,44 & chr8 \\
\hline NRCAM & 4897 & neuronal cell adhesion molecule & 8,28 & chr7 \\
\hline NRG1 & 3084 & neuregulin 1 & 10,33 & chr8 \\
\hline NRIP1 & 8204 & nuclear receptor interacting protein 1 & 7,91 & chr21 \\
\hline NRIP3 & 56675 & nuclear receptor interacting protein 3 & 2,04 & chr11 \\
\hline NRP1 & 8829 & neuropilin 1 & 3,18 & chr10 \\
\hline NRP2 & 8828 & Neuropilin 2 & 4,20 & chr2 \\
\hline NSUN6 & 221078 & NOL1/NOP2/Sun domain family, member 6 & 6,89 & chr10 \\
\hline NTF3 & 4908 & neurotrophin 3 & 2,80 & chr12 \\
\hline NTRK2 & 4915 & neurotrophic tyrosine kinase, receptor, type 2 & 2,92 & chr9 \\
\hline NUAK1 & 9891 & NUAK family, SNF1-like kinase, 1 & 3,58 & chr12 \\
\hline NUDT5 & 11164 & Nudix (nucleoside diphosphate linked moiety X)-type motif 5 & 2,43 & chr10 \\
\hline NUDT6 & 11162 & nudix (nucleoside diphosphate linked moiety X)-type motif 6 & 2,77 & chr4 \\
\hline NUPL1 & 9818 & Nucleoporin like 1 & 2,59 & chr13 \\
\hline OBSL1 & 23363 & obscurin-like 1 & 2,53 & chr2 \\
\hline ODZ2 & 57451 & odz, odd Oz/ten-m homolog 2 (Drosophila) & 5,57 & chr5 \\
\hline ODZ4 & 26011 & odz, odd Oz/ten-m homolog 4 (Drosophila) & 3,54 & chr11 \\
\hline OGT & 8473 & O-linked N-acetylglucosamine (GlcNAc) transferase (UDP-N-acetylglucosamine:polyper & 2,02 & $\operatorname{chr} X$ \\
\hline OIP106 & 22906 & OGT(O-Glc-NAc transferase)-interacting protein $106 \mathrm{KDa}$ & 2,06 & chr3 \\
\hline OLFML2A & 169611 & olfactomedin-like $2 \mathrm{~A}$ & 2,05 & chr9 \\
\hline OPN1SW & 611 & Opsin 1 (cone pigments), short-wave-sensitive (color blindness, tritan) & 2,27 & chr7 \\
\hline OPN3 & 23596 & opsin 3 (encephalopsin, panopsin) & 5,67 & chr1 \\
\hline OPTN & 10133 & optineurin & 2,42 & chr10 \\
\hline OSBPL5 & 114879 & oxysterol binding protein-like 5 & 2,79 & chr11 \\
\hline OSBPL9 & 114883 & Oxysterol binding protein-like 9 & 2,60 & chr1 \\
\hline OTX2 & 5015 & orthodenticle homolog 2 (Drosophila) & 2,24 & chr14 \\
\hline ovos2 & 144203 & ovostatin 2 & 2,70 & chr12 \\
\hline OXR1 & 55074 & oxidation resistance 1 & 2,28 & chr8 \\
\hline P18SRP & 285672 & p18 splicing regulatory protein & 2,20 & chr5 \\
\hline PABPN1 & 8106 & poly $(\mathrm{A})$ binding protein, nuclear 1 & 4,03 & chr14 \\
\hline PAFAH1B1 & 5048 & platelet-activating factor acetylhydrolase, isoform lb, alpha subunit $45 \mathrm{kDa}$ & 2,08 & chr17 \\
\hline PAG1 & 55824 & phosphoprotein associated with glycosphingolipid microdomains 1 & 13,80 & chr8 \\
\hline PAN3 & 255967 & PABP1-dependent poly A-specific ribonuclease subunit PAN3 & 2,12 & chr13 \\
\hline PAPD4 & 167153 & PAP associated domain containing 4 & 2,34 & chr5 \\
\hline PAPOLA & 10914 & poly(A) polymerase alpha & 2,22 & chr14 \\
\hline PAPPA & 5069 & pregnancy-associated plasma protein A, pappalysin 1 & 3,38 & chr9 \\
\hline
\end{tabular}




\begin{tabular}{|c|c|c|c|c|}
\hline Gene Symbol & Entrez Gene & Gene Title & NPC_up & Chromosome Number(Avadis) \\
\hline PAQR8 & 85315 & progestin and adipoQ receptor family member VIII & 2,76 & chr6 \\
\hline PARC & 23113 & p53-associated parkin-like cytoplasmic protein & 2,04 & chr6 \\
\hline PARD6G & 84552 & par-6 partitioning defective 6 homolog gamma (C. elegans) & 2,10 & chr18 \\
\hline PARP6 & 56965 & poly (ADP-ribose) polymerase family, member 6 & 2,49 & chr15 \\
\hline PARVA & 55742 & parvin, alpha & 3,62 & chr11 \\
\hline PAWR & 5074 & PRKC, apoptosis, WT1, regulator & 2,01 & chr20 \\
\hline PAX3 & 5077 & paired box gene 3 (Waardenburg syndrome 1 ) & 7,02 & chr2 \\
\hline PAX6 & 5080 & paired box gene 6 (aniridia, keratitis) & 92,78 & chr11 \\
\hline PCAF & 8850 & p300/CBP-associated factor & 3,29 & chr3 \\
\hline PCBP2 & 5094 & Poly $(\mathrm{rC})$ binding protein 2 & 2,28 & chr12 \\
\hline PCDH17 & 27253 & Protocadherin 17 & 6,86 & chr13 \\
\hline PCDH18 & 54510 & protocadherin 18 & 2,59 & chr4 \\
\hline PCDH9 & 5101 & protocadherin 9 & 8,49 & chr13 \\
\hline PCDHB10 & 56126 & protocadherin beta 10 & 3,63 & chr5 \\
\hline PCDHB14 & 56122 & protocadherin beta 14 & 2,58 & chr5 \\
\hline PCGF3 & 10336 & Polycomb group ring finger 3 & 2,57 & chr4 \\
\hline PCGF4 & 648 & polycomb group ring finger 4 & 2,51 & chr10 \\
\hline PCGF5 & 84333 & polycomb group ring finger 5 & 2,92 & chr10 \\
\hline PCM1 & 5108 & Pericentriolar material 1 & 2,91 & chr8 \\
\hline PCMTD1 & 115294 & Protein-L-isoaspartate (D-aspartate) O-methyltransferase domain containing 1 & 3,12 & chr8 \\
\hline PCSK5 & 5125 & Proprotein convertase subtilisin/kexin type 5 & 4,59 & chr9 \\
\hline PCTK2 & 5128 & PCTAIRE protein kinase 2 & 3,18 & chr12 \\
\hline PDCD4 & 27250 & programmed cell death 4 (neoplastic transformation inhibitor) & 2,77 & chr10 \\
\hline PDE7A & 5150 & phosphodiesterase 7A & 2,00 & chr8 \\
\hline PDGFC & 56034 & platelet derived growth factor C & 7,92 & chr4 \\
\hline PDGFRB & 5159 & platelet-derived growth factor receptor, beta polypeptide & 2,36 & chr5 \\
\hline PDZRN3 & 23024 & PDZ domain containing RING finger 3 & 3,06 & chr3 \\
\hline PEG10 & 23089 & paternally expressed 10 & 2,75 & chr7 \\
\hline PFAAP5 & 10443 & Hypothetical gene CG012 & 4,32 & chr13 \\
\hline PGAP1 & 80055 & GPI deacylase & 6,04 & chr2 \\
\hline PGM5 & 5239 & phosphoglucomutase 5 & 3,61 & chr9 \\
\hline PHC2 & 1912 & polyhomeotic-like 2 (Drosophila) & 11,78 & chr1 \\
\hline PHF10 & 55274 & PHD finger protein 10 & 2,31 & chr6 \\
\hline PHF2 & 5253 & PHD finger protein 2 & 2,74 & chr9 \\
\hline PHF20L1 & 51105 & PHD finger protein 20-like 1 & 2,35 & chr8 \\
\hline PHF21B & 112885 & PHD finger protein 21B & 2,15 & chr22 \\
\hline PHTF1 & 10745 & putative homeodomain transcription factor 1 & 2,09 & chr1 \\
\hline PIAS1 & 8554 & protein inhibitor of activated STAT, 1 & 2,92 & chr15 \\
\hline PIK3C2A & 5286 & Phosphoinositide-3-kinase, class 2, alpha polypeptide & 2,80 & chr11 \\
\hline |PIK3C3 & 5289 & Phosphoinositide-3-kinase, class 3 & 2,58 & chr18 \\
\hline |PIK3R1 & 5295 & phosphoinositide-3-kinase, regulatory subunit 1 (p85 alpha) & 3,61 & chr5 \\
\hline PIK3R3 & 8503 & phosphoinositide-3-kinase, regulatory subunit 3 (p55, gamma) & 3,93 & chr1 \\
\hline PIK4CA & 5297 & phosphatidylinositol 4-kinase, catalytic, alpha polypeptide & 2,09 & chr22 \\
\hline PIK4CA & $20686 / / / 529$ & phosphatidylinositol 4-kinase, catalytic, alpha polypeptide /// hypothetical protein LOC22 & 2,84 & chr22 \\
\hline PILRB & 29990 & paired immunoglobin-like type 2 receptor beta & 2,31 & chr7 \\
\hline PITPNA & 5306 & phosphatidylinositol transfer protein, alpha & 2,52 & chr17 \\
\hline PITPNB & 23760 & Phosphatidylinositol transfer protein, beta & 2,04 & chr22 \\
\hline PKIA & 5569 & Protein kinase (cAMP-dependent, catalytic) inhibitor alpha & 3,14 & chr8 \\
\hline PKNOX2 & 63876 & PBX/knotted 1 homeobox 2 & 5,62 & chr11 \\
\hline PKP4 & 8502 & Plakophilin 4 & 2,36 & chr2 \\
\hline PLAGL1 & 5325 & pleiomorphic adenoma gene-like 1 & 5,59 & chr6 \\
\hline PLAT & 5327 & plasminogen activator, tissue & 3,10 & chr8 \\
\hline PLCB1 & 23236 & phospholipase C, beta 1 (phosphoinositide-specific) & 2,44 & chr20 \\
\hline PLCE1 & 51196 & phospholipase C, epsilon 1 & 2,01 & chr10 \\
\hline PLCL3 & 23007 & phospholipase C-like 3 & 2,01 & chr3 \\
\hline PLEKHA1 & 59338 & pleckstrin homology domain containing, family A (phosphoinositide binding specific) mer & 2,26 & chr10 \\
\hline PLEKHA5 & 54477 & Pleckstrin homology domain containing, family A member 5 & 2,21 & chr12 \\
\hline PLEKHG1 & 57480 & pleckstrin homology domain containing, family G (with RhoGef domain) member 1 & 9,86 & chr6 \\
\hline PLK1 & 5347 & Polo-like kinase 1 (Drosophila) & 3,42 & chr16 \\
\hline PLXNA1 & 5361 & plexin A1 & 2,39 & chr3 \\
\hline PLXNA2 & 5362 & plexin A2 & 3,82 & chr1 \\
\hline PLXNB2 & 23654 & plexin B2 & 2,42 & chr22 \\
\hline PLXNC1 & 10154 & plexin C1 & 3,11 & chr12 \\
\hline PMP22 & 5376 & peripheral myelin protein 22 & 7,19 & chr17 \\
\hline PMS2L1 & $5379 / / / 5383$ & postmeiotic segregation increased 2-like $1 / / /$ postmeiotic segregation increased 2-like 5 & 2,13 & chr7 \\
\hline POFUT2 & 23275 & protein O-fucosyltransferase 2 & 2,18 & chr21 \\
\hline POLR2J2 & 246721 & DNA directed RNA polymerase II polypeptide J-related gene & 2,34 & chr7 \\
\hline |РОMT1 & 10585 & protein-O-mannosyltransferase 1 & 2,28 & chr9 \\
\hline
\end{tabular}




\begin{tabular}{|c|c|c|c|c|}
\hline Gene Symbol & Entrez Gene & Gene Title & NPC_up & Chromosome Number(Avadis) \\
\hline PON2 & 5445 & Paraoxonase 2 & 2,12 & chr7 \\
\hline POSTN & 10631 & periostin, osteoblast specific factor & 45,10 & chr13 \\
\hline POU3F2 & 5454 & POU domain, class 3 , transcription factor 2 & 3,76 & chr6 \\
\hline POU4F1 & 5457 & POU domain, class 4, transcription factor 1 & 3,70 & chr13 \\
\hline PPFIBP1 & $440091 / / / 8496$ & PTPRF interacting protein, binding protein 1 (liprin beta 1) /// similar to PTPRF interactin & 3,77 & chr12 \\
\hline PPM1F & 9647 & protein phosphatase $1 \mathrm{~F}$ (PP2C domain containing) & 2,46 & chr22 \\
\hline PPM1L & 151742 & Protein phosphatase 1 (formerly $2 \mathrm{C}$ )-like & 2,71 & chr3 \\
\hline PPOX & 5498 & protoporphyrinogen oxidase & 3,33 & chr1 \\
\hline PPP2R5C & 5527 & protein phosphatase 2, regulatory subunit B (B56), gamma isoform & 2,17 & chr14 \\
\hline PPP3CA & 5530 & Protein phosphatase 3 (formerly 2B), catalytic subunit, alpha isoform (calcineurin A alph & 2,06 & chr4 \\
\hline PPT2 /// EGFL8 & $80864 / / / 9374$ & palmitoyl-protein thioesterase 2 /// EGF-like-domain, multiple 8 & 3,63 & chr6 \\
\hline PRDM16 & 63976 & PR domain containing 16 & 6,13 & chr1 \\
\hline PREX1 & 57580 & phosphatidylinositol 3,4,5-trisphosphate-dependent RAC exchanger 1 & 2,79 & chr20 \\
\hline PRKAA2 & 5563 & Protein kinase, AMP-activated, alpha 2 catalytic subunit & 2,74 & chr1 \\
\hline PRKD1 & 5587 & protein kinase D1 & 2,52 & chr14 \\
\hline PRKRA & 8575 & protein kinase, interferon-inducible double stranded RNA dependent activator & 2,22 & chr2 \\
\hline PRO2852 & 114224 & hypothetical protein PRO2852 & 2,03 & chr9 \\
\hline PROS1 & 5627 & protein S (alpha) & 5,53 & chr3 \\
\hline PRPF40A & 55660 & PRP40 pre-mRNA processing factor 40 homolog A (yeast) & 2,56 & chr2 \\
\hline PRR3 & 80742 & proline rich 3 & 2,05 & chr6 \\
\hline PRRX1 & 5396 & paired related homeobox 1 & 5,41 & chr1 \\
\hline PRSS23 & 11098 & protease, serine, 23 & 36,19 & chr11 \\
\hline PRTG & 283659 & Protogenin homolog (Gallus gallus) & 38,57 & chr15 \\
\hline PSCD2 & 9266 & Pleckstrin homology, Sec7 and coiled-coil domains 2 (cytohesin-2) & 2,30 & chr19 \\
\hline PSD3 & 23362 & pleckstrin and Sec7 domain containing 3 & 2,42 & chr8 \\
\hline PSEN1 & 5663 & Presenilin 1 (Alzheimer disease 3) & 2,50 & chr14 \\
\hline PSMB7 & 5695 & Proteasome (prosome, macropain) subunit, beta type, 7 & 2,13 & chr9 \\
\hline PSME4 & 23198 & Proteasome (prosome, macropain) activator subunit 4 & 2,16 & chr2 \\
\hline PTBP2 & 58155 & Polypyrimidine tract binding protein 2 & 2,41 & chr1 \\
\hline PTEN & 5728 & Phosphatase and tensin homolog (mutated in multiple advanced cancers 1) & 2,23 & chr10 \\
\hline PTK2 & 5747 & PTK2 protein tyrosine kinase 2 & 2,47 & chr8 \\
\hline PTN & 5764 & pleiotrophin (heparin binding growth factor 8, neurite growth-promoting factor 1) & 3,49 & chr7 \\
\hline PTPN13 & 5783 & Protein tyrosine phosphatase, non-receptor type 13 (APO-1/CD95 (Fas)-associated phd & 2,57 & chr4 \\
\hline PTPN21 & 11099 & Protein tyrosine phosphatase, non-receptor type 21 & 2,75 & chr14 \\
\hline PTPN5 & 84867 & protein tyrosine phosphatase, non-receptor type 5 (striatum-enriched) & 3,51 & chr11 \\
\hline PTPRD & 5789 & Protein tyrosine phosphatase, receptor type, D & 4,30 & chr9 \\
\hline PTPRG & 5793 & Protein tyrosine phosphatase, receptor type, $\mathrm{G}$ & 2,25 & chr3 \\
\hline PTPRJ & 5795 & Protein tyrosine phosphatase, receptor type, J & 3,32 & chr11 \\
\hline PTPRM & 5797 & protein tyrosine phosphatase, receptor type, M & 4,86 & chr7 \\
\hline PTX3 & 5806 & pentraxin-related gene, rapidly induced by IL-1 beta & 5,15 & chr3 \\
\hline PUM2 & 23369 & Pumilio homolog 2 (Drosophila) & 3,87 & chr2 \\
\hline PUNC & 9543 & putative neuronal cell adhesion molecule & 4,01 & chr15 \\
\hline PURB & 5814 & purine-rich element binding protein $B$ & 2,23 & chr7 \\
\hline PURG & 29942 & purine-rich element binding protein $\mathrm{G}$ & 4,14 & chr8 \\
\hline PVRL3 & 25945 & Poliovirus receptor-related 3 & 2,81 & chr3 \\
\hline PXMP3 & 5828 & Peroxisomal membrane protein $3,35 \mathrm{kDa}$ (Zellweger syndrome) & 2,02 & chr8 \\
\hline QKI & 9444 & quaking homolog, $\mathrm{KH}$ domain RNA binding (mouse) & 2,74 & chr6 \\
\hline RAB22A & 57403 & RAB22A, member RAS oncogene family & 2,15 & chr20 \\
\hline RAB27B & 5874 & RAB27B, member RAS oncogene family & 3,53 & chr18 \\
\hline RAB30 & 27314 & RAB30, member RAS oncogene family & 2,10 & chr11 \\
\hline RAB31 & 11031 & RAB31, member RAS oncogene family & 2,18 & chr18 \\
\hline RAB40B & 10966 & RAB40B, member RAS oncogene family & 3,04 & chr17 \\
\hline RAB6B & 51560 & RAB6B, member RAS oncogene family & 2,51 & chr3 \\
\hline RAB6IP1 & 23258 & RAB6 interacting protein 1 & 2,27 & chr11 \\
\hline RABGAP1 & 23637 & RAB GTPase activating protein 1 & 2,73 & chr9 \\
\hline RABL2B & $11158 / / / 1115$ & RAB, member of RAS oncogene family-like $2 B / / / R A B$, member of RAS oncogene fami & 2,34 & chr2 \\
\hline RAF1 & 5894 & V-raf-1 murine leukemia viral oncogene homolog 1 & 2,34 & chr3 \\
\hline RAl1 & 10743 & retinoic acid induced 1 & 4,81 & $\operatorname{chr} 17$ \\
\hline RAl17 & 57178 & retinoic acid induced 17 & 2,34 & chr10 \\
\hline RALGDS & 5900 & ral guanine nucleotide dissociation stimulator & 2,97 & chr9 \\
\hline RANBP2L2 & 440872 & Ran binding protein 2-like 2 & 2,63 & chr2 \\
\hline RAP140 & 23272 & retinoblastoma-associated protein 140 & 2,22 & chr3 \\
\hline RARB & 5915 & retinoic acid receptor, beta & 2,60 & chr3 \\
\hline RASA2 & 5922 & RAS p21 protein activator 2 & 2,88 & chr3 \\
\hline RASA4 & $0156 / / / 40133$ & RAS p21 protein activator 4 /// hypothetical protein FLJ21767 & 2,08 & chr7 \\
\hline RASGRP1 & 10125 & RAS guanyl releasing protein 1 (calcium and DAG-regulated) & 3,62 & chr15 \\
\hline RASSF3 & 283349 & Ras association (RalGDS/AF-6) domain family 3 & 2,25 & chr14 \\
\hline RBBP6 & 5930 & retinoblastoma binding protein 6 & 2,47 & chr16 \\
\hline
\end{tabular}




\begin{tabular}{|c|c|c|c|c|}
\hline Gene Symbol & Entrez Gene & Gene Title & NPC_up & Chromosome Number(Avadis) \\
\hline RBM24 & 221662 & RNA binding motif protein 24 & 8,43 & chr6 \\
\hline RBM25 & 58517 & RNA binding motif protein 25 & 2,10 & chr14 \\
\hline RBM33 & 155435 & RNA binding motif protein 33 & 2,38 & chr7 \\
\hline RBM4B & 83759 & RNA binding motif protein 4B & 2,49 & chr11 \\
\hline RBM5 & 10181 & RNA binding motif protein 5 & 2,63 & chr3 \\
\hline RBM6 & 10180 & RNA binding motif protein 6 & 3,28 & chr3 \\
\hline RBMS1 & 5937 & RNA binding motif, single stranded interacting protein 1 & 2,61 & chr2 \\
\hline RBMS3 & 27303 & RNA binding motif, single stranded interacting protein & 2,50 & chr3 \\
\hline RCBTB2 & 1102 & regulator of chromosome condensation ( $\mathrm{RCC} 1$ ) and $\mathrm{BTB}$ (POZ) domain containing prot & 2,95 & chr13 \\
\hline RCN1 & 5954 & reticulocalbin 1, EF-hand calcium binding domain & 2,29 & chr11 \\
\hline RCOR3 & 55758 & REST corepressor 3 & 2,47 & chr1 \\
\hline RELN & 5649 & reelin & 15,67 & chr7 \\
\hline RERE & 473 & Arginine-glutamic acid dipeptide (RE) repeats & 2,11 & chr1 \\
\hline REV3L & 5980 & REV3-like, catalytic subunit of DNA polymerase zeta (yeast) & 5,11 & chr6 \\
\hline RFC3 & 5983 & Replication factor C (activator 1) 3, 38kDa & 2,37 & chr13 \\
\hline RFX3 & 5991 & Regulatory factor X, 3 (influences HLA class II expression) & 2,83 & chr9 \\
\hline RGL1 & 23179 & ral guanine nucleotide dissociation stimulator-like 1 & 7,87 & chr1 \\
\hline RGS12 & 6002 & regulator of G-protein signalling 12 & 2,04 & $\operatorname{chr} 4$ \\
\hline RGS20 & 8601 & regulator of G-protein signalling 20 & 2,60 & chr8 \\
\hline RGS3 & 5998 & regulator of G-protein signalling 3 & 2,24 & chr9 \\
\hline RGS4 & 5999 & regulator of G-protein signalling 4 & 8,95 & chr1 \\
\hline RHOBTB1 & 9886 & Rho-related BTB domain containing 1 & 2,75 & chr10 \\
\hline RHOBTB3 & 22836 & Rho-related BTB domain containing 3 & 6,84 & chr5 \\
\hline RHOU & 58480 & ras homolog gene family, member $U$ & 6,94 & chr1 \\
\hline RIN2 & 54453 & Ras and Rab interactor 2 & 6,62 & chr20 \\
\hline RIPX & 22902 & rap2 interacting protein $\mathrm{x}$ & 5,22 & chr4 \\
\hline RIT1 & 6016 & Ras-like without CAAX 1 & 2,44 & chr1 \\
\hline RNF103 & 7844 & ring finger protein 103 & 2,85 & chr2 \\
\hline RNF130 & 55819 & Ring finger protein 130 & 3,33 & chr5 \\
\hline RNF144 & 9781 & ring finger protein 144 & 2,53 & chr2 \\
\hline RNF165 & 494470 & ring finger protein 165 & 9,91 & $\operatorname{chr} 18$ \\
\hline RNF175 & 285533 & ring finger protein 175 & 3,51 & chr4 \\
\hline RNF19 & 25897 & Ring finger protein 19 & 2,02 & chr8 \\
\hline ROBO1 & 6091 & Roundabout, axon guidance receptor, homolog 1 (Drosophila) & 2,78 & chr3 \\
\hline ROBO2 & 6092 & roundabout, axon guidance receptor, homolog 2 (Drosophila) & 3,59 & chr3 \\
\hline ROCK2 & 9475 & Rho-associated, coiled-coil containing protein kinase 2 & 2,12 & chr2 \\
\hline ROR2 & 4920 & receptor tyrosine kinase-like orphan receptor 2 & 2,65 & chr9 \\
\hline RP11-343N15.3 & 440608 & Similar to Formin binding protein 2 (srGAP2) & 2,87 & chr1 \\
\hline RP3-473B4.1 & 159091 & Hypothetical protein BC017868 & 2,80 & $\operatorname{chr} X$ \\
\hline RPL28 & 6158 & ribosomal protein L28 & 2,02 & chr19 \\
\hline RPL31 & 6160 & ribosomal protein L31 & 3,69 & chr2 \\
\hline RPRC1 & 55700 & arginine/proline rich coiled-coil 1 & 3,64 & chr1 \\
\hline RPS15A & 6210 & Ribosomal protein S15a & 2,67 & chr16 \\
\hline RPS23 & 6228 & ribosomal protein S23 & 3,13 & chr5 \\
\hline RPS29 & 6235 & Ribosomal protein S29 & 2,38 & chr14 \\
\hline RPS6KA3 & 6197 & ribosomal protein S6 kinase, 90kDa, polypeptide 3 & 3,80 & $\operatorname{chrX}$ \\
\hline RPS6KA5 & 9252 & ribosomal protein $\mathrm{S} 6$ kinase, $90 \mathrm{kDa}$, polypeptide 5 & 2,11 & chr14 \\
\hline RRN3 & 54700 & RRN3 RNA polymerase I transcription factor homolog (yeast) & 2,13 & chr16 \\
\hline RSBN1 & 54665 & round spermatid basic protein 1 & 2,23 & chr1 \\
\hline RSL1D1 & 26156 & Ribosomal L1 domain containing 1 & 4,33 & chr16 \\
\hline RSNL2 & 79745 & restin-like 2 & 4,55 & chr2 \\
\hline RSPO3 & 84870 & R-spondin 3 homolog (Xenopus laevis) & 5,79 & chr6 \\
\hline RTN1 & 6252 & reticulon 1 & 6,98 & chr14 \\
\hline RUNX1 & 861 & runt-related transcription factor 1 (acute myeloid leukemia 1; aml1 oncogene) & 2,59 & chr21 \\
\hline RUTBC1 & 9905 & RUN and TBC1 domain containing 1 & 2,40 & chr17 \\
\hline SAPS2 & 9701 & SAPS domain family, member 2 & 2,14 & chr22 \\
\hline SBF2 & 81846 & SET binding factor 2 & 2,11 & chr11 \\
\hline SBLF & 11037 & stoned B-like factor & 3,81 & chr2 \\
\hline SCC-112 & 23244 & SCC-112 protein & 2,34 & chr4 \\
\hline SCD5 & 79966 & stearoyl-CoA desaturase 5 & 18,32 & chr4 \\
\hline SCFD1 & 23256 & Sec1 family domain containing 1 & 2,07 & chr14 \\
\hline SCHIP1 & 29970 & schwannomin interacting protein 1 & 2,20 & chr3 \\
\hline SCMH1 & 22955 & sex comb on midleg homolog 1 (Drosophila) & 2,04 & chr1 \\
\hline SCML1 & 6322 & sex comb on midleg-like 1 (Drosophila) & 3,10 & $\operatorname{chrX}$ \\
\hline SCRG1 & 11341 & scrapie responsive protein 1 & 3,50 & $\operatorname{chr} 4$ \\
\hline SCUBE2 & 57758 & signal peptide, CUB domain, EGF-like 2 & 2,88 & chr11 \\
\hline SCUBE3 & 222663 & signal peptide, CUB domain, EGF-like 3 & 14,58 & chr6 \\
\hline C1 & 6382 & indecan 1 & 2,08 & chr2 \\
\hline
\end{tabular}




\begin{tabular}{|c|c|c|c|c|}
\hline Gene Symbol & Entrez Gene & Gene Title & NPC_up & Chromosome Number(Avadis) \\
\hline SDC2 & 6383 & syndecan 2 (heparan sulfate proteoglycan 1, cell surface-associated, fibroglycan) & 4,54 & chr8 \\
\hline SDCCAG33 & 10194 & serologically defined colon cancer antigen 33 & 4,76 & chr18 \\
\hline SDHAL2 & 255812 & succinate dehydrogenase complex, subunit A, flavoprotein-like 2 & 3,76 & chr3 \\
\hline SDK1 & 221935 & sidekick homolog 1 (chicken) & 2,89 & chr7 \\
\hline SDK2 & 54549 & sidekick homolog 2 (chicken) & 13,25 & chr17 \\
\hline SEC15L1 & 54536 & SEC15-like 1 (S. cerevisiae) & 2,41 & chr10 \\
\hline SEC6L1 & 11336 & SEC6-like 1 (S. cerevisiae) & 2,53 & chr5 \\
\hline SELENBP1 & 8991 & selenium binding protein $1 / / /$ selenium binding protein 1 & 2,12 & chr1 \\
\hline SEMA3A & 10371 & sema domain, immunoglobulin domain (lg), short basic domain, secreted, (semaphorin) & 2,56 & chr7 \\
\hline SEMA3C & 10512 & sema domain, immunoglobulin domain (lg), short basic domain, secreted, (semaphorin) & 8,75 & chr7 \\
\hline SEMA3F & 6405 & sema domain, immunoglobulin domain (Ig), short basic domain, secreted, (semaphorin) & 2,29 & chr3 \\
\hline SEMA4F & 10505 & sema domain, immunoglobulin domain (Ig), transmembrane domain (TM) and short cytd & 2,34 & chr2 \\
\hline SEMA5A & 9037 & sema domain, seven thrombospondin repeats (type 1 and type 1 -like), transmembrane & 8,08 & chr5 \\
\hline SEMA6D & 80031 & sema domain, transmembrane domain (TM), and cytoplasmic domain, (semaphorin) $6 \mathrm{D}$ & 4,35 & chr15 \\
\hline SENP6 & 26054 & SUMO1/sentrin specific peptidase 6 & 2,82 & chr6 \\
\hline SENP7 & 57337 & SUMO1/sentrin specific peptidase 7 & 2,13 & chr3 \\
\hline SERTAD2 & 9792 & SERTA domain containing 2 & 4,46 & chr2 \\
\hline SERTAD4 & 56256 & SERTA domain containing 4 & 2,68 & chr1 \\
\hline SESN3 & 143686 & Sestrin 3 & 5,17 & chr11 \\
\hline SESTD1 & 91404 & SEC14 and spectrin domains 1 & 3,24 & chr2 \\
\hline SET7 & 80854 & SET domain-containing protein 7 & 3,94 & chr4 \\
\hline SETBP1 & 26040 & SET binding protein 1 & 3,15 & chr18 \\
\hline SETD5 & 55209 & SET domain containing 5 & 2,25 & chr3 \\
\hline SETD6 & 79918 & SET domain containing 6 & 2,02 & $\operatorname{chr} 16$ \\
\hline SEZ6L & 23544 & Seizure related 6 homolog (mouse)-like & 3,21 & chr22 \\
\hline SFI1 & 9814 & Sfi1 homolog, spindle assembly associated (yeast) & 2,49 & chr22 \\
\hline SFPQ & 6421 & Splicing factor proline/glutamine-rich (polypyrimidine tract binding protein associated) & 3,94 & chr1 \\
\hline SFRP2 & 6423 & secreted frizzled-related protein 2 & 5,36 & chr4 \\
\hline SFRS1 & 6426 & Splicing factor, arginine/serine-rich 1 (splicing factor 2, alternate splicing factor) & 2,65 & chr17 \\
\hline SFRS11 & 9295 & splicing factor, arginine/serine-rich 11 & 4,06 & chr1 \\
\hline SFRS14 & 10147 & Splicing factor, arginine/serine-rich 14 & 2,20 & chr19 \\
\hline SFRS2IP & 9169 & Splicing factor, arginine/serine-rich 2, interacting protein & 3,45 & chr12 \\
\hline SFRS4 & 6429 & Splicing factor, arginine/serine-rich 4 & 2,26 & chr1 \\
\hline SFXN1 & 94081 & sideroflexin 1 & 2,14 & chr5 \\
\hline SGSH & 6448 & N-sulfoglucosamine sulfohydrolase (sulfamidase) & 2,36 & chr17 \\
\hline SH3BGRL2 & 83699 & SH3 domain binding glutamic acid-rich protein like 2 & 2,84 & chr6 \\
\hline SH3MD1 & 9644 & SH3 multiple domains 1 & 2,26 & chr10 \\
\hline SHANK3 & 85358 & SH3 and multiple ankyrin repeat domains 3 & 2,51 & chr22 \\
\hline $\mathrm{SHC} 2$ & 25759 & SHC (Src homology 2 domain containing) transforming protein 2 & 2,99 & chr19 \\
\hline SHRM & 57619 & Shroom & 2,20 & chr4 \\
\hline SIDT2 & 51092 & SID1 transmembrane family, member 2 & 2,52 & chr11 \\
\hline SILV & 6490 & silver homolog (mouse) & 3,28 & chr12 \\
\hline SIPA1L2 & 57568 & signal-induced proliferation-associated 1 like 2 & 3,19 & chr1 \\
\hline SIX3 & 6496 & Sine oculis homeobox homolog 3 (Drosophila) & 37,88 & chr2 \\
\hline SIX6 & 4990 & sine oculis homeobox homolog 6 (Drosophila) & 6,24 & chr14 \\
\hline SLC16A14 & 151473 & solute carrier family 16 (monocarboxylic acid transporters), member 14 & 3,79 & chr2 \\
\hline SLC16A4 & 9122 & Solute carrier family 16 (monocarboxylic acid transporters), member 4 & 2,35 & chr1 \\
\hline SLC1A2 & 6506 & solute carrier family 1 (glial high affinity glutamate transporter), member 2 & 3,01 & chr11 \\
\hline SLC1A3 & 6507 & solute carrier family 1 (glial high affinity glutamate transporter), member 3 & 3,22 & chr5 \\
\hline SLC1A4 & 6509 & solute carrier family 1 (glutamate/neutral amino acid transporter), member 4 & 2,27 & chr2 \\
\hline SLC25A16 & 8034 & Solute carrier family 25 (mitochondrial carrier; Graves disease autoantigen), member $1 €$ & 2,37 & chr10 \\
\hline SLC25A27 & 9481 & solute carrier family 25 , member 27 & 4,25 & chr6 \\
\hline SLC25A37 & 51312 & solute carrier family 25 , member 37 & 3,10 & chr8 \\
\hline SLC26A2 & 1836 & solute carrier family 26 (sulfate transporter), member 2 & 2,29 & chr5 \\
\hline SLC30A1 & 7779 & Solute carrier family 30 (zinc transporter), member 1 & 2,12 & chr1 \\
\hline SLC35D1 & 23169 & solute carrier family 35 (UDP-glucuronic acid/UDP-N-acetylgalactosamine dual transpor & 2,31 & chr1 \\
\hline SLC35E2 & 9906 & solute carrier family 35, member E2 & 3,24 & chr1 \\
\hline SLC35F1 & 222553 & solute carrier family 35 , member $\mathrm{F} 1$ & 2,10 & chr6 \\
\hline SLC39A10 & 57181 & Solute carrier family 39 (zinc transporter), member 10 & 2,98 & chr2 \\
\hline SLC4A7 & 9497 & solute carrier family 4 , sodium bicarbonate cotransporter, member 7 & 2,04 & chr3 \\
\hline SLC5A3 & 6526 & solute carrier family 5 (inositol transporters), member 3 & 4,29 & chr21 \\
\hline SLC6A16 & 28968 & Solute carrier family 6 , member 16 & 7,77 & chr19 \\
\hline SLC7A6 & 9057 & solute carrier family 7 (cationic amino acid transporter, y+ system), member 6 & 2,31 & chr16 \\
\hline SLC8A1 & 6546 & solute carrier family 8 (sodium/calcium exchanger), member 1 & 2,12 & chr2 \\
\hline SLC9A3 & 6550 & Solute carrier family 9 (sodium/hydrogen exchanger), member 3 & 2,28 & chr5 \\
\hline SLIT2 & 9353 & slit homolog 2 (Drosophila) & 7,63 & chr4 \\
\hline SLITRK4 & 139065 & SLIT and NTRK-like family, member 4 & 2,70 & $\operatorname{chr} X$ \\
\hline SLITRK5 & 26050 & SLIT and NTRK-like family, member 5 & 3,43 & $\operatorname{chr} 13$ \\
\hline
\end{tabular}




\begin{tabular}{|c|c|c|c|c|}
\hline \multicolumn{5}{|c|}{ erexpressed in NPC compared to hES (Fold Change $>2 ; \alpha<0.05$ ) } \\
\hline Gene Symbol & Entrez Gene & Gene Title & NPC_up & Chromosome Number(Avadis) \\
\hline SLITRK6 & 84189 & SLIT and NTRK-like family, member 6 & 6,19 & chr13 \\
\hline SLN & 6588 & sarcolipin & 4,50 & chr11 \\
\hline SMAD3 & 4088 & SMAD, mothers against DPP homolog 3 (Drosophila) & 3,50 & chr15 \\
\hline SMAD5 & 4090 & SMAD, mothers against DPP homolog 5 (Drosophila) & 2,22 & chr5 \\
\hline SMARCC1 & 6599 & SWI/SNF related, matrix associated, actin dependent regulator of chromatin, subfamily & 3,05 & chr3 \\
\hline SMARCD3 & 6604 & SWI/SNF related, matrix associated, actin dependent regulator of chromatin, subfamily & 2,58 & chr7 \\
\hline SMARCE1 & 6605 & SWI/SNF related, matrix associated, actin dependent regulator of chromatin, subfamily & 2,05 & chr17 \\
\hline SMBP & 56889 & SM-11044 binding protein & 2,38 & chr10 \\
\hline SMPDL3A & 10924 & sphingomyelin phosphodiesterase, acid-like $3 \mathrm{~A}$ & 2,24 & chr6 \\
\hline SMU1 & 55234 & Smu-1 suppressor of mec-8 and unc-52 homolog (C. elegans) & 2,41 & chr9 \\
\hline SNAI2 & 6591 & snail homolog 2 (Drosophila) & 9,44 & chr8 \\
\hline SNCA & 6622 & synuclein, alpha (non A4 component of amyloid precursor) /// synuclein, alpha (non $\mathrm{A} 4 \mathrm{~d}$ & 2,70 & $\operatorname{chr} 4$ \\
\hline SNF1LK & 150094 & SNF1-like kinase /// SNF1-like kinase & 2,56 & chr10 \\
\hline SNF8 & 11267 & SNF8, ESCRT-II complex subunit, homolog (S. cerevisiae) & 2,33 & chr17 \\
\hline SNRPA 1 & 6627 & Small nuclear ribonucleoprotein polypeptide A' & 2,47 & chr15 \\
\hline SNX1 & 6642 & sorting nexin 1 & 2,14 & chr15 \\
\hline socs3 & 9021 & suppressor of cytokine signaling 3 & 2,60 & chr17 \\
\hline SORBS2 & 8470 & sorbin and $\mathrm{SH} 3$ domain containing 2 & 2,69 & chr4 \\
\hline SORCS1 & 114815 & sortilin-related VPS10 domain containing receptor 1 & 3,02 & chr10 \\
\hline SORCS2 & 57537 & sortilin-related VPS10 domain containing receptor 2 & 3,39 & chr4 \\
\hline SOSTDC1 & 25928 & sclerostin domain containing 1 & 2,01 & $\operatorname{chr} 7$ \\
\hline sox 1 & 6656 & SRY (sex determining region Y)-box 1 & 8,01 & chr13 \\
\hline sox10 & 6663 & SRY (sex determining region Y)-box 10 & 2,90 & chr22 \\
\hline sox11 & 6664 & SRY (sex determining region Y)-box 11 & 8,85 & chr2 \\
\hline sox3 & 6658 & SRY (sex determining region Y)-box 3 & 3,38 & $\operatorname{chr} X$ \\
\hline sox5 & 6660 & SRY (sex determining region Y)-box 5 & 28,58 & chr12 \\
\hline sox6 & 55553 & SRY (sex determining region Y)-box 6 & 7,05 & chr11 \\
\hline soxa & 6662 & SRY (sex determining region Y)-box 9 (campomelic dysplasia, autosomal sex-reversal) & 5,01 & chr17 \\
\hline SP8 & 221833 & Sp8 transcription factor & 16,95 & chr7 \\
\hline SPA17 & 53340 & sperm autoantigenic protein 17 & 3,09 & chr11 \\
\hline SPAG9 & 9043 & sperm associated antigen 9 & 2,23 & chr17 \\
\hline SPARC & 6678 & secreted protein, acidic, cysteine-rich (osteonectin) /// secreted protein, acidic, cysteine & 2,25 & $\operatorname{chr} 16$ \\
\hline SPHK1 & 8877 & sphingosine kinase 1 & 6,46 & chr17 \\
\hline SPOCK & 6695 & sparc/osteonectin, cwcv and kazal-like domains proteoglycan (testican) & 4,73 & chr5 \\
\hline SPON1 & 10418 & spondin 1 , extracellular matrix protein & 9,34 & chr11 \\
\hline SPPL3 & 121665 & signal peptide peptidase 3 & 2,02 & chr12 \\
\hline SPSB4 & 92369 & splA/ryanodine receptor domain and SOCS box containing 4 & 2,16 & chr3 \\
\hline SPTBN1 & 6711 & Spectrin, beta, non-erythrocytic 1 & 2,29 & chr2 \\
\hline SREBF1 & 6720 & Sterol regulatory element binding transcription factor 1 & 2,06 & chr17 \\
\hline SRGAP2 & 23380 & SLIT-ROBO Rho GTPase activating protein 2 & 3,08 & chr1 \\
\hline SRGAP3 & 9901 & SLIT-ROBO Rho GTPase activating protein 3 & 6,11 & chr3 \\
\hline SRP54 & 6729 & Signal recognition particle $54 \mathrm{kDa}$ & 2,10 & chr14 \\
\hline SRR & 63826 & serine racemase & 4,61 & chr17 \\
\hline SSBP2 & 23635 & Single-stranded DNA binding protein 2 & 7,89 & chr5 \\
\hline SSFA2 & 6744 & sperm specific antigen 2 & 4,38 & chr2 \\
\hline $\mathrm{SSH} 1$ & 54434 & slingshot homolog 1 (Drosophila) & 2,21 & chr12 \\
\hline SSPO & 23145 & SCO-spondin homolog (Bos taurus) & 2,06 & $\operatorname{chr} 7$ \\
\hline SSR1 & 6745 & Signal sequence receptor, alpha (translocon-associated protein alpha) & 3,14 & chr6 \\
\hline SST & 6750 & somatostatin & 5,09 & chr3 \\
\hline ST3GAL3 & 6487 & ST3 beta-galactoside alpha-2,3-sialyltransferase 3 & 2,35 & chr1 \\
\hline ST3GAL5 & 8869 & ST3 beta-galactoside alpha-2,3-sialyltransferase 5 & 2,13 & chr2 \\
\hline ST5 & 6764 & suppression of tumorigenicity 5 & 2,17 & chr11 \\
\hline ST6GALNAC5 & 81849 & ST6 (alpha-N-acetyl-neuraminyl-2,3-beta-galactosyl-1,3)-N-acetylgalactosaminide alpha & 8,46 & chr1 \\
\hline ST7L & 54879 & Suppression of tumorigenicity 7 like & 7,03 & chr1 \\
\hline ST8SIA2 & 8128 & ST8 alpha-N-acetyl-neuraminide alpha-2,8-sialyltransferase 2 & 7,30 & (vide) \\
\hline STK17A & 9263 & Serine/threonine kinase 17a (apoptosis-inducing) & 2,04 & $\operatorname{chr} 7$ \\
\hline STMN2 & 11075 & stathmin-like 2 & 12,77 & chr8 \\
\hline STMN4 & 81551 & stathmin-like 4 /// stathmin-like 4 & 3,21 & chr8 \\
\hline STOX1 & 219736 & storkhead box 1 & 2,04 & chr10 \\
\hline STT3B & 201595 & STT3, subunit of the oligosaccharyltransferase complex, homolog B (S. cerevisiae) & 2,12 & chr3 \\
\hline STX12 & 23673 & syntaxin 12 & 2,42 & chr1 \\
\hline STX7 & 8417 & Syntaxin 7 & 2,33 & chr6 \\
\hline SULF1 & 23213 & sulfatase 1 & 20,55 & chr8 \\
\hline SUPT7L & 9913 & suppressor of Ty 7 (S. cerevisiae)-like & 2,06 & chr2 \\
\hline SUV420H1 & 51111 & Suppressor of variegation 4-20 homolog 1 (Drosophila) & 2,41 & chr11 \\
\hline SVIL & 6840 & supervillin & 2,59 & chr10 \\
\hline SYNE1 1 & 23345 & spectrin repeat containing, nuclear envelope 1 & 2,07 & chr6 \\
\hline SYT11 & 23208 & synaptotagmin XI & 3,88 & chr1 \\
\hline
\end{tabular}




\begin{tabular}{|c|c|c|c|c|}
\hline Gene Symbol & Entrez Gene & Gene Title & NPC_up & Chromosome Number(Avadis) \\
\hline SYT17 & 51760 & Synaptotagmin XVII & 3,76 & chr16 \\
\hline SYTL2 & 54843 & synaptotagmin-like 2 & 5,56 & chr11 \\
\hline TAF15 & 8148 & TAF15 RNA polymerase II, TATA box binding protein (TBP)-associated factor, $68 \mathrm{kDa}$ & 2,84 & chr17 \\
\hline TAGLN3 & 29114 & transgelin 3 & 3,36 & chr3 \\
\hline TAIP-2 & 80034 & TGF-beta induced apotosis protein 2 & 2,28 & chr2 \\
\hline TANC & 85461 & TPR domain, ankyrin-repeat and coiled-coil-containing & 2,91 & chr2 \\
\hline TARDBP & 23435 & TAR DNA binding protein /// TAR DNA binding protein & 2,19 & chr1 \\
\hline TBC1D12 & 23232 & TBC1 domain family, member 12 & 2,51 & chr10 \\
\hline TBC1D3 & $14060 / / / 8421$ & TBC1 domain family, member $3 / / /$ TBC1 domain family, member 3C & 6,00 & chr17_random \\
\hline TBC1D8 & 11138 & TBC1 domain family, member 8 (with GRAM domain) & 2,20 & chr2 \\
\hline TBL1X & 6907 & transducin (beta)-like 1X-linked & 2,71 & $\operatorname{chr} X$ \\
\hline TBRG1 & 84897 & transforming growth factor beta regulator 1 & 2,48 & chr11 \\
\hline TCF12 & 6938 & Transcription factor 12 (HTF4, helix-loop-helix transcription factors 4) & 2,55 & chr15 \\
\hline TCF8 & 6935 & transcription factor 8 (represses interleukin 2 expression) & 6,03 & chr10 \\
\hline TDRD7 & 23424 & tudor domain containing 7 & 3,05 & chr9 \\
\hline TEAD1 & 7003 & TEA domain family member 1 (SV40 transcriptional enhancer factor) & 2,63 & chr11 \\
\hline TERF2 & 7014 & telomeric repeat binding factor 2 & 2,26 & chr16 \\
\hline TEX10 & 54881 & Testis expressed sequence 10 & 2,17 & chr9 \\
\hline TFAP2A & 7020 & transcription factor AP-2 alpha (activating enhancer binding protein 2 alpha) & 20,12 & chr6 \\
\hline TFAP2B & 7021 & transcription factor AP-2 beta (activating enhancer binding protein 2 beta) & 13,06 & chr6 \\
\hline TFDP2 & 7029 & Transcription factor Dp-2 (E2F dimerization partner 2) & 2,21 & chr3 \\
\hline TFPI & 7035 & tissue factor pathway inhibitor (lipoprotein-associated coagulation inhibitor) & 4,00 & chr2 \\
\hline TGFB2 & 7042 & Transforming growth factor, beta 2 & 2,81 & chr1 \\
\hline TGFBI & 7045 & transforming growth factor, beta-induced, $68 \mathrm{kDa}$ & 7,02 & chr5 \\
\hline TGFBR1 & 7046 & Transforming growth factor, beta receptor I (activin A receptor type II-like kinase, 53kDa & 2,88 & chr9 \\
\hline TGFBR3 & 7049 & transforming growth factor, beta receptor III (betaglycan, 300kDa) & 5,34 & chr1 \\
\hline THBS3 & 7059 & thrombospondin 3 & 4,71 & chr1 \\
\hline THRAP2 & 23389 & Thyroid hormone receptor associated protein 2 & 2,79 & chr12 \\
\hline THSD1 & $74500 / / / 5590$ & thrombospondin, type I, domain containing $1 / / /$ thrombospondin, type I, domain containi & 3,05 & chr13 \\
\hline TIA1 & 7072 & TIA1 cytotoxic granule-associated RNA binding protein & 2,69 & chr2 \\
\hline TIAM1 & 7074 & T-cell lymphoma invasion and metastasis 1 & 3,51 & chr21 \\
\hline TIFA & 92610 & TRAF-interacting protein with a forkhead-associated domain & 2,81 & chr4 \\
\hline TIGA1 & 114915 & TIGA1 & 2,74 & chr5 \\
\hline TIMP2 & 7077 & TIMP metallopeptidase inhibitor 2 & 2,40 & chr17 \\
\hline TIMP3 & 7078 & TIMP metallopeptidase inhibitor 3 (Sorsby fundus dystrophy, pseudoinflammatory) & 6,97 & chr22 \\
\hline TIPRL & 261726 & TIP41, TOR signalling pathway regulator-like (S. cerevisiae) & 2,24 & chr1 \\
\hline TK2 & 7084 & thymidine kinase 2, mitochondrial & 2,16 & chr16 \\
\hline TLE4 & 7091 & transducin-like enhancer of split 4 (E(sp1) homolog, Drosophila) & 4,03 & chr9 \\
\hline TM2D1 & 83941 & TM2 domain containing 1 & 2,09 & chr1 \\
\hline TMCC1 & 23023 & transmembrane and coiled-coil domain family 1 & 2,08 & chr3 \\
\hline TMCC3 & 57458 & Transmembrane and coiled-coil domain family 3 & 2,53 & chr12 \\
\hline TMED4 & 222068 & transmembrane emp24 protein transport domain containing 4 & 2,59 & chr7 \\
\hline TMEFF2 & 23671 & transmembrane protein with EGF-like and two follistatin-like domains 2 & 9,12 & chr2 \\
\hline TMEM118 & 84900 & Transmembrane protein 118 & 3,17 & chr12 \\
\hline TMEM2 & 23670 & transmembrane protein 2 & 2,97 & chr9 \\
\hline TMEM20 & 159371 & transmembrane protein 20 & 2,31 & chr10 \\
\hline TMEM29 & 29057 & transmembrane protein 29 & 2,03 & $\operatorname{chr} X$ \\
\hline TMEM46 & 387914 & transmembrane protein 46 & 4,82 & chr13 \\
\hline TMEM5 & 10329 & transmembrane protein 5 & 2,67 & chr12 \\
\hline TMEM50B & 757 & transmembrane protein $50 \mathrm{~B}$ & 2,01 & chr21 \\
\hline TMEM76 & 138050 & transmembrane protein 76 & 4,68 & chr8 \\
\hline TMSL8 & 11013 & thymosin-like 8 & 2,67 & $\operatorname{chr} X$ \\
\hline TMTC2 & 160335 & Transmembrane and tetratricopeptide repeat containing 2 & 2,11 & chr12 \\
\hline TNC & 3371 & tenascin C (hexabrachion) & 6,97 & chr9 \\
\hline TncRNA & 283131 & trophoblast-derived noncoding RNA & 4,46 & chr11 \\
\hline TNFAIP1 & 7126 & tumor necrosis factor, alpha-induced protein 1 (endothelial) & 3,35 & chr17 \\
\hline TNFAIP3 & 7128 & tumor necrosis factor, alpha-induced protein 3 & 3,65 & chr6 \\
\hline TNFRSF19 & 55504 & tumor necrosis factor receptor superfamily, member 19 & 8,90 & chr9 \\
\hline TNS1 & 7145 & tensin $1 / / /$ tensin 1 & 3,69 & chr2 \\
\hline TP53INP1 & 94241 & tumor protein p53 inducible nuclear protein 1 & 3,60 & chr8 \\
\hline TPBG & 7162 & trophoblast glycoprotein & 6,71 & chr6 \\
\hline TPM1 & 7168 & Tropomyosin 1 (alpha) & 11,80 & chr15 \\
\hline TRA2A & 29896 & Transformer-2 alpha & 4,88 & chr7 \\
\hline TRAM2 & 9697 & translocation associated membrane protein 2 & 2,05 & chr6 \\
\hline TRIB2 & 28951 & tribbles homolog 2 (Drosophila) & 2,63 & chr2 \\
\hline TRIM44 & 54765 & tripartite motif-containing 44 & 2,44 & chr11 \\
\hline TRIM45 & 80263 & Tripartite motif-containing 45 & 2,69 & chr1 \\
\hline TRIM50B & 375593 & tripartite motif-containing 50B & 3,84 & chr7 \\
\hline
\end{tabular}




\begin{tabular}{|c|c|c|c|c|}
\hline Gene Symbol & Entrez Gene & Gene Title & NPC_up & Chromosome Number(Avadis) \\
\hline TRO & 7216 & trophinin /// trophinin & 2,25 & $\operatorname{chrX}$ \\
\hline TRPM3 & 80036 & transient receptor potential cation channel, subfamily $\mathrm{M}$, member 3 & 3,58 & chr9 \\
\hline TRPM8 & 79054 & transient receptor potential cation channel, subfamily $\mathrm{M}$, member 8 & 2,24 & chr2 \\
\hline TSGA14 & 95681 & testis specific, 14 & 3,15 & chr7 \\
\hline TSPAN14 & 81619 & tetraspanin 14 & 2,32 & chr10 \\
\hline TSPAN18 & 90139 & tetraspanin 18 & 3,72 & chr11 \\
\hline TSPAN3 & 10099 & Tetraspanin 3 & 2,18 & chr15 \\
\hline TSPAN5 & 10098 & tetraspanin $5 / / /$ tetraspanin 5 & 2,53 & chr4 \\
\hline TSPYL2 & 64061 & TSPY-like 2 & 2,03 & $\operatorname{chrX}$ \\
\hline TSPYL4 & 23270 & TSPY-like 4 & 2,30 & chr6 \\
\hline TTBK2 & 146057 & tau tubulin kinase 2 & 2,27 & chr15 \\
\hline TTC10 & 8100 & tetratricopeptide repeat domain 10 & 2,70 & chr13 \\
\hline TTC12 & 54970 & tetratricopeptide repeat domain 12 & 2,99 & chr11 \\
\hline TTC14 & 151613 & Tetratricopeptide repeat domain 14 & 2,11 & chr3 \\
\hline TTC17 & 55761 & tetratricopeptide repeat domain 17 & 2,90 & chr11 \\
\hline TTC3 & 7267 & tetratricopeptide repeat domain 3 & 5,88 & chr21 \\
\hline TTYH1 & 57348 & tweety homolog 1 (Drosophila) & 2,45 & chr19 \\
\hline TXNIP & 10628 & thioredoxin interacting protein & 3,89 & chr1 \\
\hline U2AF1 & 7307 & U2(RNU2) small nuclear RNA auxiliary factor 1 & 2,01 & chr15 \\
\hline UBE2E3 & 10477 & Ubiquitin-conjugating enzyme E2E 3 (UBC4/5 homolog, yeast) & 2,28 & chr2 \\
\hline UBE2H & 7328 & Ubiquitin-conjugating enzyme E2H (UBC8 homolog, yeast) & 2,75 & chr7 \\
\hline |UBE2I & 7329 & Ubiquitin-conjugating enzyme E2I (UBC9 homolog, yeast) & 2,47 & chr16 \\
\hline UBE2J1 & 51465 & ubiquitin-conjugating enzyme E2, J1 (UBC6 homolog, yeast) & 2,97 & chr6 \\
\hline UBE2R2 & 54926 & ubiquitin-conjugating enzyme E2R 2 & 2,65 & chr9 \\
\hline UBE3B & 89910 & ubiquitin protein ligase E3B & 2,05 & chr12 \\
\hline UBE3C & 9690 & Ubiquitin protein ligase E3C & 2,38 & $\operatorname{chr} 7$ \\
\hline UCP2 & 7351 & uncoupling protein 2 (mitochondrial, proton carrier) & 2,13 & chr11 \\
\hline |UGCGL2 & 55757 & UDP-glucose ceramide glucosyltransferase-like 2 & 2,46 & chr13 \\
\hline UHRF1 & 29128 & ubiquitin-like, containing PHD and RING finger domains, 1 & 2,29 & chr12 \\
\hline ULK2 & 9706 & |unc-51-like kinase 2 (C. elegans) & 3,51 & chr17 \\
\hline UNC84B & 25777 & unc-84 homolog B (C. elegans) & 2,07 & chr22 \\
\hline USP13 & 8975 & Ubiquitin specific peptidase 13 (isopeptidase T-3) & 3,56 & chr5 \\
\hline USP3 & 9960 & ubiquitin specific peptidase 3 & 10,29 & chr15 \\
\hline USP30 & 84749 & Ubiquitin specific peptidase 30 & 3,30 & chr12 \\
\hline |USP34 & 9736 & Ubiquitin specific peptidase 34 & 2,80 & chr2 \\
\hline |USP47 & 55031 & ubiquitin specific peptidase 47 & 3,03 & chr11 \\
\hline |USP49 & 25862 & Ubiquitin specific peptidase 49 & 3,11 & chr6 \\
\hline VAMP4 & 8674 & vesicle-associated membrane protein 4 & 2,40 & chr1 \\
\hline VASH1 & 22846 & vasohibin 1 & 2,51 & chr14 \\
\hline VCAM1 & 7412 & vascular cell adhesion molecule 1 & 3,00 & chr1 \\
\hline VEGFC & 7424 & vascular endothelial growth factor $\mathrm{C}$ & 2,77 & chr4 \\
\hline VGLL4 & 9686 & vestigial like 4 (Drosophila) & 2,40 & chr3 \\
\hline VprBP & 9730 & Vpr-binding protein & 2,07 & chr3 \\
\hline VPS13B & 157680 & vacuolar protein sorting 13B (yeast) & 2,56 & chr8 \\
\hline VPS13C & 54832 & Vacuolar protein sorting 13C (yeast) & 2,82 & chr15 \\
\hline |VPS24 & 51652 & vacuolar protein sorting 24 (yeast) & 3,12 & chr2 \\
\hline VTCN1 & 79679 & V-set domain containing $T$ cell activation inhibitor 1 & 5,12 & chr1 \\
\hline WASF1 & 8936 & WAS protein family, member 1 & 2,05 & chr6 \\
\hline WASF3 & 10810 & WAS protein family, member 3 & 4,31 & chr13 \\
\hline WASPIP & 7456 & Wiskott-Aldrich syndrome protein interacting protein & 4,75 & chr2 \\
\hline WDR1 & 9948 & WD repeat domain 1 & 2,59 & $\operatorname{chr} 4$ \\
\hline WDR13 & 64743 & WD repeat domain 13 & 2,20 & $\operatorname{chr} X$ \\
\hline WDR19 & 57728 & WD repeat domain 19 & 2,60 & chr4 \\
\hline WDR27 & 253769 & CDNA FLJ46815 fis, clone TRACH3036897 /// WD repeat domain 27 & 2,37 & chr6 \\
\hline WDR32 & 79269 & WD repeat domain 32 & 2,92 & chr9 \\
\hline WDR33 & 55339 & WD repeat domain 33 & 2,76 & chr2 \\
\hline WDR42A & 50717 & WD repeat domain 42A & 2,05 & chr1 \\
\hline WDR5B & 54554 & WD repeat domain $5 B$ & 2,08 & chr3 \\
\hline WDR61 & 80349 & WD repeat domain 61 & 2,14 & chr15 \\
\hline WDR68 & 10238 & WD repeat domain 68 & 2,07 & chr17 \\
\hline WDTC1 & 23038 & WD and tetratricopeptide repeats 1 & 2,17 & chr1 \\
\hline WDTC2 & 9742 & WD and tetratricopeptide repeats 2 & 2,08 & chr16 \\
\hline WFIKKN1 & 117166 & WAP, follistatin/kazal, immunoglobulin, kunitz and netrin domain containing 1 & 2,98 & chr16 \\
\hline WHSC1 & 7468 & Wolf-Hirschhorn syndrome candidate 1 & 5,42 & chr4 \\
\hline WHSC1L1 & 54904 & Wolf-Hirschhorn syndrome candidate 1 -like 1 & 2,23 & chr8 \\
\hline WNT2B & 7482 & wingless-type MMTV integration site family, member $2 \mathrm{~B}$ & 3,80 & chr1 \\
\hline WNT5A & 7474 & wingless-type MMTV integration site family, member $5 \mathrm{~A}$ & 5,15 & chr3 \\
\hline IT5B & 029 & wingless-type MMTV integration site family, member $5 B$, & 12,12 & chr12 \\
\hline
\end{tabular}




\begin{tabular}{|c|c|c|c|c|}
\hline Gene Symbol & Entrez Gene & Gene Title & NPC_up & Chromosome Number(Avadis) \\
\hline WSB1 & 26118 & WD repeat and SOCS box-containing 1 & 7,05 & chr17 \\
\hline WWOX & 51741 & B-box and SPRY domain containing & 2,51 & $\operatorname{chr} 16$ \\
\hline XPA & 7507 & xeroderma pigmentosum, complementation group $\mathrm{A}$ & 4,10 & chr9 \\
\hline XPR1 & 9213 & Xenotropic and polytropic retrovirus receptor & 2,32 & chr1 \\
\hline YAF2 & 10138 & YY1 associated factor 2 & 4,72 & chr12 \\
\hline YEATS2 & 55689 & YEATS domain containing 2 & 3,40 & chr3 \\
\hline YPEL1 & 29799 & yippee-like 1 (Drosophila) & 2,76 & chr22 \\
\hline YPEL2 & 388403 & yippee-like 2 (Drosophila) & 3,51 & chr17 \\
\hline YPEL5 & 51646 & yippee-like 5 (Drosophila) & 2,92 & chr2 \\
\hline ZADH2 & 284273 & zinc binding alcohol dehydrogenase, domain containing 2 & 6,70 & $\operatorname{chr} 18$ \\
\hline ZAK & 51776 & sterile alpha motif and leucine zipper containing kinase AZK & 2,45 & chr2 \\
\hline ZBTB10 & 65986 & Zinc finger and BTB domain containing 10 & 2,33 & chr8 \\
\hline ZBTB16 & 7704 & zinc finger and BTB domain containing 16 & 12,49 & chr11 \\
\hline ZBTB20 & 26137 & zinc finger and BTB domain containing 20 & 2,97 & chr3 \\
\hline ZBTB33 & 10009 & zinc finger and BTB domain containing 33 & 2,01 & $\operatorname{chr} X$ \\
\hline ZBTB4 & 57659 & zinc finger and BTB domain containing 4 & 2,08 & chr17 \\
\hline $\mathrm{ZC} 3 \mathrm{H} 11 \mathrm{~A}$ & 9877 & zinc finger $\mathrm{CCCH}$-type containing $11 \mathrm{~A}$ & 2,08 & chr1 \\
\hline $\mathrm{ZC} 3 \mathrm{H} 12 \mathrm{~B}$ & 340554 & zinc finger $\mathrm{CCCH}$-type containing 12B & 2,18 & $\operatorname{chrX}$ \\
\hline $\mathrm{ZC} 3 \mathrm{H} 12 \mathrm{C}$ & 85463 & zinc finger $\mathrm{CCCH}$-type containing $12 \mathrm{C}$ & 2,07 & chr11 \\
\hline $\mathrm{ZC} 3 \mathrm{H} 8$ & 84524 & Zinc finger $\mathrm{CCCH}$-type containing 8 & 2,17 & chr2 \\
\hline $\mathrm{ZCCHC} 11$ & 23318 & Zinc finger, $\mathrm{CCHC}$ domain containing 11 & 3,81 & chr1 \\
\hline ZCSL2 & 285381 & zinc finger, CSL-type containing 2 & 2,00 & chr3 \\
\hline ZCSL3 & 120526 & Zinc finger, CSL-type containing 3 & 2,67 & chr11 \\
\hline ZFAND3 & 60685 & Zinc finger, AN1-type domain 3 & 2,77 & chr6 \\
\hline ZFHX1B & 9839 & zinc finger homeobox $1 \mathrm{~b}$ & 3,92 & chr2 \\
\hline ZFHX4 & 79776 & zinc finger homeodomain 4 & 7,11 & chr8 \\
\hline ZFP90 & 146198 & Zinc finger protein 90 homolog (mouse) & 3,17 & chr16 \\
\hline ZFPM2 & 23414 & zinc finger protein, multitype 2 & 3,09 & chr8 \\
\hline ZFR & 51663 & Zinc finger RNA binding protein & 2,01 & chr5 \\
\hline ZFYVE16 & 9765 & zinc finger, FYVE domain containing 16 & 4,24 & chr5 \\
\hline $\mathrm{ZHX} 1$ & 11244 & zinc fingers and homeoboxes 1 & 5,85 & chr8 \\
\hline ZIC1 & 7545 & Zic family member 1 (odd-paired homolog, Drosophila) & 24,39 & chr3 \\
\hline ZKSCAN1 & 7586 & Zinc finger with KRAB and SCAN domains 1 & 4,07 & chr7 \\
\hline ZMYND11 & 10771 & zinc finger, MYND domain containing 11 & 2,46 & chr10 \\
\hline ZNF117 & 7670 & Zinc finger protein 117 (HPF9) & 5,39 & chr7 \\
\hline ZNF124 & 7678 & zinc finger protein 124 (HZF-16) & 3,45 & chr1 \\
\hline ZNF131 & 7690 & Zinc finger protein 131 (clone pHZ-10) & 2,04 & chr5 \\
\hline ZNF141 & 7700 & Zinc finger protein 141 (clone pHZ-44) & 2,37 & chr4 \\
\hline ZNF148 & 7707 & Zinc finger protein $148(\mathrm{pHZ}-52)$ & 3,57 & chr3 \\
\hline ZNF161 & 7716 & zinc finger protein 161 & 2,75 & chr3 \\
\hline ZNF177 & 7730 & zinc finger protein 177 & 2,53 & chr19 \\
\hline ZNF185 & 7739 & zinc finger protein 185 (LIM domain) & 2,31 & $\operatorname{chr} X$ \\
\hline ZNF193 & 7746 & zinc finger protein 193 & 2,42 & chr6 \\
\hline ZNF218 & 128553 & Zinc finger protein 218 & 2,30 & chr20 \\
\hline ZNF22 & 7570 & zinc finger protein 22 (KOX 15) & 2,16 & chr10 \\
\hline ZNF226 & 7769 & zinc finger protein 226 & 2,21 & chr19 \\
\hline ZNF264 & 9422 & zinc finger protein 264 & 2,17 & chr19 \\
\hline ZNF266 & 10781 & zinc finger protein 266 & 2,46 & chr19 \\
\hline ZNF274 & 10782 & zinc finger protein 274 & 2,12 & chr19 \\
\hline ZNF297B & 23099 & zinc finger protein 297B & 2,24 & chr9 \\
\hline ZNF302 & 55900 & zinc finger protein 302 & 2,20 & $\operatorname{chr} 19$ \\
\hline ZNF334 & 55713 & zinc finger protein 334 & 2,12 & chr20 \\
\hline ZNF343 & 79175 & zinc finger protein 343 & 2,21 & chr20 \\
\hline ZNF346 & 23567 & zinc finger protein 346 & 3,63 & chr5 \\
\hline ZNF354B & 117608 & Zinc finger protein 354B & 2,27 & chr5 \\
\hline ZNF37B & 256112 & zinc finger protein 37b (KOX 21) & 2,25 & chr10 \\
\hline ZNF390 & 222696 & zinc finger protein 390 & 2,16 & chr6 \\
\hline ZNF395 & 55893 & zinc finger protein 395 & 2,17 & chr8 \\
\hline ZNF410 & 57862 & zinc finger protein 410 & 2,36 & chr14 \\
\hline ZNF42 & 7593 & zinc finger protein 42 (myeloid-specific retinoic acid-responsive) & 2,01 & chr19 \\
\hline ZNF436 & 80818 & zinc finger protein 436 & 4,19 & chr1 \\
\hline ZNF44 & 51710 & Zinc finger protein 44 (KOX 7) & 3,06 & chr19 \\
\hline ZNF447 & 65982 & zinc finger protein 447 & 3,12 & chr19 \\
\hline ZNF452 & 114821 & zinc finger protein 452 & 2,01 & (vide) \\
\hline ZNF471 & 57573 & Zinc finger protein 471 & 2,20 & chr19 \\
\hline ZNF503 & 84858 & zinc finger protein 503 & 16,13 & chr10 \\
\hline ZNF510 & 22869 & Zinc finger protein 510 & 2,15 & chr9 \\
\hline ZNF514 & 4 & er prote & 3,44 & chr2 \\
\hline
\end{tabular}




\begin{tabular}{|l|c|l|c|c|}
\hline \multicolumn{2}{|l|}{ Table S2 : Genes overexpressed in NPC compared to hES (Fold Change > 2; $\alpha<0.05)$} & NPC_up & Chromosome Number(Avadis) \\
\hline Gene Symbol & Entrez Gene & Gene Title & 4,43 & chr18 \\
ZNF516 & 9658 & Zinc finger protein 516 & 2,33 & (vide) \\
ZNF518 & 9849 & Zinc finger protein 518 & 4,03 & chr18 \\
ZNF519 & 162655 & Zinc finger protein 519 & 13,21 & chr18 \\
ZNF521 & 25925 & Zinc finger protein 521 & 4,31 & chr2 \\
ZNF533 & 151126 & Zinc finger protein 533 & 3,33 & chr19 \\
ZNF544 & 27300 & Zinc finger protein 544 & 2,16 & chr19 \\
ZNF548 & 147694 & Zinc finger protein 548 & 2,39 & chr19 \\
ZNF580 & 51157 & Zinc finger protein 580 & 2,25 & chr19 \\
ZNF606 & 80095 & Zinc finger protein 606 & 2,55 & chr5 \\
ZNF608 & 57507 & Zinc finger protein 608 & 2,82 & chr9 \\
ZNF618 & 114991 & Zinc finger protein 618 & 2,13 & chr3 \\
ZNF621 & 285268 & zinc finger protein 621 & 2,66 & chr16 \\
ZNF629 & 23361 & Zinc finger protein 629 & 3,75 & chr12 \\
ZNF641 & 121274 & Zinc finger protein 641 & 4,39 & chr12 \\
ZNF664 & 144348 & Zinc finger protein 664 & 3,67 & chr22 \\
ZNF70 & 7621 & Zinc finger protein 70 (Cos17) & 4,24 & chr8 \\
ZNF703 & 80139 & Zinc finger protein 703 & 2,32 & chr19 \\
ZNF708 & 7562 & Zinc finger protein 708 (KOX8) & 2,86 & chr19 \\
ZNF709 & 163051 & Zinc finger protein 709 & 8,82 & chr15 \\
ZNF710 & 374655 & Zinc finger protein 710 & 3,79 & chr19 \\
ZNF83 & 55769 & Zinc finger protein 83 (HPF1) & 4,42 & chr12 \\
ZNF84 & 7637 & Zinc finger protein 84 (HPF2) & 2,89 & chr19 \\
ZNF91 & 7644 & Zinc finger protein 91 (HPF7, HTF10) & 2,10 & chr16 \\
ZNRF1 & 84937 & Zinc and ring finger 1 & 2,93 & chr7 \\
ZRF1 & 27000 & Zuotin related factor 1 & 5,08 & chr5 \\
ZSWIM6 & 57688 & Zinc finger, SWIM-type containing 6 & & \\
\hline
\end{tabular}




\begin{tabular}{|c|c|c|c|c|}
\hline Gene Symbol & Entrez Gene & Gene Title & NPC_down & Chromosome Number(Avadis) \\
\hline 37316 & 92935 & methionine-tRNA synthetase 2 (mitochondrial) & 2,04 & chr2 \\
\hline 37500 & 4735 & septin 2 & 2,17 & chr2 \\
\hline 38961 & 23157 & septin 6 & 2,96 & $\operatorname{chrX}$ \\
\hline AAMP & 14 & angio-associated, migratory cell protein & 2,36 & chr2 \\
\hline AARS & 16 & alanyl-tRNA synthetase & 2,24 & chr16 \\
\hline AASS & 10157 & aminoadipate-semialdehyde synthase & 6,49 & chr7 \\
\hline ABHD4 & 63874 & abhydrolase domain containing 4 & 2,19 & chr14 \\
\hline ABHD5 & 51099 & abhydrolase domain containing 5 & 3,19 & chr3 \\
\hline ABHD9 & 79852 & abhydrolase domain containing 9 & 5,63 & chr19 \\
\hline ACAA2 & 10449 & acetyl-Coenzyme A acyltransferase 2 (mitochondrial 3-oxoacyl-Coenzyme & 2,51 & chr18 \\
\hline ACAD8 & 27034 & acyl-Coenzyme A dehydrogenase family, member 8 & 2,11 & chr11 \\
\hline ACADSB & 36 & acyl-Coenzyme A dehydrogenase, short/branched chain & 2,18 & chr10 \\
\hline ACOT7 & 11332 & acyl-CoA thioesterase 7 & 2,37 & chr1 \\
\hline АСОТ & 10005 & Acyl-CoA thioesterase 8 & 3,12 & chr20 \\
\hline ACOX1 & 51 & acyl-Coenzyme A oxidase 1, palmitoyl & 3,16 & chr17 \\
\hline ACSL1 & 2180 & acyl-CoA synthetase long-chain family member 1 & 2,48 & chr4 \\
\hline ACTB & 60 & actin, beta & 2,34 & (vide) \\
\hline ACY1 & 95 & aminoacylase 1 & 2,59 & chr3 \\
\hline AD-003 & 28989 & AD-003 protein & 2,48 & chr9 \\
\hline ADAMTS8 & 11095 & ADAM metallopeptidase with thrombospondin type 1 motif, 8 & 4,79 & chr11 \\
\hline ADCY2 & 108 & adenylate cyclase 2 (brain) & 15,50 & chr5 \\
\hline ADD2 & 119 & adducin 2 (beta) & 4,26 & chr2 \\
\hline ADM & 133 & adrenomedullin & 2,02 & chr11 \\
\hline ADRA2A & 150 & adrenergic, alpha-2A-, receptor /// adrenergic, alpha-2A-, receptor & 3,05 & chr10 \\
\hline ADRM1 & 11047 & adhesion regulating molecule 1 & 3,40 & chr20 \\
\hline AER61 & 285203 & AER61 glycosyltransferase & 2,95 & chr3 \\
\hline AFG3L2 & 10939 & AFG3 ATPase family gene 3 -like 2 (yeast) & 2,56 & chr18 \\
\hline AGPS & 8540 & Alkylglycerone phosphate synthase & 2,08 & chr2 \\
\hline AGTRAP & 57085 & angiotensin II receptor-associated protein & 2,26 & chr1 \\
\hline AIM1 & 202 & absent in melanoma 1 & 8,04 & chr6 \\
\hline AK2 & 204 & adenylate kinase 2 & 2,32 & chr1 \\
\hline AK3L1 & 205 & adenylate kinase 3-like 1 & 3,20 & chr1 \\
\hline AK3L1 & $205 / / / 387851$ & adenylate kinase 3 -like $1 / / /$ adenylate kinase 3 -like 2 & 4,00 & chr1 \\
\hline AKAP1 & 8165 & A kinase (PRKA) anchor protein 1 & 2,21 & chr17 \\
\hline AKAP7 & 9465 & A kinase (PRKA) anchor protein 7 & 2,66 & chr6 \\
\hline ALDOA & 226 & aldolase $\mathrm{A}$, fructose-bisphosphate & 2,39 & chr16 \\
\hline ALDOC & 230 & aldolase C, fructose-bisphosphate & 3,69 & chr17 \\
\hline ALG3 & 10195 & asparagine-linked glycosylation 3 homolog (yeast, alpha-1,3-mannosyltrar & 2,21 & chr3 \\
\hline ALMS1 & 7840 & Alstrom syndrome 1 & 3,31 & chr2 \\
\hline AMID & 84883 & apoptosis-inducing factor (AIF)-like mitochondrion-associated inducer of $d$ & 2,13 & chr10 \\
\hline AMMECR1 & 9949 & Alport syndrome, mental retardation, midface hypoplasia and elliptocytosi & 2,30 & $\operatorname{chr} \mathrm{X}$ \\
\hline AMSH-LP & 57559 & associated molecule with the SH3 domain of STAM (AMSH) like protein & 2,86 & chr10 \\
\hline ANGEL2 & 90806 & Angel homolog 2 (Drosophila) & 8,58 & chr1 \\
\hline ANKHD1 & 54882 & ankyrin repeat and $\mathrm{KH}$ domain containing 1 & 3,44 & chr5 \\
\hline ANKRD11 & 29123 & ankyrin repeat domain 11 & 2,09 & chr16 \\
\hline ANKRD39 & 51239 & ankyrin repeat domain 39 & 2,78 & chr2 \\
\hline ANKRD41 & 126549 & ankyrin repeat domain 41 & 2,22 & chr19 \\
\hline ANP32A & 8125 & Acidic (leucine-rich) nuclear phosphoprotein 32 family, member A & 2,14 & chr15 \\
\hline ANXA4 & 307 & annexin A4 & 2,67 & chr2 \\
\hline AP1M2 & 10053 & adaptor-related protein complex 1 , mu 2 subunit & 6,96 & chr19 \\
\hline AP1S3 & 130340 & Adaptor-related protein complex 1 , sigma 3 subunit & 2,14 & chr2 \\
\hline AP2S1 & 1175 & adaptor-related protein complex 2 , sigma 1 subunit & 2,04 & chr19 \\
\hline APBB2 & 323 & amyloid beta (A4) precursor protein-binding, family B, member 2 (Fe65-lik & 2,24 & chr4 \\
\hline AРОВЕСЗВ & 9582 & apolipoprotein B mRNA editing enzyme, catalytic polypeptide-like $3 B$ & 2,97 & chr22 \\
\hline APOC1 & 341 & apolipoprotein C-I & 3,35 & chr19 \\
\hline APOE & 348 & apolipoprotein E & 3,65 & chr19 \\
\hline APRT & 353 & adenine phosphoribosyltransferase & 2,63 & chr16 \\
\hline ARF4 & 378 & ADP-ribosylation factor 4 & 2,25 & chr3 \\
\hline ARF6 & 382 & ADP-ribosylation factor 6 & 2,63 & chr14 \\
\hline ARFGEF1 & 10565 & ADP-ribosylation factor guanine nucleotide-exchange factor 1 (brefeldin $A$ & 2,46 & chr8 \\
\hline ARHGAP8 & $23779 / / / 553158$ & Rho GTPase activating protein 8 /// PRR5-ARHGAP8 fusion & 4,84 & chr22 \\
\hline ARHGEF5 & 7984 & Rho guanine nucleotide exchange factor (GEF) 5 & 2,72 & chr7 \\
\hline ARHGEF9 & 23229 & Cdc42 guanine nucleotide exchange factor (GEF) 9 & 3,08 & $\operatorname{chr} X$ \\
\hline ARIH2 & 10425 & ariadne homolog 2 (Drosophila) & 2,08 & chr3 \\
\hline ARL8 & 221079 & ADP-ribosylation factor-like 8 & 2,15 & chr10 \\
\hline ARPC5L & 81873 & actin related protein $2 / 3$ complex, subunit 5 -like & 2,33 & chr9 \\
\hline
\end{tabular}




\begin{tabular}{|c|c|c|c|c|}
\hline Gene Symbol & Entrez Gene & Gene Title & NPC_down & Chromosome Number(Avadis) \\
\hline ASAHL & 27163 & $\mathrm{~N}$-acylsphingosine amidohydrolase (acid ceramidase)-like & 2,29 & chr4 \\
\hline ASS & 445 & argininosuccinate synthetase & 7,38 & chr5 \\
\hline ATF1 & 466 & activating transcription factor 1 & 2,38 & chr12 \\
\hline ATG3 & 64422 & ATG3 autophagy related 3 homolog (S. cerevisiae) & 2,62 & chr3 \\
\hline ATG5 & 9474 & ATG5 autophagy related 5 homolog (S. cerevisiae) & 2,22 & chr6 \\
\hline ATP5G1 & 516 & ATP synthase, $\mathrm{H}_{+}$transporting, mitochondrial F0 complex, subunit c (sub & 2,93 & chr17 \\
\hline ATP5G3 & 518 & ATP synthase, $\mathrm{H}_{+}$transporting, mitochondrial F0 complex, subunit c (sub & 2,50 & chr2 \\
\hline ATP5H & 10476 & ATP synthase, $\mathrm{H}+$ transporting, mitochondrial F0 complex, subunit $d$ & 2,40 & chr9 \\
\hline ATP5I & 521 & ATP synthase, $\mathrm{H}+$ transporting, mitochondrial F0 complex, subunit e & 2,51 & chr4 \\
\hline ATP6V1C1 & 528 & ATPase, $\mathrm{H}+$ transporting, lysosomal $42 \mathrm{kDa}$, V1 subunit $\mathrm{C}$, isoform 1 & 3,29 & chr8 \\
\hline ATPBD1B & 54707 & ATP binding domain 1 family, member $B$ & 2,23 & chr1 \\
\hline AURKAIP1 & 54998 & aurora kinase $\mathrm{A}$ interacting protein 1 & 2,94 & chr1_random \\
\hline AVPI1 & 60370 & arginine vasopressin-induced 1 & 2,08 & chr10 \\
\hline B3GNT7 & 93010 & |UDP-GIcNAc:betaGal beta-1,3-N-acetylglucosaminyltransferase 7 & 7,97 & chr2 \\
\hline B4GALT4 & 8702 & UDP-Gal:betaGIcNAc beta 1,4- galactosyltransferase, polypeptide 4 & 2,26 & chr3 \\
\hline BAG2 & 9532 & BCL2-associated athanogene 2 & 4,88 & chr6 \\
\hline BAG4 & 9530 & BCL2-associated athanogene 4 & 2,02 & chr8 \\
\hline BAT2D1 & 23215 & BAT2 domain containing 1 & 2,02 & chr1 \\
\hline BAX & 581 & BCL2-associated $X$ protein & 3,17 & chr19 \\
\hline BCCIP & 56647 & BRCA2 and CDKN1A interacting protein & 2,37 & chr10 \\
\hline BCL11A & 53335 & B-cell CLL/lymphoma 11A (zinc finger protein) & 4,92 & chr2 \\
\hline BCL11B & 64919 & B-cell CLL/lymphoma 11B (zinc finger protein) & 3,88 & chr14 \\
\hline BCL2L11 & 10018 & BCL2-like 11 (apoptosis facilitator) & 3,29 & chr2 \\
\hline BCL2L12 & 83596 & BCL2-like 12 (proline rich) & 2,47 & chr19 \\
\hline BCOR & 54880 & BCL6 co-repressor & 3,24 & $\operatorname{chr} X$ \\
\hline BDP1 & 55814 & B double prime 1 , subunit of RNA polymerase III transcription initiation fad & 2,53 & chr5 \\
\hline BICD1 & 636 & bicaudal D homolog 1 (Drosophila) & 4,31 & chr12 \\
\hline BLVRA & 644 & biliverdin reductase $\mathrm{A}$ & 2,02 & chr7 \\
\hline BLVRB & 645 & biliverdin reductase $B$ (flavin reductase (NADPH)) & 2,47 & chr19 \\
\hline BMPR2 & 659 & bone morphogenetic protein receptor, type II (serine/threonine kinase) & 2,18 & chr2 \\
\hline BNC2 & 54796 & Basonuclin 2 & 2,54 & chr9 \\
\hline BOLA2 & 552900 & bolA-like 2 (E. coli) & 2,82 & chr16 \\
\hline BOLA3 & 388962 & bolA-like 3 (E. coli) & 2,09 & chr2 \\
\hline BOP1 & 23246 & block of proliferation 1 & 2,58 & chr8 \\
\hline BRAF & 673 & v-raf murine sarcoma viral oncogene homolog B1 & 2,03 & chr7 \\
\hline BRWD3 & 254065 & bromodomain and WD repeat domain containing 3 & 2,22 & $\operatorname{chr} X$ \\
\hline BSCL2 & 221092 /// 26580 & Bernardinelli-Seip congenital lipodystrophy 2 (seipin) /// heterogeneous nu & 2,32 & chr11 \\
\hline BSPRY & 54836 & B-box and SPRY domain containing & 2,33 & chr9 \\
\hline BTBD15 & 29068 & BTB (POZ) domain containing 15 & 2,17 & chr11 \\
\hline BTBD7 & 55727 & BTB (POZ) domain containing 7 & 3,07 & chr14 \\
\hline BTD & 686 & biotinidase & 2,34 & chr3 \\
\hline BUB1 & 699 & BUB1 budding uninhibited by benzimidazoles 1 homolog (yeast) & 2,78 & chr2 \\
\hline BXDC1 & 84154 & brix domain containing 1 & 2,04 & chr6 \\
\hline BYSL & 705 & bystin-like & 2,15 & chr6 \\
\hline C10orf42 & 90550 & chromosome 10 open reading frame 42 & 2,05 & chr10 \\
\hline C10orf47 & 254427 & chromosome 10 open reading frame 47 & 2,10 & chr10 \\
\hline C10orf70 & 55847 & chromosome 10 open reading frame 70 & 2,11 & chr10 \\
\hline C10orf77 & 79847 & chromosome 10 open reading frame 77 & 2,08 & chr10 \\
\hline C10orf95 & $54808 / / / 79946$ & Chromosome 10 open reading frame $95 / / /$ Dymeclin & 3,30 & chr10 \\
\hline C11orf32 & 442871 & chromosome 11 open reading frame 32 & 2,69 & chr11 \\
\hline C12orf10 & 60314 & chromosome 12 open reading frame 10 & 2,16 & chr12 \\
\hline C12orf11 & 55726 & chromosome 12 open reading frame 11 & 2,08 & chr12 \\
\hline C13orf3 & 221150 & chromosome 13 open reading frame 3 & 2,32 & chr13 \\
\hline C14orf115 & 55237 & chromosome 14 open reading frame 115 & 7,70 & chr14 \\
\hline C14orf122 & 51016 & chromosome 14 open reading frame 122 & 2,28 & chr14 \\
\hline C14orf150 & 112840 & chromosome 14 open reading frame 150 & 2,08 & chr14 \\
\hline C14orf151 & 84800 & chromosome 14 open reading frame $151 / / /$ chromosome 14 open readin & 2,04 & chr14 \\
\hline C14orf156 & 81892 & chromosome 14 open reading frame $156 / / /$ chromosome 14 open readin & 2,26 & chr14 \\
\hline C15orf25 & 55142 & chromosome 15 open reading frame 25 & 2,25 & chr15 \\
\hline C16orf35 & 8131 & chromosome 16 open reading frame 35 & 2,07 & chr16 \\
\hline C17orf39 & 79018 & chromosome 17 open reading frame 39 & 2,20 & chr17 \\
\hline C19orf25 & 148223 & chromosome 19 open reading frame 25 & 2,08 & chr19 \\
\hline C19orf33 & 64073 & chromosome 19 open reading frame 33 & 2,81 & chr19 \\
\hline C1GALT1 & 56913 & core 1 synthase, glycoprotein-N-acetylgalactosamine 3-beta-galactosyltra & 2,88 & chr7 \\
\hline C1orf108 & 79647 & chromosome 1 open reading frame 108 & 4,91 & chr1 \\
\hline C1orf121 & 51029 & |chromosome 1 open reading frame 121 & 4,24 & chr1 \\
\hline
\end{tabular}




\begin{tabular}{|c|c|c|c|c|}
\hline Gene Symbol & Entrez Gene & Gene Title & NPC_down & Chromosome Number(Avadis) \\
\hline C1orf135 & 79000 & chromosome 1 open reading frame 135 & 2,13 & chr1 \\
\hline C1orf163 & 65260 & chromosome 1 open reading frame 163 & 3,02 & chr1 \\
\hline C1orf25 & 81627 & chromosome 1 open reading frame 25 & 2,25 & chr1 \\
\hline C1orf31 & 388753 & chromosome 1 open reading frame 31 & 2,19 & chr1 \\
\hline C1orf43 & 25912 & chromosome 1 open reading frame 43 & 6,30 & chr1 \\
\hline C1orf59 & 113802 & chromosome 1 open reading frame 59 & 3,56 & chr1 \\
\hline C1orf67 & 200095 & chromosome 1 open reading frame 67 & 3,05 & chr1 \\
\hline C1orf69 & 200205 & chromosome 1 open reading frame 69 & 2,30 & chr1 \\
\hline C1orf77 & 26097 & chromosome 1 open reading frame 77 & 2,04 & chr1 \\
\hline C1orf97 & 84791 & chromosome 1 open reading frame $97 / / /$ chromosome 1 open reading fra & 3,59 & chr1 \\
\hline C1QBP & 708 & Complement component 1 , q subcomponent binding protein & 2,92 & chr17 \\
\hline C20orf11 & 54994 & chromosome 20 open reading frame 11 & 2,16 & chr20 \\
\hline C20orf118 & 140711 & Chromosome 20 open reading frame 118 & 8,42 & chr20 \\
\hline C20orf19 & 55857 & chromosome 20 open reading frame 19 & 2,65 & chr20 \\
\hline C20orf31 & 55741 & chromosome 20 open reading frame 31 & 2,68 & chr20 \\
\hline C20orf35 & 55861 & chromosome 20 open reading frame 35 & 3,34 & chr20 \\
\hline C20orf42 & 55612 & chromosome 20 open reading frame 42 & 7,93 & chr20 \\
\hline C20orf6 & 51575 & chromosome 20 open reading frame 6 & 2,57 & chr20 \\
\hline C20orf7 & 79133 & chromosome 20 open reading frame 7 & 2,08 & chr20 \\
\hline C21 orf45 & 54069 & chromosome 21 open reading frame 45 & 2,05 & chr21 \\
\hline C22orf16 & 400916 & chromosome 22 open reading frame 16 & 3,78 & chr22 \\
\hline $\mathrm{C} 2 \mathrm{~F}$ & 10436 & C2f protein & 3,21 & chr12 \\
\hline $\mathrm{C} 3 \mathrm{~F}$ & 10162 & putative protein similar to nessy (Drosophila) & 2,56 & chr12 \\
\hline C3orf14 & 57415 & chromosome 3 open reading frame 14 & 2,29 & chr3 \\
\hline C5orf18 & 7905 & chromosome 5 open reading frame 18 & 2,65 & chr5 \\
\hline C6orf117 & 112609 & chromosome 6 open reading frame 117 & 9,75 & chr6 \\
\hline C6orf150 & 115004 & chromosome 6 open reading frame 150 & 2,12 & chr6 \\
\hline C6orf189 & 221303 & chromosome 6 open reading frame 189 & 2,21 & chr6 \\
\hline C6orf192 & 116843 & chromosome 6 open reading frame 192 & 2,41 & chr6 \\
\hline C6orf211 & 79624 & chromosome 6 open reading frame 211 & 2,19 & chr6 \\
\hline C6orf55 & 51534 & chromosome 6 open reading frame 55 & 2,34 & chr6 \\
\hline C6orf62 & 81688 & chromosome 6 open reading frame 62 & 2,14 & chr6 \\
\hline C6orf66 & 29078 & chromosome 6 open reading frame 66 & 2,97 & chr6 \\
\hline C7orf20 & 51608 & chromosome 7 open reading frame 20 & 2,00 & chr7 \\
\hline C7orf28A & $221960 / / / 51622$ & chromosome 7 open reading frame $28 \mathrm{~A} / / /$ chromosome 7 open reading f & 2,42 & chr3 \\
\hline C7orf30 & 115416 & chromosome 7 open reading frame 30 & 2,05 & chr7 \\
\hline C8orf38 & 137682 & chromosome 8 open reading frame 38 & 2,34 & chr8 \\
\hline C8orf42 & 157695 & Chromosome 8 open reading frame 42 & 2,07 & chr8 \\
\hline C9orf41 & 138199 & chromosome 9 open reading frame 41 & 2,26 & chr9 \\
\hline C9orf82 & 79886 & chromosome 9 open reading frame 82 & 2,01 & chr9 \\
\hline CABYR & 26256 & calcium binding tyrosine-(Y)-phosphorylation regulated (fibrousheathin 2) & 2,27 & chr18 \\
\hline CACYBP & 27101 & calcyclin binding protein & 3,07 & chr1 \\
\hline CADPS2 & 93664 & Ca2+-dependent activator protein for secretion 2 & 2,86 & chr7 \\
\hline CALB1 & 793 & calbindin $1,28 \mathrm{kDa}$ & 6,00 & chr8 \\
\hline CALM3 & 808 & calmodulin 3 (phosphorylase kinase, delta) & 2,37 & chr19 \\
\hline CALN1 & 83698 & Calneuron 1 & 3,54 & chr7 \\
\hline CALU & 813 & calumenin & 2,00 & chr7 \\
\hline CAMKV & 79012 & CaM kinase-like vesicle-associated & 2,99 & chr3 \\
\hline CANX & 821 & calnexin & 2,24 & chr5 \\
\hline CAPG & 822 & capping protein (actin filament), gelsolin-like & 6,00 & chr2 \\
\hline CAPN1 & 823 & calpain 1 , (mu/l) large subunit & 2,60 & chr11 \\
\hline CAPZB & 832 & capping protein (actin filament) muscle Z-line, beta & 2,03 & chr1 \\
\hline CARD11 & 84433 & caspase recruitment domain family, member 11 & 3,90 & chr7 \\
\hline CARHSP1 & 23589 & calcium regulated heat stable protein $1,24 \mathrm{kDa}$ & 2,59 & chr16 \\
\hline CASP3 & 836 & caspase 3 , apoptosis-related cysteine peptidase & 2,31 & chr4 \\
\hline CBR1 & 873 & carbonyl reductase 1 & 6,13 & chr21 \\
\hline CBR3 & 874 & carbonyl reductase 3 & 4,42 & chr21 \\
\hline CCDC4 & 389206 & coiled-coil domain containing 4 & 4,98 & chr4 \\
\hline CCNA1 & 8900 & cyclin A1 & 2,06 & chr13 \\
\hline CCNA2 & 890 & cyclin A2 & 2,16 & chr4 \\
\hline CCND2 & 894 & Cyclin D2 & 2,79 & chr12 \\
\hline CCNF & 899 & cyclin F & 2,60 & chr16 \\
\hline CCRN4L & 25819 & CCR4 carbon catabolite repression 4-like (S. cerevisiae) & 2,27 & chr4 \\
\hline ССТ2 & 10576 & chaperonin containing TCP1, subunit 2 (beta) & 2,11 & chr12 \\
\hline CD164 & 8763 & CD164 antigen, sialomucin & 2,47 & chr6 \\
\hline CD24 & 934 & CD24 antigen (small cell lung carcinoma cluster 4 antigen) & 15,01 & chr5 \\
\hline
\end{tabular}




\begin{tabular}{|c|c|c|c|c|}
\hline Gene Symbol & Entrez Gene & Gene Title & NPC_down & Chromosome Number(Avadis) \\
\hline CD2AP & 23607 & CD2-associated protein & 2,45 & chr6 \\
\hline CD320 & 51293 & CD320 antigen & 2,22 & chr19 \\
\hline CD3EAP & 10849 & CD3E antigen, epsilon polypeptide associated protein & 2,65 & chr19 \\
\hline CD9 & 928 & CD9 antigen (p24) & 9,84 & chr12 \\
\hline CDC37L1 & 55664 & CDC37 cell division cycle 37 homolog (S. cerevisiae)-like 1 & 2,81 & chr9 \\
\hline CDCA2 & 157313 & cell division cycle associated 2 & 2,05 & chr8 \\
\hline CDCA7L & 55536 & cell division cycle associated 7 -like & 2,71 & $\operatorname{chr} 7$ \\
\hline CDCP1 & 64866 & CUB domain containing protein 1 & 3,37 & chr3 \\
\hline $\mathrm{CDH} 1$ & 999 & cadherin 1 , type 1, E-cadherin (epithelial) & 3,71 & chr16 \\
\hline $\mathrm{CDH} 3$ & 1001 & cadherin 3 , type 1 , P-cadherin (placental) & 2,37 & chr16 \\
\hline CDK2AP2 & 10263 & CDK2-associated protein 2 & 2,37 & chr11 \\
\hline CDK5 & 1020 & cyclin-dependent kinase 5 & 2,01 & chr8 \\
\hline CDK6 & 1021 & cyclin-dependent kinase 6 & 3,10 & chr7 \\
\hline CDK7 & 1022 & cyclin-dependent kinase 7 (MO15 homolog, Xenopus laevis, cdk-activatin & 2,23 & chr5 \\
\hline CDKL5 & 6792 & Cyclin-dependent kinase-like 5 & 3,07 & $\operatorname{chr} x$ \\
\hline CEBPZ & 10153 & CCAAT/enhancer binding protein zeta & 3,32 & chr2 \\
\hline CECR2 & 27443 & cat eye syndrome chromosome region, candidate 2 & 2,39 & chr22 \\
\hline CENTB1 & 9744 & centaurin, beta 1 & 2,05 & chr17 \\
\hline CGI-115 & 51018 & CGI-115 protein & 2,41 & chr1 \\
\hline CGI-37 & 51388 & comparative gene identification transcript 37 & 2,04 & chr16 \\
\hline CGI-69 & 51629 & CGI-69 protein & 2,15 & chr17 \\
\hline CGNL1 & 84952 & cingulin-like 1 & 4,15 & chr15 \\
\hline $\mathrm{CHCHD} 4$ & 131474 & coiled-coil-helix-coiled-coil-helix domain containing 4 & 2,36 & chr3 \\
\hline $\mathrm{CHCHD7}$ & 79145 & coiled-coil-helix-coiled-coil-helix domain containing 7 & 3,05 & chr8 \\
\hline CHEK1 & 1111 & CHK1 checkpoint homolog (S. pombe) & 2,23 & chr11 \\
\hline CHEK2 & 11200 & CHK2 checkpoint homolog (S. pombe) & 2,31 & chr22 \\
\hline CHES1 & 1112 & checkpoint suppressor 1 & 2,22 & chr14 \\
\hline CHFR & 55743 & checkpoint with forkhead and ring finger domains & 2,40 & chr12 \\
\hline CHGB & 1114 & chromogranin B (secretogranin 1) & 2,32 & chr20 \\
\hline CHIC2 & 26511 & cysteine-rich hydrophobic domain 2 & 2,33 & chr4 \\
\hline CHKA & 1119 & choline kinase alpha & 2,08 & chr11 \\
\hline CHPT1 & 56994 & choline phosphotransferase 1 & 2,01 & chr12 \\
\hline CHST4 & 10164 & carbohydrate (N-acetylglucosamine 6-O) sulfotransferase 4 & 7,93 & chr16 \\
\hline CHST7 & 56548 & carbohydrate (N-acetylglucosamine 6-O) sulfotransferase 7 & 5,10 & $\operatorname{chr} X$ \\
\hline CHURC1 & 91612 & churchill domain containing 1 & 2,05 & chr1 \\
\hline CIRH1A & 84916 & cirrhosis, autosomal recessive $1 \mathrm{~A}$ (cirhin) & 2,18 & chr16 \\
\hline CKMT1B & $1159 / / / 548596$ & creatine kinase, mitochondrial 1B /// creatine kinase, mitochondrial $1 \mathrm{~A}$ & 10,16 & chr15 \\
\hline CLCC1 & 23155 & chloride channel CLIC-like 1 & 2,38 & chr1 \\
\hline CLDN10 & 9071 & claudin 10 & 2,04 & chr13 \\
\hline CLDN23 & 137075 & claudin 23 & 2,16 & chr8 \\
\hline CLDN6 & 9074 & claudin 6 & 6,34 & chr1 \\
\hline CLDN7 & 1366 & claudin 7 & 4,38 & chr17 \\
\hline CLIC4 & 25932 & chloride intracellular channel 4 & 4,79 & chr1 \\
\hline CLN8 & 2055 & ceroid-lipofuscinosis, neuronal 8 (epilepsy, progressive with mental retard & 2,50 & chr8 \\
\hline COBL & 23242 & cordon-bleu homolog (mouse) & 6,85 & chr7 \\
\hline COL9A3 & 1299 & collagen, type IX, alpha 3 & 2,94 & chr20 \\
\hline COMMD7 & 149951 & COMM domain containing 7 & 2,26 & chr20 \\
\hline COMTD1 & 118881 & catechol-O-methyltransferase domain containing 1 & 4,63 & chr10 \\
\hline COQ3 & 51805 & coenzyme Q3 homolog, methyltransferase (yeast) & 2,28 & chr6 \\
\hline COX5B & 1329 & cytochrome $\mathrm{c}$ oxidase subunit $\mathrm{Vb}$ & 2,15 & chr2 \\
\hline COX6A1 & 1337 & cytochrome c oxidase subunit Vla polypeptide 1 /// cytochrome c oxidase & 9,62 & (vide) \\
\hline Cox7B & 1349 & cytochrome c oxidase subunit VIllb & 2,17 & $\operatorname{chr} x$ \\
\hline CPT1A & 1374 & carnitine palmitoyltransferase $1 \mathrm{~A}$ (liver) & 3,19 & chr11 \\
\hline CRB3 & 92359 & crumbs homolog 3 (Drosophila) & 2,25 & chr19 \\
\hline CRK7 & 51755 & CDC2-related protein kinase 7 & 2,06 & chr17 \\
\hline CRSP2 & 9282 & cofactor required for $\mathrm{Sp} 1$ transcriptional activation, subunit 2, 150kDa & 2,01 & $\operatorname{chr} \mathrm{X}$ \\
\hline CRTAP & 10491 & cartilage associated protein & 2,22 & chr3 \\
\hline CRYM & 1428 & crystallin, mu & 3,28 & chr16 \\
\hline CSDA & 8531 & cold shock domain protein $\mathrm{A}$ & 2,46 & chr12 \\
\hline CSNK1E & 1454 & Casein kinase 1 , epsilon & 2,32 & chr22 \\
\hline CSNK2A1 & 1457 & casein kinase 2, alpha 1 polypeptide & 3,50 & chr20 \\
\hline CSPG5 & 10675 & chondroitin sulfate proteoglycan 5 (neuroglycan C) & 2,00 & chr3 \\
\hline CSTF1 & 1477 & cleavage stimulation factor, 3 pre-RNA, subunit $1,50 \mathrm{kDa}$ & 2,27 & chr20 \\
\hline CSTF2 & 1478 & cleavage stimulation factor, 3 ' pre-RNA, subunit $2,64 \mathrm{kDa}$ & 2,49 & $\operatorname{chr} x$ \\
\hline CTBP2 & 1488 & C-terminal binding protein 2 & 2,12 & chr5 \\
\hline CTNNA1 & 1495 & Catenin (cadherin-associated protein), alpha 1, 102kDa & 2,16 & chr5 \\
\hline
\end{tabular}




\begin{tabular}{|c|c|c|c|c|}
\hline Gene Symbol & Entrez Gene & Gene Title & NPC_down & Chromosome Number(Avadis) \\
\hline CTPS2 & 56474 & CTP synthase II & 3,06 & $\operatorname{chr} \mathrm{X}$ \\
\hline CTSC & 1075 & cathepsin C & 5,33 & chr11 \\
\hline CTSD & 1509 & cathepsin D (lysosomal aspartyl peptidase) & 2,07 & chr11 \\
\hline CTTN & 2017 & cortactin & 2,25 & chr11 \\
\hline CUL4A & 8451 & Cullin 4A & 2,30 & chr13 \\
\hline CXADR & 1525 & coxsackie virus and adenovirus receptor & 2,26 & chr21 \\
\hline CXCL12 & 6387 & chemokine (C-X-C motif) ligand 12 (stromal cell-derived factor 1) & 4,19 & $\operatorname{chr} 10$ \\
\hline CXCL5 & 6374 & chemokine (C-X-C motif) ligand 5 & 5,46 & chr4 \\
\hline CXCL6 & 6372 & chemokine (C-X-C motif) ligand 6 (granulocyte chemotactic protein 2) & 2,39 & chr4 \\
\hline CXorf15 & 55787 & chromosome $\mathrm{X}$ open reading frame 15 & 2,21 & $\operatorname{chr} X$ \\
\hline cXorf26 & 51260 & chromosome $\mathrm{X}$ open reading frame 26 & 2,38 & $\operatorname{chr} X$ \\
\hline cXorf39 & 139231 & Chromosome $\mathrm{X}$ open reading frame 39 & 2,36 & $\operatorname{chr} \mathrm{X}$ \\
\hline CYB5 & 1528 & cytochrome b-5 & 2,55 & chr18 \\
\hline CYB5R2 & 51700 & cytochrome b5 reductase 2 & 2,28 & chr11 \\
\hline CYBA & 1535 & cytochrome b-245, alpha polypeptide & 2,07 & chr16 \\
\hline CYP2B7P1 & 1556 & cytochrome P450, family 2, subfamily B, polypeptide 7 pseudogene 1 & 2,44 & chr19 \\
\hline CYP2S1 & 29785 & cytochrome P450, family 2, subfamily S, polypeptide 1 & 8,51 & chr19 \\
\hline CYP4X1 & 260293 & cytochrome P450, family 4, subfamily X, polypeptide 1 & 2,77 & chr1 \\
\hline D15Wsu75e & 27351 & DNA segment, Chr 15, Wayne State University 75, expressed & 2,20 & chr22 \\
\hline D4S234E & 27065 & DNA segment on chromosome 4 (unique) 234 expressed sequence & 2,46 & chr4 \\
\hline DAB1 & $115209 / / / 1600$ & disabled homolog 1 (Drosophila) /// OMA1 homolog, zinc metallopeptidase & 2,78 & chr1 \\
\hline DAF & 1604 & decay accelerating factor for complement (CD55, Cromer blood group sys & 3,89 & chr1 \\
\hline DATF1 & 11083 & death associated transcription factor 1 & 3,24 & chr20 \\
\hline DAZAP1 & 26528 & DAZ associated protein 1 & 2,19 & chr19 \\
\hline DBC1 & 1620 & deleted in bladder cancer 1 & 7,99 & chr9 \\
\hline DCAMKL1 & 9201 & doublecortin and CaM kinase-like 1 & 5,60 & chr13 \\
\hline DCPS & 28960 & decapping enzyme, scavenger & 2,54 & chr11 \\
\hline DCUN1D5 & 84259 & DCN1, defective in cullin neddylation 1 , domain containing 5 (S. cerevisia & 2,03 & chr11 \\
\hline DCXR & 51181 & dicarbonyl/L-xylulose reductase & 2,53 & chr17 \\
\hline DDT & 1652 & D-dopachrome tautomerase & 4,84 & chr22 \\
\hline DDX18 & 8886 & DEAD (Asp-Glu-Ala-Asp) box polypeptide 18 & 2,50 & chr2 \\
\hline DDX25 & 29118 & DEAD (Asp-Glu-Ala-Asp) box polypeptide 25 & 2,32 & chr11 \\
\hline DDX3X & 1654 & DEAD (Asp-Glu-Ala-Asp) box polypeptide 3, X-linked & 2,68 & $\operatorname{chr} \mathrm{X}$ \\
\hline DDX58 & 23586 & DEAD (Asp-Glu-Ala-Asp) box polypeptide 58 & 2,05 & chr9 \\
\hline DENND2C & 163259 & DENN/MADD domain containing $2 \mathrm{C}$ & 2,83 & chr1 \\
\hline DENR & 8562 & density-regulated protein & 2,68 & chr12 \\
\hline DERL1 & 79139 & Der1-like domain family, member 1 & 2,00 & chr8 \\
\hline DFFA & 1676 & DNA fragmentation factor, $45 \mathrm{kDa}$, alpha polypeptide & 2,10 & chr1 \\
\hline DHCR7 & 1717 & 7-dehydrocholesterol reductase & 2,49 & chr11 \\
\hline DHFR & 1719 & dihydrofolate reductase & 2,19 & chr4 \\
\hline DIAPH2 & 1730 & diaphanous homolog 2 (Drosophila) & 3,20 & $\operatorname{chr} X$ \\
\hline DIPA & 11007 & hepatitis delta antigen-interacting protein A & 2,71 & chr11 \\
\hline DKC1 & 1736 & dyskeratosis congenita 1 , dyskerin & 2,46 & $\operatorname{chr} \mathrm{X}$ \\
\hline DKFZP564F0 & 25940 & DKFZP564F0522 protein & 2,66 & chr2 \\
\hline DKFZP564J0 & 25923 & DKFZP564J0863 protein & 3,00 & chr11 \\
\hline DKFZP564J1 & 25854 & DKFZP564J102 protein & 2,97 & chr4 \\
\hline DKFZp564J19 & 54458 & DKFZp564J157 protein & 2,71 & chr12 \\
\hline DKFZP564Md & 25906 & DKFZP564M082 protein & 2,21 & chr11 \\
\hline DMXL1 & 1657 & Dmx-like 1 & 2,13 & chr5 \\
\hline DNAJA2 & 10294 & DnaJ (Hsp40) homolog, subfamily A, member 2 & 4,57 & chr16 \\
\hline DNAJA4 & 55466 & DnaJ (Hsp40) homolog, subfamily A, member 4 & 2,45 & chr15 \\
\hline DNAJB6 & 10049 & DnaJ (Hsp40) homolog, subfamily B, member 6 & 4,52 & chr7 \\
\hline DNCL1 & 8655 & dynein, cytoplasmic, light polypeptide 1 & 2,05 & chr14 \\
\hline DNMT3A & 1788 & DNA (cytosine-5-)-methyltransferase 3 alpha & 2,08 & chr2 \\
\hline DNMT3B & 1789 & DNA (cytosine-5-)-methyltransferase 3 beta & 7,56 & chr20 \\
\hline DNPEP & 23549 & aspartyl aminopeptidase & 3,20 & chr2 \\
\hline DOCK4 & 9732 & dedicator of cytokinesis 4 & 2,02 & chr7 \\
\hline DOCK5 & 80005 & dedicator of cytokinesis 5 & 3,08 & chr8 \\
\hline $\mathrm{DPH} 2$ & 1802 & DPH2 homolog (S. cerevisiae) & 2,22 & chr1 \\
\hline DPM1 & 8813 & Dolichyl-phosphate mannosyltransferase polypeptide 1 , catalytic subunit & 2,15 & chr20 \\
\hline DPP3 & 10072 & dipeptidylpeptidase 3 & 2,22 & chr11 \\
\hline DPPA2 & 151871 & developmental pluripotency associated 2 & 11,70 & chr3 \\
\hline DPPA3 & 359787 & developmental pluripotency associated 3 & 7,34 & chr14 \\
\hline DPPA4 & 55211 & developmental pluripotency associated 4 & 6,72 & chr3 \\
\hline DPPA5 & 340168 & developmental pluripotency associated 5 & 4,32 & chr6 \\
\hline DPYSL3 & 1809 & dihydropyrimidinase-like 3 & 4,18 & chr5 \\
\hline
\end{tabular}




\begin{tabular}{|c|c|c|c|c|}
\hline Gene Symbol & Entrez Gene & Gene Title & NPC_down & Chromosome Number(Avadis) \\
\hline DR1 & 1810 & down-regulator of transcription 1, TBP-binding (negative cofactor 2) & 2,71 & chr1 \\
\hline DRCTNNB1A & 84668 & down-regulated by Ctnnb1, a & 2,92 & chr7 \\
\hline DRG2 & 1819 & developmentally regulated GTP binding protein 2 & 2,02 & chr17 \\
\hline DRIM & 27340 & down-regulated in metastasis & 2,03 & chr12 \\
\hline DSTN & 11034 & Destrin (actin depolymerizing factor) & 2,37 & chr20 \\
\hline DSU & 55686 & dilute suppressor & 2,53 & chr2 \\
\hline DTWD2 & 285605 & DTW domain containing 2 & 2,07 & chr5 \\
\hline |DTX3L & 151636 & deltex 3-like (Drosophila) & 2,52 & chr3 \\
\hline DUS3L & 56931 & dihydrouridine synthase 3-like (S. cerevisiae) & 2,02 & chr19 \\
\hline DUSP5 & 1847 & dual specificity phosphatase 5 & 2,48 & chr10 \\
\hline DUT & 1854 & dUTP pyrophosphatase & 2,95 & chr15 \\
\hline DXS9879E & 8270 & DNA segment on chromosome X (unique) 9879 expressed sequence & 3,11 & $\operatorname{chrX}$ \\
\hline E2IG5 & 26355 & growth and transformation-dependent protein & 2,08 & chr3 \\
\hline EAF2 & 55840 & ELL associated factor 2 & 3,45 & chr3 \\
\hline EBNA1BP2 & 10969 & EBNA1 binding protein 2 & 2,28 & chr1 \\
\hline ECAT11 & 54596 & hypothetical protein FLJ10884 & 7,60 & chr1 \\
\hline EDG7 & 23566 & Endothelial differentiation, Iysophosphatidic acid G-protein-coupled recept & 4,52 & chr1 \\
\hline EHD4 & 30844 & EH-domain containing 4 & 2,20 & chr15 \\
\hline EIF1AX & 1964 & eukaryotic translation initiation factor $1 \mathrm{~A}, \mathrm{X}$-linked & 2,49 & chr1 \\
\hline EIF2AK4 & 440275 & eukaryotic translation initiation factor 2 alpha kinase 4 & 3,08 & chr15 \\
\hline EIF2C2 & 27161 & Eukaryotic translation initiation factor $2 \mathrm{C}, 2$ & 2,23 & chr15 \\
\hline EIF3S12 & 27335 & eukaryotic translation initiation factor 3 , subunit 12 & 2,17 & chr19 \\
\hline EIF3S4 & 8666 & eukaryotic translation initiation factor 3 , subunit 4 delta, $44 \mathrm{kDa}$ & 2,18 & chr19 \\
\hline EIF4E3 & 317649 & eukaryotic translation initiation factor $4 \mathrm{E}$ member 3 & 2,61 & chr3 \\
\hline EIF4EBP1 & 1978 & eukaryotic translation initiation factor $4 \mathrm{E}$ binding protein 1 & 3,37 & chr8 \\
\hline EIF5 & 1983 & eukaryotic translation initiation factor 5 & 2,36 & chr14 \\
\hline EIF5A & 1984 & eukaryotic translation initiation factor $5 \mathrm{~A}$ & 4,16 & chr10 \\
\hline ELL2 & 22936 & elongation factor, RNA polymerase II, 2 & 3,13 & chr1 \\
\hline ELL3 & 80237 & elongation factor RNA polymerase II-like 3 & 2,49 & chr15 \\
\hline ENPP1 & 5167 & ectonucleotide pyrophosphatase/phosphodiesterase 1 & 4,84 & chr6 \\
\hline ENSA & 2029 & endosulfine alpha & 2,22 & chr1 \\
\hline EPB41L4B & 54566 & erythrocyte membrane protein band 4.1 like 4B & 7,33 & chr9 \\
\hline EPB41L5 & 57669 & erythrocyte membrane protein band 4.1 like 5 & 3,65 & chr2 \\
\hline EPHA1 & 2041 & EPH receptor A1 & 8,89 & chr7 \\
\hline EPS8L1 & 54869 & EPS8-like 1 & 2,46 & chr19 \\
\hline EPS8L2 & 64787 & EPS8-like 2 & 3,98 & chr11 \\
\hline ERBB2 & 2064 & v-erb-b2 erythroblastic leukemia viral oncogene homolog 2, neuro/glioblas & 2,54 & chr17 \\
\hline ESCO1 & 114799 & establishment of cohesion 1 homolog 1 (S. cerevisiae) & 2,39 & chr18 \\
\hline ESRRA & 2101 & estrogen-related receptor alpha & 2,10 & chr11 \\
\hline ETF1 & 2107 & eukaryotic translation termination factor 1 & 2,07 & chr5 \\
\hline ETV4 & 2118 & ets variant gene 4 ( $E 1 \mathrm{~A}$ enhancer binding protein, $\mathrm{E} 1 \mathrm{AF}$ ) /// ets variant ge & 3,34 & chr22 \\
\hline EXOSC2 & 23404 & exosome component 2 & 2,62 & chr9 \\
\hline EXOSC3 & 51010 & exosome component 3 & 3,11 & chr9 \\
\hline EXOSC4 & 54512 & exosome component 4 & 2,09 & chr8 \\
\hline EXOSC5 & 56915 & exosome component 5 & 2,95 & chr19 \\
\hline F11R & 50848 & F11 receptor & 3,53 & chr1 \\
\hline F2RL1 & 2150 & coagulation factor II (thrombin) receptor-like 1 & 5,03 & chr5 \\
\hline F8A1 & 8263 & coagulation factor VIII-associated (intronic transcript) 1 & 2,09 & $\operatorname{chr} X$ \\
\hline FABP5 & 2171 & fatty acid binding protein 5 (psoriasis-associated) & 2,87 & chr7 \\
\hline FADS3 & 3995 & fatty acid desaturase 3 & 3,05 & chr11 \\
\hline FAIM & 55179 & Fas apoptotic inhibitory molecule & 2,14 & chr3 \\
\hline FALZ & 2186 & fetal Alzheimer antigen & 3,35 & chr17 \\
\hline FAM29A & 54801 & family with sequence similarity 29 , member A & 2,01 & chr7 \\
\hline FAM36A & 116228 & family with sequence similarity 36 , member $A$ & 2,46 & chr1 \\
\hline FAM46B & 115572 & family with sequence similarity 46 , member $B$ & 8,50 & chr1 \\
\hline FAM80A & 284716 & family with sequence similarity 80 , member $\mathrm{A}$ & 2,18 & chr1 \\
\hline FAM91A2 & $388685 / / / 57234$ & family with sequence similarity 91, member A2 /// FLJ39739 protein & 3,61 & chr1 \\
\hline FANCF & 2188 & Fanconi anemia, complementation group $\mathrm{F}$ & 2,23 & chr11 \\
\hline FARSLA & 2193 & phenylalanine-tRNA synthetase-like, alpha subunit & 2,95 & chr19 \\
\hline FBXL15 & 79176 & F-box and leucine-rich repeat protein 15 & 2,31 & chr10 \\
\hline FBXL16 & 146330 & F-box and leucine-rich repeat protein 16 & 4,14 & chr16 \\
\hline FBXO2 & 26232 & F-box protein 2 & 2,96 & chr1 \\
\hline FBXO22 & 26263 & F-box protein 22 & 2,85 & chr15 \\
\hline FBXO25 & 26260 & F-box protein 25 & 2,82 & chr8 \\
\hline FBXO28 & 23219 & F-box protein 28 & 2,55 & chr1 \\
\hline FEM1B & 10116 & fem-1 homolog b (C. elegans) & 2,07 & chr15 \\
\hline
\end{tabular}




\begin{tabular}{|c|c|c|c|c|}
\hline Gene Symbol & Entrez Gene & Gene Title & NPC_down & Chromosome Number(Avadis) \\
\hline FGD6 & 55785 & FYVE, RhoGEF and PH domain containing 6 & 2,32 & chr12 \\
\hline FGF19 & 9965 & fibroblast growth factor 19 & 2,77 & chr11 \\
\hline FGF2 & 2247 & fibroblast growth factor 2 (basic) & 3,80 & chr4 \\
\hline FGF4 & 2249 & fibroblast growth factor 4 (heparin secretory transforming protein 1, Kapos & 10,26 & chr11 \\
\hline FGFR4 & 2264 & fibroblast growth factor receptor 4 & 3,21 & chr5 \\
\hline FKBP11 & 51303 & FK506 binding protein $11,19 \mathrm{kDa}$ & 2,86 & chr12 \\
\hline FKBP1A & 2280 & FK506 binding protein $1 \mathrm{~A}, 12 \mathrm{kDa}$ & 2,43 & chr20 \\
\hline FKBP1B & 2281 & FK506 binding protein 1B, $12.6 \mathrm{kDa}$ & 2,72 & chr2 \\
\hline FKBP4 & 2288 & FK506 binding protein $4,59 \mathrm{kDa}$ & 2,72 & chr12 \\
\hline FLAD1 & 80308 & Fad1, flavin adenine dinucleotide synthetase, homolog (yeast) & 2,26 & chr1 \\
\hline FLJ10006 & 55677 & hypothetical protein FLJ10006 & 2,02 & chr2 \\
\hline FLJ10534 & 55720 & hypothetical protein FLJ10534 & 3,22 & chr17 \\
\hline FLJ10652 & 55196 & hypothetical protein FLJ10652 & 4,55 & chr12 \\
\hline FLJ10719 & 55215 & hypothetical protein FLJ10719 & 2,08 & chr15 \\
\hline FLJ10826 & 55239 & hypothetical protein FLJ10826 & 2,98 & chr16 \\
\hline FLJ11151 & 55313 & Hypothetical protein FLJ11151 & 2,06 & chr16 \\
\hline FLJ11184 & 55319 & hypothetical protein FLJ11184 & 2,04 & chr4 \\
\hline FLJ11286 & 55337 & hypothetical protein FLJ11286 & 6,62 & chr19 \\
\hline FLJ12505 & 79805 & hypothetical protein FLJ12505 & 6,81 & chr1 \\
\hline FLJ12684 & 79584 & hypothetical protein FLJ12684 & 4,79 & chr4 \\
\hline FLJ13220 & 60558 & hypothetical protein FLJ13220 & 2,17 & chr4 \\
\hline FLJ13491 & 79676 & hypothetical protein FLJ13491 & 2,33 & chr12 \\
\hline FLJ13984 & 79828 & hypothetical protein FLJ13984 & 3,50 & chr2 \\
\hline FLJ20105 & 54821 & FLJ20105 protein & 2,18 & $\operatorname{chrX}$ \\
\hline FLJ20273 & 54502 & RNA-binding protein & 4,07 & chr4 \\
\hline FLJ20449 & 54937 & hypothetical protein FLJ20449 & 7,04 & chr13 \\
\hline FLJ20512 & 54958 & hypothetical protein FLJ20512 & 2,68 & chr19 \\
\hline FLJ20516 & 54962 & timeless-interacting protein & 2,02 & chr15 \\
\hline FLJ20582 & 54989 & hypothetical protein FLJ20582 & 3,03 & $\operatorname{chr} \mathrm{X}$ \\
\hline FLJ20641 & 55010 & hypothetical protein FLJ20641 & 2,08 & chr12 \\
\hline FLJ20674 & 54621 & Hypothetical protein FLJ20674 & 4,41 & chr12 \\
\hline FLJ21924 & 79832 & hypothetical protein FLJ21924 & 2,21 & chr11 \\
\hline FLJ22318 & 64777 & hypothetical protein FLJ22318 & 2,25 & chr5 \\
\hline FLJ22662 & 79887 & hypothetical protein FLJ22662 & 3,99 & chr12 \\
\hline FLJ30656 & 124801 & hypothetical protein FLJ30656 & 2,66 & chr8 \\
\hline FLJ30707 & 220108 & hypothetical protein FLJ30707 & 3,79 & chr13 \\
\hline FLJ32810 & 143872 & hypothetical protein FLJ32810 & 2,30 & chr11 \\
\hline FLJ36116 & 388666 & hypothetical locus LOC388666 & 9,37 & chr1 \\
\hline FLJ44186 & 346689 & FLJ44186 protein & 4,00 & chr7 \\
\hline FLJ46419 & 388507 & FLJ46419 protein & 2,93 & chr19 \\
\hline FLJ90086 & 389389 & Similar to Al661453 protein & 2,60 & chr6 \\
\hline FLJ90231 & 283176 & hypothetical protein FLJ90231 & 14,09 & chr11 \\
\hline FLNB & 2317 & filamin B, beta (actin binding protein 278) & 2,37 & chr3 \\
\hline FLT1 & 2321 & Fms-related tyrosine kinase 1 (vascular endothelial growth factor/vascular & 6,90 & chr13 \\
\hline FOXA3 & 3171 & forkhead box A3 & 4,46 & chr19 \\
\hline FOXD3 & 27022 & Forkhead box D3 & 2,70 & chr1 \\
\hline FOXH1 & 8928 & Forkhead box H1 & 8,54 & chr8 \\
\hline FOXO1A & 2308 & forkhead box O1A (rhabdomyosarcoma) & 5,73 & chr13 \\
\hline FRAG1 & 27315 & FGF receptor activating protein 1 & 2,40 & chr11 \\
\hline FRAT2 & 23401 & frequently rearranged in advanced T-cell lymphomas 2 & 8,12 & chr10 \\
\hline FTSJ1 & 24140 & FtsJ homolog 1 (E. coli) & 2,45 & $\operatorname{chr} \mathrm{X}$ \\
\hline FXYD5 & 53827 & FXYD domain containing ion transport regulator 5 & 6,47 & chr19 \\
\hline FZD7 & 8324 & frizzled homolog 7 (Drosophila) & 2,91 & chr2 \\
\hline G3BP2 & 9908 & Ras-GTPase activating protein $\mathrm{SH} 3$ domain-binding protein 2 & 3,48 & chr4 \\
\hline GABRA5 & 2558 & gamma-aminobutyric acid (GABA) A receptor, alpha 5 & 3,52 & chr15 \\
\hline GABRB3 & 2562 & gamma-aminobutyric acid (GABA) A receptor, beta 3 & 14,57 & chr15 \\
\hline GABRB3 & $1653 / / / 2562$ & Gamma-aminobutyric acid (GABA) A receptor, beta 3 /// DEAD (Asp-Glu- & 16,90 & chr15 \\
\hline GADD45GIP1 & 90480 & growth arrest and DNA-damage-inducible, gamma interacting protein 1 & 3,18 & chr19 \\
\hline GAL & 51083 & galanin & 37,74 & chr11 \\
\hline GALNT3 & 2591 & |UDP-N-acetyl-alpha-D-galactosamine:polypeptide $\mathrm{N}$-acetylgalactosaminy & 12,66 & chr2 \\
\hline GALNT7 & 51809 & |UDP-N-acetyl-alpha-D-galactosamine:polypeptide $\mathrm{N}$-acetylgalactosaminy & 2,44 & chr4 \\
\hline GAP43 & 2596 & growth associated protein 43 & 2,40 & chr3 \\
\hline GARNL4 & 23108 & GTPase activating Rap/RanGAP domain-like 4 & 5,50 & chr17 \\
\hline GART & 2618 & Phosphoribosylglycinamide formyltransferase, phosphoribosylglycinamide & 2,70 & chr21 \\
\hline GCAT & 23464 & glycine C-acetyltransferase (2-amino-3-ketobutyrate coenzyme A ligase) & 2,19 & chr22 \\
\hline GCHFR & 2644 & GTP cyclohydrolase I feedback regulator & 2,65 & chr15 \\
\hline
\end{tabular}




\begin{tabular}{|c|c|c|c|c|}
\hline Gene Symbol & Entrez Gene & Gene Title & NPC_down & Chromosome Number(Avadis) \\
\hline GCLC & 2729 & glutamate-cysteine ligase, catalytic subunit & 2,51 & chr6 \\
\hline GCNT2 & 2651 & glucosaminyl (N-acetyl) transferase 2, I-branching enzyme & 3,49 & chr6 \\
\hline GCSH & 2653 & glycine cleavage system protein $\mathrm{H}$ (aminomethyl carrier) & 2,31 & chr5 \\
\hline GDAP1L1 & 78997 & ganglioside-induced differentiation-associated protein 1 -like 1 & 2,09 & chr20 \\
\hline GDF3 & 9573 & growth differentiation factor 3 & 17,78 & chr12 \\
\hline GEMIN5 & 25929 & gem (nuclear organelle) associated protein 5 & 2,09 & chr5 \\
\hline GEMIN7 & 79760 & gem (nuclear organelle) associated protein 7 & 2,69 & chr19 \\
\hline GFM1 & 85476 & G elongation factor, mitochondrial 1 & 2,00 & chr3 \\
\hline GFPT1 & 2673 & glutamine-fructose-6-phosphate transaminase 1 & 2,20 & chr2 \\
\hline GGT1 & 2678 & gamma-glutamyltransferase 1 & 2,11 & chr22 \\
\hline GLDC & 2731 & glycine dehydrogenase (decarboxylating; glycine decarboxylase, glycine & 2,00 & chr4 \\
\hline GLOXD1 & 84842 & glyoxalase domain containing 1 & 4,34 & chr1 \\
\hline GLS & 2744 & glutaminase & 2,14 & chr2 \\
\hline GLS2 & 27165 & glutaminase 2 (liver, mitochondrial) & 3,88 & chr12 \\
\hline GMDS & 2762 & GDP-mannose 4,6-dehydratase & 2,64 & chr6 \\
\hline GMFB & 2764 & glia maturation factor, beta & 2,40 & chr3 \\
\hline GMPPA & 29926 & GDP-mannose pyrophosphorylase A & 2,89 & chr2 \\
\hline GNAS & 2778 & GNAS complex locus & 4,11 & chr20 \\
\hline GNB1 & 2782 & guanine nucleotide binding protein ( $G$ protein), beta polypeptide 1 & 2,06 & chr1 \\
\hline GNG4 & 2786 & guanine nucleotide binding protein ( $G$ protein), gamma 4 & 2,03 & chr1 \\
\hline GNPTAB & 79158 & $\mathrm{~N}$-acetylglucosamine-1-phosphate transferase, alpha and beta subunits & 5,38 & chr12 \\
\hline GOT1 & 2805 & glutamic-oxaloacetic transaminase 1, soluble (aspartate aminotransferase & 2,19 & chr10 \\
\hline GPC4 & 2239 & glypican 4 & 8,40 & $\operatorname{chr} X$ \\
\hline GPI & 2821 & glucose phosphate isomerase & 2,97 & chr19 \\
\hline GPM6B & 2824 & glycoprotein M6B & 2,51 & $\operatorname{chr} X$ \\
\hline GPR & 11245 & putative $\mathrm{G}$ protein coupled receptor & 5,24 & chr15 \\
\hline GPR160 & 26996 & G protein-coupled receptor 160 & 10,38 & chr3 \\
\hline GPR27 & 2850 & G protein-coupled receptor 27 & 2,67 & chr3 \\
\hline GPR54 & 84634 & G protein-coupled receptor 54 & 2,86 & chr19 \\
\hline GPR64 & 10149 & G protein-coupled receptor 64 & 11,31 & $\operatorname{chr} X$ \\
\hline GPRC5B & 51704 & G protein-coupled receptor, family C, group 5 , member B & 2,59 & chr16 \\
\hline GPS2 & 2874 & G protein pathway suppressor 2 & 2,20 & chr17 \\
\hline GPT2 & 84706 & glutamic pyruvate transaminase (alanine aminotransferase) 2 & 2,11 & chr16 \\
\hline GPX1 & 2876 & glutathione peroxidase 1 & 2,13 & chr3 \\
\hline GPX3 & 2878 & glutathione peroxidase 3 (plasma) & 3,16 & chr5 \\
\hline GRB10 & 2887 & growth factor receptor-bound protein 10 & 2,74 & chr7 \\
\hline GRPEL1 & 80273 & GrpE-like 1, mitochondrial (E. coli) & 3,06 & chr4 \\
\hline GRPEL2 & 134266 & GrpE-like 2, mitochondrial (E. coli) & 2,43 & chr5 \\
\hline GRSF1 & 2926 & G-rich RNA sequence binding factor 1 & 2,44 & chr4 \\
\hline GRTP1 & 79774 & Growth hormone regulated TBC protein 1 & 3,51 & chr13 \\
\hline GSDMDC1 & 79792 & gasdermin domain containing 1 & 2,21 & chr8 \\
\hline GSR & 2936 & glutathione reductase & 2,58 & chr8 \\
\hline GSTO1 & 9446 & glutathione S-transferase omega 1 & 2,50 & chr3 \\
\hline GSTO2 & 119391 & glutathione S-transferase omega 2 & 2,09 & chr10 \\
\hline GSTP1 & 2950 & glutathione S-transferase pi & 3,02 & chr11 \\
\hline GTF2H2 & 2966 & general transcription factor IIH, polypeptide 2, 44kDa & 3,50 & chr5 \\
\hline GTF3C2 & 2976 & general transcription factor IIIC, polypeptide 2 , beta $110 \mathrm{kDa}$ & 2,12 & chr2 \\
\hline GUCA1A & 2978 & guanylate cyclase activator $1 \mathrm{~A}$ (retina) & 3,15 & chr6 \\
\hline GULP1 & 51454 & GULP, engulfment adaptor PTB domain containing 1 & 3,61 & chr2 \\
\hline GYG & 2992 & glycogenin & 2,46 & chr3 \\
\hline GYLTL1B & 120071 & glycosyltransferase-like 1B & 3,65 & chr11 \\
\hline H2AFJ & 55766 & H2A histone family, member $\mathrm{J}$ & 2,04 & chr12 \\
\hline HAS3 & 3038 & hyaluronan synthase 3 & 4,99 & chr16 \\
\hline HBII-437 & 338427 /// 347686 & HBII-437 C/D box snoRNA /// HBII-13 snoRNA & 4,34 & chr15 \\
\hline HDAC8 & 55869 & histone deacetylase 8 & 4,13 & $\operatorname{chrX}$ \\
\hline HDCMA18P & 51574 & HDCMA18P protein & 4,97 & chr4 \\
\hline HEATR1 & 55127 & HEAT repeat containing 1 & 2,02 & chr1 \\
\hline HERC5 & 51191 & hect domain and RLD 5 & 10,06 & chr4 \\
\hline HERC6 & 55008 & hect domain and RLD 6 & 3,70 & chr4 \\
\hline HEY2 & 23493 & hairy/enhancer-of-split related with YRPW motif 2 & 8,67 & chr6 \\
\hline HIP2 & 3093 & Huntingtin interacting protein 2 & 2,11 & chr4 \\
\hline HIST1H1A & 3024 & histone 1, H1a & 4,87 & chr6 \\
\hline HIST1H1C & 3006 & histone $1, \mathrm{H} 1 \mathrm{C}$ & 3,52 & chr6 \\
\hline HIST1H1D & 3007 & histone $1, \mathrm{H} 1 \mathrm{~d}$ & 2,30 & chr6 \\
\hline HIST3H2A & 92815 & histone $3, \mathrm{H} 2 \mathrm{a}$ & 2,04 & chr1 \\
\hline |HK1 & 3098 & hexokinase 1 & 2,55 & chr10 \\
\hline
\end{tabular}




\begin{tabular}{|c|c|c|c|c|}
\hline Gene Symbol & Entrez Gene & Gene Title & NPC_down & Chromosome Number(Avadis) \\
\hline HKE2 & 10471 & HLA class II region expressed gene KE2 & 2,29 & chr6 \\
\hline HLA-DOA & 3111 & major histocompatibility complex, class II, DO alpha & 2,61 & chr6 \\
\hline HLA-DPB1 & 3115 & major histocompatibility complex, class II, DP beta 1 & 2,29 & chr6 \\
\hline HLA-DPB2 & 3116 & major histocompatibility complex, class II, DP beta 2 (pseudogene) & 48,66 & chr6 \\
\hline HLA-F & 3134 & major histocompatibility complex, class I, F & 2,14 & chr6 \\
\hline HLRC1 & 83475 & HEAT-like (PBS lyase) repeat containing $1 / / /$ HEAT-like (PBS lyase) repe & 2,15 & chr19 \\
\hline HMG4L & 128872 & high-mobility group (nonhistone chromosomal) protein 4-like & 2,76 & $\operatorname{chr} \mathrm{X}$ \\
\hline HMGB1 & $10357 / / / 3146$ & high-mobility group box $1 / / /$ high-mobility group (nonhistone chromosoma & 2,04 & chr3 \\
\hline HMOX2 & 3163 & heme oxygenase (decycling) 2 & 2,86 & chr16 \\
\hline HN1 & 51155 & hematological and neurological expressed 1 & 3,78 & chr17 \\
\hline HNRPF & 3185 & heterogeneous nuclear ribonucleoprotein $\mathrm{F}$ & 2,38 & chr10 \\
\hline HPCL2 & 26061 & 2-hydroxyphytanoyl-CoA lyase & 2,00 & chr3 \\
\hline HPRT1 & 3251 & hypoxanthine phosphoribosyltransferase 1 (Lesch-Nyhan syndrome) & 2,14 & $\operatorname{chr} \mathrm{X}$ \\
\hline HRASLS & 57110 & HRAS-like suppressor & 2,04 & chr3 \\
\hline HRASLS3 & 11145 & HRAS-like suppressor 3 & 18,51 & chr11 \\
\hline HRB & 3267 & HIV-1 Rev binding protein & 2,22 & chr2 \\
\hline |HRLP5 & 117245 & H-rev107-like protein 5 & 3,45 & chr11 \\
\hline HRMT1L3 & 10196 & HMT1 hnRNP methyltransferase-like 3 (S. cerevisiae) & 2,04 & chr11 \\
\hline HRMT1L6 & 55170 & HMT1 hnRNP methyltransferase-like 6 (S. cerevisiae) & 2,00 & chr1 \\
\hline |HSA9761 & 27292 & dimethyladenosine transferase & 2,86 & chr5 \\
\hline HSD11B2 & 3291 & hydroxysteroid (11-beta) dehydrogenase 2 & 2,04 & chr16 \\
\hline HSD17B4 & 3295 & hydroxysteroid (17-beta) dehydrogenase 4 & 2,89 & chr5 \\
\hline |HSGT1 & 11319 & suppressor of S. cerevisiae gcr2 & 2,03 & chr10 \\
\hline HSPA2 & 3306 & heat shock $70 \mathrm{kDa}$ protein 2 & 10,00 & chr14 \\
\hline HSPA4 & 3308 & Heat shock $70 \mathrm{kDa}$ protein 4 & 2,74 & chr5 \\
\hline HSPA8 & 3312 & heat shock $70 \mathrm{kDa}$ protein 8 & 2,75 & chr3 \\
\hline HSPA9B & 3313 & heat shock 70kDa protein 9B (mortalin-2) & 2,24 & chr2 \\
\hline HSPB1 & 3315 & heat shock 27kDa protein 1 & 2,93 & chr7 \\
\hline HSPC111 & 51491 & hypothetical protein HSPC111 & 4,96 & chr5 \\
\hline HSPC176 & 51693 & hematopoietic stem/progenitor cells 176 & 2,39 & chr16 \\
\hline HSPCB & 3326 & heat shock $90 \mathrm{kDa}$ protein 1 , beta & 3,26 & chr6 \\
\hline |HSPD1 & 3329 & heat shock $60 \mathrm{kDa}$ protein 1 (chaperonin) & 2,61 & chr5 \\
\hline HSPE1 & 3336 & heat shock $10 \mathrm{kDa}$ protein 1 (chaperonin 10) & 2,25 & chr2 \\
\hline |HSUP1 & 441951 & Similar to RPE-spondin & 2,86 & chr20 \\
\hline HTATIP2 & 10553 & HIV-1 Tat interactive protein $2,30 \mathrm{kDa}$ & 4,92 & chr11 \\
\hline HTR7 & 3363 & 5-hydroxytryptamine (serotonin) receptor 7 (adenylate cyclase-coupled) & 2,17 & chr8 \\
\hline |HYLS1 & 219844 & hydrolethalus syndrome 1 & 2,14 & chr11 \\
\hline ICA1 & 3382 & islet cell autoantigen $1,69 \mathrm{kDa}$ & 3,89 & chr7 \\
\hline ICAM3 & 3385 & intercellular adhesion molecule 3 & 3,54 & chr19 \\
\hline ID1 & 3397 & inhibitor of DNA binding 1, dominant negative helix-loop-helix protein & 3,54 & chr20 \\
\hline IDE & 3416 & insulin-degrading enzyme & 2,45 & chr10 \\
\hline IDH1 & 3417 & isocitrate dehydrogenase $1(\mathrm{NADP}+)$, soluble & 2,55 & chr2 \\
\hline IDH3A & 3419 & isocitrate dehydrogenase $3(\mathrm{NAD}+)$ alpha & 3,56 & chr15 \\
\hline IFI30 & 10437 & interferon, gamma-inducible protein 30 & 4,68 & chr19 \\
\hline |IFITM1 & 8519 & interferon induced transmembrane protein $1(9-27)$ & 11,53 & chr11 \\
\hline IFITM2 & 10581 & interferon induced transmembrane protein $2(1-8 \mathrm{D})$ & 2,06 & chr11 \\
\hline IFITM3 & 10410 & interferon induced transmembrane protein $3(1-8 \mathrm{U})$ & 2,86 & chr11 \\
\hline IGFBP6 & 3489 & insulin-like growth factor binding protein 6 & 2,36 & chr12 \\
\hline |IL27RA & 9466 & interleukin 27 receptor, alpha & 2,97 & (vide) \\
\hline IMP-1 & 10642 & |GF-II mRNA-binding protein 1 & 3,10 & chr17 \\
\hline IMP3 & 55272 & IMP3, U3 small nucleolar ribonucleoprotein, homolog (yeast) & 2,85 & chr15 \\
\hline IMP4 & 92856 & IMP4, U3 small nucleolar ribonucleoprotein, homolog (yeast) & 2,70 & chr2 \\
\hline INDO & 3620 & indoleamine-pyrrole 2,3 dioxygenase & 18,56 & chr8 \\
\hline INSR & 3643 & Insulin receptor & 2,46 & chr19 \\
\hline IPW & 3653 & imprinted in Prader-Willi syndrome & 2,81 & chr15 \\
\hline IQGAP1 & 8826 & IQ motif containing GTPase activating protein 1 & 2,11 & chr15 \\
\hline IQGAP2 & 10788 & IQ motif containing GTPase activating protein 2 & 3,09 & chr5 \\
\hline ISOC2 & 79763 & isochorismatase domain containing 2 & 3,14 & chr19 \\
\hline ITCH & 83737 & itchy homolog E3 ubiquitin protein ligase (mouse) & 2,04 & chr20 \\
\hline ITGA6 & 3655 & integrin, alpha 6 & 3,40 & chr2 \\
\hline ITGB1BP3 & 27231 & integrin beta 1 binding protein 3 & 7,12 & chr19 \\
\hline ITGB4BP & 3692 & integrin beta 4 binding protein & 3,15 & chr20 \\
\hline ITM2A & 9452 & integral membrane protein $2 \mathrm{~A}$ & 8,30 & $\operatorname{chr} \mathrm{X}$ \\
\hline ITM2C & 81618 & integral membrane protein $2 \mathrm{C} / / /$ integral membrane protein $2 \mathrm{C}$ & 2,50 & chr2 \\
\hline ITPA & 3704 & inosine triphosphatase (nucleoside triphosphate pyrophosphatase) & 2,25 & chr20 \\
\hline |ITPR3 & 3710 & |inositol 1,4,5-triphosphate receptor, type 3 & 2,86 & chr6 \\
\hline
\end{tabular}




\begin{tabular}{|c|c|c|c|c|}
\hline Gene Symbol & Entrez Gene & Gene Title & NPC_down & Chromosome Number(Avadis) \\
\hline IVD & 3712 & isovaleryl Coenzyme A dehydrogenase & 2,06 & chr15 \\
\hline JAK1 & 3716 & Janus kinase 1 (a protein tyrosine kinase) & 2,20 & chr1 \\
\hline JARID2 & 3720 & Jumonji, AT rich interactive domain 2 & 3,19 & chr6 \\
\hline JAZF1 & 221895 & juxtaposed with another zinc finger gene 1 & 4,31 & chr7 \\
\hline JMJD1C & 221037 & jumonji domain containing $1 \mathrm{C}$ & 2,31 & chr10 \\
\hline JMJD2A & 9682 & jumonji domain containing $2 \mathrm{~A}$ & 2,64 & chr1 \\
\hline JMJD2C & 23081 & jumonji domain containing $2 \mathrm{C}$ & 2,10 & chr9 \\
\hline JMY & 133746 & junction-mediating and regulatory protein & 2,04 & chr5 \\
\hline JPH1 & 56704 & junctophilin 1 & 2,82 & chr8 \\
\hline KBTBD8 & 84541 & kelch repeat and BTB (POZ) domain containing 8 & 4,06 & chr3 \\
\hline KCND2 & 3751 & potassium voltage-gated channel, Shal-related subfamily, member 2 & 2,30 & chr7 \\
\hline KCNG3 & 170850 & potassium voltage-gated channel, subfamily G, member 3 & 3,93 & chr2 \\
\hline KCNK1 & 3775 & potassium channel, subfamily K, member 1 & 3,12 & chr1 \\
\hline KCNK12 & 56660 & potassium channel, subfamily K, member 12 & 2,37 & chr2 \\
\hline KCNN2 & 3781 & potassium intermediate/small conductance calcium-activated channel, sul & 3,55 & chr5 \\
\hline KCNS3 & 3790 & potassium voltage-gated channel, delayed-rectifier, subfamily S, member & 3,68 & chr2 \\
\hline KCTD14 & 65987 & potassium channel tetramerisation domain containing 14 & 5,10 & chr11 \\
\hline KDR & 3791 & kinase insert domain receptor (a type III receptor tyrosine kinase) & 3,69 & chr4 \\
\hline KHK & 3795 & ketohexokinase (fructokinase) /// ketohexokinase (fructokinase) & 2,42 & chr2 \\
\hline |KIAA0020 & 9933 & KIAA0020 & 2,20 & chr9 \\
\hline KIAA0182 & 23199 & KIAA0182 protein & 2,30 & chr16 \\
\hline |KIAA0368 & 23392 & KIAA0368 & 3,15 & chr9 \\
\hline KIAA0494 & 9813 & KIAA0494 & 2,15 & chr1 \\
\hline KIAA0859 & 51603 & KIAA0859 & 2,41 & chr1 \\
\hline KIAA0888 & 26049 & KIAA0888 protein & 2,27 & chr5 \\
\hline KIAA1008 & 22894 & KIAA1008 & 2,06 & chr13 \\
\hline KIAA1033 & 23325 & KIAA1033 & 2,07 & chr12 \\
\hline KIAA1155 & 400961 & KIAA1155 protein & 2,73 & chr2 \\
\hline |KIAA1244 & 57221 & KIAA1244 & 2,12 & chr6 \\
\hline KIAA1404 & 57169 & KIAA1404 protein & 2,16 & chr20 \\
\hline KIAA1524 & 57650 & KIAA1524 & 2,00 & chr3 \\
\hline KIAA1553 & 57673 & KIAA1553 & 2,21 & chr6 \\
\hline |KIAA1754L & 150771 & |KIAA1754-like & 2,17 & chr2 \\
\hline KIAA1804 & 84451 & mixed lineage kinase 4 & 3,66 & chr1 \\
\hline |KIAA1944 & 121256 & KIAA1944 protein & 2,67 & chr12 \\
\hline KIF1A & 547 & kinesin family member $1 \mathrm{~A}$ & 4,01 & chr2 \\
\hline KIF5A & 3798 & Kinesin family member $5 \mathrm{~A}$ & 3,02 & chr12 \\
\hline KLF4 & 9314 & Kruppel-like factor 4 (gut) & 4,13 & chr9 \\
\hline KLF7 & 8609 & Kruppel-like factor 7 (ubiquitous) & 2,26 & chr2 \\
\hline KLHL7 & 55975 & kelch-like 7 (Drosophila) & 4,38 & chr7 \\
\hline |KLKB1 & 3818 & kallikrein B, plasma (Fletcher factor) 1 & 8,18 & chr4 \\
\hline |KRTAP4-7 & 85287 & keratin associated protein 4-7 & 2,91 & chr15 \\
\hline KRTCAP3 & 200634 & keratinocyte associated protein 3 & 5,12 & chr2 \\
\hline KUB3 & 91419 & Ku70-binding protein 3 & 4,91 & chr12 \\
\hline LACTB & 114294 & lactamase, beta & 2,62 & chr15 \\
\hline LACTB2 & 51110 & lactamase, beta 2 & 2,31 & chr8 \\
\hline LAMP2 & 3920 & |ysosomal-associated membrane protein 2 & 2,16 & $\operatorname{chr} X$ \\
\hline LANCL2 & 55915 & LanC lantibiotic synthetase component C-like 2 (bacterial) & 2,50 & chr7 \\
\hline LAPTM4B & 55353 & lysosomal associated protein transmembrane 4 beta & 3,70 & chr8 \\
\hline LARP2 & 55132 & La ribonucleoprotein domain family, member 2 & 3,06 & chr4 \\
\hline LARP4 & 113251 & La ribonucleoprotein domain family, member 4 & 2,66 & chr12 \\
\hline LARS & 51520 & leucyl-tRNA synthetase & 2,47 & chr5 \\
\hline LARS2 & 23395 & leucyl-tRNA synthetase 2 , mitochondrial & 2,56 & chr3 \\
\hline LCK & 3932 & lymphocyte-specific protein tyrosine kinase & 23,95 & chr1 \\
\hline LDB2 & 9079 & LIM domain binding 2 & 4,80 & chr4 \\
\hline LECT1 1 & 11061 & leukocyte cell derived chemotaxin 1 & 17,62 & chr13 \\
\hline LEFTY1 & 10637 & left-right determination factor 1 & 60,37 & chr1 \\
\hline LEFTY2 & 7044 & left-right determination factor 2 & 11,70 & chr1 \\
\hline LGALS1 & 3956 & lectin, galactoside-binding, soluble, 1 (galectin 1) & 13,73 & chr22 \\
\hline LIAS & 11019 & lipoic acid synthetase & 2,16 & chr4 \\
\hline LISCH7 & 51599 & liver-specific bHLH-Zip transcription factor & 2,02 & chr19 \\
\hline LITAF & 9516 & lipopolysaccharide-induced TNF factor & 2,94 & chr16 \\
\hline LNK & 10019 & lymphocyte adaptor protein & 4,33 & chr12 \\
\hline LOC114977 & 114977 & hypothetical protein BC014148 & 2,07 & chr19 \\
\hline LOC116238 & 116238 & hypothetical protein BC014072 & 3,47 & chr17 \\
\hline |LOC126295 & 126295 & hypothetical protein LOC126295 & 3,07 & chr19 \\
\hline
\end{tabular}


Table S3 : Genes downregulated in NPC compared to hES (Fold Change $>2 ; \alpha<0.05$ )

\begin{tabular}{|c|c|c|c|c|}
\hline Gene Symbol & Entrez Gene & Gene Title & NPC_down & Chromosome Number(Avadis) \\
\hline LOC131076 & 131076 & hypothetical LOC131076 & 2,11 & $\operatorname{chr18}$ \\
\hline OC138255 & 138255 & OTTHUMP00000021439 & 20,90 & chr9 \\
\hline LOC150084 & 150084 & hypothetical protein LOC150084 & 2,13 & chr21 \\
\hline LOC150371 & 150371 & hypothetical LOC150371 & 2,42 & chr22 \\
\hline LOC151194 & 151194 & similar to hepatocellular carcinoma-associated antigen HCA557b & 2,47 & chr2 \\
\hline LOC151963 & 151963 & similar to BcDNA:GH11415 gene product & 2,13 & chr3 \\
\hline LOC153346 & 153346 & hypothetical protein LOC153346 & 5,83 & chr5 \\
\hline LOC153469 & 153469 & hypothetical protein LOC153469 & 3,95 & chr5 \\
\hline LOC155036 & 155036 & hypothetical protein LOC155036 & 2,02 & chr7 \\
\hline LOC157627 & 157627 & hypothetical protein LOC157627 & 6,16 & chr8 \\
\hline LOC169834 & 169834 & hypothetical protein LOC169834 & 2,01 & chr9 \\
\hline LOC201895 & 201895 & hypothetical protein LOC201895 & 2,28 & chr4 \\
\hline LOC202451 & 202451 & hypothetical protein LOC202451 & 2,63 & chr6 \\
\hline LOC253982 & 253982 & hypothetical protein LOC253982 & 2,02 & chr16 \\
\hline LOC283377 & 283377 & hypothetical protein LOC283377 & 2,41 & chr12 \\
\hline LOC283871 & 283871 & hypothetical protein LOC283871 & 2,25 & chr16 \\
\hline LOC284611 & 284611 & hypothetical protein LOC284611 & 2,04 & chr1 \\
\hline LOC284801 & 284801 & hypothetical protein LOC284801 & 4,31 & chr20 \\
\hline LOC285016 & 285016 & hypothetical protein LOC285016 & 2,90 & chr2 \\
\hline LOC285401 & 285401 & hypothetical protein LOC285401 & 2,02 & chr3 \\
\hline LOC286044 & 286044 & hypothetical protein LOC286044 & 3,25 & chr8 \\
\hline LOC342979 & 342979 & hypothetical LOC342979 & 2,77 & chr19 \\
\hline LOC388335 & 388335 & similar to RIKEN cDNA A730055C05 gene & 2,23 & $\operatorname{chr} 17$ \\
\hline LOC388610 & 388610 & hypothetical LOC388610 & 5,21 & chr1 \\
\hline LOC388638 & 388638 & hypothetical LOC388638 & 4,76 & chr1 \\
\hline LOC389362 & 389362 & hypothetical LOC389362 & 3,83 & chr6 \\
\hline LOC389541 & 389541 & similar to CG14977-PA & 2,58 & chr7 \\
\hline LOC389857 & 389857 & hypothetical protein & 2,11 & $\operatorname{chrX}$ \\
\hline LOC391020 & 391020 & similar to Interferon-induced transmembrane protein 3 (Interferon-inducibl & 2,90 & chr1 \\
\hline LOC391833 & 391833 & Similar to $40 \mathrm{~S}$ ribosomal protein $\mathrm{S} 10$ & 2,80 & chr5 \\
\hline LOC400690 & 400690 & hypothetical gene supported by AK092138 & 2,74 & $\operatorname{chr} 19$ \\
\hline LOC400948 & 400948 & similar to RIKEN cDNA $2310016 \mathrm{E} 02$ & 2,04 & chr2 \\
\hline LOC439949 & 439949 & hypothetical gene supported by AY007155 & 4,36 & chr10 \\
\hline LOC440122 & 440122 & Similar to KRAB zinc finger protein $6 \mathrm{D}$ & 2,44 & chr12 \\
\hline LOC440132 & 440132 & LOC440132 & 4,16 & chr13 \\
\hline LOC440731 & 440731 & LOC440731 & 2,20 & chr1 \\
\hline LOC440737 & 440737 & Similar to 60 S ribosomal protein L35 & 7,02 & chr16 \\
\hline LOC441164 & 441164 & Chromosome 6 open reading frame 160 & 2,08 & chr6 \\
\hline LOC441168 & 441168 & hypothetical protein LOC441168 & 2,26 & chr6 \\
\hline LOC441458 & 441458 & hypothetical gene supported by AK091930 & 4,61 & chr9 \\
\hline LOC441628 & 441628 & similar to POU domain, class 5 , transcription factor 1 (Octamer-binding tra & 18,61 & chr1 \\
\hline LOC441762 & 441762 & Similar to CG7467-PA /// Similar to CG7467-PA & 2,64 & $\operatorname{chr} 16$ \\
\hline LOC442447 & 442447 & Similar to Chloride intracellular channel protein 4 (Intracellular chloride ion & 8,73 & $\operatorname{chrX}$ \\
\hline LOC494143 & 494143 & similar to RIKEN cDNA $2510006 \mathrm{C} 20$ gene & 3,47 & chr2 \\
\hline LOC56902 & 56902 & putatative $28 \mathrm{kDa}$ protein & 2,41 & chr2 \\
\hline LOC91614 & 91614 & novel 58.3 KDA protein & 2,31 & chr11 \\
\hline LOC91661 & 91661 & Hypothetical protein BC001610 & 2,53 & (vide) \\
\hline LOC92345 & 92345 & hypothetical protein BC008207 & 2,58 & chr4 \\
\hline LRP8 & 7804 & low density lipoprotein receptor-related protein 8 , apolipoprotein e recepto & 2,69 & chr1 \\
\hline LRRC16 & 55604 & Leucine rich repeat containing 16 & 2,43 & chr6 \\
\hline LSM7 & 51690 & LSM7 homolog, U6 small nuclear RNA associated (S. cerevisiae) & 2,26 & chr19 \\
\hline LYAR & 55646 & hypothetical protein FLJ20425 & 2,45 & chr4 \\
\hline LYPLA2 & $3 / / / 285840 / / / 38$ & |ysophospholipase II /// lysophospholipase II pseudogene 1 /// similar to A & 2,02 & chr1 \\
\hline LYSMD2 & 256586 & LysM, putative peptidoglycan-binding, domain containing 2 & 2,36 & chr15 \\
\hline MAD2L2 & 10459 & MAD2 mitotic arrest deficient-like 2 (yeast) & 4,22 & chr1 \\
\hline MAGI2 & 9863 & membrane associated guanylate kinase, WW and PDZ domain containind & 3,13 & chr7 \\
\hline Magmas & 51025 & mitochondria-associated protein involved in granulocyte-macrophage cold & 2,07 & chr16 \\
\hline MAL2 & 114569 & mal, T-cell differentiation protein 2 & 4,78 & chr8 \\
\hline MAP1LC3B & 81631 & microtubule-associated protein 1 light chain 3 beta & 3,04 & chr12 \\
\hline MAP3K5 & 4217 & mitogen-activated protein kinase kinase kinase 5 & 2,98 & chr6 \\
\hline MAP4 & 4134 & microtubule-associated protein 4 & 2,64 & chr3 \\
\hline MAP4K1 & 11184 & mitogen-activated protein kinase kinase kinase kinase 1 & 2,45 & chr19 \\
\hline MAP7 & 9053 & microtubule-associated protein 7 & 4,43 & chr6 \\
\hline MAPK1 & 5594 & mitogen-activated protein kinase 1 & 2,51 & chr22 \\
\hline MAPK13 & 5603 & mitogen-activated protein kinase 13 & 3,91 & chr6 \\
\hline MAPKAP1 & 79109 & mitogen-activated protein kinase associated protein 1 & 2,96 & chr9 \\
\hline
\end{tabular}




\begin{tabular}{|c|c|c|c|c|}
\hline Gene Symbol & Entrez Gene & Gene Title & NPC_down & Chromosome Number(Avadis) \\
\hline MARCH3 & 115123 & membrane-associated ring finger (C3HC4) 3 & 3,65 & chr5 \\
\hline MARS & 4141 & methionine-tRNA synthetase & 2,11 & chr12 \\
\hline |MARVELD3 & 91862 & MARVEL domain containing 3 & 4,59 & chr16 \\
\hline MATK & 4145 & megakaryocyte-associated tyrosine kinase & 2,49 & chr19 \\
\hline MATR3 & 9782 & Matrin 3 & 2,87 & chr5 \\
\hline MCART1 & 92014 & mitochondrial carrier triple repeat 1 & 2,04 & chr9 \\
\hline MCcC2 & 64087 & methylcrotonoyl-Coenzyme A carboxylase 2 (beta) & 2,06 & chr5 \\
\hline MCL1 & 4170 & myeloid cell leukemia sequence 1 (BCL2-related) & 2,40 & chr1 \\
\hline MCM5 & 4174 & MCM5 minichromosome maintenance deficient 5 , cell division cycle 46 (S & 2,79 & chr22 \\
\hline MDH2 & 4191 & malate dehydrogenase 2, NAD (mitochondrial) & 2,26 & chr7 \\
\hline MDN1 & 23195 & MDN1, midasin homolog (yeast) & 2,13 & chr6 \\
\hline ME1 & 4199 & Malic enzyme 1, NADP(+)-dependent, cytosolic & 2,20 & chr6 \\
\hline ME2 & 4200 & malic enzyme 2, NAD(+)-dependent, mitochondrial & 2,03 & chr18 \\
\hline MEF2A & 4205 & MADS box transcription enhancer factor 2, polypeptide A (myocyte enhan & 2,22 & chr15 \\
\hline MEGF10 & 84466 & MEGF10 protein & 4,81 & chr5 \\
\hline MFSD3 & 113655 & major facilitator superfamily domain containing 3 & 3,24 & chr8 \\
\hline MGC10993 & 80775 & hypothetical protein MGC10993 & 2,73 & chr2 \\
\hline MGC11324 & 84803 & hypothetical protein MGC11324 /// hypothetical protein MGC11324 & 2,30 & chr4 \\
\hline MGC13017 & 91368 & similar to RIKEN cDNA A430101B06 gene & 2,56 & chr5 \\
\hline MGC13096 & 84306 & hypothetical protein MGC13096 /// hypothetical protein MGC13096 & 3,69 & chr19 \\
\hline MGC13114 & 84326 & hypothetical protein MGC13114 & 2,10 & chr16 \\
\hline MGC13170 & 84798 & multidrug resistance-related protein /// multidrug resistance-related protein & 2,42 & chr19 \\
\hline MGC14798 & 89978 & similar to RIKEN cDNA $5730421 \mathrm{E} 18$ gene & 3,29 & chr15 \\
\hline MGC15763 & 92106 & hypothetical protein $\mathrm{BC} 008322$ & 2,37 & chr3 \\
\hline MGC17299 & 128218 & hypothetical protein MGC17299 & 5,60 & chr1 \\
\hline MGC19604 & 112812 & similar to RIKEN cDNA B230118G17 gene & 2,14 & chr19 \\
\hline MGC21881 & 389741 & hypothetical protein MGC21881 & 2,55 & chr9 \\
\hline MGC22793 & 221908 & hypothetical protein MGC22793 & 2,48 & chr7 \\
\hline MGC2408 & 84291 & hypothetical protein MGC2408 & 2,39 & chr3 \\
\hline MGC24665 & 116028 & hypothetical protein MGC24665 & 2,16 & chr16 \\
\hline MGC2477 & 79081 & hypothetical protein MGC2477 & 2,06 & chr11 \\
\hline MGC2574 & 79080 & hypothetical protein MGC2574 & 2,69 & chr11 \\
\hline MGC40168 & 148645 & hypothetical protein MGC40168 & 2,52 & chr1 \\
\hline MGC40397 & 121053 & hypothetical protein MGC40397 & 2,54 & chr12 \\
\hline MGC4172 & 79154 & short-chain dehydrogenase/reductase & 2,51 & chr17 \\
\hline MGC4504 & 79094 & hypothetical protein MGC4504 & 2,77 & chr15 \\
\hline MGC45871 & 359845 & hypothetical protein MGC45871 & 3,23 & chr17 \\
\hline MGC5352 & 192111 & Bcl-XL-binding protein v68 & 2,20 & chr12 \\
\hline MGC5509 & 79074 & Hypothetical protein MGC5509 & 2,46 & chr2 \\
\hline MGC61571 & 152100 & hypothetical protein MGC61571 & 2,24 & chr3 \\
\hline MGST1 & 4257 & microsomal glutathione S-transferase 1 & 4,03 & chr12 \\
\hline MICB & 4277 & MHC class I polypeptide-related sequence B & 3,46 & chr6 \\
\hline MID1IP1 & 58526 & MID1 interacting protein 1 (gastrulation specific G12-like (zebrafish)) & 2,45 & $\operatorname{chr} X$ \\
\hline MKI67IP & 84365 & MKI67 (FHA domain) interacting nucleolar phosphoprotein & 2,04 & chr2 \\
\hline MKKS & 8195 & McKusick-Kaufman syndrome & 2,17 & chr20 \\
\hline MKLN1 & 4289 & muskelin 1, intracellular mediator containing kelch motifs & 3,33 & chr7 \\
\hline MOBK1B & 55233 & MOB1, Mps One Binder kinase activator-like 1B (yeast) & 2,27 & chr2 \\
\hline MOcos & 55034 & molybdenum cofactor sulfurase & 3,29 & chr18 \\
\hline MON1A & 84315 & MON1 homolog A (yeast) /// MON1 homolog A (yeast) & 2,11 & chr3 \\
\hline MPP1 & 4354 & membrane protein, palmitoylated $1,55 \mathrm{kDa}$ & 2,52 & $\operatorname{chr} X$ \\
\hline MPP6 & 51678 & membrane protein, palmitoylated 6 (MAGUK p55 subfamily member 6 ) & 3,16 & chr7 \\
\hline MRGPRF & 219928 & MAS-related GPR, member F & 2,86 & chr11 \\
\hline MRPL11 & 65003 & mitochondrial ribosomal protein L11 & 2,12 & chr11 \\
\hline MRPL12 & 6182 & mitochondrial ribosomal protein L12 & 2,31 & chr17 \\
\hline MRPL13 & 28998 & mitochondrial ribosomal protein L13 & 2,10 & chr8 \\
\hline MRPL16 & 54948 & mitochondrial ribosomal protein L16 & 2,69 & chr11 \\
\hline MRPL17 & 63875 & mitochondrial ribosomal protein L17 & 2,73 & chr11 \\
\hline MRPL18 & 29074 & mitochondrial ribosomal protein L18 & 2,04 & chr6 \\
\hline MRPL19 & 9801 & mitochondrial ribosomal protein L19 & 2,40 & chr2 \\
\hline MRPL21 & 219927 & mitochondrial ribosomal protein L21 & 2,29 & chr11 \\
\hline MRPL30 & 51263 & mitochondrial ribosomal protein L30 & 2,50 & chr2 \\
\hline MRPL32 & 64983 & mitochondrial ribosomal protein L32 & 2,72 & chr7 \\
\hline MRPL34 & 64981 & mitochondrial ribosomal protein L34 /// mitochondrial ribosomal protein L3 & 2,28 & chr19 \\
\hline MRPL35 & 51318 & mitochondrial ribosomal protein L35 & 2,74 & chr2 \\
\hline MRPL37 & 51253 & mitochondrial ribosomal protein L37 & 2,31 & chr1 \\
\hline |MRPL38 & 64978 & mitochondrial ribosomal protein L38 & 2,55 & chr17 \\
\hline
\end{tabular}




\begin{tabular}{|c|c|c|c|c|}
\hline Gene Symbol & Entrez Gene & Gene Title & NPC_down & Chromosome Number(Avadis) \\
\hline MRPL4 & 51073 & mitochondrial ribosomal protein L4 & 4,23 & chr19 \\
\hline MRPL41 & 64975 & mitochondrial ribosomal protein L41 & 2,65 & chr9 \\
\hline MRPL42 & 28977 & mitochondrial ribosomal protein L42 & 2,79 & chr12 \\
\hline MRPL43 & 84545 & mitochondrial ribosomal protein L43 /// mitochondrial ribosomal protein L4 & 2,11 & chr10 \\
\hline MRPL44 & 65080 & mitochondrial ribosomal protein L44 & 2,56 & chr2 \\
\hline MRPL50 & 54534 & mitochondrial ribosomal protein L50 & 2,45 & chr9 \\
\hline MRPL52 & 122704 & mitochondrial ribosomal protein L52 & 2,37 & chr14 \\
\hline MRPL54 & 116541 & mitochondrial ribosomal protein L54 & 2,24 & chr19 \\
\hline MRPS10 & 55173 & mitochondrial ribosomal protein $\mathrm{S} 10$ & 3,56 & chr6 \\
\hline MRPS12 & 6183 & mitochondrial ribosomal protein $\mathrm{S} 12$ & 5,07 & chr19 \\
\hline MRPS15 & 64960 & mitochondrial ribosomal protein S15 /// mitochondrial ribosomal protein S1 & 2,38 & chr1 \\
\hline MRPS16 & 51021 & mitochondrial ribosomal protein S16 & 2,53 & chr10 \\
\hline MRPS17 & 51373 & mitochondrial ribosomal protein S17 & 2,81 & chr7 \\
\hline MRPS18B & 28973 & mitochondrial ribosomal protein S18B & 2,44 & chr6 \\
\hline MRPS2 & 51116 & mitochondrial ribosomal protein S2 & 2,21 & chr9 \\
\hline MRPS23 & 51649 & mitochondrial ribosomal protein S23 & 2,29 & chr17 \\
\hline MRPS25 & 64432 & mitochondrial ribosomal protein S25 & 3,02 & chr3 \\
\hline MRPS28 & 28957 & mitochondrial ribosomal protein S28 & 2,03 & chr8 \\
\hline MRPS30 & 10884 & mitochondrial ribosomal protein $\mathrm{S} 30$ & 2,36 & chr5 \\
\hline MRPS34 & 65993 & mitochondrial ribosomal protein S34 & 3,18 & chr16 \\
\hline MRPS36 & 92259 & mitochondrial ribosomal protein S36 & 2,21 & chr5 \\
\hline MRS2L & 57380 & MRS2-like, magnesium homeostasis factor (S. cerevisiae) & 6,54 & chr6 \\
\hline MSH2 & 4436 & mutS homolog 2, colon cancer, nonpolyposis type 1 (E. coli) & 2,55 & chr2 \\
\hline MT1E & 4493 & metallothionein $1 \mathrm{E}$ (functional) & 5,73 & chr16 \\
\hline MT1F & 4494 & metallothionein $1 \mathrm{~F}$ (functional) & 7,66 & chr16 \\
\hline MT1G & 4495 & metallothionein $1 \mathrm{G}$ & 9,40 & chr16 \\
\hline MT1H & 4496 & metallothionein $1 \mathrm{H}$ & 8,77 & chr16 \\
\hline MT1M & 4499 & Metallothionein $1 \mathrm{M}$ & 4,82 & chr1 \\
\hline MT1X & 4501 & metallothionein $1 \mathrm{X}$ & 9,12 & chr16 \\
\hline MT2A & 4502 & metallothionein $2 \mathrm{~A}$ & 5,41 & chr16 \\
\hline MTA3 & 57504 & metastasis associated 1 family, member 3 & 2,74 & chr2 \\
\hline MTAC2D1 & 123036 & membrane targeting (tandem) C2 domain containing 1 & 4,93 & chr14 \\
\hline MTAP & 4507 & methylthioadenosine phosphorylase & 2,11 & chr9 \\
\hline MTHFD1 & 4522 & methylenetetrahydrofolate dehydrogenase (NADP+ dependent) 1 , mether & 2,49 & chr14 \\
\hline MTHFD1L & 25902 & methylenetetrahydrofolate dehydrogenase (NADP+ dependent) 1-like & 2,46 & chr6 \\
\hline MTHFD2 & 10797 & methylenetetrahydrofolate dehydrogenase (NADP+ dependent) 2, mether & 2,40 & chr2 \\
\hline MTHFS & 10588 & 5,10 -methenyltetrahydrofolate synthetase (5-formyltetrahydrofolate cyclo- & 2,08 & chr15 \\
\hline MTL5 & 9633 & Metallothionein-like 5 , testis-specific (tesmin) & 2,09 & chr11 \\
\hline MTP18 & 51537 & mitochondrial protein $18 \mathrm{kDa}$ & 2,31 & chr22 \\
\hline MTRF1 & 9617 & mitochondrial translational release factor 1 & 2,08 & chr13 \\
\hline |MYBL2 & 4605 & v-myb myeloblastosis viral oncogene homolog (avian)-like 2 & 2,35 & chr20 \\
\hline MYC & 4609 & v-myc myelocytomatosis viral oncogene homolog (avian) & 3,58 & chr8 \\
\hline MYLIP & 29116 & myosin regulatory light chain interacting protein & 2,07 & chr6 \\
\hline MYO1E & 4643 & myosin IE & 3,48 & chr15 \\
\hline MYOZ3 & 91977 & myozenin 3 & 2,35 & chr5 \\
\hline NALP12 & 91662 & NACHT, leucine rich repeat and PYD containing 12 & 2,68 & chr19 \\
\hline NALP2 & 55655 & NACHT, leucine rich repeat and PYD containing 2 & 4,67 & chr19 \\
\hline NANOG & 79923 & Nanog homeobox & 51,32 & chr12 \\
\hline NANS & 54187 & $\mathrm{~N}$-acetyIneuraminic acid synthase (sialic acid synthase) & 2,29 & chr9 \\
\hline NAP1L2 & 4674 & nucleosome assembly protein 1 -like 2 & 2,56 & $\operatorname{chr} X$ \\
\hline NAP1L3 & 4675 & nucleosome assembly protein 1 -like 3 & 2,03 & $\operatorname{chr} X$ \\
\hline NARG1 & 80155 & NMDA receptor regulated 1 & 2,87 & chr4 \\
\hline NBN & 4683 & nibrin & 2,09 & chr8 \\
\hline NCBP1 & 4686 & nuclear cap binding protein subunit $1,80 \mathrm{kDa}$ & 2,44 & chr9 \\
\hline NCBP2 & 22916 & Nuclear cap binding protein subunit 2, $20 \mathrm{kDa}$ & 2,33 & chr3 \\
\hline NDUFB10 & 4716 & NADH dehydrogenase (ubiquinone) 1 beta subcomplex, $10,22 \mathrm{kDa}$ & 2,22 & chr16 \\
\hline NDUFB7 & 4713 & NADH dehydrogenase (ubiquinone) 1 beta subcomplex, $7,18 \mathrm{kDa}$ & 2,20 & chr19 \\
\hline NDUFS7 & 374291 & NADH dehydrogenase (ubiquinone) Fe-S protein $7,20 \mathrm{kDa}$ (NADH-coenz & 2,87 & chr19 \\
\hline NDUFS8 & 4728 & $\mathrm{NADH}$ dehydrogenase (ubiquinone) Fe-S protein $8,23 \mathrm{kDa}$ (NADH-coenz & 2,44 & chr11 \\
\hline NDUFV2 & 4729 & NADH dehydrogenase (ubiquinone) flavoprotein $2,24 \mathrm{kDa}$ & 2,37 & chr18 \\
\hline NEDD4L & 23327 & neural precursor cell expressed, developmentally down-regulated 4-like & 2,30 & chr18 \\
\hline $\mathrm{NEFH}$ & 4744 & neurofilament, heavy polypeptide $200 \mathrm{kDa}$ & 3,99 & chr22 \\
\hline NEFL & 4747 & neurofilament, light polypeptide $68 \mathrm{kDa}$ & 3,21 & chr8 \\
\hline NETO1 & 81832 & neuropilin (NRP) and tolloid (TLL)-like 1 & 3,92 & chr18 \\
\hline NFATC2IP & 84901 & nuclear factor of activated T-cells, cytoplasmic, calcineurin-dependent 2 in & 2,62 & chr16 \\
\hline NFE2L3 & 9603 & nuclear factor (erythroid-derived 2)-like 3 & 8,22 & chr7 \\
\hline
\end{tabular}




\begin{tabular}{|c|c|c|c|c|}
\hline Gene Symbol & Entrez Gene & Gene Title & NPC_down & Chromosome Number(Avadis) \\
\hline NFIX & 4784 & nuclear factor I/X (CCAAT-binding transcription factor) & 2,76 & chr19 \\
\hline NFYB & 4801 & nuclear transcription factor $\mathrm{Y}$, beta & 2,84 & chr3 \\
\hline NHP2L1 & 4809 & |NHP2 non-histone chromosome protein 2-like 1 (S. cerevisiae) & 2,13 & chr22 \\
\hline NIFIE14 & 10430 & seven transmembrane domain protein & 2,43 & chr19 \\
\hline NLE1 & 54475 & notchless homolog 1 (Drosophila) & 3,25 & chr17 \\
\hline NLGN4X & 57502 & neuroligin 4, X-linked & 2,30 & $\operatorname{chr} x$ \\
\hline NLK & 51701 & nemo like kinase & 2,17 & chr17 \\
\hline NLN & 57486 & neurolysin (metallopeptidase M3 family) & 3,20 & chr5 \\
\hline NMB & 4828 & neuromedin B & 2,09 & chr15 \\
\hline NME3 & 4832 & non-metastatic cells 3 , protein expressed in & 2,45 & chr16 \\
\hline NMI & 9111 & $\mathrm{~N}$-myc (and STAT) interactor & 11,10 & chr2 \\
\hline NMT1 & 4836 & N-myristoyltransferase 1 & 2,10 & chr17 \\
\hline NMU & 10874 & neuromedin U & 6,95 & chr4 \\
\hline NOB1P & 28987 & nin one binding protein & 2,56 & chr4 \\
\hline NODAL & 4838 & nodal homolog (mouse) & 9,78 & chr10 \\
\hline NOL11 & 25926 & nucleolar protein 11 & 2,02 & chr17 \\
\hline NOL5A & 10528 & nucleolar protein $5 \mathrm{~A}$ (56kDa with $\mathrm{KKE} / \mathrm{D}$ repeat) & 2,00 & chr20 \\
\hline NOL7 & 51406 & Nucleolar protein $7,27 \mathrm{kDa}$ & 2,03 & chr6 \\
\hline NOLA3 & 55505 & nucleolar protein family A, member 3 (H/ACA small nucleolar RNPs) & 2,14 & chr15 \\
\hline NOLC1 & 9221 & nucleolar and coiled-body phosphoprotein 1 & 3,02 & chr10 \\
\hline NOM1 & 64434 & nucleolar protein with MIF4G domain 1 & 2,66 & chr7 \\
\hline NP & 4860 & nucleoside phosphorylase & 3,62 & chr14 \\
\hline NPM3 & 10360 & nucleophosmin/nucleoplasmin, 3 & 2,94 & chr10 \\
\hline NPTX1 & 4884 & neuronal pentraxin I & 4,53 & chr17 \\
\hline NPTX2 & 4885 & neuronal pentraxin II & 2,15 & chr7 \\
\hline NQO2 & 4835 & $\mathrm{NAD}(\mathrm{P}) \mathrm{H}$ dehydrogenase, quinone 2 & 2,08 & chr6 \\
\hline NR5A2 & 2494 & nuclear receptor subfamily 5 , group A, member 2 & 2,42 & chr1 \\
\hline NRBF2 & 29982 & nuclear receptor binding factor 2 & 2,37 & chr8 \\
\hline NSBP1 & 79366 & nucleosomal binding protein 1 & 3,04 & $\operatorname{chr} X$ \\
\hline NSD1 & 64324 & nuclear receptor binding SET domain protein 1 & 2,37 & chr5 \\
\hline NSFL1C & 55968 & NSFL1 (p97) cofactor (p47) & 2,06 & chr20 \\
\hline NTHL1 & 4913 & nth endonuclease III-like 1 (E. coli) & 2,58 & chr16 \\
\hline NTS & 4922 & neurotensin & 4,45 & chr12 \\
\hline NUDT1 & 4521 & nudix (nucleoside diphosphate linked moiety X)-type motif 1 & 2,29 & chr7 \\
\hline NUDT15 & 55270 & nudix (nucleoside diphosphate linked moiety X)-type motif 15 & 2,62 & chr13 \\
\hline NUDT21 & 11051 & nudix (nucleoside diphosphate linked moiety X)-type motif 21 & 4,50 & chr16 \\
\hline NUDT22 & 84304 & nudix (nucleoside diphosphate linked moiety X)-type motif 22 /// nudix (nu & 2,00 & chr11 \\
\hline NUP50 & 10762 & nucleoporin 50kDa & 2,36 & chr22 \\
\hline NUP88 & 4927 & nucleoporin $88 \mathrm{kDa}$ & 2,17 & chr17 \\
\hline OACT1 & 154141 & O-acyltransferase (membrane bound) domain containing 1 & 3,41 & chr6 \\
\hline ОАТ & 4942 & Ornithine aminotransferase (gyrate atrophy) & 2,41 & chr10 \\
\hline OAZ1 & 4946 & ornithine decarboxylase antizyme 1 & 2,22 & chr2 \\
\hline OAZ2 & 4947 & ornithine decarboxylase antizyme 2 & 2,48 & chr15 \\
\hline OGDHL & 55753 & oxoglutarate dehydrogenase-like & 3,01 & chr10 \\
\hline OIP5 & 11339 & Opa interacting protein 5 & 2,01 & chr15 \\
\hline OLFM1 & 10439 & olfactomedin 1 & 2,61 & chr9 \\
\hline OMA1 & 115209 & OMA1 homolog, zinc metallopeptidase (S. cerevisiae) & 3,24 & chr1 \\
\hline ORC1L & 4998 & origin recognition complex, subunit 1 -like (yeast) & 2,54 & chr1 \\
\hline OSBPL10 & 114884 & oxysterol binding protein-like 10 & 5,40 & chr3 \\
\hline OSTM1 & 28962 & osteopetrosis associated transmembrane protein 1 & 2,15 & chr6 \\
\hline OTUD6B & 51633 & OTU domain containing 6B & 2,10 & chr8 \\
\hline OVOL1 & 5017 & ovo-like 1(Drosophila) & 2,02 & chr11 \\
\hline OVOL2 & 58495 & ovo-like 2 (Drosophila) /// ovo-like 2 (Drosophila) & 4,95 & chr20 \\
\hline P2RX5 & 5026 & purinergic receptor P2X, ligand-gated ion channel, 5 & 2,62 & chr17 \\
\hline PACSIN1 & 29993 & protein kinase $C$ and casein kinase substrate in neurons 1 & 2,63 & chr6 \\
\hline PAH & 5053 & phenylalanine hydroxylase & 2,33 & chr12 \\
\hline PAICS & 10606 & phosphoribosylaminoimidazole carboxylase, phosphoribosylaminoimidazc & 2,09 & chr4 \\
\hline PAK1 & 5058 & p21/Cdc42/Rac1-activated kinase 1 (STE20 homolog, yeast) & 3,44 & chr11 \\
\hline PAK1IP1 & 55003 & PAK1 interacting protein 1 & 2,71 & chr6 \\
\hline PAPD1 & 55149 & PAP associated domain containing 1 & 2,13 & chr10 \\
\hline PAPSS2 & 9060 & 3'-phosphoadenosine 5'-phosphosulfate synthase 2 & 4,71 & chr10 \\
\hline PAQR5 & 54852 & progestin and adipoQ receptor family member V & 2,02 & chr15 \\
\hline PARD6A & 50855 & par-6 partitioning defective 6 homolog alpha (C.elegans) & 2,07 & chr16 \\
\hline PARP12 & 64761 & poly (ADP-ribose) polymerase family, member 12 & 4,74 & chr7 \\
\hline PAWR & 5074 & PRKC, apoptosis, WT1, regulator & 2,91 & chr20 \\
\hline PBP & 5037 & prostatic binding protein & 2,19 & chr12 \\
\hline
\end{tabular}




\begin{tabular}{|c|c|c|c|c|}
\hline Gene Symbol & Entrez Gene & Gene Title & NPC_down & Chromosome Number(Avadis) \\
\hline PBX1 & 5087 & Pre-B-cell leukemia transcription factor 1 & 3,32 & chr1 \\
\hline PCBD2 & 84105 & 6-pyruvoyl-tetrahydropterin synthase/dimerization cofactor of hepatocyte & 3,20 & chr5 \\
\hline |PCMT1 & 5110 & protein-L-isoaspartate (D-aspartate) O-methyltransferase & 2,11 & chr6 \\
\hline PCNXL2 & 80003 & Pecanex-like 2 (Drosophila) & 2,72 & chr1 \\
\hline PCSK9 & 255738 & proprotein convertase subtilisin/kexin type 9 & 5,11 & chr1 \\
\hline PCYT1B & 9468 & phosphate cytidylyltransferase 1 , choline, beta & 2,95 & $\operatorname{chr} X$ \\
\hline PDAP1 & 11333 & PDGFA associated protein 1 & 2,68 & chr5 \\
\hline PDCD10 & 11235 & programmed cell death 10 & 2,22 & chr3 \\
\hline PDCL3 & $285359 / / / 79031$ & phosducin-like 3 /// hypothetical protein FLJ12205 & 2,45 & chr2 \\
\hline PDF & $64146 / / / 84342$ & peptide deformylase-like protein /// component of oligomeric golgi compley & 2,00 & chr16 \\
\hline PDGFA & 5154 & platelet-derived growth factor alpha polypeptide & 2,76 & chr7_random \\
\hline PDHB & 5162 & pyruvate dehydrogenase (lipoamide) beta & 2,05 & chr3 \\
\hline PDIA5 & 10954 & protein disulfide isomerase family $\mathrm{A}$, member 5 & 2,69 & chr3 \\
\hline PDK3 & 5165 & pyruvate dehydrogenase kinase, isoenzyme 3 & 3,03 & $\operatorname{chr} \mathrm{X}$ \\
\hline PDLIM1 & 9124 & PDZ and LIM domain 1 (elfin) & 4,00 & chr10 \\
\hline PDPN & 10630 & podoplanin & 5,95 & chr1 \\
\hline |PDZK3 & 23037 & PDZ domain containing 3 & 2,53 & chr5 \\
\hline PDZK4 & 57595 & PDZ domain containing 4 & 3,94 & $\operatorname{chr} X$ \\
\hline PERP & 64065 & PERP, TP53 apoptosis effector & 2,59 & chr6 \\
\hline PFDN4 & 5203 & prefoldin 4 & 2,19 & chr20 \\
\hline PFN1 & 5216 & profilin 1 & 2,55 & chr1 \\
\hline PGBD5 & 79605 & piggyBac transposable element derived 5 & 2,80 & chr1 \\
\hline PGK1 & 5230 & phosphoglycerate kinase 1 & 2,00 & $\operatorname{chr} X$ \\
\hline PGM2L1 & 283209 & phosphoglucomutase 2 -like 1 & 2,22 & chr11 \\
\hline PGRMC1 & 10857 & progesterone receptor membrane component 1 & 2,19 & $\operatorname{chr} X$ \\
\hline PHB & 5245 & prohibitin & 2,28 & chr16 \\
\hline PHC1 & 1911 & polyhomeotic-like 1 (Drosophila) & 6,36 & chr12 \\
\hline PHF15 & 23338 & PHD finger protein 15 & 5,66 & chr5 \\
\hline PHF17 & 79960 & PHD finger protein 17 & 2,69 & chr4 \\
\hline PHF19 & 26147 & PHD finger protein 19 & 2,96 & chr9 \\
\hline PHLDA1 & 22822 & Pleckstrin homology-like domain, family A, member 1 & 3,12 & chr12 \\
\hline PIAS2 & 9063 & Protein inhibitor of activated STAT, 2 & 2,70 & chr18 \\
\hline PIGW & 284098 & phosphatidylinositol glycan, class W & 2,24 & chr17 \\
\hline PIK3CB & 5291 & phosphoinositide-3-kinase, catalytic, beta polypeptide & 2,18 & chr3 \\
\hline PIK3CD & 5293 & phosphoinositide-3-kinase, catalytic, delta polypeptide /// phosphoinositide & 2,83 & chr1 \\
\hline PIM2 & 11040 & pim-2 oncogene & 3,86 & $\operatorname{chr} X$ \\
\hline PINK1 & 65018 & PTEN induced putative kinase 1 & 2,16 & chr1 \\
\hline PINX1 & 54984 & PIN2-interacting protein 1 & 2,33 & chr8 \\
\hline PIP5K2A & 5305 & Phosphatidylinositol-4-phosphate 5-kinase, type II, alpha & 2,60 & chr10 \\
\hline PIPOX & 51268 & pipecolic acid oxidase & 2,96 & chr17 \\
\hline PITPNC1 & 26207 & phosphatidylinositol transfer protein, cytoplasmic 1 & 3,76 & chr17 \\
\hline PKIB & 5570 & protein kinase (cAMP-dependent, catalytic) inhibitor beta & 2,69 & chr6 \\
\hline PLA2G12A & 81579 & phospholipase A2, group XIIA /// phospholipase A2, group XIIA & 2,71 & chr1 \\
\hline PLAA & 9373 & phospholipase A2-activating protein & 2,01 & chr9 \\
\hline PLS1 & 5357 & plastin 1 (I isoform) & 2,30 & chr3 \\
\hline PLSCR1 & 5359 & phospholipid scramblase 1 & 3,01 & chr3 \\
\hline PMAIP1 & 5366 & phorbol-12-myristate-13-acetate-induced protein 1 & 31,01 & chr18 \\
\hline PMM2 & 5373 & phosphomannomutase 2 & 2,15 & chr16 \\
\hline PODXL & 5420 & podocalyxin-like & 4,34 & chr7 \\
\hline POLE4 & 56655 & polymerase (DNA-directed), epsilon 4 (p12 subunit) & 2,16 & chr2 \\
\hline POLR1A & 25885 & polymerase (RNA) I polypeptide A, $194 \mathrm{kDa}$ & 2,36 & chr2 \\
\hline POLR1B & 84172 & polymerase (RNA) I polypeptide B, $128 \mathrm{kDa}$ & 2,93 & chr2 \\
\hline POLR1D & 51082 & polymerase (RNA) I polypeptide D, $16 \mathrm{kDa}$ & 2,58 & chr13 \\
\hline POLR2E & 5434 & polymerase (RNA) II (DNA directed) polypeptide E, 25kDa & 2,17 & chr19 \\
\hline POLR2F & 5435 & polymerase (RNA) II (DNA directed) polypeptide F & 2,39 & chr22 \\
\hline POLR2I & 5438 & polymerase (RNA) II (DNA directed) polypeptide I, $14.5 \mathrm{kDa}$ & 2,27 & chr19 \\
\hline POLR2L & 5441 & polymerase (RNA) II (DNA directed) polypeptide L, 7.6kDa /// polymerase & 2,14 & chr11 \\
\hline POLR3D & 661 & polymerase (RNA) III (DNA directed) polypeptide D, 44kDa & 2,16 & chr8 \\
\hline POLR3G & 10622 & Polymerase (RNA) III (DNA directed) polypeptide G (32kD) & 11,89 & chr5 \\
\hline POLR3K & 51728 & polymerase (RNA) III (DNA directed) polypeptide K, $12.3 \mathrm{kDa}$ & 3,17 & chr16 \\
\hline POLRMT & 5442 & polymerase (RNA) mitochondrial (DNA directed) & 2,19 & chr17 \\
\hline POP7 & 10248 & processing of precursor 7 , ribonuclease P subunit (S. cerevisiae) & 2,16 & chr7 \\
\hline POR & 5447 & P450 (cytochrome) oxidoreductase & 2,41 & chr7 \\
\hline POU5F1 & $628 / / / 5460 / / / 5$ & POU domain, class 5 , transcription factor $1 / / /$ POU domain, class 5 , trans & 32,05 & chr1 \\
\hline PPAN & 56342 & peter pan homolog (Drosophila) & 2,06 & chr19 \\
\hline PPAP2A & 8611 & phosphatidic acid phosphatase type $2 \mathrm{~A}$ & 3,05 & chr5 \\
\hline
\end{tabular}




\begin{tabular}{|c|c|c|c|c|}
\hline Gene Symbol & Entrez Gene & Gene Title & NPC_down & Chromosome Number(Avadis) \\
\hline PPAP2C & 8612 & phosphatidic acid phosphatase type $2 \mathrm{C}$ & 8,09 & chr19 \\
\hline PPCDC & 60490 & phosphopantothenoylcysteine decarboxylase & 2,41 & chr15 \\
\hline PPGB & 5476 & protective protein for beta-galactosidase (galactosialidosis) & 2,34 & chr20 \\
\hline PPHLN1 & 51535 & periphilin 1 & 2,25 & chr12 \\
\hline PPID & 5481 & peptidylprolyl isomerase $\mathrm{D}$ (cyclophilin $\mathrm{D}$ ) & 3,51 & chr4 \\
\hline PPM1B & 5495 & protein phosphatase $1 \mathrm{~B}$ (formerly $2 \mathrm{C}$ ), magnesium-dependent, beta isofo & 2,75 & chr2 \\
\hline PPM1H & 57460 & protein phosphatase $1 \mathrm{H}$ (PP2C domain containing) & 2,85 & chr12 \\
\hline |PPM1J & 333926 & protein phosphatase $1 \mathrm{~J}$ (PP2C domain containing) & 2,79 & chr1 \\
\hline PPP1R14A & 94274 & protein phosphatase 1 , regulatory (inhibitor) subunit $14 \mathrm{~A}$ & 3,61 & chr19 \\
\hline PPP1R14B & 26472 & protein phosphatase 1 , regulatory (inhibitor) subunit 14B & 3,17 & chr22 \\
\hline PPP1R16B & 26051 & protein phosphatase 1 , regulatory (inhibitor) subunit 16B & 4,22 & chr20 \\
\hline PPP2R1B & 5519 & protein phosphatase 2 (formerly $2 A$ ), regulatory subunit $A$ (PR 65 ), beta is & 3,50 & chr11 \\
\hline PPP2R5A & 5525 & protein phosphatase 2 , regulatory subunit $B$ (B56), alpha isoform & 2,67 & chr1 \\
\hline PPP6C & 5537 & Protein phosphatase 6 , catalytic subunit & 2,01 & chr9 \\
\hline PQBP1 & 10084 & polyglutamine binding protein 1 & 2,05 & $\operatorname{chr} X$ \\
\hline PQLC3 & 130814 & PQ loop repeat containing 3 & 3,76 & chr2 \\
\hline PRDM14 & 63978 & PR domain containing 14 & 9,93 & chr8 \\
\hline PRDX6 & 9588 & peroxiredoxin 6 & 2,01 & chr1 \\
\hline PREPL & 9581 & prolyl endopeptidase-like & 2,04 & chr2 \\
\hline PRKAA1 & 5562 & protein kinase, AMP-activated, alpha 1 catalytic subunit & 2,02 & chr5 \\
\hline PRKAB2 & 5565 & protein kinase, AMP-activated, beta 2 non-catalytic subunit & 2,79 & chr1 \\
\hline PRKAR1B & 5575 & Protein kinase, cAMP-dependent, regulatory, type I, beta & 4,38 & chr21 \\
\hline PRKAR2B & 5577 & protein kinase, cAMP-dependent, regulatory, type II, beta & 2,13 & chr7 \\
\hline PRKCB1 & 5579 & protein kinase $\mathrm{C}$, beta 1 & 2,74 & chr16 \\
\hline PRKCQ & 5588 & protein kinase C, theta & 3,08 & chr10 \\
\hline PRO0149 & 29035 & PRO0149 protein & 2,19 & chr16 \\
\hline PRO1843 & 55378 & hypothetical protein PRO1843 & 2,53 & chr3 \\
\hline PRO1853 & 55471 & hypothetical protein PRO1853 & 2,39 & chr2 \\
\hline PROCR & 10544 & protein C receptor, endothelial (EPCR) & 3,03 & chr20 \\
\hline PRODH & 5625 & proline dehydrogenase (oxidase) 1 & 4,03 & chr22 \\
\hline PRPF38A & 84950 & PRP38 pre-mRNA processing factor 38 (yeast) domain containing A & 2,04 & chr1 \\
\hline PRPS1 & 5631 & phosphoribosyl pyrophosphate synthetase 1 & 3,07 & $\operatorname{chr} X$ \\
\hline PRPS2 & 5634 & Phosphoribosyl pyrophosphate synthetase 2 & 2,43 & $\operatorname{chr} X$ \\
\hline PRSS16 & 10279 & protease, serine, 16 (thymus) & 2,62 & chr6 \\
\hline PRSS8 & 5652 & protease, serine, 8 (prostasin) & 2,07 & chr16 \\
\hline PSMA5 & 5686 & proteasome (prosome, macropain) subunit, alpha type, 5 & 2,29 & chr1 \\
\hline PSMB10 & 5699 & proteasome (prosome, macropain) subunit, beta type, 10 & 3,64 & chr16 \\
\hline PSMB8 & 5696 & proteasome (prosome, macropain) subunit, beta type, 8 (large multifunctic & 7,99 & chr6 \\
\hline PSMC4 & 5704 & proteasome (prosome, macropain) 26S subunit, ATPase, 4 & 3,13 & chr19 \\
\hline PSMD12 & 5718 & proteasome (prosome, macropain) 26S subunit, non-ATPase, 12 & 2,33 & chr17 \\
\hline PSME1 & 5720 & proteasome (prosome, macropain) activator subunit 1 (PA28 alpha) & 2,57 & chr14 \\
\hline PSME2 & 5721 & proteasome (prosome, macropain) activator subunit 2 (PA28 beta) & 2,36 & chr5 \\
\hline PSME3 & 10197 & proteasome (prosome, macropain) activator subunit 3 (PA28 gamma; Ki) & 2,65 & chr17 \\
\hline PSMF1 & 9491 & proteasome (prosome, macropain) inhibitor subunit 1 (PI31) & 2,65 & chr20 \\
\hline PSPH & 5723 & phosphoserine phosphatase & 2,01 & chr7 \\
\hline PTCHD1 & 139411 & patched domain containing 1 & 2,11 & $\operatorname{chr} x$ \\
\hline PTEN & 5728 & Phosphatase and tensin homolog (mutated in multiple advanced cancers & 2,12 & chr10 \\
\hline PTK9L & 11344 & PTK9L protein tyrosine kinase 9-like (A6-related protein) & 2,27 & chr3 \\
\hline PTMA & 5757 & prothymosin, alpha (gene sequence 28 ) & 2,06 & chr2 \\
\hline PTPN2 & 5771 & protein tyrosine phosphatase, non-receptor type 2 & 2,19 & chr13 \\
\hline PTPN6 & 5777 & protein tyrosine phosphatase, non-receptor type 6 & 2,85 & chr12 \\
\hline PUS1 & 80324 & pseudouridylate synthase 1 & 2,18 & chr12 \\
\hline PWP2H & 5822 & PWP2 periodic tryptophan protein homolog (yeast) & 2,23 & chr21 \\
\hline PX19 & 27166 & px19-like protein & 3,05 & chr1 \\
\hline PYCARD & 29108 & PYD and CARD domain containing & 8,35 & chr16 \\
\hline |PYCR2 & 29920 & pyrroline-5-carboxylate reductase family, member 2 & 2,95 & chr1 \\
\hline QRSL1 & 55278 & glutaminyl-tRNA synthase (glutamine-hydrolyzing)-like 1 & 2,31 & chr6 \\
\hline RAB10 & 10890 & RAB10, member RAS oncogene family & 2,15 & chr2 \\
\hline RAB11FIP4 & 84440 & RAB11 family interacting protein 4 (class II) & 2,81 & chr17 \\
\hline RAB15 & 376267 & RAB15, member RAS onocogene family & 2,16 & chr14 \\
\hline RAB20 & 55647 & RAB20, member RAS oncogene family & 4,17 & chr13 \\
\hline RAB34 & 83871 & RAB34, member RAS oncogene family & 3,47 & chr17 \\
\hline RAB38 & 23682 & RAB38, member RAS oncogene family & 2,10 & chr11 \\
\hline RAB39B & 116442 & RAB39B, member RAS oncogene family & 2,19 & $\operatorname{chr} X$ \\
\hline RAB3B & 5865 & RAB3B, member RAS oncogene family & 2,14 & chr1 \\
\hline RAB5A & 5868 & RAB5A, member RAS oncogene family & 2,20 & chr3 \\
\hline
\end{tabular}




\begin{tabular}{|c|c|c|c|c|}
\hline Gene Symbol & Entrez Gene & Gene Title & NPC_down & Chromosome Number(Avadis) \\
\hline RAB7 & 7879 & RAB7, member RAS oncogene family & 2,08 & chr3 \\
\hline RABAC1 & 10567 & Rab acceptor 1 (prenylated) & 2,00 & chr19 \\
\hline RABEPK & 10244 & Rab9 effector protein with kelch motifs & 2,16 & chr9 \\
\hline RABGAP1L & 9910 & RAB GTPase activating protein 1-like & 9,92 & chr1 \\
\hline RALA & 5898 & v-ral simian leukemia viral oncogene homolog A (ras related) & 2,68 & chr7 \\
\hline RALGPS1 & 9649 & Ral GEF with $\mathrm{PH}$ domain and $\mathrm{SH} 3$ binding motif 1 & 2,13 & chr9 \\
\hline RARRES2 & 5919 & retinoic acid receptor responder (tazarotene induced) 2 & 14,87 & chr7 \\
\hline RASEF & 158158 & RAS and EF-hand domain containing & 2,05 & chr9 \\
\hline RASGEF1A & 221002 & RasGEF domain family, member $1 \mathrm{~A}$ & 3,22 & chr10 \\
\hline RASGRP2 & 10235 & RAS guanyl releasing protein 2 (calcium and DAG-regulated) & 2,89 & chr11 \\
\hline RASIP1 & 54922 & Ras interacting protein 1 & 2,05 & chr19 \\
\hline RASL11B & 65997 & RAS-like, family 11 , member B & 5,59 & chr4 \\
\hline RBBP7 & 5931 & Retinoblastoma binding protein 7 & 2,34 & $\operatorname{chr} X$ \\
\hline RBM13 & 84549 & RNA binding motif protein 13 /// RNA binding motif protein 13 & 2,01 & chr8 \\
\hline RBM15 & 64783 & RNA binding motif protein 15 & 2,33 & chr1 \\
\hline RBM19 & 9904 & RNA binding motif protein 19 & 2,40 & chr12 \\
\hline RBM35A & 54845 & RNA binding motif protein $35 \mathrm{~A}$ & 13,21 & chr8 \\
\hline RBM35B & 80004 & RNA binding motif protein 35B & 3,72 & chr16 \\
\hline RBM7 & 10179 & RNA binding motif protein 7 & 2,76 & chr11 \\
\hline RBP7 & 116362 & retinol binding protein 7 , cellular & 2,23 & chr1 \\
\hline RBPMS & 11030 & RNA binding protein with multiple splicing & 3,46 & chr8 \\
\hline RBPMS2 & 348093 & RNA binding protein with multiple splicing 2 & 4,02 & chr15 \\
\hline RBPSUH & 3516 & recombining binding protein suppressor of hairless (Drosophila) & 2,64 & chr4 \\
\hline $\mathrm{RCC} 1$ & 1104 & regulator of chromosome condensation 1 & 2,01 & chr1 \\
\hline $\mathrm{RCHY} 1$ & 25898 & ring finger and $\mathrm{CHY}$ zinc finger domain containing 1 & 2,21 & chr4 \\
\hline $\mathrm{RCN} 3$ & 57333 & reticulocalbin 3, EF-hand calcium binding domain & 2,75 & chr19 \\
\hline RENT1 & 5976 & regulator of nonsense transcripts 1 & 2,27 & chr19 \\
\hline REXO2 & 25996 & REX2, RNA exonuclease 2 homolog (S. cerevisiae) & 3,49 & chr11 \\
\hline RIS1 & 25907 & Ras-induced senescence 1 & 5,18 & chr3 \\
\hline RNF12 & 51132 & ring finger protein 12 & 2,14 & chr15 \\
\hline RNF125 & 54941 & ring finger protein 125 & 3,83 & chr18 \\
\hline RNF126 & 55658 & ring finger protein 126 & 2,03 & chr19 \\
\hline RNF138 & 51444 & ring finger protein 138 & 2,63 & chr18 \\
\hline RNU3IP2 & 9136 & RNA, U3 small nucleolar interacting protein 2 & 3,17 & chr3 \\
\hline RP1-112K5.2 & 90121 & hypothetical protein DT1P1A10 & 2,16 & $\operatorname{chr} X$ \\
\hline RPE & 6120 & ribulose-5-phosphate-3-epimerase & 2,34 & chr2 \\
\hline RPE & $440001 / / / 6120$ & ribulose-5-phosphate-3-epimerase /// similar to Ribulose-phosphate 3-epir & 2,75 & chr10 \\
\hline RPL22L1 & 200916 & ribosomal protein L22-like 1 & 2,52 & chr3 \\
\hline RPL36AL & 6166 & ribosomal protein L36a-like & 2,25 & chr5 \\
\hline RPRM & 56475 & reprimo, TP53 dependant G2 arrest mediator candidate & 2,30 & chr2 \\
\hline RPS24 & 6229 & Ribosomal protein S24 & 2,96 & chr10 \\
\hline RPS27L & 51065 & Ribosomal protein S27-like & 2,01 & chr15 \\
\hline RPS6KA1 & 6195 & ribosomal protein S6 kinase, 90kDa, polypeptide 1 & 2,35 & chr1 \\
\hline RPUSD2 & 27079 & RNA pseudouridylate synthase domain containing 2 & 2,27 & chr15 \\
\hline RRAS2 & 22800 & related RAS viral (r-ras) oncogene homolog 2 & 4,73 & chr1 \\
\hline RRBP1 & 6238 & ribosome binding protein 1 homolog $180 \mathrm{kDa}(\mathrm{dog})$ & 3,21 & chr20 \\
\hline RRM2B & 50484 & ribonucleotide reductase M2 B (TP53 inducible) & 2,36 & chr8 \\
\hline RRS1 & 23212 & RRS1 ribosome biogenesis regulator homolog (S. cerevisiae) & 2,69 & chr8 \\
\hline RSL1D1 & 26156 & Ribosomal L1 domain containing 1 & 2,18 & chr16 \\
\hline RSU1 & 6251 & Ras suppressor protein 1 & 2,11 & chr10 \\
\hline RTN4IP1 & 84816 & reticulon 4 interacting protein 1 & 3,90 & chr12 \\
\hline SACM1L & 22908 & SAC1 suppressor of actin mutations 1-like (yeast) & 2,03 & chr3 \\
\hline SAMD6 & 203286 & sterile alpha motif domain containing 6 & 2,51 & chr9 \\
\hline SAMHD1 & 25939 & SAM domain and HD domain 1 & 9,21 & chr20 \\
\hline SAP18 & 10284 & sin3-associated polypeptide, $18 \mathrm{kDa}$ & 3,13 & chr13 \\
\hline SAS10 & 57050 & disrupter of silencing 10 & 2,05 & chr4 \\
\hline SAV1 & 60485 & salvador homolog 1 (Drosophila) & 2,03 & chr14 \\
\hline SCAMP1 & 9522 & secretory carrier membrane protein 1 & 2,06 & chr5 \\
\hline SCAMP5 & 192683 & secretory carrier membrane protein 5 & 2,02 & chr15 \\
\hline SCAND1 & 51282 & SCAN domain containing 1 & 2,37 & chr20 \\
\hline SCARB1 & 949 & scavenger receptor class $B$, member 1 & 2,49 & chr12 \\
\hline SCD & 6319 & stearoyl-CoA desaturase (delta-9-desaturase) /// stearoyl-CoA desaturase & 2,89 & chr10 \\
\hline SCG3 & 29106 & secretogranin III & 12,46 & chr15 \\
\hline SCGB3A2 & 117156 & secretoglobin, family $3 \mathrm{~A}$, member 2 & 10,13 & chr5 \\
\hline SCLY & 51540 & selenocysteine lyase & 3,66 & chr2 \\
\hline SCNN1A & 6337 & sodium channel, nonvoltage-gated 1 alpha & 12,10 & chr12 \\
\hline
\end{tabular}




\begin{tabular}{|c|c|c|c|c|}
\hline Gene Symbol & Entrez Gene & Gene Title & NPC_down & Chromosome Number(Avadis) \\
\hline SCNN1G & 6340 & sodium channel, nonvoltage-gated 1 , gamma & 2,24 & chr16 \\
\hline sco1 & 6341 & SCO cytochrome oxidase deficient homolog 1 (yeast) & 2,06 & chr17 \\
\hline $\mathrm{scO} 2$ & 9997 & SCO cytochrome oxidase deficient homolog 2 (yeast) & 2,04 & chr22 \\
\hline SCP2 & 6342 & Sterol carrier protein 2 & 2,01 & chr1 \\
\hline SDHB & 6390 & succinate dehydrogenase complex, subunit B, iron sulfur (Ip) & 3,40 & chr1 \\
\hline SDHD & 6392 & succinate dehydrogenase complex, subunit $\mathrm{D}$, integral membrane protein & 2,92 & chr1 \\
\hline SEC11L3 & 90701 & SEC11-like 3 (S. cerevisiae) & 2,19 & chr18 \\
\hline SEC22L3 & 9117 & SEC22 vesicle trafficking protein-like 3 (S. cerevisiae) /// SEC22 vesicle tr & 2,79 & chr3 \\
\hline SEC24D & 9871 & SEC24 related gene family, member D (S. cerevisiae) & 2,89 & chr4 \\
\hline SEC5L1 & 55770 & SEC5-like 1 (S. cerevisiae) & 2,21 & chr6 \\
\hline SEH1L & 81929 & SEH1-like (S. cerevisiae) & 2,13 & chr18 \\
\hline SELT & 51714 & selenoprotein T & 2,03 & chr3 \\
\hline SEMAGA & 57556 & sema domain, transmembrane domain (TM), and cytoplasmic domain, (s & 2,25 & chr5 \\
\hline SENP2 & 59343 & SUMO1/sentrin/SMT3 specific peptidase 2 & 2,03 & chr3 \\
\hline SEPHS1 & 22929 & Selenophosphate synthetase 1 & 7,65 & chr2 \\
\hline SERF1A & 56617 /// 8293 & small EDRK-rich factor 1A (telomeric) /// small EDRK-rich factor 1B (centre & 2,09 & chr5 \\
\hline SERF2 & 10169 & small EDRK-rich factor 2 & 2,06 & chr15 \\
\hline SERF2 & $10169 / / / 25764$ & small EDRK-rich factor $2 / / /$ Huntingtin interacting protein $\mathrm{K}$ & 2,41 & chr15 \\
\hline SERP1 & 27230 & stress-associated endoplasmic reticulum protein 1 & 2,05 & chr3 \\
\hline SERPINB9 & 5272 & serpin peptidase inhibitor, clade B (ovalbumin), member 9 & 2,01 & chr6 \\
\hline SERPINE2 & 5270 & serpin peptidase inhibitor, clade $E$ (nexin, plasminogen activator inhibitor $t$ & 2,34 & chr2 \\
\hline SERPINI1 & 5274 & serpin peptidase inhibitor, clade I (neuroserpin), member 1 & 3,43 & chr3 \\
\hline SET & $389168 / / / 6418$ & SET translocation (myeloid leukemia-associated) /// similar to SET protein & 3,25 & $\operatorname{chrX}$ \\
\hline SFRS2 & 6427 & splicing factor, arginine/serine-rich 2 & 2,21 & chr17 \\
\hline SFT2D1 & 113402 & SFT2 domain containing 1 & 2,78 & chr6 \\
\hline SGK & 6446 & serum/glucocorticoid regulated kinase & 4,88 & chr6 \\
\hline SGK3 & 23678 & serum/glucocorticoid regulated kinase family, member 3 & 2,52 & chr8 \\
\hline SH3GL2 & 6456 & SH3-domain GRB2-like 2 & 2,63 & chr9 \\
\hline SHANK2 & 22941 & SH3 and multiple ankyrin repeat domains 2 & 2,64 & chr11 \\
\hline SHMT2 & 6472 & serine hydroxymethyltransferase 2 (mitochondrial) & 2,23 & chr12 \\
\hline SIGIRR & 59307 & single immunoglobulin and toll-interleukin 1 receptor (TIR) domain & 3,49 & chr11 \\
\hline SIPA1L1 & 26037 & signal-induced proliferation-associated 1 like 1 & 2,03 & chr14 \\
\hline SIRT1 & 23411 & sirtuin (silent mating type information regulation 2 homolog) 1 (S. cerevisia & 4,58 & chr10 \\
\hline SITPEC & 51295 & signaling intermediate in Toll pathway, evolutionarily conserved & 2,23 & $\operatorname{chr} 19$ \\
\hline SIVA & 10572 & CD27-binding (Siva) protein & 2,30 & chr14 \\
\hline SKIL & 6498 & SKI-like & 4,29 & chr3 \\
\hline SKP2 & 6502 & S-phase kinase-associated protein $2(\mathrm{p} 45)$ & 2,29 & chr5 \\
\hline SLC12A8 & 84561 & solute carrier family 12 (potassium/chloride transporters), member 8 & 2,72 & chr3 \\
\hline SLC13A3 & 64849 & solute carrier family 13 (sodium-dependent dicarboxylate transporter), me & 2,03 & chr20 \\
\hline SLC16A1 & 6566 & solute carrier family 16 (monocarboxylic acid transporters), member 1 & 3,59 & chr1 \\
\hline SLC16A10 & 117247 & solute carrier family 16 (monocarboxylic acid transporters), member 10 & 3,47 & chr6 \\
\hline SLC18A2 & 6571 & Solute carrier family 18 (vesicular monoamine), member 2 & 2,02 & chr10 \\
\hline SLC1A5 & 6510 & solute carrier family 1 (neutral amino acid transporter), member 5 & 2,28 & chr19 \\
\hline SLC25A19 & 60386 & solute carrier family 25 (mitochondrial deoxynucleotide carrier), member 1 & 3,14 & chr17 \\
\hline SLC25A21 & 89874 & solute carrier family 25 (mitochondrial oxodicarboxylate carrier), member 2 & 2,27 & chr14 \\
\hline SLC25A4 & 291 & solute carrier family 25 (mitochondrial carrier; adenine nucleotide transloce & 3,22 & chr4 \\
\hline SLC27A2 & 11001 & solute carrier family 27 (fatty acid transporter), member 2 & 2,29 & chr15 \\
\hline SLC27A3 & 11000 & solute carrier family 27 (fatty acid transporter), member 3 & 2,88 & chr1 \\
\hline SLC37A1 & 54020 & solute carrier family 37 (glycerol-3-phosphate transporter), member 1 & 2,49 & chr21 \\
\hline SLC38A5 & 92745 & solute carrier family 38 , member 5 & 2,53 & $\operatorname{chr} \mathrm{X}$ \\
\hline SLC39A1 & 27173 & solute carrier family 39 (zinc transporter), member 1 & 2,07 & chr1 \\
\hline SLC39A14 & 23516 & solute carrier family 39 (zinc transporter), member 14 & 2,95 & chr8 \\
\hline SLC39A8 & 64116 & solute carrier family 39 (zinc transporter), member 8 & 2,75 & chr4 \\
\hline SLC43A1 & 8501 & solute carrier family 43 , member 1 & 2,87 & chr11 \\
\hline SLC44A1 & 23446 & solute carrier family 44, member 1 & 3,12 & chr9 \\
\hline SLC4A11 & 83959 & solute carrier family 4, sodium bicarbonate transporter-like, member 11 & 4,80 & chr20 \\
\hline SLC4A5 & 57835 & Solute carrier family 4, sodium bicarbonate cotransporter, member 5 & 2,17 & chr2 \\
\hline SLC7A3 & 84889 & solute carrier family 7 (cationic amino acid transporter, $y+$ system), memb & 6,00 & $\operatorname{chrX}$ \\
\hline SLCO4A1 & 28231 & solute carrier organic anion transporter family, member 4A1 & 2,77 & chr20 \\
\hline SLCO4C1 & 353189 & solute carrier organic anion transporter family, member 4C1 & 4,54 & chr5 \\
\hline SMC6L1 & 79677 & SMC6 structural maintenance of chromosomes 6-like 1 (yeast) & 2,05 & chr2 \\
\hline SMILE & 160418 & SMILE protein & 2,14 & chr12 \\
\hline SMN1 & $6606 / / / 6607$ & survival of motor neuron 1 , telomeric /// survival of motor neuron 2 , centro & 2,16 & chr5 \\
\hline SMPDL3B & 27293 & sphingomyelin phosphodiesterase, acid-like 3B & 5,26 & chr1 \\
\hline SNRPC & 6631 & small nuclear ribonucleoprotein polypeptide C & 2,02 & chr5 \\
\hline SNRPN & $6638 / / / 8926$ & small nuclear ribonucleoprotein polypeptide $\mathrm{N} / / /$ SNRPN upstream readin & 5,38 & chr15 \\
\hline
\end{tabular}




\begin{tabular}{|c|c|c|c|c|}
\hline Gene Symbol & Entrez Gene & Gene Title & NPC_down & Chromosome Number(Avadis) \\
\hline SNRPN & 6638 & Small nuclear ribonucleoprotein polypeptide $\mathrm{N}$ & 4,20 & $\operatorname{chr15}$ \\
\hline SNX5 & 27131 & sorting nexin 5 & 2,03 & chr20 \\
\hline SOCS1 & 8651 & suppressor of cytokine signaling 1 & 3,51 & chr16 \\
\hline SOD2 & 6648 & superoxide dismutase 2 , mitochondrial & 3,16 & chr6 \\
\hline SORBS1 & 10580 & sorbin and $\mathrm{SH} 3$ domain containing 1 & 3,46 & chr10 \\
\hline SORL1 & 6653 & sortilin-related receptor, L(DLR class) A repeats-containing & 2,41 & chr11 \\
\hline SP110 & 3431 & SP110 nuclear body protein & 2,15 & chr2 \\
\hline SPATA11 & 84266 & spermatogenesis associated 11 & 2,05 & chr19 \\
\hline SPBC25 & 57405 & spindle pole body component 25 homolog (S. cerevisiae) & 2,14 & chr2 \\
\hline SPG20 & 23111 & spastic paraplegia 20, spartin (Troyer syndrome) & 2,62 & chr13 \\
\hline SPIB & 6689 & Spi-B transcription factor (Spi-1/PU.1 related) /// Spi-B transcription factor & 2,73 & chr19 \\
\hline SPINT1 & 6692 & serine peptidase inhibitor, Kunitz type 1 & 4,77 & chr15 \\
\hline SPINT2 & 10653 & serine peptidase inhibitor, Kunitz type, 2 & 2,18 & chr19 \\
\hline SPRY4 & 81848 & Sprouty homolog 4 (Drosophila) & 5,13 & chr11 \\
\hline SPSB2 & 84727 & splA/ryanodine receptor domain and SOCS box containing 2 & 2,17 & chr12 \\
\hline SPTLC2 & 9517 & serine palmitoyltransferase, long chain base subunit 2 & 2,91 & chr14 \\
\hline SRD5A1 & 6715 & steroid-5-alpha-reductase, alpha polypeptide 1 (3-0xo-5 alpha-steroid delt & 2,15 & chr5 \\
\hline SRM & 6723 & spermidine synthase & 2,48 & chr1 \\
\hline SRPRB & 58477 & signal recognition particle receptor, B subunit & 2,18 & chr3 \\
\hline SRY & 6736 & sex determining region $Y$ & 2,02 & chrY \\
\hline SSNA1 & 8636 & Sjogren's syndrome nuclear autoantigen 1 & 2,21 & chr9 \\
\hline SSR1 & 6745 & Signal sequence receptor, alpha (translocon-associated protein alpha) & 2,24 & chr6 \\
\hline SSR3 & 6747 & signal sequence receptor, gamma (translocon-associated protein gamma & 2,11 & chr3 \\
\hline SSSCA1 & 10534 & Sjogren's syndrome/scleroderma autoantigen 1 & 2,31 & chr11 \\
\hline SSU72 & 29101 & Ssu72 RNA polymerase II CTD phosphatase homolog (yeast) & 2,11 & chr1 \\
\hline ST14 & 6768 & suppression of tumorigenicity 14 (colon carcinoma, matriptase, epithin) & 2,03 & chr11 \\
\hline ST8SIA3 & 51046 & ST8 alpha-N-acetyl-neuraminide alpha-2,8-sialyltransferase 3 & 2,36 & chr18 \\
\hline STAT3 & 6774 & signal transducer and activator of transcription 3 (acute-phase response f & 3,17 & chr17 \\
\hline STC2 & 8614 & stanniocalcin 2 & 3,44 & chr5 \\
\hline STEAP1 & 26872 & six transmembrane epithelial antigen of the prostate 1 & 3,08 & chr7 \\
\hline STEAP2 & 261729 & six transmembrane epithelial antigen of the prostate 2 & 2,72 & chr7 \\
\hline STEAP3 & 55240 & STEAP family member 3 & 3,37 & chr2 \\
\hline STIP1 & 10963 & stress-induced-phosphoprotein 1 (Hsp70/Hsp90-organizing protein) & 2,17 & chr11 \\
\hline STMN3 & 50861 & stathmin-like 3 & 4,51 & chr20 \\
\hline STRBP & 55342 & Spermatid perinuclear RNA binding protein & 2,09 & chr9 \\
\hline STRN & 6801 & Striatin, calmodulin binding protein & 2,41 & chr2 \\
\hline STX6 & 10228 & syntaxin 6 & 2,42 & chr1 \\
\hline SUB1 & 10923 & SUB1 homolog (S. cerevisiae) & 2,47 & chr5 \\
\hline SUGT1 & 10910 & SGT1, suppressor of G2 allele of SKP1 (S. cerevisiae) & 2,02 & chr13 \\
\hline SUPT3H & 8464 & suppressor of Ty 3 homolog (S. cerevisiae) & 2,19 & chr6 \\
\hline SURB7 & 9412 & SRB7 suppressor of RNA polymerase B homolog (yeast) & 2,88 & chr12 \\
\hline SURF4 & 6836 & surfeit 4 & 2,70 & chr9 \\
\hline SWAP70 & 23075 & SWAP-70 protein & 2,21 & chr11 \\
\hline SYAP1 & 94056 & synapse associated protein 1, SAP47 homolog (Drosophila) & 2,15 & $\operatorname{chr} \mathrm{X}$ \\
\hline SYNCRIP & 10492 & synaptotagmin binding, cytoplasmic RNA interacting protein & 3,18 & chr20 \\
\hline SYPL1 & 6856 & synaptophysin-like 1 & 2,46 & chr7 \\
\hline SYT6 & 148281 & synaptotagmin VI & 3,29 & chr1 \\
\hline TACSTD1 & 4072 & tumor-associated calcium signal transducer 1 & 4,44 & chr2 \\
\hline TAF12 & 6883 & TAF12 RNA polymerase II, TATA box binding protein (TBP)-associated fa & 2,08 & chr1 \\
\hline TAF4B & 6875 & TAF4b RNA polymerase II, TATA box binding protein (TBP)-associated fa & 2,93 & chr18 \\
\hline TAF5L & 27097 & TAF5-like RNA polymerase II, p300/CBP-associated factor (PCAF)-assoc & 2,11 & chr1 \\
\hline TALDO1 & 6888 & transaldolase 1 & 2,42 & chr11 \\
\hline TAP1 & 6890 & transporter 1, ATP-binding cassette, sub-family B (MDR/TAP) & 4,51 & chr6 \\
\hline TAP2 & 6891 & transporter 2, ATP-binding cassette, sub-family B (MDR/TAP) & 4,37 & chr6 \\
\hline TARS & 6897 & threonyl-tRNA synthetase & 3,28 & chr5 \\
\hline TBC1D23 & 55773 & TBC1 domain family, member 23 & 2,68 & chr3 \\
\hline TBC1D4 & 9882 & TBC1 domain family, member 4 & 2,13 & chr13 \\
\hline TBC1D8 & 11138 & TBC1 domain family, member 8 (with GRAM domain) & 2,75 & chr2 \\
\hline TBCC & 6903 & tubulin-specific chaperone $\mathrm{c}$ & 2,02 & chr6 \\
\hline TBCE & 6905 & tubulin-specific chaperone e & 2,04 & chr1 \\
\hline TBRG4 & 9238 & transforming growth factor beta regulator 4 & 2,67 & chr7 \\
\hline TCEB3 & 6924 & transcription elongation factor B (SIII), polypeptide $3(110 \mathrm{kDa}$, elongin A) & 2,52 & chr1 \\
\hline TCF7L1 & 83439 & transcription factor 7-like 1 (T-cell specific, HMG-box) /// transcription facto & 2,14 & chr2 \\
\hline TCFL5 & 10732 & Transcription factor-like 5 (basic helix-loop-helix) & 2,26 & chr20 \\
\hline TDGF1 & 6997 & teratocarcinoma-derived growth factor 1 & 45,48 & chr3 \\
\hline TDRKH & 11022 & fudor and $\mathrm{KH}$ domain containing & 2,02 & chr1 \\
\hline
\end{tabular}




\begin{tabular}{|c|c|c|c|c|}
\hline Gene Symbol & Entrez Gene & Gene Title & NPC_down & Chromosome Number(Avadis) \\
\hline TEAD4 & 7004 & TEA domain family member 4 & 5,14 & chr12 \\
\hline TEGT & 7009 & testis enhanced gene transcript (BAX inhibitor 1) & 2,05 & chr12 \\
\hline TERF1 & 7013 & telomeric repeat binding factor (NIMA-interacting) 1 & 10,42 & chr8 \\
\hline TEX15 & 56154 & testis expressed sequence 15 & 2,17 & chr8 \\
\hline TFB1M & 51106 & transcription factor B1, mitochondrial & 2,59 & chr6 \\
\hline TFDP2 & 7029 & Transcription factor Dp-2 (E2F dimerization partner 2) & 2,27 & chr3 \\
\hline TFRC & 7037 & transferrin receptor (p90, CD71) & 2,69 & chr3 \\
\hline THAP11 & 57215 & THAP domain containing 11 & 2,01 & chr16 \\
\hline THAP4 & 51078 & THAP domain containing 4 & 2,03 & chr2_random \\
\hline THBS2 & 7058 & thrombospondin 2 & 5,05 & chr6 \\
\hline THEM2 & 55856 & thioesterase superfamily member 2 & 4,10 & chr6 \\
\hline THOC4 & 10189 & THO complex 4 & 2,15 & chr17_random \\
\hline THY1 & 7070 & Thy -1 cell surface antigen & 5,02 & chr11 \\
\hline |THY28 & 29087 & thymocyte protein thy28 & 2,63 & chr11 \\
\hline TIGD7 & 91151 & tigger transposable element derived 7 /// tigger transposable element deri & 2,36 & chr16 \\
\hline TIMM13 & 26517 & translocase of inner mitochondrial membrane 13 homolog (yeast) & 2,71 & chr19 \\
\hline TIMM22 & 29928 & translocase of inner mitochondrial membrane 22 homolog (yeast) & 2,01 & chr17 \\
\hline TIMM50 & 92609 & translocase of inner mitochondrial membrane 50 homolog (yeast) & 2,32 & chr19 \\
\hline TIMM8A & 1678 & translocase of inner mitochondrial membrane 8 homolog A (yeast) & 2,20 & chr2 \\
\hline TIMM8B & 26521 & translocase of inner mitochondrial membrane 8 homolog B (yeast) & 2,04 & chr11 \\
\hline TIMP4 & 7079 & TIMP metallopeptidase inhibitor 4 & 2,96 & chr3 \\
\hline TJP2 & 9414 & tight junction protein 2 (zona occludens 2 ) & 2,06 & chr9 \\
\hline TKT & 7086 & transketolase (Wernicke-Korsakoff syndrome) & 5,33 & chr3 \\
\hline TLE2 & 7089 & transducin-like enhancer of split 2 (E(sp1) homolog, Drosophila) & 3,41 & $\operatorname{chr} 19$ \\
\hline TMED2 & 10959 & transmembrane emp24 domain trafficking protein 2 & 2,35 & chr12 \\
\hline TMED5 & 50999 & transmembrane emp24 protein transport domain containing 5 & 2,53 & chr1 \\
\hline TMEM11 & 8834 & transmembrane protein 11 & 2,04 & chr17 \\
\hline TMEM23 & 259230 & transmembrane protein 23 & 3,59 & chr10 \\
\hline TMEM28 & 27112 & transmembrane protein 28 & 2,27 & $\operatorname{chrX}$ \\
\hline TMEMЗОВ & 161291 & transmembrane protein $30 \mathrm{~B}$ & 3,79 & chr14 \\
\hline TMEM33 & 55161 & Transmembrane protein 33 & 2,13 & chr4 \\
\hline TMEM37 & 140738 & transmembrane protein 37 & 3,40 & chr2 \\
\hline TMEM4 & 10330 & transmembrane protein 4 & 2,06 & chr12 \\
\hline TMEM48 & 55706 & transmembrane protein 48 & 2,46 & chr1 \\
\hline TMEM64 & 169200 & transmembrane protein 64 & 2,64 & chr8 \\
\hline TMEPAI & 56937 & transmembrane, prostate androgen induced RNA & 3,98 & chr20 \\
\hline TMPRSS2 & 7113 & transmembrane protease, serine 2 & 3,12 & chr21 \\
\hline TNFRSF12A & 51330 & tumor necrosis factor receptor superfamily, member $12 \mathrm{~A}$ & 3,08 & chr16 \\
\hline TNFSF11 & 8600 & tumor necrosis factor (ligand) superfamily, member 11 & 5,08 & chr13 \\
\hline TNNI3 & 7137 & troponin I type 3 (cardiac) & 2,03 & chr19 \\
\hline TNPO3 & 23534 & transportin 3 & 2,11 & chr7 \\
\hline TOMM7 & $201725 / / / 54543$ & |translocase of outer mitochondrial membrane 7 homolog (yeast) /// hypoth & 2,15 & chr7 \\
\hline TOMM70A & 9868 & translocase of outer mitochondrial membrane 70 homolog A (yeast) & 2,90 & chr3 \\
\hline TOP1MT & 116447 & topoisomerase (DNA) I, mitochondrial & 2,31 & chr8 \\
\hline TOPORS & 10210 & topoisomerase I binding, arginine/serine-rich & 2,17 & chr9 \\
\hline TOR3A & 64222 & torsin family 3 , member A & 2,04 & chr1 \\
\hline TP53RK & 112858 & TP53 regulating kinase & 4,09 & chr20 \\
\hline TPARL & 55858 & TPA regulated locus & 2,60 & chr4 \\
\hline TPD52 & 7163 & tumor protein D52 & 7,84 & chr8 \\
\hline TPST2 & 8459 & tyrosylprotein sulfotransferase 2 & 4,76 & chr22 \\
\hline TRAF3IP2 & 10758 & TRAF3 interacting protein 2 & 3,82 & chr6 \\
\hline TRAPPC5 & 126003 & trafficking protein particle complex 5 & 2,27 & chr19 \\
\hline TREX2 & $11219 / / / 55559$ & three prime repair exonuclease $2 / / / 26 \mathrm{~S}$ proteasome-associated $\mathrm{UCH}$ inte & 2,23 & $\operatorname{chr} X$ \\
\hline TRHDE & 29953 & thyrotropin-releasing hormone degrading enzyme & 2,12 & chr12 \\
\hline TRIM14 & 9830 & tripartite motif-containing 14 & 9,94 & chr9 \\
\hline TRIM22 & 10346 & tripartite motif-containing 22 & 4,12 & chr11 \\
\hline TRIM37 & 4591 & |tripartite motif-containing 37 & 2,28 & chr17 \\
\hline TRIM59 & 286827 & tripartite motif-containing 59 & 2,63 & chr3 \\
\hline TRIM6 & 117854 & tripartite motif-containing 6 & 4,48 & chr11 \\
\hline TRIP10 & 9322 & thyroid hormone receptor interactor 10 & 2,61 & chr19 \\
\hline TSTA3 & 7264 & tissue specific transplantation antigen P35B & 2,39 & chr8 \\
\hline TTC9 & 23508 & tetratricopeptide repeat domain 9 & 2,22 & chr14 \\
\hline TTF2 & 8458 & |transcription termination factor, RNA polymerase II & 2,70 & chr1 \\
\hline TTMP & 79669 & TPA-induced transmembrane protein & 2,20 & chr3 \\
\hline TUBA1 & 7277 & tubulin, alpha 1 (testis specific) & 4,99 & chr2 \\
\hline |TUBB2 & 7280 & tubulin, beta 2 & 3,04 & chr6 \\
\hline
\end{tabular}




\begin{tabular}{|c|c|c|c|c|}
\hline Gene Symbol & Entrez Gene & Gene Title & NPC_down & Chromosome Number(Avadis) \\
\hline TUBB2 & $347733 / / / 7280$ & tubulin, beta 2 /// tubulin, beta polypeptide paralog & 2,66 & chr6 \\
\hline TUBB3 & 10381 & tubulin, beta 3 & 2,53 & chr16 \\
\hline TUBB6 & 84617 & tubulin, beta 6 & 2,92 & chr18 \\
\hline TUBG1 & 7283 & tubulin, gamma 1 & 2,11 & chr7 \\
\hline |TUSC2 & 11334 & tumor suppressor candidate 2 & 2,21 & chr3 \\
\hline TXN & 7295 & thioredoxin & 2,18 & chr17 \\
\hline TXNL2 & 10539 & thioredoxin-like 2 & 2,43 & chr6 \\
\hline U2AF2 & 11338 & U2 (RNU2) small nuclear RNA auxiliary factor 2 & 2,58 & chr19 \\
\hline UBE2D3 & 7323 & ubiquitin-conjugating enzyme E2D 3 (UBC4/5 homolog, yeast) & 2,20 & chr4 \\
\hline UBE2G1 & 7326 & ubiquitin-conjugating enzyme E2G 1 (UBC7 homolog, yeast) & 3,12 & chr17 \\
\hline UBE2M & 9040 & ubiquitin-conjugating enzyme E2M (UBC12 homolog, yeast) & 2,59 & chr16 \\
\hline UBE2N & 7334 & ubiquitin-conjugating enzyme E2N (UBC13 homolog, yeast) & 2,61 & chr12 \\
\hline UBE2S & 27338 & ubiquitin-conjugating enzyme E2S & 3,04 & chr17 \\
\hline UBE3B & 89910 & ubiquitin protein ligase $\mathrm{E} 3 \mathrm{~B}$ & 2,37 & chr12 \\
\hline UBE4B & 10277 & ubiquitination factor E4B (UFD2 homolog, yeast) & 2,30 & chr1 \\
\hline UBQLN1 & 29979 & ubiquilin 1 & 2,04 & chr9 \\
\hline UCHL1 & 7345 & ubiquitin carboxyl-terminal esterase L1 (ubiquitin thiolesterase) & 2,10 & chr4 \\
\hline UCHL3 & 7347 & ubiquitin carboxyl-terminal esterase L3 (ubiquitin thiolesterase) & 2,04 & chr13 \\
\hline UCK2 & 7371 & uridine-cytidine kinase 2 & 2,34 & chr1 \\
\hline UGCGL2 & 55757 & UDP-glucose ceramide glucosyltransferase-like 2 & 2,49 & chr13 \\
\hline UGP2 & 7360 & UDP-glucose pyrophosphorylase 2 & 4,36 & chr2 \\
\hline UGT8 & 7368 & UDP glycosyltransferase 8 (UDP-galactose ceramide galactosyltransferas & 7,99 & chr4 \\
\hline UIP1 & 55559 & 26S proteasome-associated UCH interacting protein 1 & 2,13 & $\operatorname{chr} X$ \\
\hline UMPS & 7372 & uridine monophosphate synthetase (orotate phosphoribosyl transferase a & 2,27 & chr3 \\
\hline UNG & 7374 & uracil-DNA glycosylase & 2,01 & chr12 \\
\hline UNQ501 & 374882 & MBC3205 & 2,05 & chr19 \\
\hline UQCR & 10975 & ubiquinol-cytochrome $\mathrm{c}$ reductase, $6.4 \mathrm{kDa}$ subunit & 2,08 & chr19 \\
\hline USP28 & 57646 & ubiquitin specific peptidase 28 & 5,28 & chr11 \\
\hline USP31 & 57478 & ubiquitin specific peptidase 31 & 2,04 & chr16 \\
\hline USP44 & 84101 & ubiquitin specific peptidase 44 & 6,56 & chr12 \\
\hline USP48 & 84196 & ubiquitin specific peptidase 48 & 2,42 & chr1 \\
\hline USP53 & 54532 & ubiquitin specific peptidase 53 & 2,10 & chr4 \\
\hline USP9X & 8239 & ubiquitin specific peptidase 9, X-linked (fat facets-like, Drosophila) & 2,32 & $\operatorname{chr} X$ \\
\hline UTP11L & 51118 & UTP11-like, U3 small nucleolar ribonucleoprotein, (yeast) & 2,12 & chr1 \\
\hline UXS1 & 80146 & UDP-glucuronate decarboxylase 1 & 2,30 & chr2 \\
\hline VAMP8 & 8673 & vesicle-associated membrane protein 8 (endobrevin) & 3,21 & chr2 \\
\hline VASP & 7408 & vasodilator-stimulated phosphoprotein & 2,58 & chr19 \\
\hline VDP & 8615 & Vesicle docking protein $\mathrm{p} 115$ & 4,00 & chr4 \\
\hline VIL2 & 7430 & villin 2 (ezrin) & 3,36 & chr6 \\
\hline VSNL1 & 7447 & visinin-like 1 & 4,85 & chr2 \\
\hline WBSCR17 & 64409 & Williams-Beuren syndrome chromosome region 17 & 2,10 & $\operatorname{chr} 7$ \\
\hline WDFY1 & 57590 & WD repeat and FYVE domain containing 1 & 2,23 & chr2 \\
\hline WDR1 & 9948 & WD repeat domain 1 & 2,30 & chr4 \\
\hline WDR12 & 55759 & WD repeat domain 12 & 2,28 & chr2 \\
\hline WDR21A & 26094 & WD repeat domain $21 \mathrm{~A}$ & 2,62 & chr14 \\
\hline WDR33 & 55339 & WD repeat domain 33 & 2,02 & chr2 \\
\hline WDR39 & 9391 & WD repeat domain 39 & 2,15 & chr2 \\
\hline WDR40B & 139170 & WD repeat domain $40 B$ & 3,17 & $\operatorname{chr} X$ \\
\hline WDR44 & 54521 & WD repeat domain 44 & 2,79 & $\operatorname{chr} X$ \\
\hline WDR46 & 9277 & WD repeat domain 46 & 2,00 & chr6 \\
\hline WDR5 & 11091 & WD repeat domain 5 & 2,19 & chr9 \\
\hline WDR67 & 93594 & WD repeat domain 67 & 2,27 & chr8 \\
\hline WDR72 & 256764 & WD repeat domain 72 & 4,96 & chr15 \\
\hline WDR74 & 54663 & WD repeat domain $74 / / /$ WD repeat domain 74 & 2,58 & chr11 \\
\hline WDR77 & 79084 & WD repeat domain 77 & 2,70 & chr1 \\
\hline WDR8 & 49856 & WD repeat domain 8 & 2,05 & chr1 \\
\hline WFDC2 & 10406 & WAP four-disulfide core domain 2 & 2,79 & chr20 \\
\hline WIF1 & 11197 & WNT inhibitory factor 1 & 10,39 & chr12 \\
\hline WNK1 & 65125 & WNK lysine deficient protein kinase 1 /// WNK lysine deficient protein kina & 2,25 & chr12 \\
\hline WWTR1 & 25937 & WW domain containing transcription regulator 1 & 2,11 & chr3 \\
\hline XPO5 & 57510 & exportin 5 & 2,35 & chr6 \\
\hline YIF1B & 90522 & Yip1 interacting factor homolog B (S. cerevisiae) & 3,47 & chr19 \\
\hline YTHDC2 & 64848 & YTH domain containing 2 & 2,34 & chr5 \\
\hline YTHDF3 & 253943 & YTH domain family, member 3 & 2,03 & chr8 \\
\hline YWHAB & 7529 & tyrosine 3-monooxygenase/tryptophan 5-monooxygenase activation prote & 2,36 & chr20 \\
\hline |YWHAE & 7531 & |tyrosine 3-monooxygenase/tryptophan 5-monooxygenase activation prote & 2,22 & chr7 \\
\hline
\end{tabular}




\begin{tabular}{|c|c|c|c|c|}
\hline Gene Symbol & Entrez Gene & Gene Title & NPC_down & Chromosome Number(Avadis) \\
\hline ZA20D2 & 7763 & zinc finger, A20 domain containing 2 & 2,93 & chr9 \\
\hline ZADH1 & 145482 & zinc binding alcohol dehydrogenase, domain containing 1 & 2,13 & chr14 \\
\hline ZBTB3 & 79842 & zinc finger and BTB domain containing 3 & 5,03 & chr11 \\
\hline ZBTB8OS & 339487 & zinc finger and BTB domain containing 8 opposite strand & 2,03 & chr1 \\
\hline ZC3HAV1 & 56829 & zinc finger $\mathrm{CCCH}$-type, antiviral 1 & 3,88 & chr7 \\
\hline ZCSL2 & 285381 & zinc finger, CSL-type containing 2 & 2,68 & chr3 \\
\hline ZD52F10 & 93099 & dermokine & 3,52 & chr19 \\
\hline ZDHHC22 & 283576 & zinc finger, DHHC-type containing 22 & 2,67 & chr14 \\
\hline ZDHHC23 & 254887 & zinc finger, DHHC-type containing 23 & 5,17 & chr3 \\
\hline ZFP42 & 132625 & zinc finger protein 42 & 11,68 & chr4 \\
\hline ZIC3 & 7547 & Zic family member 3 heterotaxy 1 (odd-paired homolog, Drosophila) & 4,06 & $\operatorname{chrX}$ \\
\hline ZIK1 & 284307 & zinc finger protein interacting with $\mathrm{K}$ protein 1 & 2,17 & chr19 \\
\hline ZNF101 & 94039 & zinc finger protein 101 & 3,08 & chr19 \\
\hline ZNF114 & 163071 & zinc finger protein 114 & 2,49 & chr19 \\
\hline ZNF134 & 7693 & Zinc finger protein 134 (clone pHZ-15) & 2,26 & chr19 \\
\hline ZNF138 & 7697 & zinc finger protein 138 & 2,13 & chr7 \\
\hline ZNF146 & 7705 & zinc finger protein 146 & 2,08 & chr19 \\
\hline ZNF165 & 7718 & zinc finger protein 165 & 5,51 & chr6 \\
\hline ZNF204 & 7754 & zinc finger protein 204 & 2,57 & chr6 \\
\hline ZNF206 & 84891 & zinc finger protein 206 & 8,70 & chr16 \\
\hline ZNF217 & 7764 & zinc finger protein 217 & 2,05 & chr20 \\
\hline ZNF239 & 8187 & zinc finger protein 239 & 2,45 & chr10 \\
\hline ZNF253 & $114977 / / / 56242$ & Zinc finger protein 253 /// hypothetical protein BC014148 & 2,15 & chr19 \\
\hline ZNF259 & 8882 & zinc finger protein 259 & 2,68 & chr6 \\
\hline ZNF267 & 10308 & zinc finger protein 267 & 2,46 & chr16 \\
\hline ZNF281 & 23528 & zinc finger protein 281 & 2,53 & chr1 \\
\hline ZNF313 & 55905 & zinc finger protein 313 & 2,10 & chr20 \\
\hline ZNF342 & 162979 & zinc finger protein 342 & 2,30 & chr19 \\
\hline ZNF398 & 57541 & zinc finger protein 398 & 4,29 & chr7 \\
\hline ZNF483 & 158399 & zinc finger protein 483 & 2,04 & chr9 \\
\hline ZNF488 & 118738 & zinc finger protein 488 & 2,04 & chr10 \\
\hline ZNF581 & 51545 & zinc finger protein 581 & 2,10 & chr19 \\
\hline ZNF588 & 51427 & zinc finger protein 588 & 2,31 & chr7 \\
\hline ZNF589 & 51385 & zinc finger protein 589 & 3,76 & chr3 \\
\hline ZNF600 & 162966 & zinc finger protein 600 & 2,61 & chr19 \\
\hline ZNF614 & 80110 & zinc finger protein 614 & 2,21 & chr19 \\
\hline ZNF616 & 90317 & zinc finger protein 616 & 2,10 & chr19 \\
\hline ZNF649 & 65251 & zinc finger protein 649 & 6,04 & chr19 \\
\hline ZNF691 & 51058 & zinc finger protein 691 & 2,19 & chr1 \\
\hline ZNF90 & 7643 & zinc finger protein 90 (HTF9) & 2,04 & chr19 \\
\hline ZRF1 & 27000 & Zuotin related factor 1 & 2,10 & chr7 \\
\hline ZSCAN2 & 54993 & zinc finger and SCAN domain containing 2 & 2,83 & chr15 \\
\hline ZSWIM3 & 140831 & zinc finger, SWIM-type containing 3 & 2,40 & chr20 \\
\hline ZYG11A & 440590 & zyg-11 homolog A (C. elegans) & 2,93 & chr1 \\
\hline $\mathrm{ZYX}$ & 7791 & zyxin & 2,23 & chr7 \\
\hline & & Primary neuroblastoma cDNA, clone:Nbla11652 & 39,16 & (vide) \\
\hline & & carbohydrate (N-acetylgalactosamine 4-0) sulfotransferase 9 /// carbohyd & 3,73 & (vide) \\
\hline & & HESB like domain containing 2 /// HESB like domain containing 2 & 2,91 & chr14 \\
\hline & & ubiquitin specific peptidase 32 /// ubiquitin specific peptidase 32 & 2,78 & chr11 \\
\hline & & dual specificity phosphatase $16 / / /$ dual specificity phosphatase 16 & 2,65 & chr21 \\
\hline & & G1 to $S$ phase transition $1 / / / G 1$ to $S$ phase transition 1 & 2,64 & chrY \\
\hline & & ubiquitin specific peptidase 45 /// ubiquitin specific peptidase 45 & 2,54 & chr6_random \\
\hline & & proteasome (prosome, macropain) subunit, beta type, $2 / / /$ proteasome $(\mathfrak{p}$ & 2,27 & chr19 \\
\hline & & mitochondrial ribosomal protein S18A /// mitochondrial ribosomal protein $\$$ & 2,15 & chr8 \\
\hline
\end{tabular}


Table S4 : Genes overexpressed in MPC compared to hES (Fold Change $>2$; $a<0.05$ )

\begin{tabular}{|c|c|c|c|c|}
\hline Gene Symbol & Entrez Gene & Gene Title & MPC_up & Chromosome Number(Avadis) \\
\hline 38961 & 23157 & septin 6 & 2,09 & chrX \\
\hline 39326 & 989 & Septin 7 & 2,10 & chr7 \\
\hline 40787 & 55752 & Septin 11 & 73,88 & chr4 \\
\hline MICB & $4276 / / / / 4277$ & MHC class I polypeptide-related sequence A /// MHC class I polypeptide-related & 8,37 & chr6 \\
\hline AADACL1 & 57552 & arylacetamide deacetylase-like 1 & 4,26 & chr3 \\
\hline ABHD2 & 11057 & abhydrolase domain containing 2 & 2,32 & chr15 \\
\hline ABI1 & 10006 & abl-interactor 1 & 2,24 & chr10 \\
\hline ABI3BP & 25890 & ABI gene family, member 3 (NESH) binding protein & 5,82 & chr3 \\
\hline ABLIM3 & 22885 & actin binding LIM protein family, member 3 & 2,00 & chr5 \\
\hline ABR & 29 & active BCR-related gene & 2,64 & chr17 \\
\hline ACBD3 & 64746 & acyl-Coenzyme A binding domain containing 3 & 2,33 & chr1 \\
\hline ACOX3 & 8310 & acyl-Coenzyme A oxidase 3, pristanoyl & 6,12 & chr4 \\
\hline ACP2 & 53 & acid phosphatase 2, lysosomal & 2,32 & chr11 \\
\hline ACSL1 & 2180 & acyl-CoA synthetase long-chain family member 1 & 4,65 & chr4 \\
\hline ACSL4 & 2182 & acyl-CoA synthetase long-chain family member 4 & 3,23 & $\operatorname{chr} \mathrm{X}$ \\
\hline ACSS2 & 55902 & acyl-CoA synthetase short-chain family member 2 & 2,59 & chr20 \\
\hline ACTA2 & 59 & actin, alpha 2, smooth muscle, aorta & 100,38 & chr10 \\
\hline ACTG2 & 72 & actin, gamma 2, smooth muscle, enteric & 92,67 & chr2 \\
\hline ACTN1 & 87 & actinin, alpha 1 & 5,49 & chr14 \\
\hline ACTN4 & 81 & actinin, alpha 4 & 3,50 & chr19 \\
\hline ACTR10 & 55860 & actin-related protein 10 homolog (S. cerevisiae) & 2,52 & chr14 \\
\hline ACTR1A & 10121 & ARP1 actin-related protein 1 homolog A, centractin alpha (yeast) & 2,60 & chr10 \\
\hline ACTR2 & 10097 & ARP2 actin-related protein 2 homolog (yeast) & 4,01 & chr2 \\
\hline ACTR3 & 10096 & ARP3 actin-related protein 3 homolog (yeast) & 3,56 & chr2 \\
\hline ACVR1 & 90 & activin A receptor, type I & 2,11 & chr2 \\
\hline ADA & 100 & adenosine deaminase & 2,90 & chr20 \\
\hline ADAM10 & 102 & ADAM metallopeptidase domain 10 & 2,14 & chr15 \\
\hline ADAM12 & 8038 & ADAM metallopeptidase domain 12 (meltrin alpha) & 18,98 & chr10 \\
\hline ADAM19 & 8728 & ADAM metallopeptidase domain 19 (meltrin beta) & 17,94 & chr5 \\
\hline ADAM23 & 8745 & ADAM metallopeptidase domain 23 & 3,67 & chr2 \\
\hline ADAM9 & 8754 & ADAM metallopeptidase domain 9 (meltrin gamma) & 18,84 & chr8 \\
\hline ADAMTS1 & 9510 & ADAM metallopeptidase with thrombospondin type 1 motif, 1 & 4,91 & chr21 \\
\hline ADAMTS5 & 11096 & ADAM metallopeptidase with thrombospondin type 1 motif, 5 (aggrecanase-2) & 9,73 & (vide) \\
\hline ADAMTS6 & 11174 & ADAM metallopeptidase with thrombospondin type 1 motif, 6 & 23,20 & chr5 \\
\hline ADARB1 & 104 & adenosine deaminase, RNA-specific, B1 (RED1 homolog rat) & 2,66 & chr21 \\
\hline ADCY6 & 112 & adenylate cyclase 6 & 2,68 & chr12 \\
\hline ADCY7 & 113 & adenylate cyclase 7 & 4,04 & chr16 \\
\hline ADCY9 & 115 & adenylate cyclase 9 & 2,59 & chr16 \\
\hline ADD1 & 118 & |adducin 1 (alpha) & 6,50 & chr4 \\
\hline ADK & 132 & adenosine kinase & 4,23 & chr10 \\
\hline ADM & 133 & adrenomedullin & 2,37 & chr11 \\
\hline AEBP1 & 165 & AE binding protein 1 & 3,30 & chr7 \\
\hline AER61 & 285203 & AER61 glycosyltransferase & 2,05 & chr3 \\
\hline AFAP & 60312 & actin filament associated protein & 6,64 & chr4 \\
\hline AFF3 & 3899 & AF4/FMR2 family, member 3 & 17,41 & chr2 \\
\hline AG1 & 440673 & AG1 protein & 2,73 & chr1 \\
\hline AGA & 175 & aspartylglucosaminidase & 3,03 & chr4 \\
\hline AGPAT3 & 56894 & 1-acylglycerol-3-phosphate O-acyltransferase 3 & 18,83 & chr21 \\
\hline AGTR1 & 185 & angiotensin II receptor, type 1 & 6,52 & chr3 \\
\hline AHI1 & 54806 & Abelson helper integration site & 2,70 & chr6 \\
\hline AHNAK & 79026 & AHNAK nucleoprotein (desmoyokin) & 16,67 & chr11 \\
\hline AHR & 196 & aryl hydrocarbon receptor & 8,35 & chr7 \\
\hline AK1 & 203 & adenylate kinase 1 & 5,98 & chr9 \\
\hline AK2 & 204 & adenylate kinase 2 & 5,50 & chr1 \\
\hline AK5 & 26289 & adenylate kinase 5 & 4,34 & chr1 \\
\hline AKAP12 & 9590 & A kinase (PRKA) anchor protein (gravin) 12 & 3,01 & chr6 \\
\hline AKAP2 & $11217 / / / 445815$ & A kinase (PRKA) anchor protein 2 /// PALM2-AKAP2 protein & 2,80 & chr9 \\
\hline AKT1 & 207 & $v$-akt murine thymoma viral oncogene homolog 1 & 3,57 & chr14 \\
\hline AKT3 & 10000 & V-akt murine thymoma viral oncogene homolog 3 (protein kinase B, gamma) & 6,86 & chr1 \\
\hline ALCAM & 214 & activated leukocyte cell adhesion molecule & 14,41 & chr3 \\
\hline ALDH1L2 & 160428 & aldehyde dehydrogenase 1 family, member L2 & 15,17 & chr12 \\
\hline ALG14 & 199857 & asparagine-linked glycosylation 14 homolog (yeast) & 2,56 & chr1 \\
\hline ALPK2 & 115701 & alpha-kinase 2 & 13,60 & chr18 \\
\hline ALS2 & 57679 & amyotrophic lateral sclerosis 2 (juvenile) & 2,48 & chr2 \\
\hline ALS2CR19 & 117583 & amyotrophic lateral sclerosis 2 (juvenile) chromosome region, candidate 19 & 2,17 & chr1 \\
\hline ALS2CR3 & 66008 & amyotrophic lateral sclerosis 2 (juvenile) chromosome region, candidate 3 & 4,36 & chr2 \\
\hline
\end{tabular}


Table S4 : Genes overexpressed in MPC compared to hES (Fold Change $>2$; $\mathrm{a}<0.05$ )

\begin{tabular}{|c|c|c|c|c|}
\hline Gene Symbol & Entrez Gene & Gene Title & MPC_up & Chromosome Number(Avadis) \\
\hline ALS2CR4 & 65062 & amyotrophic lateral sclerosis 2 (juvenile) chromosome region, candidate 4 & 2,26 & chr2 \\
\hline ALS2CR7 & 65061 & amyotrophic lateral sclerosis 2 (juvenile) chromosome region, candidate 7 & 5,16 & chr2 \\
\hline AMFR & 267 & autocrine motility factor receptor & 3,63 & chr16 \\
\hline AMIGO2 & 347902 & adhesion molecule with Ig-like domain 2 & 60,78 & chr12 \\
\hline AMOTL1 & 154810 & angiomotin like 1 & 3,56 & chr11 \\
\hline AMOTL2 & 51421 & angiomotin like 2 & 4,97 & chr3 \\
\hline AMPD2 & 271 & adenosine monophosphate deaminase 2 (isoform $\mathrm{L}$ ) & 2,02 & chr1 \\
\hline ANAPC13 & 25847 & anaphase promoting complex subunit 13 & 2,48 & chr3 \\
\hline ANGPTL2 & 23452 & angiopoietin-like 2 & 2,49 & chr9 \\
\hline ANKH & 56172 & ankylosis, progressive homolog (mouse) & 2,33 & chr5 \\
\hline ANKRA2 & 57763 & ankyrin repeat, family A (RFXANK-like), 2 & 2,58 & chr5 \\
\hline ANKRD1 & 27063 & ankyrin repeat domain 1 (cardiac muscle) & 9,51 & chr10 \\
\hline ANKRD13 & 88455 & ankyrin repeat domain 13 & 10,10 & chr12 \\
\hline ANKRD25 & 25959 & ankyrin repeat domain 25 & 2,08 & chr19 \\
\hline ANKRD28 & 23243 & ankyrin repeat domain 28 & 6,26 & chr3 \\
\hline ANKRD44 & 91526 & Ankyrin repeat domain 44 & 4,98 & chr2 \\
\hline ANTXR1 & 84168 & anthrax toxin receptor 1 & 4,37 & chr2 \\
\hline ANTXR2 & 118429 & Anthrax toxin receptor 2 & 17,52 & chr4 \\
\hline ANXA1 & 301 & annexin A1 & 50,64 & chr9 \\
\hline ANXA11 & 311 & annexin A11 & 2,15 & chr10 \\
\hline ANXA2 & 302 & annexin A2 & 7,32 & chr9 \\
\hline ANXA2P1 & 303 & annexin A2 pseudogene 1 & 3,65 & chr4 \\
\hline ANXA2P2 & 304 & annexin A2 pseudogene 2 & 7,03 & chr9 \\
\hline ANXA4 & 307 & annexin A4 & 2,99 & chr2 \\
\hline ANXA5 & 308 & annexin A5 & 2,64 & chr4 \\
\hline ANXA6 & 309 & annexin A6 & 7,19 & chr5 \\
\hline AOF1 & 221656 & amine oxidase (flavin containing) domain 1 & 2,47 & chr6 \\
\hline AP1M1 & 8907 & adaptor-related protein complex 1 , mu 1 subunit & 2,57 & chr19 \\
\hline AP1S1 & 1174 & adaptor-related protein complex 1 , sigma 1 subunit & 2,71 & chr7 \\
\hline AP2M1 & 1173 & adaptor-related protein complex 2 , mu 1 subunit & 2,55 & chr3 \\
\hline AP2S1 & 1175 & adaptor-related protein complex 2 , sigma 1 subunit & 2,12 & chr19 \\
\hline APBA2 & 321 & amyloid beta (A4) precursor protein-binding, family A, member 2 (X11-like) & 2,11 & chr15 \\
\hline АРОВЕСЗС & 27350 & apolipoprotein B mRNA editing enzyme, catalytic polypeptide-like $3 \mathrm{C}$ & 3,18 & chr22 \\
\hline APPBP2 & 10513 & amyloid beta precursor protein (cytoplasmic tail) binding protein 2 & 5,39 & chr17 \\
\hline ARAF & 369 & v-raf murine sarcoma 3611 viral oncogene homolog & 2,20 & $\operatorname{chrX}$ \\
\hline ARF4 & 378 & ADP-ribosylation factor 4 & 2,73 & chr3 \\
\hline ARF4L & 379 & ADP-ribosylation factor 4-like & 2,70 & chr17 \\
\hline ARFGAP1 & 55738 & ADP-ribosylation factor GTPase activating protein 1 & 2,63 & chr20 \\
\hline ARFGAP3 & 26286 & ADP-ribosylation factor GTPase activating protein 3 & 4,76 & chr22 \\
\hline ARHGAP1 & 392 & Rho GTPase activating protein 1 & 2,35 & chr11 \\
\hline ARHGAP18 & 93663 & Rho GTPase activating protein 18 & 4,52 & chr6 \\
\hline ARHGAP23 & 57636 & Rho GTPase activating protein 23 & 4,45 & chr17 \\
\hline ARHGAP24 & 83478 & Rho GTPase activating protein 24 & 10,45 & chr4 \\
\hline ARHGAP29 & 9411 & Rho GTPase activating protein 29 & 6,50 & chr1 \\
\hline ARHGAP5 & 394 & Rho GTPase activating protein 5 & 2,61 & chr14 \\
\hline ARHGAP6 & 395 & Rho GTPase activating protein 6 & 2,11 & $\operatorname{chrX}$ \\
\hline ARHGDIA & 396 & Rho GDP dissociation inhibitor (GDI) alpha /// Rho GDP dissociation inhibitor (G & 3,69 & chr17_random \\
\hline ARHGDIB & 397 & Rho GDP dissociation inhibitor (GDI) beta & 20,28 & chr12 \\
\hline ARHGEF12 & 23365 & Rho guanine nucleotide exchange factor (GEF) 12 & 3,21 & chr11 \\
\hline ARHGEF17 & 9828 & Rho guanine nucleotide exchange factor (GEF) 17 & 2,09 & chr11 \\
\hline ARID5B & 84159 & AT rich interactive domain 5B (MRF1-like) & 135,65 & chr10 \\
\hline ARL1 & 400 & ADP-ribosylation factor-like 1 & 2,71 & chr12 \\
\hline ARL2BP & 23568 & ADP-ribosylation factor-like 2 binding protein & 3,01 & chr16 \\
\hline ARL6IP5 & 10550 & ADP-ribosylation-like factor 6 interacting protein 5 & 6,20 & chr3 \\
\hline ARL7 & 10123 & ADP-ribosylation factor-like 7 & 4,22 & chr2 \\
\hline ARMCX3 & 51566 & armadillo repeat containing, $X$-linked 3 & 6,67 & $\operatorname{chrX}$ \\
\hline ARPC1B & 10095 & actin related protein $2 / 3$ complex, subunit $1 \mathrm{~B}, 41 \mathrm{kDa}$ & 2,24 & chr7 \\
\hline ARPC2 & 10109 & actin related protein $2 / 3$ complex, subunit $2,34 \mathrm{kDa}$ & 2,13 & chr2 \\
\hline ARPC5 & 10092 & actin related protein $2 / 3$ complex, subunit $5,16 \mathrm{kDa}$ & 3,48 & chr1 \\
\hline ARRDC4 & 91947 & arrestin domain containing 4 & 7,86 & chr15 \\
\hline ARSB & 411 & arylsulfatase B & 3,20 & chr5 \\
\hline ARSI & 340075 & arylsulfatase I & 2,15 & chr5 \\
\hline ARSJ & 79642 & arylsulfatase $\mathrm{J}$ & 70,99 & chr4 \\
\hline ARTS-1 & 51752 & type 1 tumor necrosis factor receptor shedding aminopeptidase regulator & 3,05 & chr5 \\
\hline ASAH1 & 427 & N-acylsphingosine amidohydrolase (acid ceramidase) 1 & 2,03 & chr8 \\
\hline ASAM & 79827 & Adipocyte-specific adhesion molecule & 5,27 & chr11 \\
\hline
\end{tabular}


Table S4 : Genes overexpressed in MPC compared to hES (Fold Change > 2; $a<0.05$ )

\begin{tabular}{|c|c|c|c|c|}
\hline Gene Symbol & Entrez Gene & Gene Title & MPC_up & Chromosome Number(Avadis) \\
\hline ASB8 & 140461 & ankyrin repeat and SOCS box-containing 8 & 4,22 & chr12 \\
\hline ASPH & 444 & Aspartate beta-hydroxylase & 7,54 & chr8 \\
\hline ASXL1 & 171023 & additional sex combs like 1 (Drosophila) & 3,56 & chr20 \\
\hline ATBF1 & 463 & AT-binding transcription factor 1 & 4,23 & chr16 \\
\hline ATF6 & 22926 & Activating transcription factor 6 & 4,17 & chr1 \\
\hline ATG4A & 115201 & ATG4 autophagy related 4 homolog A (S. cerevisiae) & 3,34 & $\operatorname{chrX}$ \\
\hline ATG7 & 10533 & ATG7 autophagy related 7 homolog (S. cerevisiae) & 2,99 & chr3 \\
\hline ATM & 472 & ataxia telangiectasia mutated (includes complementation groups $\mathrm{A}, \mathrm{C}$ and $\mathrm{D}$ ) & 2,49 & chr11 \\
\hline ATP10A & 57194 & ATPase, Class V, type $10 \mathrm{~A}$ & 2,95 & chr15 \\
\hline ATP10D & 57205 & ATPase, Class V, type 10D & 32,40 & chr4 \\
\hline ATP11B & 23200 & ATPase, Class VI, type 11B & 4,34 & chr3 \\
\hline ATP13A3 & 79572 & ATPase type 13 A3 & 4,08 & chr3 \\
\hline ATP2A2 & 488 & ATPase, Ca++ transporting, cardiac muscle, slow twitch 2 & 2,04 & chr12 \\
\hline ATP2B4 & 493 & ATPase, Ca++ transporting, plasma membrane 4 & 11,50 & chr1 \\
\hline ATP2C1 & 27032 & ATPase, $\mathrm{Ca}++$ transporting, type $2 \mathrm{C}$, member 1 & 4,09 & chr3 \\
\hline ATP5E & 514 & ATP synthase, $H+$ transporting, mitochondrial F1 complex, epsilon subunit & 2,17 & chr20 \\
\hline ATP6AP2 & 10159 & ATPase, $\mathrm{H}+$ transporting, lysosomal accessory protein 2 & 2,17 & $\operatorname{chrX}$ \\
\hline ATP6V0D1 & 9114 & ATPase, $\mathrm{H}+$ transporting, lysosomal $38 \mathrm{kDa}$, $\mathrm{V} 0$ subunit $\mathrm{d}$ isoform 1 & 2,09 & chr16 \\
\hline ATP6VOE & 8992 & ATPase, $\mathrm{H}+$ transporting, lysosomal 9kDa, $\mathrm{V} 0$ subunit e & 3,70 & chr5 \\
\hline ATP7A & 538 & ATPase, $\mathrm{Cu}_{++}$transporting, alpha polypeptide (Menkes syndrome) & 6,56 & $\operatorname{chrX}$ \\
\hline ATP8B1 & 5205 & ATPase, Class I, type 8B, member 1 & 24,44 & chr18 \\
\hline ATP9A & 10079 & ATPase, Class II, type 9A & 3,17 & chr20 \\
\hline ATXN1 & 6310 & ataxin 1 & 10,66 & chr6 \\
\hline AVO3 & 253260 & TORC2-specific protein AVO3 & 2,26 & chr5 \\
\hline AXL & 558 & $A X L$ receptor tyrosine kinase & 10,29 & chr19 \\
\hline AZI2 & 64343 & 5-azacytidine induced 2 & 3,19 & chr3 \\
\hline B2M & 567 & beta-2-microglobulin & 7,86 & chr15 \\
\hline B3GALT6 & 126792 & UDP-Gal:betaGal beta 1,3-galactosyltransferase polypeptide 6 & 2,12 & chr1 \\
\hline B3GTL & 145173 & beta 3-glycosyltransferase-like & 3,13 & chr13 \\
\hline B4GALT1 & 2683 & UDP-Gal:betaGIcNAc beta 1,4- galactosyltransferase, polypeptide 1 & 2,38 & chr9 \\
\hline bA16L21.2.1 & 548645 & DnaJ-like protein & 2,04 & chr9 \\
\hline BACE1 & 23621 & beta-site APP-cleaving enzyme 1 & 9,01 & chr11 \\
\hline BACE2 & 25825 & beta-site APP-cleaving enzyme 2 & 4,07 & chr21 \\
\hline BACH1 & 571 & BTB and CNC homology 1 , basic leucine zipper transcription factor 1 & 4,61 & chr21 \\
\hline BAG2 & 9532 & BCL2-associated athanogene 2 & 2,08 & chr6 \\
\hline BAG3 & 9531 & BCL2-associated athanogene 3 & 4,88 & chr10 \\
\hline BAZ1A & 11177 & bromodomain adjacent to zinc finger domain, $1 \mathrm{~A}$ & 2,35 & chr14 \\
\hline BBX & 56987 & Bobby sox homolog (Drosophila) & 2,15 & chr3 \\
\hline BC002942 & 91289 & hypothetical protein BC002942 & 4,19 & chr22 \\
\hline BCAP29 & 55973 & B-cell receptor-associated protein 29 & 3,69 & chr7 \\
\hline BCAR3 & 8412 & Breast cancer anti-estrogen resistance 3 & 5,25 & chr1 \\
\hline BCAT1 & 586 & branched chain aminotransferase 1 , cytosolic & 2,15 & chr12 \\
\hline BCL10 & 8915 & B-cell CLL/lymphoma 10 & 2,12 & chr1 \\
\hline BCL2L13 & 23786 & BCL2-like 13 (apoptosis facilitator) & 2,47 & chr22 \\
\hline BCL2L2 & 599 & BCL2-like 2 & 2,53 & chr14 \\
\hline BCL3 & 602 & B-cell CLL/ymphoma 3 & 3,22 & chr19 \\
\hline BCL6 & 604 & B-cell CLL/yymphoma 6 (zinc finger protein 51) /// B-cell CLL/ymphoma 6 (zinc & 4,77 & chr3 \\
\hline BDKRB1 & 623 & bradykinin receptor B1 & 4,87 & chr14 \\
\hline BDKRB2 & 624 & bradykinin receptor B2 & 2,23 & chr14 \\
\hline BDNF & 627 & brain-derived neurotrophic factor & 25,72 & chr11 \\
\hline BET1L & 51272 & blocked early in transport 1 homolog (S. cerevisiae)-like & 2,02 & chr11 \\
\hline BGN & 633 & biglycan & 23,88 & $\operatorname{chr} X$ \\
\hline BGN & $10194 / / / 633$ & biglycan /// serologically defined colon cancer antigen 33 & 39,55 & $\operatorname{chrX}$ \\
\hline BHLHB2 & 8553 & basic helix-loop-helix domain containing, class B, 2 & 6,35 & chr3 \\
\hline BHLHB3 & 79365 & basic helix-loop-helix domain containing, class B, 3 & 4,59 & chr12 \\
\hline $\mathrm{BIC}$ & 114614 & BIC transcript & 2,42 & chr21 \\
\hline BICD2 & 23299 & bicaudal D homolog 2 (Drosophila) & 3,05 & chr9 \\
\hline BID & 637 & BH3 interacting domain death agonist & 2,33 & chr22 \\
\hline BIRC2 & 329 & baculoviral IAP repeat-containing 2 & 2,55 & chr11 \\
\hline BIRC4 & 331 & baculoviral IAP repeat-containing 4 & 2,01 & $\operatorname{chr} X$ \\
\hline BIVM & 54841 & basic, immunoglobulin-like variable motif containing & 2,86 & chr13 \\
\hline Bles03 & 83638 & basophilic leukemia expressed protein BLES03 & 2,16 & chr11 \\
\hline BLZF1 & 8548 & basic leucine zipper nuclear factor 1 (JEM-1) & 3,21 & chr1 \\
\hline BMP1 & 649 & bone morphogenetic protein 1 & 8,18 & chr8 \\
\hline BMPR2 & 659 & bone morphogenetic protein receptor, type II (serine/threonine kinase) & 11,78 & chr2 \\
\hline BNC1 & 646 & basonuclin 1 & 9,76 & chr15 \\
\hline
\end{tabular}


Table S4 : Genes overexpressed in MPC compared to hES (Fold Change $>2 ; \mathrm{a}<0.05$ )

\begin{tabular}{|c|c|c|c|c|}
\hline Gene Symbol & Entrez Gene & Gene Title & MPC_up & Chromosome Number(Avadis) \\
\hline BNIP2 & 663 & BCL2/adenovirus E1B 19kDa interacting protein 2 & 2,71 & chr15 \\
\hline BNIP3L & 665 & BCL2/adenovirus E1B 19kDa interacting protein 3-like /// BCL2/adenovirus E1A & 4,10 & chr8 \\
\hline BPGM & 669 & 2,3-bisphosphoglycerate mutase /// 2,3-bisphosphoglycerate mutase & 3,32 & chr7 \\
\hline BPNT1 & 10380 & $3^{\prime}\left(2^{\prime}\right), 5$ '-bisphosphate nucleotidase 1 & 2,40 & chr1 \\
\hline BRP44L & 51660 & brain protein 44 -like & 2,70 & chr6 \\
\hline BTBD6 & 90135 & BTB (POZ) domain containing 6 & 4,67 & chr14 \\
\hline BTBD7 & 55727 & BTB (POZ) domain containing 7 & 2,88 & chr14 \\
\hline BTG2 & 7832 & BTG family, member 2 & 3,36 & chr1 \\
\hline BTN2A1 & 11120 & butyrophilin, subfamily 2, member A1 & 2,04 & chr6 \\
\hline BTN3A1 & 11119 & butyrophilin, subfamily 3 , member A1 & 2,23 & chr6 \\
\hline BTN3A2 & 11118 & butyrophilin, subfamily 3 , member A2 & 3,77 & chr6 \\
\hline BTN3A3 & $10384 / / / 11118$ & butyrophilin, subfamily 3 , member A3 /// butyrophilin, subfamily 3 , member A2 & 5,21 & chr6 \\
\hline BVES & 11149 & blood vessel epicardial substance & 6,10 & chr6 \\
\hline BZRP & 706 & benzodiazapine receptor (peripheral) & 9,01 & chr22 \\
\hline C10orf10 & 11067 & chromosome 10 open reading frame 10 & 9,04 & chr10 \\
\hline C10orf32 & 119032 & chromosome 10 open reading frame 32 & 3,34 & chr10 \\
\hline C10orf45 & 83641 & chromosome 10 open reading frame 45 & 2,91 & chr10 \\
\hline C10orf56 & 219654 & chromosome 10 open reading frame 56 & 4,53 & chr10 \\
\hline C10orf88 & 80007 & chromosome 10 open reading frame 88 & 3,76 & chr10 \\
\hline C10orf97 & 80013 & chromosome 10 open reading frame 97 & 2,62 & chr10 \\
\hline C11orf17 & 56672 & chromosome 11 open reading frame 17 & 2,94 & chr11 \\
\hline C11orf17 /// NUA & $56672 / / / 81788$ & chromosome 11 open reading frame $17 / / /$ chromosome 11 open reading fram & 2,23 & chr1 \\
\hline C11orf24 & 53838 & chromosome 11 open reading frame 24 & 2,57 & chr11 \\
\hline C11orf41 & 25758 & chromosome 11 open reading frame 41 & 3,95 & chr11 \\
\hline C11orf9 & 745 & chromosome 11 open reading frame 9 & 3,13 & chr11 \\
\hline C13orf1 & 57213 & chromosome 13 open reading frame 1 & 2,52 & chr13 \\
\hline C13orf12 & 51371 & chromosome 13 open reading frame 12 & 4,45 & chr13 \\
\hline C14orf125 & 25938 & chromosome 14 open reading frame 125 & 2,98 & chr14 \\
\hline C14orf139 & 79686 & chromosome 14 open reading frame 139 & 16,45 & chr14 \\
\hline C14orf149 & 112849 & chromosome 14 open reading frame 149 & 4,64 & chr14 \\
\hline C14orf24 & 283635 & chromosome 14 open reading frame 24 & 4,17 & chr14 \\
\hline C14orf28 & 122525 & chromosome 14 open reading frame 28 & 4,52 & chr14 \\
\hline C14orf34 & 55673 & chromosome 14 open reading frame 34 & 2,07 & chr17 \\
\hline C14orf37 & 145407 & chromosome 14 open reading frame 37 & 2,65 & chr14 \\
\hline C14orf43 & 91748 & chromosome 14 open reading frame 43 & 2,47 & chr14 \\
\hline C14orf44 & 145483 & Chromosome 14 open reading frame 44 & 2,01 & chr14 \\
\hline C14orf45 & 80127 & chromosome 14 open reading frame 45 & 5,28 & chr14 \\
\hline C14orf78 & 113146 & chromosome 14 open reading frame 78 & 8,90 & chr14 \\
\hline C14orf92 & 9878 & chromosome 14 open reading frame 92 & 2,32 & chr4 \\
\hline C15orf21 & 283651 & Chromosome 15 open reading frame 21 & 21,94 & chr15 \\
\hline C15orf38 & 348110 & Chromosome 15 open reading frame 38 & 2,46 & chr15 \\
\hline C16orf30 & 79652 & chromosome 16 open reading frame 30 & 2,19 & chr16 \\
\hline C18orf10 & 25941 & Chromosome 18 open reading frame 10 & 2,08 & chr18 \\
\hline C18orf4 & 92126 & chromosome 18 open reading frame 4 & 3,23 & chr18 \\
\hline C19orf10 & 56005 & chromosome 19 open reading frame 10 & 4,24 & chr19 \\
\hline C1orf119 & 56900 & chromosome 1 open reading frame 119 & 2,93 & chr1 \\
\hline C1orf139 & 79971 & chromosome 1 open reading frame 139 & 26,40 & chr1 \\
\hline C1orf144 & 26099 & chromosome 1 open reading frame 144 & 8,89 & chr1 \\
\hline C1orf22 & 80267 & chromosome 1 open reading frame 22 & 6,25 & chr1 \\
\hline C1orf24 & 116496 & chromosome 1 open reading frame 24 & 3,10 & chr1 \\
\hline C1orf53 & 388722 & chromosome 1 open reading frame 53 & 2,25 & chr1 \\
\hline C1orf54 & 79630 & chromosome 1 open reading frame 54 & 2,49 & chr1 \\
\hline C1orf71 & 163882 & chromosome 1 open reading frame 71 & 3,06 & chr1 \\
\hline C1orf78 & 55194 & chromosome 1 open reading frame $78 / / /$ chromosome 1 open reading frame 7 & 2,33 & chr1 \\
\hline C1orf85 & 112770 & Chromosome 1 open reading frame 85 & 7,06 & chr1 \\
\hline C1orf91 & 56063 & chromosome 1 open reading frame 91 & 2,42 & chr1 \\
\hline C20orf100 & 84969 & chromosome 20 open reading frame 100 & 2,46 & chr20 \\
\hline C20orf117 & 140710 & chromosome 20 open reading frame 117 & 2,41 & chr20 \\
\hline C20orf142 & 128486 & chromosome 20 open reading frame 142 & 4,11 & chr20 \\
\hline C20orf18 & 10616 & chromosome 20 open reading frame 18 & 2,16 & chr20 \\
\hline C20orf194 & 25943 & chromosome 20 open reading frame 194 & 2,47 & chr20 \\
\hline C20orf22 & 26090 & chromosome 20 open reading frame 22 & 2,43 & chr20 \\
\hline C20orf29 & 55317 & chromosome 20 open reading frame 29 & 2,43 & chr20 \\
\hline C21 orf51 & 54065 & chromosome 21 open reading frame 51 & 2,22 & chr21 \\
\hline C21 orf7 & 56911 & chromosome 21 open reading frame 7 & 24,00 & chr21 \\
\hline C21 orf86 & 257103 & Chromosome 21 open reading frame 86 & 2,11 & chr21 \\
\hline
\end{tabular}


Table S4 : Genes overexpressed in MPC compared to hES (Fold Change > 2; $\mathrm{a}<0.05$ )

\begin{tabular}{|c|c|c|c|c|}
\hline Gene Symbol & Entrez Gene & Gene Title & MPC_up & Chromosome Number(Avadis) \\
\hline C2orf10 & 91752 & chromosome 2 open reading frame 10 & 4,29 & chr2 \\
\hline C2orf17 & 79137 & chromosome 2 open reading frame 17 & 3,82 & chr2 \\
\hline C2orf18 & 54978 & chromosome 2 open reading frame 18 & 3,27 & chr2 \\
\hline C2orf27 & 29798 & Chromosome 2 open reading frame 27 & 6,46 & chr2 \\
\hline C2orf30 & 27248 & chromosome 2 open reading frame 30 & 3,50 & chr2 \\
\hline C2orf32 & 25927 & chromosome 2 open reading frame 32 & 13,68 & chr2 \\
\hline C2orf7 & 84279 & chromosome 2 open reading frame 7 & 2,50 & chr2 \\
\hline C3orf6 & 152137 & chromosome 3 open reading frame 6 & 6,50 & chr3 \\
\hline C5orf13 & 9315 & chromosome 5 open reading frame 13 & 3,39 & chr5 \\
\hline C5orf14 & 79770 & chromosome 5 open reading frame 14 & 2,56 & chr5 \\
\hline C5orf3 & 10827 & chromosome 5 open reading frame 3 & 4,97 & chr5 \\
\hline C6orf145 & 221749 & chromosome 6 open reading frame 145 & 28,85 & chr6 \\
\hline C6orf155 & 79940 & Chromosome 6 open reading frame 155 & 31,16 & chr6 \\
\hline C6orf188 & 254228 & chromosome 6 open reading frame 188 & 2,10 & chr6 \\
\hline C6orf48 & 50854 & chromosome 6 open reading frame 48 & 2,22 & chr6 \\
\hline C6orf62 & 81688 & chromosome 6 open reading frame 62 & 2,02 & chr6 \\
\hline C6orf65 & 221336 & chromosome 6 open reading frame 65 & 4,70 & chr6 \\
\hline C6orf69 & 222658 & chromosome 6 open reading frame 69 & 8,87 & chr6 \\
\hline C6orf72 & 116254 & chromosome 6 open reading frame 72 & 2,34 & chr6 \\
\hline C6orf89 & 221477 & Chromosome 6 open reading frame 89 & 5,92 & chr6 \\
\hline C7orf10 & 79783 & chromosome 7 open reading frame 10 & 3,44 & chr7 \\
\hline C7orf19 & 80228 & chromosome 7 open reading frame 19 & 2,02 & chr7 \\
\hline C7orf25 & 79020 & chromosome 7 open reading frame 25 & 2,09 & chr7 \\
\hline C9orf10 & 23196 & chromosome 9 open reading frame 10 & 5,77 & chr9 \\
\hline C9orf150 & 286343 & chromosome 9 open reading frame 150 & 6,47 & chr9 \\
\hline C9orf19 & 152007 & chromosome 9 open reading frame 19 & 2,43 & chr9 \\
\hline C9orf3 & 84909 & chromosome 9 open reading frame 3 & 3,37 & chr9 \\
\hline C9orf80 & 58493 & Chromosome 9 open reading frame 80 & 2,67 & chr9 \\
\hline C9orf88 & 64855 & chromosome 9 open reading frame 88 & 6,54 & chr9 \\
\hline C9orf89 & 84270 & chromosome 9 open reading frame 89 & 2,09 & chr9 \\
\hline C9orf94 & 206938 & chromosome 9 open reading frame 94 & 6,26 & chr9 \\
\hline C9orf95 & 54981 & chromosome 9 open reading frame 95 & 3,37 & chr9 \\
\hline CA12 & 771 & carbonic anhydrase XII & 11,03 & chr15 \\
\hline CAB39L & 81617 & calcium binding protein 39-like & 2,08 & chr13 \\
\hline CACNB3 & 784 & calcium channel, voltage-dependent, beta 3 subunit & 2,91 & chr12 \\
\hline CALCOCO1 & 57658 & calcium binding and coiled-coil domain 1 & 3,05 & chr12 \\
\hline CALD1 & 800 & caldesmon 1 & 18,09 & chr7 \\
\hline CALU & 813 & calumenin & 5,44 & chr7 \\
\hline CAMK2D & 817 & Calcium/calmodulin-dependent protein kinase (CaM kinase) II delta & 9,74 & chr4 \\
\hline CAMK2N1 & 55450 & calcium/calmodulin-dependent protein kinase II inhibitor 1 & 10,98 & chr1 \\
\hline CAMTA2 & 23125 & calmodulin binding transcription activator 2 & 3,19 & chr17 \\
\hline CANT1 & 124583 & calcium activated nucleotidase 1 & 2,11 & chr17 \\
\hline CAP1 & 10487 & CAP, adenylate cyclase-associated protein 1 (yeast) & 2,51 & chr1 \\
\hline CAP2 & 10486 & CAP, adenylate cyclase-associated protein, 2 (yeast) & 7,26 & chr6 \\
\hline CAPN2 & 824 & calpain 2, (m/ll) large subunit & 13,95 & chr1 \\
\hline CAPN7 & 23473 & calpain 7 & 3,02 & chr3 \\
\hline CAPNS1 & 826 & calpain, small subunit 1 /// calpain, small subunit 1 & 3,94 & chr19 \\
\hline CART1 & 8092 & cartilage paired-class homeoprotein 1 & 2,51 & chr12 \\
\hline CASC4 & 113201 & cancer susceptibility candidate 4 & 3,50 & chr15 \\
\hline CASP4 & 837 & caspase 4 , apoptosis-related cysteine peptidase & 3,52 & chr11 \\
\hline CASP7 & 840 & caspase 7 , apoptosis-related cysteine peptidase & 3,07 & chr10 \\
\hline CASP8 & 841 & caspase 8 , apoptosis-related cysteine peptidase & 4,36 & chr2 \\
\hline CAST & 831 & calpastatin & 6,80 & chr5 \\
\hline CAV1 & 857 & caveolin 1 , caveolae protein, $22 \mathrm{kDa}$ & 27,31 & chr7 \\
\hline CAV2 & 858 & caveolin 2 & 46,79 & chr7 \\
\hline CBFB & 865 & core-binding factor, beta subunit & 2,72 & chr16 \\
\hline CBR3 & 874 & carbonyl reductase 3 & 3,59 & chr21 \\
\hline CBX4 & 8535 & chromobox homolog 4 (Pc class homolog, Drosophila) & 6,13 & chr17 \\
\hline CBX6 & 23466 & Chromobox homolog 6 & 3,65 & chr22 \\
\hline CCBE1 & 147372 & collagen and calcium binding EGF domains 1 & 2,79 & chr18 \\
\hline CCDC6 & 8030 & coiled-coil domain containing 6 & 2,23 & chr10 \\
\hline CCDC75 & 253635 & Coiled-coil domain containing 75 & 2,67 & chr2 \\
\hline CCND1 & 595 & cyclin D1 & 6,46 & chr11 \\
\hline CCND3 & 896 & cyclin D3 & 2,20 & chr6 \\
\hline CCNG2 & 901 & Cyclin G2 & 2,05 & chr4 \\
\hline CCPG1 & 9236 & cell cycle progression 1 & 9,01 & chr15 \\
\hline
\end{tabular}


Table S4 : Genes overexpressed in MPC compared to hES (Fold Change $>2 ; \mathrm{a}<0.05$ )

\begin{tabular}{|c|c|c|c|c|}
\hline Gene Symbol & Entrez Gene & Gene Title & MPC_up & Chromosome Number(Avadis) \\
\hline CD109 & 135228 & CD109 antigen (Gov platelet alloantigens) & 10,06 & chr6 \\
\hline CD151 & 977 & CD151 antigen & 6,14 & chr11 \\
\hline CD164 & 8763 & CD164 antigen, sialomucin & 2,57 & chr6 \\
\hline CD24 & 934 & CD24 antigen (small cell lung carcinoma cluster 4 antigen) & 2,83 & chr5 \\
\hline CD248 & 57124 & CD248 antigen, endosialin & 12,50 & chr11 \\
\hline CD274 & 29126 & CD274 antigen & 40,81 & chr9 \\
\hline CD44 & 960 & CD44 antigen (homing function and Indian blood group system) & 111,41 & chr11 \\
\hline CD47 & 961 & (CD47 antigen (Rh-related antigen, integrin-associated signal transducer) & 10,25 & chr3 \\
\hline CD58 & 965 & CD58 antigen, (lymphocyte function-associated antigen 3) & 2,04 & chr1 \\
\hline CD59 & 966 & CD59 antigen p18-20 (antigen identified by monoclonal antibodies 16.3A5, EJ1 & 15,10 & chr11 \\
\hline CD99 & 4267 & CD99 antigen & 13,94 & $\operatorname{chrX}$ \\
\hline CD99L2 & 83692 & CD99 antigen-like 2 & 5,02 & $\operatorname{chrX}$ \\
\hline CDA08 & 81533 & T-cell immunomodulatory protein & 3,36 & chr16 \\
\hline CDC27 & 996 & Cell division cycle 27 & 2,65 & chr17 \\
\hline CDC2L6 & 23097 & cell division cycle 2-like 6 (CDK8-like) & 3,15 & chr6 \\
\hline CDC42 & 998 & cell division cycle 42 (GTP binding protein, 25kDa) & 2,39 & chr1 \\
\hline CDC42BPA & 8476 & CDC42 binding protein kinase alpha (DMPK-like) & 2,33 & chr1 \\
\hline CDC42EP3 & 10602 & CDC42 effector protein (Rho GTPase binding) 3 & 40,24 & chr2 \\
\hline CDC42EP5 & 148170 & CDC42 effector protein (Rho GTPase binding) 5 & 26,82 & chr19 \\
\hline CDGAP & 57514 & Cdc42 GTPase-activating protein & 2,48 & chr3 \\
\hline $\mathrm{CDH} 10$ & 1008 & cadherin 10, type 2 (T2-cadherin) & 4,25 & chr5 \\
\hline $\mathrm{CDH} 11$ & 1009 & cadherin 11 , type 2, OB-cadherin (osteoblast) & 30,34 & chr16 \\
\hline $\mathrm{CDH} 13$ & 1012 & cadherin $13, \mathrm{H}$-cadherin (heart) & 14,00 & chr16 \\
\hline CDH2 & 1000 & cadherin 2 , type $1, \mathrm{~N}$-cadherin (neuronal) & 6,83 & chr18 \\
\hline $\mathrm{CDH} 6$ & 1004 & cadherin 6, type 2, K-cadherin (fetal kidney) & 4,81 & chr5 \\
\hline CDK6 & 1021 & cyclin-dependent kinase 6 & 24,64 & chr7 \\
\hline CDKN1A & 1026 & cyclin-dependent kinase inhibitor $1 \mathrm{~A}$ (p21, Cip1) & 55,11 & chr6 \\
\hline CDKN2A & 1029 & cyclin-dependent kinase inhibitor 2A (melanoma, p16, inhibits CDK4) & 22,27 & chr9 \\
\hline CDKN2B & 1030 & cyclin-dependent kinase inhibitor 2B (p15, inhibits CDK4) & 45,10 & chr9 \\
\hline CDKN2C & 1031 & cyclin-dependent kinase inhibitor $2 \mathrm{C}$ (p18, inhibits CDK4) & 6,71 & chr1 \\
\hline CEBPD & 1052 & |CCAAT/enhancer binding protein (C/EBP), delta & 5,63 & chr8 \\
\hline CEECAM1 & 51148 & cerebral endothelial cell adhesion molecule 1 & 6,20 & chr9 \\
\hline CENTB5 & 116983 & centaurin, beta 5 & 2,06 & chr1 \\
\hline CES2 & 8824 & carboxylesterase 2 (intestine, liver) & 2,29 & chr16 \\
\hline $\mathrm{CFH}$ & 3075 & complement factor $\mathrm{H}$ & 6,49 & chr1 \\
\hline CFHL1 & $3075 / / / 3078$ & complement factor $\mathrm{H} / / /$ complement factor $\mathrm{H}$-related 1 & 10,31 & chr1 \\
\hline CFL1 & 1072 & cofilin 1 (non-muscle) & 2,23 & chr1 \\
\hline CFL2 & 1073 & cofilin 2 (muscle) & 13,08 & chr14 \\
\hline CFLAR & 8837 & CASP8 and FADD-like apoptosis regulator & 3,61 & chr2 \\
\hline CGI-116 & 51019 & CGI-116 protein & 3,64 & chr12 \\
\hline $\mathrm{CHD} 2$ & 1106 & chromodomain helicase DNA binding protein 2 & 2,17 & chr15 \\
\hline CHD3 & 1107 & chromodomain helicase DNA binding protein 3 & 3,27 & chr17 \\
\hline CHES1 & 1112 & checkpoint suppressor 1 & 2,07 & chr14 \\
\hline CHID1 & 66005 & Chitinase domain containing 1 & 3,05 & chr11 \\
\hline CHM & 1121 & choroideremia (Rab escort protein 1) & 2,13 & $\operatorname{chrX}$ \\
\hline CHMP1B & 57132 & chromatin modifying protein $1 \mathrm{~B}$ & 2,19 & chr18 \\
\hline CHMP5 & 51510 & chromatin modifying protein 5 & 2,35 & chr9 \\
\hline CHPF & 79586 & chondroitin polymerizing factor & 2,40 & chr2 \\
\hline CHST3 & 9469 & carbohydrate (chondroitin 6) sulfotransferase 3 & 3,32 & chr10 \\
\hline CHURC1 & 91612 & churchill domain containing 1 & 2,50 & chr1 \\
\hline CIB1 & 10519 & calcium and integrin binding 1 (calmyrin) & 3,77 & chr15 \\
\hline CIRBP & 1153 & cold inducible RNA binding protein & 2,41 & chr19 \\
\hline CITED2 & 10370 & Cbp/p300-interacting transactivator, with Glu/Asp-rich carboxy-terminal domain & 3,77 & chr6 \\
\hline CKAP4 & 10970 & cytoskeleton-associated protein 4 & 4,50 & chr12 \\
\hline CKIP-1 & 51177 & CK2 interacting protein $1 ; \mathrm{HQ} 0024 \mathrm{C}$ protein & 2,67 & chr1 \\
\hline CLCC1 & 23155 & chloride channel CLIC-like 1 & 2,05 & chr1 \\
\hline CLCN3 & 1182 & chloride channel 3 & 2,07 & chr4 \\
\hline CLDN1 & 9076 & claudin 1 & 10,12 & chr3 \\
\hline CLIPR-59 & 25999 & CLIP-170-related protein & 3,38 & chr19 \\
\hline CLN5 & 1203 & ceroid-lipofuscinosis, neuronal 5 & 4,59 & chr13 \\
\hline CLSTN2 & 64084 & calsyntenin 2 & 2,86 & chr3 \\
\hline CLTB & 1212 & clathrin, light polypeptide (Lcb) /// clathrin, light polypeptide (Lcb) & 2,22 & chr5 \\
\hline CMPK & 51727 & cytidylate kinase & 8,32 & chr1 \\
\hline $\mathrm{CNIH}$ & 10175 & Cornichon homolog (Drosophila) & 3,43 & chr14 \\
\hline CNKSR3 & 154043 & CNKSR family member 3 & 2,08 & chr6 \\
\hline CNN1 & 1264 & calponin 1, basic, smooth muscle & 12,00 & chr19 \\
\hline
\end{tabular}


Table S4 : Genes overexpressed in MPC compared to hES (Fold Change $>2 ; \mathrm{a}<0.05$ )

\begin{tabular}{|c|c|c|c|c|}
\hline Gene Symbol & Entrez Gene & Gene Title & MPC_up & Chromosome Number(Avadis) \\
\hline CNN2 & 1265 & calponin 2 & 3,51 & chr19 \\
\hline CNN3 & 1266 & calponin 3 , acidic & 2,21 & chr1 \\
\hline CNTNAP1 & 8506 & contactin associated protein 1 & 3,78 & chr17 \\
\hline COBLL1 & 22837 & COBL-like 1 & 3,42 & chr2 \\
\hline COG1 & 9382 & component of oligomeric golgi complex 1 & 2,27 & chr17 \\
\hline coG5 & 10466 & component of oligomeric golgi complex 5 & 3,88 & chr7 \\
\hline COG6 & 57511 & component of oligomeric golgi complex 6 & 4,62 & chr13 \\
\hline COL11A1 & 1301 & collagen, type $\mathrm{XI}$, alpha 1 & 36,87 & chr1 \\
\hline COL12A1 & 1303 & collagen, type XII, alpha 1 & 118,63 & chr6 \\
\hline COL13A1 & 1305 & collagen, type XIII, alpha 1 & 8,13 & chr10 \\
\hline COL1A1 & 1277 & collagen, type I, alpha 1 & 99,56 & chr17 \\
\hline COL1A2 & 1278 & Collagen, type I, alpha 2 & 52,81 & chr7 \\
\hline COL25A1 & 84570 & collagen, type XXV, alpha 1 & 2,08 & chr4 \\
\hline COL3A1 & 1281 & collagen, type III, alpha 1 (Ehlers-Danlos syndrome type IV, autosomal domina & 80,39 & (vide) \\
\hline COL4A1 & 1282 & collagen, type IV, alpha 1 & 18,41 & chr13 \\
\hline COL4A2 & 1284 & collagen, type IV, alpha 2 & 21,01 & chr13 \\
\hline COL4A5 & 1287 & collagen, type IV, alpha 5 (Alport syndrome) & 2,58 & $\operatorname{chrX}$ \\
\hline COL5A1 & 1289 & collagen, type $\mathrm{V}$, alpha 1 & 118,26 & chr9 \\
\hline COL5A2 & 1290 & collagen, type $\mathrm{V}$, alpha 2 & 82,78 & chr2 \\
\hline COL6A1 & 1291 & collagen, type VI, alpha 1 & 3,20 & chr21 \\
\hline COL6A2 & 1292 & collagen, type VI, alpha 2 & 51,34 & chr21 \\
\hline COL6A3 & 1293 & collagen, type VI, alpha 3 & 82,16 & chr2 \\
\hline COL8A1 & 1295 & Collagen, type VIII, alpha 1 & 291,36 & chr3 \\
\hline COLEC12 & 81035 & collectin sub-family member 12 /// collectin sub-family member 12 & 8,37 & chr18 \\
\hline COMMD3 & 23412 & COMM domain containing 3 & 9,33 & chr10 \\
\hline COMMD8 & 54951 & COMM domain containing 8 & 3,62 & chr4 \\
\hline СOMT & 1312 & catechol-O-methyltransferase & 5,97 & chr22 \\
\hline COPA & 1314 & coatomer protein complex, subunit alpha & 2,51 & chr1 \\
\hline COPB & 1315 & coatomer protein complex, subunit beta & 3,08 & chr11 \\
\hline СОРB2 & 9276 & coatomer protein complex, subunit beta 2 (beta prime) & 2,27 & chr3 \\
\hline COPE & 11316 & coatomer protein complex, subunit epsilon & 2,19 & chr19 \\
\hline COPG & 22820 & coatomer protein complex, subunit gamma & 2,74 & chr3 \\
\hline COPZ1 & 22818 & coatomer protein complex, subunit zeta 1 & 2,20 & chr12 \\
\hline COPZ2 & 51226 & coatomer protein complex, subunit zeta 2 & 20,86 & chr17 \\
\hline CORO1C & 23603 & coronin, actin binding protein, $1 \mathrm{C}$ & 7,82 & chr12 \\
\hline COTL1 & 23406 & coactosin-like 1 (Dictyostelium) & 2,66 & chr16 \\
\hline CPA4 & 51200 & carboxypeptidase A4 & 9,29 & chr7 \\
\hline CPE & 1363 & carboxypeptidase E & 5,86 & chr4 \\
\hline CPEB2 & 132864 & cytoplasmic polyadenylation element binding protein 2 & 8,67 & chr4 \\
\hline CPEB4 & 80315 & cytoplasmic polyadenylation element binding protein 4 & 2,96 & chr5 \\
\hline CRAT & 1384 & carnitine acetyltransferase & 3,90 & chr9 \\
\hline CREB3 & 10488 & CAMP responsive element binding protein 3 & 4,52 & chr9 \\
\hline CREB3L1 & 90993 & CAMP responsive element binding protein 3 -like 1 & 13,03 & chr11 \\
\hline CREB3L2 & 64764 & CAMP responsive element binding protein 3 -like 2 & 3,27 & chr7 \\
\hline CREB5 & 9586 & CAMP responsive element binding protein 5 & 6,36 & chr7 \\
\hline CRELD1 & 78987 & cysteine-rich with EGF-like domains 1 & 2,88 & chr3 \\
\hline CRI1 & 23741 & CREBBP/EP300 inhibitor 1 /// CREBBP/EP300 inhibitor 1 & 2,73 & chr15 \\
\hline CRIM1 & 51232 & cysteine rich transmembrane BMP regulator 1 (chordin-like) & 22,12 & chr2 \\
\hline CROT & 54677 & carnitine O-octanoyltransferase & 2,82 & chr7 \\
\hline CRTAP & 10491 & cartilage associated protein & 3,03 & chr3 \\
\hline CRYL1 & 51084 & crystallin, lambda 1 & 2,12 & chr13 \\
\hline CSAD & 51380 & cysteine sulfinic acid decarboxylase & 2,96 & chr12 \\
\hline CSGICA-T & 54480 & chondroitin sulfate glucuronyltransferase & 7,26 & chr7 \\
\hline CSNK1D & 1453 & casein kinase 1 , delta & 2,07 & chr17 \\
\hline CSNK1G1 & 53944 & casein kinase 1 , gamma 1 & 2,41 & chr15 \\
\hline CSPG4 & 1464 & Chondroitin sulfate proteoglycan 4 (melanoma-associated) & 13,86 & chr15 \\
\hline CSRP1 & 1465 & cysteine and glycine-rich protein 1 & 14,42 & chr1 \\
\hline $\operatorname{css} 3$ & 337876 & chondroitin sulfate synthase 3 & 24,41 & chr5 \\
\hline CST3 & 1471 & cystatin C (amyloid angiopathy and cerebral hemorrhage) & 6,45 & chr20 \\
\hline CTBS & 1486 & chitobiase, di-N-acetyl- & 10,27 & chr1 \\
\hline CTGF & 1490 & connective tissue growth factor & 20,66 & chr6 \\
\hline CTSB & 1508 & cathepsin B & 17,12 & chr8 \\
\hline CTSC & 1075 & cathepsin C & 2,73 & chr11 \\
\hline CTTN & 2017 & cortactin & 4,24 & chr11 \\
\hline CTTNBP2NL & 55917 & CTTNBP2 $\mathrm{N}$-terminal like & 2,01 & chr1 \\
\hline CUEDC2 & 79004 & CUE domain containing 2 & 2,04 & chr10 \\
\hline
\end{tabular}


Table S4 : Genes overexpressed in MPC compared to hES (Fold Change $>2 ; \mathrm{a}<0.05$ )

\begin{tabular}{|c|c|c|c|c|}
\hline Gene Symbol & Entrez Gene & Gene Title & MPC_up & Chromosome Number(Avadis) \\
\hline CUL4B & 8450 & cullin 4B & 2,80 & chr10 \\
\hline CUL5 & 8065 & cullin 5 & 2,16 & chr11 \\
\hline CUL7 & 9820 & cullin 7 & 3,91 & chr6 \\
\hline CUTL1 & 1523 & Cut-like 1, CCAAT displacement protein (Drosophila) & 2,08 & chr7 \\
\hline CXCL1 & 2919 & chemokine (C-X-C motif) ligand 1 (melanoma growth stimulating activity, alpha) & 3,08 & chr4 \\
\hline CXCL12 & 6387 & chemokine (C-X-C motif) ligand 12 (stromal cell-derived factor 1) & 2,80 & chr10 \\
\hline CXorf33 & 139322 & chromosome $\mathrm{X}$ open reading frame 33 & 3,22 & $\operatorname{chrX}$ \\
\hline CXorf39 & 139231 & Chromosome $\mathrm{X}$ open reading frame 39 & 2,37 & $\operatorname{chrX}$ \\
\hline CXorf6 & 10046 & chromosome $\mathrm{X}$ open reading frame 6 & 2,22 & $\operatorname{chrX}$ \\
\hline $\mathrm{CXX} 1$ & 8933 & CAAX box 1 & 6,79 & $\operatorname{chrX}$ \\
\hline CYB5R1 & 51706 & cytochrome b5 reductase 1 & 5,37 & chr1 \\
\hline CYB5R3 & 1727 & cytochrome b5 reductase 3 & 4,06 & chr22 \\
\hline CYBASC3 & 220002 & cytochrome b, ascorbate dependent 3 & 3,34 & chr11 \\
\hline CYBRD1 & 79901 & cytochrome b reductase 1 & 31,09 & chr2 \\
\hline CYLD & 1540 & cylindromatosis (turban tumor syndrome) & 4,52 & chr16 \\
\hline CYP1B1 & 1545 & cytochrome P450, family 1 , subfamily B, polypeptide 1 & 8,47 & chr2 \\
\hline CYP2U1 & 113612 & cytochrome P450, family 2, subfamily U, polypeptide 1 & 3,83 & chr4 \\
\hline |CYR61 & 3491 & cysteine-rich, angiogenic inducer, 61 & 11,63 & chr1 \\
\hline DAAM2 & 23500 & dishevelled associated activator of morphogenesis 2 & 4,30 & chr6 \\
\hline DAB2 & 1601 & Disabled homolog 2, mitogen-responsive phosphoprotein (Drosophila) & 72,26 & chr5 \\
\hline DAP & 1611 & death-associated protein & 6,45 & chr5 \\
\hline DAZAP2 & 9802 & DAZ associated protein 2 & 4,86 & chr12 \\
\hline DAZAP2 & 401029 /// 9802 & DAZ associated protein $2 / / /$ similar to DAZ-associated protein 2 (Deleted in azd & 3,35 & chr12 \\
\hline DBNL & 28988 & drebrin-like & 2,02 & chr7 \\
\hline DCBLD1 & 285761 & discoidin, CUB and LCCL domain containing 1 & 6,28 & chr6 \\
\hline DCBLD2 & 131566 & discoidin, CUB and LCCL domain containing 2 & 5,97 & chr3 \\
\hline DCTD & 1635 & dCMP deaminase & 2,57 & chr4 \\
\hline DCTN3 & 11258 & dynactin 3 (p22) & 4,54 & chr9 \\
\hline DCUN1D4 & 23142 & DCN1, defective in cullin neddylation 1, domain containing 4 (S. cerevisiae) & 5,80 & chr4 \\
\hline DDAH1 & 23576 & dimethylarginine dimethylaminohydrolase 1 & 3,91 & chr1 \\
\hline DDEF1 & 50807 & development and differentiation enhancing factor 1 & 5,49 & chr8 \\
\hline DDEF2 & 8853 & development and differentiation enhancing factor 2 & 5,62 & chr2 \\
\hline DDHD2 & 23259 & DDHD domain containing 2 & 3,81 & chr8 \\
\hline DDIT3 & 1649 & DNA-damage-inducible transcript 3 & 3,99 & chr12 \\
\hline DDR2 & 4921 & Discoidin domain receptor family, member 2 & 51,24 & chr1 \\
\hline DECR1 & 1666 & 2,4-dienoyl CoA reductase 1 , mitochondrial & 2,43 & chr8 \\
\hline DEGS1 & 8560 & degenerative spermatocyte homolog 1 , lipid desaturase (Drosophila) & 2,49 & chr1 \\
\hline DFNA5 & 1687 & deafness, autosomal dominant 5 & 17,62 & chr7 \\
\hline DGKA & 1606 & diacylglycerol kinase, alpha $80 \mathrm{kDa}$ & 5,45 & chr12 \\
\hline DHRS1 & 115817 & dehydrogenase/reductase (SDR family) member 1 & 2,31 & chr14 \\
\hline DIO2 & 1734 & Deiodinase, iodothyronine, type II & 14,77 & chr14 \\
\hline DIP13B & 55198 & DIP13 beta & 4,09 & chr12 \\
\hline DJ328E19.C1.1 & $55 / / / 440670 / / / / 4$ & hypothetical protein DJ328E19.C1.1 /// hypothetical protein FLJ20719 /// hypoth & 3,73 & chr1_random \\
\hline DJ328E19.C1.1 & $401967 / / / 44067$ & hypothetical protein DJ328E19.C1.1 /// hypothetical protein FLJ20719 /// hypoth & 2,88 & chr1_random \\
\hline DKFZP434B033 & 25851 & DKFZP434B0335 protein & 2,44 & chr7 \\
\hline DKFZp434C032 & 54762 & hypothetical protein DKFZp434C0328 & 3,29 & chr3 \\
\hline DKFZP434F031 & 81575 & hypothetical protein DKFZp434F0318 /// hypothetical protein DKFZp434F0318 & 2,96 & chr12 \\
\hline DKFZp434K243 & 84216 & hypothetical protein DKFZp434K2435 & 4,09 & chr12 \\
\hline DKFZp434L142 & 51313 & hypothetical protein DKFZp434L142 & 11,72 & chr4 \\
\hline DKFZP564D166 & 26115 & putative ankyrin-repeat containing protein & 3,49 & chr17 \\
\hline DKFZP564D172 & 83989 & hypothetical protein DKFZp564D172 & 3,35 & chr5 \\
\hline DKFZP564J012 & 25915 & nuclear protein E3-3 & 2,15 & chr3 \\
\hline DKFZP564J086 & 25923 & DKFZP564J0863 protein & 3,99 & chr11 \\
\hline DKFZp564K142 & 84061 & implantation-associated protein & 2,46 & chrX_random \\
\hline DKFZP586D091 & 25895 & hepatocellularcarcinoma-associated antigen HCA557a & 3,18 & chr12 \\
\hline DKFZP586H212 & 25891 & regeneration associated muscle protease & 5,16 & chr11 \\
\hline DKFZp686K161 & 388957 & Similar to BMP2 inducible kinase & 3,12 & chr2 \\
\hline DKFZp761B107 & 91050 & Hypothetical protein DKFZp761B107 /// Similar to DKFZP434L187 protein & 2,03 & chr4 \\
\hline DKFZp761D112 & 84257 & hypothetical protein DKFZp761D112 & 2,33 & chr8 \\
\hline DKK1 & 22943 & dickkopf homolog 1 (Xenopus laevis) & 17,54 & chr10 \\
\hline DKK3 & 27122 & dickkopf homolog 3 (Xenopus laevis) & 19,69 & chr11 \\
\hline DLC1 & 10395 & deleted in liver cancer 1 & 69,90 & chr8 \\
\hline DLG1 & 1739 & discs, large homolog 1 (Drosophila) & 3,05 & chr3 \\
\hline DLX1 & 1745 & distal-less homeo box 1 & 35,47 & chr2 \\
\hline DLX2 & 1746 & distal-less homeo box 2 & 25,71 & chr2 \\
\hline DMN & 23336 & desmuslin & 7,70 & chr15 \\
\hline
\end{tabular}


Table S4 : Genes overexpressed in MPC compared to hES (Fold Change > 2; $\mathrm{a}<0.05$ )

\begin{tabular}{|c|c|c|c|c|}
\hline Gene Symbol & Entrez Gene & Gene Title & MPC_up & Chromosome Number(Avadis) \\
\hline DNAJB12 & 54788 & DnaJ (Hsp40) homolog, subfamily B, member 12 & 2,60 & chr10 \\
\hline DNAJB14 & 79982 & DnaJ (Hsp40) homolog, subfamily B, member 14 & 2,74 & chr4 \\
\hline DNAJB4 & 11080 & DnaJ (Hsp40) homolog, subfamily B, member 4 & 7,82 & chr1 \\
\hline DNAJB9 & 4189 & DnaJ (Hsp40) homolog, subfamily B, member 9 & 3,73 & chr7 \\
\hline DNAJC1 & 64215 & DnaJ (Hsp40) homolog, subfamily C, member 1 & 2,93 & chr10 \\
\hline DNAJC13 & 23317 & DnaJ (Hsp40) homolog, subfamily C, member 13 & 2,71 & chr3 \\
\hline DNAJC3 & 5611 & Hypothetical protein LOC144871 & 4,16 & chr13 \\
\hline DNAJC6 & 9829 & DnaJ (Hsp40) homolog, subfamily C, member 6 & 3,03 & chr1 \\
\hline DNAPTP6 & 26010 & DNA polymerase-transactivated protein 6 & 2,23 & chr2 \\
\hline DNASE1L1 & 1774 & deoxyribonuclease I-like 1 & 7,70 & $\operatorname{chrX}$ \\
\hline DNCLI2 & 1783 & dynein, cytoplasmic, light intermediate polypeptide 2 & 2,08 & chr16 \\
\hline DOC1 & 11259 & downregulated in ovarian cancer 1 & 120,26 & chr3 \\
\hline DOCK1 & 1793 & dedicator of cytokinesis 1 & 4,20 & chr10 \\
\hline DOCK10 & 55619 & dedicator of cytokinesis 10 & 9,82 & chr2 \\
\hline DOCK2 & 1794 & Dedicator of cytokinesis 2 & 16,79 & chr5 \\
\hline DOCK5 & 80005 & dedicator of cytokinesis 5 & 3,84 & chr8 \\
\hline DPF3 & 8110 & D4, zinc and double PHD fingers, family 3 & 6,47 & chr14 \\
\hline DPP8 & 54878 & Dipeptidyl-peptidase 8 & 2,27 & chr15 \\
\hline DPY19L1 & 23333 & dpy-19-like 1 (C. elegans) & 2,44 & chr7 \\
\hline DPY19L4 & 286148 & dpy-19-like 4 (C. elegans) & 2,64 & chr8 \\
\hline DPYD & 1806 & dihydropyrimidine dehydrogenase & 33,29 & chr1 \\
\hline DRAP1 & 10589 & DR1-associated protein 1 (negative cofactor 2 alpha) & 2,70 & chr11 \\
\hline DSC3 & 1825 & desmocollin 3 & 7,15 & chr18 \\
\hline DSCR1 & 1827 & Down syndrome critical region gene 1 & 68,24 & chr21 \\
\hline DSP & 1832 & desmoplakin & 3,83 & chr6 \\
\hline DSTN & 11034 & Destrin (actin depolymerizing factor) & 2,81 & chr20 \\
\hline DUSP1 & 1843 & dual specificity phosphatase 1 & 9,65 & chr5 \\
\hline DUSP10 & 11221 & dual specificity phosphatase 10 & 3,51 & chr1 \\
\hline DUSP14 & 11072 & dual specificity phosphatase 14 & 2,33 & chr17 \\
\hline DUSP18 & 150290 & dual specificity phosphatase 18 & 2,04 & chr22 \\
\hline DVL3 & 1857 & dishevelled, dsh homolog 3 (Drosophila) & 3,21 & chr3 \\
\hline DYM & 54808 & Dymeclin & 2,10 & chr18 \\
\hline DYRK4 & 8798 & dual-specificity tyrosine-(Y)-phosphorylation regulated kinase 4 & 3,97 & chr12 \\
\hline DYSF & 8291 & dysferlin, limb girdle muscular dystrophy 2B (autosomal recessive) & 2,48 & chr2 \\
\hline DZIP1 & 22873 & DAZ interacting protein 1 & 2,82 & chr13 \\
\hline EBF & 1879 & Early B-cell factor & 2,40 & chr5 \\
\hline $\mathrm{EBI} 2$ & 1880 & Epstein-Barr virus induced gene 2 (lymphocyte-specific G protein-coupled rece & 2,06 & chr13 \\
\hline ECE1 & 1889 & Endothelin converting enzyme 1 & 2,38 & chr1 \\
\hline ECM1 & 1893 & extracellular matrix protein 1 & 8,48 & chr1 \\
\hline ECM2 & 1842 & extracellular matrix protein 2 , female organ and adipocyte specific & 2,46 & chr9 \\
\hline EDEM1 & 9695 & ER degradation enhancer, mannosidase alpha-like 1 & 4,99 & chr3 \\
\hline EDG2 & 1902 & endothelial differentiation, Iysophosphatidic acid G-protein-coupled receptor, 2 & 13,99 & chr9 \\
\hline EDG3 & 1903 & endothelial differentiation, sphingolipid G-protein-coupled receptor, 3 & 5,42 & chr9 \\
\hline EDIL3 & 10085 & EGF-like repeats and discoidin I-like domains 3 & 6,80 & chr5 \\
\hline EDN1 & 1906 & endothelin 1 & 3,78 & chr6 \\
\hline EDNRA & 1909 & endothelin receptor type A & 5,23 & chr4 \\
\hline EEA1 & 8411 & early endosome antigen $1,162 \mathrm{kD}$ & 2,68 & chr12 \\
\hline EEF1D & 1936 & Eukaryotic translation elongation factor 1 delta (guanine nucleotide exchange $\mathrm{p}$ & 2,69 & chr8 \\
\hline EFEMP1 & 2202 & EGF-containing fibulin-like extracellular matrix protein 1 & 23,35 & chr2 \\
\hline EFEMP2 & 30008 & EGF-containing fibulin-like extracellular matrix protein 2 & 15,26 & chr11 \\
\hline EFHA2 & 286097 & EF-hand domain family, member A2 & 5,77 & chr8 \\
\hline EGFL5 & 1955 & EGF-like-domain, multiple 5 & 3,04 & chr9 \\
\hline EGFR & 1956 & epidermal growth factor receptor (erythroblastic leukemia viral (v-erb-b) oncoge & 15,05 & chr7 \\
\hline EGR1 & 1958 & Early growth response 1 & 3,24 & chr5 \\
\hline EHD1 & 10938 & EH-domain containing 1 & 4,00 & chr11 \\
\hline EHD2 & 30846 & EH-domain containing 2 & 24,04 & chr1 \\
\hline EHD3 & 30845 & EH-domain containing 3 & 4,30 & chr2 \\
\hline EHD4 & 30844 & EH-domain containing 4 & 3,75 & chr15 \\
\hline EIF1 & 10209 & Eukaryotic translation initiation factor 1 & 2,81 & chr17 \\
\hline EIF2C2 & 27161 & Eukaryotic translation initiation factor 2C, 2 & 6,05 & chr15 \\
\hline EIF2S1 & 1965 & eukaryotic translation initiation factor 2 , subunit 1 alpha, $35 \mathrm{kDa}$ & 4,40 & chr14 \\
\hline EIF5A2 & 56648 & eukaryotic translation initiation factor $5 \mathrm{~A} 2$ & 4,68 & chr3 \\
\hline ELF1 & 1997 & E74-like factor 1 (ets domain transcription factor) & 2,32 & chr13 \\
\hline ELF4 & 2000 & E74-like factor 4 (ets domain transcription factor) & 12,20 & $\operatorname{chrX}$ \\
\hline ELK3 & 2004 & ELK3, ETS-domain protein (SRF accessory protein 2) & 10,83 & chr12 \\
\hline ELL2 & 22936 & elongation factor, RNA polymerase II, 2 & 4,14 & chr1 \\
\hline
\end{tabular}


Table S4 : Genes overexpressed in MPC compared to hES (Fold Change > 2; $\mathrm{a}<0.05$ )

\begin{tabular}{|c|c|c|c|c|}
\hline Gene Symbol & Entrez Gene & Gene Title & MPC_up & Chromosome Number(Avadis) \\
\hline ELMOD2 & 255520 & ELMO domain containing 2 & 2,78 & chr4 \\
\hline ELOVL1 & 64834 & elongation of very long chain fatty acids (FEN1/Elo2, SUR4/Elo3, yeast)-like 1 & 5,70 & chr1 \\
\hline ELOVL2 & 54898 & elongation of very long chain fatty acids (FEN1/Elo2, SUR4/Elo3, yeast)-like 2 & 2,14 & chr6 \\
\hline ELTD1 & 64123 & EGF, latrophilin and seven transmembrane domain containing 1 & 4,27 & chr1 \\
\hline EMILIN1 & 11117 & elastin microfibril interfacer 1 & 12,99 & chr2 \\
\hline EMP1 & 2012 & epithelial membrane protein 1 & 52,02 & chr12 \\
\hline EMP3 & 2014 & epithelial membrane protein 3 & 14,12 & chr19 \\
\hline ENAH & 55740 & enabled homolog (Drosophila) & 4,24 & chr1 \\
\hline ENC1 & 8507 & ectodermal-neural cortex (with BTB-like domain) & 13,67 & chr5 \\
\hline ENG & 2022 & endoglin (Osler-Rendu-Weber syndrome 1) & 3,87 & chr9 \\
\hline ENTPD4 & 9583 & ectonucleoside triphosphate diphosphohydrolase 4 & 2,82 & chr8 \\
\hline ENTPD5 & 957 & Ectonucleoside triphosphate diphosphohydrolase 5 & 2,93 & chr14 \\
\hline EPAS1 & 2034 & endothelial PAS domain protein 1 & 51,27 & chr2 \\
\hline EPB41L1 & 2036 & Erythrocyte membrane protein band 4.1 -like 1 & 4,95 & chr5 \\
\hline EPB41L3 & 23136 & erythrocyte membrane protein band 4.1 -like 3 & 6,18 & chr18 \\
\hline EPHA2 & 1969 & EPH receptor A2 & 4,81 & chr1 \\
\hline EPHA5 & 2044 & EPH receptor A5 & 6,44 & chr4 \\
\hline EPHB2 & 2048 & EPH receptor B2 & 4,63 & chr1 \\
\hline EPIM & 2054 & epimorphin & 2,12 & chr12 \\
\hline EPLIN & 51474 & epithelial protein lost in neoplasm beta & 6,59 & chr12 \\
\hline EPM2AIP1 & 9852 & EPM2A (laforin) interacting protein 1 & 3,88 & chr3 \\
\hline EPRS & 2058 & glutamyl-prolyl-tRNA synthetase & 2,44 & chr1 \\
\hline EPS15L1 & 58513 & epidermal growth factor receptor pathway substrate 15 -like 1 & 2,32 & chr19 \\
\hline EPS8 & 2059 & epidermal growth factor receptor pathway substrate 8 & 11,93 & chr12 \\
\hline ERBB2IP & 55914 & erbb2 interacting protein & 4,29 & chr5 \\
\hline ERO1L & 30001 & ERO1-like (S. cerevisiae) & 2,78 & chr14 \\
\hline ERRFI1 & 54206 & ERBB receptor feedback inhibitor 1 & 3,50 & chr1 \\
\hline ETHE1 & 23474 & ethylmalonic encephalopathy 1 & 3,35 & chr19 \\
\hline ETS1 & 2113 & v-ets erythroblastosis virus E26 oncogene homolog 1 (avian) & 2,07 & chr11 \\
\hline ETV5 & 2119 & ets variant gene 5 (ets-related molecule) & 2,34 & chr3 \\
\hline ETV6 & 2120 & ets variant gene 6 ( $T E L$ oncogene) & 2,17 & chr12 \\
\hline EVC & 2121 & Ellis van Creveld syndrome & 3,60 & chr4 \\
\hline EVI1 & 2122 & ecotropic viral integration site 1 & 9,76 & chr3 \\
\hline EVI5 & 7813 & ecotropic viral integration site 5 & 8,09 & chr1 \\
\hline EXT1 & 2131 & Exostoses (multiple) 1 & 6,55 & chr8 \\
\hline EXT2 & 2132 & exostoses (multiple) 2 & 2,27 & chr11 \\
\hline F25965 & 55957 & protein F25965 & 3,09 & chr19 \\
\hline $\mathrm{F} 2 \mathrm{R}$ & 2149 & coagulation factor II (thrombin) receptor & 7,70 & chr5 \\
\hline F2RL2 & 2151 & coagulation factor II (thrombin) receptor-like 2 & 79,14 & chr5 \\
\hline FAM14A & 83982 & family with sequence similarity 14 , member $A$ & 2,10 & chr14 \\
\hline FAM18B & 51030 & family with sequence similarity 18 , member $B$ & 3,53 & chr16 \\
\hline FAM38A & 9780 & family with sequence similarity 38 , member $A$ & 3,85 & chr16 \\
\hline FAM43A & 131583 & family with sequence similarity 43 , member $A$ & 2,78 & chr3 \\
\hline FAM46A & 55603 & family with sequence similarity 46 , member $A$ & 9,30 & chr6 \\
\hline FAM54B & 56181 & family with sequence similarity 54 , member B & 2,01 & chr1 \\
\hline FAM55C & 91775 & family with sequence similarity 55 , member $\mathrm{C}$ & 7,99 & chr3 \\
\hline FAM62B & 57488 & family with sequence similarity 62 (C2 domain containing) member B & 2,04 & chr7 \\
\hline FAM65A & 79567 & family with sequence similarity 65 , member $A$ & 2,40 & chr16 \\
\hline FAM73A & 374986 & family with sequence similarity 73 , member $A$ & 3,98 & chr1 \\
\hline FAM79A & 127262 & family with sequence similarity 79 , member $A$ & 2,26 & chr1 \\
\hline FAM89B & 23625 & family with sequence similarity 89 , member $B$ & 3,96 & chr11 \\
\hline FAM8A1 & 51439 & family with sequence similarity 8, member $A 1$ & 3,51 & chr6 \\
\hline FAM91A1 & 157769 & family with sequence similarity 91 , member A1 & 2,12 & chr8 \\
\hline FAP & 2191 & fibroblast activation protein, alpha & 50,54 & chr2 \\
\hline FAS & 355 & Fas (TNF receptor superfamily, member 6) & 12,93 & chr10 \\
\hline FAT4 & 79633 & FAT tumor suppressor homolog 4 (Drosophila) & 2,38 & chr4 \\
\hline FBLIM1 & 54751 & filamin binding LIM protein 1 & 3,76 & chr1 \\
\hline FBLN1 & 2192 & fibulin 1 & 2,80 & chr22 \\
\hline FBLN2 & 2199 & fibulin 2 & 5,51 & chr3 \\
\hline FBLN5 & 10516 & fibulin 5 & 4,89 & chr14 \\
\hline FBN1 & 2200 & fibrillin 1 (Marfan syndrome) & 181,73 & chr15 \\
\hline FBN2 & 2201 & fibrillin 2 (congenital contractural arachnodactyly) & 12,59 & chr5 \\
\hline FBXL17 & 64839 & F-box and leucine-rich repeat protein 17 & 4,70 & chr5 \\
\hline FBXL2 & 25827 & F-box and leucine-rich repeat protein 2 & 2,70 & chr3 \\
\hline FBXL3 & 26224 & F-box and leucine-rich repeat protein 3 & 3,82 & chr13 \\
\hline FBXL5 & 26234 & F-box and leucine-rich repeat protein 5 & 3,24 & chr4 \\
\hline
\end{tabular}


Table S4 : Genes overexpressed in MPC compared to hES (Fold Change $>2 ; \mathrm{a}<0.05$ )

\begin{tabular}{|c|c|c|c|c|}
\hline Gene Symbol & Entrez Gene & Gene Title & MPC_up & Chromosome Number(Avadis) \\
\hline FBXO3 & 26273 & F-box protein 3 & 2,32 & chr11 \\
\hline FBXO32 & 114907 & F-box protein 32 & 4,52 & chr8 \\
\hline FBXO6 & 26270 & F-box protein 6 & 2,71 & chr1 \\
\hline FBXO8 & 26269 & F-box protein 8 & 3,50 & chr4 \\
\hline FCGRT & 2217 & Fc fragment of lgG, receptor, transporter, alpha & 2,18 & chr19 \\
\hline FCHSD2 & 9873 & FCH and double $\mathrm{SH} 3$ domains 2 & 5,48 & chr11 \\
\hline FCMD & 2218 & Fukuyama type congenital muscular dystrophy (fukutin) & 2,48 & chr9 \\
\hline FER1L3 & 26509 & fer-1-like 3, myoferlin (C. elegans) & 125,57 & chr10 \\
\hline FGF1 & 2246 & fibroblast growth factor 1 (acidic) & 4,58 & chr5 \\
\hline FGF5 & 2250 & fibroblast growth factor 5 & 2,61 & chr4 \\
\hline FGFR1OP2 & 26127 & FGFR1 oncogene partner 2 & 4,33 & chr12 \\
\hline FHL1 & 2273 & four and a half LIM domains 1 & 4,26 & $\operatorname{chrX}$ \\
\hline FHL2 & 2274 & four and a half LIM domains 2 & 6,90 & chr2 \\
\hline FHL3 & 2275 & four and a half LIM domains 3 & 2,28 & chr1 \\
\hline FKBP14 & 55033 & FK506 binding protein 14, $22 \mathrm{kDa}$ & 10,09 & chr7 \\
\hline FKBP7 & 51661 & FK506 binding protein 7 & 12,51 & chr2 \\
\hline FKBP9 & 11328 & FK506 binding protein 9, $63 \mathrm{kDa}$ & 11,51 & chr7 \\
\hline FLI1 & 2313 & Friend leukemia virus integration 1 & 17,02 & chr11 \\
\hline FLJ10260 & 55106 & likely ortholog of mouse schlafen 3 & 2,48 & chr17 \\
\hline FLJ10357 & 55701 & hypothetical protein FLJ10357 & 2,57 & chr14 \\
\hline FLJ10808 & 55236 & hypothetical protein FLJ10808 & 3,01 & chr4 \\
\hline FLJ10980 & 56204 & hypothetical protein FLJ10980 & 3,23 & chr15 \\
\hline FLJ11151 & 55313 & Hypothetical protein FLJ11151 & 3,59 & chr16 \\
\hline FLJ11259 & 55332 & hypothetical protein FLJ11259 & 7,14 & chr12 \\
\hline FLJ11273 & 54664 & hypothetical protein FLJ11273 & 2,96 & chr7 \\
\hline FLJ12649 & 79649 & hypothetical protein FLJ12649 & 2,23 & $\operatorname{chrX}$ \\
\hline FLJ12681 & 64788 & hypothetical protein FLJ12681 & 3,33 & chr16 \\
\hline FLJ13391 & 84141 & hypothetical protein FLJ13391 & 7,47 & chr2 \\
\hline FLJ13448 & 80219 & hypothetical protein FLJ13448 & 2,20 & chr2 \\
\hline FLJ13710 & 79875 & hypothetical protein FLJ13710 & 5,47 & chr15 \\
\hline FLJ13855 & 65264 & hypothetical protein FLJ13855 & 2,93 & chr17 \\
\hline FLJ13868 & 64755 & hypothetical protein FLJ13868 & 2,43 & chr16 \\
\hline FLJ14001 & 79730 & Hypothetical protein FLJ14001 & 3,12 & chr4 \\
\hline FLJ14054 & 79614 & hypothetical protein FLJ14054 & 10,80 & chr5 \\
\hline FLJ14213 & 79899 & hypothetical protein FLJ14213 & 4,97 & chr11 \\
\hline FLJ14525 & 84886 & hypothetical protein FLJ14525 & 4,69 & chr1 \\
\hline FLJ14640 & 84902 & hypothetical protein FLJ14640 & 2,58 & chr19 \\
\hline FLJ14800 & 84926 & hypothetical protein FLJ14800 & 2,20 & chr12 \\
\hline FLJ20054 & 54530 & hypothetical protein FLJ20054 & 3,11 & chr1 \\
\hline FLJ20186 & 54849 & hypothetical protein FLJ20186 & 2,31 & chr16 \\
\hline FLJ20254 & 54867 & Hypothetical protein FLJ20254 & 3,45 & chr2 \\
\hline FLJ20294 & 55626 & Hypothetical protein FLJ20294 & 2,25 & chr6 \\
\hline FLJ20298 & 54885 & FLJ20298 protein & 11,01 & $\operatorname{chr} X$ \\
\hline FLJ20481 & 54947 & hypothetical protein FLJ20481 & 11,80 & chr16 \\
\hline FLJ20507 & 55654 & hypothetical protein FLJ20507 & 2,58 & chr2 \\
\hline FLJ20719 & $440673 / / / 55672$ & hypothetical protein FLJ20719 /// AG1 protein & 2,76 & chr1_random \\
\hline FLJ20719 & $440670 / / / 44067$ & hypothetical protein FLJ20719 /// hypothetical protein LOC200030 /// hypothetid & 2,42 & chr1 \\
\hline FLJ20719 & $70 / / / 440673 / / / 4$ & hypothetical protein FLJ20719 /// hypothetical protein MGC8902 /// AE01 mRN/ & 2,33 & chr1 \\
\hline FLJ20920 & 80221 & hypothetical protein FLJ20920 & 3,25 & $\operatorname{chr} 17$ \\
\hline FLJ21075 & 80099 & hypothetical protein FLJ21075 & 2,23 & chr7 \\
\hline FLJ21127 & 79600 & hypothetical protein FLJ21127 & 2,08 & chr12 \\
\hline FLJ21159 & 79884 & ASAP & 2,55 & chr4 \\
\hline FLJ21657 & 64417 & hypothetical protein FLJ21657 & 2,99 & chr5 \\
\hline FLJ21827 & 56912 & hypothetical protein FLJ21827 & 2,35 & chr11 \\
\hline FLJ21986 & 79974 & hypothetical protein FLJ21986 & 20,43 & chr7 \\
\hline FLJ22028 & 79912 & hypothetical protein FLJ22028 & 3,38 & chr12 \\
\hline FLJ22222 & 79701 & hypothetical protein FLJ22222 & 2,66 & chr17 \\
\hline FLJ22313 & 64224 & hypothetical protein FLJ22313 & 2,21 & chr7 \\
\hline FLJ22471 & 80212 & limkain beta 2 & 18,90 & chr12 \\
\hline FLJ22833 & 64859 & hypothetical protein FLJ22833 & 29,06 & chr2 \\
\hline FLJ22965 & 63932 & hypothetical protein FLJ22965 & 2,02 & $\operatorname{chrX}$ \\
\hline FLJ23514 & 60494 & hypothetical protein FLJ23514 & 2,32 & chr11 \\
\hline FLJ23518 & 79780 & hypothetical protein FLJ23518 & 2,51 & chr11 \\
\hline FLJ23867 & 200058 & hypothetical protein FLJ23867 & 2,65 & chr1 \\
\hline FLJ30594 & 150622 & hypothetical locus FLJ30594 & 3,70 & chr17 \\
\hline FLJ30596 & 133686 & hypothetical protein FLJ30596 & 2,77 & chr5 \\
\hline
\end{tabular}


Table S4 : Genes overexpressed in MPC compared to hES (Fold Change $>2$; $\mathrm{a}<0.05$ )

\begin{tabular}{|c|c|c|c|c|}
\hline Gene Symbol & Entrez Gene & Gene Title & MPC_up & Chromosome Number(Avadis) \\
\hline FLJ30851 & 375190 & FLJ30851 protein & 2,03 & chr2 \\
\hline FLJ31033 & 91351 & hypothetical protein FLJ31033 & 2,62 & chr4 \\
\hline FLJ34236 & 283373 & hypothetical protein FLJ34236 & 2,11 & chr12 \\
\hline FLJ34922 & 91607 & likely ortholog of mouse schlafen $8 / 9$ & 3,11 & chr17 \\
\hline FLJ36031 & 168455 & Hypothetical protein FLJ36031 & 10,77 & chr7 \\
\hline FLJ36748 & 134265 & hypothetical protein FLJ36748 & 2,42 & chr5 \\
\hline FLJ37562 & 134553 & hypothetical protein FLJ37562 & 14,25 & chr5 \\
\hline FLJ38101 & 255919 & hypothetical protein FLJ38101 & 2,28 & chr16 \\
\hline FLJ38725 & 144811 & hypothetical protein FLJ38725 & 7,54 & chr13 \\
\hline FLJ39370 & 132720 & hypothetical protein FLJ39370 & 6,14 & $\operatorname{chr} 4$ \\
\hline FLJ39378 & 353116 & hypothetical protein FLJ39378 & 3,15 & chr12 \\
\hline FLJ39441 & 144108 & hypothetical protein FLJ39441 & 2,18 & chr11 \\
\hline FLJ42709 & 441094 & hypothetical gene supported by AK 124699 & 2,30 & chr5 \\
\hline FLJ43339 & 388115 & FLJ43339 protein & 4,21 & chr15 \\
\hline FLJ44635 & 392490 & TPT1-like protein & 2,26 & $\operatorname{chrX}$ \\
\hline FLJ45831 & 400576 & FLJ45831 protein /// MRNA; cDNA DKFZp564A222 (from clone DKFZp564A22 & 4,50 & chr2 \\
\hline FLJ90166 & 164284 & hypothetical protein FLJ90166 & 7,80 & chr20 \\
\hline FLNA & 2316 & filamin A, alpha (actin binding protein 280) & 6,93 & $\operatorname{chrX}$ \\
\hline FLNB & 2317 & filamin B, beta (actin binding protein 278) & 2,06 & chr3 \\
\hline FLNC & 2318 & filamin C, gamma (actin binding protein 280) & 9,72 & chr7 \\
\hline FLYWCH1 & 84256 & FLYWCH-type zinc finger 1 & 3,14 & chr16 \\
\hline FMN2 & 56776 & formin 2 & 6,91 & chr1 \\
\hline FN1 & 2335 & fibronectin 1 & 178,10 & chr2 \\
\hline FNDC3B & 64778 & fibronectin type III domain containing 3B & 7,30 & chr3 \\
\hline FOSL1 & 8061 & FOS-like antigen 1 & 3,19 & chr11 \\
\hline FOSL2 & 2355 & FOS-like antigen 2 & 15,44 & chr2 \\
\hline FOXC1 & 2296 & forkhead box C1 & 8,78 & chr6 \\
\hline FOXD1 & 2297 & forkhead box D1 & 25,91 & chr5 \\
\hline FOXF1 & 2294 & forkhead box F1 & 8,38 & chr16 \\
\hline FOXF2 & 2295 & forkhead box F2 & 3,12 & chr6 \\
\hline FOXJ2 & 55810 & forkhead box J2 & 2,42 & chr12 \\
\hline FOXK1 & 221937 & Forkhead box K1 & 2,53 & chr7 \\
\hline FOXL1 & 2300 & Forkhead box L1 & 6,23 & chr16 \\
\hline FOXP1 & 27086 & Forkhead box P1 & 5,07 & chr3 \\
\hline FREQ & 23413 & Frequenin homolog (Drosophila) & 2,16 & chr9 \\
\hline FRMD4A & 55691 & FERM domain containing $4 \mathrm{~A}$ & 6,77 & chr10 \\
\hline FRMD6 & 122786 & FERM domain containing 6 & 49,90 & chr14 \\
\hline FRS2 & 10818 & Fibroblast growth factor receptor substrate 2 & 2,35 & chr12 \\
\hline FSTL1 & 11167 & follistatin-like 1 & 4,78 & chr3 \\
\hline FSTL3 & 10272 & follistatin-like 3 (secreted glycoprotein) & 6,37 & chr19 \\
\hline FTH1 & 2495 & ferritin, heavy polypeptide 1 & 2,38 & chr11 \\
\hline FTL & 2512 & Ferritin, light polypeptide & 2,50 & $\operatorname{chrX}$ \\
\hline FUT6 & 2528 & Fucosyltransferase 6 (alpha $(1,3)$ fucosyltransferase) & 2,79 & chr16 \\
\hline FVT1 & 2531 & Follicular lymphoma variant translocation 1 & 3,31 & chr18 \\
\hline FXYD5 & 53827 & FXYD domain containing ion transport regulator 5 & 3,33 & chr19 \\
\hline FYCO1 & 79443 & FYVE and coiled-coil domain containing 1 & 3,80 & chr3 \\
\hline FYTTD1 & 84248 & forty-two-three domain containing 1 & 6,14 & chr3 \\
\hline FZD1 & 8321 & frizzled homolog 1 (Drosophila) & 5,79 & chr7 \\
\hline FZD2 & 2535 & frizzled homolog 2 (Drosophila) & 7,48 & chr17 \\
\hline FZD6 & 8323 & frizzled homolog 6 (Drosophila) & 5,16 & chr8 \\
\hline GABARAP & 11337 & GABA(A) receptor-associated protein & 2,04 & chr17 \\
\hline GABRB1 & 2560 & gamma-aminobutyric acid (GABA) A receptor, beta 1 & 2,41 & chr4 \\
\hline GADD45A & 1647 & growth arrest and DNA-damage-inducible, alpha & 11,34 & chr1 \\
\hline GADD45B & 4616 & growth arrest and DNA-damage-inducible, beta & 13,14 & chr19 \\
\hline GALNACT-2 & 55454 & chondroitin sulfate GalNAcT-2 & 6,52 & chr10 \\
\hline GALNT10 & 55568 & UDP-N-acetyl-alpha-D-galactosamine:polypeptide $\mathrm{N}$-acetylgalactosaminyltrans & 21,41 & chr5 \\
\hline GALNT2 & 2590 & UDP-N-acetyl-alpha-D-galactosamine:polypeptide $\mathrm{N}$-acetylgalactosaminyltrans & 6,45 & chr1 \\
\hline GALNT4 & 8693 & UDP-N-acetyl-alpha-D-galactosamine:polypeptide $\mathrm{N}$-acetylgalactosaminyltrans & 3,36 & chr12 \\
\hline GALNT5 & 11227 & UDP-N-acetyl-alpha-D-galactosamine:polypeptide $\mathrm{N}$-acetylgalactosaminyltrans & 6,70 & chr2 \\
\hline GANAB & 23193 & glucosidase, alpha; neutral AB & 2,08 & chr11 \\
\hline GAS2L1 & 10634 & growth arrest-specific 2 like 1 & 3,98 & chr22 \\
\hline GAS6 & 2621 & growth arrest-specific 6 & 13,68 & chr13 \\
\hline GATA2 & 2624 & GATA binding protein 2 & 5,09 & chr3 \\
\hline GATA6 & 2627 & GATA binding protein 6 & 9,85 & chr18 \\
\hline GBA & 2629 & Glucosidase, beta; acid (includes glucosylceramidase) & 4,52 & chr1 \\
\hline GBA /// GBAP & $2629 / / / 2630$ & glucosidase, beta; acid (includes glucosylceramidase) /// glucosidase, beta; aci & 2,77 & chr1 \\
\hline
\end{tabular}


Table S4 : Genes overexpressed in MPC compared to hES (Fold Change $>2 ; \mathrm{a}<0.05$ )

\begin{tabular}{|c|c|c|c|c|}
\hline Gene Symbol & Entrez Gene & Gene Title & MPC_up & Chromosome Number(Avadis) \\
\hline GBA2 & 57704 & Glucosidase, beta (bile acid) 2 & 3,15 & chr9 \\
\hline GBE1 & 2632 & glucan (1,4-alpha-), branching enzyme 1 (glycogen branching enzyme, Anders & 5,34 & chr3 \\
\hline GBP1 & 2633 & guanylate binding protein 1 , interferon-inducible, $67 \mathrm{kDa}$ & 28,18 & chr1 \\
\hline GBP2 & 2634 & guanylate binding protein 2 , interferon-inducible /// guanylate binding protein 2 & 3,60 & chr1 \\
\hline GBP3 & 2635 & guanylate binding protein 3 & 34,83 & chr1 \\
\hline GDF15 & 9518 & growth differentiation factor 15 & 17,75 & chr19 \\
\hline GEM & 2669 & GTP binding protein overexpressed in skeletal muscle & 4,85 & chr8 \\
\hline GFPT1 & 2673 & glutamine-fructose-6-phosphate transaminase 1 & 2,82 & chr2 \\
\hline GGH & 8836 & gamma-glutamyl hydrolase (conjugase, folylpolygammaglutamyl hydrolase) & 2,13 & chr8 \\
\hline GHR & 2690 & growth hormone receptor & 2,30 & chr5 \\
\hline GIT2 & 9815 & G protein-coupled receptor kinase interactor 2 & 3,76 & chr12 \\
\hline GLIPR1 & 11010 & GLI pathogenesis-related 1 (glioma) & 110,49 & chr12 \\
\hline GLIS1 & 148979 & GLIS family zinc finger 1 & 2,87 & chr1 \\
\hline GLIS3 & 169792 & GLIS family zinc finger 3 & 11,17 & chr9 \\
\hline GLRB & 2743 & glycine receptor, beta & 3,68 & chr4 \\
\hline GLRX & 2745 & glutaredoxin (thioltransferase) & 2,97 & chr14 \\
\hline GLRX2 & 51022 & glutaredoxin 2 & 3,23 & chr1 \\
\hline GLS & 2744 & glutaminase & 17,00 & chr2 \\
\hline GLT8D1 & 55830 & glycosyltransferase 8 domain containing 1 & 2,60 & chr3 \\
\hline GLT8D2 & 83468 & glycosyltransferase 8 domain containing 2 & 12,49 & chr12 \\
\hline GLTP & 51228 & glycolipid transfer protein & 2,84 & chr12 \\
\hline GlyBP & 9731 & glycine-, glutamate-, thienylcyclohexylpiperidine-binding protein & 3,62 & chr1 \\
\hline GNA11 & 2767 & Guanine nucleotide binding protein (G protein), alpha 11 (Gq class) & 2,96 & chr19 \\
\hline GNAl1 & 2770 & guanine nucleotide binding protein ( $G$ protein), alpha inhibiting activity polypept & 2,56 & chr7 \\
\hline GNAI2 & 2771 & guanine nucleotide binding protein ( $G$ protein), alpha inhibiting activity polypept & 2,96 & chr3 \\
\hline GNAO1 & 2775 & guanine nucleotide binding protein ( $G$ protein), alpha activating activity polypep & 2,19 & chr16 \\
\hline GNB1 & 2782 & guanine nucleotide binding protein ( $G$ protein), beta polypeptide 1 & 3,97 & chr1 \\
\hline GNB4 & 59345 & guanine nucleotide binding protein (G protein), beta polypeptide 4 & 2,63 & chr3 \\
\hline GNE & 10020 & glucosamine (UDP-N-acetyl)-2-epimerase/N-acetylmannosamine kinase & 2,37 & chr9 \\
\hline GNG11 & 2791 & guanine nucleotide binding protein ( $G$ protein), gamma 11 & 4,07 & chr7 \\
\hline GNG12 & 55970 & guanine nucleotide binding protein ( $G$ protein), gamma 12 & 28,04 & chr1 \\
\hline GNPDA2 & 132789 & glucosamine-6-phosphate deaminase 2 & 4,78 & chr4 \\
\hline GNS & 2799 & glucosamine (N-acetyl)-6-sulfatase (Sanfilippo disease IIID) & 6,57 & chr12 \\
\hline GOLGA2 & 2801 & golgi autoantigen, golgin subfamily a, 2 & 2,99 & chr9 \\
\hline GOLGA3 & 2802 & golgi autoantigen, golgin subfamily a, 3 & 3,30 & chr12 \\
\hline GOLGB1 & 2804 & Golgi autoantigen, golgin subfamily b, macrogolgin (with transmembrane signal & 2,12 & chr3 \\
\hline GOLPH4 & 27333 & golgi phosphoprotein 4 & 3,03 & chr3 \\
\hline GOLT1B & 51026 & golgi transport 1 homolog $\mathrm{B}$ (S. cerevisiae) & 7,34 & chr12 \\
\hline GOPC & 57120 & Golgi associated PDZ and coiled-coil motif containing & 3,65 & chr6 \\
\hline GOSR1 & 9527 & golgi SNAP receptor complex member 1 & 2,31 & chr17 \\
\hline GOSR2 & 9570 & Golgi SNAP receptor complex member 2 & 2,39 & chr17 \\
\hline GPC1 & 2817 & glypican 1 & 5,86 & chr2 \\
\hline GPR124 & 25960 & G protein-coupled receptor 124 & 6,41 & chr8 \\
\hline GPR126 & 57211 & G protein-coupled receptor 126 & 3,17 & chr6 \\
\hline GPR155 & 151556 & G protein-coupled receptor 155 & 5,24 & chr2 \\
\hline GPR161 & 23432 & G protein-coupled receptor 161 & 3,67 & chr1 \\
\hline GPRC5A & 9052 & G protein-coupled receptor, family C, group 5 , member A & 17,86 & chr12 \\
\hline GPSM1 & 26086 & G-protein signalling modulator 1 (AGS3-like, C. elegans) & 5,25 & chr9 \\
\hline GREM1 & 26585 & gremlin 1, cysteine knot superfamily, homolog (Xenopus laevis) & 400,20 & chr15 \\
\hline GRK5 & 2869 & G protein-coupled receptor kinase 5 & 5,38 & chr10 \\
\hline GRN & 2896 & granulin & 2,32 & chr17 \\
\hline GSK3B & 2932 & Glycogen synthase kinase 3 beta & 4,02 & chr3 \\
\hline GSN & 2934 & gelsolin (amyloidosis, Finnish type) & 5,06 & chr9 \\
\hline GSTK1 & 373156 & glutathione S-transferase kappa 1 & 2,08 & chr7 \\
\hline GSTM2 & 2946 & glutathione S-transferase M2 (muscle) & 2,11 & chr1 \\
\hline GSTM3 & 2947 & glutathione S-transferase M3 (brain) & 21,84 & chr1 \\
\hline GTF2H1 & 2965 & general transcription factor IIH, polypeptide $1,62 \mathrm{kDa}$ & 2,29 & chr11 \\
\hline GTPBP5 & 26164 & GTP binding protein 5 (putative) & 2,86 & chr20 \\
\hline GUK1 & 2987 & guanylate kinase $1 / / /$ guanylate kinase 1 & 2,41 & chr1 \\
\hline H2AFY & 9555 & H2A histone family, member $Y$ & 3,70 & chr5 \\
\hline H6PD & 9563 & hexose-6-phosphate dehydrogenase (glucose 1-dehydrogenase) & 4,63 & chr1 \\
\hline HABP4 & 22927 & hyaluronan binding protein 4 & 2,84 & chr9 \\
\hline HAPLN1 & 1404 & Hyaluronan and proteoglycan link protein 1 & 5,76 & chr5 \\
\hline HBEGF & 1839 & heparin-binding EGF-like growth factor & 11,83 & chr5 \\
\hline HBP1 & 26959 & HMG-box transcription factor 1 & 2,71 & chr7 \\
\hline HCFC2 & 29915 & host cell factor $\mathrm{C} 2$ & 3,62 & chr12 \\
\hline
\end{tabular}


Table S4 : Genes overexpressed in MPC compared to hES (Fold Change $>2 ; \mathrm{a}<0.05$ )

\begin{tabular}{|c|c|c|c|c|}
\hline Gene Symbol & Entrez Gene & Gene Title & MPC_up & Chromosome Number(Avadis) \\
\hline HDLBP & 3069 & high density lipoprotein binding protein (vigilin) & 3,06 & chr2 \\
\hline HECA & 51696 & headcase homolog (Drosophila) & 2,68 & chr6 \\
\hline HECTD2 & 143279 & HECT domain containing 2 & 2,37 & chr10 \\
\hline HECW2 & 57520 & HECT, $C 2$ and WW domain containing E3 ubiquitin protein ligase 2 & 3,40 & chr2 \\
\hline HEG1 & 57493 & HEG homolog 1 (zebrafish) & 25,65 & chr3 \\
\hline HERC4 & 26091 & hect domain and RLD 4 & 2,76 & chr10 \\
\hline HERPUD1 & 9709 & homocysteine-inducible, endoplasmic reticulum stress-inducible, ubiquitin-like $\mathrm{d}$ & 9,52 & chr16 \\
\hline HEXIM1 & 10614 & hexamethylene bis-acetamide inducible 1 & 2,18 & chr17 \\
\hline HEY1 & 23462 & hairy/enhancer-of-split related with YRPW motif 1 & 2,36 & chr8 \\
\hline HIF1A & 3091 & hypoxia-inducible factor 1 , alpha subunit (basic helix-loop-helix transcription fac & 2,04 & chr14 \\
\hline HIPK2 & 28996 & Homeodomain interacting protein kinase 2 & 3,01 & chr7 \\
\hline HIST1H2BC & 8347 & histone $1, \mathrm{H} 2 \mathrm{bc}$ & 2,42 & chr6 \\
\hline HIST1H4H & 8365 & histone $1, \mathrm{H} 4 \mathrm{~h}$ & 2,57 & chr6 \\
\hline HIVEP3 & 59269 & Human immunodeficiency virus type I enhancer binding protein 3 & 2,40 & chr1 \\
\hline HLA-B & 3106 & major histocompatibility complex, class I, B & 2,92 & chr6 \\
\hline HLA-C & 3107 & major histocompatibility complex, class I, C & 2,61 & chr6 \\
\hline HLA-E & 3133 & major histocompatibility complex, class I, E & 5,94 & chr6 \\
\hline HLX1 & 3142 & H2.0-like homeo box 1 (Drosophila) & 2,35 & chr1 \\
\hline HMCN1 & 83872 & hemicentin 1 & 12,20 & chr1 \\
\hline HNRPLL & 92906 & heterogeneous nuclear ribonucleoprotein L-like & 2,32 & chr2 \\
\hline HNRPUL2 & 221092 & heterogeneous nuclear ribonucleoprotein U-like 2 & 2,01 & chr11 \\
\hline HNT & 50863 & neurotrimin & 14,49 & chr11 \\
\hline HOMER3 & 9454 & homer homolog 3 (Drosophila) & 2,37 & chr19 \\
\hline HOM-TES-103 & 25900 & HOM-TES-103 tumor antigen-like & 2,52 & chr12 \\
\hline HOOK3 & 84376 & Hook homolog 3 (Drosophila) & 2,95 & chr8 \\
\hline HOXB2 & 3212 & homeo box B2 & 4,42 & chr17 \\
\hline HOXB7 & 3217 & homeo box B7 & 2,76 & chr17 \\
\hline HP1BP3 & 50809 & heterochromatin protein 1 , binding protein 3 & 2,30 & chr1 \\
\hline HPCAL1 & 3241 & hippocalcin-like 1 & 3,08 & chr2 \\
\hline $\mathrm{HRH} 1$ & 3269 & histamine receptor $\mathrm{H} 1$ & 9,88 & chr3 \\
\hline HRMT1L1 & 3275 & HMT1 hnRNP methyltransferase-like 1 (S. cerevisiae) & 3,10 & chr21 \\
\hline HSPA5 & 3309 & heat shock $70 \mathrm{kDa}$ protein 5 (glucose-regulated protein, $78 \mathrm{kDa}$ ) & 5,63 & chr9 \\
\hline HSPB1 & 3315 & heat shock 27kDa protein 1 & 11,96 & chr7 \\
\hline HSPB8 & 26353 & heat shock 22kDa protein 8 & 9,67 & chr12 \\
\hline HSPG2 & 3339 & heparan sulfate proteoglycan 2 (perlecan) & 4,94 & chr1 \\
\hline HT008 & 55852 & uncharacterized hypothalamus protein HT008 & 2,11 & chr17 \\
\hline HTRA1 & 5654 & HtrA serine peptidase 1 & 7,83 & chr10 \\
\hline IBRDC1 & 154214 & IBR domain containing 1 & 3,31 & chr6 \\
\hline ICHTHYIN & 348938 & ichthyin protein & 7,55 & chr5 \\
\hline ICK & 22858 & intestinal cell (MAK-like) kinase & 2,16 & chr6 \\
\hline ID3 & 3399 & inhibitor of DNA binding 3, dominant negative helix-loop-helix protein & 2,79 & chr1 \\
\hline IDH2 & 3418 & isocitrate dehydrogenase $2(\mathrm{NADP}+)$, mitochondrial & 2,20 & chr15 \\
\hline IDS & 3423 & iduronate 2-sulfatase (Hunter syndrome) & 6,12 & $\operatorname{chrX}$ \\
\hline IER3 & 8870 & |immediate early response 3 & 22,38 & chr6 \\
\hline IER3IP1 & 51124 & immediate early response 3 interacting protein 1 & 2,60 & chr18 \\
\hline IER5 & 51278 & immediate early response 5 & 5,96 & chr1 \\
\hline IER5L & 389792 & immediate early response 5 -like & 6,44 & chr9 \\
\hline IFI16 & 3428 & interferon, gamma-inducible protein 16 & 36,68 & chr1 \\
\hline IFIT2 & 3433 & interferon-induced protein with tetratricopeptide repeats 2 & 2,94 & chr10 \\
\hline IFIT3 & 3437 & Interferon-induced protein with tetratricopeptide repeats 3 & 3,61 & chr10 \\
\hline IFIT5 & 24138 & interferon-induced protein with tetratricopeptide repeats 5 & 4,05 & chr10 \\
\hline IFNAR1 & 3454 & interferon (alpha, beta and omega) receptor 1 & 4,25 & chr21 \\
\hline IFNGR2 & 3460 & interferon gamma receptor 2 (interferon gamma transducer 1) & 2,13 & chr21 \\
\hline IFT20 & 90410 & intraflagellar transport protein IFT20 & 3,44 & chr17 \\
\hline IGFBP3 & 3486 & insulin-like growth factor binding protein 3 & 28,09 & chr7 \\
\hline IGFBP4 & 3487 & insulin-like growth factor binding protein 4 & 4,71 & chr17 \\
\hline IGFBP5 & 3488 & insulin-like growth factor binding protein 5 & 142,96 & chr2 \\
\hline IGFBP6 & 3489 & insulin-like growth factor binding protein 6 & 7,75 & chr12 \\
\hline IGFBP7 & 3490 & insulin-like growth factor binding protein 7 & 343,83 & chr4 \\
\hline IKBKE & 9641 & inhibitor of kappa light polypeptide gene enhancer in B-cells, kinase epsilon & 2,48 & chr1 \\
\hline IKIP & 121457 & IKK interacting protein & 18,59 & chr12 \\
\hline IL10RB & 3588 & interleukin 10 receptor, beta & 6,65 & chr21 \\
\hline IL11 & 3589 & interleukin 11 & 5,73 & chr19 \\
\hline IL11RA & 3590 & interleukin 11 receptor, alpha & 2,79 & chr9 \\
\hline |L13RA1 & 3597 & interleukin 13 receptor, alpha 1 & 11,07 & $\operatorname{chrX}$ \\
\hline |L15 & 3600 & |interleukin 15 & 3,11 & chr4 \\
\hline
\end{tabular}




\begin{tabular}{|c|c|c|c|c|}
\hline Gene Symbol & Entrez Gene & Gene Title & MPC_up & Chromosome Number(Avadis) \\
\hline |L1R1 & 3554 & interleukin 1 receptor, type I & 20,71 & chr2 \\
\hline IL1RAP & 3556 & interleukin 1 receptor accessory protein & 2,59 & chr3 \\
\hline |IL6 & 3569 & interleukin 6 (interferon, beta 2) & 3,36 & chr7 \\
\hline |IL6ST & 3572 & interleukin 6 signal transducer (gp130, oncostatin M receptor) & 18,73 & chr17 \\
\hline |L7R & 3575 & Interleukin 7 receptor & 9,35 & chr5 \\
\hline |L8 & 3576 & |interleukin 8 & 14,71 & chr4 \\
\hline |ILK & 3611 & integrin-linked kinase & 3,30 & chr11 \\
\hline IMPAD1 & 54928 & inositol monophosphatase domain containing 1 & 4,98 & chr8 \\
\hline IMPDH1 & 3614 & IMP (inosine monophosphate) dehydrogenase 1 & 2,30 & chr7 \\
\hline ING4 & 51147 & inhibitor of growth family, member 4 & 3,14 & chr12 \\
\hline INHBA & 3624 & inhibin, beta $\mathrm{A}$ (activin $\mathrm{A}$, activin $\mathrm{AB}$ alpha polypeptide) & 113,49 & chr7 \\
\hline |INPP1 & 3628 & inositol polyphosphate-1-phosphatase & 4,14 & chr2 \\
\hline INPP4B & 8821 & inositol polyphosphate-4-phosphatase, type II, $105 \mathrm{kDa}$ & 7,24 & chr4 \\
\hline INPP5A & 3632 & inositol polyphosphate-5-phosphatase, $40 \mathrm{kDa}$ & 2,00 & chr10 \\
\hline IPP & 3652 & intracisternal A particle-promoted polypeptide & 4,28 & chr1 \\
\hline |IQWD1 & 55827 & IQ motif and WD repeats 1 & 3,48 & chr1 \\
\hline |RAK2 & 3656 & interleukin-1 receptor-associated kinase 2 & 4,82 & chr3 \\
\hline IRF2BP2 & 359948 & interferon regulatory factor 2 binding protein 2 & 3,69 & chr1 \\
\hline ISGF3G & 10379 & interferon-stimulated transcription factor 3 , gamma 48kDa & 2,21 & chr14 \\
\hline ITCH & 83737 & itchy homolog E3 ubiquitin protein ligase (mouse) & 2,85 & chr20 \\
\hline |ITGA11 & 22801 & lintegrin, alpha 11 & 12,78 & chr15 \\
\hline ITGA2 & 3673 & Integrin, alpha 2 (CD49B, alpha 2 subunit of VLA-2 receptor) & 8,92 & chr5 \\
\hline ITGA3 & 3675 & integrin, alpha 3 (antigen CD49C, alpha 3 subunit of VLA-3 receptor) & 6,45 & chr17 \\
\hline ITGA4 & 3676 & integrin, alpha 4 (antigen CD49D, alpha 4 subunit of VLA-4 receptor) & 9,27 & chr2 \\
\hline ITGA5 & 3678 & integrin, alpha 5 (fibronectin receptor, alpha polypeptide) & 14,20 & chr12 \\
\hline ITGAV & 3685 & Integrin, alpha V (vitronectin receptor, alpha polypeptide, antigen CD51) & 10,17 & chr2 \\
\hline ITGB1 & 3688 & integrin, beta 1 (fibronectin receptor, beta polypeptide, antigen CD29 includes $N$ & 8,89 & chr10 \\
\hline ITGB1BP1 & 9270 & integrin beta 1 binding protein 1 & 3,04 & chr2 \\
\hline ITGB3 & 3690 & integrin, beta 3 (platelet glycoprotein IIla, antigen CD61) & 3,21 & chr17 \\
\hline ITM2B & 9445 & integral membrane protein $2 \mathrm{~B}$ & 2,02 & chr13 \\
\hline ITPR2 & 3709 & Family with sequence similarity 20 , member $C$ & 2,21 & chr12 \\
\hline JAK1 & $3716 / / / 391045$ & Janus kinase 1 (a protein tyrosine kinase) /// similar to Solute carrier family 2, fa & 9,31 & chr1 \\
\hline JAK1 & 3716 & Janus kinase 1 (a protein tyrosine kinase) & 5,81 & chr1 \\
\hline JAK2 & 3717 & Janus kinase 2 (a protein tyrosine kinase) & 2,41 & chr9 \\
\hline JAZF1 & 221895 & juxtaposed with another zinc finger gene 1 & 2,18 & chr7 \\
\hline JRKL & 8690 & jerky homolog-like (mouse) & 2,56 & chr11 \\
\hline JUB & 84962 & jub, ajuba homolog (Xenopus laevis) & 4,38 & chr14 \\
\hline JUN & 3725 & v-jun sarcoma virus 17 oncogene homolog (avian) & 5,16 & chr1 \\
\hline JUND & 3727 & jun $\mathrm{D}$ proto-oncogene & 2,01 & chr19 \\
\hline KATNAL1 & 84056 & katanin $\mathrm{p} 60$ subunit A-like 1 & 3,79 & chr13 \\
\hline KBTBD9 & 114818 & kelch repeat and BTB (POZ) domain containing 9 & 5,22 & chr2 \\
\hline KCNG1 & 3755 & potassium voltage-gated channel, subfamily G, member 1 & 3,92 & chr20 \\
\hline KCNMA1 & 3778 & potassium large conductance calcium-activated channel, subfamily $\mathrm{M}$, alpha $\mathrm{m}$ | & 18,21 & chr10 \\
\hline KCTD10 & 83892 & potassium channel tetramerisation domain containing 10 & 3,83 & chr12 \\
\hline KCTD11 & 147040 & potassium channel tetramerisation domain containing 11 & 2,09 & chr17 \\
\hline KCTD18 & 130535 & potassium channel tetramerisation domain containing 18 & 4,78 & chr2 \\
\hline KCTD9 & 54793 & potassium channel tetramerisation domain containing 9 & 2,44 & chr9 \\
\hline KDELR2 & 11014 & KDEL (Lys-Asp-Glu-Leu) endoplasmic reticulum protein retention receptor 2 & 4,73 & chr7 \\
\hline KDELR3 & 11015 & KDEL (Lys-Asp-Glu-Leu) endoplasmic reticulum protein retention receptor 3 & 43,78 & chr22 \\
\hline KIAA0063 & 9929 & KIAA0063 gene product & 2,75 & chr22 \\
\hline KIAA0090 & 23065 & KIAA0090 & 2,14 & chr1 \\
\hline KIAA0143 & 23167 & KIAA0143 protein & 2,03 & chr8 \\
\hline KIAA0247 & 9766 & KIAA0247 & 3,91 & chr14 \\
\hline KIAA0256 & 9728 & KIAA0256 gene product & 2,42 & chr15 \\
\hline KIAA0265 & 23008 & KIAA0265 protein & 2,95 & chr7 \\
\hline KIAA0268 & $77 / / / 375056 / / / 4$ & C219-reactive peptide /// AAAP6077 /// similar to C219-reactive peptide & 2,32 & chr1_random \\
\hline KIAA0310 & 9919 & KIAA0310 & 2,07 & chr9 \\
\hline KIAA0323 & 23351 & KIAA0323 & 2,69 & chr14 \\
\hline |KIAA0372 & 9652 & KIAA0372 & 4,17 & chr5 \\
\hline KIAA0427 & 9811 & |KIAA0427 & 4,02 & chr18 \\
\hline |KIAA0470 & 9859 & KIAA0470 & 2,15 & chr4 \\
\hline |KIAA0527 & 26032 & KIAA0527 protein & 2,69 & chr3 \\
\hline KIAA0543 & 23145 & likely ortholog of mouse SCO-spondin & 2,03 & chr7 \\
\hline |KIAA0652 & 9776 & KIAA0652 gene product & 3,19 & chr11 \\
\hline KIAA0690 & 23223 & KIAA0690 & 4,25 & chr10 \\
\hline |KIAA0692 & 23141 & |KIAA0692 protein & 2,19 & chr12 \\
\hline
\end{tabular}




\begin{tabular}{|c|c|c|c|c|}
\hline Gene Symbol & Entrez Gene & Gene Title & MPC_up & Chromosome Number(Avadis) \\
\hline KIAA0738 & 9747 & KIAA0738 gene product & 3,83 & chr7 \\
\hline |KIAA0776 & 23376 & |KIAA0776 & 2,88 & chr6 \\
\hline |KIAA0802 & 23255 & |KIAA0802 & 2,25 & chr18 \\
\hline |KIAA0830 & 23052 & KIAA0830 protein & 20,43 & chr11 \\
\hline |KIAA0882 & 23158 & KIAA0882 protein & 28,31 & chr4 \\
\hline |KIAA0934 & 22982 & |KIAA0934 & 4,12 & chr10 \\
\hline |KIAA0992 & 23022 & palladin & 17,41 & chr4 \\
\hline |KIAA1040 & 23041 & KIAA1040 protein & 2,82 & chr12 \\
\hline |KIAA1043 & 23331 & KIAA1043 protein & 2,12 & chr22 \\
\hline |KIAA1055 & 23102 & KIAA1055 protein & 2,42 & chr15 \\
\hline KIAA1128 & 54462 & KIAA1128 & 3,63 & chr10 \\
\hline |KIAA1181 & 57222 & endoplasmic reticulum-golgi intermediate compartment $32 \mathrm{kDa}$ protein & 6,03 & chr5 \\
\hline |KIAA1199 & 57214 & |KIAA1199 & 90,25 & chr15 \\
\hline |KIAA1404 & 57169 & KIAA1404 protein & 2,00 & chr20 \\
\hline |KIAA1432 & 57589 & |KIAA1432 & 4,51 & chr9 \\
\hline |KIAA1434 & 56261 & hypothetical protein KIAA1434 & 2,23 & chr20 \\
\hline |KIAA1458 & 57606 & KIAA1458 protein & 2,67 & chr4 \\
\hline |KIAA1462 & 57608 & |KIAA1462 & 4,78 & chr10 \\
\hline |KIAA1539 & 80256 & |KIAA1539 & 4,18 & chr9 \\
\hline |KIAA1546 & 57667 & KIAA1546 protein & 4,97 & chr4 \\
\hline KIAA1600 & 57700 & |KIAA1600 & 4,24 & chr10 \\
\hline |KIAA1632 & 57724 & |KIAA1632 & 3,06 & chr18 \\
\hline |KIAA1704 & 55425 & |KIAA1704 & 2,31 & chr13 \\
\hline KIAA1715 & 80856 & KIAA1715 & 5,84 & chr2 \\
\hline |KIAA1729 & 85460 & KIAA1729 protein & 4,80 & chr4 \\
\hline |KIAA1754 & 85450 & |KIAA1754 & 2,75 & chr10 \\
\hline KIAA1838 & 84498 & KIAA1838 & 2,23 & chr6 \\
\hline |KIAA1856 & 84629 & KIAA1856 protein & 2,95 & chr7 \\
\hline |KIAA1912 & 114800 & KIAA1912 protein & 6,59 & chr2 \\
\hline KIAA1913 & 114801 & KIAA1913 & 2,60 & chr6 \\
\hline KIAA1949 & 170954 & KIAA1949 & 7,66 & chr6 \\
\hline |KIAA1961 & 96459 & |KIAA1961 gene & 2,24 & chr5 \\
\hline |KIAA1971 & 123720 & similar to junction-mediating and regulatory protein p300 JMY & 2,72 & chr15 \\
\hline |KIAA1972 & 89970 & KIAA1972 protein & 2,26 & chr16 \\
\hline KIF5B & 3799 & kinesin family member $5 B$ & 3,38 & chr10 \\
\hline KIFAP3 & 22920 & kinesin-associated protein 3 & 4,57 & chr1 \\
\hline KIRREL & 55243 & kin of IRRE like (Drosophila) & 4,40 & chr1 \\
\hline KITLG & 4254 & KIT ligand & 12,81 & chr12 \\
\hline |KLF10 & 7071 & Kruppel-like factor 10 & 2,88 & chr8 \\
\hline KLF12 & 11278 & Kruppel-like factor 12 & 3,71 & chr13 \\
\hline KLF2 & 10365 & Kruppel-like factor 2 (lung) & 2,68 & chr19 \\
\hline KLF3 & 51274 & Kruppel-like factor 3 (basic) & 2,07 & chr4 \\
\hline KLF6 & 1316 & Kruppel-like factor 6 & 6,00 & chr10 \\
\hline KLF7 & 8609 & Kruppel-like factor 7 (ubiquitous) & 3,30 & chr2 \\
\hline KLF9 & 687 & Kruppel-like factor 9 & 11,19 & chr9 \\
\hline KLHDC5 & 57542 & kelch domain containing 5 & 3,14 & chr12 \\
\hline KLHL20 & 27252 & kelch-like 20 (Drosophila) & 2,41 & chr1 \\
\hline KLHL5 & 51088 & Kelch-like 5 (Drosophila) & 5,51 & chr4 \\
\hline |KLHL8 & 57563 & kelch-like 8 (Drosophila) & 2,32 & chr4 \\
\hline KLHL9 & 55958 & kelch-like 9 (Drosophila) & 3,53 & chr9 \\
\hline KPNA1 & 3836 & karyopherin alpha 1 (importin alpha 5) & 3,03 & chr3 \\
\hline |KPNB1 & 3837 & Karyopherin (importin) beta 1 & 2,47 & chr17 \\
\hline |KRT10 & 3858 & keratin 10 (epidermolytic hyperkeratosis; keratosis palmaris et plantaris) & 2,53 & chr17 \\
\hline KRTAP2-1 & 81872 & keratin associated protein 2-1 & 10,76 & chr17_random \\
\hline L3MBTL3 & 84456 & |(3)mbt-like 3 (Drosophila) & 2,26 & chr6 \\
\hline LACTB & 114294 & lactamase, beta & 2,37 & chr15 \\
\hline LAMA4 & 3910 & laminin, alpha 4 & 8,96 & chr6 \\
\hline LAMB1 & 3912 & laminin, beta 1 & 3,05 & chr7 \\
\hline LAMB2 & 3913 & laminin, beta 2 (laminin S) & 6,62 & chr3 \\
\hline LAMC1 & 3915 & laminin, gamma 1 (formerly LAMB2) & 4,84 & chr1 \\
\hline LAMP2 & 3920 & lysosomal-associated membrane protein 2 & 6,02 & $\operatorname{chrX}$ \\
\hline LARP6 & 55323 & La ribonucleoprotein domain family, member 6 & 7,55 & chr15 \\
\hline LASP1 & 3927 & LIM and SH3 protein 1 & 3,61 & chr17 \\
\hline LATS2 & 26524 & LATS, large tumor suppressor, homolog 2 (Drosophila) & 2,71 & chr13 \\
\hline LDB3 & 11155 & LIM domain binding 3 & 14,73 & chr10 \\
\hline |LENG4 & 79143 & leukocyte receptor cluster (LRC) member 4 & 2,11 & chr19 \\
\hline
\end{tabular}


Table S4 : Genes overexpressed in MPC compared to hES (Fold Change > 2; a $<0.05$ )

\begin{tabular}{|c|c|c|c|c|}
\hline Gene Symbol & Entrez Gene & Gene Title & MPC_up & Chromosome Number(Avadis) \\
\hline LEPR & 3953 & Leptin receptor & 4,39 & chr1 \\
\hline LEPR & $3953 / / / 54741$ & leptin receptor /// leptin receptor overlapping transcript & 3,49 & chr1 \\
\hline LEPRE1 & 64175 & leucine proline-enriched proteoglycan (leprecan) 1 & 4,05 & chr1 \\
\hline LEPREL2 & 10536 & leprecan-like 2 & 2,51 & chr12 \\
\hline LEPROT & 54741 & leptin receptor overlapping transcript & 3,50 & chr1 \\
\hline LGALS1 & 3956 & lectin, galactoside-binding, soluble, 1 (galectin 1) & 52,69 & chr22 \\
\hline LGALS3 & $3958 / / / 81625$ & lectin, galactoside-binding, soluble, 3 (galectin 3) /// galectin-3 internal gene & 10,80 & chr14 \\
\hline LGALS3BP & 3959 & lectin, galactoside-binding, soluble, 3 binding protein & 7,85 & chr17 \\
\hline LGALS8 & 3964 & lectin, galactoside-binding, soluble, 8 (galectin 8) & 5,12 & chr1 \\
\hline LGMN & 5641 & legumain & 2,55 & chr13 \\
\hline LHCGR & 3973 & Luteinizing hormone/choriogonadotropin receptor & 4,36 & chr2 \\
\hline LHFP & 10186 & lipoma HMGIC fusion partner & 8,04 & chr13 \\
\hline LHX8 & 431707 & LIM homeobox 8 & 5,89 & chr1 \\
\hline LIF & 3976 & leukemia inhibitory factor (cholinergic differentiation factor) & 8,10 & chr22 \\
\hline LIG4 & 3981 & ligase IV, DNA, ATP-dependent & 3,28 & chr13 \\
\hline LIMS1 & 3987 & LIM and senescent cell antigen-like domains 1 & 3,84 & chr2 \\
\hline LIMS3 & 96626 & LIM and senescent cell antigen-like domains 3 & 7,42 & chr2 \\
\hline LIX1L & 128077 & Lix1 homolog (mouse) like & 22,18 & chr1 \\
\hline LMAN1 & 3998 & lectin, mannose-binding, 1 & 3,18 & chr18 \\
\hline LMBRD2 & 92255 & LMBR1 domain containing 2 & 3,11 & chr5 \\
\hline LMCD1 & 29995 & LIM and cysteine-rich domains 1 & 11,48 & chr3 \\
\hline LMNA & 4000 & $\operatorname{lamin} \mathrm{A} / \mathrm{C}$ & 11,58 & chr1 \\
\hline LMO7 & 4008 & LIM domain 7 & 22,68 & chr13 \\
\hline LMOD1 & 25802 & leiomodin 1 (smooth muscle) & 9,05 & chr1 \\
\hline LNPEP & 4012 & leucyl/cystinyl aminopeptidase & 4,33 & chr5 \\
\hline LOC126917 & 126917 & hypothetical protein LOC126917 & 3,01 & chr1 \\
\hline LOC133308 & 133308 & hypothetical protein BC009732 & 2,30 & chr4 \\
\hline LOC134147 & 134147 & similar to mouse 2310016A09Rik gene & 2,24 & chr5 \\
\hline LOC143381 & 143381 & hypothetical protein LOC143381 & 3,76 & chr10 \\
\hline LOC143903 & 143903 & layilin & 19,22 & chr11 \\
\hline LOC144363 & 144363 & hypothetical protein LOC144363 & 3,55 & chr12 \\
\hline LOC144871 & 144871 & Hypothetical protein LOC144871 & 3,58 & chr13 \\
\hline LOC148898 & 148898 & hypothetical protein $\mathrm{BC} 007899$ & 2,06 & chr1 \\
\hline LOC149478 & 149478 & Hypothetical protein LOC149478 & 2,32 & chr1 \\
\hline LOC151194 & 151194 & similar to hepatocellular carcinoma-associated antigen HCA557b & 2,09 & chr2 \\
\hline LOC153222 & 153222 & adult retina protein & 2,11 & chr5 \\
\hline LOC162073 & 162073 & Hypothetical protein LOC162073 & 10,19 & chr16 \\
\hline LOC168850 & 168850 & hypothetical protein LOC168850 & 3,81 & chr7 \\
\hline LOC196463 & 196463 & Hypothetical protein LOC196463 & 2,79 & chr12 \\
\hline LOC200030 & 200030 & hypothetical protein LOC200030 & 2,35 & chr1 \\
\hline LOC200030 & $200030 / / / 40078$ & hypothetical protein LOC200030 /// hypothetical LOC400781 & 3,71 & chr1 \\
\hline LOC201895 & 201895 & hypothetical protein LOC201895 & 4,58 & chr4 \\
\hline LOC203411 & 203411 & hypothetical protein LOC203411 & 2,87 & $\operatorname{chrX}$ \\
\hline LOC203427 & 203427 & similar to solute carrier family 25 , member 16 & 5,18 & $\operatorname{chrX}$ \\
\hline LOC222070 & 222070 & hypothetical protein LOC222070 & 2,14 & chr7 \\
\hline LOC253981 & 253981 & hypothetical protein LOC253981 & 2,35 & chr4 \\
\hline LOC255783 & 255783 & hypothetical protein LOC255783 & 6,04 & chr19 \\
\hline LOC283130 & 283130 & Hypothetical protein LOC283130 & 2,40 & chr11 \\
\hline LOC283219 & 283219 & hypothetical protein LOC283219 & 4,57 & chr11 \\
\hline LOC283464 & 283464 & hypothetical protein LOC283464 & 3,05 & chr12 \\
\hline LOC283480 & 283480 & Hypothetical protein LOC283480 & 3,34 & chr13 \\
\hline LOC283508 & 283508 & hypothetical protein LOC283508 & 2,61 & chr13 \\
\hline LOC283537 & 283537 & hypothetical protein LOC283537 & 5,46 & chr13 \\
\hline LOC283677 & 283677 & hypothetical LOC283677 & 43,29 & chr4 \\
\hline LOC283687 & 283687 & hypothetical protein LOC283687 & 2,61 & chr15 \\
\hline LOC283824 & 283824 & hypothetical protein LOC283824 & 4,06 & chr16 \\
\hline LOC284454 & 284454 & hypothetical protein LOC284454 & 3,16 & chr19 \\
\hline LOC285382 & 285382 & hypothetical gene supported by AK091454 & 3,00 & chr3 \\
\hline LOC285431 & 285431 & hypothetical protein LOC285431 & 3,94 & chr4 \\
\hline LOC285550 & 285550 & hypothetical protein LOC285550 & 3,21 & chr4 \\
\hline LOC285831 & 285831 & hypothetical protein LOC285831 & 2,40 & chr6 \\
\hline LOC286144 & 286144 & Hypothetical protein LOC286144 & 2,83 & chr8 \\
\hline LOC286167 & 286167 & hypothetical protein LOC286167 & 6,83 & chr8 \\
\hline LOC286170 & 286170 & hypothetical protein LOC286170 & 3,73 & chr8 \\
\hline LOC286437 & 286437 & hypothetical protein LOC286437 & 2,02 & $\operatorname{chr} X$ \\
\hline |LOC338620 & 338620 & hypothetical protein LOC338620 & 7,74 & chr10 \\
\hline
\end{tabular}


Table S4 : Genes overexpressed in MPC compared to hES (Fold Change $>2 ; \mathrm{a}<0.05$ )

\begin{tabular}{|c|c|c|c|c|}
\hline Gene Symbol & Entrez Gene & Gene Title & MPC_up & Chromosome Number(Avadis) \\
\hline LOC339005 & 339005 /// 44025 & hypothetical protein LOC339005 /// hypothetical protein LOC440253 & 2,68 & chr15 \\
\hline LOC340061 & 340061 & hypothetical protein LOC340061 & 9,37 & chr5 \\
\hline LOC346887 & 346887 & similar to solute carrier family 16 (monocarboxylic acid transporters), member 1 & 2,12 & chr8 \\
\hline LOC374395 & 374395 & similar to RIKEN cDNA $1810059 \mathrm{G} 22$ & 2,00 & chr11 \\
\hline LOC387758 & 387758 & similar to RIKEN cDNA $1110018 \mathrm{M} 03$ & 5,96 & chr11 \\
\hline LOC387882 & 387882 & hypothetical protein & 12,25 & chr12 \\
\hline LOC388114 & 388114 & Hypothetical LOC388114 & 2,51 & chr15 \\
\hline LOC388620 & 388620 & Similar to implantation-associated protein & 3,92 & chr1 \\
\hline LOC389129 & 389129 & similar to CG9996-PA & 10,22 & chr3 \\
\hline LOC389432 & 389432 & SAM domain containing 1 & 3,51 & chr6 \\
\hline LOC399959 & 399959 & Hypothetical gene supported by BX647608 & 74,36 & chr11 \\
\hline LOC400843 & 400843 & hypothetical LOC400843 & 4,40 & chr20 \\
\hline LOC401093 & 401093 & hypothetical LOC401093 & 4,14 & chr3 \\
\hline LOC401115 & 401115 & hypothetical gene supported by BC038466; BC062790 & 2,14 & chr4 \\
\hline LOC401212 & 401212 & hypothetical gene supported by BX640700 & 2,71 & chr5 \\
\hline LOC401394 & $401394 / / / 402578$ & hypothetical LOC401394 /// hypothetical LOC402578 & 5,06 & chr7 \\
\hline LOC402560 & 402560 & Hypothetical LOC401384 & 9,69 & chr7 \\
\hline LOC440460 & 440460 & SH3-domain GRB2-like pseudogene 3 & 3,94 & chr17 \\
\hline LOC440536 & 440536 & hypothetical gene supported by AK098812 & 3,65 & chr19 \\
\hline LOC440885 & 440885 & LOC440885 & 5,28 & chr2 \\
\hline LOC440886 & 440886 & Similar to lymphocyte-specific protein 1 & 8,46 & chr2 \\
\hline LOC440928 & 440928 & hypothetical gene supported by AK096649 & 4,77 & chr2 \\
\hline LOC440995 & 440995 & hypothetical gene supported by BC034933; BC068085 & 3,35 & chr3 \\
\hline LOC441022 & 441022 & similar to RUN and FYVE domain-containing 2; Run- and FYVE-domain contain & 2,29 & chr4 \\
\hline LOC441212 & 441212 & PNAS-13 & 2,18 & chr7 \\
\hline LOC441428 & 441428 & Hypothetical gene supported by BX641014 & 2,73 & chr9 \\
\hline LOC441461 & 441461 & hypothetical gene supported by $\mathrm{BC} 030123$ & 12,72 & chr9 \\
\hline LOC441762 & 441762 & Similar to CG7467-PA /// Similar to CG7467-PA & 2,16 & chr16 \\
\hline LOC492311 & 492311 & similar to bovine IgA regulatory protein & 2,31 & chr5 \\
\hline LOC493869 & 493869 & similar to RIKEN cDNA $2310016 \mathrm{C} 16$ & 3,01 & chr5 \\
\hline LOC51315 & 51315 & hypothetical protein LOC51315 & 3,50 & chr2 \\
\hline LOC51334 & 51334 & mesenchymal stem cell protein DSC54 & 14,29 & chr5 \\
\hline LOC554202 & 554202 & hypothetical LOC554202 & 2,23 & chr9 \\
\hline LOC90693 & 90693 & LOC90693 protein & 3,01 & chr7 \\
\hline LOC91137 & 91137 & Hypothetical protein BC017169 & 9,30 & chr5 \\
\hline LOC92689 & 92689 & hypothetical protein BC001096 & 39,06 & chr4 \\
\hline LOC93349 & 93349 & hypothetical protein BC004921 & 9,80 & chr2 \\
\hline LOC96610 & 96610 & Hypothetical protein similar to KIAA0187 gene product & 3,16 & chr9 \\
\hline LONPL & 83752 & Peroxisomal LON protease like & 5,70 & chr16 \\
\hline LOX & 4015 & |lysyl oxidase & 388,97 & chr5 \\
\hline LOXL1 & 4016 & lysyl oxidase-like 1 & 24,19 & chr15 \\
\hline LOXL2 & 4017 & lysyl oxidase-like 2 & 51,18 & chr8 \\
\hline LOXL4 & 84171 & lysyl oxidase-like 4 & 2,54 & chr10 \\
\hline LPGAT1 & 9926 & Lysophosphatidylglycerol acyltransferase 1 & 3,99 & chr1 \\
\hline LPIN1 & 23175 & lipin 1 & 4,92 & chr2 \\
\hline LPIN2 & 9663 & lipin 2 & 2,21 & chr18 \\
\hline LPP & 4026 & LIM domain containing preferred translocation partner in lipoma & 4,68 & chr3 \\
\hline LRAP & 64167 & Leukocyte-derived arginine aminopeptidase & 4,38 & chr5 \\
\hline LRIG3 & 121227 & leucine-rich repeats and immunoglobulin-like domains 3 & 3,72 & chr12 \\
\hline LRP1 & 4035 & low density lipoprotein-related protein 1 (alpha-2-macroglobulin receptor) & 4,26 & chr12 \\
\hline LRP10 & 26020 & low density lipoprotein receptor-related protein 10 & 15,41 & chr14 \\
\hline LRP11 & 84918 & low density lipoprotein receptor-related protein 11 & 3,70 & chr6 \\
\hline LRP12 & 29967 & low density lipoprotein-related protein 12 & 7,16 & chr8 \\
\hline LRPAP1 & 4043 & Low density lipoprotein receptor-related protein associated protein 1 & 3,03 & chr4 \\
\hline LRRC15 & 131578 & leucine rich repeat containing 15 & 2,69 & chr3 \\
\hline LRRC17 & 10234 & leucine rich repeat containing 17 & 40,11 & chr7 \\
\hline LRRC35 & 219899 & Leucine rich repeat containing 35 & 2,02 & chr11 \\
\hline LRRC41 & 10489 & leucine rich repeat containing 41 & 2,31 & chr6 \\
\hline LRRC8C & 84230 & leucine rich repeat containing 8 family, member $\mathrm{C}$ & 3,01 & chr1 \\
\hline LRRFIP1 & 9208 & leucine rich repeat (in FLII) interacting protein 1 & 2,48 & chr2 \\
\hline LTB4DH & 22949 & leukotriene B4 12-hydroxydehydrogenase & 2,20 & chr9 \\
\hline LTBP1 & 4052 & latent transforming growth factor beta binding protein 1 & 8,13 & chr2 \\
\hline LTBP2 & 4053 & latent transforming growth factor beta binding protein 2 & 13,08 & chr14 \\
\hline LTBP3 & 4054 & latent transforming growth factor beta binding protein 3 & 9,77 & chr11 \\
\hline LXN & 56925 & latexin & 4,64 & chr3 \\
\hline LY96 & 23643 & |lymphocyte antigen 96 & 3,86 & chr8 \\
\hline
\end{tabular}


Table S4 : Genes overexpressed in MPC compared to hES (Fold Change > 2; $a<0.05$ )

\begin{tabular}{|c|c|c|c|c|}
\hline Gene Symbol & Entrez Gene & Gene Title & MPC_up & Chromosome Number(Avadis) \\
\hline LYPD1 & 116372 & LYG/PLAUR domain containing 1 & 7,27 & chr2 \\
\hline LYPLA3 & 23659 & lysophospholipase 3 (lysosomal phospholipase A2) & 2,15 & chr16 \\
\hline LYSMD3 & 116068 & LysM, putative peptidoglycan-binding, domain containing 3 & 2,57 & chr5 \\
\hline LYST & 1130 & lysosomal trafficking regulator & 6,29 & chr1 \\
\hline LZTFL1 & 54585 & leucine zipper transcription factor-like 1 & 2,62 & chr3 \\
\hline LZTR2 & 89866 & leucine zipper transcription regulator 2 & 3,05 & chr1 \\
\hline LZTS1 & 11178 & leucine zipper, putative tumor suppressor 1 & 3,77 & chr8 \\
\hline M6PRBP1 & 10226 & mannose-6-phosphate receptor binding protein 1 & 3,61 & chr19 \\
\hline MAB21L2 & 10586 & mab-21-like 2 (C. elegans) & 45,95 & chr4 \\
\hline MAFF & 23764 & v-maf musculoaponeurotic fibrosarcoma oncogene homolog $\mathrm{F}$ (avian) & 13,02 & chr22 \\
\hline MAGED1 & 9500 & melanoma antigen family $\mathrm{D}, 1$ & 2,62 & $\operatorname{chrX}$ \\
\hline MAGED2 & 10916 & melanoma antigen family $\mathrm{D}, 2$ & 2,62 & $\operatorname{chrX}$ \\
\hline MAGEH1 & 28986 & melanoma antigen family $\mathrm{H}, 1$ & 2,30 & $\operatorname{chrX}$ \\
\hline MALAT1 & 378938 & metastasis associated lung adenocarcinoma transcript 1 (non-coding RNA) & 3,37 & chr11 \\
\hline MAML2 & 84441 & Mastermind-like 2 (Drosophila) & 6,19 & chr11 \\
\hline MAN1A1 & 4121 & Mannosidase, alpha, class $1 \mathrm{~A}$, member 1 & 4,54 & chr6 \\
\hline MAN1A2 & 10905 & Mannosidase, alpha, class $1 \mathrm{~A}$, member 2 & 2,77 & chr1 \\
\hline MAN1B1 & 11253 & mannosidase, alpha, class 1B, member 1 & 2,03 & chr9 \\
\hline MAN2B2 & 23324 & mannosidase, alpha, class 2B, member 2 & 12,03 & chr4 \\
\hline MANEA & 79694 & mannosidase, endo-alpha & 2,28 & chr6 \\
\hline MAP1A & 4130 & microtubule-associated protein $1 \mathrm{~A}$ & 16,47 & chr15 \\
\hline MAP1B & 4131 & microtubule-associated protein 1B & 6,41 & chr5 \\
\hline MAP1LC3B & 81631 & microtubule-associated protein 1 light chain 3 beta & 4,53 & chr12 \\
\hline MAP3K5 & 4217 & mitogen-activated protein kinase kinase kinase 5 & 2,25 & chr6 \\
\hline MAP4 & 4134 & microtubule-associated protein 4 & 5,40 & chr3 \\
\hline MAP4K5 & 11183 & mitogen-activated protein kinase kinase kinase kinase 5 & 3,18 & chr14 \\
\hline MAPK1 & 5594 & mitogen-activated protein kinase 1 & 2,26 & chr22 \\
\hline MAPKAP1 & 79109 & mitogen-activated protein kinase associated protein 1 & 2,72 & chr9 \\
\hline MAPKBP1 & 23005 & mitogen activated protein kinase binding protein 1 & 2,06 & chr15 \\
\hline MARCH4 & 57574 & membrane-associated ring finger (C3HC4) 4 & 2,94 & chr2 \\
\hline MARCKS & 4082 & Myristoylated alanine-rich protein kinase $C$ substrate & 3,23 & chr6 \\
\hline MARVELD1 & 83742 & MARVEL domain containing 1 & 3,21 & chr10 \\
\hline MAST4 & 23227 & microtubule associated serine/threonine kinase family member 4 & 3,48 & chr5 \\
\hline MAT2A & 4144 & Methionine adenosyltransferase II, alpha & 2,05 & chr2 \\
\hline MAWBP & 64081 & MAWD binding protein & 2,62 & chr10 \\
\hline MAX & 4149 & MYC associated factor $X$ & 2,35 & chr14 \\
\hline MBD2 & 8932 & methyl-CpG binding domain protein 2 & 30,82 & chr18 \\
\hline MBD5 & 55777 & methyl-CpG binding domain protein 5 & 3,24 & chr2 \\
\hline MBNL1 & 4154 & muscleblind-like (Drosophila) & 22,48 & chr3 \\
\hline MBNL2 & 10150 & Muscleblind-like 2 (Drosophila) & 32,00 & chr13 \\
\hline MBTPS1 & 8720 & membrane-bound transcription factor peptidase, site 1 & 2,51 & chr16 \\
\hline MCAM & 4162 & melanoma cell adhesion molecule & 5,47 & chr11 \\
\hline MCFD2 & 90411 & multiple coagulation factor deficiency 2 & 2,98 & chr2 \\
\hline MDFIC & 29969 & MyoD family inhibitor domain containing /// MyoD family inhibitor domain contaif & 5,53 & chr7 \\
\hline MDM2 & 4193 & Mdm2, transformed $3 T 3$ cell double minute 2, p53 binding protein (mouse) & 2,82 & chr12 \\
\hline ME1 & 4199 & Malic enzyme 1, NADP(+)-dependent, cytosolic & 12,94 & chr6 \\
\hline MED28 & 80306 & mediator of RNA polymerase II transcription, subunit 28 homolog (yeast) & 2,03 & chr1 \\
\hline MED8 & 112950 & mediator of RNA polymerase II transcription, subunit 8 homolog (yeast) & 3,65 & chr1 \\
\hline MEF2A & 4205 & MADS box transcription enhancer factor 2 , polypeptide $A$ (myocyte enhancer fo & 2,45 & chr15 \\
\hline MEF2C & 4208 & MADS box transcription enhancer factor 2 , polypeptide $C$ (myocyte enhancer ff & 5,07 & chr5 \\
\hline MEF2D & 4209 & MADS box transcription enhancer factor 2 , polypeptide $\mathrm{D}$ (myocyte enhancer ff & 2,11 & chr1 \\
\hline MEIS2 & 4212 & Meis1, myeloid ecotropic viral integration site 1 homolog 2 (mouse) & 21,69 & chr15 \\
\hline MET & 4233 & met proto-oncogene (hepatocyte growth factor receptor) & 6,83 & chr7 \\
\hline MFAP5 & 8076 & microfibrillar associated protein 5 & 13,66 & chr12 \\
\hline MFI2 & 4241 & antigen p97 (melanoma associated) identified by monoclonal antibodies 133.2 & 3,08 & chr3 \\
\hline MFSD1 & 64747 & major facilitator superfamily domain containing 1 & 5,41 & chr3 \\
\hline MGAT1 & 4245 & mannosyl (alpha-1,3-)-glycoprotein beta-1,2-N-acetylglucosaminyltransferase & 2,68 & chr5 \\
\hline MGAT2 & 4247 & mannosyl (alpha-1,6-)-glycoprotein beta-1,2-N-acetylglucosaminyltransferase & 3,34 & chr14 \\
\hline MGC10850 & 84736 & hypothetical protein MGC10850 & 4,33 & chr11 \\
\hline MGC10854 & 84260 & trichoplein & 3,24 & chr12 \\
\hline MGC11324 & 84803 & hypothetical protein MGC11324 /// hypothetical protein MGC11324 & 2,89 & chr4 \\
\hline MGC14376 & 84981 & hypothetical protein MGC14376 & 14,20 & chr17 \\
\hline MGC15429 & 84836 & hypothetical protein MGC15429 & 2,72 & chr3 \\
\hline MGC15476 & 147906 & thymus expressed gene 3-like & 2,75 & chr19 \\
\hline MGC15523 & 124565 & hypothetical protein MGC15523 & 3,26 & chr17 \\
\hline |MGC16121 & 84848 & Hypothetical protein MGC16121 & 2,74 & $\operatorname{chr} X$ \\
\hline
\end{tabular}


Table S4 : Genes overexpressed in MPC compared to hES (Fold Change $>2 ; \mathrm{a}<0.05$ )

\begin{tabular}{|c|c|c|c|c|}
\hline Gene Symbol & Entrez Gene & Gene Title & MPC_up & Chromosome Number(Avadis) \\
\hline MGC17330 & 113791 & HGFL gene /// HGFL gene & 3,20 & chr22 \\
\hline MGC17337 & 91283 & similar to RIKEN cDNA 5730528L13 gene & 2,30 & chr9 \\
\hline MGC17943 & 90488 & hypothetical protein MGC17943 & 15,82 & chr12 \\
\hline MGC19764 & 162394 & likely ortholog of mouse schlafen 5 & 11,78 & chr17 \\
\hline MGC20235 & 113277 & hypothetical protein MGC20235 & 3,86 & chr17 \\
\hline MGC23985 & 389336 & similar to AVLV472 & 8,56 & chr5 \\
\hline MGC24039 & 160518 & hypothetical protein MGC24039 & 2,79 & chr12 \\
\hline MGC26963 & 166929 & hypothetical protein MGC26963 & 49,90 & chr4 \\
\hline MGC2752 & 65996 & hypothetical protein MGC2752 & 2,23 & chr19 \\
\hline MGC3123 & 79089 & hypothetical protein MGC3123 & 2,23 & chr17 \\
\hline MGC34646 & 157807 & Hypothetical protein MGC34646 & 10,17 & chr8 \\
\hline MGC34830 & 120196 & hypothetical protein MGC34830 & 4,48 & chr11 \\
\hline MGC39900 & 286527 & hypothetical protein MGC39900 & 2,10 & $\operatorname{chrX}$ \\
\hline MGC4677 & 112597 & hypothetical protein MGC4677 & 34,47 & chr2 \\
\hline MGC5370 & 84825 & hypothetical protein MGC5370 & 3,31 & chr12 \\
\hline MGC5508 & 79073 & hypothetical protein MGC5508 & 2,29 & chr11 \\
\hline MGC5618 & 79099 & hypothetical protein MGC5618 & 2,25 & chr6 \\
\hline MGLL & 11343 & monoglyceride lipase /// monoglyceride lipase & 10,84 & chr3 \\
\hline MICA & 4276 & MHC class I polypeptide-related sequence A & 7,74 & chr6 \\
\hline MICAL2 & 9645 & microtubule associated monoxygenase, calponin and LIM domain containing 2 & 127,34 & chr11 \\
\hline MINA & 84864 & MYC induced nuclear antigen & 2,01 & chr3 \\
\hline MIRN21 & 406991 & microRNA 21 & 7,85 & chr9 \\
\hline MITF & 4286 & microphthalmia-associated transcription factor & 4,29 & chr3 \\
\hline MKRN2 & 23609 & makorin, ring finger protein, 2 & 2,93 & chr3 \\
\hline MLLT11 & 10962 & myeloid/lymphoid or mixed-lineage leukemia (trithorax homolog, Drosophila); tr & 2,34 & chr1 \\
\hline MLLT6 & 4302 & myeloid/lymphoid or mixed-lineage leukemia (trithorax homolog, Drosophila); tr & 2,44 & chr17 \\
\hline MLPH & 79083 & melanophilin & 8,12 & chr2 \\
\hline MLR2 & 84458 & ligand-dependent corepressor & 2,52 & chr10 \\
\hline MLXIP & 22877 & MLX interacting protein & 2,75 & chr12 \\
\hline MME & 4311 & membrane metallo-endopeptidase (neutral endopeptidase, enkephalinase, CA & 7,11 & chr3 \\
\hline MMP1 & 4312 & matrix metallopeptidase 1 (interstitial collagenase) & 114,26 & chr11 \\
\hline MMP14 & 4323 & matrix metallopeptidase 14 (membrane-inserted) & 2,53 & chr14 \\
\hline MMP16 & 4325 & matrix metallopeptidase 16 (membrane-inserted) & 3,45 & chr8 \\
\hline MMP19 & 4327 & matrix metallopeptidase 19 & 2,74 & chr12 \\
\hline MMP2 & 4313 & matrix metallopeptidase 2 (gelatinase $\mathrm{A}, 72 \mathrm{kDa}$ gelatinase, $72 \mathrm{kDa}$ type IV colla & 20,82 & chr16 \\
\hline MN1 & 4330 & meningioma (disrupted in balanced translocation) 1 & 4,01 & chr22 \\
\hline MOBKL2A & 126308 & MOB1, Mps One Binder kinase activator-like 2A (yeast) & 6,42 & chr19 \\
\hline MOCS2 & 4338 & molybdenum cofactor synthesis 2 & 2,85 & chr5 \\
\hline MONDOA & 22877 & MondoA & 2,18 & chr12 \\
\hline MORC4 & 79710 & MORC family CW-type zinc finger 4 & 12,70 & $\operatorname{chrX}$ \\
\hline MOSPD1 & 56180 & motile sperm domain containing 1 & 2,48 & $\operatorname{chrX}$ \\
\hline MOSPD2 & 158747 & motile sperm domain containing 2 & 2,23 & $\operatorname{chrX}$ \\
\hline MOXD1 & 26002 & monooxygenase, DBH-like 1 & 6,96 & chr6 \\
\hline MPPE1 & 65258 & metallophosphoesterase 1 & 2,48 & chr18 \\
\hline MPZL1 & 9019 & myelin protein zero-like 1 & 3,30 & chr1 \\
\hline MRAS & 22808 & muscle RAS oncogene homolog & 5,80 & $\operatorname{chr} 3$ \\
\hline MRC2 & 9902 & mannose receptor, C type 2 & 8,50 & chr17 \\
\hline MRCL3 & 10627 & myosin regulatory light chain MRCL3 & 2,21 & chr18 \\
\hline MRCL3 & $103910 / / / 10627$ & myosin regulatory light chain MRCL3 /// myosin regulatory light chain MRLC2 & 2,50 & chr18 \\
\hline MRLC2 & 103910 & myosin regulatory light chain MRLC2 & 2,26 & chr18 \\
\hline MRPL2 & 51069 & mitochondrial ribosomal protein L2 & 2,94 & chr6 \\
\hline MRPS22 & 56945 & Mitochondrial ribosomal protein S22 & 3,62 & chr3 \\
\hline MRVI1 & 10335 & Murine retrovirus integration site 1 homolog & 3,75 & chr11 \\
\hline MSN & 4478 & moesin & 4,80 & chr5 \\
\hline MSRB3 & 253827 & methionine sulfoxide reductase $\mathrm{B} 3$ & 138,84 & chr12 \\
\hline MSX1 & 4487 & msh homeo box homolog 1 (Drosophila) & 9,21 & chr4 \\
\hline MSX2 & 4488 & msh homeo box homolog 2 (Drosophila) & 3,44 & chr5 \\
\hline MT2A & 4502 & metallothionein $2 \mathrm{~A}$ & 3,32 & chr16 \\
\hline MTCBP-1 & 55256 & membrane-type 1 matrix metalloproteinase cytoplasmic tail binding protein- 1 & 2,21 & chr2 \\
\hline MTCH2 & 23788 & mitochondrial carrier homolog 2 (C. elegans) & 4,26 & chr11 \\
\hline MTDH & 92140 & Metadherin & 4,46 & chr8 \\
\hline MTMR6 & 9107 & myotubularin related protein 6 & 4,36 & chr13 \\
\hline MVP & 9961 & major vault protein & 18,90 & chr16 \\
\hline MXD1 & 4084 & MAX dimerization protein 1 & 5,94 & chr2 \\
\hline MXD4 & 10608 & MAX dimerization protein 4 & 2,28 & chr4 \\
\hline MXI1 & 4601 & MAX interactor $1 / / / \mathrm{MAX}$ interactor 1 & 2,00 & chr10 \\
\hline
\end{tabular}


Table S4 : Genes overexpressed in MPC compared to hES (Fold Change $>2 ; \mathrm{a}<0.05$ )

\begin{tabular}{|c|c|c|c|c|}
\hline Gene Symbol & Entrez Gene & Gene Title & MPC_up & Chromosome Number(Avadis) \\
\hline MXRA5 & 25878 & matrix-remodelling associated 5 & 15,40 & $\operatorname{chrX}$ \\
\hline MXRA7 & 439921 & matrix-remodelling associated 7 & 9,58 & chr17 \\
\hline MXRA8 & 54587 & matrix-remodelling associated 8 & 9,81 & chr1_random \\
\hline MYADM & 91663 & myeloid-associated differentiation marker & 3,81 & chr19 \\
\hline MYBL1 & 4603 & v-myb myeloblastosis viral oncogene homolog (avian)-like 1 & 7,15 & chr8 \\
\hline MYH9 & 4627 & myosin, heavy polypeptide 9 , non-muscle & 6,41 & chr22 \\
\hline MYL9 & 10398 & myosin, light polypeptide 9 , regulatory & 21,07 & chr20 \\
\hline MYLK & 4638 & myosin, light polypeptide kinase & 19,76 & chr3 \\
\hline MYO1C & 4641 & myosin IC & 4,16 & chr17 \\
\hline MYO5A & 4644 & myosin VA (heavy polypeptide 12 , myoxin) & 2,29 & chr15 \\
\hline MYOCD & 93649 & myocardin & 25,49 & chr17 \\
\hline NAGK & 55577 & $\mathrm{~N}$-acetylglucosamine kinase /// N-acetylglucosamine kinase & 2,55 & chr2 \\
\hline NALP1 & 22861 & NACHT, leucine rich repeat and PYD (pyrin domain) containing 1 & 7,16 & chr17 \\
\hline NAP5 & 344148 & Nck-associated protein 5 & 4,32 & chr2 \\
\hline NAPA & 8775 & N-ethylmaleimide-sensitive factor attachment protein, alpha & 3,08 & chr19 \\
\hline NAV1 & 89796 & neuron navigator 1 & 7,97 & chr1 \\
\hline NAV3 & 89795 & neuron navigator 3 & 13,42 & chr12 \\
\hline NBEA & 26960 & neurobeachin & 4,14 & chr13 \\
\hline NBL1 & 4681 & neuroblastoma, suppression of tumorigenicity 1 & 3,22 & chr1 \\
\hline NBPF8 & 641559 & Neuroblastoma breakpoint family, member 20 & 6,97 & chr1 \\
\hline NBR1 & 4077 & neighbor of BRCA1 gene 1 & 2,15 & chr17 \\
\hline NCBP2 & 22916 & Nuclear cap binding protein subunit $2,20 \mathrm{kDa}$ & 2,14 & chr3 \\
\hline NCOA3 & 8202 & nuclear receptor coactivator 3 & 2,43 & chr20 \\
\hline NCOA7 & 135112 & nuclear receptor coactivator 7 & 7,07 & chr6 \\
\hline NCSTN & 23385 & nicastrin & 2,37 & chr1 \\
\hline NDEL1 & 81565 & nudE nuclear distribution gene $E$ homolog like 1 (A. nidulans) /// nudE nuclear & 3,19 & chr17 \\
\hline NDFIP1 & 80762 & Nedd4 family interacting protein 1 & 3,72 & chr5 \\
\hline NDFIP2 & 54602 & Nedd4 family interacting protein 2 & 4,80 & chr13 \\
\hline NDP52 & 10241 & nuclear domain 10 protein & 2,54 & chr17 \\
\hline NDRG1 & 10397 & $\mathrm{~N}$-myc downstream regulated gene 1 & 7,39 & chr8 \\
\hline NDUFS1 & 4719 & NADH dehydrogenase (ubiquinone) Fe-S protein 1, 75kDa (NADH-coenzyme & 2,29 & chr2 \\
\hline NECAP2 & 55707 & NECAP endocytosis associated 2 & 2,18 & chr1 \\
\hline NEDD4 & 4734 & neural precursor cell expressed, developmentally down-regulated 4 & 15,69 & chr15 \\
\hline NEDD9 & 4739 & neural precursor cell expressed, developmentally down-regulated 9 & 9,65 & chr6 \\
\hline NEGR1 & 257194 & neuronal growth regulator 1 & 7,89 & chr1 \\
\hline NEK6 & 10783 & NIMA (never in mitosis gene a)-related kinase 6 & 6,67 & chr9 \\
\hline NEK7 & 140609 & NIMA (never in mitosis gene a)-related kinase 7 & 19,50 & chr1 \\
\hline NEXN & 91624 & nexilin ( $\mathrm{F}$ actin binding protein) & 56,40 & chr1 \\
\hline NF1 & 4763 & Neurofibromin 1 (neurofibromatosis, von Recklinghausen disease, Watson dise & 2,22 & chr17 \\
\hline NF2 & 4771 & neurofibromin 2 (bilateral acoustic neuroma) & 3,06 & chr22 \\
\hline NFAT5 & 10725 & nuclear factor of activated T-cells 5 , tonicity-responsive & 8,73 & chr16 \\
\hline NFATC4 & 4776 & nuclear factor of activated T-cells, cytoplasmic, calcineurin-dependent 4 & 2,27 & chr14 \\
\hline NFE2L2 & 4780 & nuclear factor (erythroid-derived 2)-like 2 & 3,06 & chr2 \\
\hline NFIC & 4782 & nuclear factor I/C (CCAAT-binding transcription factor) & 4,04 & chr19 \\
\hline NFKB1 & 4790 & nuclear factor of kappa light polypeptide gene enhancer in B-cells 1 (p105) & 4,13 & chr4 \\
\hline NFKBIZ & 64332 & nuclear factor of kappa light polypeptide gene enhancer in B-cells inhibitor, zeta & 5,86 & chr3 \\
\hline NGFB & 4803 & nerve growth factor, beta polypeptide & 2,34 & chr1 \\
\hline NID1 & 4811 & nidogen 1 & 6,56 & chr1 \\
\hline NID2 & 22795 & nidogen 2 (osteonidogen) & 16,49 & chr14 \\
\hline NIN & 51199 & ninein (GSK3B interacting protein) & 5,22 & chr14 \\
\hline NIPSNAP3A & 25934 & nipsnap homolog 3A (C. elegans) /// nipsnap homolog 3A (C. elegans) & 5,15 & chr9 \\
\hline NKIRAS1 & 28512 & NFKB inhibitor interacting Ras-like 1 & 2,40 & chr3 \\
\hline NKIRAS2 & 28511 & NFKB inhibitor interacting Ras-like 2 & 2,35 & chr17 \\
\hline NKX3-1 & 4824 & NK3 transcription factor related, locus 1 (Drosophila) & 2,35 & chr8 \\
\hline NMT2 & 9397 & $\mathrm{~N}$-myristoyltransferase 2 & 5,09 & chr10 \\
\hline NNMT & 4837 & nicotinamide N-methyltransferase & 261,83 & chr11 \\
\hline NNT & 23530 & nicotinamide nucleotide transhydrogenase & 3,92 & chr5 \\
\hline NOD27 & 84166 & nucleotide-binding oligomerization domains 27 & 2,94 & chr16 \\
\hline NOL3 & 8996 & nucleolar protein 3 (apoptosis repressor with CARD domain) & 2,25 & chr16 \\
\hline NOTCH2 & 4853 & Notch homolog 2 (Drosophila) & 3,29 & chr1 \\
\hline $\mathrm{NOTCH} 2 \mathrm{NL}$ & 388677 & Notch homolog 2 (Drosophila) N-terminal like & 4,37 & chr1 \\
\hline NOX4 & 50507 & NADPH oxidase 4 & 10,24 & chr11 \\
\hline NPAL3 & 57185 & NIPA-like domain containing 3 & 9,52 & chr1 \\
\hline NPAS2 & 4862 & neuronal PAS domain protein 2 & 3,09 & chr2 \\
\hline NPHP3 & 27031 & nephronophthisis 3 (adolescent) & 4,45 & chr3 \\
\hline NPR3 & 4883 & natriuretic peptide receptor $\mathrm{C} /$ guanylate cyclase $\mathrm{C}$ (atrionatriuretic peptide rece| & 4,56 & chr5 \\
\hline
\end{tabular}


Table S4 : Genes overexpressed in MPC compared to hES (Fold Change $>2 ; \mathrm{a}<0.05$ )

\begin{tabular}{|c|c|c|c|c|}
\hline Gene Symbol & Entrez Gene & Gene Title & MPC_up & Chromosome Number(Avadis) \\
\hline NQO1 & 1728 & $\mathrm{NAD}(\mathrm{P}) \mathrm{H}$ dehydrogenase, quinone 1 & 6,69 & chr16 \\
\hline NR2F2 & 7026 & nuclear receptor subfamily 2, group $\mathrm{F}$, member 2 & 49,89 & chr15 \\
\hline NR3C1 & 2908 & nuclear receptor subfamily 3 , group C, member 1 (glucocorticoid receptor) /// nu & 7,55 & chr5 \\
\hline NRBP2 & 340371 & nuclear receptor binding protein 2 & 3,83 & chr8 \\
\hline NRIP1 & 8204 & nuclear receptor interacting protein 1 & 4,48 & chr21 \\
\hline NRIP3 & 56675 & nuclear receptor interacting protein 3 & 3,46 & chr11 \\
\hline NRP1 & 8829 & neuropilin 1 & 37,96 & chr10 \\
\hline NRP2 & 8828 & Neuropilin 2 & 12,17 & chr2 \\
\hline NRXN3 & 9369 & neurexin 3 & 3,03 & chr14 \\
\hline NS3TP2 & 65983 & HCV NS3-transactivated protein 2 & 2,89 & chr5 \\
\hline NS5ATP13TP2 & 220323 & NS5ATP13TP2 protein & 5,13 & chr11 \\
\hline NSF & 4905 & $\mathrm{~N}$-ethylmaleimide-sensitive factor & 2,57 & chr17 \\
\hline NT5E & 4907 & 5'-nucleotidase, ecto (CD73) & 83,19 & chr6 \\
\hline NTF3 & 4908 & neurotrophin 3 & 2,15 & chr12 \\
\hline NTN4 & 59277 & netrin 4 & 68,83 & chr12 \\
\hline NUAK1 & 9891 & NUAK family, SNF1-like kinase, 1 & 4,24 & chr12 \\
\hline NUCB1 & 4924 & nucleobindin 1 & 2,67 & chr19 \\
\hline NUCB2 & 4925 & nucleobindin 2 & 4,76 & chr11 \\
\hline NUDT4 & 11163 & Nudix (nucleoside diphosphate linked moiety X)-type motif 4 pseudogene 2 & 4,85 & chr5 \\
\hline NUMB & 8650 & numb homolog (Drosophila) & 2,52 & chr14 \\
\hline ОАСТ2 & 129642 & O-acyltransferase (membrane bound) domain containing 2 & 2,58 & chr2 \\
\hline OCRL & 4952 & oculocerebrorenal syndrome of Lowe & 2,34 & $\operatorname{chrX}$ \\
\hline ODZ2 & 57451 & odz, odd Oz/ten-m homolog 2 (Drosophila) & 8,53 & chr5 \\
\hline OGFRL1 & 79627 & opioid growth factor receptor-like 1 & 2,63 & chr6 \\
\hline OIP106 & 22906 & OGT(O-Glc-NAc transferase)-interacting protein $106 \mathrm{KDa}$ & 2,47 & chr3 \\
\hline OLFML2A & 169611 & olfactomedin-like 2A & 3,57 & chr9 \\
\hline OPN1SW & 611 & Opsin 1 (cone pigments), short-wave-sensitive (color blindness, tritan) & 4,37 & chr7 \\
\hline OPN3 & 23596 & opsin 3 (encephalopsin, panopsin) & 7,42 & chr1 \\
\hline OPTN & 10133 & optineurin & 12,82 & chr10 \\
\hline ORMDL1 & 94101 & ORM1-like 1 (S. cerevisiae) & 2,79 & chr2 \\
\hline Os9 & 10956 & amplified in osteosarcoma & 4,49 & chr12 \\
\hline OSAP & 84709 & ovary-specific acidic protein & 11,35 & chr4 \\
\hline OSBPL5 & 114879 & oxysterol binding protein-like 5 & 3,43 & chr11 \\
\hline OSMR & 9180 & Oncostatin M receptor & 49,48 & chr5 \\
\hline OSTF1 & 26578 & osteoclast stimulating factor 1 & 2,21 & chr9 \\
\hline OXR1 & 55074 & oxidation resistance 1 & 3,94 & chr8 \\
\hline OXTR & 5021 & oxytocin receptor & 94,66 & chr3 \\
\hline P4HA1 & 5033 & procollagen-proline, 2-oxoglutarate 4-dioxygenase (proline 4-hydroxylase), alph & 5,06 & chr10 \\
\hline P4HA2 & 8974 & procollagen-proline, 2-oxoglutarate 4-dioxygenase (proline 4-hydroxylase), alph & 21,34 & chr5 \\
\hline P4HA3 & 283208 & procollagen-proline, 2-oxoglutarate 4-dioxygenase (proline 4-hydroxylase), alph & 3,18 & chr11 \\
\hline $\mathrm{P} 4 \mathrm{HB}$ & 5034 & procollagen-proline, 2-oxoglutarate 4-dioxygenase (proline 4-hydroxylase), beta & 3,93 & chr17 \\
\hline PACS1 & 55690 & phosphofurin acidic cluster sorting protein 1 & 2,20 & chr11 \\
\hline PAEP & 5047 & progestagen-associated endometrial protein (placental protein 14, pregnancy-a & 3,72 & chr19 \\
\hline PAFAH1B1 & 5048 & platelet-activating factor acetylhydrolase, isoform Ib, alpha subunit $45 \mathrm{kDa}$ & 2,20 & chr17 \\
\hline PAG1 & 55824 & phosphoprotein associated with glycosphingolipid microdomains 1 & 6,23 & chr8 \\
\hline PAK2 & 5062 & p21 (CDKN1A)-activated kinase 2 & 2,34 & chr3 \\
\hline PALM2 & 114299 & paralemmin 2 & 2,50 & chr9 \\
\hline PALM2-AKAP2 & 445815 & PALM2-AKAP2 protein & 2,95 & chr9 \\
\hline PAM & 5066 & peptidylglycine alpha-amidating monooxygenase & 4,53 & chr5 \\
\hline PANX1 & 24145 & Pannexin 1 & 2,46 & chr11 \\
\hline PAPPA & 5069 & pregnancy-associated plasma protein A, pappalysin 1 & 172,39 & chr9 \\
\hline PAPSS2 & 9060 & 3'-phosphoadenosine 5'-phosphosulfate synthase 2 & 6,50 & chr10 \\
\hline PARVA & 55742 & parvin, alpha & 24,65 & chr11 \\
\hline PBXIP1 & 57326 & pre-B-cell leukemia transcription factor interacting protein 1 & 2,67 & chr1 \\
\hline PCAF & 8850 & p300/CBP-associated factor & 3,33 & chr3 \\
\hline PCDH9 & 5101 & protocadherin 9 & 7,10 & chr13 \\
\hline PCDHB10 & 56126 & protocadherin beta 10 & 4,26 & chr5 \\
\hline PCDHGC3 & / 56105 /// 56106 & protocadherin gamma subfamily C, $3 / / /$ protocadherin gamma subfamily $C, 3 /$ | & 3,34 & chr5 \\
\hline PCGF1 & 84759 & polycomb group ring finger 1 & 2,08 & chr2 \\
\hline PCGF4 & 648 & polycomb group ring finger 4 & 3,20 & chr10 \\
\hline PCGF5 & 84333 & polycomb group ring finger 5 & 19,14 & chr10 \\
\hline PCOLCE & 5118 & procollagen C-endopeptidase enhancer & 7,16 & chr7 \\
\hline PCSK7 & 9159 & Proprotein convertase subtilisin/kexin type 7 & 10,70 & chr11 \\
\hline PCTK2 & 5128 & PCTAIRE protein kinase 2 & 3,01 & chr12 \\
\hline PCYOX1 & 51449 & prenylcysteine oxidase 1 & 2,05 & chr2 \\
\hline PCYT1A & 5130 & Phosphate cytidylyltransferase 1 , choline, alpha & 3,18 & chr3 \\
\hline
\end{tabular}


Table S4 : Genes overexpressed in MPC compared to hES (Fold Change > 2; $\mathrm{a}<0.05$ )

\begin{tabular}{|c|c|c|c|c|}
\hline Gene Symbol & Entrez Gene & Gene Title & MPC_up & Chromosome Number(Avadis) \\
\hline PDCD4 & 27250 & programmed cell death 4 (neoplastic transformation inhibitor) & 3,33 & chr10 \\
\hline PDCD6 & $10016 / / / / 57491$ & programmed cell death $6 / / /$ aryl-hydrocarbon receptor repressor & 8,20 & chr5 \\
\hline PDE1C & 5137 & phosphodiesterase 1C, calmodulin-dependent 70kDa & 3,29 & chr7 \\
\hline PDE4B & 5142 & phosphodiesterase 4B, cAMP-specific (phosphodiesterase E4 dunce homolog, & 2,67 & chr1 \\
\hline PDE4DIP & 9659 & phosphodiesterase 4D interacting protein (myomegalin) & 5,79 & chr1 \\
\hline PDE5A & 8654 & phosphodiesterase 5A, cGMP-specific & 2,11 & chr4 \\
\hline PDE7B & 27115 & phosphodiesterase 7B & 2,68 & chr6 \\
\hline PDE8A & 5151 & phosphodiesterase 8A & 5,18 & chr15 \\
\hline PDGFC & 56034 & platelet derived growth factor $\mathrm{C}$ & 24,38 & chr4 \\
\hline PDGFRB & 5159 & platelet-derived growth factor receptor, beta polypeptide & 10,51 & chr5 \\
\hline PDGFRL & 5157 & platelet-derived growth factor receptor-like & 5,77 & chr8 \\
\hline PDK2 & 5164 & pyruvate dehydrogenase kinase, isoenzyme 2 & 2,17 & chr17 \\
\hline PDLIM2 & 64236 & PDZ and LIM domain 2 (mystique) & 2,68 & chr8 \\
\hline PDLIM3 & 27295 & PDZ and LIM domain 3 & 6,40 & chr4 \\
\hline PDLIM4 & 8572 & PDZ and LIM domain 4 & 5,04 & chr5 \\
\hline PDLIM5 & 10611 & PDZ and LIM domain 5 & 7,12 & chr4 \\
\hline PDLIM7 & 9260 & PDZ and LIM domain 7 (enigma) & 7,69 & chr5 \\
\hline PDZRN3 & 23024 & PDZ domain containing RING finger 3 & 3,20 & chr3 \\
\hline PEA15 & 8682 & phosphoprotein enriched in astrocytes 15 & 8,19 & chr1 \\
\hline PEAR1 & 375033 & platelet endothelial aggregation receptor 1 & 9,29 & chr1 \\
\hline PEX10 & 5192 & Peroxisome biogenesis factor 10 & 3,21 & chr1 \\
\hline PEX11B & 8799 & peroxisomal biogenesis factor $11 \mathrm{~B}$ & 2,81 & chr1 \\
\hline PEX13 & 5194 & peroxisome biogenesis factor 13 & 2,27 & chr2 \\
\hline PGCP & 10404 & plasma glutamate carboxypeptidase & 5,60 & chr8 \\
\hline PGM3 & 5238 & phosphoglucomutase 3 & 3,07 & chr6 \\
\hline PGRMC2 & 10424 & progesterone receptor membrane component 2 & 2,08 & chr4 \\
\hline PHC2 & 1912 & polyhomeotic-like 2 (Drosophila) & 8,80 & chr1 \\
\hline $\mathrm{PHC} 3$ & 80012 & polyhomeotic like 3 (Drosophila) & 2,09 & chr3 \\
\hline PHF20L1 & 51105 & PHD finger protein 20-like 1 & 4,35 & chr8 \\
\hline PHLDA1 & 22822 & Pleckstrin homology-like domain, family A, member 1 & 3,23 & chr12 \\
\hline PHLDA2 & 7262 & pleckstrin homology-like domain, family A, member 2 & 15,10 & chr11 \\
\hline PHLDA3 & 23612 & pleckstrin homology-like domain, family A, member 3 & 4,83 & chr1 \\
\hline PHLDB2 & 90102 & pleckstrin homology-like domain, family B, member 2 & 14,96 & chr3 \\
\hline PHTF2 & 57157 & putative homeodomain transcription factor 2 & 2,69 & chr7 \\
\hline PICALM & 8301 & phosphatidylinositol binding clathrin assembly protein & 4,52 & chr11 \\
\hline PIGX & 54965 & phosphatidylinositol glycan, class X & 2,28 & chr3 \\
\hline PIK3С3 & 5289 & Phosphoinositide-3-kinase, class 3 & 3,10 & chr18 \\
\hline PIK4CB & 5298 & phosphatidylinositol 4-kinase, catalytic, beta polypeptide & 3,53 & chr1 \\
\hline PINK1 & 65018 & PTEN induced putative kinase 1 & 2,72 & chr1 \\
\hline PIP5K1C & 23396 & phosphatidylinositol-4-phosphate 5-kinase, type I, gamma & 2,21 & chr19 \\
\hline PITPNA & 5306 & phosphatidylinositol transfer protein, alpha & 2,42 & chr17 \\
\hline PITRM1 & 10531 & pitrilysin metallopeptidase 1 & 2,67 & chr10 \\
\hline PJA2 & 9867 & praja 2, RING-H2 motif containing & 3,02 & chr5 \\
\hline PKIB & 5570 & protein kinase (cAMP-dependent, catalytic) inhibitor beta & 2,33 & chr6 \\
\hline PKIG & 11142 & protein kinase (cAMP-dependent, catalytic) inhibitor gamma & 2,08 & chr20 \\
\hline PLA2G4A & 5321 & phospholipase A2, group IVA (cytosolic, calcium-dependent) & 3,38 & chr1 \\
\hline PLAGL1 & 5325 & pleiomorphic adenoma gene-like 1 & 17,03 & chr6 \\
\hline PLAT & 5327 & plasminogen activator, tissue & 23,22 & chr8 \\
\hline PLAU & 5328 & plasminogen activator, urokinase & 16,09 & chr10 \\
\hline PLAUR & 5329 & plasminogen activator, urokinase receptor & 18,67 & chr19 \\
\hline PLCB1 & 23236 & phospholipase C, beta 1 (phosphoinositide-specific) & 2,42 & chr20 \\
\hline PLD1 & 5337 & phospholipase D1, phophatidylcholine-specific & 4,36 & chr3 \\
\hline PLD3 & 23646 & phospholipase D family, member 3 & 3,91 & chr19 \\
\hline PLDN & 26258 & pallidin homolog (mouse) & 2,39 & chr15 \\
\hline PLEC1 & 5339 & plectin 1 , intermediate filament binding protein $500 \mathrm{kDa}$ & 2,66 & chr8 \\
\hline PLEKHA2 & 59339 & pleckstrin homology domain containing, family A (phosphoinositide binding spe & 18,71 & chr8 \\
\hline PLEKHA3 & 65977 & pleckstrin homology domain containing, family A (phosphoinositide binding spe & 2,58 & chr2 \\
\hline PLEKHC1 & 10979 & pleckstrin homology domain containing, family C (with FERM domain) member & 2,27 & chr14 \\
\hline PLK1 & 5347 & Polo-like kinase 1 (Drosophila) & 2,32 & chr16 \\
\hline PLK2 & 10769 & polo-like kinase 2 (Drosophila) & 18,51 & chr5 \\
\hline PLK3 & 1263 & polo-like kinase 3 (Drosophila) & 2,02 & chr1 \\
\hline PLOD1 & 5351 & procollagen-lysine 1, 2-oxoglutarate 5-dioxygenase 1 & 9,76 & chr1 \\
\hline PLOD2 & 5352 & procollagen-lysine, 2-oxoglutarate 5-dioxygenase 2 & 10,14 & chr3 \\
\hline PLOD3 & 8985 & procollagen-lysine, 2-oxoglutarate 5-dioxygenase 3 & 3,27 & chr7 \\
\hline PLP2 & 5355 & proteolipid protein 2 (colonic epithelium-enriched) & 20,06 & $\operatorname{chrX}$ \\
\hline PLSCR3 & $254863 / / / 57$ & phospholipid scramblase $3 / / /$ hypothetical protein MGC40107 & 3,10 & chr17 \\
\hline
\end{tabular}




\begin{tabular}{|c|c|c|c|c|}
\hline Gene Symbol & Entrez Gene & Gene Title & MPC_up & Chromosome Number(Avadis) \\
\hline PLSCR4 & 57088 & phospholipid scramblase 4 & 2,86 & chr3 \\
\hline PLXNA2 & 5362 & plexin A2 & 2,19 & chr1 \\
\hline PLXNA3 & 55558 & plexin A3 & 2,83 & $\operatorname{chrX}$ \\
\hline PLXNB2 & 23654 & plexin B2 & 3,30 & chr22 \\
\hline PLXND1 & 23129 & plexin D1 & 3,22 & chr3 \\
\hline PME-1 & 51400 & protein phosphatase methylesterase- 1 & 3,23 & chr11 \\
\hline PMM1 & 5372 & phosphomannomutase 1 & 3,75 & chr22 \\
\hline |PMP22 & 5376 & peripheral myelin protein 22 & 8,35 & chr17 \\
\hline POFUT2 & 23275 & protein O-fucosyltransferase 2 & 3,22 & chr21 \\
\hline POLH & 5429 & Polymerase (DNA directed), eta & 3,42 & chr6 \\
\hline POLK & 51426 & polymerase (DNA directed) kappa & 3,54 & chr5 \\
\hline POLR3GL & 84265 & polymerase (RNA) III (DNA directed) polypeptide G (32kD) like & 6,75 & chr1 \\
\hline PON2 & 5445 & Paraoxonase 2 & 2,47 & chr7 \\
\hline POPDC3 & 64208 & popeye domain containing 3 & 4,44 & chr6 \\
\hline |PORIMIN & 114908 & pro-oncosis receptor inducing membrane injury gene & 2,81 & chr11 \\
\hline POSTN & 10631 & periostin, osteoblast specific factor & 132,94 & chr13 \\
\hline PPAPDC1A & 196051 & phosphatidic acid phosphatase type 2 domain containing $1 \mathrm{~A}$ & 14,97 & chr10 \\
\hline PPARA & 5465 & peroxisome proliferative activated receptor, alpha & 2,14 & chr22 \\
\hline PPARD & 5467 & peroxisome proliferative activated receptor, delta & 2,94 & chr6 \\
\hline PPARG & 5468 & Peroxisome proliferative activated receptor, gamma & 2,00 & chr3 \\
\hline PPFIBP1 & 8496 & PTPRF interacting protein, binding protein 1 (liprin beta 1) & 5,22 & chr12 \\
\hline PPGB & 5476 & protective protein for beta-galactosidase (galactosialidosis) & 2,61 & chr20 \\
\hline PPIC & 5480 & peptidylprolyl isomerase C (cyclophilin C) & 4,59 & chr5 \\
\hline PPM1F & 9647 & protein phosphatase $1 \mathrm{~F}$ (PP2C domain containing) & 2,59 & chr22 \\
\hline PPM1K & 152926 & protein phosphatase $1 \mathrm{~K}$ (PP2C domain containing) & 2,38 & chr4 \\
\hline PPP1R12A & 4659 & protein phosphatase 1 , regulatory (inhibitor) subunit 12A & 2,00 & chr12 \\
\hline |PPP1R12B & 4660 & protein phosphatase 1 , regulatory (inhibitor) subunit 12B & 2,04 & chr1 \\
\hline PPP1R15A & 23645 & protein phosphatase 1 , regulatory (inhibitor) subunit 15A & 3,29 & chr19 \\
\hline PPP1R2 & 5504 & protein phosphatase 1, regulatory (inhibitor) subunit 2 & 2,07 & chr5 \\
\hline PPP1R3C & 5507 & protein phosphatase 1 , regulatory (inhibitor) subunit $3 \mathrm{C}$ & 6,33 & chr10 \\
\hline PРP3СB & 5532 & protein phosphatase 3 (formerly 2B), catalytic subunit, beta isoform (calcineurin & 2,37 & chr10 \\
\hline PPP3CC & 5533 & protein phosphatase 3 (formerly 2B), catalytic subunit, gamma isoform (calcine & 6,33 & chr8 \\
\hline PRAF2 & 11230 & PRA1 domain family, member 2 & 5,78 & $\operatorname{chrX}$ \\
\hline PRB1 & $440083 / / / 5542$ & proline-rich protein BstNI subfamily 1 /// proline-rich protein BstNI subfamily 2 & 2,66 & chr12 \\
\hline PRDM16 & 63976 & PR domain containing 16 & 2,61 & chr1 \\
\hline PRDM2 & 7799 & PR domain containing 2, with ZNF domain & 2,02 & chr1 \\
\hline PRG1 & 5552 & proteoglycan 1 , secretory granule & 268,53 & chr10 \\
\hline PRICKLE2 & 166336 & prickle-like 2 (Drosophila) & 5,66 & chr3 \\
\hline PRKAA1 & 5562 & protein kinase, AMP-activated, alpha 1 catalytic subunit & 3,78 & chr5 \\
\hline PRKACB & 5567 & protein kinase, cAMP-dependent, catalytic, beta & 2,71 & chr1 \\
\hline PRKAG1 & 5571 & protein kinase, AMP-activated, gamma 1 non-catalytic subunit & 2,55 & chr12 \\
\hline PRKAG2 & 51422 & protein kinase, AMP-activated, gamma 2 non-catalytic subunit & 6,38 & chr7 \\
\hline PRKAR1A & 5573 & protein kinase, cAMP-dependent, regulatory, type I, alpha (tissue specific exting & 2,27 & chr17 \\
\hline PRKCDBP & 112464 & protein kinase $\mathrm{C}$, delta binding protein & 5,23 & chr11 \\
\hline PRKCE & 5581 & protein kinase C, epsilon & 4,13 & chr2 \\
\hline PRKCSH & 5589 & protein kinase $\mathrm{C}$ substrate $80 \mathrm{~K}-\mathrm{H}$ & 2,24 & chr19 \\
\hline PRNP & 5621 & prion protein (p27-30) (Creutzfeld-Jakob disease, Gerstmann-Strausler-Schein & 8,86 & chr20 \\
\hline PRO1073 & 29005 & PRO1073 protein & 3,57 & chr11 \\
\hline PRO1855 & 55379 & hypothetical protein PRO1855 & 2,19 & chr17 \\
\hline PRRG1 & 5638 & proline rich Gla (G-carboxyglutamic acid) 1 & 3,54 & $\operatorname{chrX}$ \\
\hline PRRX1 & 5396 & paired related homeobox 1 & 119,70 & chr1 \\
\hline PRSS12 & 8492 & Protease, serine, 12 (neurotrypsin, motopsin) & 8,46 & chr4 \\
\hline PRSS23 & 11098 & protease, serine, 23 & 114,10 & chr11 \\
\hline PSCD3 & 9265 & pleckstrin homology, Sec7 and coiled-coil domains 3 & 2,71 & chr7 \\
\hline PSD3 & 23362 & pleckstrin and Sec7 domain containing 3 & 2,42 & chr8 \\
\hline PSMB2 & 5690 & proteasome (prosome, macropain) subunit, beta type, 2 & 2,58 & chr1 \\
\hline PSMB7 & 5695 & Proteasome (prosome, macropain) subunit, beta type, 7 & 3,97 & chr9 \\
\hline PSTPIP2 & 9050 & proline-serine-threonine phosphatase interacting protein 2 & 3,08 & chr18 \\
\hline PTEN & 5728 & Phosphatase and tensin homolog (mutated in multiple advanced cancers 1) & 2,70 & chr10 \\
\hline PTGER2 & 5732 & prostaglandin E receptor 2 (subtype EP2), 53kDa & 2,68 & chr14 \\
\hline PTGER4 & 5734 & prostaglandin E receptor 4 (subtype EP4) & 4,84 & chr5 \\
\hline PTGFR & 5737 & prostaglandin $\mathrm{F}$ receptor (FP) & 2,67 & chr1 \\
\hline PTGFRN & 5738 & prostaglandin F2 receptor negative regulator & 3,47 & chr1 \\
\hline PTGS2 & 5743 & prostaglandin-endoperoxide synthase 2 (prostaglandin $\mathrm{G} / \mathrm{H}$ synthase and cycld & 8,89 & chr1 \\
\hline PTHLH & 5744 & parathyroid hormone-like hormone /// parathyroid hormone-like hormone & 8,74 & chr12 \\
\hline PTK9 & 5756 & PTK9 protein tyrosine kinase 9 & 4,53 & chr12 \\
\hline
\end{tabular}


Table S4 : Genes overexpressed in MPC compared to hES (Fold Change $>2 ; \mathrm{a}<0.05$ )

\begin{tabular}{|c|c|c|c|c|}
\hline Gene Symbol & Entrez Gene & Gene Title & MPC_up & Chromosome Number(Avadis) \\
\hline PTP4A1 & 7803 & protein tyrosine phosphatase type IVA, member 1 & 3,48 & chr6 \\
\hline PTP4A2 & 8073 & protein tyrosine phosphatase type IVA, member 2 & 2,64 & chr1 \\
\hline PTPLA & 9200 & protein tyrosine phosphatase-like (proline instead of catalytic arginine), membe & 2,53 & chr10 \\
\hline PTPN11 & 5781 & protein tyrosine phosphatase, non-receptor type 11 (Noonan syndrome 1) & 3,00 & chr12 \\
\hline PTPN21 & 11099 & Protein tyrosine phosphatase, non-receptor type 21 & 4,13 & chr14 \\
\hline PTPN9 & 5780 & protein tyrosine phosphatase, non-receptor type 9 & 2,21 & chr15 \\
\hline PTPNS1 & 140885 & protein tyrosine phosphatase, non-receptor type substrate 1 & 2,90 & chr20 \\
\hline PTPRJ & 5795 & Protein tyrosine phosphatase, receptor type, J & 2,78 & chr11 \\
\hline PTPRM & 5797 & protein tyrosine phosphatase, receptor type, M & 14,22 & chr7 \\
\hline PTRF & 284119 & polymerase I and transcript release factor & 22,23 & chr17 \\
\hline PTX1 & 51290 & PTX1 protein & 2,21 & chr12 \\
\hline PTX3 & 5806 & pentraxin-related gene, rapidly induced by IL-1 beta & 116,99 & chr3 \\
\hline PURB & 5814 & purine-rich element binding protein $B$ & 2,34 & chr7 \\
\hline PVR & 5817 & poliovirus receptor & 3,52 & chr19 \\
\hline PXDN & 7837 & peroxidasin homolog (Drosophila) & 3,26 & chr2 \\
\hline PXK & 54899 & PX domain containing serine/threonine kinase & 5,67 & chr3 \\
\hline PXN & 5829 & paxillin & 2,21 & chr12 \\
\hline PYCR1 & 5831 & pyrroline-5-carboxylate reductase 1 & 2,10 & chr17 \\
\hline PYGB & 5834 & phosphorylase, glycogen; brain & 3,06 & chr20 \\
\hline QIL1 & 125988 & QIL1 protein & 2,87 & chr19 \\
\hline QKI & 9444 & quaking homolog, $\mathrm{KH}$ domain RNA binding (mouse) & 2,04 & chr6 \\
\hline QSCN6 & 5768 & quiescin Q6 & 5,39 & chr1 \\
\hline RAB11FIP2 & 22841 & RAB11 family interacting protein 2 (class I) & 2,07 & chr10 \\
\hline RAB11FIP5 & 26056 & RAB11 family interacting protein 5 (class I) & 4,01 & chr2 \\
\hline RAB12 & 201475 & RAB12, member RAS oncogene family & 6,19 & chr18 \\
\hline RAB18 & 22931 & RAB18, member RAS oncogene family & 2,36 & chr10 \\
\hline RAB2 & 5862 & RAB2, member RAS oncogene family & 2,97 & chr8 \\
\hline RAB22A & 57403 & RAB22A, member RAS oncogene family & 3,54 & chr20 \\
\hline RAB23 & 51715 & RAB23, member RAS oncogene family & 4,32 & chr6 \\
\hline RAB27A & 5873 & RAB27A, member RAS oncogene family & 4,20 & chr15 \\
\hline RAB27B & 5874 & RAB27B, member RAS oncogene family & 9,99 & chr18 \\
\hline RAB30 & 27314 & RAB30, member RAS oncogene family & 11,08 & chr11 \\
\hline RAB31 & 11031 & RAB31, member RAS oncogene family & 4,87 & chr18 \\
\hline RAB32 & 10981 & RAB32, member RAS oncogene family & 15,55 & chr6 \\
\hline RAB33A & 9363 & RAB33A, member RAS oncogene family & 2,53 & $\operatorname{chrX}$ \\
\hline RAB3B & 5865 & RAB3B, member RAS oncogene family & 3,19 & chr1 \\
\hline RAB3GAP1 & 22930 & RAB3 GTPase activating protein subunit 1 (catalytic) & 2,33 & chr2 \\
\hline RAB40B & 10966 & RAB40B, member RAS oncogene family & 6,49 & chr17 \\
\hline RAB6A /// RAB6 & $5870 / / / 84084$ & RAB6A, member RAS oncogene family /// RAB6C, member RAS oncogene farl & 2,01 & chr2 \\
\hline RAB6IP2 & 23085 & RAB6 interacting protein 2 & 2,73 & chr12 \\
\hline RAB9B & 51209 & RAB9B, member RAS oncogene family & 2,03 & $\operatorname{chrX}$ \\
\hline RABGEF1 & 27342 & RAB guanine nucleotide exchange factor (GEF) 1 & 2,80 & chr7 \\
\hline RAFTLIN & 23180 & raft-linking protein & 42,35 & chr3 \\
\hline RAGE & 5891 & renal tumor antigen & 7,59 & chr14 \\
\hline RAl1 & 10743 & retinoic acid induced 1 & 3,94 & chr17 \\
\hline RAl14 & 26064 & retinoic acid induced 14 & 3,36 & chr5 \\
\hline RALB & 5899 & v-ral simian leukemia viral oncogene homolog B (ras related; GTP binding prote & 3,79 & chr2 \\
\hline RaLP & 399694 & rai-like protein & 3,49 & chr15 \\
\hline RAP1A & 5906 & RAP1A, member of RAS oncogene family & 2,74 & chr1 \\
\hline RAP1GDS1 & 5910 & RAP1, GTP-GDP dissociation stimulator 1 & 3,76 & chr4 \\
\hline RAP2C & 57826 & RAP2C, member of RAS oncogene family & 2,01 & $\operatorname{chrX}$ \\
\hline RAPH1 & 65059 & Ras association (RalGDS/AF-6) and pleckstrin homology domains 1 & 5,11 & chr2 \\
\hline RARB & 5915 & retinoic acid receptor, beta & 9,28 & chr3 \\
\hline RASA1 & 5921 & RAS p21 protein activator (GTPase activating protein) 1 & 2,72 & chr5 \\
\hline RASSF3 & 283349 & Ras association (RalGDS/AF-6) domain family 3 & 3,54 & chr14 \\
\hline RASSF4 & 83937 & Ras association (RalGDS/AF-6) domain family 4 & 2,07 & chr10 \\
\hline RASSF8 & 11228 & Ras association (RalGDS/AF-6) domain family 8 & 2,01 & chr12 \\
\hline RB1 & 5925 & retinoblastoma 1 (including osteosarcoma) & 2,97 & chr13 \\
\hline RBL2 & 5934 & retinoblastoma-like 2 (p130) & 3,17 & chr16 \\
\hline RBM18 & 92400 & RNA binding motif protein 18 & 3,58 & chr9 \\
\hline RBM24 & 221662 & RNA binding motif protein 24 & 28,95 & chr6 \\
\hline RBM9 & 23543 & RNA binding motif protein 9 & 3,26 & chr22 \\
\hline RBMS2 & 5939 & RNA binding motif, single stranded interacting protein 2 & 3,74 & chr12 \\
\hline RBMS3 & 27303 & RNA binding motif, single stranded interacting protein & 18,12 & chr3 \\
\hline RCN1 & 5954 & reticulocalbin 1, EF-hand calcium binding domain & 2,85 & chr11 \\
\hline RCN3 & 57333 & reticulocalbin 3, EF-hand calcium binding domain & 3,50 & chr19 \\
\hline
\end{tabular}




\begin{tabular}{|c|c|c|c|c|}
\hline Gene Symbol & Entrez Gene & Gene Title & MPC_up & Chromosome Number(Avadis) \\
\hline RECK & 8434 & reversion-inducing-cysteine-rich protein with kazal motifs & 7,09 & chr9 \\
\hline RECQL & 5965 & RecQ protein-like (DNA helicase Q1-like) & 3,08 & chr12 \\
\hline REEP3 & 221035 & Receptor accessory protein 3 & 4,45 & chr10 \\
\hline RELA & 5970 & v-rel reticuloendotheliosis viral oncogene homolog A, nuclear factor of kappa lig & 2,84 & chr11 \\
\hline REV3L & 5980 & REV3-like, catalytic subunit of DNA polymerase zeta (yeast) & 2,41 & chr6 \\
\hline REXO2 & 25996 & REX2, RNA exonuclease 2 homolog (S. cerevisiae) & 2,08 & chr11 \\
\hline RFK & 55312 & |riboflavin kinase & 2,94 & chr9 \\
\hline RGL1 & 23179 & ral guanine nucleotide dissociation stimulator-like 1 & 7,51 & chr1 \\
\hline RGMB & 285704 & RGM domain family, member B & 5,25 & chr5 \\
\hline RGNEF & 64283 & Rho-guanine nucleotide exchange factor & 3,79 & chr5 \\
\hline RGS10 & 6001 & regulator of G-protein signalling 10 & 2,66 & chr10 \\
\hline RGS20 & 8601 & regulator of G-protein signalling 20 & 2,53 & chr8 \\
\hline RGS3 & 5998 & regulator of G-protein signalling 3 & 8,93 & chr9 \\
\hline RGS4 & 5999 & regulator of G-protein signalling 4 & 133,63 & chr1 \\
\hline RHBDL7 & 57414 & rhomboid, veinlet-like 7 (Drosophila) & 2,65 & chr7 \\
\hline RHOB & 388 & ras homolog gene family, member B & 2,95 & chr2 \\
\hline RHOBTB1 & 9886 & Rho-related BTB domain containing 1 & 3,16 & chr10 \\
\hline RHOBTB3 & 22836 & Rho-related BTB domain containing 3 & 26,55 & chr5 \\
\hline RHOC & 389 & ras homolog gene family, member $\mathrm{C}$ & 10,36 & chr1 \\
\hline RHOG & 391 & ras homolog gene family, member G (rho G) & 2,44 & chr11 \\
\hline RHOJ & 57381 & ras homolog gene family, member $\mathrm{J}$ & 13,07 & chr14 \\
\hline RHOQ & 23433 & ras homolog gene family, member $Q$ & 2,45 & chr2 \\
\hline RHOQ /// LOC2\& & 23433 /// 284988 & ras homolog gene family, member $Q / / /$ similar to $A R H Q$ protein & 3,10 & chr2 \\
\hline RIG & 10530 & regulated in glioma & 4,17 & chr11 \\
\hline RIN2 & 54453 & Ras and Rab interactor 2 & 37,07 & chr20 \\
\hline RIOK3 & 8780 & RIO kinase 3 (yeast) /// RIO kinase 3 (yeast) & 2,29 & chr18 \\
\hline RIPK1 & 8737 & receptor (TNFRSF)-interacting serine-threonine kinase 1 & 3,05 & chr6 \\
\hline RIPX & 22902 & rap2 interacting protein $\mathrm{x}$ & 2,08 & chr4 \\
\hline RIT1 & 6016 & Ras-like without CAAX 1 & 2,38 & chr1 \\
\hline RNASE4 & 6038 & ribonuclease, RNase A family, 4 & 10,14 & chr14 \\
\hline RND3 & 390 & Rho family GTPase 3 & 7,69 & chr2 \\
\hline RNF103 & 7844 & ring finger protein 103 & 3,75 & chr2 \\
\hline RNF11 & 26994 & ring finger protein 11 & 2,28 & chr1 \\
\hline RNF14 & 9604 & ring finger protein 14 & 3,12 & chr5 \\
\hline RNF150 & 57484 & ring finger protein 150 & 2,03 & chr4 \\
\hline RNF185 & 91445 & |ring finger protein 185 & 2,60 & chr22 \\
\hline RNF19 & 25897 & Ring finger protein 19 & 3,02 & chr8 \\
\hline RNF6 & 6049 & ring finger protein ( $(\mathrm{C} 3 \mathrm{H} 2 \mathrm{C} 3$ type $) 6$ & 3,18 & chr13 \\
\hline RNH1 & 6050 & ribonuclease/angiogenin inhibitor 1 & 3,39 & chr11 \\
\hline RNPC2 & 9584 & RNA-binding region (RNP1, RRM) containing 2 & 2,14 & chr20 \\
\hline ROCK2 & 9475 & Rho-associated, coiled-coil containing protein kinase 2 & 2,65 & chr2 \\
\hline RP11-343N15.3 & 440608 & Similar to Formin binding protein 2 (srGAP2) & 2,41 & chr1 \\
\hline RP11-378J18.4 & 375056 & C219-reactive peptide & 2,95 & chr1 \\
\hline RP2 & 6102 & retinitis pigmentosa 2 (X-linked recessive) & 3,03 & $\operatorname{chrX}$ \\
\hline RPL23AP7 & 118433 & ribosomal protein L23a pseudogene 7 & 3,44 & chr1 \\
\hline RPRC1 & 55700 & arginine/proline rich coiled-coil 1 & 6,11 & chr1 \\
\hline RPS23 & 6228 & ribosomal protein S23 & 4,98 & chr5 \\
\hline RPS27L & 51065 & Ribosomal protein S27-like & 4,27 & chr15 \\
\hline RPS6KA2 & 6196 & ribosomal protein S6 kinase, 90kDa, polypeptide 2 & 4,08 & chr6 \\
\hline RPS6KA3 & 6197 & ribosomal protein $\mathrm{S} 6$ kinase, 90kDa, polypeptide 3 & 3,21 & $\operatorname{chrX}$ \\
\hline RRAD & 6236 & Ras-related associated with diabetes & 2,65 & chr16 \\
\hline RRAGB & 10325 & Ras-related GTP binding B & 2,23 & $\operatorname{chrX}$ \\
\hline RRAS & 6237 & related RAS viral (r-ras) oncogene homolog & 16,59 & chr19 \\
\hline RRBP1 & 6238 & ribosome binding protein 1 homolog $180 \mathrm{kDa}$ (dog) & 2,01 & chr20 \\
\hline RSL1D1 & 26156 & Ribosomal L1 domain containing 1 & 4,27 & chr16 \\
\hline RSN & 6249 & restin (Reed-Steinberg cell-expressed intermediate filament-associated protein) & 5,89 & chr12 \\
\hline RSNL2 & 79745 & restin-like 2 & 37,98 & chr2 \\
\hline RSU1 & 6251 & Ras suppressor protein 1 & 2,56 & chr10 \\
\hline RTN4 & 57142 & reticulon 4 & 2,24 & chr2 \\
\hline RUNX1 & 861 & runt-related transcription factor 1 (acute myeloid leukemia 1 ; aml1 oncogene) & 37,16 & chr21 \\
\hline RUNX2 & 860 & runt-related transcription factor 2 & 10,68 & chr6 \\
\hline RUSC2 & 9853 & $\mathrm{RUN}$ and $\mathrm{SH} 3$ domain containing 2 & 3,56 & chr9 \\
\hline RXRB & 6257 & retinoid $X$ receptor, beta & 2,65 & chr6 \\
\hline S100A11 & 6282 & S100 calcium binding protein $\mathrm{A} 11$ (calgizzarin) & 11,04 & chr1 \\
\hline S100A13 & 6284 & S100 calcium binding protein $\mathrm{A} 13$ & 2,75 & chr1 \\
\hline S100A16 & 140576 & S100 calcium binding protein A16 & 14,87 & chr1 \\
\hline
\end{tabular}


Table S4 : Genes overexpressed in MPC compared to hES (Fold Change $>2$; $a<0.05$ )

\begin{tabular}{|c|c|c|c|c|}
\hline Gene Symbol & Entrez Gene & Gene Title & MPC_up & Chromosome Number(Avadis) \\
\hline SACS & 26278 & spastic ataxia of Charlevoix-Saguenay (sacsin) & 2,33 & chr13 \\
\hline SAMD4 & 23034 & sterile alpha motif domain containing 4 & 5,95 & chr14 \\
\hline SAMD9 & 54809 & sterile alpha motif domain containing 9 & 6,51 & chr7 \\
\hline SAR1B & 51128 & SAR1 gene homolog B (S. cerevisiae) & 3,44 & chr5 \\
\hline SAT & 6303 & Spermidine/spermine N1-acetyltransferase & 6,26 & $\operatorname{chrX}$ \\
\hline SATB2 & 23314 & SATB family member 2 & 2,94 & chr2 \\
\hline SATL1 & 340562 & Spermidine/spermine N1-acetyl transferase-like 1 & 4,19 & $\operatorname{chrX}$ \\
\hline SBDS & 51119 & Shwachman-Bodian-Diamond syndrome & 3,63 & chr7 \\
\hline SBDS /// SBDSA & $155370 / / / 51119$ & Shwachman-Bodian-Diamond syndrome /// Shwachman-Bodian-Diamond synd & 3,67 & chr7 \\
\hline SBLF & 11037 & stoned B-like factor & 3,58 & chr2 \\
\hline SC65 & 10609 & synaptonemal complex protein SC65 & 5,32 & chr17 \\
\hline SCAP2 & 8935 & src family associated phosphoprotein 2 & 2,24 & chr7 \\
\hline SCARB2 & 950 & scavenger receptor class $\mathrm{B}$, member 2 & 3,11 & chr4 \\
\hline SCARF2 & 91179 & scavenger receptor class $F$, member 2 & 3,06 & chr22 \\
\hline SCD5 & 79966 & stearoyl-CoA desaturase 5 & 23,07 & chr4 \\
\hline SCFD2 & 152579 & $\sec 1$ family domain containing 2 & 2,52 & chr4 \\
\hline SCG2 & 7857 & secretogranin II (chromogranin C) & 17,83 & chr2 \\
\hline SCHIP1 & 29970 & schwannomin interacting protein 1 & 2,68 & chr3 \\
\hline SCML1 & 6322 & sex comb on midleg-like 1 (Drosophila) & 4,91 & $\operatorname{chrX}$ \\
\hline SCN9A & 6335 & sodium channel, voltage-gated, type IX, alpha & 2,32 & chr2 \\
\hline $\operatorname{scoc}$ & 60592 & short coiled-coil protein & 2,51 & chr4 \\
\hline SCRG1 & 11341 & scrapie responsive protein 1 & 3,04 & chr4 \\
\hline SCRN3 & 79634 & secernin 3 & 2,23 & chr2 \\
\hline SCUBE3 & 222663 & signal peptide, CUB domain, EGF-like 3 & 12,44 & chr6 \\
\hline SDC1 & 6382 & syndecan 1 & 2,43 & chr2 \\
\hline SDC2 & 6383 & syndecan 2 (heparan sulfate proteoglycan 1 , cell surface-associated, fibroglyca & 6,03 & chr8 \\
\hline SDC3 & 9672 & syndecan 3 ( $\mathrm{N}$-syndecan) & 3,71 & chr1 \\
\hline SDCCAG33 & 10194 & serologically defined colon cancer antigen 33 & 6,84 & chr18 \\
\hline SDF4 & 51150 & stromal cell derived factor 4 & 3,46 & chr1 \\
\hline SDSL & 113675 & serine dehydratase-like & 2,61 & chr12 \\
\hline SEC14L1 & 6397 & SEC14-like 1 (S. cerevisiae) & 2,54 & chr17 \\
\hline SEC22L1 & 9554 & SEC22 vesicle trafficking protein-like 1 (S. cerevisiae) & 12,92 & chr1 \\
\hline SEC23A & 10484 & Sec23 homolog A (S. cerevisiae) & 5,11 & chr14 \\
\hline SEC24A & 10802 & SEC24 related gene family, member A (S. cerevisiae) & 2,31 & chr5 \\
\hline SEC24D & 9871 & SEC24 related gene family, member D (S. cerevisiae) & 5,55 & chr4 \\
\hline SEC31L1 & 22872 & SEC31-like 1 (S. cerevisiae) & 3,56 & chr4 \\
\hline SEC61A1 & 29927 & Sec61 alpha 1 subunit (S. cerevisiae) & 3,65 & chr3 \\
\hline SEL1L & 6400 & sel-1 suppressor of lin-12-like (C. elegans) & 8,09 & chr14 \\
\hline SELM & 140606 & selenoprotein M & 9,68 & chr22 \\
\hline SELPLG & 6404 & selectin P ligand & 2,08 & chr12 \\
\hline SEMA3C & 10512 & sema domain, immunoglobulin domain (Ig), short basic domain, secreted, (sem & 27,09 & chr7 \\
\hline SEMA4F & 10505 & sema domain, immunoglobulin domain (Ig), transmembrane domain (TM) and & 2,50 & chr2 \\
\hline SEMA5A & 9037 & sema domain, seven thrombospondin repeats (type 1 and type 1 -like), transme & 5,01 & chr5 \\
\hline SEPT6 /// N-PAd & $23157 / / / 84656$ & septin 6 /// cytokine-like nuclear factor $n-p a c$ & 2,66 & $\operatorname{chrX}$ \\
\hline SERINC1 & 57515 & serine incorporator 1 & 4,99 & chr6 \\
\hline SERPINB6 & 5269 & serpin peptidase inhibitor, clade B (ovalbumin), member 6 & 4,35 & chr1 \\
\hline SERPINB7 & 8710 & serpin peptidase inhibitor, clade B (ovalbumin), member 7 & 14,00 & chr18 \\
\hline SERPINB8 & 5271 & serpin peptidase inhibitor, clade B (ovalbumin), member 8 & 2,86 & chr18 \\
\hline SERPINE1 & 5054 & serpin peptidase inhibitor, clade $E$ (nexin, plasminogen activator inhibitor type 1 & 201,36 & chr7 \\
\hline SERTAD1 & 29950 & SERTA domain containing 1 & 2,14 & chr19 \\
\hline SERTAD2 & 9792 & SERTA domain containing 2 & 6,31 & chr2 \\
\hline SESTD1 & 91404 & SEC14 and spectrin domains 1 & 2,06 & chr2 \\
\hline SET7 & 80854 & SET domain-containing protein 7 & 18,95 & chr4 \\
\hline SEZ6L2 & 26470 & seizure related 6 homolog (mouse)-like 2 & 2,28 & chr16 \\
\hline SFRS11 & 9295 & splicing factor, arginine/serine-rich 11 & 2,96 & chr1 \\
\hline SFT2D2 & 375035 & SFT2 domain containing 2 & 2,44 & chr1 \\
\hline SFXN1 & 94081 & sideroflexin 1 & 2,41 & chr5 \\
\hline SFXN3 & 81855 & sideroflexin 3 /// sideroflexin 3 & 7,99 & chr10 \\
\hline SGCB & 6443 & sarcoglycan, beta (43kDa dystrophin-associated glycoprotein) & 2,43 & chr4 \\
\hline$S G C D$ & 6444 & Sarcoglycan, delta (35kDa dystrophin-associated glycoprotein) & 2,03 & chr5 \\
\hline SGIP1 & 84251 & SH3-domain GRB2-like (endophilin) interacting protein 1 & 6,24 & chr1 \\
\hline SGK & 6446 & serum/glucocorticoid regulated kinase & 2,20 & chr6 \\
\hline SGPP1 & 81537 & sphingosine-1-phosphate phosphatase 1 & 3,12 & chr14 \\
\hline SGSH & 6448 & N-sulfoglucosamine sulfohydrolase (sulfamidase) & 2,81 & chr17 \\
\hline SH3BGRL & 6451 & SH3 domain binding glutamic acid-rich protein like & 2,87 & $\operatorname{chrX}$ \\
\hline SH3BGRL3 & 83442 & SH3 domain binding glutamic acid-rich protein like 3 /// SH3 domain bindir & 8,11 & chr1 \\
\hline
\end{tabular}


Table S4 : Genes overexpressed in MPC compared to hES (Fold Change $>2 ; \mathrm{a}<0.05$ )

\begin{tabular}{|c|c|c|c|c|}
\hline Gene Symbol & Entrez Gene & Gene Title & MPC_up & Chromosome Number(Avadis) \\
\hline SH3GLB1 & 51100 & SH3-domain GRB2-like endophilin B1 & 3,88 & chr1 \\
\hline SH3KBP1 & 30011 & SH3-domain kinase binding protein 1 & 2,83 & $\operatorname{chrX}$ \\
\hline SH3MD1 & 9644 & SH3 multiple domains 1 & 4,39 & chr10 \\
\hline SH3MD2 & 57630 & SH3 multiple domains 2 & 5,41 & chr4 \\
\hline SH3MD4 & 344558 & SH3 multiple domains 4 & 5,15 & chr2 \\
\hline SH3RF2 & 153769 & SH3 domain containing ring finger 2 & 3,56 & chr5 \\
\hline SHB & 6461 & Src homology 2 domain containing adaptor protein B & 2,10 & chr9 \\
\hline $\mathrm{SHC1}$ & 6464 & SHC (Src homology 2 domain containing) transforming protein 1 & 2,21 & chr1 \\
\hline SIDT2 & 51092 & SID1 transmembrane family, member 2 & 3,03 & chr11 \\
\hline SIL1 & 64374 & SIL1 homolog, endoplasmic reticulum chaperone (S. cerevisiae) & 7,07 & chr5 \\
\hline SIRT2 & 22933 & sirtuin (silent mating type information regulation 2 homolog) 2 (S. cerevisiae) & 2,87 & chr19 \\
\hline SIX1 & 6495 & Sine oculis homeobox homolog 1 (Drosophila) & 112,74 & chr14 \\
\hline SLC10A3 & 8273 & solute carrier family 10 (sodium/bile acid cotransporter family), member 3 & 2,72 & $\operatorname{chrX}$ \\
\hline SLC12A2 & 6558 & solute carrier family 12 (sodium/potassium/chloride transporters), member 2 & 2,07 & chr5 \\
\hline SLC12A4 & 6560 & solute carrier family 12 (potassium/chloride transporters), member 4 & 2,84 & chr16 \\
\hline SLC16A4 & 9122 & Solute carrier family 16 (monocarboxylic acid transporters), member 4 & 10,73 & chr1 \\
\hline SLC16A7 & 9194 & solute carrier family 16 (monocarboxylic acid transporters), member 7 & 4,54 & chr12 \\
\hline SLC17A5 & 26503 & solute carrier family 17 (anion/sugar transporter), member 5 & 7,10 & chr6 \\
\hline SLC18A2 & 6571 & Solute carrier family 18 (vesicular monoamine), member 2 & 2,49 & chr10 \\
\hline SLC1A4 & 6509 & solute carrier family 1 (glutamate/neutral amino acid transporter), member 4 & 23,28 & chr2 \\
\hline SLC20A2 & 6575 & solute carrier family 20 (phosphate transporter), member 2 & 2,33 & chr8 \\
\hline SLC22A18 & 5002 & solute carrier family 22 (organic cation transporter), member 18 & 4,89 & chr11 \\
\hline SLC22A4 & 6583 & solute carrier family 22 (organic cation transporter), member 4 & 2,24 & chr5 \\
\hline SLC25A16 & 8034 & Solute carrier family 25 (mitochondrial carrier; Graves disease autoantigen), $\mathrm{mb}$ & 4,55 & chr10 \\
\hline SLC25A20 & 788 & solute carrier family 25 (carnitine/acylcarnitine translocase), member 20 & 2,63 & chr3 \\
\hline SLC25A24 & 29957 & solute carrier family 25 (mitochondrial carrier; phosphate carrier), member 24 & 2,07 & chr1 \\
\hline SLC25A32 & 81034 & solute carrier family 25 , member 32 /// solute carrier family 25 , member 32 & 2,69 & chr8 \\
\hline SLC26A2 & 1836 & solute carrier family 26 (sulfate transporter), member 2 & 2,04 & chr5 \\
\hline SLC26A5 & 375611 & Solute carrier family 26 , member 5 (prestin) & 2,72 & chr7 \\
\hline SLC2A10 & 81031 & solute carrier family 2 (facilitated glucose transporter), member $10 / / /$ solute car & 5,13 & chr20 \\
\hline SLC30A5 & 64924 & solute carrier family 30 (zinc transporter), member 5 & 2,22 & chr5 \\
\hline SLC30A7 & 148867 & solute carrier family 30 (zinc transporter), member 7 & 4,96 & chr1 \\
\hline SLC31A2 & 1318 & solute carrier family 31 (copper transporters), member 2 & 3,06 & chr9 \\
\hline SLC35A3 & 23443 & solute carrier family 35 (UDP-N-acetylglucosamine (UDP-GlcNAc) transporter), & 2,31 & chr1 \\
\hline SLC35B2 & 347734 & solute carrier family 35, member B2 & 2,47 & chr6 \\
\hline SLC35B3 & 51000 & solute carrier family 35, member B3 & 4,68 & chr6 \\
\hline SLC35C1 & 55343 & solute carrier family 35, member $\mathrm{C} 1$ & 2,03 & chr11 \\
\hline SLC35D1 & 23169 & solute carrier family 35 (UDP-glucuronic acid/UDP-N-acetylgalactosamine dual & 6,07 & chr1 \\
\hline SLC35E1 & 79939 & solute carrier family 35, member $\mathrm{E} 1$ & 3,25 & chr19 \\
\hline SLC35F5 & 80255 & solute carrier family 35, member F5 & 4,00 & chr2 \\
\hline SLC39A 10 & 57181 & Solute carrier family 39 (zinc transporter), member 10 & 2,23 & chr2 \\
\hline SLC39A13 & 91252 & solute carrier family 39 (zinc transporter), member 13 & 2,82 & chr11 \\
\hline SLC39A6 & 25800 & solute carrier family 39 (zinc transporter), member 6 & 2,72 & chr18 \\
\hline SLC41A2 & 84102 & solute carrier family 41 , member 2 & 2,05 & chr12 \\
\hline SLC41A3 & 54946 & solute carrier family 41 , member 3 & 2,02 & chr3 \\
\hline SLC4A4 & 8671 & solute carrier family 4, sodium bicarbonate cotransporter, member 4 & 5,47 & chr4 \\
\hline SLC7A11 & 23657 & solute carrier family 7, (cationic amino acid transporter, $y+$ system) member 11 & 3,15 & chr4 \\
\hline SLC7A6 & 9057 & solute carrier family 7 (cationic amino acid transporter, $y+$ system), member 6 & 2,01 & chr16 \\
\hline SLC8A1 & 6546 & solute carrier family 8 (sodium/calcium exchanger), member 1 & 21,76 & chr2 \\
\hline SLIT3 & 6586 & slit homolog 3 (Drosophila) & 3,10 & chr5 \\
\hline SLITL2 & 114990 & slit-like 2 (Drosophila) & 3,55 & chr16 \\
\hline SMAD3 & 4088 & SMAD, mothers against DPP homolog 3 (Drosophila) & 9,44 & chr15 \\
\hline SMAP1L & 64744 & stromal membrane-associated protein 1 -like & 4,65 & chr1 \\
\hline SMARCA2 & 6595 & SWI/SNF related, matrix associated, actin dependent regulator of chromatin, s4 & 3,65 & chr9 \\
\hline SMARCD3 & 6604 & SWI/SNF related, matrix associated, actin dependent regulator of chromatin, s4 & 9,97 & chr7 \\
\hline SMBP & 56889 & SM-11044 binding protein & 2,39 & chr10 \\
\hline SMC5L1 & 23137 & SMC5 structural maintenance of chromosomes 5 -like 1 (yeast) & 2,42 & chr9 \\
\hline SMILE & 160418 & SMILE protein & 2,59 & chr12 \\
\hline SMPD1 & 6609 & sphingomyelin phosphodiesterase 1, acid lysosomal (acid sphingomyelinase) & 3,77 & chr11 \\
\hline SMPDL3A & 10924 & sphingomyelin phosphodiesterase, acid-like $3 \mathrm{~A}$ & 5,13 & chr6 \\
\hline SMURF2 & 64750 & SMAD specific E3 ubiquitin protein ligase 2 & 12,21 & chr17 \\
\hline SMYD2 & 56950 & SET and MYND domain containing 2 & 2,18 & chr1 \\
\hline SNAG1 & 112574 & Sorting nexin associated golgi protein 1 & 2,44 & chr5 \\
\hline SNAI2 & 6591 & snail homolog 2 (Drosophila) & 52,09 & chr8 \\
\hline SNF1LK & 150094 & SNF1-like kinase /// SNF1-like kinase & 4,50 & chr10 \\
\hline SNTB2 & 6645 & syntrophin, beta 2 (dystrophin-associated protein A1, 59kDa, basic component| & 2,26 & chr16 \\
\hline
\end{tabular}


Table S4 : Genes overexpressed in MPC compared to hES (Fold Change > 2; $\mathrm{a}<0.05$ )

\begin{tabular}{|c|c|c|c|c|}
\hline Gene Symbol & Entrez Gene & Gene Title & MPC_up & Chromosome Number(Avadis) \\
\hline SNX13 & 23161 & Sorting nexin 13 & 2,65 & chr7 \\
\hline SNX14 & 57231 & sorting nexin 14 & 2,58 & chr6 \\
\hline SNX19 & 399979 & sorting nexin 19 & 3,68 & chr11 \\
\hline SNX3 & 8724 & sorting nexin 3 & 3,05 & chr6 \\
\hline SNX9 & 51429 & sorting nexin 9 & 2,01 & chr6 \\
\hline SOAT1 & 6646 & sterol O-acyltransferase (acyl-Coenzyme A: cholesterol acyltransferase) 1 & 6,88 & chr1 \\
\hline socs3 & 9021 & suppressor of cytokine signaling 3 & 4,08 & chr17 \\
\hline socs5 & 9655 & suppressor of cytokine signaling 5 & 4,44 & chr2 \\
\hline SOD3 & 6649 & superoxide dismutase 3 , extracellular & 2,35 & chr4 \\
\hline SORBS2 & 8470 & sorbin and $\mathrm{SH} 3$ domain containing 2 & 4,04 & chr4 \\
\hline SOX9 & 6662 & SRY (sex determining region Y)-box 9 (campomelic dysplasia, autosomal sex-r & 2,60 & chr17 \\
\hline SP100 & 6672 & nuclear antigen Sp100 & 24,52 & chr2 \\
\hline SP110 & 3431 & SP110 nuclear body protein & 2,20 & chr2 \\
\hline SPAG9 & 9043 & sperm associated antigen 9 & 2,10 & chr17 \\
\hline SPARC & 6678 & secreted protein, acidic, cysteine-rich (osteonectin) & 42,54 & chr5 \\
\hline SPATA18 & 132671 & spermatogenesis associated 18 homolog (rat) & 7,26 & chr4 \\
\hline SPATA2O & 64847 & spermatogenesis associated 20 & 2,71 & chr17 \\
\hline SPATS2 & 65244 & spermatogenesis associated, serine-rich 2 & 3,85 & chr12 \\
\hline SPCS3 & 60559 & signal peptidase complex subunit 3 homolog (S. cerevisiae) & 2,77 & chr4 \\
\hline SPHK1 & 8877 & sphingosine kinase 1 & 23,77 & chr17 \\
\hline SPIRE1 & 56907 & spire homolog 1 (Drosophila) & 2,55 & chr18 \\
\hline SPOCD1 & 90853 & SPOC domain containing 1 & 39,33 & chr1 \\
\hline SPOCK & 6695 & sparc/osteonectin, cwcv and kazal-like domains proteoglycan (testican) & 6,42 & chr5 \\
\hline SPOP & 8405 & speckle-type POZ protein & 2,45 & chr17 \\
\hline SPSB1 & 80176 & spIA/ryanodine receptor domain and SOCS box containing 1 & 4,31 & chr1 \\
\hline SPTAN1 & 6709 & Spectrin, alpha, non-erythrocytic 1 (alpha-fodrin) /// CDNA FLJ44613 fis, clone & 5,43 & chr9 \\
\hline SPTBN1 & 6711 & Spectrin, beta, non-erythrocytic 1 & 25,86 & chr2 \\
\hline SQRDL & 58472 & sulfide quinone reductase-like (yeast) & 8,57 & chr15 \\
\hline SQSTM1 & 8878 & sequestosome 1 & 3,18 & chr5 \\
\hline SRA1 & 10011 & steroid receptor RNA activator 1 & 2,05 & chr5 \\
\hline SRGAP1 & 57522 & SLIT-ROBO Rho GTPase activating protein 1 & 3,81 & chr12 \\
\hline SRGAP2 & 23380 & SLIT-ROBO Rho GTPase activating protein 2 & 3,23 & chr1 \\
\hline SRPR & 6734 & signal recognition particle receptor ('docking protein') & 5,02 & chr11 \\
\hline SRPRB & 58477 & signal recognition particle receptor, B subunit & 2,52 & chr3 \\
\hline SRPX & 8406 & sushi-repeat-containing protein, $\mathrm{X}$-linked & 7,97 & $\operatorname{chrX}$ \\
\hline SRPX2 & 27286 & sushi-repeat-containing protein, X-linked 2 & 18,18 & $\operatorname{chrX}$ \\
\hline SRR & 63826 & serine racemase & 3,70 & chr17 \\
\hline SRXN1 & 140809 & sulfiredoxin 1 homolog (S. cerevisiae) & 2,09 & chr20 \\
\hline SSFA2 & 6744 & sperm specific antigen 2 & 29,01 & chr2 \\
\hline $\mathrm{SSH} 1$ & 54434 & slingshot homolog 1 (Drosophila) & 7,13 & chr12 \\
\hline SSPN & 8082 & sarcospan (Kras oncogene-associated gene) & 16,19 & chr12 \\
\hline SSR1 & 6745 & Signal sequence receptor, alpha (translocon-associated protein alpha) & 3,33 & chr6 \\
\hline SSR2 & 6746 & signal sequence receptor, beta (translocon-associated protein beta) & 2,83 & chr1 \\
\hline SSR3 & 6747 & signal sequence receptor, gamma (translocon-associated protein gamma) & 7,07 & chr3 \\
\hline ST3GAL1 & 6482 & ST3 beta-galactoside alpha-2,3-sialyttransferase 1 & 5,49 & chr8 \\
\hline ST3GAL3 & 6487 & ST3 beta-galactoside alpha-2,3-sialyttransferase 3 & 2,44 & chr1 \\
\hline ST3GAL5 & 8869 & ST3 beta-galactoside alpha-2,3-sialyttransferase 5 & 15,77 & chr2 \\
\hline ST5 & 6764 & suppression of tumorigenicity 5 & 2,16 & chr11 \\
\hline ST7 & 7982 & suppression of tumorigenicity 7 & 2,02 & chr7 \\
\hline STAM2 & 10254 & signal transducing adaptor molecule (SH3 domain and ITAM motif) 2 & 3,97 & chr2 \\
\hline STARD13 & 90627 & START domain containing 13 & 7,46 & chr13 \\
\hline STAT1 & 6772 & signal transducer and activator of transcription $1,91 \mathrm{kDa}$ & 2,12 & (vide) \\
\hline STAT2 & 6773 & signal transducer and activator of transcription $2,113 \mathrm{kDa}$ & 2,11 & chr12 \\
\hline STAT3 & 6774 & signal transducer and activator of transcription 3 (acute-phase response factor) & 2,17 & chr17 \\
\hline STAT6 & 6778 & signal transducer and activator of transcription 6 , interleukin-4 induced & 2,49 & chr12 \\
\hline STC2 & 8614 & stanniocalcin 2 & 6,38 & chr5 \\
\hline STCH & 6782 & stress 70 protein chaperone, microsome-associated, $60 \mathrm{kDa}$ & 2,70 & chr21 \\
\hline STK10 & 6793 & serine/threonine kinase 10 & 2,21 & chr5 \\
\hline STK17A & 9263 & Serine/threonine kinase 17a (apoptosis-inducing) & 6,55 & chr7 \\
\hline STK17B & 9262 & Basic leucine zipper and W2 domains 1 & 13,41 & chr2 \\
\hline STK32B & 55351 & serine/threonine kinase $32 \mathrm{~B}$ & 4,59 & chr4 \\
\hline STOML1 & 9399 & stomatin (EPB72)-like 1 & 3,40 & chr15 \\
\hline STS & 412 & steroid sulfatase (microsomal), arylsulfatase $\mathrm{C}$, isozyme $\mathrm{S}$ & 2,37 & $\operatorname{chrX}$ \\
\hline STS-1 & 84959 & Cbl-interacting protein Sts-1 & 13,09 & chr11 \\
\hline STX12 & 23673 & syntaxin 12 & 8,02 & chr1 \\
\hline STX5A & 6811 & syntaxin 5A & 2,81 & chr11 \\
\hline
\end{tabular}




\begin{tabular}{|c|c|c|c|c|}
\hline Gene Symbol & Entrez Gene & Gene Title & MPC_up & Chromosome Number(Avadis) \\
\hline STXBP1 & 6812 & syntaxin binding protein 1 & 2,79 & chr9 \\
\hline SULF1 & 23213 & sulfatase 1 & 89,16 & chr8 \\
\hline SUMF1 & 285362 & sulfatase modifying factor 1 & 2,32 & chr3 \\
\hline SUPT6H & 6830 & suppressor of Ty 6 homolog (S. cerevisiae) & 2,27 & chr17 \\
\hline SURF4 & 6836 & surfeit 4 & 2,15 & chr9 \\
\hline SUSD1 & 64420 & sushi domain containing 1 & 2,64 & chr9 \\
\hline SYBL1 & 6845 & synaptobrevin-like 1 & 2,90 & $\operatorname{chrX}$ \\
\hline SYDE1 & 85360 & synapse defective 1 , Rho GTPase, homolog 1 (C. elegans) & 3,99 & chr19 \\
\hline SYNE1 & 23345 & spectrin repeat containing, nuclear envelope 1 & 14,30 & chr6 \\
\hline SYNJ1 & 8867 & synaptojanin 1 & 2,29 & chr21 \\
\hline SYNJ2 & 8871 & synaptojanin 2 & 7,52 & chr6 \\
\hline SYNPO & 11346 & synaptopodin & 25,03 & chr5 \\
\hline SYNPO2 & 171024 & synaptopodin 2 & 32,72 & chr4 \\
\hline SYTL2 & 54843 & synaptotagmin-like 2 & 5,76 & chr11 \\
\hline SYTL4 & 94121 & Synaptotagmin-like 4 (granuphilin-a) & 3,38 & $\operatorname{chrX}$ \\
\hline TACC1 & 6867 & transforming, acidic coiled-coil containing protein 1 & 8,32 & chr8 \\
\hline TAF13 & 6884 & TAF13 RNA polymerase II, TATA box binding protein (TBP)-associated factor, & 2,18 & chr1 \\
\hline TAGLN & 6876 & |transgelin & 25,80 & chr11 \\
\hline TAGLN2 & 8407 & |transgelin 2 & 6,46 & chr1 \\
\hline TANC & 85461 & TPR domain, ankyrin-repeat and coiled-coil-containing & 9,37 & chr2 \\
\hline TANK & 10010 & TRAF family member-associated NFKB activator & 4,16 & chr2 \\
\hline TAPBP & 6892 & TAP binding protein (tapasin) & 2,27 & chr6 \\
\hline TAX1BP3 & 30851 & Tax1 (human T-cell leukemia virus type I) binding protein 3 & 3,35 & chr17 \\
\hline TBC1D10B & 26000 & TBC1 domain family, member 10B & 2,05 & chr16 \\
\hline TBC1D12 & 23232 & TBC1 domain family, member 12 & 4,02 & chr10 \\
\hline TBC1D19 & 55296 & TBC1 domain family, member 19 & 2,57 & chr4 \\
\hline TBC1D2 & 55357 & TBC1 domain family, member 2 & 3,50 & chr9 \\
\hline TBC1D20 & 128637 & TBC1 domain family, member 20 & 2,22 & chr20 \\
\hline TBL1X & 6907 & transducin (beta)-like 1X-linked & 2,04 & $\operatorname{chrX}$ \\
\hline TBL2 & 26608 & transducin (beta)-like 2 & 2,31 & chr7 \\
\hline TBX2 & 6909 & T-box 2 & 3,54 & chr17 \\
\hline TBX3 & 6926 & T-box 3 (ulnar mammary syndrome) & 5,02 & chr12 \\
\hline TCEA3 & 6920 & transcription elongation factor A (SII), 3 & 5,15 & chr1 \\
\hline TCEAL1 & 9338 & transcription elongation factor A (SII)-like 1 & 2,94 & $\operatorname{chrX}$ \\
\hline TCEAL3 & 85012 & transcription elongation factor A (SII)-like 3 & 3,89 & $\operatorname{chrX}$ \\
\hline TCEB1 & 6921 & transcription elongation factor B (SIII), polypeptide 1 (15kDa, elongin $\mathrm{C}$ ) & 2,02 & chr8 \\
\hline TCF8 & 6935 & transcription factor 8 (represses interleukin 2 expression) & 15,59 & chr10 \\
\hline TCIRG1 & 10312 & T-cell, immune regulator 1 , ATPase, $\mathrm{H}+$ transporting, lysosomal V0 protein a is & 2,21 & chr11 \\
\hline TCP11L1 & 55346 & t-complex 11 (mouse) like 1 & 3,21 & chr11 \\
\hline TCTA & 6988 & T-cell leukemia translocation altered gene & 2,52 & chr3 \\
\hline TCTE1L & 6990 & t-complex-associated-testis-expressed 1 -like & 4,95 & chr20 \\
\hline TCTE3 & 6991 & t-complex-associated-testis-expressed 3 & 2,17 & chr6 \\
\hline TDE1 & 10955 & tumor differentially expressed 1 & 3,61 & chr20 \\
\hline TEAD1 & 7003 & TEA domain family member 1 (SV40 transcriptional enhancer factor) & 2,79 & chr11 \\
\hline TERF2 & 7014 & telomeric repeat binding factor 2 & 2,51 & chr16 \\
\hline TERF2IP & 54386 & telomeric repeat binding factor 2 , interacting protein & 3,89 & chr16 \\
\hline TES & 26136 & testis derived transcript (3 LIM domains) & 4,38 & chr7 \\
\hline TESK1 & 7016 & testis-specific kinase 1 & 2,11 & chr9 \\
\hline TEX261 & 113419 & testis expressed sequence 261 & 3,48 & chr2 \\
\hline TFAP2A & 7020 & transcription factor AP-2 alpha (activating enhancer binding protein 2 alpha) & 16,14 & chr6 \\
\hline TFG & 10342 & TRK-fused gene & 2,05 & chr3 \\
\hline TFPI & 7035 & tissue factor pathway inhibitor (lipoprotein-associated coagulation inhibitor) & 3,89 & chr2 \\
\hline TGFB1 & 7040 & transforming growth factor, beta 1 (Camurati-Engelmann disease) & 2,33 & chr19 \\
\hline TGFB111 & 7041 & transforming growth factor beta 1 induced transcript 1 & 32,03 & chr16 \\
\hline TGFB2 & 7042 & Transforming growth factor, beta 2 & 86,91 & chr1 \\
\hline TGFBI & 7045 & transforming growth factor, beta-induced, $68 \mathrm{kDa}$ & 112,99 & chr5 \\
\hline TGFBR1 & 7046 & Transforming growth factor, beta receptor I (activin A receptor type II-like kinas & 2,27 & chr9 \\
\hline TGFBR2 & 7048 & transforming growth factor, beta receptor II $(70 / 80 \mathrm{kDa})$ & 11,19 & chr3 \\
\hline TGM2 & 7052 & transglutaminase 2 (C polypeptide, protein-glutamine-gamma-glutamyltransferd & 24,74 & chr20 \\
\hline TGOLN2 & 10618 & trans-golgi network protein 2 & 4,37 & chr2 \\
\hline THBD & 7056 & thrombomodulin & 6,10 & chr20 \\
\hline THBS1 & 7057 & thrombospondin 1 & 76,57 & chr15 \\
\hline THBS2 & 7058 & thrombospondin 2 & 22,66 & chr6 \\
\hline THBS3 & 7059 & thrombospondin 3 & 4,99 & chr1 \\
\hline THRAP2 & 23389 & Thyroid hormone receptor associated protein 2 & 7,06 & chr12 \\
\hline |THSD4 & 79875 & Thrombospondin, type I, domain containing 4 & 4,83 & chr15 \\
\hline
\end{tabular}


Table S4 : Genes overexpressed in MPC compared to hES (Fold Change > 2; $a<0.05$ )

\begin{tabular}{|c|c|c|c|c|}
\hline Gene Symbol & Entrez Gene & Gene Title & MPC_up & Chromosome Number(Avadis) \\
\hline TICAM2 & 353376 & toll-like receptor adaptor molecule 2 & 8,89 & chr5 \\
\hline TIFA & 92610 & TRAF-interacting protein with a forkhead-associated domain & 5,27 & chr4 \\
\hline TIMM17A & 10440 & translocase of inner mitochondrial membrane 17 homolog A (yeast) & 2,14 & chr1 \\
\hline TIMP1 & 7076 & TIMP metallopeptidase inhibitor 1 & 6,85 & $\operatorname{chrX}$ \\
\hline TIMP2 & 7077 & TIMP metallopeptidase inhibitor 2 & 14,70 & chr17 \\
\hline TIMP3 & 7078 & TIMP metallopeptidase inhibitor 3 (Sorsby fundus dystrophy, pseudoinflammatd & 52,32 & chr22 \\
\hline TIPARP & 25976 & TCDD-inducible poly(ADP-ribose) polymerase & 10,37 & chr3 \\
\hline TIPRL & 261726 & TIP41, TOR signalling pathway regulator-like (S. cerevisiae) & 3,33 & chr1 \\
\hline TK2 & 7084 & thymidine kinase 2 , mitochondrial & 5,02 & chr16 \\
\hline TLOC1 & 7095 & translocation protein 1 & 3,35 & chr3 \\
\hline TLR4 & 7099 & toll-like receptor 4 /// toll-like receptor 4 & 4,58 & chr9 \\
\hline TM2D1 & 83941 & TM2 domain containing 1 & 3,12 & chr1 \\
\hline TM4SF1 & 4071 & transmembrane $4 \mathrm{~L}$ six family member 1 & 48,84 & chr3 \\
\hline TM7SF1 & 7107 & transmembrane 7 superfamily member 1 (upregulated in kidney) & 2,27 & chr1 \\
\hline TM9SF1 & 10548 & transmembrane 9 superfamily member 1 & 2,73 & chr14 \\
\hline TMBIM1 & 64114 & transmembrane BAX inhibitor motif containing 1 & 2,78 & chr2 \\
\hline TMCO1 & 54499 & transmembrane and coiled-coil domains 1 & 2,37 & chr1 \\
\hline TMCO3 & 55002 & transmembrane and coiled-coil domains 3 & 4,78 & chr13 \\
\hline TMED3 & 23423 & transmembrane emp24 protein transport domain containing 3 & 2,28 & chr15 \\
\hline TMED4 & 222068 & transmembrane emp24 protein transport domain containing 4 & 2,57 & chr7 \\
\hline TMED5 & 50999 & transmembrane emp24 protein transport domain containing 5 & 2,16 & chr1 \\
\hline TMED7 & 51014 & transmembrane emp24 protein transport domain containing 7 & 2,44 & chr5 \\
\hline TMEM14A & 28978 & transmembrane protein 14A & 2,43 & chr6 \\
\hline TMEM16D & 121601 & transmembrane protein 16D & 2,46 & chr12 \\
\hline TMEM16F & 196527 & transmembrane protein $16 \mathrm{~F}$ & 2,61 & chr12 \\
\hline TMEM17 & 200728 & transmembrane protein 17 & 2,04 & chr2 \\
\hline TMEMЗОА & 55754 & transmembrane protein $30 \mathrm{~A}$ & 7,85 & chr6 \\
\hline TMEM43 & 79188 & transmembrane protein 43 & 2,80 & chr3 \\
\hline TMEM45A & 55076 & transmembrane protein $45 \mathrm{~A}$ & 2,67 & chr3 \\
\hline TMEM47 & 83604 & transmembrane protein 47 & 2,45 & $\operatorname{chrX}$ \\
\hline TMEM49 & 81671 & transmembrane protein 49 & 4,57 & chr17 \\
\hline TMEM5 & 10329 & transmembrane protein 5 & 4,10 & chr12 \\
\hline TMEM50B & 757 & transmembrane protein 50B & 4,91 & chr21 \\
\hline TMEM55A & 55529 & transmembrane protein 55A & 2,21 & chr8 \\
\hline TMEM65 & 157378 & transmembrane protein 65 & 3,26 & chr8 \\
\hline TMEM76 & 138050 & transmembrane protein 76 & 10,31 & chr8 \\
\hline TMEM87B & 84910 & Transmembrane protein 87B & 4,46 & chr2 \\
\hline TMEM9B & 56674 & TMEM9 domain family, member B & 2,23 & chr11 \\
\hline TMF1 & 7110 & TATA element modulatory factor 1 & 2,82 & chr3 \\
\hline TMOD3 & 29766 & tropomodulin 3 (ubiquitous) & 3,17 & chr15 \\
\hline TMSB10 & 9168 & thymosin, beta 10 & 2,57 & chr2 \\
\hline TNC & 3371 & tenascin C (hexabrachion) & 56,43 & chr9 \\
\hline TncRNA & 283131 & trophoblast-derived noncoding RNA & 16,78 & chr11 \\
\hline TNFAIP1 & 7126 & tumor necrosis factor, alpha-induced protein 1 (endothelial) & 7,01 & chr17 \\
\hline TNFAIP3 & 7128 & tumor necrosis factor, alpha-induced protein 3 & 6,10 & chr6 \\
\hline TNFAIP6 & 7130 & tumor necrosis factor, alpha-induced protein 6 & 2,49 & chr2 \\
\hline TNFRSF10B & 8795 & tumor necrosis factor receptor superfamily, member $10 \mathrm{~b}$ & 4,42 & chr8 \\
\hline TNFRSF10D & 8793 & tumor necrosis factor receptor superfamily, member $10 \mathrm{~d}$, decoy with truncated & 17,91 & chr8 \\
\hline TNFRSF1A & 7132 & tumor necrosis factor receptor superfamily, member $1 \mathrm{~A}$ & 6,21 & chr12 \\
\hline TNFSF4 & 7292 & tumor necrosis factor (ligand) superfamily, member 4 (tax-transcriptionally activ & 8,61 & chr1 \\
\hline TNIP1 & 10318 & TNFAIP3 interacting protein 1 & 2,96 & chr5 \\
\hline TNS1 & 7145 & tensin $1 / / /$ tensin 1 & 72,39 & chr2 \\
\hline TNS3 & 64759 & Tensin 3 & 4,74 & chr7 \\
\hline TOR1AIP2 & 163590 & Torsin A interacting protein 2 & 2,79 & chr1 \\
\hline TP53INP1 & 94241 & tumor protein p53 inducible nuclear protein 1 & 2,62 & chr8 \\
\hline TP53INP2 & 58476 & tumor protein p53 inducible nuclear protein 2 & 2,64 & chr20 \\
\hline TPCN1 & 53373 & two pore segment channel 1 & 2,75 & chr12 \\
\hline TPM1 & 7168 & Tropomyosin 1 (alpha) & 7,72 & chr15 \\
\hline TPM2 & 7169 & tropomyosin 2 (beta) & 7,37 & chr9 \\
\hline TPM3 & $7170 / / / 7171$ & tropomyosin $3 / / /$ tropomyosin 4 & 7,84 & chr3 \\
\hline TPM4 & 7171 & tropomyosin 4 & 6,47 & chr3 \\
\hline TPST1 & 8460 & tyrosylprotein sulfotransferase 1 & 3,35 & chr7 \\
\hline TRA1 & 7184 & tumor rejection antigen (gp96) 1 & 2,05 & (vide) \\
\hline TRADD & 8717 & TNFRSF1A-associated via death domain & 4,41 & chr16 \\
\hline TRAF3 & 7187 & TNF receptor-associated factor 3 & 3,00 & chr14 \\
\hline TRAM1 & 23471 & translocation associated membrane protein 1 & 2,74 & chr8 \\
\hline
\end{tabular}




\begin{tabular}{|c|c|c|c|c|}
\hline Gene Symbol & Entrez Gene & Gene Title & MPC_up & Chromosome Number(Avadis) \\
\hline TRAM2 & 9697 & translocation associated membrane protein 2 & 13,77 & chr6 \\
\hline TRAPPC1 & 58485 & |trafficking protein particle complex 1 & 3,18 & chr17 \\
\hline TRAPPC3 & 27095 & trafficking protein particle complex 3 & 2,12 & chr1 \\
\hline TRHDE & 29953 & thyrotropin-releasing hormone degrading enzyme & 10,58 & chr12 \\
\hline TRIB3 & 57761 & tribbles homolog 3 (Drosophila) & 5,27 & chr20 \\
\hline TRIM16 & $10626 / / / 147166$ & |tripartite motif-containing $16 / / /$ similar to tripartite motif-containing 16 ; estrogen- & 6,36 & chr17 \\
\hline TRIM22 & 10346 & |tripartite motif-containing 22 & 5,53 & chr11 \\
\hline TRIM34 & $445372 / / / 53840$ & tripartite motif-containing 34 /// tripartite motif-containing 6 and tripartite motif-co & 2,06 & chr11 \\
\hline TRIM44 & 54765 & |tripartite motif-containing 44 & 2,29 & chr11 \\
\hline TRIM5 & 85363 & tripartite motif-containing 5 & 2,89 & chr11 \\
\hline TRIM50B & 375593 & tripartite motif-containing 50B & 2,24 & chr7 \\
\hline TRIM56 & 81844 & Tripartite motif-containing 56 & 2,32 & chr7 \\
\hline TRIM58 & 25893 & |tripartite motif-containing 58 & 4,25 & chr1 \\
\hline TRIM62 & 55223 & |tripartite motif-containing 62 & 2,54 & chr1 \\
\hline TRIM8 & 81603 & tripartite motif-containing $8 / / /$ tripartite motif-containing 8 & 3,71 & chr10 \\
\hline TRIO & 7204 & triple functional domain (PTPRF interacting) & 3,18 & chr5 \\
\hline TRIOBP & 11078 & TRIO and F-actin binding protein & 2,09 & chr22 \\
\hline TRIP11 & 9321 & thyroid hormone receptor interactor 11 & 2,29 & chr14 \\
\hline TRPC4 & 7223 & transient receptor potential cation channel, subfamily C, member 4 & 3,31 & chr13 \\
\hline |TRPS1 & 7227 & trichorhinophalangeal syndrome I & 4,44 & chr8 \\
\hline TSC22D2 & 9819 & TSC22 domain family, member 2 & 2,33 & chr3 \\
\hline TSPAN10 & 83882 & tetraspanin 10 & 2,35 & chr17 \\
\hline TSPAN31 & 6302 & tetraspanin 31 & 2,16 & chr12 \\
\hline TSPAN5 & 10098 & tetraspanin $5 / / /$ tetraspanin 5 & 4,10 & chr4 \\
\hline TTC3 & 7267 & tetratricopeptide repeat domain 3 & 26,78 & chr21 \\
\hline TTC8 & 123016 & tetratricopeptide repeat domain 8 & 2,88 & chr14 \\
\hline TTYH2 & 94015 & tweety homolog 2 (Drosophila) & 2,18 & chr17 \\
\hline TTYH3 & 80727 & tweety homolog 3 (Drosophila) & 2,65 & chr7 \\
\hline TUBA3 & 7846 & tubulin, alpha 3 & 2,00 & chr12 \\
\hline TUFT1 & 7286 & tuftelin 1 & 4,04 & chr1 \\
\hline TULP3 & 7289 & tubby like protein 3 & 2,11 & chr12 \\
\hline TWIST1 & 7291 & twist homolog 1 (acrocephalosyndactyly 3; Saethre-Chotzen syndrome) (Droso & 10,84 & chr7 \\
\hline TWSG1 & 57045 & twisted gastrulation homolog 1 (Drosophila) & 3,64 & chr18 \\
\hline TXNDC10 & 54495 & thioredoxin domain containing 10 & 3,71 & chr18 \\
\hline TXNDC11 & 51061 & thioredoxin domain containing 11 & 3,94 & chr16 \\
\hline TXNDC13 & 56255 & thioredoxin domain containing 13 & 2,65 & chr20 \\
\hline TXNIP & 10628 & thioredoxin interacting protein & 11,94 & chr1 \\
\hline TXNRD1 & 7296 & thioredoxin reductase 1 & 3,06 & chr12 \\
\hline TXNRD3 & 114112 & thioredoxin reductase 3 & 2,06 & chr3 \\
\hline UACA & 55075 & uveal autoantigen with coiled-coil domains and ankyrin repeats & 5,87 & chr15 \\
\hline UAP1 & 6675 & UDP-N-acteylglucosamine pyrophosphorylase 1 & 5,83 & chr1 \\
\hline UBE2B & 7320 & ubiquitin-conjugating enzyme E2B (RAD6 homolog) /// ubiquitin-conjugating en & 2,59 & chr5 \\
\hline UBE2H & 7328 & Ubiquitin-conjugating enzyme E2H (UBC8 homolog, yeast) & 4,72 & chr7 \\
\hline UBE2J1 & 51465 & ubiquitin-conjugating enzyme E2, J1 (UBC6 homolog, yeast) & 10,53 & chr6 \\
\hline UBE2Q2 & 92912 & ubiquitin-conjugating enzyme $\mathrm{E} 2 \mathrm{Q}$ (putative) 2 & 2,76 & chr15 \\
\hline UBE2W & 55284 & ubiquitin-conjugating enzyme E2W (putative) & 2,32 & chr8 \\
\hline UBL3 & 5412 & ubiquitin-like 3 & 2,60 & chr13 \\
\hline UBXD1 & 80700 & UBX domain containing 1 & 3,08 & chr19 \\
\hline UEV3 & 55293 & Ubiquitin-conjugating enzyme E2-like & 8,22 & chr11 \\
\hline UFM1 & 51569 & ubiquitin-fold modifier 1 & 3,62 & chr13 \\
\hline UGCG & 7357 & UDP-glucose ceramide glucosyltransferase & 4,24 & chr9 \\
\hline UGCGL1 & 56886 & UDP-glucose ceramide glucosyltransferase-like 1 & 2,14 & chr2 \\
\hline UGCGL2 & 55757 & UDP-glucose ceramide glucosyltransferase-like 2 & 4,64 & chr13 \\
\hline UHMK1 & 127933 & U2AF homology motif (UHM) kinase 1 & 6,04 & chr1 \\
\hline ULBP2 & 80328 & UL16 binding protein 2 & 24,08 & chr6 \\
\hline ULK2 & 9706 & unc-51-like kinase 2 (C. elegans) & 2,16 & chr17 \\
\hline UNC84B & 25777 & unc-84 homolog B (C. elegans) & 2,72 & chr22 \\
\hline |UNQ1912 & 345757 & HGS_RE408 & 5,13 & chr5 \\
\hline URB & 151887 & steroid sensitive gene 1 & 147,43 & chr3 \\
\hline UROS & 7390 & uroporphyrinogen III synthase (congenital erythropoietic porphyria) & 2,02 & chr10 \\
\hline USP15 & 9958 & ubiquitin specific peptidase 15 & 2,63 & chr12 \\
\hline USP3 & 9960 & ubiquitin specific peptidase 3 & 8,33 & chr15 \\
\hline USP30 & 84749 & Ubiquitin specific peptidase 30 & 2,13 & chr12 \\
\hline USP38 & 84640 & ubiquitin specific peptidase 38 & 2,19 & chr4 \\
\hline USP40 & 55230 & ubiquitin specific peptidase 40 & 2,38 & chr2 \\
\hline |USP47 & 55031 & |ubiquitin specific peptidase 47 & 2,44 & chr11 \\
\hline
\end{tabular}


Table S4 : Genes overexpressed in MPC compared to hES (Fold Change $>2 ; \mathrm{a}<0.05$ )

\begin{tabular}{|c|c|c|c|c|}
\hline Gene Symbol & Entrez Gene & Gene Title & MPC_up & Chromosome Number(Avadis) \\
\hline USP53 & 54532 & ubiquitin specific peptidase 53 & 3,74 & chr4 \\
\hline VAMP3 & 9341 & vesicle-associated membrane protein 3 (cellubrevin) /// vesicle-associated men & 3,14 & chr1 \\
\hline VAMP4 & 8674 & vesicle-associated membrane protein 4 & 2,99 & chr1 \\
\hline VCAM1 1 & 7412 & vascular cell adhesion molecule 1 & 18,34 & chr1 \\
\hline VCPIP1 & 80124 & Valosin containing protein (p97)/p47 complex interacting protein 1 & 2,83 & chr8 \\
\hline VDR & 7421 & vitamin $\mathrm{D}(1,25$ - dihydroxyvitamin D3) receptor & 3,81 & chr12 \\
\hline VEGF & 7422 & vascular endothelial growth factor & 3,89 & chr6 \\
\hline VEGFC & 7424 & vascular endothelial growth factor $\mathrm{C}$ & 28,06 & chr4 \\
\hline VEPH1 & 79674 & ventricular zone expressed PH domain homolog 1 (zebrafish) & 16,65 & chr3 \\
\hline VGCNL1 & 259232 & Voltage gated channel like 1 & 6,11 & chr13 \\
\hline VGL-3 & 389136 & vestigial-like 3 & 26,25 & chr3 \\
\hline VIM & 7431 & vimentin & 4,65 & chr10 \\
\hline VPS13B & 157680 & vacuolar protein sorting 13B (yeast) & 2,03 & chr8 \\
\hline VPS13C & 54832 & Vacuolar protein sorting $13 \mathrm{C}$ (yeast) & 2,20 & chr15 \\
\hline VPS24 & 51652 & vacuolar protein sorting 24 (yeast) & 5,78 & chr2 \\
\hline WARS & 7453 & tryptophanyl-tRNA synthetase & 2,30 & chr14 \\
\hline WASPIP & 7456 & Wiskott-Aldrich syndrome protein interacting protein & 12,00 & chr2 \\
\hline WBP5 & 51186 & WW domain binding protein 5 & 2,61 & $\operatorname{chrX}$ \\
\hline WDR1 & 9948 & WD repeat domain 1 & 2,33 & chr4 \\
\hline WDR13 & 64743 & WD repeat domain 13 & 3,13 & $\operatorname{chr} X$ \\
\hline WDR26 & 80232 & WD repeat domain 26 & 3,10 & chr1 \\
\hline WDR32 & 79269 & WD repeat domain 32 & 3,48 & chr9 \\
\hline WDR41 & 55255 & WD repeat domain 41 & 3,20 & chr5 \\
\hline WDR47 & 22911 & WD repeat domain 47 & 4,32 & chr1 \\
\hline WDR5B & 54554 & WD repeat domain $5 B$ & 2,58 & chr3 \\
\hline WDR68 & 10238 & WD repeat domain 68 & 3,46 & chr17 \\
\hline WHDC1L2 & 440253 & WAS protein homology region 2 domain containing 1 -like 1 & 2,46 & chr15 \\
\hline WIG1 & 64393 & p53 target zinc finger protein & 14,99 & chr3 \\
\hline WIPI49 & 55062 & WD40 repeat protein Interacting with phospholnositides of $49 \mathrm{kDa}$ & 9,13 & chr17 \\
\hline WNT5B & 81029 & wingless-type MMTV integration site family, member $5 \mathrm{~B} / / /$ wingless-type MMTV & 11,48 & chr12 \\
\hline WSB2 & 55884 & WD repeat and SOCS box-containing 2 & 2,97 & chr12 \\
\hline WWTR1 & 25937 & WW domain containing transcription regulator 1 & 6,13 & chr3 \\
\hline XPA & 7507 & xeroderma pigmentosum, complementation group $\mathrm{A}$ & 2,26 & chr9 \\
\hline XRN1 & 54464 & 5'-3' exoribonuclease 1 & 2,40 & chr3 \\
\hline YIF1A & 10897 & Yip1 interacting factor homolog A (S. cerevisiae) & 2,35 & chr11 \\
\hline YIPF3 & 25844 & Yip1 domain family, member 3 & 2,44 & chr6 \\
\hline YIPF4 & 84272 & Yip1 domain family, member 4 & 3,37 & chr2 \\
\hline YIPF5 & 81555 & Yip1 domain family, member 5 & 8,82 & chr5 \\
\hline YKT6 & 10652 & SNARE protein Ykt6 & 2,31 & chr7 \\
\hline YME1L1 & 10730 & YME1-like 1 (S. cerevisiae) & 2,11 & chr10 \\
\hline YPEL2 & 388403 & yippee-like 2 (Drosophila) & 3,21 & chr17 \\
\hline YPEL5 & 51646 & yippee-like 5 (Drosophila) & 5,35 & chr2 \\
\hline YSG2 & 54414 & Ysg2 homolog (mouse) /// Ysg2 homolog (mouse) & 6,08 & chr11 \\
\hline YWHAZ & 7534 & Tyrosine 3-monooxygenase/tryptophan 5-monooxygenase activation protein, z| & 2,63 & chr8 \\
\hline ZADH2 & 284273 & zinc binding alcohol dehydrogenase, domain containing 2 & 9,43 & chr18 \\
\hline ZAK & 51776 & sterile alpha motif and leucine zipper containing kinase AZK & 24,60 & chr2 \\
\hline ZBTB1 & 22890 & zinc finger and BTB domain containing 1 & 3,05 & chr14 \\
\hline ZBTB20 & 26137 & zinc finger and BTB domain containing 20 & 4,00 & chr3 \\
\hline ZBTB38 & 253461 & zinc finger and BTB domain containing 38 & 4,45 & chr3 \\
\hline ZBTB4 & 57659 & zinc finger and BTB domain containing 4 & 3,29 & chr17 \\
\hline ZBTB41 & 360023 & zinc finger and BTB domain containing 41 & 4,03 & chr1 \\
\hline $\mathrm{ZC} 3 \mathrm{H} 11 \mathrm{~A}$ & 9877 & zinc finger $\mathrm{CCCH}$-type containing $11 \mathrm{~A}$ & 8,18 & chr1 \\
\hline $\mathrm{ZC} 3 \mathrm{H} 7 \mathrm{~A}$ & 29066 & zinc finger $\mathrm{CCCH}$-type containing 7A & 2,09 & chr16 \\
\hline ZCSL2 & 285381 & zinc finger, CSL-type containing 2 & 3,05 & chr3 \\
\hline ZDHHC24 & 254359 & zinc finger, DHHC-type containing 24 & 2,06 & chr19 \\
\hline ZDHHC9 & 51114 & Zinc finger, DHHC-type containing 9 & 2,07 & $\operatorname{chrX}$ \\
\hline ZFHX1B & 9839 & zinc finger homeobox $1 \mathrm{~b}$ & 7,83 & chr2 \\
\hline ZFHX4 & 79776 & zinc finger homeodomain 4 & 3,04 & chr8 \\
\hline ZFP91 & 80829 & zinc finger protein 91 homolog (mouse) & 2,38 & chr11 \\
\hline ZFPL1 & 7542 & zinc finger protein-like 1 & 4,15 & chr11 \\
\hline ZFPM2 & 23414 & zinc finger protein, multitype 2 & 7,09 & chr8 \\
\hline ZHX1 & 11244 & zinc fingers and homeoboxes 1 & 5,41 & chr8 \\
\hline ZHX3 & 23051 & zinc fingers and homeoboxes 3 & 2,66 & chr20 \\
\hline ZKSCAN1 & 7586 & Zinc finger with KRAB and SCAN domains 1 & 5,07 & chr7 \\
\hline ZMYM6 & 9204 & zinc finger, MYM-type 6 & 2,79 & chr1 \\
\hline ZMYND11 & 10771 & zinc finger, MYND domain containing 11 & 2,49 & chr10 \\
\hline
\end{tabular}




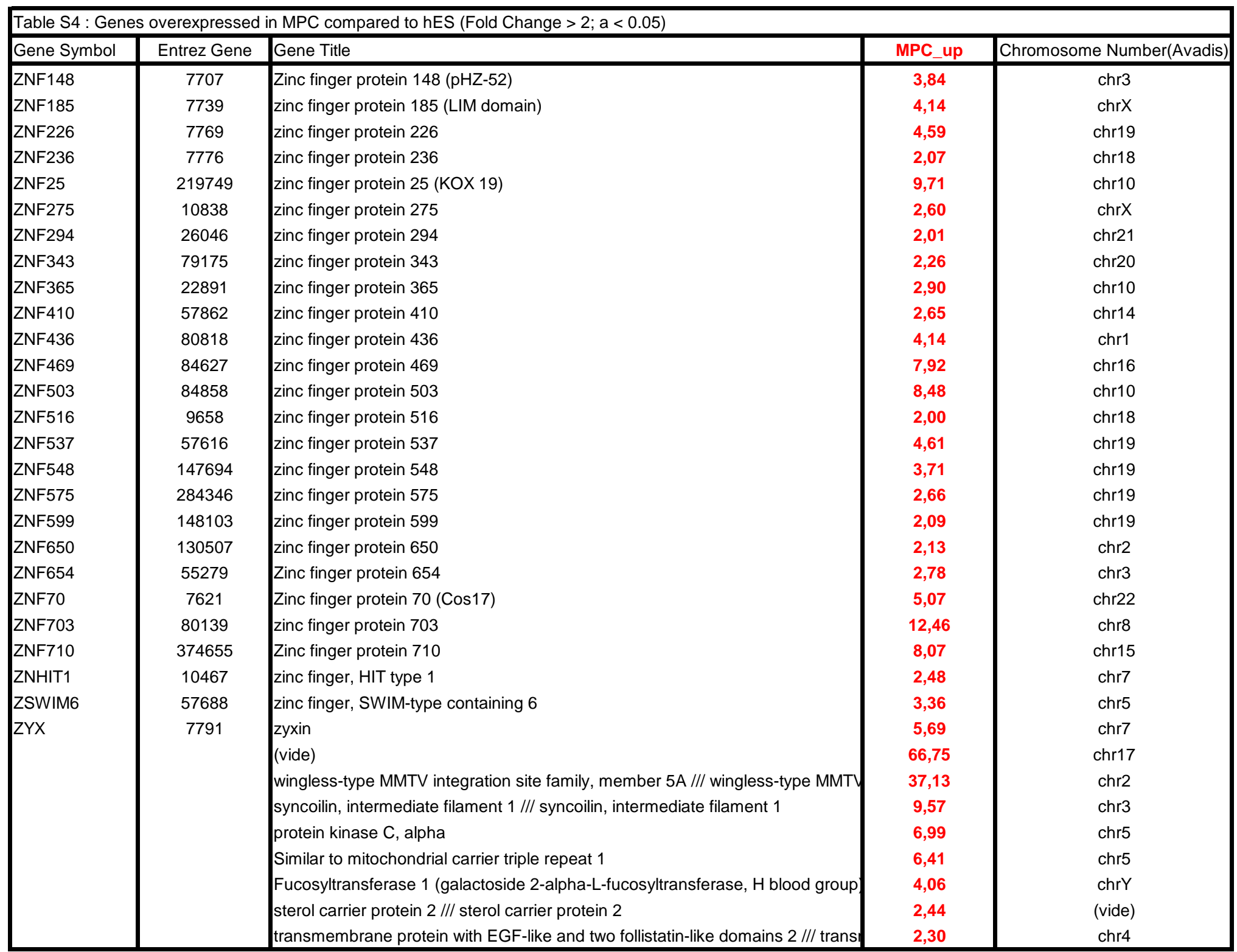




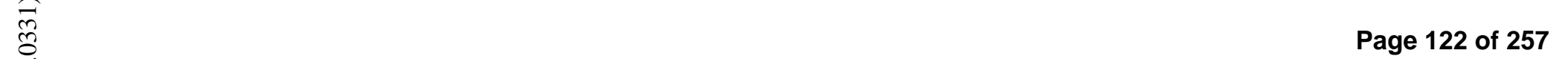

\begin{tabular}{|c|c|c|c|c|}
\hline Gene Symbol & Entrez Gene & Gene Title & MPC_down & Chromosome Number(Avadis) \\
\hline 37316 & 92935 & methionine-tRNA synthetase 2 (mitochondrial) & 2,93 & chr2 \\
\hline 37865 & 55964 & septin 3 & 4,36 & chr22 \\
\hline 38961 & 23157 & septin 6 & 2,72 & $\operatorname{chr} X$ \\
\hline AACS & 65985 & acetoacetyl-CoA synthetase & 2,48 & chr12 \\
\hline AAMP & 14 & angio-associated, migratory cell protein & 3,76 & chr2 \\
\hline AARS & 16 & alanyl-tRNA synthetase & 2,10 & chr16 \\
\hline AARSL & 57505 & alanyl-tRNA synthetase like & 2,64 & chr6 \\
\hline AASDH & 132949 & 2-aminoadipic 6-semialdehyde dehydrogenase & 2,21 & chr4 \\
\hline AASS & 10157 & aminoadipate-semialdehyde synthase & 49,82 & chr7 \\
\hline AATF & 26574 & apoptosis antagonizing transcription factor & 2,30 & chr17 \\
\hline ABCB6 & 10058 & ATP-binding cassette, sub-family B (MDR/TAP), member 6 & 2,71 & chr2 \\
\hline ABCB7 & 22 & ATP-binding cassette, sub-family B (MDR/TAP), member 7 & 4,48 & $\operatorname{chr} X$ \\
\hline ABCC5 & 10057 & ATP-binding cassette, sub-family C (CFTR/MRP), member 5 & 2,18 & chr3 \\
\hline$A B C D 4$ & 5826 & ATP-binding cassette, sub-family D (ALD), member 4 & 3,01 & $\operatorname{chr} 14$ \\
\hline ABCF1 & 23 & ATP-binding cassette, sub-family F (GCN20), member 1 /// ATP-binding cassettd & 2,00 & chr6 \\
\hline ABCG2 & 9429 & ATP-binding cassette, sub-family G (WHITE), member 2 & 2,81 & chr4 \\
\hline ABHD11 & 83451 & abhydrolase domain containing 11 & 2,24 & chr7 \\
\hline ABHD5 & 51099 & abhydrolase domain containing 5 & 2,25 & chr3 \\
\hline ABHD9 & 79852 & abhydrolase domain containing 9 & 8,16 & chr19 \\
\hline ABLIM1 & 3983 & actin binding LIM protein 1 & 2,41 & chr10 \\
\hline ABT1 & 29777 & activator of basal transcription 1 & 2,77 & chr6 \\
\hline ACAA2 & 10449 & acetyl-Coenzyme A acyltransferase 2 (mitochondrial 3-oxoacyl-Coenzyme A thid & 3,35 & chr18 \\
\hline ACACA & 31 & acetyl-Coenzyme A carboxylase alpha & 2,97 & chr17 \\
\hline ACACB & 32 & acetyl-Coenzyme A carboxylase beta & 5,86 & chr12 \\
\hline ACAD8 & 27034 & acyl-Coenzyme A dehydrogenase family, member 8 & 3,81 & chr11 \\
\hline ACADSB & 36 & acyl-Coenzyme A dehydrogenase, short/branched chain & 2,51 & chr10 \\
\hline АСАТ2 & 39 & acetyl-Coenzyme A acetyltransferase 2 (acetoacetyl Coenzyme A thiolase) & 5,22 & chr6 \\
\hline ACIN1 & 22985 & Apoptotic chromatin condensation inducer 1 & 3,16 & chr14 \\
\hline ACOT7 & 11332 & acyl-CoA thioesterase 7 & 2,12 & chr1 \\
\hline АСОТв & 10005 & Acyl-CoA thioesterase 8 & 2,29 & chr20 \\
\hline ACP1 & 52 & acid phosphatase 1 , soluble & 2,44 & chr2 \\
\hline ACP6 & 51205 & Acid phosphatase 6, lysophosphatidic & 2,60 & chr1 \\
\hline ACPL2 & 92370 & acid phosphatase-like 2 & 3,91 & chr3 \\
\hline ACTA1 & 58 & actin, alpha 1 , skeletal muscle & 5,03 & chr1 \\
\hline ACTN3 & 89 & actinin, alpha 3 & 4,03 & chr11 \\
\hline ACTR3B & 57180 & ARP3 actin-related protein 3 homolog B (yeast) & 4,29 & chr7 \\
\hline ACTR5 & 79913 & ARP5 actin-related protein 5 homolog (yeast) & 4,14 & chr20 \\
\hline ACVR2B & 93 & activin A receptor, type IIIB & 19,71 & chr3 \\
\hline ACY1 & 95 & aminoacylase 1 & 2,85 & chr3 \\
\hline ACYP1 & 97 & acylphosphatase 1 , erythrocyte (common) type & 2,22 & chr14 \\
\hline AD031 & 83935 & AD031 protein & 2,20 & chr11 \\
\hline ADAMTS19 & 171019 & ADAM metallopeptidase with thrombospondin type 1 motif, 19 & 6,19 & chr5 \\
\hline ADAMTS8 & 11095 & ADAM metallopeptidase with thrombospondin type 1 motif, 8 & 9,00 & chr11 \\
\hline ADCK1 & 57143 & aarF domain containing kinase 1 & 2,21 & chr14 \\
\hline ADCY1 & 107 & adenylate cyclase 1 (brain) & 6,53 & chr7 \\
\hline ADCY2 & 108 & adenylate cyclase 2 (brain) & 45,84 & chr5 \\
\hline ADCY7 & 113 & adenylate cyclase 7 & 2,39 & chr16 \\
\hline ADD2 & 119 & adducin 2 (beta) & 35,17 & chr2 \\
\hline ADFP & 123 & adipose differentiation-related protein & 2,01 & chr9 \\
\hline ADNP & 23394 & Activity-dependent neuroprotector & 6,89 & chr20 \\
\hline ADPN & 80339 & adiponutrin & 2,91 & chr22 \\
\hline ADRA2A & 150 & adrenergic, alpha-2A-, receptor /// adrenergic, alpha-2A-, receptor & 3,18 & chr10 \\
\hline ADRBK2 & 157 & adrenergic, beta, receptor kinase 2 & 17,21 & chr22 \\
\hline ADSL & 158 & adenylosuccinate lyase & 3,47 & chr22 \\
\hline AFF1 & 4299 & AF4/FMR2 family, member 1 & 3,80 & chr4 \\
\hline AFG3L1 & 172 & AFG3 ATPase family gene 3 -like 1 (yeast) & 3,66 & chr16 \\
\hline AFG3L2 & 10939 & AFG3 ATPase family gene 3 -like 2 (yeast) & 4,72 & chr18 \\
\hline AGPAT4 & 56895 & 1-acylglycerol-3-phosphate O-acyltransferase 4 (lysophosphatidic acid acyltrans & 2,37 & chr6 \\
\hline AGPAT5 & 55326 & 1-acylglycerol-3-phosphate O-acyltransferase 5 (lysophosphatidic acid acyltrans & 5,10 & chr8 \\
\hline AGTPBP1 & 23287 & ATP/GTP binding protein 1 & 5,09 & chr9 \\
\hline AGTRAP & 57085 & angiotensin II receptor-associated protein & 3,41 & chr1 \\
\hline AHCY & 191 & S-adenosylhomocysteine hydrolase & 3,12 & $\operatorname{chr} 20$ \\
\hline AHSA1 & 10598 & AHA1, activator of heat shock 90kDa protein ATPase homolog 1 (yeast) & 2,20 & chr14 \\
\hline AIM1 & 202 & absent in melanoma 1 & 7,08 & chr6 \\
\hline AIM1L & 55057 & absent in melanoma 1 -like & 4,73 & chr1 \\
\hline AK2 & 204 & adenylate kinase 2 & 2,17 & chr1 \\
\hline
\end{tabular}




\section{Pagge 123 of 257}

\begin{tabular}{|c|c|c|c|c|}
\hline AK3L1 & 205 & adenylate kinase 3-like 1 & 3,59 & chr1 \\
\hline AK3L1 & $205 / / / 387851$ & adenylate kinase 3 -like $1 / / /$ adenylate kinase 3 -like 2 & 2,67 & chr1 \\
\hline AKAP1 & 8165 & A kinase (PRKA) anchor protein 1 & 10,58 & chr17 \\
\hline AKAP10 & 11216 & A kinase (PRKA) anchor protein 10 & 3,59 & chr17 \\
\hline AKAP13 & 11214 & A kinase (PRKA) anchor protein 13 & 2,67 & chr15 \\
\hline AKAP7 & 9465 & A kinase (PRKA) anchor protein 7 & 2,57 & chr6 \\
\hline AKAP8 & 10270 & A kinase (PRKA) anchor protein 8 & 2,31 & chr19 \\
\hline AKR1A1 & 10327 & aldo-keto reductase family 1 , member $\mathrm{A} 1$ (aldehyde reductase) & 2,02 & chr1 \\
\hline ALDH16A1 & 126133 & aldehyde dehydrogenase 16 family, member A1 & 3,46 & chr19 \\
\hline ALDH3A2 & 224 & aldehyde dehydrogenase 3 family, member A2 & 6,74 & chr17 \\
\hline ALDH6A1 & 4329 & Aldehyde dehydrogenase 6 family, member A1 & 2,17 & chr14 \\
\hline ALDH7A1 & 501 & aldehyde dehydrogenase 7 family, member A1 & 4,60 & chr5 \\
\hline ALDOC & 230 & aldolase C, fructose-bisphosphate & 2,52 & chr17 \\
\hline ALG6 & 29929 & asparagine-linked glycosylation 6 homolog (yeast, alpha-1,3-glucosyltransferase & 4,69 & chr1 \\
\hline ALG8 & 79053 & asparagine-linked glycosylation 8 homolog (yeast, alpha-1,3-glucosyltransferase & 2,38 & chr11 \\
\hline ALMS1 & 7840 & Alstrom syndrome 1 & 9,58 & chr2 \\
\hline ALPL & 249 & alkaline phosphatase, liver/bone/kidney & 2,26 & chr1 \\
\hline ALS2CR13 & 150864 & amyotrophic lateral sclerosis 2 (juvenile) chromosome region, candidate 13 & 7,12 & chr2 \\
\hline ALS2CR19 & 117583 & amyotrophic lateral sclerosis 2 (juvenile) chromosome region, candidate 19 & 2,76 & chr1 \\
\hline AMD1 & 262 & adenosylmethionine decarboxylase 1 & 2,79 & chr6 \\
\hline AMPH & 273 & amphiphysin (Stiff-Man syndrome with breast cancer 128kDa autoantigen) & 2,91 & chr7 \\
\hline AMT & 275 & aminomethyltransferase (glycine cleavage system protein $\mathrm{T}$ ) & 9,65 & chr3 \\
\hline ANAPC1 & 64682 & Anaphase promoting complex subunit 1 & 5,21 & chr2 \\
\hline ANAPC10 & 10393 & anaphase promoting complex subunit 10 & 2,08 & chr4 \\
\hline ANAPC4 & 29945 & anaphase promoting complex subunit 4 & 2,88 & chr4 \\
\hline ANAPC7 & 51434 & anaphase promoting complex subunit 7 & 2,60 & chr12 \\
\hline ANGEL2 & 90806 & Angel homolog 2 (Drosophila) & 27,73 & chr1 \\
\hline ANK3 & 288 & ankyrin 3, node of Ranvier (ankyrin G) & 6,60 & chr10 \\
\hline ANKHD1 & 54882 & ankyrin repeat and $\mathrm{KH}$ domain containing 1 & 9,32 & chr5 \\
\hline ANKHD1 & $404734 / / / 54882$ & ankyrin repeat and $\mathrm{KH}$ domain containing $1 / / / \mathrm{MASK}-4 \mathrm{E}-\mathrm{BP} 3$ alternate reading & 2,16 & chr5 \\
\hline ANKRD10 & 55608 & Ankyrin repeat domain 10 & 9,14 & chr13 \\
\hline ANKRD16 & 54522 & ankyrin repeat domain 16 & 2,90 & chr10 \\
\hline ANKRD27 & 84079 & ankyrin repeat domain 27 (VPS9 domain) & 2,91 & chr19 \\
\hline ANKRD32 & 84250 & ankyrin repeat domain 32 & 4,56 & chr5 \\
\hline ANKRD35 & 148741 & ankyrin repeat domain 35 & 7,30 & chr1 \\
\hline ANKRD39 & 51239 & ankyrin repeat domain 39 & 2,19 & chr2 \\
\hline ANKRD41 & 126549 & ankyrin repeat domain 41 & 2,47 & chr19 \\
\hline ANKRD5 & 63926 & ankyrin repeat domain 5 & 5,66 & chr20 \\
\hline ANKRD9 & 122416 & ankyrin repeat domain 9 & 2,29 & chr14 \\
\hline ANKS1 & 23294 & ankyrin repeat and sterile alpha motif domain containing 1 & 2,50 & chr6 \\
\hline ANP32A & 8125 & Acidic (leucine-rich) nuclear phosphoprotein 32 family, member A & 3,43 & chr15 \\
\hline ANP32E & 81611 & Acidic (leucine-rich) nuclear phosphoprotein 32 family, member $\mathrm{E}$ & 3,97 & chr1 \\
\hline AOF2 & 23028 & amine oxidase (flavin containing) domain 2 & 4,12 & chr1 \\
\hline AP1G2 & 8906 & adaptor-related protein complex 1 , gamma 2 subunit & 3,44 & chr3 \\
\hline AP1M2 & 10053 & adaptor-related protein complex 1 , mu 2 subunit & 16,15 & chr19 \\
\hline AP1S2 & 8905 & Adaptor-related protein complex 1 , sigma 2 subunit & 50,35 & $\operatorname{chrX}$ \\
\hline AP4S1 & 11154 & adaptor-related protein complex 4 , sigma 1 subunit & 2,61 & chr6 \\
\hline APEH & 327 & $\mathrm{~N}$-acylaminoacyl-peptide hydrolase & 3,42 & chr3 \\
\hline APEX1 & 328 & APEX nuclease (multifunctional DNA repair enzyme) 1 & 2,48 & chr14 \\
\hline API5 & 8539 & Apoptosis inhibitor 5 & 2,49 & chr11 \\
\hline APITD1 & 378708 & apoptosis-inducing, TAF9-like domain 1 & 2,74 & chr1 \\
\hline APOC1 & 341 & apolipoprotein C-I & 14,76 & chr19 \\
\hline APOE & 348 & apolipoprotein E & 40,37 & chr19 \\
\hline APPBP1 & 8883 & amyloid beta precursor protein binding protein 1 & 2,61 & chr16 \\
\hline APRIN & 23047 & Androgen-induced proliferation inhibitor & 2,48 & chr13 \\
\hline APRT & 353 & adenine phosphoribosyltransferase & 3,77 & chr16 \\
\hline APTX & 54840 & aprataxin & 2,21 & chr9 \\
\hline AQR & 9716 & aquarius homolog (mouse) & 3,51 & chr15 \\
\hline ARFGEF1 & 10565 & ADP-ribosylation factor guanine nucleotide-exchange factor 1 (brefeldin A-inhibit, & 3,49 & chr8 \\
\hline ARG2 & 384 & arginase, type II & 8,83 & chr14 \\
\hline ARG99 & 83857 & ARG99 protein /// ARG99 protein & 2,95 & chr12 \\
\hline ARHGAP11A & 9824 & Rho GTPase activating protein $11 \mathrm{~A}$ & 2,61 & chr15 \\
\hline ARHGAP12 & 94134 & Rho GTPase activating protein 12 & 2,49 & chr10 \\
\hline ARHGAP19 & 84986 & Rho GTPase activating protein 19 & 7,54 & chr10 \\
\hline ARHGAP26 & 23092 & Rho GTPase activating protein 26 & 2,93 & chr5 \\
\hline ARHGAP28 & 79822 & Rho GTPase activating protein 28 & 5,70 & chr18 \\
\hline ARHGAP5 & 394 & Rho GTPase activating protein 5 & 2,54 & chr14 \\
\hline
\end{tabular}




\begin{tabular}{|c|c|c|c|c|}
\hline ARHGAP8 /// & $23779 / / / 553158$ & Rho GTPase activating protein 8 /// PRR5-ARHGAP8 fusion & 27,11 & chr22 \\
\hline ARHGEF10 & 9639 & Rho guanine nucleotide exchange factor (GEF) 10 & 5,62 & chr8 \\
\hline ARHGEF19 & 128272 & Rho guanine nucleotide exchange factor (GEF) 19 & 3,40 & chr1 \\
\hline ARHGEF5 & 7984 & Rho guanine nucleotide exchange factor (GEF) 5 & 5,24 & chr7 \\
\hline ARHGEF7 & 8874 & Rho guanine nucleotide exchange factor (GEF) 7 & 3,83 & chr13 \\
\hline ARHGEF9 & 23229 & Cdc42 guanine nucleotide exchange factor (GEF) 9 & 7,87 & $\operatorname{chrX}$ \\
\hline ARID1A & 8289 & AT rich interactive domain $1 \mathrm{~A}$ (SWI- like) & 2,50 & chr1 \\
\hline ARID1B & 57492 & AT rich interactive domain 1B (SWI1-like) & 3,16 & chr6 \\
\hline ARID2 & 196528 & AT rich interactive domain 2 (ARID, RFX-like) & 3,78 & chr12 \\
\hline ARID3A & 1820 & AT rich interactive domain 3A (BRIGHT- like) & 2,87 & chr19 \\
\hline ARID3B & 10620 & AT rich interactive domain $3 B$ (BRIGHT- like) & 30,08 & chr1 \\
\hline ARIH2 & 10425 & ariadne homolog 2 (Drosophila) & 3,32 & chr3 \\
\hline ARL2 & 402 & ADP-ribosylation factor-like 2 & 2,36 & chr11 \\
\hline ARL4 & 10124 & ADP-ribosylation factor-like 4 & 2,85 & chr6 \\
\hline ARLGIP & 23204 & ADP-ribosylation factor-like 6 interacting protein & 2,93 & chr16 \\
\hline ARL6IP2 & 64225 & ADP-ribosylation factor-like 6 interacting protein 2 & 2,72 & chr2 \\
\hline ARL6IP6 & 151188 & ADP-ribosylation-like factor 6 interacting protein 6 & 2,48 & chr2 \\
\hline ARL8 & 221079 & ADP-ribosylation factor-like 8 & 2,48 & chr10 \\
\hline ARMC6 & 93436 & armadillo repeat containing 6 & 2,23 & chr19 \\
\hline ARMC8 & 25852 & armadillo repeat containing 8 & 2,79 & chr3 \\
\hline ARS2 & 51593 & arsenate resistance protein ARS2 & 3,20 & chr7 \\
\hline $\mathrm{ASClZ}$ & 23300 & ATM/ATR-Substrate Chk2-Interacting Zn2+-finger protein & 2,15 & chr16 \\
\hline ASF1A & 25842 & ASF1 anti-silencing function 1 homolog A (S. cerevisiae) & 2,16 & chr6 \\
\hline ASK & 10926 & activator of $\mathrm{S}$ phase kinase & 6,58 & chr7 \\
\hline ASMTL & 8623 & acetylserotonin O-methyltransferase-like & 2,01 & chrY \\
\hline ASPM & 259266 & asp (abnormal spindle)-like, microcephaly associated (Drosophila) & 5,77 & chr1 \\
\hline ASRGL1 & 80150 & asparaginase like 1 & 9,62 & chr11 \\
\hline ASTN2 & 23245 & astrotactin 2 & 2,12 & chr9 \\
\hline ASXL2 & 55252 & additional sex combs like 2 (Drosophila) & 2,31 & chr2 \\
\hline ATAD2 & 29028 & ATPase family, AAA domain containing 2 & 2,43 & chr8 \\
\hline ATAD3B & 83858 & ATPase family, AAA domain containing $3 \mathrm{~B}$ & 2,08 & chr1_random \\
\hline ATCAY & 85300 & ataxia, cerebellar, Cayman type (caytaxin) & 2,13 & chr19 \\
\hline ATF7IP2 & 80063 & activating transcription factor 7 interacting protein 2 & 3,56 & chr16 \\
\hline ATG10 & 83734 & ATG10 autophagy related 10 homolog (S. cerevisiae) & 2,57 & chr5 \\
\hline ATG4D & 84971 & ATG4 autophagy related 4 homolog $\mathrm{D}$ (S. cerevisiae) & 2,45 & chr19 \\
\hline ATIC & 471 & 5-aminoimidazole-4-carboxamide ribonucleotide formyltransferase/IMP cyclohyd & 4,19 & chr2 \\
\hline ATP11A & 23250 & ATPase, Class VI, type 11A & 2,66 & $\operatorname{chr} 13$ \\
\hline ATP1A2 & 477 & ATPase, $\mathrm{Na}+/ \mathrm{K}+$ transporting, alpha $2(+)$ polypeptide & 8,72 & chr1 \\
\hline ATP1B3 & 483 & ATPase, $\mathrm{Na}+/ \mathrm{K}+$ transporting, beta 3 polypeptide & 2,26 & chr3 \\
\hline ATP2A1 & 487 & ATPase, $\mathrm{Ca}++$ transporting, cardiac muscle, fast twitch 1 & 3,76 & chr16 \\
\hline ATP5G1 & 516 & ATP synthase, $\mathrm{H}+$ transporting, mitochondrial F0 complex, subunit c (subunit 9), & 2,54 & chr17 \\
\hline ATP5G2 & 517 & ATP synthase, $\mathrm{H}+$ transporting, mitochondrial F0 complex, subunit c (subunit 9), & 2,44 & chr1 \\
\hline ATP5G3 & 518 & ATP synthase, $\mathrm{H}+$ transporting, mitochondrial F0 complex, subunit c (subunit 9) & 3,20 & chr2 \\
\hline ATP5H & 10476 & ATP synthase, $\mathrm{H}+$ transporting, mitochondrial F0 complex, subunit $d$ & 2,20 & chr9 \\
\hline ATP5I & 521 & ATP synthase, $\mathrm{H}+$ transporting, mitochondrial F0 complex, subunit e & 2,22 & chr4 \\
\hline ATP5O & 539 & ATP synthase, $\mathrm{H}+$ transporting, mitochondrial $\mathrm{F} 1$ complex, $\mathrm{O}$ subunit (oligomycir & 2,25 & chr21 \\
\hline ATP5S & 27109 & ATP synthase, $\mathrm{H}+$ transporting, mitochondrial F0 complex, subunit s (factor B) & 2,45 & chr14 \\
\hline ATP6AP1 & 537 & ATPase, $\mathrm{H}+$ transporting, lysosomal accessory protein 1 & 3,59 & chr16 \\
\hline ATP6VOA2 & 23545 & ATPase, $\mathrm{H}+$ transporting, lysosomal V0 subunit a isoform 2 & 2,26 & chr12 \\
\hline ATP6V0E2L & 155066 & ATPase, $\mathrm{H}+$ transporting V0 subunit $\mathrm{E}$ isoform 2-like (rat) & 2,07 & chr7 \\
\hline ATP8A1 & 10396 & ATPase, aminophospholipid transporter (APLT), Class I, type 8A, member 1 & 8,09 & chr4 \\
\hline ATPAF1 & 64756 & ATP synthase mitochondrial F1 complex assembly factor 1 & 3,00 & chr1 \\
\hline ATPBD1B & 54707 & ATP binding domain 1 family, member $B$ & 2,16 & chr1 \\
\hline ATPBD1C & 51184 & ATP binding domain 1 family, member $\mathrm{C}$ & 2,17 & chr12 \\
\hline ATRNL1 & 26033 & attractin-like 1 & 3,58 & chr10 \\
\hline ATRX & 546 & Alpha thalassemia/mental retardation syndrome X-linked (RAD54 homolog, S. c & 6,07 & $\operatorname{chr} x$ \\
\hline ATXN3 & 4287 & $\operatorname{ataxin} 3$ & 3,01 & chr14 \\
\hline ATXN7L1 & 57485 & ataxin 7-like 1 & 2,43 & chr7 \\
\hline AUH & 549 & AU RNA binding protein/enoyl-Coenzyme A hydratase & 4,13 & chr9 \\
\hline AURKAIP1 & 54998 & aurora kinase $\mathrm{A}$ interacting protein 1 & 2,90 & chr1_random \\
\hline AURKB & 9212 & aurora kinase B & 7,83 & chr17 \\
\hline AUTS2 & 26053 & autism susceptibility candidate 2 & 7,81 & chr7 \\
\hline AXIN2 & 8313 & axin 2 (conductin, axil) & 18,50 & chr17 \\
\hline AZI1 & 22994 & 5 -azacytidine induced 1 & 2,06 & chr17 \\
\hline AZIN1 & 51582 & Antizyme inhibitor 1 & 3,10 & chr8 \\
\hline B3GAT1 & 27087 & beta-1,3-glucuronyltransferase 1 (glucuronosyltransferase P) & 3,33 & chr11 \\
\hline B3GNT1 & 10678 & UDP-GIcNAc:betaGal beta-1,3-N-acetylglucosaminyltransferase 1 & 2,69 & chr2 \\
\hline
\end{tabular}




\section{Pagge 125 of 257}

\begin{tabular}{|c|c|c|c|c|}
\hline B3GNT7 & 93010 & UDP-GICNAc:betaGal beta-1,3-N-acetylglucosaminyltransferase 7 & 11,65 & chr2 \\
\hline B4GALT5 & 9334 & UDP-Gal:betaGlcNAc beta 1,4- galactosyltransferase, polypeptide 5 & 5,41 & chr20 \\
\hline B4GALT6 & 9331 & UDP-Gal:betaGlcNAc beta 1,4- galactosyltransferase, polypeptide 6 & 2,35 & chr18 \\
\hline BACE1 & 23621 & beta-site APP-cleaving enzyme 1 & 2,00 & chr11 \\
\hline BAG1 & 573 & BCL2-associated athanogene /// BCL2-associated athanogene & 3,13 & chr9 \\
\hline BAHD1 & 22893 & bromo adjacent homology domain containing 1 & 2,36 & chr15 \\
\hline BAIAP2L1 & 55971 & BAl1-associated protein 2-like 1 & 2,78 & chr7 \\
\hline BANF1 & 8815 & barrier to autointegration factor 1 & 2,18 & chr11 \\
\hline BANP & 54971 & BTG3 associated nuclear protein & 2,28 & chr16 \\
\hline BARD1 & 580 & BRCA1 associated RING domain 1 & 6,00 & chr2 \\
\hline BAT2D1 & 23215 & BAT2 domain containing 1 & 3,61 & chr1 \\
\hline BAT3 & 7917 & HLA-B associated transcript 3 & 2,33 & chr6 \\
\hline BAT4 & 7918 & HLA-B associated transcript 4 & 2,78 & chr6 \\
\hline BAX & 581 & BCL2-associated $X$ protein & 2,18 & chr19 \\
\hline BC036928 & 386758 & hypothetical protein $\mathrm{BC} 036928$ & 2,68 & (vide) \\
\hline BCCIP & 56647 & BRCA2 and CDKN1A interacting protein & 2,95 & chr10 \\
\hline BCDIN3 & 56257 & bin3, bicoid-interacting 3, homolog (Drosophila) & 2,60 & $\operatorname{chr} 7$ \\
\hline BCKDHB & 594 & branched chain keto acid dehydrogenase $E 1$, beta polypeptide (maple syrup urif & 3,86 & chr6 \\
\hline BCL11A & 53335 & B-cell CLL/lymphoma 11A (zinc finger protein) & 6,43 & chr2 \\
\hline BCL11B & 64919 & B-cell CLL/lymphoma 11B (zinc finger protein) & 5,29 & chr14 \\
\hline BCL2L11 & 10018 & BCL2-like 11 (apoptosis facilitator) & 9,23 & chr2 \\
\hline BCL2L12 & 83596 & BCL2-like 12 (proline rich) & 5,13 & chr19 \\
\hline BCOR & 54880 & BCL6 co-repressor & 8,07 & $\operatorname{chr} \mathrm{X}$ \\
\hline$B C R$ & 613 & breakpoint cluster region & 2,97 & chr22 \\
\hline BDH & 622 & 3-hydroxybutyrate dehydrogenase (heart, mitochondrial) /// 3-hydroxybutyrate df & 2,33 & $\operatorname{chr} 3$ \\
\hline BDP1 & 55814 & B double prime 1 , subunit of RNA polymerase III transcription initiation factor IIIB & 2,47 & chr5 \\
\hline BEX1 & 55859 & brain expressed, $\mathrm{X}$-linked 1 & 3,78 & $\operatorname{chr} \mathrm{X}$ \\
\hline BEX2 & 84707 & brain expressed X-linked $2 / / /$ brain expressed X-linked 2 & 16,92 & $\operatorname{chr} X$ \\
\hline BHLHB9 & 80823 & basic helix-loop-helix domain containing, class B, 9 & 4,92 & $\operatorname{chr} \mathrm{X}$ \\
\hline BICD1 & 636 & bicaudal D homolog 1 (Drosophila) & 6,67 & chr12 \\
\hline BIRC5 & 332 & baculoviral IAP repeat-containing 5 (survivin) & 7,65 & chr17 \\
\hline BIRC6 & 57448 & Splicing factor, arginine/serine-rich 12 & 2,37 & chr2 \\
\hline BLM & 641 & Bloom syndrome & 13,61 & chr15 \\
\hline BLMH & 642 & bleomycin hydrolase & 3,27 & chr17 \\
\hline ВМ039 & 55839 & uncharacterized bone marrow protein $\mathrm{BM} 039$ & 6,19 & chr16 \\
\hline BMP2K & 55589 & BMP2 inducible kinase & 2,08 & chr4 \\
\hline BMP7 & 655 & Bone morphogenetic protein 7 (osteogenic protein 1) & 4,44 & chr20 \\
\hline BMPR1A & 657 & bone morphogenetic protein receptor, type IA & 3,07 & chr10 \\
\hline BMS1L & 9790 & BMS1-like, ribosome assembly protein (yeast) & 2,81 & chr10 \\
\hline BNC2 & 54796 & Basonuclin 2 & 3,38 & chr9 \\
\hline BOLA1 & 51027 & bolA-like 1 (E. coli) & 2,19 & chr1 \\
\hline BOLA2 & 552900 & bolA-like 2 (E. coli) & 2,82 & chr16 \\
\hline BOLA3 & 388962 & bolA-like 3 (E. coli) & 2,14 & chr2 \\
\hline BOP1 & 23246 & block of proliferation 1 & 2,36 & chr8 \\
\hline BRAF & 673 & v-raf murine sarcoma viral oncogene homolog B1 & 2,24 & chr7 \\
\hline BRCA1 & 672 & breast cancer 1 , early onset & 6,06 & chr17 \\
\hline BRCA2 & 675 & breast cancer 2, early onset & 2,96 & chr13 \\
\hline BRD3 & 8019 & Bromodomain containing 3 & 2,72 & chr9 \\
\hline BRD4 & 23476 & Bromodomain containing 4 & 2,19 & chr19 \\
\hline BRD7 & 29117 & bromodomain containing 7 & 4,60 & chr3 \\
\hline BRD8 & 10902 & bromodomain containing 8 & 2,69 & chr5 \\
\hline BRRN1 & 23397 & barren homolog (Drosophila) & 6,89 & chr2 \\
\hline BRUNOL5 & 60680 & bruno-like 5, RNA binding protein (Drosophila) & 6,41 & chr19 \\
\hline BRWD1 & 54014 & bromodomain and WD repeat domain containing 1 & 4,38 & chr21 \\
\hline BRWD3 & 254065 & bromodomain and WD repeat domain containing 3 & 4,52 & $\operatorname{chr} X$ \\
\hline BSCL2 & $221092 / / / 26580$ & Bernardinelli-Seip congenital lipodystrophy 2 (seipin) /// heterogeneous nuclear & 4,85 & chr11 \\
\hline BSPRY & 54836 & B-box and SPRY domain containing & 2,63 & chr9 \\
\hline BST2 & 684 & bone marrow stromal cell antigen 2 & 13,21 & chr19 \\
\hline BTBD15 & 29068 & BTB (POZ) domain containing 15 & 7,74 & chr11 \\
\hline BTBD3 & 22903 & BTB (POZ) domain containing 3 & 2,36 & chr20 \\
\hline BTBD4 & 140685 & BTB (POZ) domain containing 4 & 2,62 & chr20 \\
\hline BTBD7 & 55727 & BTB (POZ) domain containing 7 & 2,75 & chr14 \\
\hline BTF3L4 & 91408 & Basic transcription factor 3-like 4 & 2,27 & chr1 \\
\hline BUB1 & 699 & BUB1 budding uninhibited by benzimidazoles 1 homolog (yeast) & 18,20 & chr2 \\
\hline BUB1B & 701 & BUB1 budding uninhibited by benzimidazoles 1 homolog beta (yeast) & 15,91 & chr15 \\
\hline BUB3 & 9184 & BUB3 budding uninhibited by benzimidazoles 3 homolog (yeast) & 2,87 & chr10 \\
\hline BXDC1 & 84154 & brix domain containing 1 & 2,29 & chr6 \\
\hline
\end{tabular}




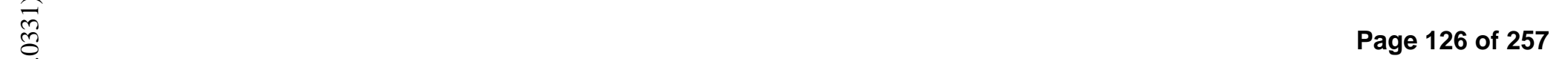

\begin{tabular}{|c|c|c|c|c|}
\hline \multicolumn{5}{|c|}{ Table S5: Genes downregulated in MPC compared to hES (Fold Change $>2 ; \alpha<0.05$ ) } \\
\hline BXDC2 & 55299 & brix domain containing 2 & 2,11 & chr5 \\
\hline BYSL & 705 & bystin-like & 2,42 & chr6 \\
\hline C10orf119 & 79892 & chromosome 10 open reading frame 119 & 2,11 & chr10 \\
\hline C10orf13 & 143282 & chromosome 10 open reading frame 13 & 4,13 & chr10 \\
\hline C10orf137 & 26098 & chromosome 10 open reading frame 137 & 2,33 & $\operatorname{chr} 10$ \\
\hline C10orf22 & 84890 & chromosome 10 open reading frame 22 & 3,13 & $\operatorname{chr} 10$ \\
\hline C10orf35 & 219738 & chromosome 10 open reading frame 35 & 5,21 & $\operatorname{chr} 10$ \\
\hline C10orf47 & 254427 & chromosome 10 open reading frame 47 & 2,87 & $\operatorname{chr} 10$ \\
\hline C10orf59 & 55328 & chromosome 10 open reading frame 59 & 3,31 & chr10 \\
\hline C10orf7 & 8872 & chromosome 10 open reading frame 7 & 2,17 & chr10 \\
\hline C10orf77 & 79847 & chromosome 10 open reading frame 77 & 2,13 & chr10 \\
\hline C10orf78 & 119392 & chromosome 10 open reading frame 78 & 2,70 & chr10 \\
\hline C10orf82 & 143379 & chromosome 10 open reading frame 82 & 2,09 & chr10 \\
\hline C10orf86 & 54780 & chromosome 10 open reading frame 86 & 6,19 & chr10 \\
\hline C10orf95 & $54808 / / / / 79946$ & Chromosome 10 open reading frame 95 /// Dymeclin & 5,60 & chr10 \\
\hline C11orf1 & 64776 & chromosome 11 open reading frame 1 & 2,86 & chr11 \\
\hline C11orf2 & 738 & chromosome 11 open reading frame2 & 2,16 & chr11 \\
\hline C11orf31 & 280636 & chromosome 11 open reading frame 31 & 3,06 & chr11 \\
\hline C11orf32 & 442871 & chromosome 11 open reading frame 32 & 66,43 & chr11 \\
\hline C12orf10 & 60314 & chromosome 12 open reading frame 10 & 2,78 & chr12 \\
\hline C13orf10 & 64062 & Chromosome 13 open reading frame 10 & 3,75 & chr13 \\
\hline C13orf23 & 80209 & chromosome 13 open reading frame 23 & 4,17 & $\operatorname{chr} 13$ \\
\hline C13orf25 & 407975 & chromosome 13 open reading frame 25 & 4,22 & $\operatorname{chr} 13$ \\
\hline C13orf3 & 221150 & chromosome 13 open reading frame 3 & 11,94 & $\operatorname{chr} 13$ \\
\hline C13orf7 & 79596 & chromosome 13 open reading frame 7 & 2,39 & $\operatorname{chr} 13$ \\
\hline C14orf1 & 11161 & chromosome 14 open reading frame 1 & 2,15 & chr14 \\
\hline C14orf104 & 55172 & chromosome 14 open reading frame 104 & 4,22 & chr14 \\
\hline C14orf106 & 55320 & chromosome 14 open reading frame 106 & 8,32 & chr14 \\
\hline C14orf109 & 26175 & chromosome 14 open reading frame 109 & 2,49 & chr14 \\
\hline C14orf115 & 55237 & chromosome 14 open reading frame 115 & 20,80 & chr14 \\
\hline C14orf120 & 25983 & chromosome 14 open reading frame 120 & 3,13 & chr14 \\
\hline C14orf122 & 51016 & chromosome 14 open reading frame 122 & 3,45 & chr14 \\
\hline C14orf130 & 55148 & chromosome 14 open reading frame 130 & 2,14 & chr14 \\
\hline C14orf135 & 64430 & Chromosome 14 open reading frame 135 & 2,07 & (vide) \\
\hline C14orf143 & 90141 & chromosome 14 open reading frame 143 & 2,47 & chr14 \\
\hline C14orf145 & 145508 & chromosome 14 open reading frame 145 & 2,77 & chr14 \\
\hline C14orf150 & 112840 & chromosome 14 open reading frame 150 & 3,53 & $\operatorname{chr} 14$ \\
\hline C14orf156 & 81892 & chromosome 14 open reading frame $156 / / /$ chromosome 14 open reading fram & 3,56 & chr14 \\
\hline C14orf159 & 80017 & chromosome 14 open reading frame 159 & 3,11 & chr14 \\
\hline C14orf169 & 79697 & chromosome 14 open reading frame 169 & 3,44 & chr14 \\
\hline C14orf94 & 54930 & chromosome 14 open reading frame 94 & 7,54 & chr14 \\
\hline C15orf20 & 80119 & chromosome 15 open reading frame 20 & 12,30 & chr15 \\
\hline C15orf23 & 90417 & chromosome 15 open reading frame 23 & 2,65 & chr15 \\
\hline C16orf33 & 79622 & chromosome 16 open reading frame 33 & 3,19 & chr16 \\
\hline C16orf34 & 90861 & chromosome 16 open reading frame 34 & 2,34 & chr16 \\
\hline C16orf5 & 29965 & chromosome 16 open reading frame 5 & 2,94 & chr16 \\
\hline C16orf51 & 25880 & chromosome 16 open reading frame 51 & 2,08 & chr16 \\
\hline C16orf53 & 79447 & chromosome 16 open reading frame 53 & 5,47 & chr16 \\
\hline C17orf25 & 51031 & chromosome 17 open reading frame 25 & 2,09 & chr17 \\
\hline C17orf32 & 147007 & chromosome 17 open reading frame 32 & 2,04 & chr17 \\
\hline C17orf39 & 79018 & chromosome 17 open reading frame 39 & 2,03 & chr17 \\
\hline C17orf41 & 79915 & chromosome 17 open reading frame 41 & 3,37 & chr17 \\
\hline C17orf63 & 55731 & Chromosome 17 open reading frame 63 & 3,39 & $\operatorname{chr} 17$ \\
\hline C18orf18 & 147525 & chromosome 18 open reading frame 18 & 2,31 & $\operatorname{chr} 18$ \\
\hline C18orf19 & 125228 & chromosome 18 open reading frame 19 & 2,19 & $\operatorname{chr} 18$ \\
\hline C18orf22 & 79863 & chromosome 18 open reading frame 22 & 2,68 & $\operatorname{chr} 18$ \\
\hline C18orf37 & 125476 & chromosome 18 open reading frame 37 & 3,07 & chr18 \\
\hline C18orf54 & 162681 & Chromosome 18 open reading frame 54 & 6,97 & chr18 \\
\hline C18orf55 & 29090 & chromosome 18 open reading frame 55 & 2,28 & chr18 \\
\hline C18orf9 & 79959 & chromosome 18 open reading frame 9 & 3,29 & chr18 \\
\hline C19orf32 & 92840 & chromosome 19 open reading frame 32 & 2,03 & chr19 \\
\hline C19orf33 & 64073 & chromosome 19 open reading frame 33 & 3,02 & chr19 \\
\hline C19orf7 & 23211 & chromosome 19 open reading frame 7 & 2,31 & chr19 \\
\hline C1GALT1 & 56913 & core 1 synthase, glycoprotein-N-acetylgalactosamine 3-beta-galactosyltransfera & 2,17 & $\mathrm{chr} 7$ \\
\hline C1orf104 & 284618 & Chromosome 1 open reading frame 104 & 3,02 & chr1 \\
\hline C1orf106 & 55765 & chromosome 1 open reading frame 106 & 23,01 & chr1 \\
\hline C1orf108 & 79647 & chromosome 1 open reading frame 108 & 4,08 & chr1 \\
\hline
\end{tabular}




\section{Pagge 127 of 257}

\begin{tabular}{|c|c|c|c|c|}
\hline C1orf112 & 55732 & Chromosome 1 open reading frame 112 & 4,43 & chr1 \\
\hline C1orf114 & 57821 & chromosome 1 open reading frame 114 & 3,44 & chr1 \\
\hline C1orf115 & 79762 & chromosome 1 open reading frame 115 & 2,67 & chr1 \\
\hline C1orf121 & 51029 & chromosome 1 open reading frame 121 & 7,68 & chr1 \\
\hline C1orf128 & 57095 & chromosome 1 open reading frame 128 & 2,51 & chr1 \\
\hline C1orf135 & 79000 & chromosome 1 open reading frame 135 & 7,70 & chr1 \\
\hline C1orf156 & 92342 & chromosome 1 open reading frame 156 & 3,28 & chr1 \\
\hline C1orf163 & 65260 & chromosome 1 open reading frame 163 & 4,70 & chr1 \\
\hline C1orf164 & 55182 & chromosome 1 open reading frame 164 & 2,73 & chr1 \\
\hline C1orf165 & 79656 & chromosome 1 open reading frame 165 & 3,70 & chr1 \\
\hline C1orf171 & 127253 & chromosome 1 open reading frame 171 & 2,60 & chr1 \\
\hline C1orf172 & 126695 & chromosome 1 open reading frame 172 & 2,27 & chr1 \\
\hline C1orf187 & 374946 & chromosome 1 open reading frame 187 & 9,38 & chr1 \\
\hline C1orf192 & 257177 & Chromosome 1 open reading frame 192 & 2,06 & chr1 \\
\hline C1orf31 & 388753 & chromosome 1 open reading frame 31 & 2,38 & chr1 \\
\hline C1orf37 & 92703 & chromosome 1 open reading frame 37 & 3,17 & chr1 \\
\hline C1orf43 & 25912 & chromosome 1 open reading frame 43 & 7,95 & chr1 \\
\hline C1orf48 & 25936 & chromosome 1 open reading frame 48 & 2,29 & chr1 \\
\hline C1orf57 & 84284 & chromosome 1 open reading frame 57 & 3,85 & chr1 \\
\hline C1orf59 & 113802 & chromosome 1 open reading frame 59 & 5,01 & chr1 \\
\hline C1orf67 & 200095 & chromosome 1 open reading frame 67 & 2,50 & chr1 \\
\hline C1orf69 & 200205 & chromosome 1 open reading frame 69 & 2,80 & chr1 \\
\hline C1orf77 & 26097 & chromosome 1 open reading frame 77 & 4,26 & chr1 \\
\hline C1orf79 & 85028 & chromosome 1 open reading frame 79 & 3,21 & chr1 \\
\hline C1orf93 & 127281 & chromosome 1 open reading frame 93 & 2,11 & chr1 \\
\hline C1orf97 & 84791 & chromosome 1 open reading frame $97 / / /$ chromosome 1 open reading frame 97 & 5,22 & chr1 \\
\hline C1QBP & 708 & Complement component $1, q$ subcomponent binding protein & 5,06 & chr17 \\
\hline C20orf11 & 54994 & chromosome 20 open reading frame 11 & 3,03 & chr20 \\
\hline C20orf118 & 140711 & Chromosome 20 open reading frame 118 & 6,38 & chr20 \\
\hline C20orf119 & 80336 & Similar to embryonic poly(A) binding protein & 5,64 & chr20 \\
\hline C20orf12 & 55184 & Chromosome 20 open reading frame 12 & 3,39 & $\operatorname{chr} 13$ \\
\hline C20orf129 & 81610 & chromosome 20 open reading frame 129 & 10,15 & chr20 \\
\hline C20orf14 & 24148 & chromosome 20 open reading frame 14 & 2,12 & chr20 \\
\hline C20orf160 & 140706 & chromosome 20 open reading frame 160 & 4,36 & chr20 \\
\hline C20orf172 & 79980 & chromosome 20 open reading frame 172 & 3,65 & chr20 \\
\hline C20orf177 & 63939 & chromosome 20 open reading frame 177 & 2,41 & chr20 \\
\hline C20orf19 & 55857 & chromosome 20 open reading frame 19 & 4,40 & chr20 \\
\hline C20orf35 & 55861 & chromosome 20 open reading frame 35 & 5,36 & chr20 \\
\hline C20orf39 & 79953 & chromosome 20 open reading frame 39 & 2,12 & chr20 \\
\hline C20orf42 & 55612 & chromosome 20 open reading frame 42 & 8,00 & chr20 \\
\hline C20orf6 & 51575 & chromosome 20 open reading frame 6 & 3,54 & chr20 \\
\hline C20orf7 & 79133 & chromosome 20 open reading frame 7 & 3,09 & chr20 \\
\hline C20orf72 & 92667 & chromosome 20 open reading frame 72 & 6,41 & chr20 \\
\hline C20orf74 & 57186 & chromosome 20 open reading frame 74 & 4,13 & chr20 \\
\hline C20orf94 & 128710 & chromosome 20 open reading frame 94 & 2,73 & chr20 \\
\hline C21orf18 & 54093 & chromosome 21 open reading frame 18 & 4,26 & chr21 \\
\hline C21orf33 & 8209 & chromosome 21 open reading frame 33 & 2,05 & chr21 \\
\hline C21orf45 & 54069 & chromosome 21 open reading frame 45 & 12,84 & chr21 \\
\hline C21orf57 & 54059 & Chromosome 21 open reading frame 57 & 3,48 & (vide) \\
\hline C21orf58 & 54058 & chromosome 21 open reading frame 58 & 2,02 & chr21 \\
\hline C21 orf59 & 56683 & chromosome 21 open reading frame 59 & 5,56 & chr21 \\
\hline C21orf66 & 94104 & Chromosome 21 open reading frame 66 & 3,56 & chr21 \\
\hline C22orf16 & 400916 & chromosome 22 open reading frame 16 & 3,74 & chr22 \\
\hline C22orf18 & 79019 & chromosome 22 open reading frame 18 & 6,28 & chr22 \\
\hline C22orf3 & 25807 & chromosome 22 open reading frame 3 & 3,41 & chr22 \\
\hline $\mathrm{C} 2 \mathrm{~F}$ & 10436 & C2f protein & 3,57 & chr12 \\
\hline C2orf31 & 81561 & chromosome 2 open reading frame $31 / / /$ chromosome 2 open reading frame 31 & 249,90 & chr2 \\
\hline C2orf34 & 79823 & chromosome 2 open reading frame 34 & 2,32 & chr2 \\
\hline $\mathrm{C} 3 \mathrm{~F}$ & 10162 & putative protein similar to nessy (Drosophila) & 2,79 & chr12 \\
\hline C3orf14 & 57415 & chromosome 3 open reading frame 14 & 2,85 & chr3 \\
\hline C3orf17 & 25871 & Chromosome 3 open reading frame 17 & 2,04 & chr3 \\
\hline C4orf14 & 84273 & chromosome 4 open reading frame 14 & 2,49 & $\operatorname{chr} 4$ \\
\hline C4orf15 & 79441 & chromosome 4 open reading frame 15 & 2,18 & chr4 \\
\hline C6orf108 & 10591 & chromosome 6 open reading frame 108 & 2,61 & chr6 \\
\hline C6orf111 & 25957 & chromosome 6 open reading frame 111 & 3,93 & chr6 \\
\hline C6orf115 & 58527 & chromosome 6 open reading frame 115 & 3,75 & $\operatorname{chr} 6$ \\
\hline C6orf117 & 112609 & chromosome 6 open reading frame 117 & 13,54 & chr6 \\
\hline
\end{tabular}

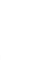

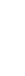

(1)

-




\begin{tabular}{|c|c|c|c|c|}
\hline C6orf130 & 221443 & chromosome 6 open reading frame 130 & 4,71 & chr6 \\
\hline C6orf136 & 221545 & chromosome 6 open reading frame 136 & 2,49 & chr6 \\
\hline C6orf139 & 55166 & chromosome 6 open reading frame 139 & 2,48 & chr6 \\
\hline C6orf149 & 57128 & chromosome 6 open reading frame 149 & 2,52 & chr6 \\
\hline C6orf157 & 90025 & chromosome 6 open reading frame 157 & 3,55 & chr6 \\
\hline C6orf166 & 55122 & Chromosome 6 open reading frame 166 & 2,31 & chr6 \\
\hline C6orf168 & 84553 & chromosome 6 open reading frame 168 & 3,31 & chr6 \\
\hline C6orf173 & 387103 & chromosome 6 open reading frame 173 & 5,96 & chr6 \\
\hline C6orf189 & 221303 & chromosome 6 open reading frame 189 & 2,26 & chr6 \\
\hline C6orf192 & 116843 & chromosome 6 open reading frame 192 & 7,62 & chr6 \\
\hline C6orf210 & 57107 & chromosome 6 open reading frame 210 & 2,06 & chr6 \\
\hline C6orf211 & 79624 & chromosome 6 open reading frame 211 & 2,17 & chr6 \\
\hline C6orf49 & 29964 & Chromosome 6 open reading frame 49 & 5,50 & chr6 \\
\hline C6orf60 & 79632 & chromosome 6 open reading frame 60 & 5,09 & chr6 \\
\hline C6orf66 & 29078 & chromosome 6 open reading frame 66 & 3,13 & chr6 \\
\hline C6orf75 & 60487 & chromosome 6 open reading frame 75 & 2,13 & chr6 \\
\hline C6orf84 & 22832 & chromosome 6 open reading frame 84 & 2,02 & chr6 \\
\hline C6orf85 & 63027 & chromosome 6 open reading frame 85 & 8,52 & chr6 \\
\hline C6orf93 & 84946 & chromosome 6 open reading frame 93 & 2,84 & chr6 \\
\hline C7orf16 & 10842 & chromosome 7 open reading frame 16 & 2,27 & chr7 \\
\hline C7orf20 & 51608 & chromosome 7 open reading frame 20 & 3,90 & chr7 \\
\hline C7orf24 & 79017 & chromosome 7 open reading frame 24 & 3,93 & chr7 \\
\hline C7orf27 & 221927 & chromosome 7 open reading frame 27 & 2,49 & chr7 \\
\hline C7orf29 & 113763 & chromosome 7 open reading frame 29 & 2,59 & chr7 \\
\hline C7orf30 & 115416 & chromosome 7 open reading frame 30 & 3,05 & chr7 \\
\hline C7orf36 & 57002 & chromosome 7 open reading frame 36 & 2,27 & chr7 \\
\hline C8orf38 & 137682 & chromosome 8 open reading frame 38 & 3,39 & chr8 \\
\hline C8orf40 & 114926 & chromosome 8 open reading frame 40 & 2,20 & chr8 \\
\hline C8orf41 & 80185 & chromosome 8 open reading frame 41 & 2,49 & chr8 \\
\hline C8orf42 & 157695 & Chromosome 8 open reading frame 42 & 8,19 & chr8 \\
\hline C8orf52 & 55656 & chromosome 8 open reading frame 52 & 2,42 & chr8 \\
\hline C9orf100 & 84904 & chromosome 9 open reading frame 100 & 2,01 & $\operatorname{chr} 9$ \\
\hline C9orf112 & 92715 & chromosome 9 open reading frame 112 & 2,30 & chr9 \\
\hline C9orf114 & 51490 & chromosome 9 open reading frame 114 & 3,41 & $\operatorname{chr} 9$ \\
\hline C9orf123 & 90871 & chromosome 9 open reading frame 123 & 3,74 & chr9 \\
\hline C9orf125 & 84302 & chromosome 9 open reading frame $125 / / /$ chromosome 9 open reading frame 1 & 2,01 & $\operatorname{chr} 9$ \\
\hline C9orf126 & 286205 & chromosome 9 open reading frame 126 & 2,53 & chr9 \\
\hline C9orf140 & 89958 & chromosome 9 open reading frame 140 & 6,65 & $\operatorname{chr} 9$ \\
\hline C9orf42 & 116224 & chromosome 9 open reading frame 42 & 2,23 & chr9 \\
\hline C9orf45 & 81571 & chromosome 9 open reading frame 45 & 11,84 & chr9 \\
\hline C9orf58 & 83543 & chromosome 9 open reading frame 58 & 26,18 & $\operatorname{chr} 9$ \\
\hline C9orf64 & 84267 & chromosome 9 open reading frame 64 & 6,54 & chr9 \\
\hline C9orf72 & 203228 & chromosome 9 open reading frame 72 & 3,91 & chr9 \\
\hline C9orf76 & 80010 & chromosome 9 open reading frame 76 & 5,34 & $\operatorname{chr} 9$ \\
\hline C9orf77 & 51104 & chromosome 9 open reading frame 77 & 2,76 & chr9 \\
\hline C9orf80 & 58493 & Chromosome 9 open reading frame 80 & 2,65 & chr9 \\
\hline C9orf81 & 84131 & chromosome 9 open reading frame 81 & 3,46 & chr9 \\
\hline C9orf86 & 55684 & chromosome 9 open reading frame 86 & 3,03 & chr9 \\
\hline CA11 & 770 & carbonic anhydrase XI & 4,10 & chr19 \\
\hline CA14 & 23632 & carbonic anhydrase XIV & 12,91 & chr1 \\
\hline CA2 & 760 & carbonic anhydrase II & 12,39 & chr8 \\
\hline CA5BL & 340591 & Carbonic anhydrase VB-like & 2,40 & $\operatorname{chrX}$ \\
\hline CABC1 & 56997 & chaperone, $\mathrm{ABC} 1$ activity of bc1 complex like (S. pombe) & 5,20 & chr1 \\
\hline CABP7 & 164633 & calcium binding protein 7 & 2,52 & chr22 \\
\hline CABYR & 26256 & calcium binding tyrosine-(Y)-phosphorylation regulated (fibrousheathin 2) & 2,11 & chr18 \\
\hline CACHD1 & 57685 & cache domain containing 1 & 11,98 & chr1 \\
\hline CACNA1D & 776 & Calcium channel, voltage-dependent, $L$ type, alpha 1D subunit & 2,58 & chr3 \\
\hline CACNA2D2 & 9254 & calcium channel, voltage-dependent, alpha $2 /$ delta subunit 2 & 7,30 & chr3 \\
\hline CACYBP & 27101 & calcyclin binding protein & 3,81 & chr1 \\
\hline CAD & 790 & carbamoyl-phosphate synthetase 2 , aspartate transcarbamylase, and dihydroor & 3,64 & chr2 \\
\hline CADPS & 8618 & Ca2+-dependent secretion activator & 4,52 & chr3 \\
\hline CADPS2 & 93664 & $\mathrm{Ca} 2+-$ dependent activator protein for secretion 2 & 11,67 & chr7 \\
\hline CALB1 & 793 & calbindin $1,28 \mathrm{kDa}$ & 17,68 & chr8 \\
\hline CALCA & 796 & calcitonin/calcitonin-related polypeptide, alpha & 2,42 & chr11 \\
\hline CALM3 & 808 & calmodulin 3 (phosphorylase kinase, delta) & 3,81 & chr19 \\
\hline CALML4 & 91860 & calmodulin-like 4 & 2,05 & chr15 \\
\hline CALN1 & 83698 & Calneuron 1 & 4,17 & chr7 \\
\hline
\end{tabular}




\section{Pagge 129 of 257}

\begin{tabular}{|c|c|c|c|c|}
\hline CAMK2G & 818 & Calcium/calmodulin-dependent protein kinase (CaM kinase) II gamma & 4,18 & chr10 \\
\hline CAMKV & 79012 & CaM kinase-like vesicle-associated & 5,11 & chr3 \\
\hline CAMTA1 & 23261 & calmodulin binding transcription activator 1 & 2,05 & chr1 \\
\hline CANP & 374393 & cancer-associated nucleoprotein & 3,53 & chr11 \\
\hline CARD11 & 84433 & caspase recruitment domain family, member 11 & 3,96 & $\operatorname{chr} 7$ \\
\hline CARHSP1 & 23589 & calcium regulated heat stable protein $1,24 \mathrm{kDa}$ & 4,08 & chr16 \\
\hline CARKL & 23729 & carbohydrate kinase-like & 2,23 & chr17 \\
\hline CARM1 & 10498 & coactivator-associated arginine methyltransferase 1 & 2,25 & chr19 \\
\hline CASC3 & 22794 & cancer susceptibility candidate 3 & 2,91 & chr17 \\
\hline CASC5 & 57082 & cancer susceptibility candidate 5 & 3,74 & chr15 \\
\hline CASP3 & 836 & caspase 3 , apoptosis-related cysteine peptidase & 2,04 & chr4 \\
\hline CASP8AP2 & 9994 & CASP8 associated protein 2 & 2,49 & chr6 \\
\hline CASP9 & 842 & caspase 9 , apoptosis-related cysteine peptidase & 4,78 & chr1 \\
\hline CASQ1 & 844 & Calsequestrin 1 (fast-twitch, skeletal muscle) & 4,79 & chr1 \\
\hline CAST1 & 26059 & CAZ-associated structural protein & 3,19 & chr3 \\
\hline CBFA2T2 & 9139 & core-binding factor, runt domain, alpha subunit 2; translocated to, 2 & 2,83 & chr20 \\
\hline CBL & 867 & Cas-Br-M (murine) ecotropic retroviral transforming sequence & 2,93 & chr11 \\
\hline CBLL1 & 79872 & Cas-Br-M (murine) ecotropic retroviral transforming sequence-like 1 & 2,18 & chr7 \\
\hline CBR1 & 873 & carbonyl reductase 1 & 3,41 & chr21 \\
\hline CBR4 & 84869 & carbonic reductase 4 & 4,76 & chr4 \\
\hline CBS & 875 & cystathionine-beta-synthase & 7,01 & chr21 \\
\hline CBX1 & 10951 & chromobox homolog 1 (HP1 beta homolog Drosophila) & 2,34 & chr17 \\
\hline СвХ2 & 84733 & chromobox homolog 2 (Pc class homolog, Drosophila) & 17,18 & chr17 \\
\hline CBX3 & 11335 & Chromobox homolog 3 (HP1 gamma homolog, Drosophila) & 3,04 & chr7 \\
\hline CBX5 & 23468 & Chromobox homolog 5 (HP1 alpha homolog, Drosophila) & 2,76 & chr12 \\
\hline CCAR1 & 55749 & Cell division cycle and apoptosis regulator 1 & 3,12 & chr10 \\
\hline CCBL1 & 883 & cysteine conjugate-beta lyase; cytoplasmic (glutamine transaminase $\mathrm{K}$, kyneure & 2,98 & chr9 \\
\hline CCDC12 & 151903 & coiled-coil domain containing 12 & 2,41 & chr3 \\
\hline CCDC14 & 64770 & coiled-coil domain containing 14 & 2,16 & chr3 \\
\hline CCDC18 & 343099 & coiled-coil domain containing 18 & 3,97 & chr1 \\
\hline CCDC21 & 64793 & coiled-coil domain containing 21 & 3,56 & chr1 \\
\hline $\mathrm{CCDC} 23$ & 374969 & coiled-coil domain containing 23 & 2,38 & chr1 \\
\hline CCDC25 & 55246 & coiled-coil domain containing 25 & 2,02 & chr8 \\
\hline CCDC4 & 389206 & coiled-coil domain containing 4 & 103,58 & chr4 \\
\hline CCDC5 & 115106 & coiled-coil domain containing 5 (spindle associated) & 5,49 & chr18 \\
\hline CCHCR1 & 54535 & coiled-coil alpha-helical rod protein 1 & 2,42 & chr6 \\
\hline CCNA1 & 8900 & cyclin A1 & 2,01 & chr13 \\
\hline CCNA2 & 890 & cyclin A2 & 13,97 & chr4 \\
\hline CCNB1 & 891 & cyclin B1 & 6,04 & chr5 \\
\hline CCNB1IP1 & 57820 & cyclin B1 interacting protein 1 & 3,68 & chr1 \\
\hline CCNB2 & 9133 & cyclin B2 & 7,90 & chr15 \\
\hline CCNE1 & 898 & cyclin E1 & 7,20 & chr19 \\
\hline CCNF & 899 & cyclin F & 6,53 & chr16 \\
\hline CCNJ & 54619 & cyclin J & 3,81 & chr10 \\
\hline CCNL1 & 57018 & cyclin L1 & 2,35 & chr3 \\
\hline CCNT2 & 905 & cyclin T2 & 2,51 & chr2 \\
\hline ССТ2 & 10576 & chaperonin containing TCP1, subunit 2 (beta) & 4,11 & chr12 \\
\hline ССТ3 & 7203 & chaperonin containing TCP1, subunit 3 (gamma) & 2,53 & chr1 \\
\hline ССT5 & 22948 & chaperonin containing TCP1, subunit 5 (epsilon) & 2,39 & chr5 \\
\hline CD200 & 4345 & CD200 antigen & 10,93 & chr3 \\
\hline CD24 & 934 & CD24 antigen (small cell lung carcinoma cluster 4 antigen) & 48,71 & chr5 \\
\hline CD2AP & 23607 & CD2-associated protein & 2,58 & chr6 \\
\hline CD3EAP & 10849 & CD3E antigen, epsilon polypeptide associated protein & 2,16 & chr19 \\
\hline CD9 & 928 & CD9 antigen (p24) & 6,23 & chr12 \\
\hline CDC14B & 8555 & CDC14 cell division cycle 14 homolog B (S. cerevisiae) & 3,44 & chr9 \\
\hline CDC2 & 983 & cell division cycle $2, \mathrm{G} 1$ to $\mathrm{S}$ and $\mathrm{G} 2$ to $\mathrm{M}$ & 8,81 & chr10 \\
\hline CDC20 & 991 & CDC20 cell division cycle 20 homolog (S. cerevisiae) & 13,22 & chr1 \\
\hline CDC25A & 993 & cell division cycle $25 \mathrm{~A}$ & 14,93 & chr3 \\
\hline CDC25C & 995 & cell division cycle $25 \mathrm{C}$ & 9,86 & chr5 \\
\hline CDC26 & 246184 & cell division cycle 26 & 2,09 & $\operatorname{chr} 7$ \\
\hline CDC2L5 & 8621 & Cell division cycle 2-like 5 (cholinesterase-related cell division controller) & 2,44 & chr7 \\
\hline CDC2L6 & 23097 & cell division cycle 2 -like 6 (CDK8-like) & 2,05 & chr6 \\
\hline CDC45L & 8318 & CDC45 cell division cycle 45 -like (S. cerevisiae) & 4,29 & chr22 \\
\hline CDC6 & 990 & CDC6 cell division cycle 6 homolog (S. cerevisiae) & 8,11 & chr17 \\
\hline $\mathrm{CDC7}$ & 8317 & CDC7 cell division cycle 7 (S. cerevisiae) & 24,79 & chr1 \\
\hline CDCA1 & 83540 & cell division cycle associated 1 & 4,89 & chr1 \\
\hline CDCA2 & 157313 & cell division cycle associated 2 & 6,46 & chr8 \\
\hline
\end{tabular}




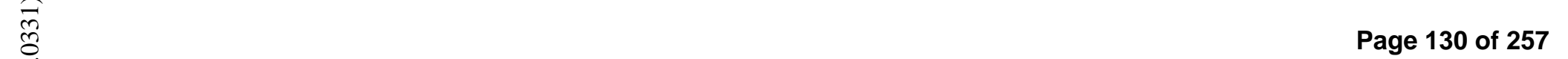

\begin{tabular}{|c|c|c|c|c|}
\hline CDCA3 & 83461 & cell division cycle associated 3 & 3,75 & chr12 \\
\hline CDCA4 & 55038 & cell division cycle associated 4 & 2,82 & chr14 \\
\hline CDCA5 & 113130 & cell division cycle associated 5 & 10,54 & chr11 \\
\hline CDCA7 & 83879 & cell division cycle associated $7 / / /$ cell division cycle associated 7 & 5,84 & chr2 \\
\hline CDCA7L & 55536 & cell division cycle associated 7 -like & 10,10 & chr7 \\
\hline CDCA8 & 55143 & cell division cycle associated 8 & 6,49 & chr1 \\
\hline $\mathrm{CDH} 1$ & 999 & cadherin 1, type 1, E-cadherin (epithelial) & 422,37 & $\operatorname{chr} 16$ \\
\hline $\mathrm{CDH} 3$ & 1001 & cadherin 3, type 1, P-cadherin (placental) & 27,30 & $\operatorname{chr} 16$ \\
\hline CDK3 & 1018 & cyclin-dependent kinase 3 & 2,70 & chr17 \\
\hline CDK5R1 & 8851 & cyclin-dependent kinase 5 , regulatory subunit 1 (p35) & 4,05 & chr17 \\
\hline CDK5RAP1 & 51654 & CDK5 regulatory subunit associated protein 1 & 3,53 & chr20 \\
\hline CDK5RAP2 & 55755 & CDK5 regulatory subunit associated protein 2 & 2,10 & chr9 \\
\hline CDK6 & 1021 & cyclin-dependent kinase 6 & 2,03 & $\operatorname{chr} 7$ \\
\hline CDKN1C & 1028 & Cyclin-dependent kinase inhibitor 1C (p57, Kip2) & 2,83 & chr11 \\
\hline CDKN3 & 1033 & cyclin-dependent kinase inhibitor 3 (CDK2-associated dual specificity phosphata & 6,24 & chr14 \\
\hline CDO1 & 1036 & cysteine dioxygenase, type I & 7,08 & chr5 \\
\hline CDON & 50937 & Cdon homolog (mouse) & 6,95 & chr11 \\
\hline CDS1 & 1040 & CDP-diacylglycerol synthase (phosphatidate cytidylyltransferase) 1 & 11,28 & chr4 \\
\hline CDS2 & 8760 & CDP-diacylglycerol synthase (phosphatidate cytidylyltransferase) 2 & 5,30 & chr20 \\
\hline CDT1 & 81620 & DNA replication factor & 7,21 & chr16 \\
\hline CDYL & 9425 & chromodomain protein, Y-like & 3,06 & chr6 \\
\hline CEBPZ & 10153 & CCAAT/enhancer binding protein zeta & 5,61 & chr2 \\
\hline CECR1 & 51816 & cat eye syndrome chromosome region, candidate 1 & 11,82 & chr22 \\
\hline CECR2 & 27443 & cat eye syndrome chromosome region, candidate 2 & 12,73 & chr22 \\
\hline CECR5 & 27440 & cat eye syndrome chromosome region, candidate 5 & 7,64 & chr22 \\
\hline CELSR1 & 9620 & cadherin, EGF LAG seven-pass G-type receptor 1 (flamingo homolog, Drosophi & 3,01 & chr22 \\
\hline CELSR2 & 1952 & cadherin, EGF LAG seven-pass G-type receptor 2 (flamingo homolog, Drosophi & 3,19 & chr1 \\
\hline CELSR3 & 1951 & cadherin, EGF LAG seven-pass G-type receptor 3 (flamingo homolog, Drosophi & 3,93 & chr3 \\
\hline CENPA & 1058 & centromere protein $\mathrm{A}, 17 \mathrm{kDa}$ & 4,72 & chr2 \\
\hline CENPE & 1062 & centromere protein $\mathrm{E}, 312 \mathrm{kDa}$ & 5,80 & chr4 \\
\hline CENPF & 1063 & centromere protein $\mathrm{F}, 350 / 400 \mathrm{ka}$ (mitosin) & 8,67 & chr1 \\
\hline CENPH & 64946 & centromere protein $\mathrm{H}$ & 10,07 & chr5 \\
\hline CENPJ & 55835 & centromere protein $\mathrm{J}$ & 4,55 & chr13 \\
\hline CENTB1 & 9744 & centaurin, beta 1 & 2,28 & chr17 \\
\hline CENTD1 & 116984 & centaurin, delta 1 & 10,10 & $\operatorname{chr} 4$ \\
\hline CENTG2 & 116987 & Centaurin, gamma 2 & 2,77 & chr2 \\
\hline Cep152 & 22995 & KIAA0912 protein & 3,56 & chr15 \\
\hline Cep192 & 55125 & centrosomal protein $192 \mathrm{kDa}$ & 2,21 & $\operatorname{chr} 18$ \\
\hline CEP4 & 9662 & centrosomal protein 4 & 2,67 & chr4 \\
\hline CEP68 & 23177 & Centrosomal protein $68 \mathrm{kDa}$ & 2,49 & chr2 \\
\hline Cep70 & 80321 & p10-binding protein & 2,51 & chr3 \\
\hline Cep72 & 55722 & centrosomal protein $72 \mathrm{kDa}$ & 4,69 & chr5 \\
\hline CETN3 & 1070 & centrin, EF-hand protein, 3 (CDC31 homolog, yeast) & 2,85 & chr5 \\
\hline CFC1 & 55997 & cripto, FRL-1, cryptic family 1 & 2,22 & chr2 \\
\hline CFDP1 & 10428 & craniofacial development protein 1 & 2,40 & chr16 \\
\hline CGI-09 & 51605 & CGI-09 protein & 2,88 & chr20 \\
\hline CGI-115 & 51018 & CGI-115 protein & 2,97 & chr1 \\
\hline CGI-121 & 51002 & CGI-121 protein & 2,11 & chr2 \\
\hline CGI-14 & 51005 & CGI-14 protein & 2,25 & chr16 \\
\hline CGI-37 & 51388 & comparative gene identification transcript 37 & 2,02 & chr16 \\
\hline CGI-69 & 51629 & CGI-69 protein & 2,61 & chr17 \\
\hline CGN & 57530 & cingulin & 11,26 & chr1 \\
\hline CGNL1 & 84952 & cingulin-like 1 & 37,79 & chr15 \\
\hline CHAF1A & 10036 & chromatin assembly factor 1 , subunit $A(p 150)$ & 6,90 & chr19 \\
\hline CHAF1B & 8208 & chromatin assembly factor 1 , subunit B (p60) & 3,03 & chr21 \\
\hline $\mathrm{CHCHD} 1$ & 118487 & coiled-coil-helix-coiled-coil-helix domain containing 1 & 2,72 & $\operatorname{chr} 10$ \\
\hline $\mathrm{CHCHD} 3$ & 54927 & coiled-coil-helix-coiled-coil-helix domain containing 3 & 2,32 & chr1 \\
\hline $\mathrm{CHCHD} 4$ & 131474 & coiled-coil-helix-coiled-coil-helix domain containing 4 & 2,78 & chr3 \\
\hline $\mathrm{CHCHD8}$ & 51287 & coiled-coil-helix-coiled-coil-helix domain containing 8 & 2,15 & chr11 \\
\hline CHD1 & 1105 & chromodomain helicase DNA binding protein 1 & 2,65 & chr5 \\
\hline CHD1L & 9557 & Chromodomain helicase DNA binding protein 1-like & 3,07 & chr1 \\
\hline CHD4 & 1108 & chromodomain helicase DNA binding protein 4 & 2,28 & chr12 \\
\hline CHD7 & 55636 & chromodomain helicase DNA binding protein 7 & 5,99 & chr8 \\
\hline CHD8 & 57680 & chromodomain helicase DNA binding protein 8 & 2,29 & chr14 \\
\hline CHEK1 & 1111 & CHK1 checkpoint homolog (S. pombe) & 5,62 & chr11 \\
\hline CHEK2 & 11200 & CHK2 checkpoint homolog (S. pombe) & 14,75 & chr22 \\
\hline CHERP & 10523 & calcium homeostasis endoplasmic reticulum protein & 2,65 & chr19 \\
\hline
\end{tabular}




\section{Pagge 131 of 257}

\begin{tabular}{|c|c|c|c|c|}
\hline CHES1 & 1112 & checkpoint suppressor 1 & 2,72 & chr14 \\
\hline CHGB & 1114 & chromogranin B (secretogranin 1) & 5,86 & chr20 \\
\hline CHKA & 1119 & choline kinase alpha & 4,38 & chr11 \\
\hline CHKB & $1120 / / / 1375$ & choline kinase beta /// carnitine palmitoyltransferase 1B (muscle) & 2,45 & chr22 \\
\hline CHML & 1122 & choroideremia-like (Rab escort protein 2) & 2,28 & chr1 \\
\hline CHN1 & 1123 & chimerin (chimaerin) 1 & 4,05 & chr2 \\
\hline CHST10 & 9486 & carbohydrate sulfotransferase 10 & 2,21 & chr2 \\
\hline CHST11 & 50515 & carbohydrate (chondroitin 4) sulfotransferase 11 & 2,35 & chr12 \\
\hline CHST4 & 10164 & carbohydrate ( $\mathrm{N}$-acetylglucosamine 6-O) sulfotransferase 4 & 9,16 & chr16 \\
\hline CHST6 & 4166 & carbohydrate ( $\mathrm{N}$-acetylglucosamine $6-\mathrm{O}$ ) sulfotransferase 6 & 2,06 & chr16 \\
\hline CHST7 & 56548 & carbohydrate ( $\mathrm{N}$-acetylglucosamine 6-O) sulfotransferase 7 & 2,02 & $\operatorname{chrX}$ \\
\hline CHST9 & 83539 & carbohydrate ( $\mathrm{N}$-acetylgalactosamine 4-0) sulfotransferase 9 & 7,97 & chr18 \\
\hline CHTF18 & 63922 & CTF18, chromosome transmission fidelity factor 18 homolog (S. cerevisiae) & 5,24 & chr16 \\
\hline CIAPIN1 & 57019 & cytokine induced apoptosis inhibitor 1 & 2,29 & chr18 \\
\hline CIB2 & 10518 & calcium and integrin binding family member 2 & 5,80 & chr15 \\
\hline CIRH1A & 84916 & cirrhosis, autosomal recessive $1 \mathrm{~A}$ (cirhin) & 3,32 & chr16 \\
\hline CIT & 11113 & citron (rho-interacting, serine/threonine kinase 21) & 2,43 & chr12 \\
\hline CKAP5 & 9793 & cytoskeleton associated protein 5 & 2,36 & chr11 \\
\hline CKB & 1152 & creatine kinase, brain & 9,17 & chr14 \\
\hline CKLFSF4 & 146223 & chemokine-like factor superfamily 4 & 3,42 & chr16 \\
\hline CKLFSF8 & 152189 & chemokine-like factor superfamily 8 & 4,48 & chr3 \\
\hline CKMT1B & $1159 / / / 548596$ & creatine kinase, mitochondrial $1 \mathrm{~B} / / /$ creatine kinase, mitochondrial $1 \mathrm{~A}$ & 37,87 & chr15 \\
\hline CKS1B & 1163 & CDC28 protein kinase regulatory subunit $1 \mathrm{~B}$ & 8,07 & chr1 \\
\hline CKS2 & 1164 & CDC28 protein kinase regulatory subunit 2 & 4,93 & chr9 \\
\hline CLASP1 & 23332 & Cytoplasmic linker associated protein 1 & 3,05 & chr2 \\
\hline CLCN4 & 1183 & Chloride channel 4 & 2,66 & $\operatorname{chrX}$ \\
\hline CLDN10 & 9071 & claudin 10 & 26,20 & chr13 \\
\hline CLDN23 & 137075 & claudin 23 & 3,42 & chr8 \\
\hline CLDN3 & 1365 & claudin 3 & 2,34 & chr7 \\
\hline CLDN6 & 9074 & claudin 6 & 67,55 & chr1 \\
\hline CLDN7 & 1366 & claudin 7 & 10,65 & chr17 \\
\hline CLGN & 1047 & calmegin & 3,12 & chr4 \\
\hline CLK2 & 1196 & CDC-like kinase 2 & 2,63 & chr1 \\
\hline CLN6 & 54982 & ceroid-lipofuscinosis, neuronal 6 , late infantile, variant & 2,50 & chr15 \\
\hline CLNS1A & $1207 / / / 56650$ & chloride channel, nucleotide-sensitive, $1 \mathrm{~A} / / /$ chromosome 3 open reading frame & 2,23 & chr11 \\
\hline CLPX & 10845 & ClpX caseinolytic peptidase X homolog (E. coli) & 2,00 & chr15 \\
\hline CLTC & 1213 & Clathrin, heavy polypeptide $(\mathrm{Hc})$ & 2,08 & chr17 \\
\hline CLYBL & 171425 & citrate lyase beta like & 3,59 & chr13 \\
\hline CMAS & 55907 & cytidine monophosphate $\mathrm{N}$-acetylneuraminic acid synthetase & 2,08 & chr12 \\
\hline CNAP1 & 9918 & chromosome condensation-related SMC-associated protein 1 & 3,95 & chr12 \\
\hline CNDP2 & 55748 & CNDP dipeptidase 2 (metallopeptidase M20 family) & 3,07 & chr18 \\
\hline $\mathrm{CNIH} 4$ & 29097 & cornichon homolog 4 (Drosophila) & 2,64 & chr1 \\
\hline CNKSR2 & 22866 & Connector enhancer of kinase suppressor of Ras 2 & 24,77 & $\operatorname{chrX}$ \\
\hline CNNM2 & 54805 & cyclin M2 & 2,90 & chr10 \\
\hline CNNM3 & 26505 & Cyclin M3 & 4,25 & chr2 \\
\hline CNOT1 & 23019 & CCR4-NOT transcription complex, subunit 1 & 2,78 & chr16 \\
\hline CNOT10 & 25904 & CCR4-NOT transcription complex, subunit 10 & 2,98 & chr3 \\
\hline CNOT2 & 4848 & CCR4-NOT transcription complex, subunit 2 & 2,09 & chr12 \\
\hline CNOT7 & 29883 & CCR4-NOT transcription complex, subunit 7 & 2,44 & chr1 \\
\hline CNTNAP2 & 26047 & contactin associated protein-like 2 & 56,91 & chr7 \\
\hline CNTNAP3 & $389734 / / / 79937$ & contactin associated protein-like 3 /// contactin associated protein-like 3B & 3,27 & chr9_random \\
\hline COBL & 23242 & cordon-bleu homolog (mouse) & 18,16 & chr7 \\
\hline $\mathrm{COCH}$ & 1690 & coagulation factor $\mathrm{C}$ homolog, cochlin (Limulus polyphemus) & 13,70 & chr14 \\
\hline COIL & 8161 & coilin & 2,22 & chr14 \\
\hline COL4A3BP & 10087 & collagen, type IV, alpha 3 (Goodpasture antigen) binding protein & 2,55 & chr5 \\
\hline COL9A1 & 1297 & collagen, type IX, alpha 1 & 4,24 & chr6 \\
\hline COL9A3 & 1299 & collagen, type IX, alpha 3 & 15,51 & chr20 \\
\hline COMMD4 & 54939 & COMM domain containing 4 & 3,34 & chr15 \\
\hline COMMD5 & 28991 & COMM domain containing $5 / / /$ COMM domain containing 5 & 2,05 & chr8 \\
\hline COMTD1 & 118881 & catechol-O-methyltransferase domain containing 1 & 4,56 & chr10 \\
\hline COPG2 & 26958 & Coatomer protein complex, subunit gamma 2 & 2,62 & chr7 \\
\hline COPS3 & 8533 & COP9 constitutive photomorphogenic homolog subunit 3 (Arabidopsis) & 2,11 & chr17 \\
\hline COQ2 & 27235 & coenzyme Q2 homolog, prenyltransferase (yeast) & 5,04 & chr4 \\
\hline COQ3 & 51805 & coenzyme Q3 homolog, methyltransferase (yeast) & 3,95 & chr6 \\
\hline COQ7 & 10229 & coenzyme Q7 homolog, ubiquinone (yeast) & 2,92 & chr16 \\
\hline CORO2A & 7464 & coronin, actin binding protein, $2 \mathrm{~A}$ & 2,07 & chr9 \\
\hline $\operatorname{cox} 15$ & 1355 & coX15 homolog, cytochrome c oxidase assembly protein (yeast) & 2,29 & chr10 \\
\hline
\end{tabular}




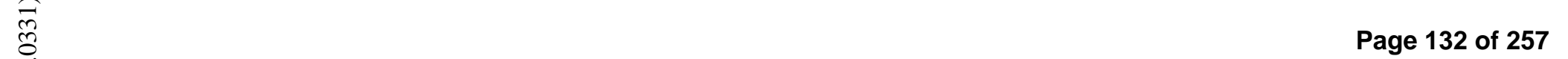

\begin{tabular}{|c|c|c|c|c|}
\hline $\operatorname{cox} 17$ & 10063 & COX17 homolog, cytochrome c oxidase assembly protein (yeast) & 2,46 & $\operatorname{chr} 13$ \\
\hline COX5A & 9377 & cytochrome $\mathrm{c}$ oxidase subunit $\mathrm{Va}$ & 3,32 & chr15 \\
\hline Cox5B & 1329 & cytochrome $\mathrm{c}$ oxidase subunit $\mathrm{Vb}$ & 2,22 & chr2 \\
\hline COX6A1 & 1337 & cytochrome $\mathrm{c}$ oxidase subunit Vla polypeptide $1 / / /$ cytochrome $\mathrm{c}$ oxidase subun & 12,36 & (vide) \\
\hline $\operatorname{cox} 7 \mathrm{C}$ & 1350 & cytochrome c oxidase subunit VIIC & 2,56 & chr5 \\
\hline CPSF2 & 53981 & cleavage and polyadenylation specific factor $2,100 \mathrm{kDa}$ & 2,29 & $\operatorname{chr} 14$ \\
\hline CPSF3 & 51692 & cleavage and polyadenylation specific factor $3,73 \mathrm{kDa}$ & 3,79 & chr2 \\
\hline CPSF4 & 10898 & cleavage and polyadenylation specific factor $4,30 \mathrm{kDa}$ & 2,45 & chr7 \\
\hline CPSF6 & 11052 & Cleavage and polyadenylation specific factor $6,68 \mathrm{kDa}$ & 4,99 & chr12 \\
\hline CPT1A & 1374 & carnitine palmitoyltransferase $1 \mathrm{~A}$ (liver) & 2,97 & chr11 \\
\hline CPT1C & 126129 & carnitine palmitoyttransferase $1 \mathrm{C}$ & 3,59 & chr19 \\
\hline СРT2 & 1376 & carnitine palmitoyltransferase II & 2,64 & chr1 \\
\hline CPVL & 54504 & carboxypeptidase, vitellogenic-like /// carboxypeptidase, vitellogenic-like & 10,44 & $\operatorname{chr} 7$ \\
\hline CPXM & 56265 & carboxypeptidase X (M14 family) & 4,77 & chr20 \\
\hline CRABP1 & 1381 & cellular retinoic acid binding protein 1 & 12,52 & chr15 \\
\hline CRB3 & 92359 & crumbs homolog 3 (Drosophila) & 2,27 & chr19 \\
\hline CREB3L4 & 148327 & CAMP responsive element binding protein 3 -like 4 & 5,92 & chr1 \\
\hline CRISPLD1 & 83690 & cysteine-rich secretory protein LCCL domain containing 1 & 4,72 & chr8 \\
\hline CRK7 & 51755 & CDC2-related protein kinase 7 & 2,77 & chr17 \\
\hline CRKL & 1399 & v-crk sarcoma virus CT10 oncogene homolog (avian)-like & 2,31 & chr22 \\
\hline CRLF3 & 51379 & Cytokine receptor-like factor 3 & 2,78 & chr17 \\
\hline CRMP1 & 1400 & collapsin response mediator protein 1 & 3,00 & chr4 \\
\hline CROP & 51747 & cisplatin resistance-associated overexpressed protein & 2,10 & chr17 \\
\hline CRSP2 & 9282 & cofactor required for $\mathrm{Sp} 1$ transcriptional activation, subunit 2, 150kDa & 2,63 & $\operatorname{chr} X$ \\
\hline CRSP9 & 9443 & cofactor required for Sp1 transcriptional activation, subunit 9, 33kDa & 2,11 & chr5 \\
\hline CRYM & 1428 & crystallin, mu & 11,70 & chr16 \\
\hline CSE1L & 1434 & CSE1 chromosome segregation 1 -like (yeast) & 5,22 & chr20 \\
\hline CSNK1A1 & 1452 & Casein kinase 1 , alpha 1 & 3,54 & chr5 \\
\hline CSNK2A1 & 1457 & casein kinase 2, alpha 1 polypeptide & 3,30 & chr20 \\
\hline CSNK2A2 & 1459 & Casein kinase 2 , alpha prime polypeptide & 2,10 & chr16 \\
\hline CSPG5 & 10675 & chondroitin sulfate proteoglycan 5 (neuroglycan C) & 6,19 & chr3 \\
\hline CSPG6 & 9126 & chondroitin sulfate proteoglycan 6 (bamacan) & 4,00 & chr10 \\
\hline CSPP1 & 79848 & centrosome and spindle pole associated protein 1 & 2,23 & chr8 \\
\hline CSRP2BP & 57325 & CSRP2 binding protein & 3,32 & chr20 \\
\hline CSTF1 & 1477 & cleavage stimulation factor, 3 pre-RNA, subunit $1,50 \mathrm{kDa}$ & 3,64 & chr20 \\
\hline CSTF2 & 1478 & cleavage stimulation factor, 3 pre-RNA, subunit 2, 64kDa & 3,39 & $\operatorname{chrX}$ \\
\hline CSTF2T & 23283 & cleavage stimulation factor, 3 ' pre-RNA, subunit $2,64 \mathrm{kDa}$, tau variant & 3,60 & $\operatorname{chr} 10$ \\
\hline СТВР2 & 1488 & C-terminal binding protein 2 & 3,20 & chr5 \\
\hline СТCF & 10664 & CCCTC-binding factor (zinc finger protein) & 4,68 & chr16 \\
\hline CTDP1 & 9150 & CTD (carboxy-terminal domain, RNA polymerase II, polypeptide A) phosphatase & 2,38 & $\operatorname{chr} 18$ \\
\hline CTDSPL2 & 51496 & CTD (carboxy-terminal domain, RNA polymerase II, polypeptide A) small phosph & 2,97 & chr15 \\
\hline CTNNA2 & 1496 & catenin (cadherin-associated protein), alpha 2 & 3,17 & chr2 \\
\hline CTNNBL1 & 56259 & catenin, beta like $1 / / /$ catenin, beta like 1 & 2,82 & chr20 \\
\hline CTPS2 & 56474 & CTP synthase II & 5,15 & $\operatorname{chrX}$ \\
\hline CTSC & 1075 & cathepsin C & 4,28 & chr11 \\
\hline CTSL2 & 1515 & cathepsin L2 & 62,34 & chr9 \\
\hline CTTNBP2 & 83992 & Cortactin binding protein 2 & 3,52 & chr7 \\
\hline CUGBP1 & 10658 & CUG triplet repeat, RNA binding protein 1 & 4,93 & chr11 \\
\hline CUGBP2 & 10659 & CUG triplet repeat, RNA binding protein 2 & 7,20 & chr10 \\
\hline CUL1 & 8454 & Cullin 1 & 5,56 & chr7 \\
\hline CUL3 & 8452 & Cullin 3 & 2,10 & chr2 \\
\hline CUL4A & 8451 & Cullin 4A & 2,61 & chr13 \\
\hline CUTC & 51076 & cutC copper transporter homolog (E.coli) & 2,57 & $\operatorname{chr} 10$ \\
\hline CUTL1 & 1523 & Cut-like 1, CCAAT displacement protein (Drosophila) & 2,90 & $\operatorname{chr} 7$ \\
\hline CUTL2 & 23316 & cut-like 2 (Drosophila) & 5,06 & chr12 \\
\hline CWF19L1 & 55280 & CWF19-like 1, cell cycle control (S. pombe) & 2,16 & $\operatorname{chr} 10$ \\
\hline CWF19L2 & 143884 & CWF19-like 2, cell cycle control (S. pombe) & 2,59 & chr11 \\
\hline CXADR & 1525 & coxsackie virus and adenovirus receptor & 113,79 & chr21 \\
\hline CXCR4 & 7852 & chemokine (C-X-C motif) receptor 4 & 13,89 & chr2 \\
\hline CXorf15 & 55787 & chromosome $\mathrm{X}$ open reading frame 15 & 2,39 & $\operatorname{chr} X$ \\
\hline CXorf26 & 51260 & chromosome $\mathrm{X}$ open reading frame 26 & 3,17 & $\operatorname{chr} X$ \\
\hline CXorf39 & 139231 & Chromosome $\mathrm{X}$ open reading frame 39 & 2,02 & $\operatorname{chr} X$ \\
\hline CXorf45 & 79868 & chromosome $\mathrm{X}$ open reading frame 45 & 2,26 & $\operatorname{chr} X$ \\
\hline CXorf50 & 203429 & chromosome $\mathrm{X}$ open reading frame 50 & 3,11 & $\operatorname{chr} X$ \\
\hline CXorf53 & 79184 & chromosome $\mathrm{X}$ open reading frame 53 & 2,93 & chr5 \\
\hline $\mathrm{CXXC1}$ & 30827 & CXXC finger 1 (PHD domain) & 2,02 & chr18 \\
\hline |cxxc6 & 80312 & CXXC finger 6 & 31,21 & $\operatorname{chr} 10$ \\
\hline
\end{tabular}




\begin{tabular}{|c|c|c|c|c|}
\hline CYB5 & 1528 & cytochrome b-5 & 3,80 & $\operatorname{chr} 18$ \\
\hline CYB561 & 1534 & cytochrome b-561 & 7,06 & chr17 \\
\hline CYB5R2 & 51700 & cytochrome b5 reductase 2 & 9,26 & chr11 \\
\hline CYC1 & 1537 & cytochrome c-1 & 2,14 & chr8 \\
\hline CYP26A1 & 1592 & cytochrome P450, family 26 , subfamily A, polypeptide 1 & 61,72 & chr10 \\
\hline CYP2B7P1 & 1556 & cytochrome P450, family 2, subfamily B, polypeptide 7 pseudogene 1 & 2,66 & chr19 \\
\hline CYP2S1 & 29785 & cytochrome P450, family 2, subfamily S, polypeptide 1 & 9,85 & chr19 \\
\hline CYP4X1 & 260293 & cytochrome P450, family 4, subfamily X, polypeptide 1 & 3,88 & chr1 \\
\hline CYP51A1 & 1595 & cytochrome P450, family 51, subfamily A, polypeptide 1 & 2,10 & chr3 \\
\hline CYYR1 & 116159 & cysteine/tyrosine-rich 1 & 21,13 & chr21 \\
\hline D21S2056E & 8568 & DNA segment on chromosome 21 (unique) 2056 expressed sequence & 3,02 & chr21 \\
\hline D4S234E & 27065 & DNA segment on chromosome 4 (unique) 234 expressed sequence & 6,78 & $\operatorname{chr} 4$ \\
\hline DAB1 & 1600 & Disabled homolog 1 (Drosophila) & 11,13 & chr1 \\
\hline DAB1 & $115209 / / / 1600$ & disabled homolog 1 (Drosophila) /// OMA1 homolog, zinc metallopeptidase (S. ce & 3,40 & chr1 \\
\hline DAG1 & 1605 & dystroglycan 1 (dystrophin-associated glycoprotein 1) & 2,11 & chr3 \\
\hline DAPK1 & 1612 & death-associated protein kinase 1 & 17,36 & chr9 \\
\hline DARS & 1615 & aspartyl-tRNA synthetase & 3,10 & chr2 \\
\hline DATF1 & 11083 & death associated transcription factor 1 & 8,74 & chr20 \\
\hline DAZAP1 & 26528 & DAZ associated protein 1 & 3,34 & chr19 \\
\hline DBC1 & 1620 & deleted in bladder cancer 1 & 25,86 & chr9 \\
\hline DBI & 1622 & diazepam binding inhibitor (GABA receptor modulator, acyl-Coenzyme A binding & 2,46 & chr2 \\
\hline DCAMKL1 & 9201 & doublecortin and CaM kinase-like 1 & 16,27 & chr13 \\
\hline DCC1 & 79075 & defective in sister chromatid cohesion homolog 1 (S. cerevisiae) & 16,86 & chr8 \\
\hline $\mathrm{DCl}$ & 1632 & dodecenoyl-Coenzyme $\mathrm{A}$ delta isomerase ( 3,2 trans-enoyl-Coenzyme $\mathrm{A}$ isomer & 3,26 & chr16 \\
\hline DCLRE1A & 9937 & DNA cross-link repair 1A (PSO2 homolog, S. cerevisiae) & 2,90 & chr10 \\
\hline DCLRE1C & 64421 & DNA cross-link repair 1C (PSO2 homolog, S. cerevisiae) & 3,15 & chr10 \\
\hline DCP2 & 167227 & DCP2 decapping enzyme homolog (S. cerevisiae) & 7,68 & chr5 \\
\hline DCPS & 28960 & decapping enzyme, scavenger & 3,49 & chr11 \\
\hline DCUN1D2 & 55208 & DCN1, defective in cullin neddylation 1 , domain containing 2 (S. cerevisiae) & 2,02 & chr13 \\
\hline DCUN1D4 & 23142 & DCN1, defective in cullin neddylation 1, domain containing 4 (S. cerevisiae) & 2,30 & chr4 \\
\hline DCX & 1641 & doublecortex; lissencephaly, X-linked (doublecortin) & 2,51 & $\operatorname{chr} X$ \\
\hline DDEFL1 & 55616 & development and differentiation enhancing factor-like 1 & 2,15 & chr1 \\
\hline DDIT4 & 54541 & DNA-damage-inducible transcript 4 & 3,04 & chr10 \\
\hline DDT & 1652 & D-dopachrome tautomerase & 6,00 & chr22 \\
\hline DDX11 & 1663 & DEAD/H (Asp-Glu-Ala-Asp/His) box polypeptide 11 (CHL1-like helicase homolog & 4,99 & chr12 \\
\hline DDX17 & 10521 & DEAD (Asp-Glu-Ala-Asp) box polypeptide 17 & 3,06 & chr22 \\
\hline DDX18 & 8886 & DEAD (Asp-Glu-Ala-Asp) box polypeptide 18 & 2,79 & chr2 \\
\hline DDX20 & 11218 & DEAD (Asp-Glu-Ala-Asp) box polypeptide 20 & 3,12 & chr1 \\
\hline DDX21 & 9188 & DEAD (Asp-Glu-Ala-Asp) box polypeptide $21 / / /$ DEAD (Asp-Glu-Ala-Asp) box pd & 2,16 & chr10 \\
\hline DDX25 & 29118 & DEAD (Asp-Glu-Ala-Asp) box polypeptide 25 & 37,88 & chr11 \\
\hline DDX27 & 55661 & DEAD (Asp-Glu-Ala-Asp) box polypeptide 27 & 3,41 & chr20 \\
\hline DDX28 & 55794 & DEAD (Asp-Glu-Ala-Asp) box polypeptide 28 & 3,81 & chr16 \\
\hline DDX31 & 64794 & DEAD (Asp-Glu-Ala-Asp) box polypeptide 31 & 2,67 & chr9 \\
\hline DDX39 & 10212 & DEAD (Asp-Glu-Ala-Asp) box polypeptide 39 & 4,18 & chr19 \\
\hline DDX3X & 1654 & DEAD (Asp-Glu-Ala-Asp) box polypeptide 3, X-linked & 3,08 & $\operatorname{chr} X$ \\
\hline DDX3Y & 8653 & DEAD (Asp-Glu-Ala-Asp) box polypeptide 3, Y-linked & 2,21 & chrY \\
\hline DDX46 & 9879 & DEAD (Asp-Glu-Ala-Asp) box polypeptide 46 & 2,70 & chr5 \\
\hline DDX48 & 9775 & DEAD (Asp-Glu-Ala-Asp) box polypeptide 48 & 3,19 & chr17 \\
\hline DDX54 & 79039 & DEAD (Asp-Glu-Ala-Asp) box polypeptide 54 & 2,39 & chr12 \\
\hline DDX55 & 57696 & DEAD (Asp-Glu-Ala-Asp) box polypeptide 55 & 2,69 & chr12 \\
\hline DDX6 & 1656 & DEAD (Asp-Glu-Ala-Asp) box polypeptide 6 & 5,03 & chr11 \\
\hline DEDD2 & 162989 & death effector domain containing 2 & 2,03 & chr19 \\
\hline DEK & 7913 & DEK oncogene (DNA binding) & 2,45 & chr6 \\
\hline DELGEF & 26297 & deafness locus associated putative guanine nucleotide exchange factor & 2,28 & chr11 \\
\hline DENND2C & 163259 & DENN/MADD domain containing $2 \mathrm{C}$ & 3,33 & chr1 \\
\hline DENR & 8562 & density-regulated protein & 2,37 & chr12 \\
\hline DEPDC1 & 55635 & DEP domain containing 1 & 3,85 & chr9 \\
\hline DEPDC1B & 55789 & DEP domain containing $1 \mathrm{~B}$ & 18,80 & chr5 \\
\hline DEPDC5 & 9681 & DEP domain containing 5 & 2,02 & chr22 \\
\hline DFFA & 1676 & DNA fragmentation factor, $45 \mathrm{kDa}$, alpha polypeptide & 2,52 & chr1 \\
\hline DFFB & 1677 & DNA fragmentation factor, $40 \mathrm{kDa}$, beta polypeptide (caspase-activated DNase) & 2,21 & chr1 \\
\hline DGKZ & 8525 & diacylglycerol kinase, zeta 104kDa & 2,17 & chr11 \\
\hline DHCR24 & 1718 & 24-dehydrocholesterol reductase & 6,02 & chr1 \\
\hline DHCR7 & 1717 & 7-dehydrocholesterol reductase & 2,82 & chr11 \\
\hline DHFR & 1719 & dihydrofolate reductase & 7,16 & chr4 \\
\hline DHODH & 1723 & dihydroorotate dehydrogenase & 3,08 & chr16 \\
\hline DHTKD1 & 55526 & dehydrogenase $\mathrm{E} 1$ and transketolase domain containing 1 & 4,50 & chr10 \\
\hline
\end{tabular}




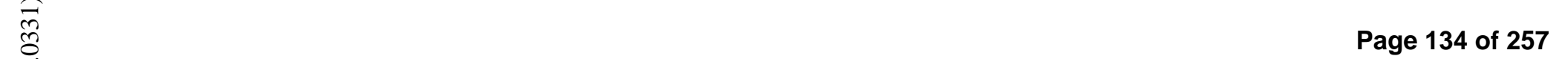

\begin{tabular}{|c|c|c|c|c|}
\hline DHX15 & 1665 & DEAH (Asp-Glu-Ala-His) box polypeptide 15 & 3,06 & chr4 \\
\hline DHX30 & 22907 & DEAH (Asp-Glu-Ala-His) box polypeptide 30 & 2,09 & chr3 \\
\hline DHX33 & 56919 & DEAH (Asp-Glu-Ala-His) box polypeptide 33 & 4,52 & chr17 \\
\hline DHX35 & 60625 & DEAH (Asp-Glu-Ala-His) box polypeptide 35 & 4,73 & chr20 \\
\hline DHX36 & 170506 & DEAH (Asp-Glu-Ala-His) box polypeptide 36 & 3,14 & chr3 \\
\hline DHX37 & 57647 & DEAH (Asp-Glu-Ala-His) box polypeptide 37 & 2,74 & chr12 \\
\hline DHX9 & 1660 & DEAH (Asp-Glu-Ala-His) box polypeptide 9 & 5,17 & chr1 \\
\hline DIAPH2 & 1730 & diaphanous homolog 2 (Drosophila) & 5,84 & $\operatorname{chrX}$ \\
\hline DKC1 & 1736 & dyskeratosis congenita 1 , dyskerin & 4,97 & $\operatorname{chr} \mathrm{X}$ \\
\hline DKFZp434K1 & 222229 & hypothetical protein DKFZp434K1815 & 2,01 & chr7 \\
\hline DKFZp434P0| & 91531 & hypothetical protein DKFZp434P055 & 4,60 & chr2 \\
\hline DKFZp547E0 & 283846 & hypothetical gene LOC283846 & 7,44 & chr16 \\
\hline DKFZP564J1 & 25854 & DKFZP564J102 protein & 6,02 & chr4 \\
\hline DKFZp564J14 & 54458 & DKFZp564J157 protein & 2,14 & chr12 \\
\hline DKFZP586A0 & 25840 & DKFZP586A0522 protein & 11,85 & chr12 \\
\hline DKFZp586114 & 222161 & hypothetical protein DKFZp58611420 & 20,32 & chr7 \\
\hline DKFZP761M1 & 54492 & hypothetical protein DKFZP761M1511 & 2,83 & chr5 \\
\hline DKFZp761P0 & 157285 & Hypothetical protein DKFZp761P0423 & 4,43 & chr8 \\
\hline DKFZp762E1 & 55355 & hypothetical protein DKFZp762E1312 & 6,88 & chr2 \\
\hline DKFZp762H1] & 256306 & hypothetical protein DKFZp762H185 & 2,09 & chr17 \\
\hline DKFZp762113 & 136051 & hypothetical protein DKFZp762I137 & 2,87 & chr7 \\
\hline DKFZp77901 & 374899 & hypothetical protein DKFZp7790175 & 2,87 & chr19 \\
\hline DLAT & 1737 & dihydrolipoamide S-acetyltransferase (E2 component of pyruvate dehydrogenas & 3,28 & chr11 \\
\hline DLD & 1738 & Dihydrolipoamide dehydrogenase (E3 component of pyruvate dehydrogenase $c$ & 2,65 & chr7 \\
\hline DLEU1 & 10301 & deleted in lymphocytic leukemia, 1 & 2,07 & chr13 \\
\hline DLG3 & 1741 & discs, large homolog 3 (neuroendocrine-dlg, Drosophila) & 5,20 & $\operatorname{chrX}$ \\
\hline DLG7 & 9787 & discs, large homolog 7 (Drosophila) & 11,38 & chr14 \\
\hline DLL1 & 28514 & delta-like 1 (Drosophila) & 2,48 & chr6 \\
\hline DLNB14 & 338657 & similar to DLNB14 & 3,17 & chr11 \\
\hline DMAP1 & 55929 & DNA methyltransferase 1 associated protein 1 & 2,59 & chr1 \\
\hline DMXL1 & 1657 & Dmx-like 1 & 2,08 & chr5 \\
\hline DNA2L & 1763 & DNA2 DNA replication helicase 2-like (yeast) & 11,44 & chr10 \\
\hline DNAJA2 & 10294 & DnaJ (Hsp40) homolog, subfamily A, member 2 & 3,03 & chr16 \\
\hline DNAJA4 & 55466 & DnaJ (Hsp40) homolog, subfamily A, member 4 & 2,66 & chr15 \\
\hline DNAJB6 & 10049 & DnaJ (Hsp40) homolog, subfamily B, member 6 & 3,26 & chr7 \\
\hline DNAJC17 & 55192 & DnaJ (Hsp40) homolog, subfamily C, member 17 & 2,38 & chr15 \\
\hline DNAJC19 & 131118 & DnaJ (Hsp40) homolog, subfamily C, member 19 & 2,65 & chr14 \\
\hline DNAJC7 & 7266 & DnaJ (Hsp40) homolog, subfamily C, member 7 & 3,31 & chr17 \\
\hline DNAJC9 & 23234 & DnaJ (Hsp40) homolog, subfamily C, member 9 & 3,02 & chr10 \\
\hline DNCH2 & 79659 & dynein, cytoplasmic, heavy polypeptide 2 & 2,71 & chr11 \\
\hline DND1 & 373863 & dead end homolog 1 (zebrafish) & 5,67 & chr17 \\
\hline DNMT1 & 1786 & DNA (cytosine-5-)-methyltransferase 1 & 2,76 & chr19 \\
\hline DNMT2 & 1787 & DNA (cytosine-5-)-methyltransferase 2 & 4,47 & chr10 \\
\hline DNMT3A & 1788 & DNA (cytosine-5-)-methyltransferase 3 alpha & 17,15 & chr2 \\
\hline DNMT3B & 1789 & DNA (cytosine-5-)-methyltransferase 3 beta & 162,94 & chr20 \\
\hline DNPEP & 23549 & aspartyl aminopeptidase & 2,07 & chr2 \\
\hline DOCK3 & 1795 & dedicator of cytokinesis 3 & 2,22 & chr3 \\
\hline DOCK4 & 9732 & dedicator of cytokinesis 4 & 2,49 & chr7 \\
\hline DONSON & 29980 & downstream neighbor of SON & 3,03 & chr21 \\
\hline DOT1L & 84444 & DOT1-like, histone H3 methyltransferase (S. cerevisiae) & 2,17 & chr19 \\
\hline DPH2 & 1802 & DPH2 homolog (S. cerevisiae) & 3,48 & chr1 \\
\hline DPH5 & 51611 & DPH5 homolog (S. cerevisiae) & 2,40 & chr1 \\
\hline DPP3 & 10072 & dipeptidylpeptidase 3 & 2,39 & chr11 \\
\hline DPPA2 & 151871 & developmental pluripotency associated 2 & 13,48 & chr3 \\
\hline DPPA3 & 359787 & developmental pluripotency associated 3 & 8,51 & chr14 \\
\hline DPPA4 & 55211 & developmental pluripotency associated 4 & 175,49 & chr3 \\
\hline DPPA5 & 340168 & developmental pluripotency associated 5 & 4,27 & chr6 \\
\hline DPYSL3 & 1809 & dihydropyrimidinase-like 3 & 6,42 & chr5 \\
\hline DRIM & 27340 & down-regulated in metastasis & 2,11 & chr12 \\
\hline DSC2 & 1824 & desmocollin 2 & 18,30 & chr18 \\
\hline DSU & 55686 & dilute suppressor & 14,11 & chr2 \\
\hline DTL & 51514 & denticleless homolog (Drosophila) & 13,63 & chr1 \\
\hline DTNA & 1837 & dystrobrevin, alpha & 4,74 & chr18 \\
\hline DTX3 & 196403 & deltex 3 homolog (Drosophila) & 2,75 & chr12 \\
\hline DTX4 & 23220 & deltex 4 homolog (Drosophila) & 2,25 & chr11 \\
\hline DTYMK & 1841 & deoxythymidylate kinase (thymidylate kinase) & 2,48 & chr2_random \\
\hline DUS1L & 64118 & dihydrouridine synthase 1-like (S. cerevisiae) & 2,02 & chr17 \\
\hline
\end{tabular}




\begin{tabular}{|c|c|c|c|c|}
\hline DUS3L & 56931 & dihydrouridine synthase 3-like (S. cerevisiae) & 4,04 & chr19 \\
\hline DUS4L & 11062 & dihydrouridine synthase 4 -like (S. cerevisiae) & 3,95 & $\operatorname{chr} 7$ \\
\hline DUSP16 & 80824 & dual specificity phosphatase 16 & 5,74 & chr12 \\
\hline DUSP6 & 1848 & dual specificity phosphatase 6 & 2,83 & chr12 \\
\hline DUT & 1854 & dUTP pyrophosphatase & 13,41 & chr15 \\
\hline DVL2 & 1856 & dishevelled, dsh homolog 2 (Drosophila) & 2,32 & chr17 \\
\hline DXS9879E & 8270 & DNA segment on chromosome X (unique) 9879 expressed sequence & 3,77 & $\operatorname{chrX}$ \\
\hline DYNLL2 & 140735 & Dynein, light chain, LC8-type 2 & 2,10 & chr17 \\
\hline DZIP3 & 9666 & zinc finger DAZ interacting protein 3 & 4,45 & chr3 \\
\hline E2-230K & 63893 & likely ortholog of mouse ubiquitin-conjugating enzyme E2-230K & 3,21 & chr17 \\
\hline E2F1 & 1869 & E2F transcription factor 1 & 2,23 & chr20 \\
\hline E2F3 & 1871 & E2F transcription factor 3 & 2,45 & chr6 \\
\hline E2F5 & 1875 & E2F transcription factor 5, p130-binding & 6,01 & chr8 \\
\hline E2F8 & 79733 & E2F transcription factor 8 & 6,77 & chr11 \\
\hline E2IG5 & 26355 & growth and transformation-dependent protein & 3,50 & chr3 \\
\hline EAF2 & 55840 & ELL associated factor 2 & 2,93 & chr3 \\
\hline EBAG9 & 9166 & estrogen receptor binding site associated, antigen, 9 & 2,22 & chr8 \\
\hline EBP & 10682 & emopamil binding protein (sterol isomerase) & 3,70 & $\operatorname{chrX}$ \\
\hline EBPL & 84650 & emopamil binding protein-like & 2,70 & $\operatorname{chr} 13$ \\
\hline ECAT11 & 54596 & hypothetical protein FLJ10884 & 671,16 & chr1 \\
\hline $\mathrm{ECH} 1$ & 1891 & enoyl Coenzyme A hydratase 1, peroxisomal & 2,55 & chr19 \\
\hline ECHDC2 & 55268 & enoyl Coenzyme A hydratase domain containing 2 & 2,49 & chr1 \\
\hline ECHDC3 & 79746 & enoyl Coenzyme A hydratase domain containing 3 & 2,64 & $\operatorname{chr} 10$ \\
\hline ECT2 & 1894 & epithelial cell transforming sequence 2 oncogene & 3,24 & chr3 \\
\hline EDD1 & 51366 & E3 ubiquitin protein ligase, HECT domain containing, 1 & 3,16 & chr8 \\
\hline EDG4 & 9170 & endothelial differentiation, lysophosphatidic acid G-protein-coupled receptor, 4 & 13,14 & chr19 \\
\hline EDG7 & 23566 & Endothelial differentiation, lysophosphatidic acid G-protein-coupled receptor, 7 & 9,01 & chr1 \\
\hline EDNRB & 1910 & endothelin receptor type B & 22,33 & chr13 \\
\hline EED & 8726 & embryonic ectoderm development & 2,55 & chr11 \\
\hline EEF1E1 & 9521 & eukaryotic translation elongation factor 1 epsilon 1 & 2,97 & chr6 \\
\hline EFHC1 & 114327 & EF-hand domain (C-terminal) containing 1 & 2,26 & chr6 \\
\hline EFNA4 & 1945 & ephrin-A4 & 2,45 & chr1 \\
\hline EFNA5 & 1946 & Ephrin-A5 & 3,06 & chr5 \\
\hline EFNB3 & 1949 & ephrin-B3 & 2,29 & chr17 \\
\hline EFTUD1 & 79631 & elongation factor Tu GTP binding domain containing 1 & 3,56 & chr15 \\
\hline EGLN3 & 112399 & egl nine homolog 3 (C. elegans) & 3,49 & chr14 \\
\hline EHHADH & 1962 & enoyl-Coenzyme A, hydratase/3-hydroxyacyl Coenzyme A dehydrogenase & 2,01 & chr3 \\
\hline EHMT1 & 79813 & euchromatic histone-lysine $\mathrm{N}$-methyltransferase 1 & 3,31 & chr9 \\
\hline EHMT2 & 10919 & euchromatic histone-lysine $\mathrm{N}$-methyltransferase 2 & 4,70 & chr6 \\
\hline EID3 & 493861 & E1A-like inhibitor of differentiation 3 & 2,08 & chr12 \\
\hline EIF1AX & 1964 & eukaryotic translation initiation factor $1 \mathrm{~A}, \mathrm{X}$-linked & 3,04 & chr1 \\
\hline EIF2AK4 & 440275 & eukaryotic translation initiation factor 2 alpha kinase 4 & 2,59 & chr15 \\
\hline EIF2B4 & 8890 & eukaryotic translation initiation factor $2 \mathrm{~B}$, subunit 4 delta, $67 \mathrm{kDa}$ & 2,18 & chr2 \\
\hline EIF2C2 & 27161 & Eukaryotic translation initiation factor $2 \mathrm{C}, 2$ & 3,65 & chr15 \\
\hline EIF2S2 & 8894 & Eukaryotic translation initiation factor 2 , subunit 2 beta, $38 \mathrm{kDa}$ & 2,19 & chr2 \\
\hline EIF3S10 & 8661 & eukaryotic translation initiation factor 3 , subunit 10 theta, $150 / 170 \mathrm{kDa}$ & 2,07 & $\operatorname{chr} 10$ \\
\hline EIF3S12 & 27335 & eukaryotic translation initiation factor 3 , subunit 12 & 2,73 & chr19 \\
\hline EIF3S4 & 8666 & eukaryotic translation initiation factor 3 , subunit 4 delta, $44 \mathrm{kDa}$ & 2,04 & chr19 \\
\hline EIF3S8 & 8663 & eukaryotic translation initiation factor 3 , subunit $8,110 \mathrm{kDa}$ & 2,74 & chr16 \\
\hline EIF3S9 & 8662 & eukaryotic translation initiation factor 3 , subunit 9 eta, $116 \mathrm{kDa}$ & 5,05 & chr7 \\
\hline EIF4A1 & 1973 & eukaryotic translation initiation factor $4 \mathrm{~A}$, isoform $1 / / /$ eukaryotic translation initia & 2,05 & chr12 \\
\hline EIF4E & 1977 & eukaryotic translation initiation factor $4 \mathrm{E}$ & 2,83 & chr4 \\
\hline EIF4E3 & 317649 & eukaryotic translation initiation factor $4 \mathrm{E}$ member 3 & 3,95 & chr3 \\
\hline EIF4EBP2 & 1979 & eukaryotic translation initiation factor $4 \mathrm{E}$ binding protein 2 & 7,55 & $\operatorname{chr} 10$ \\
\hline EIF4G3 & 8672 & Eukaryotic translation initiation factor 4 gamma, 3 & 2,36 & chr1 \\
\hline EIF5 & 1983 & eukaryotic translation initiation factor 5 & 2,34 & chr14 \\
\hline ELAC2 & 60528 & elaC homolog 2 (E. coli) & 2,36 & chr17 \\
\hline ELAVL1 & 1994 & ELAV (embryonic lethal, abnormal vision, Drosophila)-like 1 (Hu antigen R) & 2,03 & chr19 \\
\hline ELAVL2 & 1993 & ELAV (embryonic lethal, abnormal vision, Drosophila)-like 2 (Hu antigen B) & 8,46 & chr9 \\
\hline ELL3 & 80237 & elongation factor RNA polymerase II-like 3 & 2,15 & chr15 \\
\hline ELOVL6 & 79071 & ELOVL family member 6 , elongation of long chain fatty acids (FEN1/Elo2, SUR4 & 5,58 & chr4 \\
\hline ELOVL7 & 79993 & ELOVL family member 7 , elongation of long chain fatty acids (yeast) & 14,29 & chr5 \\
\hline ELP4 & 26610 & elongation protein 4 homolog (S. cerevisiae) & 2,12 & chr11 \\
\hline EME1 & 146956 & essential meiotic endonuclease 1 homolog 1 (S. pombe) & 2,93 & chr17 \\
\hline EMID2 & 136227 & EMI domain containing 2 & 5,51 & chr7 \\
\hline EML4 & 27436 & echinoderm microtubule associated protein like 4 & 2,26 & chr2 \\
\hline ENO1 & $2023 / / / 6636$ & enolase 1, (alpha) /// small nuclear ribonucleoprotein polypeptide F & 4,19 & chr12 \\
\hline
\end{tabular}




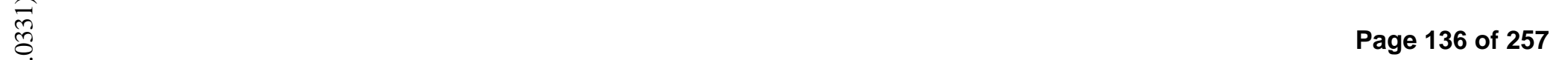

\begin{tabular}{|c|c|c|c|c|}
\hline ENO2 & 2026 & Lonlace 2 (a amma neurnnal) & & \\
\hline ENO3 & & & & \\
\hline ENOSF1 & & & & \\
\hline EINUST I & 55556 & enolase superfamily member 1 & 2,85 & chr18 \\
\hline ENPP1 & 5167 & ectonucleotide pyrophosphatase/phosphodiesterase 1 & 4,19 & chr6 \\
\hline ENPP2 & 5168 & ectonucleotide pyrophosphatase/phosphodiesterase 2 (autotaxin) & 3,22 & chr8 \\
\hline ENPP4 & 22875 & ectonucleotide pyrophosphatase/phosphodiesterase 4 (putative function) & 4,56 & chr6 \\
\hline ENPP5 & 59084 & ectonucleotide pyrophosphatase/phosphodiesterase 5 (putative function) & 3,14 & chr6 \\
\hline ENSA & 2029 & endosulfine alpha & 3,90 & chr1 \\
\hline ENY2 & 56943 & enhancer of yellow 2 homolog (Drosophila) & 2,13 & chr8 \\
\hline EOMES & 8320 & eomesodermin homolog (Xenopus laevis) & 3,86 & chr3 \\
\hline EP300 & 2033 & E1A binding protein $\mathrm{p} 300$ & 2,85 & chr22 \\
\hline EP400 & 57634 & E1A binding protein $\mathrm{p} 400$ & 2,39 & chr12 \\
\hline EPB41 & 2035 & Erythrocyte membrane protein band 4.1 (elliptocytosis $1, \mathrm{RH}$-linked) & 8,93 & chr1 \\
\hline EPB41L2 & 2037 & erythrocyte membrane protein band 4.1 -like 2 & 2,38 & chr6 \\
\hline EPB41L4B & 54566 & erythrocyte membrane protein band 4.1 like $4 \mathrm{~B}$ & 8,75 & chr9 \\
\hline EPB41L5 & 57669 & erythrocyte membrane protein band 4.1 like 5 & 9,76 & chr2 \\
\hline EPC2 & 26122 & enhancer of polycomb homolog 2 (Drosophila) & 2,34 & chr2 \\
\hline EPHA1 & 2041 & EPH receptor A1 & 14,27 & $\mathrm{chr} 7$ \\
\hline EPHA7 & 2045 & EPH receptor A7 & 5,60 & chr6 \\
\hline EPHB1 & 2047 & EPH receptor B1 & 3,65 & chr3 \\
\hline EPHB4 & 2050 & EPH receptor B4 & 2,21 & chr7 \\
\hline EPPK1 & 83481 & epiplakin 1 & 7,19 & chr8 \\
\hline EPS8L1 & 54869 & EPS8-like 1 & 5,16 & chr19 \\
\hline EPS8L2 & 64787 & EPS8-like 2 & 5,61 & chr11 \\
\hline ERBB2 & 2064 & v-erb-b2 erythroblastic leukemia viral oncogene homolog 2, neuro/glioblastoma & 4,29 & chr17 \\
\hline ERBB3 & 2065 & v-erb-b2 erythroblastic leukemia viral oncogene homolog 3 (avian) & 33,97 & chr12 \\
\hline ERBP & 30836 & estrogen receptor binding protein & 2,63 & chr1 \\
\hline ERCC3 & 2071 & excision repair cross-complementing rodent repair deficiency, complementation & 2,30 & chr2 \\
\hline ERH & 2079 & enhancer of rudimentary homolog (Drosophila) /// enhancer of rudimentary hom & 2,41 & chr14 \\
\hline ERP29 & 10961 & endoplasmic reticulum protein 29 & 3,32 & chr12 \\
\hline ESCO1 & 114799 & establishment of cohesion 1 homolog 1 (S. cerevisiae) & 4,22 & chr18 \\
\hline ESPL1 & 9700 & extra spindle poles like 1 (S. cerevisiae) & 13,37 & chr12 \\
\hline ESRRG & 2104 & estrogen-related receptor gamma & 2,43 & chr1 \\
\hline EST1B & 23381 & Est1p-like protein B & 2,16 & chr1 \\
\hline ETF1 & 2107 & eukaryotic translation termination factor 1 & 2,06 & chr5 \\
\hline ETNK1 & 55500 & Ethanolamine kinase 1 & 3,92 & chr12 \\
\hline ETV1 & 2115 & ets variant gene 1 & 5,25 & chr7 \\
\hline ETV4 & 2118 & ets variant gene 4 ( $E 1 \mathrm{~A}$ enhancer binding protein, $\mathrm{E} 1 \mathrm{AF}$ ) /// ets variant gene 4 ( & 3,51 & chr22 \\
\hline EWSR1 & 2130 & Ewing sarcoma breakpoint region 1 & 2,96 & chr22 \\
\hline EXO1 & 9156 & exonuclease 1 & 6,17 & chr1 \\
\hline EXOSC1 & 51013 & exosome component 1 & 2,14 & chr10 \\
\hline EXOSC10 & 5394 & exosome component 10 & 2,25 & chr1 \\
\hline EXOSC2 & 23404 & exosome component 2 & 5,05 & chr9 \\
\hline EXOSC3 & 51010 & exosome component 3 & 5,76 & chr9 \\
\hline EXOSC4 & 54512 & exosome component 4 & 2,62 & chr8 \\
\hline EXOSC5 & 56915 & exosome component 5 & 4,29 & chr19 \\
\hline EXOSC7 & 23016 & exosome component 7 & 4,46 & chr3 \\
\hline EXOSC8 & 11340 & exosome component 8 & 3,75 & chr13 \\
\hline EXOSC9 & 5393 & exosome component 9 & 3,99 & chr4 \\
\hline EZH2 & 2146 & enhancer of zeste homolog 2 (Drosophila) & 5,64 & $\mathrm{chr} 7$ \\
\hline F11R & 50848 & F11 receptor & 56,82 & chr1 \\
\hline FABP5 & 2171 & fatty acid binding protein 5 (psoriasis-associated) & 6,51 & chr7 \\
\hline FABP7 & 2173 & fatty acid binding protein 7 , brain & 12,13 & $\operatorname{chr} 6$ \\
\hline FADS1 & 3992 & fatty acid desaturase 1 & 2,67 & chr11 \\
\hline FAF1 & 11124 & Fas (TNFRSF6) associated factor 1 & 2,37 & chr1 \\
\hline FAHD2A & 51011 & fumarylacetoacetate hydrolase domain containing $2 \mathrm{~A}$ & 2,01 & chr2 \\
\hline FAIM & 55179 & Fas apoptotic inhibitory molecule & 3,11 & chr3 \\
\hline FALZ & 2186 & fetal Alzheimer antigen & 17,12 & chr17 \\
\hline FAM20A & 54757 & Family with sequence similarity 20 , member $\mathrm{A}$ & 2,63 & chr17 \\
\hline FAM29A & 54801 & family with sequence similarity 29 , member A & 5,17 & chr7 \\
\hline FAM33A & 348235 & family with sequence similarity 33 , member A & 3,05 & chr17 \\
\hline FAM44B & 91272 & family with sequence similarity 44 , member B & 2,74 & chr5 \\
\hline FAM46B & 115572 & family with sequence similarity 46 , member B & 28,85 & chr1 \\
\hline FAM48A & 55578 & family with sequence similarity 48 , member $\mathrm{A}$ & 2,31 & chr13 \\
\hline FAM49B & 51571 & Family with sequence similarity 49 , member $B$ & 9,78 & chr8 \\
\hline FAM54A & 113115 & family with sequence similarity 54 , member A & 4,24 & chr6 \\
\hline FAM59A & 64762 & family with sequence similarity 59 , member A & 4,06 & $\operatorname{chr} 18$ \\
\hline
\end{tabular}




\section{Pagge 137 of 257}

\begin{tabular}{|c|c|c|c|c|}
\hline FAM59B & 150946 & family with sequence similarity 59 , member $B$ & 2,25 & chr2 \\
\hline FAM60A & 58516 & family with sequence similarity 60 , member $A$ & 8,19 & chr1 \\
\hline FAM61A & 26065 & family with sequence similarity 61 , member $A$ & 2,22 & chr19 \\
\hline FAM61B & 149986 & family with sequence similarity 61 , member $B$ & 2,10 & chr20 \\
\hline FAM62B & 57488 & family with sequence similarity 62 (C2 domain containing) member B & 2,08 & chr7 \\
\hline FAM64A & 54478 & Family with sequence similarity 64 , member $A$ & 11,88 & chr17 \\
\hline FAM72A & 389835 & family with sequence similarity 72 , member A & 12,60 & chr1 \\
\hline FAM76A & 199870 & Family with sequence similarity 76 , member $A$ & 3,37 & chr1 \\
\hline FAM76B & 143684 & family with sequence similarity 76 , member $B$ & 2,44 & chr11 \\
\hline FAM7A2 & 89839 & Family with sequence similarity 7 , member $\mathrm{A} 2$ & 2,05 & (vide) \\
\hline FAM80A & 284716 & family with sequence similarity 80 , member $A$ & 2,35 & chr1 \\
\hline FAM80B & 57494 & Family with sequence similarity 80 , member $B$ & 2,89 & chr12 \\
\hline FAM96A & 84191 & family with sequence similarity 96 , member A & 2,59 & chr15 \\
\hline FAM98B & 283742 & Family with sequence similarity 98 , member $B$ & 2,61 & chr15 \\
\hline FANCD2 & 2177 & Fanconi anemia, complementation group D2 & 11,33 & chr3 \\
\hline FANCE & 2178 & Fanconi anemia, complementation group $\mathrm{E}$ & 2,07 & chr6 \\
\hline FANCF & 2188 & Fanconi anemia, complementation group $\mathrm{F}$ & 3,58 & chr11 \\
\hline FANCG & 2189 & Fanconi anemia, complementation group $\mathrm{G}$ & 6,29 & chr9 \\
\hline FANCL & 55120 & Fanconi anemia, complementation group L & 5,67 & chr2 \\
\hline FANCM & 57697 & Fanconi anemia, complementation group $\mathrm{M}$ & 2,86 & chr14 \\
\hline FARSLA & 2193 & phenylalanine-tRNA synthetase-like, alpha subunit & 2,12 & chr19 \\
\hline FASN & 2194 & fatty acid synthase & 2,76 & chr6 \\
\hline FBL & 2091 & fibrillarin /// fibrillarin & 3,66 & chr19 \\
\hline FBXL10 & 84678 & F-box and leucine-rich repeat protein 10 & 6,00 & chr12 \\
\hline FBXL16 & 146330 & F-box and leucine-rich repeat protein 16 & 2,65 & chr16 \\
\hline FBXL20 & 84961 & F-box and leucine-rich repeat protein 20 & 2,20 & chr17 \\
\hline FBXL6 & 26233 & F-box and leucine-rich repeat protein 6 & 2,28 & chr8 \\
\hline FBXL77 & 23194 & F-box and leucine-rich repeat protein 7 & 2,12 & chr5 \\
\hline FBXO11 & 80204 & F-box protein 11 & 2,68 & chr2 \\
\hline FBXO2 & 26232 & F-box protein 2 & 4,30 & chr1 \\
\hline FBXO25 & 26260 & F-box protein 25 & 4,62 & chr8 \\
\hline FBXO28 & 23219 & F-box protein 28 & 3,91 & chr1 \\
\hline FBXO41 & 150726 & F-box protein 41 & 2,25 & chr2 \\
\hline FBXO45 & 200933 & F-box protein 45 & 2,45 & chr3 \\
\hline FBXO5 & 26271 & F-box protein 5 & 5,11 & chr6 \\
\hline FBXW4 & 6468 & F-box and WD-40 domain protein 4 & 2,29 & chr10 \\
\hline FDFT1 & 2222 & farnesyl-diphosphate farnesyltransferase 1 & 2,88 & chr8 \\
\hline FDPS & 2224 & farnesyl diphosphate synthase (farnesyl pyrophosphate synthetase, dimethylally & 2,62 & chr1 \\
\hline FEM1A & 55527 & Fem-1 homolog a (C.elegans) & 2,10 & chr13 \\
\hline FEN1 & 2237 & flap structure-specific endonuclease 1 & 7,50 & chr11 \\
\hline FGD4 & 121512 & FYVE, RhoGEF and PH domain containing 4 & 19,15 & chr12 \\
\hline FGD5 & 152273 & FYVE, RhoGEF and PH domain containing 5 & 2,51 & chr3 \\
\hline FGD6 & 55785 & FYVE, RhoGEF and PH domain containing 6 & 2,42 & chr12 \\
\hline FGF11 & 2256 & fibroblast growth factor 11 & 2,99 & chr17 \\
\hline FGF13 & 2258 & fibroblast growth factor 13 & 32,75 & $\operatorname{chrX}$ \\
\hline FGF19 & 9965 & fibroblast growth factor 19 & 3,16 & chr11 \\
\hline FGF4 & 2249 & fibroblast growth factor 4 (heparin secretory transforming protein 1, Kaposi sarcd & 12,46 & chr11 \\
\hline FGFR1 & 2260 & Fibroblast growth factor receptor 1 (fms-related tyrosine kinase 2, Pfeiffer syndrd & 7,15 & chr8 \\
\hline FGFR1OP & 11116 & FGFR1 oncogene partner & 3,43 & chr6 \\
\hline FGFR2 & 2263 & fibroblast growth factor receptor 2 (bacteria-expressed kinase, keratinocyte grow & 28,00 & chr10 \\
\hline FGFR3 & 2261 & fibroblast growth factor receptor 3 (achondroplasia, thanatophoric dwarfism) & 13,26 & chr4 \\
\hline FGFR4 & 2264 & fibroblast growth factor receptor 4 & 4,27 & chr5 \\
\hline FIGN & 55137 & Fidgetin & 3,25 & chr2 \\
\hline FIGNL1 & 63979 & fidgetin-like 1 & 2,64 & chr7 \\
\hline FILIP1 & 27145 & filamin A interacting protein 1 & 6,89 & chr6 \\
\hline FKBP1B & 2281 & FK506 binding protein $1 \mathrm{~B}, 12.6 \mathrm{kDa}$ & 5,59 & chr2 \\
\hline FKBP3 & 2287 & FK506 binding protein $3,25 \mathrm{kDa}$ & 2,87 & chr14 \\
\hline FKBP4 & 2288 & FK506 binding protein 4, 59kDa & 3,43 & chr12 \\
\hline FKBP5 & 2289 & FK506 binding protein 5 & 5,45 & chr6 \\
\hline FKBPL & 63943 & FK506 binding protein like & 2,31 & chr6 \\
\hline FKSG14 & 64105 & leucine zipper protein FKSG14 & 3,99 & chr5 \\
\hline FLAD1 & 80308 & Fad1, flavin adenine dinucleotide synthetase, homolog (yeast) & 3,65 & chr1 \\
\hline FLJ10006 & 55677 & hypothetical protein FLJ10006 & 2,66 & chr2 \\
\hline FLJ10154 & 55082 & Hypothetical protein FLJ10154 & 2,39 & chr13 \\
\hline FLJ10213 & 55096 & hypothetical protein FLJ10213 & 2,62 & chr3 \\
\hline FLJ10374 & 55702 & hypothetical protein FLJ10374 & 2,74 & chr19 \\
\hline FLJ10379 & 55133 & hypothetical protein FLJ10379 & 2,26 & chr2 \\
\hline
\end{tabular}




\begin{tabular}{|c|c|c|c|c|c|}
\hline$\infty .0$ & Table S5: Ge & nes downregulate & din MPC compared to hES (Fold Change $>2 ; \alpha<0.05$ ) & & \\
\hline & FLJ10415 & 55139 & hypothetical protein FLJ10415 & 2,14 & chr2 \\
\hline o & FLJ10534 & 55720 & hypothetical protein FLJ10534 & 4,32 & chr17 \\
\hline & FLJ10652 & 55196 & hypothetical protein FLJ10652 & 19,55 & chr12 \\
\hline 它 & FLJ10700 & 55731 & hypothetical protein FLJ10700 & 3,52 & chr17 \\
\hline 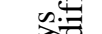 & FLJ10707 & 55209 & hypothetical protein FLJ10707 & 2,24 & chr3 \\
\hline$\lambda$ & FLJ10719 & 55215 & hypothetical protein FLJ10719 & 9,90 & chr15 \\
\hline$\Xi$ & FLJ10774 & 55226 & $\mathrm{~N}$-acetyltransferase-like protein & 4,48 & chr11 \\
\hline $\bar{E}$ & FLJ10803 & 55744 & hypothetical protein FLJ10803 & 3,73 & chr7 \\
\hline 0 & FLJ10847 & 55244 & hypothetical protein FLJ10847 & 2,03 & chr17 \\
\hline के & FLJ11021 & 65117 & similar to splicing factor, arginine/serine-rich 4 & 2,01 & chr12 \\
\hline $\mathrm{z}$ & FLJ11127 & 54491 & hypothetical protein FLJ11127 & 2,08 & chr5 \\
\hline పొర్ల & FLJ11200 & 55325 & hypothetical protein FLJ11200 & 2,10 & chr4 \\
\hline 욜 & FLJ11301 & 55341 & hypothetical protein FLJ11301 & 3,34 & chr3 \\
\hline 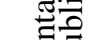 & FLJ11305 & 55795 & hypothetical protein FLJ11305 & 2,66 & chr13 \\
\hline 巴ِ & FLJ11712 & 79621 & hypothetical protein FLJ11712 & 2,68 & chr13 \\
\hline อีลส & FLJ11806 & 79882 & nuclear protein UKp68 & 3,09 & chr14 \\
\hline $0 \Xi$ & FLJ11850 & 64752 & hypothetical protein FLJ11850 & 2,38 & chr4 \\
\hline$\geq 0$ & FLJ12436 & 79714 & hypothetical protein FLJ12436 & 3,28 & chr3 \\
\hline E & FLJ12443 & 79888 & hypothetical protein FLJ12443 & 5,35 & chr5 \\
\hline$\stackrel{\dot{\Xi}}{>}$ & FLJ12505 & 79805 & hypothetical protein FLJ12505 & 13,72 & chr1 \\
\hline$\stackrel{ }{\exists}$ & FLJ12610 & 79840 & hypothetical protein FLJ12610 & 2,35 & chr2 \\
\hline ¿ & FLJ12684 & 79584 & hypothetical protein FLJ12684 & 5,45 & chr4 \\
\hline 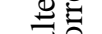 & FLJ12788 & 64427 & hypothetical protein FLJ12788 & 2,03 & chr2 \\
\hline is & FLJ12949 & 65095 & hypothetical protein FLJ12949 & 2,93 & chr19 \\
\hline 중 & FLJ13089 & 80018 & hypothetical protein FLJ13089 & 2,14 & chr12 \\
\hline 忞运 & FLJ13149 & 60493 & hypothetical protein FLJ13149 & 2,37 & chr20 \\
\hline$=$ & FLJ13213 & 79811 & modulator of estrogen induced transcription & 3,91 & chr15 \\
\hline$\tilde{z}=\tilde{z}$ & FLJ13220 & 60558 & hypothetical protein FLJ13220 & 2,52 & chr4 \\
\hline E o. & FLJ13273 & 79807 & hypothetical protein FLJ13273 & 2,61 & chr4 \\
\hline$\Xi$ & FLJ13305 & 84140 & hypothetical protein FLJ13305 & 7,27 & chr2 \\
\hline $\bar{\omega}$ & FLJ13491 & 79676 & hypothetical protein FLJ13491 & 2,20 & chr12 \\
\hline ప. & FLJ13614 & 84142 & hypothetical protein FLJ13614 & 2,51 & chr4 \\
\hline 8 & FLJ13848 & 79829 & hypothetical protein FLJ13848 & 2,69 & chr11 \\
\hline$\equiv$ & FLJ13909 & 80178 & hypothetical protein FLJ13909 & 7,82 & chr16 \\
\hline$\infty \equiv$ & FLJ13910 & 64795 & hypothetical protein FLJ13910 & 5,68 & chr2 \\
\hline ש & FLJ13912 & 64785 & hypothetical protein FLJ13912 & 3,44 & $\operatorname{chr} 16$ \\
\hline Uే & FLJ13984 & 79828 & hypothetical protein FLJ13984 & 3,46 & chr2 \\
\hline E잉 & FLJ14001 & 79730 & Hypothetical protein FLJ14001 & 6,58 & chr4 \\
\hline ๘ & FLJ14503 & 256714 & hypothetical protein FLJ14503 & 3,30 & $\operatorname{chrX}$ \\
\hline 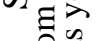 & FLJ14624 & 84899 & hypothetical protein FLJ14624 & 2,35 & chr13 \\
\hline ב & FLJ14627 & 84900 & hypothetical protein FLJ14627 & 3,49 & chr12 \\
\hline$\equiv$ & FLJ14668 & 84908 & hypothetical protein FLJ14668 & 3,18 & chr2 \\
\hline & FLJ14712 & 221806 & hypothetical protein FLJ14712 & 16,52 & chr7 \\
\hline$f$ & FLJ14827 & 84934 & hypothetical protein FLJ14827 & 2,46 & chr12 \\
\hline & FLJ14981 & 84954 & hypothetical protein FLJ14981 & 2,05 & chr19 \\
\hline ప.ల్ & FLJ20105 & 54821 & FLJ20105 protein & 7,60 & $\operatorname{chrX}$ \\
\hline$\overline{0}$ & FLJ20209 & 57245 & hypothetical protein FLJ20209 & 3,42 & chr3 \\
\hline ع & FLJ20211 & 54859 & hypothetical protein FLJ20211 & 2,75 & chr3 \\
\hline ة̈ & FLJ20273 & 54502 & RNA-binding protein & 38,26 & chr4 \\
\hline & FLJ20297 /// 月 & 150776 /// 55627 & hypothetical protein FLJ20297 /// FLJ41352 protein & 2,97 & chr2 \\
\hline ప్రు & FLJ20309 & 54891 & Hypothetical protein FLJ20309 & 3,09 & chr2 \\
\hline E्री & FLJ20323 & 54468 & hypothetical protein FLJ20323 /// hypothetical protein FLJ20323 & 2,94 & chr7 \\
\hline ల̄e्巳 & FLJ20397 & 54919 & hypothetical protein FLJ20397 & 2,88 & chr7 \\
\hline లిల్ర & FLJ20449 & 54937 & hypothetical protein FLJ20449 & 9,15 & chr13 \\
\hline$\stackrel{\Xi}{\Xi}$ & FLJ20485 & 54517 & hypothetical protein FLJ20485 & 2,75 & chr7 \\
\hline 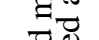 & FLJ20512 & 54958 & hypothetical protein FLJ20512 & 4,19 & $\operatorname{chr} 19$ \\
\hline$\equiv$ & FLJ20516 & 54962 & timeless-interacting protein & 4,34 & chr15 \\
\hline 巴ِ & FLJ20551 & 54977 & hypothetical protein FLJ20551 & 2,26 & chr3 \\
\hline$\exists$ & FLJ20582 & 54989 & hypothetical protein FLJ20582 & 2,97 & $\operatorname{chrX}$ \\
\hline $\bar{\Xi} \stackrel{T}{\square}$ & FLJ20628 & 55006 & hypothetical protein FLJ20628 /// hypothetical protein FLJ20628 & 2,81 & chr2 \\
\hline 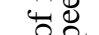 & FLJ20641 & 55010 & hypothetical protein FLJ20641 & 6,83 & chr12 \\
\hline & FLJ20643 & 55011 & hypothetical protein FLJ20643 & 2,54 & chr19 \\
\hline$\Xi \nsubseteq$ & FLJ20674 & 54621 & Hypothetical protein FLJ20674 & 10,63 & chr12 \\
\hline 0 & FLJ20758 & 55037 & FLJ20758 protein & 5,11 & chr2 \\
\hline డี & FLJ20859 & 64745 & FLJ20859 gene & 3,05 & chr14 \\
\hline $\bar{\pi}$ & FLJ21103 & 79607 & hypothetical protein FLJ21103 & 2,70 & chr11 \\
\hline$\overline{0} \cdot \bar{\Xi}$ & |FLJ21148 & 79918 & hypothetical protein FLJ21148 & 7,14 & $\operatorname{chr} 16$ \\
\hline
\end{tabular}




\section{Pagge 139 of 257}

\begin{tabular}{|c|c|c|c|c|}
\hline FLJ21168 & 80143 & hypothetical nrotein Fl 121168 // hynothetical protein FI 21168 & 205 & chr1 \\
\hline FLJ21816 & 79728 & hypothetical protein FLJ21816 & 3,96 & chr16 \\
\hline FLJ21839 & 60509 & hypothetical protein FLJ21839 & 2,65 & chr2 \\
\hline FLJ21865 & 64772 & endo-beta- $\mathrm{N}$-acetylglucosaminidase & 2,23 & chr17 \\
\hline FLJ21901 & 79675 & hypothetical protein FLJ21901 & 4,79 & chr2 \\
\hline FLJ21924 & 79832 & hypothetical protein FLJ21924 & 6,81 & chr11 \\
\hline FLJ21945 & 80304 & hypothetical protein FLJ21945 & 3,73 & chr2 \\
\hline FLJ21963 & 79611 & FLJ21963 protein & 7,45 & chr12 \\
\hline FLJ22104 & 65084 & hypothetical protein FLJ22104 & 2,64 & chr11 \\
\hline FLJ22318 & 64777 & hypothetical protein FLJ22318 & 2,99 & chr5 \\
\hline FLJ22531 & 79703 & hypothetical protein FLJ22531 & 3,97 & chr11 \\
\hline FLJ22555 & 79568 & hypothetical protein FLJ22555 & 2,57 & chr2 \\
\hline FLJ22624 & 79866 & FLJ22624 protein & 5,99 & chr13 \\
\hline FLJ22662 & 79887 & hypothetical protein FLJ22662 & 26,69 & chr12 \\
\hline FLJ22795 & 80154 & hypothetical protein FLJ22795 & 2,05 & chr15 \\
\hline FLJ23342 & 79684 & Hypothetical protein FLJ23342 & 3,02 & chr11 \\
\hline FLJ23441 & 79731 & hypothetical protein FLJ23441 & 3,91 & chr11 \\
\hline FLJ23556 & 79938 & hypothetical protein FLJ23556 & 6,18 & chr10 \\
\hline FLJ23861 & 151050 & hypothetical protein FLJ23861 & 2,76 & chr2 \\
\hline FLJ25006 & 124923 & hypothetical protein FLJ25006 & 6,04 & chr17 \\
\hline FLJ25076 & 134111 & similar to CG4502-PA & 4,90 & chr5 \\
\hline FLJ25222 & 374666 & CXYorf1-related protein & 2,71 & chr2 \\
\hline FLJ25416 & 220042 & hypothetical protein FLJ25416 & 9,94 & chr11 \\
\hline FLJ25778 & 254048 & Hypothetical protein FLJ25778 & 4,22 & chr7 \\
\hline FLJ25967 & 440823 & hypothetical gene supported by AK098833 & 27,57 & chr22 \\
\hline FLJ26175 & 388566 & FLJ26175 protein & 2,23 & chr19 \\
\hline FLJ27354 & 400761 & Hypothetical gene supported by AK 130864 & 14,74 & chr1 \\
\hline FLJ30046 & 122060 & hypothetical protein FLJ30046 & 15,55 & chr13 \\
\hline FLJ30428 & 150519 & similar to hypothetical protein A230046P18; cDNA sequence BC055759 & 8,21 & chr2 \\
\hline FLJ30656 & 124801 & hypothetical protein FLJ30656 & 2,81 & chr8 \\
\hline FLJ30707 & 220108 & hypothetical protein FLJ30707 & 12,56 & chr13 \\
\hline FLJ31204 & 158584 & hypothetical protein FLJ31204 & 2,64 & $\operatorname{chrX}$ \\
\hline FLJ31978 & 144423 & hypothetical protein FLJ31978 & 2,16 & chr12 \\
\hline FLJ32009 & 220001 & hypothetical protein FLJ32009 & 2,44 & chr11 \\
\hline FLJ32363 & 375444 & FLJ32363 protein & 3,57 & chr5 \\
\hline FLJ32452 & 93058 & hypothetical protein FLJ32452 & 3,62 & chr12 \\
\hline FLJ32499 & 124637 & hypothetical protein FLJ32499 & 2,51 & chr17 \\
\hline FLJ32745 & 165055 & hypothetical protein FLJ32745 & 4,98 & chr2 \\
\hline FLJ32810 & 143872 & hypothetical protein FLJ32810 & 2,59 & chr11 \\
\hline FLJ33008 & 145748 & hypothetical protein FLJ33008 & 2,13 & chr15 \\
\hline FLJ33318 & 162461 & hypothetical protein FLJ33318 & 2,43 & chr17 \\
\hline FLJ34208 & 401106 & Hypothetical gene supported by AK091527 & 2,22 & chr3 \\
\hline FLJ35119 & 126074 & hypothetical protein FLJ35119 & 3,15 & $\operatorname{chr} 19$ \\
\hline FLJ35348 & 266655 & FLJ35348 & 3,46 & chr9 \\
\hline FLJ35801 & 150291 & hypothetical protein FLJ35801 & 3,03 & chr22 \\
\hline FLJ35934 & $400579 / / / 40058$ & FLJ35934 protein /// hypothetical gene supported by AK093253 & 2,15 & chr17 \\
\hline FLJ35954 & 166968 & hypothetical protein FLJ35954 & 2,74 & chr5 \\
\hline FLJ36116 & 388666 & hypothetical locus LOC388666 & 9,18 & chr1 \\
\hline FLJ37478 & 339983 & Hypothetical protein LOC339983 & 2,89 & chr4 \\
\hline FLJ37953 & 129450 & hypothetical protein FLJ37953 & 2,11 & chr2 \\
\hline FLJ38426 & 283742 & hypothetical protein FLJ38426 & 2,24 & chr15 \\
\hline FLJ38973 & 205327 & hypothetical protein FLJ38973 & 4,57 & chr2 \\
\hline FLJ38991 & 285521 & mitochondrial COX18 & 2,14 & chr4 \\
\hline FLJ39616 & 51275 & apoptosis-related protein PNAS-1 & 2,64 & chr12 \\
\hline FLJ39739 & 388685 & FLJ39739 protein & 5,96 & chr1 \\
\hline FLJ40092 & 401196 & FLJ40092 protein & 6,32 & chr5 \\
\hline FLJ40142 & 400073 & FLJ40142 protein & 2,30 & chr12 \\
\hline FLJ40432 & 151195 & hypothetical protein FLJ40432 & 2,54 & chr2 \\
\hline FLJ40869 & 348654 & hypothetical protein FLJ40869 & 2,14 & chr2 \\
\hline FLJ41131 & 284325 & FLJ41131 protein & 2,32 & chr19 \\
\hline FLJ44186 & 346689 & FLJ44186 protein & 5,11 & chr7 \\
\hline FLJ46072 & 286077 & FLJ46072 protein & 7,62 & chr8 \\
\hline FLJ46419 & 388507 & FLJ46419 protein & 11,93 & chr19 \\
\hline FLJ90024 & 129303 & fasting-inducible integral membrane protein TM6P1 & 2,01 & chr2 \\
\hline FLJ90036 & 255403 & hypothetical protein FLJ90036 & 4,15 & chr4 \\
\hline FLJ90086 & 389389 & Similar to Al661453 protein & 4,72 & chr6 \\
\hline FLJ90231 & 283176 & hypothetical protein FLJ90231 & 18,33 & chr11 \\
\hline
\end{tabular}




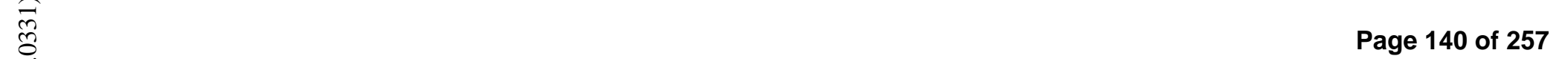

\begin{tabular}{|c|c|c|c|c|}
\hline FLJ90652 & 283899 & hypothetical protein FLJ90652 & 2,34 & chr16 \\
\hline FLT1 & 2321 & Fms-related tyrosine kinase 1 (vascular endothelial growth factor/vascular perm & 3,96 & $\operatorname{chr} 13$ \\
\hline FLVCR & 28982 & feline leukemia virus subgroup $\mathrm{C}$ cellular receptor & 15,20 & chr1 \\
\hline FN5 & 56935 & FN5 protein & 2,60 & chr11 \\
\hline FNBP1L & 54874 & Formin binding protein 1 -like & 4,18 & chr1 \\
\hline FNBP3 & 55660 & formin binding protein 3 & 2,53 & chr2 \\
\hline FOS & 2353 & v-fos FBJ murine osteosarcoma viral oncogene homolog & 3,11 & chr14 \\
\hline FOXA3 & 3171 & forkhead box A3 & 29,89 & chr19 \\
\hline FOXD3 & 27022 & Forkhead box D3 & 4,63 & chr1 \\
\hline FOXH1 & 8928 & Forkhead box H1 & 16,42 & chr8 \\
\hline FOXM1 & 2305 & forkhead box M1 & 5,94 & chr12 \\
\hline FOXO1A & 2308 & forkhead box O1A (rhabdomyosarcoma) & 10,79 & chr13 \\
\hline FOXO6 & 343552 & forkhead box protein $\mathrm{O} 6$ & 6,29 & chr1 \\
\hline FRAG1 & 27315 & FGF receptor activating protein 1 & 4,79 & chr11 \\
\hline FRAS1 & 80144 & Fraser syndrome 1 & 47,12 & chr4 \\
\hline FRAT1 & 10023 & frequently rearranged in advanced T-cell lymphomas & 4,48 & chr10 \\
\hline FRAT2 & 23401 & frequently rearranged in advanced T-cell lymphomas 2 & 28,64 & $\operatorname{chr} 10$ \\
\hline FREM2 & 341640 & FRAS1 related extracellular matrix protein 2 & 17,37 & chr13 \\
\hline FSD1 & 79187 & fibronectin type III and SPRY domain containing 1 & 3,17 & chr19 \\
\hline FSHPRH1 & 2491 & FSH primary response (LRPR1 homolog, rat) 1 & 2,45 & $\operatorname{chrX}$ \\
\hline FTSJ2 & 29960 & FtsJ homolog 2 (E. coli) & 2,61 & chr7 \\
\hline FTSJ3 & 117246 & FtsJ homolog 3 (E. coli) & 2,03 & chr17 \\
\hline FUBP1 & 8880 & Far upstream element (FUSE) binding protein 1 & 3,27 & chr1 \\
\hline FUS & 2521 & fusion (involved in $\mathrm{t}(12 ; 16)$ in malignant liposarcoma) & 6,51 & chr16 \\
\hline FUSIP1 & 10772 & FUS interacting protein (serine/arginine-rich) 1 & 2,83 & chr1_random \\
\hline FUT1 & 2523 & fucosyltransferase 1 (galactoside 2-alpha-L-fucosyltransferase) & 3,99 & chr12 \\
\hline FUT10 & 84750 & fucosyltransferase 10 (alpha $(1,3)$ fucosyltransferase) & 2,78 & chr8 \\
\hline FUT4 & 2526 & fucosyltransferase 4 (alpha $(1,3)$ fucosyltransferase, myeloid-specific) & 2,77 & chr11 \\
\hline FXN & 2395 & frataxin & 3,59 & chr9 \\
\hline FXR1 & 8087 & Fragile $\mathrm{X}$ mental retardation, autosomal homolog 1 & 3,89 & chr3 \\
\hline FXYD6 & 53826 & FXYD domain containing ion transport regulator 6 & 9,99 & chr11 \\
\hline FZD3 & 7976 & frizzled homolog 3 (Drosophila) & 11,16 & chr8 \\
\hline FZD5 & 7855 & frizzled homolog 5 (Drosophila) & 3,52 & chr2 \\
\hline G3BP & 10146 & Ras-GTPase-activating protein SH3-domain-binding protein & 3,99 & chr5 \\
\hline G3BP2 & 9908 & Ras-GTPase activating protein $\mathrm{SH} 3$ domain-binding protein 2 & 3,32 & chr4 \\
\hline GAB1 & 2549 & GRB2-associated binding protein 1 & 2,61 & chr4 \\
\hline GABBR1 & 2550 & gamma-aminobutyric acid (GABA) B receptor, 1 & 2,10 & chr6 \\
\hline GABPB2 & 2553 & GA binding protein transcription factor, beta subunit 2 & 3,21 & chr15 \\
\hline GABRB3 & 2562 & gamma-aminobutyric acid (GABA) A receptor, beta 3 & 100,50 & chr15 \\
\hline GABRB3 & $1653 / / / 2562$ & Gamma-aminobutyric acid (GABA) A receptor, beta 3 /// DEAD (Asp-Glu-Ala-As & 55,60 & chr15 \\
\hline GADD45GIP1 & 90480 & growth arrest and DNA-damage-inducible, gamma interacting protein 1 & 2,63 & chr19 \\
\hline GAJ & 84057 & GAJ protein & 13,42 & chr4 \\
\hline GAL & 51083 & galanin & 53,56 & chr11 \\
\hline GALNAC4S- 6 & 51363 & B cell RAG associated protein & 9,57 & chr10 \\
\hline GALNT12 & 79695 & UDP-N-acetyl-alpha-D-galactosamine:polypeptide $\mathrm{N}$-acetylgalactosaminyltransf & 13,20 & chr9 \\
\hline GALNT13 & 114805 & UDP-N-acetyl-alpha-D-galactosamine:polypeptide $\mathrm{N}$-acetylgalactosaminyltransf & 3,78 & chr2 \\
\hline GALNT3 & 2591 & UDP-N-acetyl-alpha-D-galactosamine:polypeptide N-acetylgalactosaminyltransfe & 12,62 & chr2 \\
\hline GAP43 & 2596 & growth associated protein 43 & 12,36 & chr3 \\
\hline GAPDHS & 26330 & Glyceraldehyde-3-phosphate dehydrogenase, spermatogenic & 4,10 & chr19 \\
\hline GARNL1 & 253959 & GTPase activating Rap/RanGAP domain-like 1 & 2,76 & chr9 \\
\hline GARNL4 & 23108 & GTPase activating Rap/RanGAP domain-like 4 & 4,66 & chr17 \\
\hline GART & 2618 & Phosphoribosylglycinamide formyltransferase, phosphoribosylglycinamide synth & 5,03 & chr21 \\
\hline GATAD1 & 57798 & GATA zinc finger domain containing 1 & 2,02 & chr7 \\
\hline GATAD2A & 54815 & GATA zinc finger domain containing $2 \mathrm{~A}$ & 2,63 & chr19 \\
\hline GATAD2B & 57459 & GATA zinc finger domain containing 2B & 2,09 & chr1 \\
\hline GATM & 2628 & glycine amidinotransferase (L-arginine:glycine amidinotransferase) & 3,72 & chr15 \\
\hline GBA2 & 57704 & Glucosidase, beta (bile acid) 2 & 3,20 & chr9 \\
\hline GCA & 25801 & grancalcin, EF-hand calcium binding protein /// grancalcin, EF-hand calcium bind & 9,28 & chr2 \\
\hline GCDH & 2639 & glutaryl-Coenzyme A dehydrogenase & 2,72 & $\operatorname{chr} 19$ \\
\hline $\mathrm{GCH} 1$ & 2643 & GTP cyclohydrolase 1 (dopa-responsive dystonia) & 3,95 & chr14 \\
\hline GCHFR & 2644 & GTP cyclohydrolase I feedback regulator & 3,42 & chr15 \\
\hline GCL & 64395 & Germ cell-less homolog 1 (Drosophila) & 2,43 & chr2 \\
\hline GCLC & 2729 & glutamate-cysteine ligase, catalytic subunit & 4,53 & chr6 \\
\hline GCNT1 & 2650 & glucosaminyl ( $\mathrm{N}$-acetyl) transferase 1 , core 2 (beta-1,6-N-acetylglucosaminyltrar & 2,73 & chr9 \\
\hline GCNT2 & 2651 & glucosaminyl (N-acetyl) transferase 2, I-branching enzyme & 51,06 & chr6 \\
\hline GCSH & 2653 & glycine cleavage system protein $\mathrm{H}$ (aminomethyl carrier) & 4,63 & chr5 \\
\hline GDAP1 & 54332 & Ganglioside-induced differentiation-associated protein 1 & 10,97 & chr8 \\
\hline
\end{tabular}




\section{Pagge 141 of 257}

\begin{tabular}{|c|c|c|c|c|}
\hline GDAP1L1 & 78997 & ganglioside-induced differentiation-associated protein 1 -like 1 & 2,36 & chr20 \\
\hline GDF1 & $10715 / / / 2657$ & growth differentiation factor 1 /// LAG1 longevity assurance homolog 1 (S. cerevi) & 2,46 & $\operatorname{chr} 19$ \\
\hline GDF3 & 9573 & growth differentiation factor 3 & 20,30 & chr12 \\
\hline GDPD2 & 54857 & glycerophosphodiester phosphodiesterase domain containing 2 & 2,16 & $\operatorname{chrX}$ \\
\hline GEMIN4 & 50628 & gem (nuclear organelle) associated protein 4 & 4,30 & chr17 \\
\hline GEMIN5 & 25929 & gem (nuclear organelle) associated protein 5 & 3,99 & chr5 \\
\hline GEMIN6 & 79833 & gem (nuclear organelle) associated protein 6 & 3,29 & chr2 \\
\hline GEMIN7 & 79760 & gem (nuclear organelle) associated protein 7 & 2,17 & chr19 \\
\hline GGA2 & 23062 & golgi associated, gamma adaptin ear containing, ARF binding protein 2 & 5,26 & chr16 \\
\hline GGA3 & 23163 & golgi associated, gamma adaptin ear containing, ARF binding protein 3 & 3,07 & chr17 \\
\hline GGT1 & 2678 & gamma-glutamyltransferase 1 & 3,51 & chr22 \\
\hline GIYD2 & $548593 / / / 79008$ & GIY-YIG domain containing $2 / / /$ GIY-YIG domain containing 1 & 2,03 & chr16 \\
\hline GJA7 & 10052 & gap junction protein, alpha $7,45 \mathrm{kDa}$ (connexin 45 ) & 3,90 & chr17 \\
\hline GK001 & 57003 & GK001 protein & 2,95 & chr17 \\
\hline GKAP1 & 80318 & G kinase anchoring protein 1 & 3,42 & chr9 \\
\hline GLCCI1 & 113263 & glucocorticoid induced transcript 1 & 3,81 & chr7 \\
\hline GLDC & 2731 & glycine dehydrogenase (decarboxylating; glycine decarboxylase, glycine cleavad & 87,67 & chr4 \\
\hline GLI2 & 2736 & GLI-Kruppel family member GLI2 & 3,07 & chr2 \\
\hline GLMN & 11146 & glomulin, FKBP associated protein & 4,11 & chr1 \\
\hline GLOXD1 & 84842 & glyoxalase domain containing 1 & 6,83 & chr1 \\
\hline GLS2 & 27165 & glutaminase 2 (liver, mitochondrial) & 4,33 & chr12 \\
\hline GLTSCR2 & 29997 & glioma tumor suppressor candidate region gene 2 & 3,40 & chr19 \\
\hline GLUL & 2752 & glutamate-ammonia ligase (glutamine synthetase) & 4,30 & chr1 \\
\hline GLYATL1 & 92292 & glycine-N-acyltransferase-like 1 & 2,12 & chr11 \\
\hline GMCL1 & 64395 & Germ cell-less homolog 1 (Drosophila) & 3,18 & chr2 \\
\hline GMDS & 2762 & GDP-mannose 4,6-dehydratase & 2,73 & chr6 \\
\hline GMEB1 & 10691 & glucocorticoid modulatory element binding protein 1 & 2,84 & chr1 \\
\hline GMNN & 51053 & geminin, DNA replication inhibitor & 5,59 & chr6 \\
\hline GMPS & 8833 & Guanine monphosphate synthetase & 2,47 & chr3 \\
\hline GNAL & 2774 & guanine nucleotide binding protein (G protein), alpha activating activity polypepti & 2,61 & chr18 \\
\hline GNAS & 2778 & GNAS complex locus & 6,61 & chr20 \\
\hline GNB2L1 & 10399 & Guanine nucleotide binding protein (G protein), beta polypeptide 2 -like 1 & 2,90 & chr5 \\
\hline GNG4 & 2786 & guanine nucleotide binding protein ( $G$ protein), gamma 4 & 3,72 & chr1 \\
\hline GNL3 & 26354 & guanine nucleotide binding protein-like 3 (nucleolar) & 2,91 & chr3 \\
\hline GNPDA1 & 10007 & glucosamine-6-phosphate deaminase 1 & 3,66 & chr5 \\
\hline GOLGA1 & 2800 & Golgi autoantigen, golgin subfamily a, 1 & 2,49 & chr9 \\
\hline GON4 & 54856 & gon-4 homolog (C.elegans) & 2,10 & chr1 \\
\hline GPAM & 57678 & glycerol-3-phosphate acyltransferase, mitochondrial & 2,38 & chr10 \\
\hline GPATC2 & 55105 & G patch domain containing 2 & 2,19 & chr1 \\
\hline GPATC4 & 54865 & G patch domain containing 4 & 4,91 & chr1 \\
\hline GPBP1 & 65056 & GC-rich promoter binding protein 1 & 3,62 & chr5 \\
\hline GPBP1L1 & 60313 & GC-rich promoter binding protein 1-like 1 & 2,17 & chr1 \\
\hline GPC2 & 221914 & glypican 2 (cerebroglycan) & 2,14 & chr7 \\
\hline GPC3 & 2719 & glypican 3 & 4,73 & $\operatorname{chrX}$ \\
\hline GPC4 & 2239 & glypican 4 & 52,71 & $\operatorname{chrX}$ \\
\hline GPD2 & 2820 & glycerol-3-phosphate dehydrogenase 2 (mitochondrial) & 2,36 & chr2 \\
\hline GPIAP1 & 4076 & GPI-anchored membrane protein 1 & 3,24 & chr11 \\
\hline GPM6B & 2824 & glycoprotein M6B & 63,43 & $\operatorname{chrX}$ \\
\hline GPR125 & 166647 & G protein-coupled receptor 125 & 3,73 & chr1 \\
\hline GPR143 & 4935 & G protein-coupled receptor 143 & 9,16 & $\operatorname{chrX}$ \\
\hline GPR160 & 26996 & G protein-coupled receptor 160 & 34,21 & chr3 \\
\hline GPR19 & 2842 & G protein-coupled receptor 19 & 28,34 & chr12 \\
\hline GPR23 & 2846 & G protein-coupled receptor 23 & 3,28 & $\operatorname{chrX}$ \\
\hline GPR27 & 2850 & G protein-coupled receptor 27 & 35,07 & chr3 \\
\hline GPR37 & 2861 & G protein-coupled receptor 37 (endothelin receptor type B-like) & 2,86 & chr7 \\
\hline GPR51 & 9568 & G protein-coupled receptor 51 & 2,04 & chr9 \\
\hline GPR54 & 84634 & G protein-coupled receptor 54 & 5,37 & chr19 \\
\hline GPR63 & 81491 & G protein-coupled receptor 63 /// G protein-coupled receptor 63 & 2,10 & chr6 \\
\hline GPR64 & 10149 & G protein-coupled receptor 64 & 24,81 & $\operatorname{chrX}$ \\
\hline GPRASP2 & 114928 & G protein-coupled receptor associated sorting protein 2 & 2,45 & $\operatorname{chrX}$ \\
\hline GPRC5B & 51704 & G protein-coupled receptor, family C, group 5 , member B & 55,30 & chr16 \\
\hline GPSN2 & 9524 & glycoprotein, synaptic 2 & 2,38 & chr19 \\
\hline GPX3 & 2878 & glutathione peroxidase 3 (plasma) & 4,57 & chr5 \\
\hline GRB10 & 2887 & growth factor receptor-bound protein 10 & 3,18 & chr7 \\
\hline GRB14 & 2888 & growth factor receptor-bound protein 14 & 5,66 & chr2 \\
\hline GREB1 & 9687 & GREB1 protein & 2,61 & chr2 \\
\hline GRHL1 & 29841 & grainyhead-like 1 (Drosophila) & 3,76 & chr2 \\
\hline
\end{tabular}




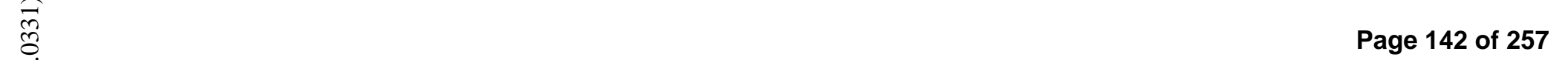

\begin{tabular}{|c|c|c|c|c|}
\hline GRHL2 & 79977 & grainyhead-like 2 (Drosophila) & 3,35 & chr8 \\
\hline GRIPAP1 & 56850 & GRIP1 associated protein 1 & 2,13 & $\operatorname{chr} X$ \\
\hline GRPEL2 & 134266 & GrpE-like 2, mitochondrial (E. coli) & 2,57 & chr5 \\
\hline GRSF1 & 2926 & G-rich RNA sequence binding factor 1 & 2,08 & chr4 \\
\hline GRTP1 & 79774 & Growth hormone regulated TBC protein 1 & 32,25 & chr13 \\
\hline GRWD1 & 83743 & glutamate-rich WD repeat containing 1 & 2,91 & chr19 \\
\hline GSDMDC1 & 79792 & gasdermin domain containing 1 & 2,70 & chr8 \\
\hline GSG2 & 83903 & germ cell associated 2 (haspin) & 2,20 & chr17 \\
\hline GSPT1 & 2935 & G1 to $S$ phase transition 1 & 2,51 & chr16 \\
\hline GSPT2 & 23708 & G1 to $S$ phase transition $2 / / / \mathrm{G} 1$ to $S$ phase transition 2 & 2,71 & $\operatorname{chr} \mathrm{X}$ \\
\hline GSR & 2936 & glutathione reductase & 2,04 & chr8 \\
\hline GSTO2 & 119391 & glutathione S-transferase omega 2 & 10,16 & chr10 \\
\hline GSTP1 & 2950 & glutathione S-transferase pi & 2,42 & chr11 \\
\hline GSTT1 & 2952 & Glutathione S-transferase theta 1 & 2,55 & chr22 \\
\hline GSTZ1 & 2954 & glutathione transferase zeta 1 (maleylacetoacetate isomerase) & 3,45 & chr14 \\
\hline GTF2E1 & 2960 & general transcription factor IIE, polypeptide 1 , alpha $56 \mathrm{kDa}$ & 3,49 & chr3 \\
\hline GTF2H2 & 2966 & general transcription factor IIH, polypeptide $2,44 \mathrm{kDa}$ & 4,18 & chr5 \\
\hline GTF2H4 & 2968 & general transcription factor IIH, polypeptide $4,52 \mathrm{kDa}$ & 4,38 & chr6 \\
\hline GTF3A & 2971 & general transcription factor IIIA & 2,66 & chr13 \\
\hline GTF3C1 & 2975 & general transcription factor IIIC, polypeptide 1 , alpha $220 \mathrm{kDa}$ & 2,41 & chr16 \\
\hline GTF3C2 & 2976 & general transcription factor IIIC, polypeptide 2 , beta $110 \mathrm{kDa}$ & 3,32 & chr2 \\
\hline GTF3C4 & 9329 & general transcription factor IIIC, polypeptide $4,90 \mathrm{kDa}$ & 2,54 & chr9 \\
\hline GTF3C5 & 9328 & general transcription factor IIIC, polypeptide $5,63 \mathrm{kDa}$ & 2,76 & chr9 \\
\hline GTPBP1 & 9567 & GTP binding protein 1 & 2,75 & chr22 \\
\hline GTPBP6 & 8225 & GTP binding protein 6 (putative) & 2,39 & chrX_random \\
\hline GTPBP7 & 92170 & GTP-binding protein 7 & 3,63 & chr10 \\
\hline GTSE1 & 51512 & G-2 and S-phase expressed 1 & 4,60 & chr22 \\
\hline GTSE1 & $440834 / / / 51512$ & G-2 and S-phase expressed $1 / / /$ hypothetical gene supported by BC069212 & 2,15 & chr22 \\
\hline GUCA1A & 2978 & guanylate cyclase activator $1 \mathrm{~A}$ (retina) & 17,64 & chr6 \\
\hline GULP1 & 51454 & GULP, engulfment adaptor PTB domain containing 1 & 3,94 & chr2 \\
\hline GUSB & 2990 & glucuronidase, beta & 2,09 & chr7 \\
\hline GYG2 & 8908 & glycogenin 2 & 9,77 & $\operatorname{chr} X$ \\
\hline GYLTL1B & 120071 & glycosyltransferase-like 1B & 5,12 & chr11 \\
\hline H17 & 55572 & hypothetical protein $\mathrm{H} 17$ & 3,97 & chr11 \\
\hline H1Fo & 3005 & $\mathrm{H} 1$ histone family, member 0 & 2,54 & chr22 \\
\hline $\mathrm{H} 1 \mathrm{FX}$ & 8971 & $H 1$ histone family, member $X$ & 2,50 & chr3 \\
\hline $\mathrm{H} 2 \mathrm{AFV}$ & 94239 & H2A histone family, member $V$ & 2,01 & $\operatorname{chr} 7$ \\
\hline H2AFX & 3014 & H2A histone family, member $X$ & 7,02 & chr11 \\
\hline H3F3А & 3020 & H3 histone, family $3 \mathrm{~A}$ & 2,68 & chr1 \\
\hline H3F3A & $3020 / / / 440926$ & $\mathrm{H} 3$ histone, family $3 \mathrm{~A} / / / \mathrm{H} 3$ histone, family $3 \mathrm{~A}$ pseudogene & 4,78 & chr2 \\
\hline HA-1 & 23526 & minor histocompatibility antigen HA-1 & 16,31 & chr19 \\
\hline HADH2 & 3028 & hydroxyacyl-Coenzyme A dehydrogenase, type II & 2,20 & $\operatorname{chrX}$ \\
\hline HADHSC & 3033 & L-3-hydroxyacyl-Coenzyme A dehydrogenase, short chain & 2,10 & chr4 \\
\hline HAS2 & 3037 & hyaluronan synthase 2 & 13,06 & chr8 \\
\hline HAS3 & 3038 & hyaluronan synthase 3 & 6,93 & chr16 \\
\hline HAT1 & 8520 & histone acetyltransferase 1 & 2,36 & chr2 \\
\hline HBII-276HG & 641638 & HBII-276 host gene & 2,33 & chr8 \\
\hline $\mathrm{HBII}-437 / / / \mathrm{H}$ & 338427 /// 347686 & HBII-437 C/D box snoRNA /// HBII-13 snoRNA & 6,22 & chr15 \\
\hline HBLD1 & 122961 & HESB like domain containing 1 & 2,22 & chr14 \\
\hline HBLD2 & 81689 & HESB like domain containing 2 & 3,00 & chr1 \\
\hline HBXAP & 51773 & hepatitis $B$ virus $\mathrm{x}$ associated protein & 2,27 & chr11 \\
\hline hCAP-D3 & 23310 & KIAA0056 protein & 3,31 & chr11 \\
\hline HCAP-G & 64151 & chromosome condensation protein $\mathrm{G}$ & 6,93 & chr4 \\
\hline hCAP-H2 & 29781 & kleisin beta & 2,98 & chr22 \\
\hline HCG12 & 493826 & HLA complex group 12 & 3,24 & chr6 \\
\hline HCG18 & 414777 & HLA complex group 18 & 2,34 & chr6 \\
\hline HCNGP & 29115 & transcriptional regulator protein & 2,16 & chr17 \\
\hline HCRP1 & 387535 & hepatocellular carcinoma-related HCRP1 & 2,38 & chr6 \\
\hline $\mathrm{HD}$ & 3064 & huntingtin (Huntington disease) & 2,24 & $\operatorname{chr} 4$ \\
\hline HDAC1 & 3065 & histone deacetylase 1 & 2,74 & chr1 \\
\hline HDAC2 & 3066 & Histone deacetylase 2 & 3,07 & chr6 \\
\hline HDAC3 & 8841 & histone deacetylase 3 & 2,19 & chr5 \\
\hline HDAC4 & 9759 & histone deacetylase 4 & 2,62 & chr2 \\
\hline HDAC5 & 10014 & histone deacetylase 5 & 3,52 & chr17 \\
\hline HDAC8 & 55869 & histone deacetylase 8 & 2,43 & $\operatorname{chr} X$ \\
\hline HDCMA18P & 51574 & HDCMA18P protein & 201,71 & chr4 \\
\hline HDDC2 & 51020 & HD domain containing 2 & 2,69 & chr6 \\
\hline
\end{tabular}




\section{Pagge 143 of 257}

\begin{tabular}{|c|c|c|c|c|}
\hline HDGF & 3068 & hepatoma-derived growth factor (high-mobility group protein 1 -like) & 203 & chr1 \\
\hline HDHD1A & 8226 & haloacid dehalogenase-like hydrolase domain containing $1 \mathrm{~A}$ & 2,01 & $\operatorname{chr} x$ \\
\hline HDHD2 & 84064 & haloacid dehalogenase-like hydrolase domain containing 2 & 3,02 & chr18 \\
\hline HEATR1 & 55127 & HEAT repeat containing 1 & 3,73 & chr1 \\
\hline HELLS & 3070 & helicase, lymphoid-specific & 22,76 & chr10 \\
\hline HERC5 & 51191 & hect domain and RLD 5 & 24,83 & chr4 \\
\hline HERC6 & 55008 & hect domain and RLD 6 & 3,91 & chr4 \\
\hline HES6 6 & 55502 & hairy and enhancer of split 6 (Drosophila) & 5,65 & chr2 \\
\hline HESX1 & 8820 & homeo box (expressed in ES cells) 1 & 2,43 & chr3 \\
\hline HEXIM2 & 124790 & hexamthylene bis-acetamide inducible 2 & 2,78 & chr17 \\
\hline HEY2 & 23493 & hairy/enhancer-of-split related with YRPW motif 2 & 13,03 & chr6 \\
\hline HIATL2 & 84278 & hippocampus abundant gene transcript-like 2 & 2,05 & chr9 \\
\hline HIC2 & 23119 & hypermethylated in cancer 2 & 7,88 & chr22 \\
\hline HIG2 & 29923 & hypoxia-inducible protein 2 & 3,72 & chr7 \\
\hline HINT2 & 84681 & histidine triad nucleotide binding protein $2 / / /$ histidine triad nucleotide binding pro & 2,27 & chr9 \\
\hline HIP1 & 3092 & Huntingtin interacting protein 1 & 4,66 & chr7 \\
\hline HIP2 & 3093 & Huntingtin interacting protein 2 & 4,67 & chr4 \\
\hline HIRIP3 & 8479 & HIRA interacting protein 3 & 2,75 & chr16 \\
\hline HIST1H1A & 3024 & histone $1, \mathrm{H} 1 \mathrm{a}$ & 9,81 & chr6 \\
\hline HIST1H1C & 3006 & histone $1, \mathrm{H} 1 \mathrm{c}$ & 2,82 & chr6 \\
\hline HIST1H1D & 3007 & histone $1, \mathrm{H} 1 \mathrm{~d}$ & 3,23 & chr6 \\
\hline HIST1H4K & $8362 / / / 8363$ & histone $1, \mathrm{H} 4 \mathrm{k} / / /$ histone $1, \mathrm{H} 4 \mathrm{j}$ & 4,42 & chr6 \\
\hline HIST2H4 & 8370 & Histone $\mathrm{H} 4 / \mathrm{O}$ & 3,09 & chr1 \\
\hline HIST3H2A & 92815 & histone 3, H2a & 2,39 & chr1 \\
\hline HK1 & 3098 & hexokinase 1 & 2,02 & chr10 \\
\hline HK2 & 3099 & hexokinase 2 & 3,51 & chr2 \\
\hline HKE2 & 10471 & HLA class II region expressed gene KE2 & 2,41 & chr6 \\
\hline HLA-DOA & 3111 & major histocompatibility complex, class II, DO alpha & 5,15 & chr6 \\
\hline HLA-DPA1 & 3113 & major histocompatibility complex, class II, DP alpha 1 & 2,60 & chr6 \\
\hline HLA-DPB1 & 3115 & major histocompatibility complex, class II, DP beta 1 & 2,74 & chr6 \\
\hline HLA-DPB2 & 3116 & major histocompatibility complex, class II, DP beta 2 (pseudogene) & 102,72 & chr6 \\
\hline HLA-DRB1 & 3123 & major histocompatibility complex, class II, DR beta $1 / / /$ major histocompatibility & 4,43 & chr6_hla_hap1 \\
\hline HLC-8 & 55028 & lung cancer-related protein 8 & 2,29 & chr17 \\
\hline HLRC1 & 83475 & HEAT-like (PBS lyase) repeat containing $1 / / /$ HEAT-like (PBS lyase) repeat cont & 4,51 & chr19 \\
\hline HMBS & 3145 & hydroxymethylbilane synthase & 3,70 & chr11 \\
\hline HMG4L & 128872 & high-mobility group (nonhistone chromosomal) protein 4-like & 4,88 & $\operatorname{chr} x$ \\
\hline HMGA1 & 3159 & high mobility group AT-hook 1 & 4,94 & chr6 \\
\hline HMGB1 & 3146 & high-mobility group box 1 & 4,12 & chr15 \\
\hline HMGB1 & $10357 / / / 3146$ & high-mobility group box 1 /// high-mobility group (nonhistone chromosomal) prote & 2,77 & chr3 \\
\hline HMGB2 & 3148 & high-mobility group box 2 & 2,31 & chr4 \\
\hline HMGB3 & 3149 & high-mobility group box 3 & 10,93 & chr1 \\
\hline HMGCR & 3156 & 3-hydroxy-3-methylglutaryl-Coenzyme A reductase & 3,67 & chr5 \\
\hline HMGCS1 & 3157 & 3-hydroxy-3-methylglutaryl-Coenzyme A synthase 1 (soluble) & 3,51 & chr5 \\
\hline HMGN3 & 9324 & high mobility group nucleosomal binding domain 3 & 4,02 & chr6 \\
\hline HMMR & 3161 & hyaluronan-mediated motility receptor (RHAMM) & 9,71 & chr5 \\
\hline HN1 & 51155 & hematological and neurological expressed 1 & 2,97 & chr17 \\
\hline HNRPAO & 10949 & Heterogeneous nuclear ribonucleoprotein $\mathrm{A} 0$ & 2,65 & chr5 \\
\hline HNRPA1 & 3178 & heterogeneous nuclear ribonucleoprotein A1 & 4,63 & chr12 \\
\hline HNRPA1 & /// $389674 / / / 39$ & heterogeneous nuclear ribonucleoprotein A1 /// similar to Hnrpa1 protein /// simil & 2,11 & chr5 \\
\hline HNRPA2B1 & 3181 & heterogeneous nuclear ribonucleoprotein A2/B1 & 2,61 & chr7 \\
\hline HNRPA3 & 220988 & heterogeneous nuclear ribonucleoprotein $\mathrm{A} 3$ & 3,44 & chr2 \\
\hline HNRPAB & 3182 & heterogeneous nuclear ribonucleoprotein $\mathrm{A} / \mathrm{B}$ & 2,84 & chr5 \\
\hline HNRPC & 3183 & heterogeneous nuclear ribonucleoprotein $\mathrm{C}(\mathrm{C} 1 / \mathrm{C} 2)$ & 2,84 & chr14 \\
\hline HNRPD & 3184 & Heterogeneous nuclear ribonucleoprotein D (AU-rich element RNA binding prote & 5,95 & chr4 \\
\hline HNRPDL & 9987 & heterogeneous nuclear ribonucleoprotein D-like & 3,45 & chr4 \\
\hline HNRPF & 3185 & heterogeneous nuclear ribonucleoprotein $\mathrm{F}$ & 4,97 & chr10 \\
\hline HNRPH1 & 3187 & heterogeneous nuclear ribonucleoprotein $\mathrm{H} 1(\mathrm{H})$ & 3,35 & chr5 \\
\hline HNRPH3 & 3189 & heterogeneous nuclear ribonucleoprotein $\mathrm{H} 3(2 \mathrm{H} 9)$ & 3,60 & chr10 \\
\hline HNRPK & 3190 & heterogeneous nuclear ribonucleoprotein $\mathrm{K}$ & 2,13 & chr3 \\
\hline HNRPM & 4670 & Heterogeneous nuclear ribonucleoprotein $\mathrm{M}$ & 5,58 & chr19 \\
\hline HNRPU & 3192 & heterogeneous nuclear ribonucleoprotein $\mathrm{U}$ (scaffold attachment factor $\mathrm{A}$ ) & 5,69 & chr1 \\
\hline HOMER1 & 9456 & homer homolog 1 (Drosophila) & 5,12 & chr5 \\
\hline HOOK1 & 51361 & Hook homolog 1 (Drosophila) & 24,88 & chr1 \\
\hline HOOK2 & 29911 & hook homolog 2 (Drosophila) & 2,68 & chr19 \\
\hline HOXA7 & 3204 & homeo box A7 & 5,12 & chr1 \\
\hline HPCL2 & 26061 & 2-hydroxyphytanoyl-CoA lyase & 5,99 & chr3 \\
\hline HPRT1 & 3251 & hypoxanthine phosphoribosyltransferase 1 (Lesch-Nyhan syndrome) & 2,88 & $\operatorname{chr} x$ \\
\hline
\end{tabular}




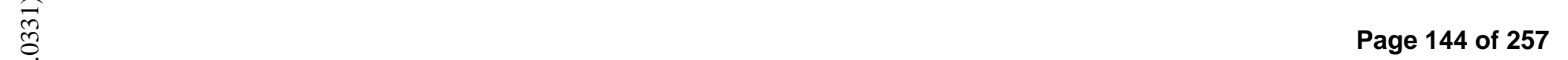

\begin{tabular}{|c|c|c|c|c|}
\hline HPS3 & 84343 & Hermansky-Pudlak syndrome 3 & 3,72 & chr3 \\
\hline HRASLS & 57110 & HRAS-like suppressor & 2,33 & chr3 \\
\hline HRASLS3 & 11145 & HRAS-like suppressor 3 & 61,46 & chr11 \\
\hline HRB & 3267 & HIV-1 Rev binding protein & 3,15 & chr2 \\
\hline HRB2 & 11103 & HIV-1 rev binding protein 2 & 2,28 & chr12 \\
\hline HRLP5 & 117245 & H-rev107-like protein 5 & 3,96 & chr11 \\
\hline HRMT1L3 & 10196 & HMT1 hnRNP methyltransferase-like 3 (S. cerevisiae) & 2,39 & $\operatorname{chr} 11$ \\
\hline HRMT1L6 & 55170 & HMT1 hnRNP methyltransferase-like 6 (S. cerevisiae) & 4,19 & chr1 \\
\hline HRSP12 & 10247 & heat-responsive protein 12 & 3,92 & chr8 \\
\hline HS3ST4 & 9951 & heparan sulfate (glucosamine) 3-O-sulfotransferase 4 & 3,19 & chr16 \\
\hline HS6ST1 & 9394 & heparan sulfate 6-O-sulfotransferase 1 & 2,30 & chr1 \\
\hline HS6ST2 & 90161 & heparan sulfate 6-O-sulfotransferase 2 & 5,56 & $\operatorname{chr} X$ \\
\hline HSCARG & 57407 & HSCARG protein & 2,02 & chr16 \\
\hline HSD11B2 & 3291 & hydroxysteroid (11-beta) dehydrogenase 2 & 2,05 & $\operatorname{chr} 16$ \\
\hline HSD17B4 & 3295 & hydroxysteroid (17-beta) dehydrogenase 4 & 6,62 & chr5 \\
\hline HSD17B7 & 51478 & hydroxysteroid (17-beta) dehydrogenase 7 & 3,01 & chr1 \\
\hline HSD17B8 & 7923 & hydroxysteroid (17-beta) dehydrogenase 8 & 2,46 & chr6 \\
\hline HSDL1 & 83693 & hydroxysteroid dehydrogenase like 1 & 2,44 & chr16 \\
\hline HSGT1 & 11319 & suppressor of S. cerevisiae gcr2 & 2,06 & chr10 \\
\hline HSPA14 & 51182 & heat shock $70 \mathrm{kDa}$ protein 14 & 4,39 & $\operatorname{chr} 10$ \\
\hline HSPA2 & 3306 & heat shock $70 \mathrm{kDa}$ protein 2 & 21,71 & chr14 \\
\hline HSPA4 & 3308 & Heat shock $70 \mathrm{kDa}$ protein 4 & 6,93 & chr5 \\
\hline HSPA8 & 3312 & heat shock $70 \mathrm{kDa}$ protein 8 & 2,23 & chr3 \\
\hline HSPA9B & 3313 & heat shock $70 \mathrm{kDa}$ protein $9 \mathrm{~B}$ (mortalin-2) & 2,34 & chr2 \\
\hline HSPBAP1 & 79663 & HSPB (heat shock $27 \mathrm{kDa}$ ) associated protein 1 & 2,50 & chr3 \\
\hline HSPC009 & 28958 & HSPC009 protein & 2,81 & chr17 \\
\hline HSPC065 & 29070 & HSPC065 protein & 2,38 & chr16 \\
\hline HSPC111 & 51491 & hypothetical protein HSPC111 & 5,41 & chr5 \\
\hline HSPC121 & 51495 & butyrate-induced transcript 1 & 3,15 & chr15 \\
\hline HSPC128 & 29080 & HSPC128 protein & 2,71 & chr12 \\
\hline HSPC138 & 51501 & hypothetical protein HSPC138 & 2,07 & chr11 \\
\hline HSPC159 & 29094 & HSPC159 protein & 3,29 & chr2 \\
\hline HSPC176 & 51693 & hematopoietic stem/progenitor cells 176 & 2,99 & chr16 \\
\hline HSPCB & 3326 & heat shock $90 \mathrm{kDa}$ protein 1 , beta & 4,16 & chr6 \\
\hline HSPD1 & 3329 & heat shock $60 \mathrm{kDa}$ protein 1 (chaperonin) & 6,10 & chr5 \\
\hline HSPE1 & 3336 & heat shock 10kDa protein 1 (chaperonin 10) & 4,12 & chr2 \\
\hline HSPH1 & 10808 & Heat shock $105 \mathrm{kDa} / 110 \mathrm{kDa}$ protein 1 & 2,38 & chr13 \\
\hline HSUP1 & 441951 & Similar to RPE-spondin & 6,50 & chr20 \\
\hline HT007 & 55863 & uncharacterized hypothalamus protein HT007 & 2,49 & chr11 \\
\hline HTATIP2 & 10553 & HIV-1 Tat interactive protein 2, 30kDa & 2,93 & chr11 \\
\hline HUNK & 30811 & hormonally upregulated Neu-associated kinase & 4,91 & chr21 \\
\hline HUWE1 & 10075 & HECT, UBA and WWE domain containing 1 & 2,40 & $\operatorname{chrX}$ \\
\hline |HYLS1 & 219844 & hydrolethalus syndrome 1 & 7,07 & chr11 \\
\hline ICA1 & 3382 & islet cell autoantigen $1,69 \mathrm{kDa}$ & 5,70 & chr7 \\
\hline ICT1 & 3396 & immature colon carcinoma transcript 1 & 2,85 & chr17 \\
\hline ID1 & 3397 & inhibitor of DNA binding 1, dominant negative helix-loop-helix protein & 5,59 & chr20 \\
\hline IDE & 3416 & insulin-degrading enzyme & 2,75 & $\operatorname{chr} 10$ \\
\hline IDH1 & 3417 & isocitrate dehydrogenase $1(\mathrm{NADP}+)$, soluble & 4,73 & chr2 \\
\hline IDH3B & 3420 & isocitrate dehydrogenase $3(\mathrm{NAD}+)$ beta & 2,73 & chr20 \\
\hline |IER2 & 9592 & immediate early response 2 & 2,99 & chr19 \\
\hline IFI30 & 10437 & interferon, gamma-inducible protein 30 & 4,77 & chr19 \\
\hline |IFITM1 & 8519 & interferon induced transmembrane protein $1(9-27)$ & 10,86 & chr11 \\
\hline |IGBP1 & 3476 & Immunoglobulin (CD79A) binding protein 1 & 2,16 & $\operatorname{chr} \mathrm{X}$ \\
\hline IGFBPL1 & 347252 & Insulin-like growth factor binding protein-like 1 & 17,18 & chr9 \\
\hline IGSF1 & 3547 & immunoglobulin superfamily, member 1 & 4,90 & $\operatorname{chr} \mathrm{X}$ \\
\hline IGSF3 & 3321 & immunoglobulin superfamily, member 3 & 25,76 & chr1 \\
\hline IGSF4D & 253559 & immunoglobulin superfamily, member 4D & 2,05 & chr3 \\
\hline IGSF9 & 57549 & immunoglobulin superfamily, member 9 & 2,96 & chr1 \\
\hline |HPK2 & 51447 & inositol hexaphosphate kinase 2 & 2,19 & chr3 \\
\hline IIIP45 & 60672 & invasion inhibitory protein 45 & 2,75 & chr1 \\
\hline |L17RB & 55540 & interleukin 17 receptor $B$ & 2,37 & chr3 \\
\hline |L17RD & 54756 & Interleukin 17 receptor D & 27,55 & chr3 \\
\hline IL19 & 29949 & Interleukin 19 & 4,99 & chr17 \\
\hline ILF2 & 3608 & interleukin enhancer binding factor $2,45 \mathrm{kDa} / / /$ interleukin enhancer binding fact & 2,26 & chr1 \\
\hline ILF3 & 3609 & interleukin enhancer binding factor $3,90 \mathrm{kDa}$ & 5,98 & chr19 \\
\hline IMMP1L & 196294 & IMP1 inner mitochondrial membrane peptidase-like (S. cerevisiae) & 2,58 & chr11 \\
\hline IMP-1 & 10642 & |GF-|| mRNA-binding protein 1 & 5,86 & chr17 \\
\hline
\end{tabular}




\section{Pagge 145 of 257}

\begin{tabular}{|c|c|c|c|c|}
\hline IMP3 & 55272 & IMP3, U3 small nucleolar ribonucleoprotein, homolog (yeast) & 6,06 & chr15 \\
\hline IMP-3 & 10643 & |GF-|| mRNA-binding protein 3 & 2,85 & chr7 \\
\hline IMPA2 & 3613 & inositol(myo)-1(or 4)-monophosphatase 2 & 23,92 & chr18 \\
\hline IMPACT & 55364 & hypothetical protein IMPACT & 2,30 & chr18 \\
\hline IMPDH2 & 3615 & IMP (inosine monophosphate) dehydrogenase 2 & 2,59 & chr3 \\
\hline INADL & 10207 & InaD-like (Drosophila) & 11,47 & chr1 \\
\hline INCENP & 3619 & Inner centromere protein antigens $135 / 155 \mathrm{kDa}$ & 5,59 & chr11 \\
\hline INDO & 3620 & indoleamine-pyrrole 2,3 dioxygenase & 29,10 & chr8 \\
\hline ING1 & 3621 & inhibitor of growth family, member 1 & 3,46 & chr13 \\
\hline ING2 & 3622 & inhibitor of growth family, member 2 & 2,03 & chr4 \\
\hline ING3 & 54556 & inhibitor of growth family, member 3 & 2,98 & chr7 \\
\hline ING5 & 84289 & inhibitor of growth family, member 5 & 5,27 & chr2_random \\
\hline INSR & 3643 & Insulin receptor & 8,58 & chr19 \\
\hline IPO11 & 51194 & importin 11 & 3,60 & chr5 \\
\hline IPO4 & 79711 & importin 4 & 2,07 & chr14 \\
\hline IPO9 & 55705 & Importin 9 & 2,28 & chr1 \\
\hline IPW & 3653 & imprinted in Prader-Willi syndrome & 6,14 & chr15 \\
\hline IQCA & 79781 & IQ motif containing with AAA domain & 3,32 & chr2 \\
\hline IQCB1 & 9657 & IQ motif containing B1 & 4,18 & chr3 \\
\hline IQGAP2 & 10788 & IQ motif containing GTPase activating protein 2 & 24,54 & chr5 \\
\hline IQGAP3 & 128239 & IQ motif containing GTPase activating protein 3 & 3,77 & chr1 \\
\hline IRAK1BP1 & 134728 & Interleukin-1 receptor-associated kinase 1 binding protein 1 & 3,80 & chr6 \\
\hline IRF3 & 3661 & interferon regulatory factor 3 & 2,23 & chr19 \\
\hline IRF6 & 3664 & interferon regulatory factor 6 & 3,51 & chr1 \\
\hline IRS4 & 8471 & Insulin receptor substrate 4 & 10,72 & $\operatorname{chrX}$ \\
\hline IRX2 & 153572 & iroquois homeobox protein 2 & 9,14 & chr5 \\
\hline ISG20L1 & 64782 & interferon stimulated exonuclease gene $20 \mathrm{kDa}$-like 1 & 2,26 & chr15 \\
\hline ISG20L2 & 81875 & interferon stimulated exonuclease gene $20 \mathrm{kDa}$-like $2 / / /$ interferon stimulated exd & 2,01 & chr1 \\
\hline ISOC2 & 79763 & isochorismatase domain containing 2 & 2,27 & chr19 \\
\hline ITGA6 & 3655 & integrin, alpha 6 & 2,95 & chr2 \\
\hline ITGA7 & 3679 & integrin, alpha 7 & 2,81 & chr12 \\
\hline ITGB1BP3 & 27231 & integrin beta 1 binding protein 3 & 11,03 & chr19 \\
\hline ITGB3BP & 23421 & integrin beta 3 binding protein (beta3-endonexin) & 5,26 & chr1 \\
\hline ITM2A & 9452 & integral membrane protein $2 \mathrm{~A}$ & 13,10 & $\operatorname{chrX}$ \\
\hline ITPK1 & 3705 & inositol 1,3,4-triphosphate $5 / 6$ kinase & 4,13 & chr14 \\
\hline ITPR3 & 3710 & inositol 1,4,5-triphosphate receptor, type 3 & 5,48 & chr6 \\
\hline ITSN1 & 6453 & intersectin 1 (SH3 domain protein) & 3,13 & chr21 \\
\hline IVD & 3712 & isovaleryl Coenzyme A dehydrogenase & 2,40 & chr15 \\
\hline JARID1B & 10765 & Jumonji, AT rich interactive domain 1B (RBP2-like) & 2,69 & chr1 \\
\hline JARID2 & 3720 & Jumonji, AT rich interactive domain 2 & 26,09 & chr6 \\
\hline JMJJ1B & 51780 & jumonji domain containing 1B & 2,34 & chr5 \\
\hline JMJD1C & 221037 & jumonji domain containing $1 \mathrm{C}$ & 4,75 & chr10 \\
\hline JMJD2A & 9682 & jumonji domain containing $2 \mathrm{~A}$ & 2,44 & chr1 \\
\hline JMJD2C & 23081 & jumonji domain containing $2 \mathrm{C}$ & 2,37 & chr9 \\
\hline JMY & 133746 & junction-mediating and regulatory protein & 3,10 & chr5 \\
\hline JPH1 & 56704 & junctophilin 1 & 10,69 & chr8 \\
\hline JPH3 & 57338 & junctophilin 3 & 6,67 & chr16 \\
\hline JPH4 & 84502 & junctophilin 4 & 2,42 & chr14 \\
\hline JTV1 & 7965 & JTV1 gene & 2,53 & chr7 \\
\hline KAL1 & 3730 & Kallmann syndrome 1 sequence & 3,97 & $\operatorname{chrX}$ \\
\hline KARCA1 & 126823 & kelch/ankyrin repeat containing cyclin $\mathrm{A} 1$ interacting protein & 3,29 & chr1 \\
\hline KARS & 3735 & |lysyl-tRNA synthetase /// lysyl-tRNA synthetase & 2,04 & chr16 \\
\hline KATNA1 & 11104 & katanin p60 (ATPase-containing) subunit A 1 & 2,07 & chr6 \\
\hline KATNB1 & 10300 & katanin p80 (WD repeat containing) subunit B 1 & 2,17 & chr16 \\
\hline KBTBD7 & 84078 & kelch repeat and BTB (POZ) domain containing 7 & 4,09 & chr13 \\
\hline KBTBD8 & 84541 & kelch repeat and BTB (POZ) domain containing 8 & 4,79 & chr3 \\
\hline KCND2 & 3751 & potassium voltage-gated channel, Shal-related subfamily, member 2 & 2,55 & chr7 \\
\hline KCNG3 & 170850 & potassium voltage-gated channel, subfamily G, member 3 & 22,68 & chr2 \\
\hline KCNK12 & 56660 & potassium channel, subfamily K, member 12 & 3,70 & chr2 \\
\hline KCNK5 & 8645 & potassium channel, subfamily K, member 5 & 8,47 & chr6 \\
\hline KCNMB4 & 27345 & potassium large conductance calcium-activated channel, subfamily $\mathrm{M}$, beta men & 2,83 & chr12 \\
\hline KCNN2 & 3781 & potassium intermediate/small conductance calcium-activated channel, subfamily & 7,20 & chr5 \\
\hline KCNQ2 & 3785 & potassium voltage-gated channel, KQT-like subfamily, member 2 & 18,62 & chr20 \\
\hline KCNS3 & 3790 & potassium voltage-gated channel, delayed-rectifier, subfamily S, member 3 & 19,35 & chr2 \\
\hline KCTD14 & 65987 & potassium channel tetramerisation domain containing 14 & 7,80 & chr11 \\
\hline KCTD2 & 23510 & potassium channel tetramerisation domain containing 2 & 2,99 & chr17 \\
\hline KCTD6 & 200845 & potassium channel tetramerisation domain containing 6 & 2,03 & chr3 \\
\hline
\end{tabular}




\begin{tabular}{|c|c|c|c|c|}
\hline KDR & 3791 & Kinase insert domain recentor (a tyne III recentor tyrosine kinase) & 1056 & chrs \\
\hline KEAP1 & 9817 & kelch-like $\mathrm{ECH}$-associated protein 1 & 2,46 & $\operatorname{chr} 19$ \\
\hline KHDRBS1 & 10657 & KH domain containing, RNA binding, signal transduction associated 1 & 2,40 & chr1 \\
\hline KHK & 3795 & ketohexokinase (fructokinase) /// ketohexokinase (fructokinase) & 6,22 & chr2 \\
\hline KIAA0020 & 9933 & KIAA0020 & 2,32 & chr9 \\
\hline KIAA0114 & 57291 & KIAA0114 gene product & 11,39 & chr4 \\
\hline KIAA0133 & 9816 & KIAA0133 & 5,15 & chr1 \\
\hline KIAA0152 & 9761 & KIAA0152 & 2,58 & chr12 \\
\hline KIAA0153 & 23170 & KIAA0153 protein & 5,73 & chr22 \\
\hline KIAA0179 & 23076 & KIAA0179 & 2,58 & chr21 \\
\hline KIAA0182 & 23199 & KIAA0182 protein & 5,08 & chr16 \\
\hline KIAA0286 & 23306 & KIAA0286 protein & 6,45 & chr12 \\
\hline KIAA0368 & 23392 & KIAA0368 & 3,18 & chr9 \\
\hline KIAA0391 & 9692 & |KIAA0391 & 2,36 & chr14 \\
\hline KIAA0406 & 9675 & KIAA0406 gene product & 3,70 & chr20 \\
\hline KIAA0495 & 57212 & KIAA0495 & 2,38 & chr1 \\
\hline KIAA0523 & 23302 & KIAA0523 protein & 13,63 & chr17 \\
\hline KIAA0528 & 9847 & KIAA0528 gene product & 4,33 & chr12 \\
\hline KIAA0555 & 9832 & Jak and microtubule interacting protein 2 & 4,25 & chr5 \\
\hline KIAA0582 & 23177 & KIAA0582 & 3,47 & chr2 \\
\hline KIAA0649 & 9858 & KIAA0649 & 2,69 & chr9 \\
\hline KIAA0664 & 23277 & |KIAA0664 protein & 2,15 & chr17 \\
\hline KIAA0672 & 9912 & KIAA0672 gene product & 4,34 & chr17 \\
\hline KIAA0683 & 9894 & KIAA0683 gene product & 2,91 & chr16 \\
\hline KIAA0828 & 23382 & |KIAA0828 protein & 2,44 & chr7 \\
\hline KIAA0841 & 23354 & |KIAA0841 & 2,08 & chr19 \\
\hline KIAA0859 & 51603 & KIAA0859 & 3,68 & chr1 \\
\hline KIAA0863 & 22850 & KIAA0863 protein & 4,60 & chr18 \\
\hline KIAA0888 & 26049 & KIAA0888 protein & 18,52 & chr5 \\
\hline KIAA0922 & 23240 & |KIAA0922 protein & 2,39 & chr4 \\
\hline KIAA0947 & 23379 & KIAA0947 protein & 2,27 & chr5 \\
\hline KIAA0971 & 22868 & |KIAA0971 & 2,13 & chr2 \\
\hline KIAA0980 & 22981 & KIAA0980 protein & 5,98 & chr20 \\
\hline KIAA0984 & 23329 & KIAA0984 protein & 2,34 & chr12 \\
\hline KIAA0999 & 23387 & KIAA0999 protein & 4,85 & chr11 \\
\hline KIAA1143 & 57456 & KIAA1143 & 3,22 & chr3 \\
\hline KIAA1155 & 400961 & KIAA1155 protein & 6,94 & chr2 \\
\hline KIAA1166 & 55906 & |KIAA1166 & 2,86 & $\operatorname{chrX}$ \\
\hline KIAA1211 & 57482 & |KIAA1211 protein & 4,71 & chr4 \\
\hline KIAA1212 & 55704 & |KIAA1212 & 4,35 & chr2 \\
\hline KIAA1219 & 57148 & |KIAA1219 protein & 2,03 & chr20 \\
\hline KIAA1240 & 54454 & KIAA1240 protein & 2,65 & chr2 \\
\hline KIAA1244 & 57221 & KIAA1244 & 2,89 & chr6 \\
\hline KIAA1274 & 27143 & KIAA1274 & 3,71 & chr10 \\
\hline KIAA1287 & 57508 & KIAA1287 & 2,29 & chr17 \\
\hline KIAA1324L & 222223 & KIAA1324-like & 4,49 & chr7 \\
\hline KIAA1333 & 55632 & |KIAA1333 & 3,51 & chr14 \\
\hline KIAA1344 & 57544 & |KIAA1344 & 3,83 & chr14 \\
\hline KIAA1411 & 57579 & |KIAA1411 & 2,94 & chr6 \\
\hline KIAA1467 & 57613 & KIAA1467 protein & 4,04 & chr12 \\
\hline KIAA1509 & 440193 & KIAA1509 & 7,86 & chr14 \\
\hline KIAA1524 & 57650 & |KIAA1524 & 4,73 & chr3 \\
\hline KIAA1533 & 57655 & |KIAA1533 & 2,09 & chr19 \\
\hline KIAA1542 & 57661 & CTD-binding SR-like protein rA9 & 2,18 & chr11 \\
\hline KIAA1545 & 57666 & KIAA1545 protein & 2,81 & chr12 \\
\hline KIAA1549 & 57670 & KIAA1549 protein & 3,58 & $\mathrm{chr} 7$ \\
\hline KIAA1553 & 57673 & KIAA1553 & 13,35 & chr6 \\
\hline KIAA1586 & 57691 & KIAA1586 & 2,27 & chr6 \\
\hline KIAA1598 & 57698 & KIAA1598 & 2,37 & chr10 \\
\hline KIAA1604 & 57703 & KIAA1604 protein & 2,89 & chr2 \\
\hline KIAA1627 & 57721 & KIAA1627 protein & 2,07 & chr4 \\
\hline KIAA1648 & 284900 & KIAA1648 protein & 5,53 & chr22 \\
\hline KIAA1671 & 85379 & KIAA1671 protein & 7,72 & chr22 \\
\hline KIAA1718 & 80853 & |KIAA1718 protein & 2,77 & $\operatorname{chr} 7$ \\
\hline KIAA1727 & 85462 & KIAA1727 protein & 8,07 & chr4 \\
\hline KIAA1731 & 85459 & |KIAA1731 & 2,26 & chr11 \\
\hline KIAA1737 & 85457 & KIAA1737 & 2,77 & chr14 \\
\hline
\end{tabular}




\section{Pagge 147 of 257}

\begin{tabular}{|c|c|c|c|c|}
\hline KIAA1754L & 150771 & KIAA1754-like & 2,53 & chr2 \\
\hline KIAA1804 & 84451 & mixed lineage kinase 4 & 22,07 & chr1 \\
\hline KIAA1815 & 79956 & |KIAA1815 & 3,86 & chr9 \\
\hline KIAA1906 & 114795 & |KIAA1906 protein & 7,00 & chr12 \\
\hline KIAA1909 & 153478 & |KIAA1909 protein & 6,79 & chr5 \\
\hline KIAA1935 & 114825 & KIAA1935 protein & 6,69 & chr5 \\
\hline KIAA1944 & 121256 & KIAA1944 protein & 2,84 & chr12 \\
\hline KIAA1958 & 158405 & |KIAA1958 & 18,10 & chr9 \\
\hline KIAA1970 & 124454 & KIAA1970 protein & 3,99 & chr16 \\
\hline KIAA1982 & 170960 & KIAA1982 protein & 5,40 & chr4 \\
\hline KIAA2010 & 55671 & KIAA2010 & 2,17 & chr14 \\
\hline KIBRA & 23286 & KIBRA protein & 4,01 & chr5 \\
\hline KIF11 & 3832 & kinesin family member 11 & 6,99 & chr10 \\
\hline KIF14 & 9928 & kinesin family member 14 & 7,32 & chr1 \\
\hline KIF15 & 56992 & kinesin family member 15 & 11,53 & chr3 \\
\hline KIF18A & 81930 & kinesin family member $18 \mathrm{~A} / / /$ kinesin family member $18 \mathrm{~A}$ & 2,36 & chr11 \\
\hline KIF1A & 547 & kinesin family member $1 \mathrm{~A}$ & 40,11 & chr2 \\
\hline KIF1B & 23095 & kinesin family member 1B & 5,00 & chr1 \\
\hline KIF20A & 10112 & kinesin family member 20A & 8,23 & chr5 \\
\hline KIF21A & 55605 & kinesin family member $21 \mathrm{~A}$ & 6,33 & chr12 \\
\hline KIF22 & 3835 & kinesin family member 22 & 10,39 & chr16 \\
\hline KIF23 & 9493 & kinesin family member 23 & 3,68 & chr15 \\
\hline KIF26A & 26153 & kinesin family member $26 \mathrm{~A}$ & 4,00 & chr14 \\
\hline KIF2C & 11004 & kinesin family member 2C & 6,72 & chr1 \\
\hline KIF4A & 24137 & kinesin family member $4 \mathrm{~A}$ & 5,74 & chr5 \\
\hline KIF5A & 3798 & Kinesin family member $5 \mathrm{~A}$ & 18,02 & chr12 \\
\hline KIF5C & 3800 & Kinesin family member $5 \mathrm{C}$ & 147,77 & chr2 \\
\hline KIF9 & 64147 & kinesin family member 9 & 2,26 & chr3 \\
\hline KIFC1 & 3833 & kinesin family member $\mathrm{C} 1$ & 2,87 & chr6 \\
\hline KIT & 3815 & v-kit Hardy-Zuckerman 4 feline sarcoma viral oncogene homolog & 12,41 & chr4 \\
\hline KLF4 & 9314 & Kruppel-like factor 4 (gut) & 6,08 & chr9 \\
\hline KLF8 & 11279 & Kruppel-like factor 8 & 6,52 & $\operatorname{chr} \mathrm{X}$ \\
\hline KLHDC4 & 54758 & kelch domain containing 4 & 2,57 & chr16 \\
\hline KLHL12 & 59349 & kelch-like 12 (Drosophila) & 2,08 & chr22 \\
\hline KLHL13 & 90293 & kelch-like 13 (Drosophila) & 3,96 & $\operatorname{chr} \mathrm{X}$ \\
\hline KLHL23 & 151230 & kelch-like 23 (Drosophila) & 8,07 & chr2 \\
\hline KLHL3 & 26249 & kelch-like 3 (Drosophila) & 4,86 & chr5 \\
\hline KLHL7 & 55975 & kelch-like 7 (Drosophila) & 7,93 & $\operatorname{chr} 7$ \\
\hline KLKB1 & 3818 & kallikrein B, plasma (Fletcher factor) 1 & 10,84 & $\operatorname{chr} 4$ \\
\hline KNTC1 & 9735 & kinetochore associated 1 & 6,70 & chr12 \\
\hline KNTC2 & 10403 & kinetochore associated 2 & 6,21 & chr18 \\
\hline KPNA2 & 3838 & karyopherin alpha 2 (RAG cohort 1 , importin alpha 1) /// karyopherin alpha 2 (RA & 2,94 & chr17 \\
\hline KPNA3 & 3839 & Karyopherin alpha 3 (importin alpha 4) & 2,83 & chr13 \\
\hline KPNA5 & 3841 & Karyopherin alpha 5 (importin alpha 6) & 2,01 & chr6 \\
\hline KPNB1 & 3837 & Karyopherin (importin) beta 1 & 2,02 & chr17 \\
\hline KPTN & 11133 & kaptin (actin binding protein) & 3,41 & chr19 \\
\hline KRTAP4-7 & 85287 & keratin associated protein 4-7 & 5,40 & chr15 \\
\hline KRTCAP3 & 200634 & keratinocyte associated protein 3 & 11,82 & chr2 \\
\hline KUB3 & 91419 & Ku70-binding protein 3 & 2,86 & chr12 \\
\hline L2HGDH & 79944 & L-2-hydroxyglutarate dehydrogenase & 3,06 & chr14 \\
\hline LACTB2 & 51110 & lactamase, beta 2 & 2,05 & chr8 \\
\hline LAMA1 & 284217 & laminin, alpha 1 & 21,06 & chr18 \\
\hline LAMC3 & 10319 & laminin, gamma 3 & 2,39 & chr9 \\
\hline LANCL2 & 55915 & LanC lantibiotic synthetase component C-like 2 (bacterial) & 2,39 & chr7 \\
\hline LAPTM4B & 55353 & lysosomal associated protein transmembrane 4 beta & 6,03 & chr8 \\
\hline LARP2 & 55132 & La ribonucleoprotein domain family, member 2 & 3,81 & chr4 \\
\hline LARP4 & 113251 & La ribonucleoprotein domain family, member 4 & 2,34 & chr12 \\
\hline LARP5 & 23185 & La ribonucleoprotein domain family, member 5 & 2,44 & chr10 \\
\hline LARS & 51520 & leucyl-tRNA synthetase & 2,62 & chr5 \\
\hline LARS2 & 23395 & leucyl-tRNA synthetase 2 , mitochondrial & 4,24 & chr3 \\
\hline LAS1L & 81887 & LAS1-like (S. cerevisiae) /// LAS1-like (S. cerevisiae) & 4,72 & $\operatorname{chr} X$ \\
\hline LASS1 & 10715 & LAG1 longevity assurance homolog 1 (S. cerevisiae) & 2,87 & $\operatorname{chr} 19$ \\
\hline LASS4 & 79603 & LAG1 longevity assurance homolog 4 (S. cerevisiae) & 2,08 & chr19 \\
\hline LASS6 & 253782 & LAG1 longevity assurance homolog 6 (S. cerevisiae) & 3,09 & chr2 \\
\hline LBR & 3930 & lamin B receptor & 2,94 & chr1 \\
\hline LCHN & 57189 & LCHN protein & 3,72 & $\operatorname{chr} 7$ \\
\hline LCK & 3932 & lymphocyte-specific protein tyrosine kinase & 37,69 & chr1 \\
\hline
\end{tabular}




\begin{tabular}{|c|c|c|c|c|}
\hline LCMT1 & 51451 & leucine carboxyl methyltransferase 1 & 2,60 & chr16 \\
\hline LCMT2 & 9836 & leucine carboxyl methyltransferase 2 & 2,40 & chr15 \\
\hline LDB2 & 9079 & LIM domain binding 2 & 3,82 & chr4 \\
\hline LECT1 & 11061 & leukocyte cell derived chemotaxin 1 & 46,66 & chr13 \\
\hline LEFTY1 & 10637 & left-right determination factor 1 & 70,89 & chr1 \\
\hline LEFTY2 & 7044 & left-right determination factor 2 & 38,07 & chr1 \\
\hline LEO1 & 123169 & Leo1, Paf1/RNA polymerase II complex component, homolog (S. cerevisiae) & 2,26 & chr15 \\
\hline LGALS1 & 3956 & lectin, galactoside-binding, soluble, 1 (galectin 1) & 76,19 & chr22 \\
\hline LIAS & 11019 & lipoic acid synthetase & 3,79 & chr4 \\
\hline LIG1 & 3978 & ligase I, DNA, ATP-dependent & 4,24 & chr19 \\
\hline LIG3 & 3980 & ligase III, DNA, ATP-dependent & 4,17 & chr17 \\
\hline LIN28 & 79727 & lin-28 homolog (C. elegans) & 660,24 & chr1 \\
\hline LIN28B & 389421 & lin-28 homolog B (C. elegans) & 143,10 & chr6 \\
\hline LIPT1 & 51601 & lipoyltransferase 1 & 3,03 & chr2 \\
\hline LISCH7 & 51599 & liver-specific bHLH-Zip transcription factor & 6,93 & chr19 \\
\hline LITAF & 9516 & lipopolysaccharide-induced TNF factor & 2,75 & chr16 \\
\hline LLGL1 & 3996 & lethal giant larvae homolog 1 (Drosophila) & 2,94 & chr17 \\
\hline LLGL2 & 3993 & lethal giant larvae homolog 2 (Drosophila) & 3,55 & chr17 \\
\hline LMNB1 & 4001 & $\operatorname{lamin} B 1$ & 11,56 & chr5 \\
\hline LMNB2 & 84823 & $\operatorname{lamin} B 2$ & 2,48 & chr19 \\
\hline LMO4 & 8543 & LIM domain only 4 & 3,64 & chr1 \\
\hline LMTK2 & 22853 & Lemur tyrosine kinase 2 & 3,05 & chr7 \\
\hline LNX & 84708 & ligand of numb-protein $X$ & 10,39 & chr4 \\
\hline LNX2 & 222484 & ligand of numb-protein $\times 2$ & 2,10 & chr13 \\
\hline LOC112703 & 112703 & hypothetical protein BC004941 & 2,78 & chr19 \\
\hline LOC112869 & 112869 & hypothetical protein BC011981 & 2,56 & chr16 \\
\hline LOC113174 & 113174 & hypothetical protein BC012010 & 4,91 & chr11 \\
\hline LOC113179 & 113179 & hypothetical protein BC011824 & 2,58 & chr19 \\
\hline LOC114977 & 114977 & hypothetical protein BC014148 & 13,14 & chr19 \\
\hline LOC115648 & 115648 & similar to hypothetical protein FLJ13659 & 3,98 & chr19 \\
\hline LOC115648/ & $\mid 148203 / / / 28444$ & similar to hypothetical protein FLJ13659 /// hypothetical protein LOC148203 /// z & 4,02 & chr19 \\
\hline LOC116238 & 116238 & hypothetical protein BC014072 & 4,06 & chr17 \\
\hline LOC124491 & 124491 & LOC124491 & 5,42 & chr16 \\
\hline LOC126295 & 126295 & hypothetical protein LOC126295 & 5,31 & chr19 \\
\hline LOC129531 & 129531 & hypothetical protein $\mathrm{BC} 018453$ & 2,53 & chr2 \\
\hline LOC130502 & 130502 & similar to CG14894-PA & 2,43 & chr2 \\
\hline LOC131076 & 131076 & hypothetical LOC131076 & 4,45 & chr18 \\
\hline LOC132241 & 132241 & hypothetical protein LOC132241 & 2,10 & chr3 \\
\hline LOC137886 & 137886 & hypothetical protein LOC137886 & 2,89 & chr8 \\
\hline LOC138255 & 138255 & OTTHUMP00000021439 & 60,20 & chr9 \\
\hline LOC139886 & 139886 & hypothetical protein LOC139886 & 3,39 & $\operatorname{chr} X$ \\
\hline LOC145786 & 145786 & hypothetical protein LOC145786 & 6,72 & chr15 \\
\hline LOC146909 & 146909 & hypothetical protein LOC146909 & 4,51 & chr17 \\
\hline LOC147645 & 147645 & hypothetical protein LOC147645 & 3,91 & chr19 \\
\hline LOC147727 & 147727 & hypothetical protein LOC147727 & 2,56 & chr19 \\
\hline LOC148203 & 148203 & hypothetical protein LOC148203 & 10,72 & chr19 \\
\hline LOC149832 & 149832 & Hypothetical protein LOC149832 & 2,17 & chr20 \\
\hline LOC150084 & 150084 & hypothetical protein LOC150084 & 2,11 & chr21 \\
\hline LOC150271 & 150271 & hypothetical protein LOC150271 & 8,28 & chr22 \\
\hline LOC150371 & 150371 & hypothetical LOC150371 & 8,35 & chr22 \\
\hline LOC151194 & 151194 & similar to hepatocellular carcinoma-associated antigen HCA557b & 10,84 & chr2 \\
\hline LOC152185 & 152185 & hypothetical protein AY099107 & 2,18 & chr3 \\
\hline LOC152217 & 152217 & hypothetical protein $\mathrm{BC} 007882$ & 2,77 & chr3 \\
\hline LOC152485 & 152485 & Hypothetical protein LOC152485 & 3,26 & chr4 \\
\hline LOC153346 & 153346 & hypothetical protein LOC153346 & 11,22 & chr5 \\
\hline LOC153469 & 153469 & hypothetical protein LOC153469 & 16,62 & chr5 \\
\hline LOC153546 & 153546 & hypothetical protein LOC153546 & 3,39 & chr5 \\
\hline LOC153561 & 153561 & Hypothetical protein LOC153561 & 11,34 & chr5 \\
\hline LOC157627 & 157627 & hypothetical protein LOC157627 & 9,48 & chr8 \\
\hline LOC159090 & 159090 & similar to hypothetical protein MGC17347 & 2,77 & $\operatorname{chr} x$ \\
\hline LOC163233 & 163233 & Similar to Zinc finger protein 93 (Zinc finger protein HTF34) & 4,03 & (vide) \\
\hline LOC169834 & 169834 & hypothetical protein LOC169834 & 13,96 & chr9 \\
\hline LOC196394 & 196394 & hypothetical protein LOC196394 & 2,24 & chr12 \\
\hline LOC200169 & 200169 & hypothetical protein LOC200169 & 3,82 & chr1 \\
\hline LOC200230 & 200230 & similar to KIAA0386 & 2,22 & chr20 \\
\hline LOC201725 & 201725 & hypothetical protein LOC201725 & 2,82 & $\operatorname{chr} 4$ \\
\hline LOC202451 & 202451 & hypothetical protein LOC202451 & 9,64 & $\operatorname{chr} 6$ \\
\hline
\end{tabular}




\section{Pagge 149 of 257}

\begin{tabular}{|c|c|c|c|c|}
\hline LOC202781 & 202781 & hypothetical protein LOC202781 & 2,54 & chr7 \\
\hline LOC203547 & 203547 & hypothetical protein LOC203547 & 2,28 & $\operatorname{chr} X$ \\
\hline LOC219638 & 219638 & Hypothetical LOC219638 & 2,22 & chr11 \\
\hline 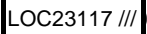 & | /// $348162 / / / 44$ & KIAA0220-like protein /// hypothetical gene LOC283846 /// hypothetical protein 3 & 4,62 & chr16 \\
\hline LOC253842 & 253842 & hypothetical protein LOC253842 & 27,88 & chr9 \\
\hline LOC254128 & 254128 & hypothetical protein LOC254128 & 2,24 & chr2 \\
\hline LOC283377 & 283377 & hypothetical protein LOC283377 & 2,07 & chr12 \\
\hline LOC283481 & 283481 & hypothetical protein LOC283481 & 2,60 & chr13 \\
\hline LOC283871 & 283871 & hypothetical protein LOC283871 & 6,51 & chr16 \\
\hline LOC284058 & 284058 & LOC284058 protein & 3,56 & chr17 \\
\hline LOC284214 & 284214 & hypothetical protein LOC284214 & 2,10 & chr18 \\
\hline LOC284373 & 284373 & Hypothetical protein LOC284373 & 2,06 & (vide) \\
\hline LOC284408 & 284408 & Hypothetical protein LOC284408 & 2,47 & chr19 \\
\hline LOC284611 & 284611 & hypothetical protein LOC284611 & 3,67 & chr1 \\
\hline LOC284701 & 284701 & hypothetical protein LOC284701 & 7,29 & chr1 \\
\hline LOC284701/| / / / & $4 / / / / 440470 / / / 4$ & hypothetical protein LOC284701 /// hypothetical gene supported by AK128780 / & 6,79 & chr1 \\
\hline LOC284702 & 284702 & hypothetical protein LOC284702 & 4,19 & chr1 \\
\hline LOC284801 & 284801 & hypothetical protein LOC284801 & 3,96 & chr20 \\
\hline LOC285016 & 285016 & hypothetical protein LOC285016 & 2,78 & chr2 \\
\hline LOC285401 & 285401 & hypothetical protein LOC285401 & 2,37 & chr3 \\
\hline LOC285958 & 285958 & hypothetical protein LOC285958 & 2,45 & chr7 \\
\hline LOC286044 & 286044 & hypothetical protein LOC286044 & 4,30 & chr8 \\
\hline 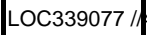 & 440549 /// 44071 & hypothetical gene supported by AK092558; AL137655; BC006361 /// similar to & 2,38 & chr1 \\
\hline LOC342892 & 342892 & Hypothetical protein LOC342892 & 8,57 & chr19 \\
\hline LOC342979 & 342979 & hypothetical LOC342979 & 8,51 & chr19 \\
\hline LOC345630 & 345630 & similar to fibrillarin & 2,26 & chr5 \\
\hline LOC347273 & 347273 & similar to RIKEN cDNA 2310039E09 & 3,68 & chr9 \\
\hline LOC348801 & 348801 & hypothetical protein LOC348801 & 3,88 & chr3 \\
\hline 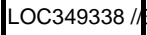 & 349338 /// 37466 & CXYorf1-related protein /// CXYorf1-related protein & 2,64 & chr2 \\
\hline |LOC388291 & 388291 & Hypothetical LOC388291 & 2,10 & chr16 \\
\hline LOC388335 & 388335 & similar to RIKEN cDNA A730055C05 gene & 2,42 & chr17 \\
\hline LOC388388 & 388388 & chromodomain helicase DNA binding protein 3 & 5,80 & chr17_random \\
\hline |LOC388494 & 388494 & hypothetical gene supported by AL365406; BC034005 & 23,78 & chr19 \\
\hline LOC388508 & 388508 & similar to ribosomal protein L17 & 3,52 & chr19 \\
\hline LOC388638 & 388638 & hypothetical LOC388638 & 8,32 & chr1 \\
\hline 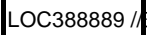 & 388889 /// 440827 & Hypothetical LOC388889 /// Hypothetical protein LOC150271 & 3,63 & chr22 \\
\hline LOC389023 & 389023 & hypothetical gene supported by BC032913; BC048425 & 2,94 & chr2 \\
\hline LOC389188 & 389188 & Hypothetical LOC389188 & 3,17 & chr3 \\
\hline LOC389295 & 389295 & Hypothetical protein LOC153561 & 5,02 & chr5 \\
\hline LOC389362 & 389362 & hypothetical LOC389362 & 2,64 & chr6 \\
\hline LOC389541 & 389541 & similar to CG14977-PA & 4,46 & chr7 \\
\hline 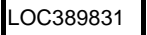 & 389831 & hypothetical gene supported by AL713796 & 5,88 & chr7_random \\
\hline LOC389857 & 389857 & hypothetical protein & 3,19 & $\operatorname{chr} X$ \\
\hline LOC390940 & 390940 & similar to R28379_1 & 3,60 & chr19 \\
\hline LOC391356 & 391356 & similar to CG14903-PA & 2,32 & chr2 \\
\hline LOC391833 & 391833 & Similar to 40 S ribosomal protein $\mathrm{S} 10$ & 3,01 & chr5 \\
\hline LOC400451 & 400451 & hypothetical gene supported by AK075564; BC060873 & 2,83 & chr15 \\
\hline LOC400506 & 400506 & Similar to TSG118.1 & 7,62 & chr16 \\
\hline LOC400680 & 400680 & Hypothetical gene supported by AK097381; BC040866 & 7,36 & chr19 \\
\hline LOC400690 & 400690 & hypothetical gene supported by AK092138 & 13,94 & chr19 \\
\hline LOC400740 & 400740 & LOC440570 & 4,08 & chr1 \\
\hline LOC400741 & 400741 & hypothetical LOC400741 & 3,60 & chr1 \\
\hline LOC400793 & 400793 & hypothetical gene supported by AK125122 & 3,07 & chr1 \\
\hline LOC401499 & 401499 & Hypothetical LOC401499 & 2,54 & chr9 \\
\hline LOC402563 & 402563 & Hypothetical LOC401385 & 2,22 & chr7 \\
\hline LOC439949 & 439949 & hypothetical gene supported by AY007155 & 7,84 & chr10 \\
\hline LOC439987 & 439987 & LOC439987 & 2,66 & chr10 \\
\hline LOC440008 & $1488 / / / 440008$ & C-terminal binding protein 2 /// LOC440008 & 4,18 & chr5 \\
\hline LOC440085 & 440085 & similar to prothymosin alpha & 3,31 & chr2 \\
\hline LOC440122 & 440122 & Similar to $K R A B$ zinc finger protein $6 D$ & 3,66 & chr12 \\
\hline LOC440132 & 440132 & LOC440132 & 4,64 & chr13 \\
\hline LOC440282 & 440282 & Hypothetical protein LOC145783 & 2,44 & chr15 \\
\hline LOC440288 & 440288 & similar to FLJ16518 protein & 4,20 & chr15 \\
\hline LOC440338 & 440338 & Hypothetical gene supported by AJ002784 & 24,11 & chr16 \\
\hline LOC440388 & 440388 & similar to coenzyme A diphosphatase & 3,79 & chr16 \\
\hline LOC440416 & 440416 & Hypothetical gene supported by BC 072410 & 3,58 & chr17 \\
\hline LOC440426 & 440426 & hypothetical gene supported by AK092922; AL831912 & 3,61 & chr12 \\
\hline
\end{tabular}




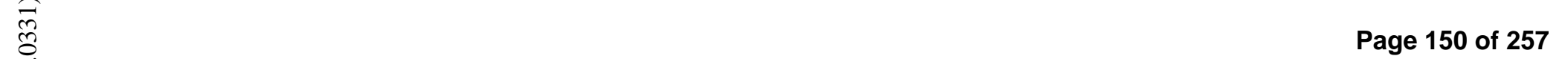

\begin{tabular}{|c|c|c|c|c|}
\hline LOC440524 & 440524 & LOC440524 & 6,67 & chr19 \\
\hline 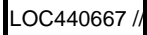 & 7 //// 440669 /// 4 & LOC440667 /// LOC440669 /// LOC440688 & 2,70 & chr1 \\
\hline LOC440669 & 440669 & LOC440669 & 3,25 & chr1 \\
\hline LOC440701 & 440701 & |LOC440701 & 2,03 & chr1 \\
\hline LOC440731 & 440731 & LOC440731 & 2,13 & chr1 \\
\hline LOC440957 & 440957 & similar to CG32736-PA & 2,41 & chr3 \\
\hline LOC440971 & 440971 & similar to Zinc finger protein Rlf (Rearranged L-myc fusion gene protein) $(\mathrm{Zn}-15$ & 3,07 & chr3 \\
\hline LOC440983 & 440983 & hypothetical gene supported by BC066916 & 3,64 & chr1 \\
\hline LOC440996 & 440996 & Hypothetical gene supported by BC 053580 & 2,45 & chr5 \\
\hline LOC441027 & 441027 & Similar to hypothetical protein LOC231503 & 4,73 & chr 4 \\
\hline LOC441114 & 441114 & Similar to acidic ribosomal protein $\mathrm{PO}$ & 2,02 & chr16 \\
\hline LOC441164 & 441164 & Chromosome 6 open reading frame 160 & 5,15 & chr6 \\
\hline 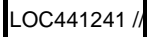 & $441241 / / / 441244$ & chaperonin containing TCP1, subunit $6 \mathrm{~A}$ (zeta 1)-like /// chaperonin containing $\mathrm{T}$ & 2,31 & chr7 \\
\hline LOC441458 & 441458 & hypothetical gene supported by AK091930 & 5,88 & chr9 \\
\hline LOC441628 & 441628 & similar to POU domain, class 5 , transcription factor 1 (Octamer-binding transcrip & 26,46 & chr1 \\
\hline LOC442075 & 442075 & weakly similar to serine/threonine protein kinase Kp78 & 3,39 & chr3 \\
\hline LOC442447 & 442447 & Similar to Chloride intracellular channel protein 4 (Intracellular chloride ion chann & 10,45 & $\operatorname{chr} X$ \\
\hline LOC442699 & 442699 & Zinc finger protein 100 & 6,69 & $\operatorname{chr} 7$ \\
\hline LOC493856 & 493856 & similar to RIKEN cDNA 1500009 M05 gene & 2,15 & chr2 \\
\hline LOC494143 & 494143 & similar to RIKEN cDNA $2510006 \mathrm{C} 20$ gene & 3,65 & chr2 \\
\hline LOC497257 & 497257 & Hypothetical LOC497257 & 12,27 & chr8 \\
\hline LOC554203 & 554203 & hypothetical LOC554203 & 2,53 & (vide) \\
\hline LOC57168 & 57168 & similar to aspartate beta hydroxylase (ASPH) & 2,85 & chr22 \\
\hline LOC58489 & 58489 & hypothetical protein from EUROIMAGE 588495 & 2,65 & chr15 \\
\hline LOC63920 & 63920 & transposon-derived Buster3 transposase-like & 2,27 & (vide) \\
\hline LOC641298 & 641298 & PI-3-kinase-related kinase SMG-1 - like locus & 2,16 & chr16 \\
\hline LOC641522 & 641522 & ADP-ribosylation factor-like 17 pseudogene 1 & 3,38 & chr17 \\
\hline LOC81558 & 81558 & C/EBP-induced protein /// C/EBP-induced protein & 9,77 & chr17 \\
\hline LOC84661 & 84661 & dpy-30-like protein & 2,82 & chr2 \\
\hline LOC89894 & 89894 & hypothetical protein $\mathrm{BC} 000282$ & 2,64 & chr12 \\
\hline LOC90321 & 90321 & hypothetical protein LOC90321 & 3,71 & chr19 \\
\hline LOC90799 & 90799 & hypothetical protein BC009518 & 3,93 & chr17 \\
\hline LOC91431 & 91431 & prematurely terminated mRNA decay factor-like & 11,02 & chr4 \\
\hline LOC91661 & 91661 & Hypothetical protein BC001610 & 2,94 & (vide) \\
\hline LOC92345 & 92345 & hypothetical protein $\mathrm{BC} 008207$ & 2,16 & chr4 \\
\hline LOC92497 & 92497 & hypothetical protein LOC92497 & 2,95 & chr12 \\
\hline LOC92558 & 92558 & hypothetical protein LOC92558 & 2,01 & chr12 \\
\hline LOC94431 & 94431 & similar to RNA polymerase I transcription factor RRN3 & 2,23 & chr16 \\
\hline LOH11CR2A & 4013 & loss of heterozygosity, 11 , chromosomal region 2 , gene $\mathrm{A}$ & 2,48 & chr11 \\
\hline LONRF1 & 91694 & LON peptidase $\mathrm{N}$-terminal domain and ring finger 1 & 4,07 & chr8 \\
\hline LPHN1 & 22859 & latrophilin 1 & 5,34 & $\operatorname{chr} 19$ \\
\hline LPHN2 & 23266 & latrophilin 2 & 5,09 & chr1 \\
\hline LPHN3 & 23284 & latrophilin 3 & 7,21 & chr4 \\
\hline |LRIG1 & 26018 & leucine-rich repeats and immunoglobulin-like domains $1 / / /$ leucine-rich repeats & 5,65 & chr3 \\
\hline |LRIG2 & 9860 & leucine-rich repeats and immunoglobulin-like domains 2 & 2,52 & chr1 \\
\hline LRP6 & 4040 & low density lipoprotein receptor-related protein 6 & 2,75 & chr12 \\
\hline LRP8 & 7804 & low density lipoprotein receptor-related protein 8 , apolipoprotein e receptor & 5,13 & chr1 \\
\hline LRPPRC & 10128 & leucine-rich PPR-motif containing & 4,08 & chr2 \\
\hline LRRC16 & 55604 & Leucine rich repeat containing 16 & 6,18 & chr6 \\
\hline LRRC34 & 151827 & leucine rich repeat containing 34 & 5,52 & chr3 \\
\hline LRRC37B & 114659 & leucine rich repeat containing 37B & 2,15 & chr17 \\
\hline LRRC45 & 201255 & leucine rich repeat containing 45 & 2,26 & chr17 \\
\hline LRRC47 & 57470 & leucine rich repeat containing 47 & 2,09 & chr1 \\
\hline LRRC8B & 23507 & leucine rich repeat containing 8 family, member $B$ & 5,41 & chr1 \\
\hline LRRC8D & 55144 & leucine rich repeat containing 8 family, member $\mathrm{D}$ & 2,32 & chr1 \\
\hline |LRRN1 & 57633 & leucine rich repeat neuronal 1 & 54,54 & chr3 \\
\hline LRRN6A & 84894 & leucine rich repeat neuronal $6 \mathrm{~A}$ & 4,78 & chr15 \\
\hline LRRTM4 & 80059 & leucine rich repeat transmembrane neuronal 4 & 3,05 & chr2 \\
\hline LSM2 & 57819 & LSM2 homolog, U6 small nuclear RNA associated (S. cerevisiae) & 2,62 & chr6 \\
\hline LSM3 & 27258 & LSM3 homolog, U6 small nuclear RNA associated (S. cerevisiae) & 3,06 & chr3 \\
\hline LSM4 & 25804 & LSM4 homolog, U6 small nuclear RNA associated (S. cerevisiae) & 3,74 & chr19 \\
\hline LSM5 & 23658 & LSM5 homolog, U6 small nuclear RNA associated (S. cerevisiae) & 3,25 & $\operatorname{chr} 7$ \\
\hline LSM6 & 11157 & LSM6 homolog, U6 small nuclear RNA associated (S. cerevisiae) & 4,62 & chr4 \\
\hline LSM7 & 51690 & LSM7 homolog, U6 small nuclear RNA associated (S. cerevisiae) & 3,30 & chr19 \\
\hline LSS & 4047 & lanosterol synthase (2,3-oxidosqualene-lanosterol cyclase) & 2,03 & chr21 \\
\hline LTA4H & 4048 & leukotriene A4 hydrolase & 2,79 & chr12 \\
\hline |LUC7L & 55692 & LUC7-like (S. cerevisiae) & 4,63 & $\operatorname{chr} 16$ \\
\hline
\end{tabular}




\begin{tabular}{|c|c|c|c|c|}
\hline LUC7L2 & 51631 & LUC7-like 2 (S. cerevisiae) & 2,02 & chr7 \\
\hline LUZP5 & 54892 & leucine zipper protein 5 & 7,76 & $\operatorname{chr} 7$ \\
\hline LYAR & 55646 & hypothetical protein FLJ20425 & 9,64 & chr4 \\
\hline LYPLA2 & 11313 & |ysophospholipase II & 2,90 & chr1 \\
\hline LYPLA2 & 3 /// $285840 / / / 38$ & |ysophospholipase II /// Iysophospholipase II pseudogene 1 /// similar to Acyl-pro & 2,47 & chr1 \\
\hline LYPLA2 & $11313 / / / 285840$ & |ysophospholipase II /// lysophospholipase II pseudogene 1 & 2,29 & chr1 \\
\hline LYPLAL1 & 127018 & Lysophospholipase-like 1 & 2,15 & chr1 \\
\hline M11S1 & 4076 & membrane component, chromosome 11, surface marker 1 & 2,59 & chr11 \\
\hline MAC30 & 27346 & hypothetical protein MAC30 & 12,85 & chr17 \\
\hline MAD2L1 & 4085 & MAD2 mitotic arrest deficient-like 1 (yeast) & 5,91 & chr14 \\
\hline MAD2L1BP & 9587 & MAD2L1 binding protein & 2,09 & chr6 \\
\hline MAD2L2 & 10459 & MAD2 mitotic arrest deficient-like 2 (yeast) & 8,42 & chr1 \\
\hline MAFB & 9935 & v-maf musculoaponeurotic fibrosarcoma oncogene homolog B (avian) & 2,53 & $\operatorname{chr} 20$ \\
\hline MAGEE1 & 57692 & melanoma antigen family E, 1 & 2,19 & $\operatorname{chrX}$ \\
\hline MAGI1 & 9223 & membrane associated guanylate kinase, WW and PDZ domain containing 1 & 4,14 & chr3 \\
\hline MAGI2 & 9863 & membrane associated guanylate kinase, WW and PDZ domain containing 2 & 5,20 & chr7 \\
\hline MAGOH & 4116 & mago-nashi homolog, proliferation-associated (Drosophila) & 4,79 & chr1 \\
\hline MAK3 & 80218 & Mak3 homolog (S. cerevisiae) & 3,96 & chr3 \\
\hline MAL2 & 114569 & mal, T-cell differentiation protein 2 & 58,76 & chr8 \\
\hline MAML1 & 9794 & mastermind-like 1 (Drosophila) & 2,39 & chr5 \\
\hline MAN2C1 & 4123 & Mannosidase, alpha, class 2C, member 1 & 2,73 & chr15 \\
\hline MAP2K6 & 5608 & mitogen-activated protein kinase kinase 6 & 20,91 & chr17 \\
\hline MAP3K1 & 4214 & Mitogen-activated protein kinase kinase kinase 1 & 3,76 & chr5 \\
\hline MAP3K4 & 4216 & mitogen-activated protein kinase kinase kinase 4 & 4,78 & chr6 \\
\hline МАРЗК9 & 4293 & mitogen-activated protein kinase kinase kinase 9 & 6,61 & chr14 \\
\hline MAP4K1 & 11184 & mitogen-activated protein kinase kinase kinase kinase 1 & 4,85 & chr19 \\
\hline MAP7 & 9053 & microtubule-associated protein 7 & 167,78 & chr6 \\
\hline MAPK1 & 5594 & mitogen-activated protein kinase 1 & 2,26 & chr22 \\
\hline MAPK13 & 5603 & mitogen-activated protein kinase 13 & 3,98 & chr6 \\
\hline MAPK7 & 5598 & mitogen-activated protein kinase 7 & 2,10 & chr17 \\
\hline MAPKAPK3 & 7867 & mitogen-activated protein kinase-activated protein kinase 3 & 4,42 & chr3 \\
\hline MAPKAPK5 & 8550 & mitogen-activated protein kinase-activated protein kinase 5 & 3,60 & chr12 \\
\hline MAPRE2 & 10982 & microtubule-associated protein, RP/EB family, member 2 & 2,47 & chr18 \\
\hline MARCKSL1 & 65108 & MARCKS-like 1 & 3,12 & chr1 \\
\hline MARK1 & 4139 & MAP/microtubule affinity-regulating kinase 1 & 6,81 & chr1 \\
\hline MARS & 4141 & methionine-tRNA synthetase & 2,15 & chr12 \\
\hline MARVELD2 & 153562 & MARVEL domain containing 2 & 18,36 & chr5 \\
\hline MARVELD3 & 91862 & MARVEL domain containing 3 & 14,15 & chr16 \\
\hline MASA & 58478 & E-1 enzyme & 2,42 & chr4 \\
\hline MASK & 51765 & Mst3 and SOK1-related kinase & 2,12 & $\operatorname{chr} X$ \\
\hline MASS1 & 84059 & monogenic, audiogenic seizure susceptibility 1 homolog (mouse) & 15,98 & chr5 \\
\hline MASTL & 84930 & microtubule associated serine/threonine kinase-like & 4,33 & chr10 \\
\hline MAT2A & 4144 & Methionine adenosyltransferase II, alpha & 3,39 & chr2 \\
\hline MATK & 4145 & megakaryocyte-associated tyrosine kinase & 5,85 & chr19 \\
\hline MATR3 & 9782 & Matrin 3 & 5,48 & chr5 \\
\hline MBD2 & 8932 & methyl-CpG binding domain protein 2 & 60,46 & chr18 \\
\hline MBD3 & 53615 & methyl-CpG binding domain protein 3 & 2,07 & chr19 \\
\hline MBD4 & 8930 & methyl-CpG binding domain protein 4 & 2,13 & $\mathrm{chr} 3$ \\
\hline MBP & 4155 & myelin basic protein & 2,20 & $\operatorname{chr} 18$ \\
\hline MBTD1 & 54799 & Mbt domain containing 1 & 7,51 & chr17 \\
\hline MCCC1 & 56922 & methylcrotonoyl-Coenzyme A carboxylase 1 (alpha) & 2,07 & chr3 \\
\hline MCcC2 & 64087 & methylcrotonoyl-Coenzyme A carboxylase 2 (beta) & 3,32 & chr5 \\
\hline MCM10 & 55388 & MCM10 minichromosome maintenance deficient 10 (S. cerevisiae) & 13,39 & chr10 \\
\hline MCM2 & 4171 & MCM2 minichromosome maintenance deficient 2, mitotin (S. cerevisiae) & 10,74 & chr3 \\
\hline MCM3 & 4172 & MCM3 minichromosome maintenance deficient 3 (S. cerevisiae) & 12,59 & chr6 \\
\hline MCMЗAPAS & 114044 & MCM3 minichromosome maintenance deficient 3 (S. cerevisiae) associated prot & 2,12 & chr21 \\
\hline MCM4 & 4173 & MCM4 minichromosome maintenance deficient 4 (S. cerevisiae) & 12,12 & chr8 \\
\hline MCM5 & 4174 & MCM5 minichromosome maintenance deficient 5 , cell division cycle 46 (S. cere) & 16,16 & chr22 \\
\hline MCM6 & 4175 & MCM6 minichromosome maintenance deficient 6 (MIS5 homolog, S. pombe) (S) & 6,30 & chr2 \\
\hline MCM7 & 4176 & MCM7 minichromosome maintenance deficient 7 (S. cerevisiae) & 10,45 & $\mathrm{chr} 7$ \\
\hline MCM8 & 84515 & MCM8 minichromosome maintenance deficient 8 (S. cerevisiae) & 5,60 & chr20 \\
\hline MCOLN3 & 55283 & mucolipin 3 & 3,86 & chr1 \\
\hline $\mathrm{MCPH} 1$ & 79648 & Microcephaly, primary autosomal recessive 1 & 2,07 & chr8 \\
\hline MDC1 & 9656 & mediator of DNA damage checkpoint 1 & 3,50 & chr6 \\
\hline MDH1 & 4190 & malate dehydrogenase 1, NAD (soluble) & 2,18 & chr2 \\
\hline MDK & 4192 & midkine (neurite growth-promoting factor 2) & 2,44 & chr11 \\
\hline MDM4 & 4194 & Mdm4, transformed $3 T 3$ cell double minute 4, p53 binding protein (mouse) & 2,37 & chr1 \\
\hline
\end{tabular}




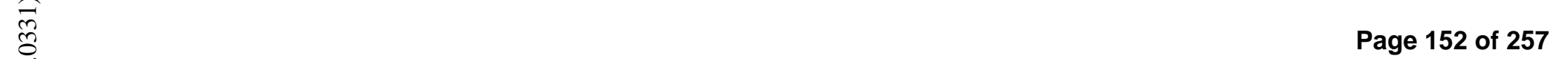

\begin{tabular}{|c|c|c|c|c|}
\hline MDN1 & 23195 & MDN1, midasin homolog (yeast) & 11,08 & chr6 \\
\hline ME2 & 4200 & malic enzyme 2, NAD(+)-dependent, mitochondrial & 2,50 & $\operatorname{chr} 18$ \\
\hline MEA1 & 4201 & male-enhanced antigen 1 & 2,12 & chr6 \\
\hline MED25 & 81857 & mediator of RNA polymerase II transcription, subunit 25 homolog (yeast) & 3,15 & chr19 \\
\hline MED28 & 80306 & mediator of RNA polymerase II transcription, subunit 28 homolog (yeast) & 5,34 & chr1 \\
\hline MED4 & 29079 & mediator of RNA polymerase II transcription, subunit 4 homolog (yeast) & 2,07 & chr13 \\
\hline MED6 & 10001 & mediator of RNA polymerase II transcription, subunit 6 homolog (yeast) & 2,26 & chr14 \\
\hline MED9 & 55090 & mediator of RNA polymerase II transcription, subunit 9 homolog (yeast) & 2,04 & chr17 \\
\hline MEF2A & 4205 & MADS box transcription enhancer factor 2, polypeptide A (myocyte enhancer fad & 2,97 & chr15 \\
\hline MEGF10 & 84466 & MEGF10 protein & 68,97 & chr5 \\
\hline MELK & 9833 & maternal embryonic leucine zipper kinase & 3,45 & chr9 \\
\hline MERTK & 10461 & c-mer proto-oncogene tyrosine kinase & 2,50 & chr2 \\
\hline METAP1 & 23173 & methionyl aminopeptidase 1 & 2,02 & chr4 \\
\hline METAP2 & 10988 & Methionyl aminopeptidase 2 & 3,16 & chr12 \\
\hline METTL3 & 56339 & methyltransferase like 3 & 3,97 & chr14 \\
\hline MFHAS1 & 9258 & malignant fibrous histiocytoma amplified sequence 1 & 11,20 & chr8 \\
\hline MFSD3 & 113655 & major facilitator superfamily domain containing 3 & 5,53 & chr8 \\
\hline MGA & 23269 & MAX gene associated & 2,97 & chr15 \\
\hline MGAT4A & 11320 & Mannosyl (alpha-1,3-)-glycoprotein beta-1,4-N-acetylglucosaminyltransferase, is & 2,70 & chr2 \\
\hline MGC10911 & 84262 & hypothetical protein MGC10911 & 2,70 & chr7 \\
\hline MGC10993 & 80775 & hypothetical protein MGC10993 & 6,41 & chr2 \\
\hline MGC11266 & 79172 & hypothetical protein MGC11266 & 3,66 & chr2 \\
\hline MGC13017 & 91368 & similar to RIKEN cDNA A430101B06 gene & 2,13 & chr5 \\
\hline MGC13096 & 84306 & hypothetical protein MGC13096 /// hypothetical protein MGC13096 & 9,22 & chr19 \\
\hline MGC13125 & 84811 & hypothetical protein MGC13125 /// hypothetical protein MGC13125 & 4,20 & chr11 \\
\hline MGC13170 & 84798 & multidrug resistance-related protein /// multidrug resistance-related protein & 3,54 & chr19 \\
\hline MGC13183 & 84318 & hypothetical protein MGC13183 /// hypothetical protein MGC13183 & 4,96 & chr12 \\
\hline MGC13204 & 83695 & hypothetical protein MGC13204 & 5,93 & chr12 \\
\hline MGC13379 & 51259 & HSPC244 & 2,66 & chr11 \\
\hline MGC14798 & 89978 & similar to RIKEN cDNA 5730421 E18 gene & 7,49 & $\operatorname{chr} 15$ \\
\hline MGC15416 & 84331 & hypothetical protein MGC15416 & 4,62 & chr16 \\
\hline MGC15634 & 84841 & hypothetical protein MGC15634 & 10,55 & chr1 \\
\hline MGC15763 & 92106 & hypothetical protein BC008322 & 2,62 & chr3 \\
\hline MGC16207 & 84853 & hypothetical protein MGC16207 & 2,09 & chr11 \\
\hline MGC16824 & 57020 & Esophageal cancer associated protein & 4,86 & chr16 \\
\hline MGC16943 & 112479 & similar to RIKEN cDNA 4933424N09 gene & 2,14 & chr16 \\
\hline MGC17299 & 128218 & hypothetical protein MGC17299 & 11,61 & chr1 \\
\hline MGC20255 & 90324 & hypothetical protein MGC20255 & 2,21 & chr19 \\
\hline MGC21881 & 389741 & hypothetical protein MGC21881 & 7,42 & chr9 \\
\hline MGC21881 & B89741 /// 401510 & hypothetical protein MGC21881 /// hypothetical LOC401510 & 4,93 & chr9 \\
\hline MGC22014 & 200424 & hypothetical protein MGC22014 & 2,12 & chr2 \\
\hline MGC22265 & 349035 & (clone CB1) mRNA fragment /// Hypothetical protein MGC22265 & 9,90 & chr5 \\
\hline MGC22793 & 221908 & hypothetical protein MGC22793 & 3,17 & $\operatorname{chr} 7$ \\
\hline MGC23280 & 147015 & hypothetical protein MGC23280 & 8,07 & chr17 \\
\hline MGC2408 & 84291 & hypothetical protein MGC2408 & 2,60 & chr3 \\
\hline MGC24103 & 158295 & hypothetical protein MGC24103 & 3,67 & chr9 \\
\hline MGC24665 & 116028 & hypothetical protein MGC24665 & 22,02 & chr16 \\
\hline MGC2477 & 79081 & hypothetical protein MGC2477 & 3,34 & chr11 \\
\hline MGC2574 & 79080 & hypothetical protein MGC2574 & 3,08 & chr11 \\
\hline MGC27345 & 157247 & hypothetical protein MGC27345 & 2,25 & chr7 \\
\hline MGC3040 & 66000 & hypothetical protein MGC3040 & 5,04 & chr3 \\
\hline MGC3196 & 79064 & hypothetical protein MGC3196 & 4,01 & chr11 \\
\hline MGC33214 & 153396 & hypothetical protein MGC33214 & 2,14 & chr5 \\
\hline MGC3329 & 79066 & hypothetical protein MGC3329 & 3,75 & chr17 \\
\hline MGC33584 & 285971 & hypothetical protein MGC33584 & 4,88 & $\operatorname{chr} 7$ \\
\hline MGC33926 & 130733 & hypothetical protein MGC33926 & 3,41 & chr2 \\
\hline MGC3731 & 79159 & hypothetical protein MGC3731 & 3,31 & chr22 \\
\hline MGC39606 & 399668 & hypothetical protein MGC39606 & 4,51 & $\operatorname{chr} X$ \\
\hline MGC40107 & 254863 & hypothetical protein MGC40107 & 2,43 & chr17 \\
\hline MGC40168 & 148645 & hypothetical protein MGC40168 & 2,80 & chr1 \\
\hline MGC40397 & 121053 & hypothetical protein MGC40397 & 3,46 & chr12 \\
\hline MGC40405 & $57415 / / / 401015$ & hypothetical protein MGC40405 /// similar to RIKEN cDNA 5830415L20 & 2,57 & chr5 \\
\hline MGC4172 & 79154 & short-chain dehydrogenase/reductase & 6,97 & chr17 \\
\hline MGC4308 & 84319 & hypothetical protein MGC4308 /// hypothetical protein MGC4308 & 4,63 & chr3 \\
\hline MGC4399 & 84275 & mitochondrial carrier protein & 2,95 & chr1 \\
\hline MGC4562 & 115752 & hypothetical protein MGC4562 & 3,53 & chr15 \\
\hline MGC45866 & 90381 & leucine-rich repeat kinase 1 & 7,82 & chr15 \\
\hline
\end{tabular}




\section{Pagge 153 of 257}

\begin{tabular}{|c|c|c|c|c|}
\hline MGC4825 & 79135 & hypothetical protein MGC4825 & $302+2>+2>0$ & $\operatorname{chr} x$ \\
\hline MGC50372 & 253143 & hypothetical protein MGC50372 & 3,80 & chr22 \\
\hline MGC5139 & 84747 & hypothetical protein MGC5139 & 2,23 & chr12 \\
\hline MGC52000 & 375260 & CXYorf1-related protein & 2,04 & chr1 \\
\hline MGC5352 & 192111 & Bcl-XL-binding protein v68 & 2,32 & chr12 \\
\hline MGC57346 & 401884 & hypothetical LOC401884 & 4,47 & chr17 \\
\hline MGC61598 & 441478 & Similar to ankyrin-repeat protein Nrarp & 19,06 & chr9 \\
\hline MGC72075 & 340277 & hypothetical protein MGC72075 & 8,77 & chr7 \\
\hline MGC90512 & 121642 & similar to hypothetical protein $9530023 \mathrm{G} 02$ & 2,93 & chr12 \\
\hline MGC9913 & 386759 & hypothetical protein MGC9913 & 2,39 & chr19 \\
\hline MGEA5 & 10724 & meningioma expressed antigen 5 (hyaluronidase) & 2,18 & chr10 \\
\hline MGST1 & 4257 & microsomal glutathione S-transferase 1 & 5,00 & chr12 \\
\hline MID1IP1 & 58526 & MID1 interacting protein 1 (gastrulation specific G12-like (zebrafish)) & 2,55 & $\operatorname{chrX}$ \\
\hline MIDN & 90007 & midnolin & 2,07 & chr19 \\
\hline MIPEP & 4285 & mitochondrial intermediate peptidase & 2,19 & chr13 \\
\hline MIZF & 25988 & MBD2 (methyl-CpG-binding protein)-interacting zinc finger protein & 2,37 & chr11 \\
\hline MKI67 & 4288 & antigen identified by monoclonal antibody Ki- 67 & 5,75 & chr10 \\
\hline MKI67IP & 84365 & MKI67 (FHA domain) interacting nucleolar phosphoprotein & 2,67 & chr2 \\
\hline MKKS & 8195 & McKusick-Kaufman syndrome & 2,48 & chr20 \\
\hline MKLN1 & 4289 & muskelin 1, intracellular mediator containing kelch motifs & 2,18 & chr7 \\
\hline MKNK2 & 2872 & MAP kinase interacting serine/threonine kinase 2 & 2,09 & chr19 \\
\hline MLC1SA & 140465 & myosin light chain 1 slow a & 2,63 & chr12 \\
\hline MLF1IP & 79682 & MLF1 interacting protein & 13,67 & chr4 \\
\hline MLH1 & 4292 & mutL homolog 1 , colon cancer, nonpolyposis type 2 (E. coli) & 2,05 & chr3 \\
\hline MLL2 & 8085 & myeloid/lymphoid or mixed-lineage leukemia 2 & 2,05 & chr12 \\
\hline MLL3 & 58508 & myeloid/lymphoid or mixed-lineage leukemia 3 & 2,37 & chr7 \\
\hline MLL5 & 55904 & Myeloid/lymphoid or mixed-lineage leukemia 5 (trithorax homolog, Drosophila) & 3,62 & chr7 \\
\hline MLLT10 & 8028 & myeloid/lymphoid or mixed-lineage leukemia (trithorax homolog, Drosophila); tra & 3,11 & chr10 \\
\hline MLLT4 & 4301 & myeloid/lymphoid or mixed-lineage leukemia (trithorax homolog, Drosophila); tra & 4,34 & chr6 \\
\hline MMD & 23531 & monocyte to macrophage differentiation-associated & 4,38 & chr17 \\
\hline MMRP19 & 51074 & likely ortholog of mouse monocyte macrophage 19 & 2,09 & chr9 \\
\hline MMS19L & 64210 & MMS19-like (MET18 homolog, S. cerevisiae) & 2,34 & chr10 \\
\hline MNAB & 54542 & membrane associated DNA binding protein & 2,82 & chr9 \\
\hline MNS1 & 55329 & meiosis-specific nuclear structural 1 & 3,74 & chr15 \\
\hline MOBKL2B & 79817 & MOB1, Mps One Binder kinase activator-like 2B (yeast) & 17,80 & chr9 \\
\hline MOSC1 & 64757 & MOCO sulphurase C-terminal domain containing 1 & 16,57 & chr1 \\
\hline MPDU1 & 9526 & mannose-P-dolichol utilization defect 1 & 2,08 & chr17 \\
\hline MPDZ & 8777 & multiple PDZ domain protein & 2,30 & chr9 \\
\hline MPHOSPH1 & 9585 & M-phase phosphoprotein 1 & 4,74 & chr10 \\
\hline MPHOSPH6 & 10200 & M-phase phosphoprotein 6 & 2,52 & chr16 \\
\hline MPHOSPH9 & 10198 & M-phase phosphoprotein 9 & 2,97 & $\operatorname{chr} 12$ \\
\hline MPP6 & 51678 & membrane protein, palmitoylated 6 (MAGUK p55 subfamily member 6 ) & 6,18 & chr7 \\
\hline MPPE1 & 65258 & metallophosphoesterase 1 & 14,58 & chr18 \\
\hline MPPED2 & 744 & metallophosphoesterase domain containing 2 & 7,34 & chr11 \\
\hline MRE11A & 4361 & MRE11 meiotic recombination 11 homolog $\mathrm{A}$ (S. cerevisiae) & 3,99 & chr11 \\
\hline MRP63 & 78988 & mitochondrial ribosomal protein 63 & 2,33 & chr13 \\
\hline MRPL10 & 124995 & mitochondrial ribosomal protein L10 & 2,65 & chr17 \\
\hline MRPL11 & 65003 & mitochondrial ribosomal protein L11 & 5,39 & chr11 \\
\hline MRPL12 & 6182 & mitochondrial ribosomal protein L12 & 2,44 & chr17 \\
\hline MRPL13 & 28998 & mitochondrial ribosomal protein L13 & 2,37 & chr8 \\
\hline MRPL14 & 64928 & mitochondrial ribosomal protein L14 & 2,09 & chr6 \\
\hline MRPL15 & 29088 & mitochondrial ribosomal protein L15 & 3,00 & chr8 \\
\hline MRPL16 & 54948 & mitochondrial ribosomal protein L16 & 4,43 & chr11 \\
\hline MRPL2 & 51069 & mitochondrial ribosomal protein $\mathrm{L} 2$ & 2,12 & chr6 \\
\hline MRPL21 & 219927 & mitochondrial ribosomal protein L21 & 3,67 & chr11 \\
\hline MRPL22 & 29093 & mitochondrial ribosomal protein L22 & 2,44 & chr5 \\
\hline MRPL27 & 51264 & mitochondrial ribosomal protein L27 /// mitochondrial ribosomal protein L27 & 2,09 & chr17 \\
\hline MRPL30 & 51263 & mitochondrial ribosomal protein L30 & 2,16 & chr2 \\
\hline MRPL32 & 64983 & mitochondrial ribosomal protein L32 & 3,38 & chr7 \\
\hline MRPL34 & 64981 & mitochondrial ribosomal protein L34 /// mitochondrial ribosomal protein L34 & 2,57 & chr19 \\
\hline MRPL35 & 51318 & mitochondrial ribosomal protein L35 & 2,30 & chr2 \\
\hline MRPL39 & 54148 & mitochondrial ribosomal protein L39 & 2,88 & chr21 \\
\hline MRPL4 & 51073 & mitochondrial ribosomal protein $\mathrm{L} 4$ & 2,26 & chr19 \\
\hline MRPL42 & 28977 & mitochondrial ribosomal protein L42 & 2,75 & chr12 \\
\hline MRPL44 & 65080 & mitochondrial ribosomal protein L44 & 3,25 & chr2 \\
\hline MRPL45 & 84311 & mitochondrial ribosomal protein L45 /// mitochondrial ribosomal protein L45 & 3,11 & chr17 \\
\hline MRPL46 & 26589 & mitochondrial ribosomal protein L46 & 2,50 & chr15 \\
\hline
\end{tabular}




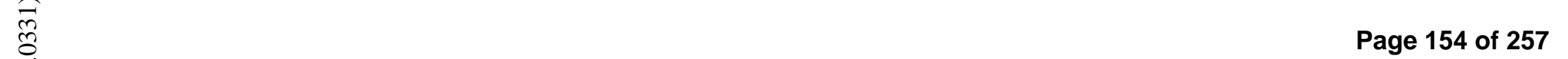

\begin{tabular}{|c|c|c|c|c|}
\hline MRPL47 & 57129 & mitochondrial ribosomal protein L47 & 2,58 & chr3 \\
\hline MRPL50 & 54534 & mitochondrial ribosomal protein L50 & 2,86 & chr9 \\
\hline MRPL51 & 51258 & mitochondrial ribosomal protein L51 /// mitochondrial ribosomal protein L51 & 2,20 & chr12 \\
\hline MRPS12 & 6183 & mitochondrial ribosomal protein S12 & 3,50 & chr19 \\
\hline MRPS17 & 51373 & mitochondrial ribosomal protein S17 & 2,53 & chr7 \\
\hline MRPS18A & 55168 & mitochondrial ribosomal protein S18A & 2,69 & chr6 \\
\hline MRPS18B & 28973 & mitochondrial ribosomal protein S18B & 3,26 & chr6 \\
\hline MRPS2 & 51116 & mitochondrial ribosomal protein S2 & 3,60 & chr9 \\
\hline MRPS23 & 51649 & mitochondrial ribosomal protein S23 & 2,28 & chr17 \\
\hline MRPS25 & 64432 & mitochondrial ribosomal protein S25 & 2,83 & chr3 \\
\hline MRPS26 & 64949 & mitochondrial ribosomal protein S26 & 4,84 & chr20 \\
\hline MRPS27 & 23107 & mitochondrial ribosomal protein S27 & 2,61 & chr5 \\
\hline MRPS28 & 28957 & mitochondrial ribosomal protein S28 & 3,85 & chr8 \\
\hline MRPS30 & 10884 & mitochondrial ribosomal protein S30 & 3,61 & chr5 \\
\hline MRPS31 & 10240 & mitochondrial ribosomal protein S31 & 3,38 & chr13 \\
\hline MRPS34 & 65993 & mitochondrial ribosomal protein S34 & 4,96 & chr16 \\
\hline |MRPS6 & 64968 & Mitochondrial ribosomal protein S6 & 4,16 & chr21 \\
\hline MRPS7 & 51081 & mitochondrial ribosomal protein S7 & 2,45 & chr17 \\
\hline MRPS9 & 64965 & mitochondrial ribosomal protein S9 & 5,66 & chr2 \\
\hline MRS2L & 57380 & MRS2-like, magnesium homeostasis factor (S. cerevisiae) & 8,79 & chr6 \\
\hline MSH2 & 4436 & mutS homolog 2, colon cancer, nonpolyposis type 1 (E. coli) & 19,28 & chr2 \\
\hline MSH5 & 4439 & mutS homolog 5 (E. coli) & 2,25 & chr6 \\
\hline MSH6 & 2956 & mutS homolog 6 (E. coli) & 5,15 & chr2 \\
\hline MSTP9 & 11223 & macrophage stimulating, pseudogene 9 & 2,13 & chr1 \\
\hline MTA1 & 9112 & metastasis associated $1 / / /$ metastasis associated 1 & 2,49 & chr14 \\
\hline MTA3 & 57504 & metastasis associated 1 family, member 3 & 8,95 & chr2 \\
\hline MTAC2D1 & 123036 & membrane targeting (tandem) C2 domain containing 1 & 7,55 & chr14 \\
\hline MTCH2 & 23788 & mitochondrial carrier homolog 2 (C. elegans) & 2,86 & chr11 \\
\hline MTERFD1 & 51001 & MTERF domain containing 1 & 3,13 & chr8 \\
\hline MTERFD2 & 130916 & MTERF domain containing 2 & 3,08 & chr2 \\
\hline MTF2 & 22823 & Metal response element binding transcription factor 2 & 12,75 & chr1 \\
\hline MTHFD1 & 4522 & methylenetetrahydrofolate dehydrogenase (NADP + dependent) 1 , methenyltetra & 4,04 & chr14 \\
\hline MTHFS & 10588 & 5,10-methenyltetrahydrofolate synthetase (5-formyltetrahydrofolate cyclo-ligase) & 2,30 & chr15 \\
\hline MTIF2 & 4528 & mitochondrial translational initiation factor 2 & 2,55 & chr2 \\
\hline MTL5 & 9633 & Metallothionein-like 5 , testis-specific (tesmin) & 2,09 & chr11 \\
\hline MTMR12 & 54545 & myotubularin related protein 12 & 2,61 & chr5 \\
\hline MTMR9 & 66036 & myotubularin related protein 9 & 2,16 & chr8 \\
\hline MTP18 & 51537 & mitochondrial protein $18 \mathrm{kDa}$ & 8,93 & chr22 \\
\hline MTRF1 & 9617 & mitochondrial translational release factor 1 & 3,66 & chr13 \\
\hline MTSS1 & 9788 & metastasis suppressor 1 & 3,63 & chr8 \\
\hline MTX2 & 10651 & metaxin 2 & 2,73 & chr2 \\
\hline MTX3 & 345778 & metaxin 3 & 2,34 & chr5 \\
\hline MUM1 & 84939 & Melanoma associated antigen (mutated) 1 & 4,63 & chr19 \\
\hline MUTYH & 4595 & mutY homolog (E. coli) & 9,11 & chr1 \\
\hline MYB & 4602 & v-myb myeloblastosis viral oncogene homolog (avian) & 8,56 & chr6 \\
\hline MYBL2 & 4605 & v-myb myeloblastosis viral oncogene homolog (avian)-like 2 & 13,60 & chr20 \\
\hline MYCBP & 26292 & c-myc binding protein & 2,40 & chr16 \\
\hline MYCL1 & 4610 & v-myc myelocytomatosis viral oncogene homolog 1 , lung carcinoma derived (av & 3,75 & chr1 \\
\hline MYCN & 4613 & v-myc myelocytomatosis viral related oncogene, neuroblastoma derived (avian) & 52,98 & chr2 \\
\hline |MYEF2 & 50804 & myelin expression factor 2 & 3,20 & chr15 \\
\hline MYLIP & 29116 & myosin regulatory light chain interacting protein & 5,48 & chr6 \\
\hline MYO10 & 4651 & myosin $\mathrm{X}$ & 2,16 & chr5 \\
\hline MYO5B & 4645 & myosin VB & 7,83 & chr18 \\
\hline MYO5C & 55930 & myosin VC & 29,17 & chr15 \\
\hline MYOHD1 & 80179 & myosin head domain containing 1 & 3,08 & chr17 \\
\hline MYOZ3 & 91977 & myozenin 3 & 2,88 & chr5 \\
\hline MYSM1 & 114803 & myb-like, SWIRM and MPN domains 1 & 2,47 & chr1 \\
\hline MYST2 & 11143 & MYST histone acetyltransferase 2 /// MYST histone acetyltransferase 2 & 8,92 & chr17 \\
\hline MYST4 & 23522 & MYST histone acetyltransferase (monocytic leukemia) 4 & 3,88 & chr10 \\
\hline N4BP2 & 55728 & Nedd4 binding protein 2 & 2,98 & chr4 \\
\hline NAALAD2 & 10003 & $\mathrm{~N}$-acetylated alpha-linked acidic dipeptidase 2 & 2,87 & chr11 \\
\hline NACA & $4666 / / / 83955$ & nascent-polypeptide-associated complex alpha polypeptide /// nascent-polypepti & 2,08 & chr12 \\
\hline NAG6 & 64753 & hypothetical protein DKFZp434G156 & 3,25 & chr7 \\
\hline NALP12 & 91662 & $\mathrm{NACHT}$, leucine rich repeat and PYD containing 12 & 2,63 & chr19 \\
\hline NALP2 & 55655 & NACHT, leucine rich repeat and PYD containing 2 & 28,94 & chr19 \\
\hline NANOG & 79923 & Nanog homeobox & 300,80 & chr12 \\
\hline NANOS1 & 340719 & nanos homolog 1 (Drosophila) & 2,08 & chr10 \\
\hline
\end{tabular}




\section{Pagge 155 of 257}

\begin{tabular}{|c|c|c|c|c|}
\hline NAP1L1 & 4673 & nucleosome assembly protein 1 -like 1 & 2,67 & chr12 \\
\hline NAP1L2 & 4674 & nucleosome assembly protein 1 -like 2 & 27,60 & $\operatorname{chr} X$ \\
\hline NAP1L3 & 4675 & nucleosome assembly protein 1 -like 3 & 2,43 & $\operatorname{chr} X$ \\
\hline NAPRT1 & 93100 & nicotinate phosphoribosyltransferase domain containing 1 & 3,57 & chr8 \\
\hline NARF & 26502 & nuclear prelamin A recognition factor & 2,13 & chr17 \\
\hline NARG1 & 80155 & NMDA receptor regulated 1 & 2,55 & chr4 \\
\hline NARG1L & 79612 & NMDA receptor regulated 1 -like & 4,61 & $\operatorname{chr} 13$ \\
\hline NARG2 & 79664 & NMDA receptor regulated 2 & 2,76 & chr3 \\
\hline NASP & 4678 & Nuclear autoantigenic sperm protein (histone-binding) & 33,83 & chr1 \\
\hline NBEA & 26960 & neurobeachin & 6,74 & $\operatorname{chr} 13$ \\
\hline NBEAL2 & 23218 & neurobeachin-like 2 & 2,30 & chr3 \\
\hline NBLA04196 & 64921 & Putative protein product of $\mathrm{Nbla04196}$ & 2,95 & chr7 \\
\hline NCBP1 & 4686 & nuclear cap binding protein subunit $1,80 \mathrm{kDa}$ & 2,94 & chr9 \\
\hline $\mathrm{NCL}$ & 4691 & nucleolin & 2,27 & chr2 \\
\hline NCOA5 & 57727 & Nuclear receptor coactivator 5 & 2,49 & chr20 \\
\hline NCOAGIP & 96764 & nuclear receptor coactivator 6 interacting protein & 3,74 & chr8 \\
\hline NCRMS & 196475 & non-coding RNA in rhabdomyosarcoma (RMS) & 27,12 & chr12 \\
\hline NDRG2 & 57447 & NDRG family member 2 & 2,42 & chr14 \\
\hline NDUFA13 & 51079 & NADH dehydrogenase (ubiquinone) 1 alpha subcomplex, 13 & 2,10 & chr19 \\
\hline NDUFA6 & 4700 & NADH dehydrogenase (ubiquinone) 1 alpha subcomplex, $6,14 \mathrm{kDa}$ & 2,13 & chr22 \\
\hline NDUFAB1 & 4706 & NADH dehydrogenase (ubiquinone) 1 , alpha/beta subcomplex, $1,8 \mathrm{kDa}$ & 2,27 & chr16 \\
\hline NDUFB10 & 4716 & NADH dehydrogenase (ubiquinone) 1 beta subcomplex, 10, 22kDa & 2,50 & chr16 \\
\hline NDUFB9 & 4715 & NADH dehydrogenase (ubiquinone) 1 beta subcomplex, $9,22 \mathrm{kDa}$ & 2,05 & chr8 \\
\hline NDUFS3 & 4722 & NADH dehydrogenase (ubiquinone) Fe-S protein $3,30 \mathrm{kDa}$ (NADH-coenzyme Q & 2,77 & chr11 \\
\hline NDUFS6 & 4726 & NADH dehydrogenase (ubiquinone) Fe-S protein $6,13 \mathrm{kDa}$ (NADH-coenzyme $\mathrm{Q}$ & 2,48 & chr5 \\
\hline NDUFS7 & 374291 & NADH dehydrogenase (ubiquinone) Fe-S protein 7, 20kDa (NADH-coenzyme $\mathrm{Q}$ & 2,57 & chr19 \\
\hline NDUFV2 & 4729 & NADH dehydrogenase (ubiquinone) flavoprotein 2, 24kDa & 2,74 & chr18 \\
\hline NEBL & 10529 & nebulette & 8,36 & chr10 \\
\hline NEF3 & 4741 & neurofilament 3 (150kDa medium) & 12,02 & chr8 \\
\hline NEFL & 4747 & neurofilament, light polypeptide $68 \mathrm{kDa}$ & 19,79 & chr8 \\
\hline NEIL3 & 55247 & nei endonuclease VIII-like 3 (E. coli) & 2,63 & chr4 \\
\hline NEK2 & 4751 & NIMA (never in mitosis gene a)-related kinase 2 & 4,74 & chr14 \\
\hline NEK4 & 6787 & NIMA (never in mitosis gene a)-related kinase 4 & 3,21 & chr3 \\
\hline NEK8 & 284086 & NIMA (never in mitosis gene a)- related kinase 8 & 2,58 & chr17 \\
\hline NELL2 & 4753 & NEL-like 2 (chicken) /// NEL-like 2 (chicken) & 69,31 & chr12 \\
\hline NEO1 & 4756 & neogenin homolog 1 (chicken) & 2,53 & chr15 \\
\hline NEXN & 91624 & nexilin ( $\mathrm{F}$ actin binding protein) & 2,74 & chr1 \\
\hline NFATC2IP & 84901 & nuclear factor of activated T-cells, cytoplasmic, calcineurin-dependent 2 interacti & 6,94 & chr16 \\
\hline NFE2L3 & 9603 & nuclear factor (erythroid-derived 2)-like 3 & 2,81 & $\mathrm{chr} 7$ \\
\hline NFRKB & 4798 & nuclear factor related to kappaB binding protein & 2,44 & chr11 \\
\hline NFX1 & 4799 & nuclear transcription factor, $\mathrm{X}$-box binding 1 & 2,10 & chr9 \\
\hline NFXL1 & 152518 & nuclear transcription factor, $\mathrm{X}$-box binding-like 1 & 3,29 & chr4 \\
\hline NFYB & 4801 & nuclear transcription factor $\mathrm{Y}$, beta & 5,78 & chr3 \\
\hline NGFRAP1L1 & 340542 & NGFRAP1-like 1 & 16,66 & $\operatorname{chr} X$ \\
\hline NGLY1 & 55768 & N-glycanase 1 & 2,30 & chr3 \\
\hline NHP2L1 & 4809 & NHP2 non-histone chromosome protein 2-like 1 (S. cerevisiae) & 2,19 & chr22 \\
\hline NHSL1 & 57224 & NHS-like 1 & 3,25 & chr6 \\
\hline NIF3L1 & 60491 & NIF3 NGG1 interacting factor 3-like 1 (S. pombe) & 4,92 & chr2 \\
\hline NIFIE14 & 10430 & seven transmembrane domain protein & 2,75 & chr19 \\
\hline NIPBL & 25836 & Nipped-B homolog (Drosophila) & 4,22 & chr5 \\
\hline NJMU-R1 & 64149 & protein kinase Njmu-R1 & 2,21 & chr17 \\
\hline NLE1 & 54475 & notchless homolog 1 (Drosophila) & 4,48 & chr17 \\
\hline NLGN4X & 57502 & neuroligin 4, X-linked & 60,63 & $\operatorname{chr} X$ \\
\hline NLK & 51701 & nemo like kinase & 3,72 & chr17 \\
\hline NLN & 57486 & neurolysin (metallopeptidase M3 family) & 4,02 & chr5 \\
\hline NME3 & 4832 & non-metastatic cells 3 , protein expressed in & 2,42 & $\operatorname{chr} 16$ \\
\hline NMI & 9111 & $\mathrm{~N}$-myc (and STAT) interactor & 2,23 & chr2 \\
\hline NMNAT2 & 23057 & nicotinamide nucleotide adenylyltransferase 2 & 2,56 & chr1 \\
\hline NMU & 10874 & neuromedin U & 53,18 & chr4 \\
\hline NOB1P & 28987 & nin one binding protein & 3,73 & chr4 \\
\hline NOC4L & 79050 & nucleolar complex associated 4 homolog (S. cerevisiae) & 2,41 & chr12 \\
\hline NODAL & 4838 & nodal homolog (mouse) & 10,18 & chr10 \\
\hline NOL1 & 4839 & nucleolar protein $1,120 \mathrm{kDa}$ & 2,23 & chr12 \\
\hline NOL11 & 25926 & nucleolar protein 11 & 4,69 & chr17 \\
\hline NOL5A & 10528 & nucleolar protein $5 \mathrm{~A}$ (56kDa with $\mathrm{KKE} / \mathrm{D}$ repeat) & 6,20 & chr20 \\
\hline NOL7 & 51406 & Nucleolar protein $7,27 \mathrm{kDa}$ & 2,15 & chr6 \\
\hline NOL9 & 79707 & nucleolar protein 9 & 2,03 & chr1 \\
\hline
\end{tabular}




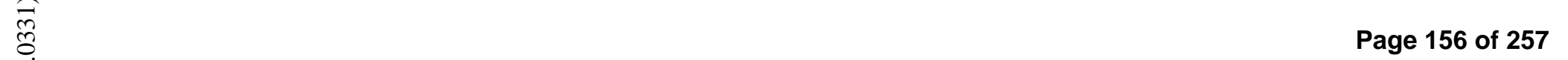

\begin{tabular}{|c|c|c|c|c|}
\hline NOLC1 & 9221 & nucleolar and coiled-body phosphoprotein 1 & 6,23 & chr10 \\
\hline NOP5/NOP58 & 51602 & nucleolar protein NOP5/NOP58 & 3,26 & chr2 \\
\hline NOSIP & 51070 & nitric oxide synthase interacting protein & 2,37 & chr19 \\
\hline NOVA1 & 4857 & neuro-oncological ventral antigen 1 & 2,65 & chr14 \\
\hline $\mathrm{NP}$ & 4860 & nucleoside phosphorylase & 2,63 & chr14 \\
\hline NPAT & 4863 & nuclear protein, ataxia-telangiectasia locus & 2,48 & chr11 \\
\hline NPM1 & 4869 & nucleophosmin (nucleolar phosphoprotein B23, numatrin) /// nucleophosmin (nu & 2,54 & chr1 \\
\hline NPM3 & 10360 & nucleophosmin/nucleoplasmin, 3 & 8,82 & $\operatorname{chr} 10$ \\
\hline NPTN & 27020 & Neuroplastin & 2,56 & chr15 \\
\hline NPTX1 & 4884 & neuronal pentraxin I & 4,21 & chr17 \\
\hline NPTX2 & 4885 & neuronal pentraxin II & 6,43 & chr7 \\
\hline NQO2 & 4835 & $\mathrm{NAD}(\mathrm{P}) \mathrm{H}$ dehydrogenase, quinone 2 & 2,60 & chr6 \\
\hline NR2C1 & 7181 & nuclear receptor subfamily 2, group C, member 1 & 2,54 & chr12 \\
\hline NR2C2 & 7182 & Nuclear receptor subfamily 2, group C, member 2 & 2,25 & chr3 \\
\hline NR2F6 & 2063 & nuclear receptor subfamily 2 , group $F$, member 6 & 4,56 & chr19 \\
\hline NR5A2 & 2494 & nuclear receptor subfamily 5 , group A, member 2 & 2,33 & chr1 \\
\hline NRF1 & 4899 & nuclear respiratory factor 1 & 2,68 & chr7 \\
\hline NRG3 & 10718 & neuregulin 3 & 2,28 & chr10 \\
\hline NRM & 11270 & nurim (nuclear envelope membrane protein) & 2,05 & chr6 \\
\hline NRXN1 & 9378 & neurexin 1 & 3,54 & chr2 \\
\hline NSBP1 & 79366 & nucleosomal binding protein 1 & 9,80 & $\operatorname{chr} X$ \\
\hline NSD1 & 64324 & nuclear receptor binding SET domain protein 1 & 4,48 & chr5 \\
\hline NSDHL & 50814 & NAD $(P)$ dependent steroid dehydrogenase-like & 2,22 & $\operatorname{chr} X$ \\
\hline NSUN2 & 54888 & NOL1/NOP2/Sun domain family, member 2 & 2,30 & chr5 \\
\hline NSUN5 & 55695 & NOL1/NOP2/Sun domain family, member 5 & 3,89 & $\operatorname{chr} 7$ \\
\hline NSUN5C & 260294 & NOL1/NOP2/Sun domain family, member $5 \mathrm{C}$ & 4,60 & $\operatorname{chr} 7$ \\
\hline NSUN6 & 221078 & NOL1/NOP2/Sun domain family, member 6 & 2,16 & chr10 \\
\hline NT5C & 30833 & 5', 3'-nucleotidase, cytosolic & 2,07 & chr7 \\
\hline NT5C2 & 22978 & 5'-nucleotidase, cytosolic II & 2,43 & chr10 \\
\hline NT5C2L1 & 221294 & 5'-nucleotidase, cytosolic II-like 1 & 7,02 & chr6 \\
\hline NTHL1 & 4913 & nth endonuclease III-like 1 (E. coli) & 4,93 & chr16 \\
\hline NTS & 4922 & neurotensin & 10,94 & chr12 \\
\hline NUBP1 & 4682 & nucleotide binding protein 1 (MinD homolog, E. coli) & 2,03 & chr16 \\
\hline NUCKS1 & 64710 & nuclear casein kinase and cyclin-dependent kinase substrate 1 & 2,78 & chr1 \\
\hline NUDC & 10726 & nuclear distribution gene $\mathrm{C}$ homolog (A. nidulans) & 3,08 & chr1 \\
\hline NUDCD1 & 84955 & NudC domain containing 1 & 2,13 & chr8 \\
\hline NUDCD2 & 134492 & NudC domain containing 2 & 3,81 & chr5 \\
\hline NUDT1 & 4521 & nudix (nucleoside diphosphate linked moiety X)-type motif 1 & 7,03 & chr7 \\
\hline NUDT10 & 170685 & nudix (nucleoside diphosphate linked moiety $\mathrm{X}$ )-type motif 10 & 3,37 & $\operatorname{chr} X$ \\
\hline NUDT11 & 55190 & nudix (nucleoside diphosphate linked moiety X)-type motif 11 & 2,89 & $\operatorname{chr} X$ \\
\hline NUDT15 & 55270 & nudix (nucleoside diphosphate linked moiety X)-type motif 15 & 6,99 & $\operatorname{chr} 13$ \\
\hline NUDT21 & 11051 & nudix (nucleoside diphosphate linked moiety X)-type motif 21 & 3,80 & chr16 \\
\hline NUDT5 & 11164 & Nudix (nucleoside diphosphate linked moiety X)-type motif 5 & 2,44 & chr10 \\
\hline NUFIP1 & 26747 & nuclear fragile $\mathrm{X}$ mental retardation protein interacting protein 1 & 4,02 & chr6 \\
\hline NUP107 & 57122 & nucleoporin $107 \mathrm{kDa}$ & 4,23 & chr12 \\
\hline NUP133 & 55746 & nucleoporin $133 \mathrm{kDa}$ & 2,28 & chr1 \\
\hline NUP153 & 9972 & nucleoporin $153 \mathrm{kDa}$ & 2,03 & chr6 \\
\hline NUP155 & 9631 & nucleoporin $155 \mathrm{kDa}$ & 2,67 & chr5 \\
\hline NUP160 & 23279 & nucleoporin $160 \mathrm{kDa}$ & 3,04 & chr11 \\
\hline NUP188 & 23511 & nucleoporin $188 \mathrm{kDa}$ & 2,37 & chr9 \\
\hline NUP205 & 23165 & nucleoporin $205 \mathrm{kDa}$ & 4,75 & $\operatorname{chr} 7$ \\
\hline NUP210 & 23225 & nucleoporin $210 \mathrm{kDa}$ & 9,21 & chr3 \\
\hline NUP214 & 8021 & nucleoporin 214kDa & 2,51 & chr9 \\
\hline NUP35 & 129401 & nucleoporin $35 \mathrm{kDa}$ & 6,49 & chr2 \\
\hline NUP37 & 79023 & nucleoporin $37 \mathrm{kDa}$ & 2,47 & chr12 \\
\hline NUP43 & 348995 & nucleoporin $43 \mathrm{kDa}$ & 2,01 & chr6 \\
\hline NUP50 & 10762 & nucleoporin $50 \mathrm{kDa}$ & 2,88 & chr22 \\
\hline NUP54 & 53371 & nucleoporin $54 \mathrm{kDa}$ & 2,89 & chr4 \\
\hline NUP62 & 23636 & nucleoporin $62 \mathrm{kDa}$ & 3,03 & chr19 \\
\hline NUP88 & 4927 & nucleoporin $88 \mathrm{kDa}$ & 3,83 & chr17 \\
\hline NUP93 & 9688 & nucleoporin $93 \mathrm{kDa}$ & 3,63 & chr16 \\
\hline NUP98 & 4928 & nucleoporin $98 \mathrm{kDa}$ & 2,14 & chr11 \\
\hline NUPL1 & 9818 & Nucleoporin like 1 & 2,48 & chr13 \\
\hline NUSAP1 & 51203 & nucleolar and spindle associated protein 1 & 7,77 & chr15 \\
\hline NVL & 4931 & nuclear VCP-like & 3,43 & chr1 \\
\hline NXT2 & 55916 & nuclear transport factor 2-like export factor 2 & 2,58 & $\operatorname{chr} X$ \\
\hline NY-REN-41 & 91057 & NY-REN-41 antigen & 6,41 & chr11 \\
\hline
\end{tabular}




\section{Pagge 157 of 257}

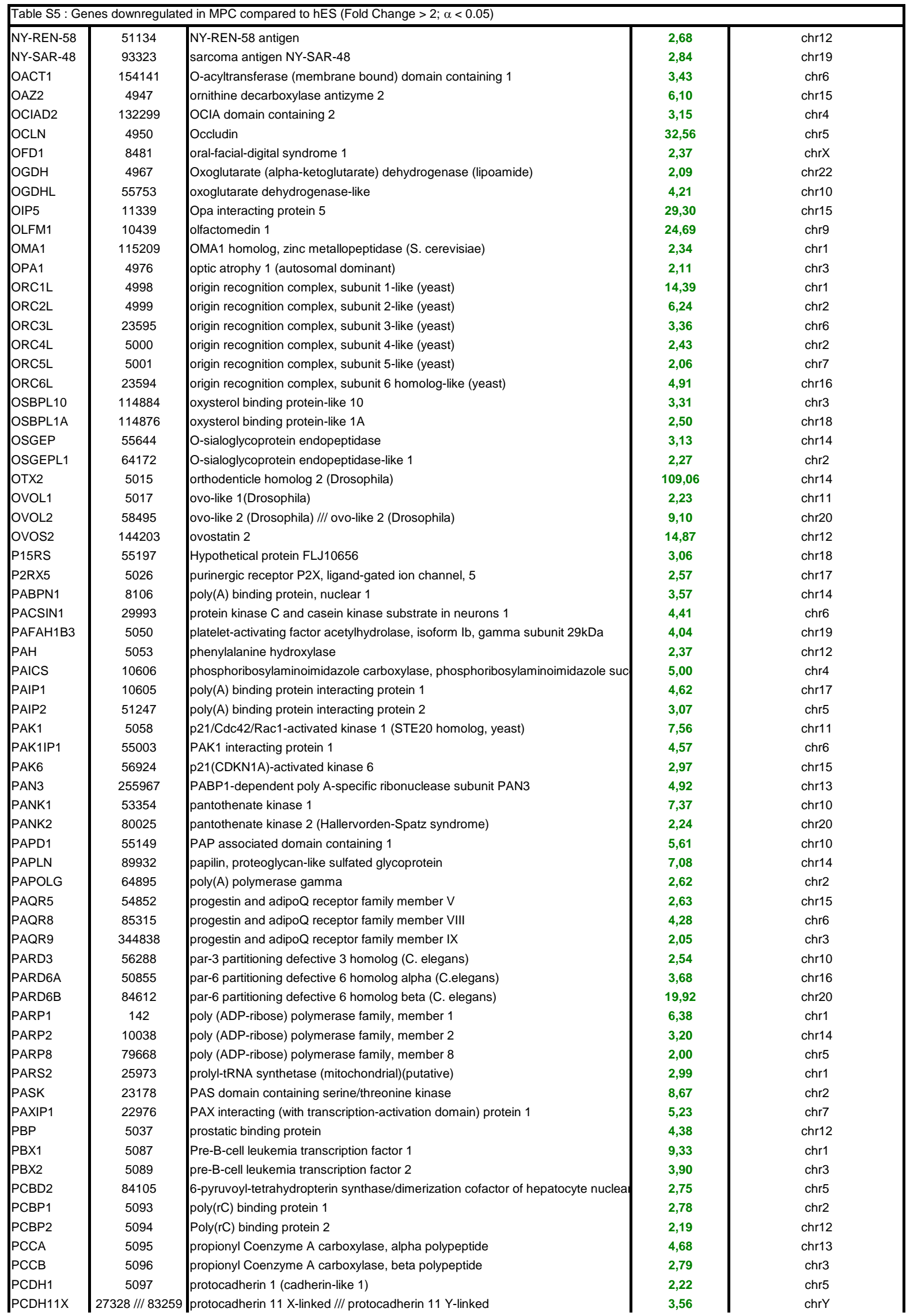




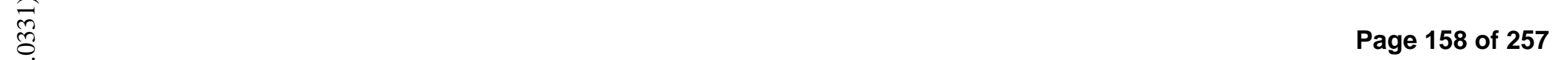

\begin{tabular}{|c|c|c|c|c|}
\hline PCDH8 & 5100 & protocadherin 8 & 4,56 & chr13 \\
\hline PCDHB3 & 56132 & protocadherin beta 3 & 2,03 & chr5 \\
\hline PCDHB5 & 26167 & protocadherin beta 5 & 6,58 & chr5 \\
\hline PCDHGA4 & 56111 & protocadherin gamma subfamily A, 4 & 2,75 & chr5 \\
\hline PCF11 & 51585 & PCF11, cleavage and polyadenylation factor subunit, homolog (S. cerevisiae) & 2,51 & chr11 \\
\hline PCGF3 & 10336 & Polycomb group ring finger 3 & 2,49 & chr4 \\
\hline PCGF6 & 84108 & polycomb group ring finger $6 / / /$ polycomb group ring finger 6 & 2,85 & chr10 \\
\hline PCLO & 27445 & piccolo (presynaptic cytomatrix protein) & 2,53 & chr7 \\
\hline PCM1 & 5108 & Pericentriolar material 1 & 2,32 & chr8 \\
\hline PCMTD2 & 55251 & protein-L-isoaspartate (D-aspartate) O-methyltransferase domain containing 2 & 2,40 & chr20 \\
\hline PCNA & 5111 & proliferating cell nuclear antigen & 3,78 & chr20 \\
\hline PCNT1 & 79902 & pericentrin 1 & 4,18 & chr17 \\
\hline PCNXL2 & 80003 & Pecanex-like 2 (Drosophila) & 2,79 & chr1 \\
\hline PCSK9 & 255738 & proprotein convertase subtilisin/kexin type 9 & 8,12 & chr1 \\
\hline PCTP & 58488 & phosphatidylcholine transfer protein & 3,42 & chr17 \\
\hline PCYT1B & 9468 & phosphate cytidylyltransferase 1 , choline, beta & 4,48 & $\operatorname{chrX}$ \\
\hline PDCD11 & 22984 & programmed cell death 11 & 4,98 & chr10 \\
\hline PDCD2 & 5134 & programmed cell death 2 & 3,52 & chr6 \\
\hline PDCD5 & 9141 & programmed cell death 5 & 2,13 & chr19 \\
\hline PDCD6 & 10016 & Programmed cell death 6 & 5,13 & chr5 \\
\hline PDCD6 & $10016 / / / 440997$ & programmed cell death $6 / / /$ hypothetical gene supported by AK055127; BC0539 & 4,21 & chr5 \\
\hline PDCL & 5082 & phosducin-like & 2,38 & chr9 \\
\hline PDCL3 & $285359 / / / 79031$ & phosducin-like 3 /// hypothetical protein FLJ12205 & 2,21 & chr2 \\
\hline PDE3B & 5140 & Phosphodiesterase 3B, cGMP-inhibited & 2,18 & chr11 \\
\hline PDE6D & 5147 & phosphodiesterase 6D, cGMP-specific, rod, delta & 2,17 & chr2 \\
\hline PDE7A & 5150 & phosphodiesterase 7A & 7,01 & chr8 \\
\hline PDE9A & 5152 & phosphodiesterase 9A & 8,87 & chr21 \\
\hline PDF & $64146 / / / 84342$ & peptide deformylase-like protein /// component of oligomeric golgi complex 8 & 3,26 & chr16 \\
\hline PDHA1 & 5160 & pyruvate dehydrogenase (lipoamide) alpha 1 & 3,32 & $\operatorname{chrX}$ \\
\hline PDK3 & 5165 & pyruvate dehydrogenase kinase, isoenzyme 3 & 6,76 & $\operatorname{chrX}$ \\
\hline PDPN & 10630 & podoplanin & 7,08 & chr1 \\
\hline PDPR & 55066 & pyruvate dehydrogenase phosphatase regulatory subunit & 3,05 & chr16 \\
\hline PDXP & 57026 & pyridoxal (pyridoxine, vitamin B6) phosphatase & 3,06 & chr22 \\
\hline PDZK3 & 23037 & PDZ domain containing 3 & 6,21 & chr5 \\
\hline PDZK4 & 57595 & PDZ domain containing 4 & 4,51 & $\operatorname{chrX}$ \\
\hline PELI1 & 57162 & pellino homolog 1 (Drosophila) & 6,44 & chr2 \\
\hline PELI2 & 57161 & pellino homolog 2 (Drosophila) & 3,17 & chr14 \\
\hline PELP1 & 27043 & proline-, glutamic acid-, leucine-rich protein 1 & 2,28 & chr17 \\
\hline PEMT & 10400 & phosphatidylethanolamine $\mathrm{N}$-methyltransferase & 2,25 & chr17 \\
\hline PEO1 & 56652 & progressive external ophthalmoplegia 1 & 4,73 & chr10 \\
\hline PET112L & 5188 & PET112-like (yeast) & 2,29 & chr4 \\
\hline PEX1 & 5189 & peroxisome biogenesis factor 1 & 2,27 & chr7 \\
\hline PEX13 & 5194 & peroxisome biogenesis factor 13 & 3,85 & chr2 \\
\hline PEX5 & 5830 & peroxisomal biogenesis factor 5 & 2,16 & chr12 \\
\hline PFAS & 5198 & phosphoribosylformylglycinamidine synthase (FGAR amidotransferase) & 7,80 & chr17 \\
\hline PFDN4 & 5203 & prefoldin 4 & 2,40 & chr20 \\
\hline PFKFB2 & 5208 & 6-phosphofructo-2-kinase/fructose-2,6-biphosphatase 2 & 2,03 & chr1 \\
\hline Pfs2 & 51659 & DNA replication complex GINS protein PSF2 & 8,81 & chr16 \\
\hline PGAP1 & 80055 & GPI deacylase & 3,02 & chr2 \\
\hline PGBD5 & 79605 & piggyBac transposable element derived 5 & 8,97 & chr1 \\
\hline PGGT1B & 5229 & Protein geranylgeranyltransferase type I, beta subunit & 2,47 & chr5 \\
\hline PGM2L1 & 283209 & phosphoglucomutase 2-like 1 & 2,53 & chr11 \\
\hline PHACTR2 & 9749 & phosphatase and actin regulator 2 & 2,86 & chr6 \\
\hline PHB & 5245 & prohibitin & 2,96 & chr16 \\
\hline PHC1 & 1911 & polyhomeotic-like 1 (Drosophila) & 34,21 & chr12 \\
\hline PHF10 & 55274 & PHD finger protein 10 & 2,83 & chr6 \\
\hline PHF13 & 148479 & PHD finger protein 13 & 2,46 & chr1 \\
\hline PHF14 & 9678 & PHD finger protein 14 & 3,30 & chr7 \\
\hline PHF15 & 23338 & PHD finger protein 15 & 5,57 & chr5 \\
\hline PHF16 & 9767 & PHD finger protein 16 & 3,37 & $\operatorname{chrX}$ \\
\hline PHF17 & 79960 & PHD finger protein 17 & 11,39 & chr4 \\
\hline PHF20 & 51230 & PHD finger protein 20 & 2,15 & chr20 \\
\hline PHF21B & 112885 & PHD finger protein 21B & 8,54 & chr22 \\
\hline PHF3 & 23469 & PHD finger protein 3 & 2,77 & chr6 \\
\hline PHF5A & 84844 & PHD finger protein $5 \mathrm{~A}$ & 4,32 & chr22 \\
\hline PHGDH & 26227 & phosphoglycerate dehydrogenase & 4,25 & chr1 \\
\hline PHIP & 55023 & pleckstrin homology domain interacting protein & 4,67 & chr6 \\
\hline
\end{tabular}




\section{Page 159 of 257}

\begin{tabular}{|c|c|c|c|c|}
\hline PHKA2 & 5256 & phosphorylase kinase, alpha 2 (liver) & 2,08 & $\operatorname{chrX}$ \\
\hline PHLPP & 23239 & PH domain and leucine rich repeat protein phosphatase & 5,94 & chr18 \\
\hline PHLPPL & 23035 & PH domain and leucine rich repeat protein phosphatase-like & 2,68 & chr16 \\
\hline PHOSPHO2 & 493911 & phosphatase, orphan 2 & 5,66 & chr2 \\
\hline PIAS2 & 9063 & Protein inhibitor of activated STAT, 2 & 4,28 & chr18 \\
\hline PIAS4 & 51588 & protein inhibitor of activated STAT, 4 & 2,46 & chr19 \\
\hline PIG8 & 9702 & translokin & 3,57 & chr11 \\
\hline PIGW & 284098 & phosphatidylinositol glycan, class W & 2,47 & chr17 \\
\hline PIK3C2A & 5286 & Phosphoinositide-3-kinase, class 2, alpha polypeptide & 2,33 & chr11 \\
\hline РІКЗСВ & 5291 & phosphoinositide-3-kinase, catalytic, beta polypeptide & 2,44 & chr3 \\
\hline PIK3R4 & 30849 & phosphoinositide-3-kinase, regulatory subunit 4, p150 & 2,72 & chr3 \\
\hline PILRB & 29990 & paired immunoglobin-like type 2 receptor beta & 3,19 & chr7 \\
\hline PIM2 & 11040 & pim-2 oncogene & 4,49 & $\operatorname{chr} X$ \\
\hline PINX1 & 54984 & PIN2-interacting protein 1 & 3,46 & chr8 \\
\hline PIP5K1B & 8395 & phosphatidylinositol-4-phosphate 5-kinase, type I, beta & 3,25 & chr9 \\
\hline PIP5K2A & 5305 & Phosphatidylinositol-4-phosphate 5-kinase, type II, alpha & 3,48 & chr10 \\
\hline PIPOX & 51268 & pipecolic acid oxidase & 10,59 & chr17 \\
\hline PIR & 8544 & pirin (iron-binding nuclear protein) & 5,24 & $\operatorname{chrX}$ \\
\hline PISD & 23761 & phosphatidylserine decarboxylase & 2,36 & chr22 \\
\hline PITPNC1 & 26207 & phosphatidylinositol transfer protein, cytoplasmic 1 & 4,84 & chr17 \\
\hline PKN3 & 29941 & protein kinase N3 & 2,76 & chr9 \\
\hline PKP4 & 8502 & Plakophilin 4 & 2,54 & chr2 \\
\hline PLA2G3 & 50487 & phospholipase A2, group III & 2,37 & chr22 \\
\hline PLA2G4B & 8681 & phospholipase A2, group IVB (cytosolic) & 2,51 & chr15 \\
\hline PLA2G7 & 7941 & phospholipase A2, group VII (platelet-activating factor acetylhydrolase, plasma) & 3,06 & chr6 \\
\hline PLAGL2 & 5326 & pleiomorphic adenoma gene-like 2 & 2,75 & chr20 \\
\hline PLCG1 & 5335 & phospholipase C, gamma 1 & 2,38 & chr20 \\
\hline PLCL2 & 23228 & phospholipase C-like 2 & 9,56 & chr3 \\
\hline PLCL3 & 23007 & phospholipase C-like 3 & 8,50 & chr3 \\
\hline PLCXD1 & 55344 & phosphatidylinositol-specific phospholipase $\mathrm{C}, \mathrm{X}$ domain containing 1 & 6,35 & $\operatorname{chrX}$ \\
\hline PLEKHA5 & 54477 & Pleckstrin homology domain containing, family A member 5 & 8,88 & chr12 \\
\hline PLEKHA8 & 84725 & Pleckstrin homology domain containing, family A (phosphoinositide binding spec & 2,94 & chr7 \\
\hline PLEKHA9 & $51054 / / / 84725$ & pleckstrin homology domain containing, family A (phosphoinositide binding spec & 2,20 & chr7 \\
\hline PLEKHB1 & 58473 & pleckstrin homology domain containing, family B (evectins) member 1 & 2,32 & chr11 \\
\hline PLEKHH1 & 57475 & pleckstrin homology domain containing, family $\mathrm{H}$ (with MyTH4 domain) member & 10,69 & chr14 \\
\hline PLEKHJ1 & 55111 & pleckstrin homology domain containing, family J member 1 & 3,88 & chr19 \\
\hline PLEKHK1 & 219790 & pleckstrin homology domain containing, family $\mathrm{K}$ member 1 & 2,19 & chr10 \\
\hline PLEKHM1 & 9842 & Pleckstrin homology domain containing, family M (with RUN domain) member 1 & 2,10 & chr17 \\
\hline PLK1 & 5347 & Polo-like kinase 1 (Drosophila) & 7,40 & chr16 \\
\hline PLK4 & 10733 & polo-like kinase 4 (Drosophila) & 13,11 & chr4 \\
\hline PLP1 & 5354 & proteolipid protein 1 (Pelizaeus-Merzbacher disease, spastic paraplegia 2, uncor & 13,04 & $\operatorname{chrX}$ \\
\hline PLS1 & 5357 & plastin 1 (I isoform) & 11,22 & chr3 \\
\hline PLSCR1 & 5359 & phospholipid scramblase 1 & 3,75 & chr3 \\
\hline PMAIP1 & 5366 & phorbol-12-myristate-13-acetate-induced protein 1 & 3,05 & chr18 \\
\hline PMF1 & 11243 & polyamine-modulated factor 1 & 3,84 & chr1 \\
\hline PMPCB & 9512 & peptidase (mitochondrial processing) beta & 2,07 & chr7 \\
\hline PMS1 & 5378 & PMS1 postmeiotic segregation increased 1 (S. cerevisiae) & 3,95 & chr2 \\
\hline PMS2L1 & $5379 / / / 5383$ & postmeiotic segregation increased 2-like $1 / / /$ postmeiotic segregation increased & 2,16 & chr7 \\
\hline PMS2L3 & 5387 & postmeiotic segregation increased 2 -like 3 & 2,04 & chr7 \\
\hline PNKP & 11284 & polynucleotide kinase 3'-phosphatase & 2,24 & chr19 \\
\hline PNN & 5411 & pinin, desmosome associated protein & 4,69 & chr14 \\
\hline PNPT1 & 87178 & polyribonucleotide nucleotidyltransferase 1 & 3,59 & chr2 \\
\hline PNRC2 & 55629 & proline-rich nuclear receptor coactivator 2 & 2,59 & chr1 \\
\hline PODXL & 5420 & podocalyxin-like & 59,45 & chr7 \\
\hline POLA & 5422 & polymerase (DNA directed), alpha & 8,59 & $\operatorname{chrX}$ \\
\hline POLA2 & 23649 & polymerase (DNA directed), alpha 2 (70kD subunit) & 2,45 & chr11 \\
\hline POLB & 5423 & polymerase (DNA directed), beta & 2,14 & chr8 \\
\hline POLD1 & 5424 & polymerase (DNA directed), delta 1 , catalytic subunit $125 \mathrm{kDa}$ & 6,28 & chr19 \\
\hline POLD2 & 5425 & polymerase (DNA directed), delta 2, regulatory subunit 50kDa & 2,34 & chr7 \\
\hline POLD3 & 10714 & polymerase (DNA-directed), delta 3, accessory subunit & 4,51 & chr11 \\
\hline POLDIP3 & 84271 & polymerase (DNA-directed), delta interacting protein 3 & 2,08 & chr22 \\
\hline POLE & 5426 & polymerase (DNA directed), epsilon & 5,23 & chr12 \\
\hline POLE2 & 5427 & polymerase (DNA directed), epsilon 2 (p59 subunit) & 10,68 & chr14 \\
\hline POLG2 & 11232 & polymerase (DNA directed), gamma 2, accessory subunit & 4,50 & chr17 \\
\hline POLI & 11201 & polymerase (DNA directed) iota & 2,73 & chr18 \\
\hline POLQ & 10721 & polymerase (DNA directed), theta & 5,72 & chr3 \\
\hline POLR1A & 25885 & polymerase (RNA) I polypeptide A, $194 \mathrm{kDa}$ & 3,58 & chr2 \\
\hline
\end{tabular}




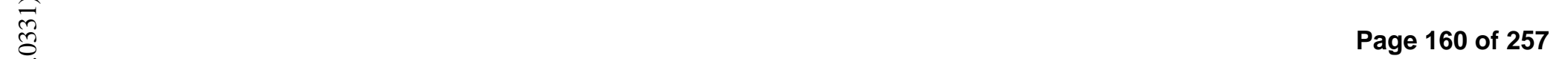

\begin{tabular}{|c|c|c|c|c|}
\hline POLR1B & 84172 & polymerase (RNA) I polypeptide B, $128 \mathrm{kDa}$ & 4,79 & chr2 \\
\hline POLR1C & 9533 & polymerase (RNA) I polypeptide C, 30kDa & 2,50 & chr6 \\
\hline POLR1D & 51082 & polymerase (RNA) I polypeptide D, $16 \mathrm{kDa}$ & 5,54 & chr13 \\
\hline POLR2D & 5433 & polymerase (RNA) II (DNA directed) polypeptide D & 3,68 & chr2 \\
\hline POLR2F & 5435 & polymerase (RNA) II (DNA directed) polypeptide F & 2,75 & chr22 \\
\hline POLR2G & 5436 & polymerase (RNA) II (DNA directed) polypeptide G & 2,07 & chr11 \\
\hline POLR2H & 5437 & polymerase (RNA) II (DNA directed) polypeptide H & 2,93 & chr3 \\
\hline POLR2I & 5438 & polymerase (RNA) II (DNA directed) polypeptide I, $14.5 \mathrm{kDa}$ & 3,54 & $\operatorname{chr} 19$ \\
\hline POLR3A & 11128 & polymerase (RNA) III (DNA directed) polypeptide A, $155 \mathrm{kDa}$ & 2,05 & chr10 \\
\hline POLR3E & 55718 & polymerase (RNA) III (DNA directed) polypeptide E (80kD) & 3,14 & chr16 \\
\hline POLR3G & 10622 & Polymerase (RNA) III (DNA directed) polypeptide G (32kD) & 8,94 & chr5 \\
\hline POLR3K & 51728 & polymerase (RNA) III (DNA directed) polypeptide K, $12.3 \mathrm{kDa}$ & 8,19 & chr16 \\
\hline POLRMT & 5442 & polymerase (RNA) mitochondrial (DNA directed) & 4,45 & chr17 \\
\hline POP4 & 10775 & Processing of precursor 4, ribonuclease P/MRP subunit (S. cerevisiae) & 2,49 & $\operatorname{chr} 19$ \\
\hline POP7 & 10248 & processing of precursor 7, ribonuclease P subunit (S. cerevisiae) & 4,58 & $\operatorname{chr} 7$ \\
\hline POR & 5447 & P450 (cytochrome) oxidoreductase & 3,75 & $\operatorname{chr} 7$ \\
\hline POU2F1 & 5451 & POU domain, class 2, transcription factor 1 & 7,47 & chr1 \\
\hline POU5F1 & $628 / / / 5460 / / / 5$ & POU domain, class 5 , transcription factor $1 / / /$ POU domain, class 5 , transcriptiol & 118,37 & chr1 \\
\hline PPA2 & 27068 & pyrophosphatase (inorganic) 2 & 2,02 & $\operatorname{chr} 4$ \\
\hline PPAN & 56342 & peter pan homolog (Drosophila) & 3,84 & chr19 \\
\hline PPAP2A & 8611 & phosphatidic acid phosphatase type $2 \mathrm{~A}$ & 2,36 & chr5 \\
\hline PPAP2C & 8612 & phosphatidic acid phosphatase type $2 \mathrm{C}$ & 9,07 & chr19 \\
\hline PPARBP & 5469 & PPAR binding protein & 2,34 & chr17 \\
\hline PPAT & 5471 & phosphoribosyl pyrophosphate amidotransferase & 6,66 & chr4 \\
\hline PPCDC & 60490 & phosphopantothenoylcysteine decarboxylase & 6,07 & chr15 \\
\hline PPFIA1 & 8500 & Protein tyrosine phosphatase, receptor type, f polypeptide (PTPRF), interacting & 2,15 & chr11 \\
\hline PPHLN1 & 51535 & periphilin 1 & 2,19 & chr12 \\
\hline PPID & 5481 & peptidylprolyl isomerase D (cyclophilin D) & 2,95 & chr4 \\
\hline PPIG & 9360 & peptidyl-prolyl isomerase G (cyclophilin G) & 2,77 & chr2 \\
\hline PPIH & 10465 & peptidyl prolyl isomerase $\mathrm{H}$ (cyclophilin $\mathrm{H}$ ) & 4,21 & chr1 \\
\hline PPIL1 & 51645 & peptidylprolyl isomerase (cyclophilin)-like 1 & 3,35 & chr6 \\
\hline PPIL5 & 122769 & peptidylprolyl isomerase (cyclophilin)-like 5 & 3,66 & chr14 \\
\hline PPL & 5493 & periplakin & 9,49 & chr16 \\
\hline PPM1B & 5495 & protein phosphatase $1 \mathrm{~B}$ (formerly $2 \mathrm{C}$ ), magnesium-dependent, beta isoform & 4,87 & chr2 \\
\hline PPM1E & 22843 & protein phosphatase 1E (PP2C domain containing) & 15,90 & chr17 \\
\hline PPM1G & 5496 & protein phosphatase $1 \mathrm{G}$ (formerly $2 \mathrm{C}$ ), magnesium-dependent, gamma isoform & 4,48 & chr2 \\
\hline PPM1H & 57460 & protein phosphatase $1 \mathrm{H}$ (PP2C domain containing) & 11,60 & chr12 \\
\hline PPM1J & 333926 & protein phosphatase 1J (PP2C domain containing) & 2,97 & chr1 \\
\hline PPM1L & 151742 & Protein phosphatase 1 (formerly 2C)-like & 3,64 & chr3 \\
\hline PPOX & 5498 & protoporphyrinogen oxidase & 2,60 & chr1 \\
\hline PPP1CC & 5501 & protein phosphatase 1 , catalytic subunit, gamma isoform & 2,51 & chr12 \\
\hline PPP1R10 & 5514 & protein phosphatase 1 , regulatory subunit 10 & 2,44 & chr6 \\
\hline PPP1R13B & 23368 & Protein phosphatase 1 , regulatory (inhibitor) subunit 13B & 23,43 & chr10 \\
\hline PPP1R14B & 26472 & protein phosphatase 1 , regulatory (inhibitor) subunit 14B & 2,64 & chr22 \\
\hline PPP1R16B & 26051 & protein phosphatase 1 , regulatory (inhibitor) subunit $16 \mathrm{~B}$ & 4,20 & chr20 \\
\hline PPP1R1A & 5502 & protein phosphatase 1 , regulatory (inhibitor) subunit $1 \mathrm{~A}$ & 2,31 & chr12 \\
\hline PPP1R9A & 55607 & Protein phosphatase 1 , regulatory (inhibitor) subunit $9 \mathrm{~A}$ & 21,83 & $\operatorname{chr} 7$ \\
\hline PPP2R1B & 5519 & protein phosphatase 2 (formerly $2 A$ ), regulatory subunit A (PR 65), beta isoform & 8,56 & chr11 \\
\hline PPP2R2A & 5520 & protein phosphatase 2 (formerly $2 A$ ), regulatory subunit $B$ ( $P R 52$ ), alpha isoform & 2,80 & chr8 \\
\hline PPP2R2B & 5521 & protein phosphatase 2 (formerly $2 A$ ), regulatory subunit $B$ ( $P R 52$ ), beta isoform & 29,75 & chr5 \\
\hline PPP2R2C & 5522 & protein phosphatase 2 (formerly $2 A$ ), regulatory subunit $B$ ( $P R 52$ ), gamma isofo & 2,15 & $\operatorname{chr} 4$ \\
\hline PPP2R3B & 28227 & protein phosphatase 2 (formerly $2 A$ ), regulatory subunit $B "$, beta & 3,04 & chrY \\
\hline PPP2R5A & 5525 & protein phosphatase 2 , regulatory subunit $B$ (B56), alpha isoform & 2,06 & chr1 \\
\hline PPP3CA & 5530 & Protein phosphatase 3 (formerly 2B), catalytic subunit, alpha isoform (calcineurir & 2,69 & chr4 \\
\hline PPP3R1 & 5534 & Protein phosphatase 3 (formerly $2 \mathrm{~B}$ ), regulatory subunit B, $19 \mathrm{kDa}$, alpha isoform & 2,65 & chr2 \\
\hline PPP6C & 5537 & Protein phosphatase 6 , catalytic subunit & 2,96 & chr9 \\
\hline PPRC1 & 23082 & peroxisome proliferative activated receptor, gamma, coactivator-related 1 & 2,98 & $\operatorname{chr} 10$ \\
\hline PPT1 & 5538 & palmitoyl-protein thioesterase 1 (ceroid-lipofuscinosis, neuronal 1 , infantile) & 3,94 & chr1 \\
\hline PPWD1 & 23398 & peptidylprolyl isomerase domain and WD repeat containing 1 & 3,43 & chr5 \\
\hline PQBP1 & 10084 & polyglutamine binding protein 1 & 2,70 & $\operatorname{chr} \mathrm{X}$ \\
\hline PRCC & 5546 & papillary renal cell carcinoma (translocation-associated) & 2,19 & chr1 \\
\hline PRDM10 & 56980 & PR domain containing 10 & 2,22 & chr11 \\
\hline PRDM14 & 63978 & PR domain containing 14 & 14,71 & chr8 \\
\hline PRDX1 & 5052 & peroxiredoxin 1 & 2,62 & chr1 \\
\hline PREP & 5550 & Prolyl endopeptidase & 2,48 & chr6 \\
\hline PRIM1 & 5557 & primase, polypeptide $1,49 \mathrm{kDa}$ & 25,84 & chr12 \\
\hline PRIMA1 & 145270 & Proline rich membrane anchor 1 & 4,43 & chr14 \\
\hline
\end{tabular}




\begin{tabular}{|c|c|c|c|c|}
\hline PRKAA2 & 5563 & Protein kinase, AMP-activated, alpha 2 catalytic subunit & 3,58 & chr1 \\
\hline PRKAR1A & 5573 & protein kinase, cAMP-dependent, regulatory, type I, alpha (tissue specific exting & 2,65 & $\operatorname{chr} 17$ \\
\hline PRKAR1B & 5575 & Protein kinase, cAMP-dependent, regulatory, type I, beta & 6,61 & chr21 \\
\hline PRKAR2B & 5577 & protein kinase, cAMP-dependent, regulatory, type II, beta & 7,32 & $\mathrm{chr} 7$ \\
\hline PRKCA & 5578 & Homo sapiens, clone IMAGE:4103364, mRNA /// Protein kinase C, alpha & 2,48 & chr15 \\
\hline PRKCB1 & 5579 & protein kinase $\mathrm{C}$, beta 1 & 7,43 & chr16 \\
\hline PRKCBP1 & 23613 & protein kinase $\mathrm{C}$ binding protein 1 & 4,07 & chr20 \\
\hline PRKCQ & 5588 & protein kinase $\mathrm{C}$, theta & 7,06 & chr10 \\
\hline PRKCZ & 5590 & protein kinase C, zeta & 11,48 & chr1 \\
\hline PRKD2 & 25865 & protein kinase D2 & 2,17 & chr19 \\
\hline PRKD3 & 23683 & protein kinase D3 /// protein kinase D3 & 2,05 & chr2 \\
\hline PRKX & 5613 & protein kinase, $X$-linked & 4,27 & $\operatorname{chrX}$ \\
\hline PRKX & $5613 / / / 5616$ & protein kinase, $X$-linked /// protein kinase, Y-linked & 3,33 & $\operatorname{chr} X$ \\
\hline PRMT7 & 54496 & protein arginine $\mathrm{N}$-methyltransferase 7 & 2,63 & chr16 \\
\hline PRO1580 & 55374 & hypothetical protein PRO1580 & 3,28 & chr5 \\
\hline PRO1843 & 55378 & hypothetical protein PRO1843 & 4,60 & chr3 \\
\hline PRO1853 & 55471 & hypothetical protein PRO1853 & 5,29 & chr2 \\
\hline PRO2852 & 114224 & hypothetical protein PRO2852 & 2,69 & chr9 \\
\hline PRODH & 5625 & proline dehydrogenase (oxidase) 1 & 7,54 & chr22 \\
\hline PROM1 & 8842 & prominin 1 & 54,82 & chr4 \\
\hline PRPF19 & 27339 & PRP19/PSO4 pre-mRNA processing factor 19 homolog (S. cerevisiae) & 2,90 & chr11 \\
\hline PRPF38A & 84950 & PRP38 pre-mRNA processing factor 38 (yeast) domain containing A & 4,62 & chr1 \\
\hline PRPF38B & 55119 & PRP38 pre-mRNA processing factor 38 (yeast) domain containing B & 2,84 & chr1 \\
\hline PRPF40A & 55660 & PRP40 pre-mRNA processing factor 40 homolog A (yeast) & 2,22 & chr2 \\
\hline PRPF4B & 8899 & PRP4 pre-mRNA processing factor 4 homolog B (yeast) & 2,16 & chr6 \\
\hline PRPS2 & 5634 & Phosphoribosyl pyrophosphate synthetase 2 & 2,22 & $\operatorname{chr} X$ \\
\hline PRPSAP2 & 5636 & phosphoribosyl pyrophosphate synthetase-associated protein 2 & 2,30 & chr17 \\
\hline PRR6 & 201161 & proline rich 6 & 11,50 & chr17 \\
\hline PRRG4 & 79056 & Proline rich Gla (G-carboxyglutamic acid) 4 (transmembrane) & 2,63 & chr11 \\
\hline PRSS15 & 9361 & protease, serine, 15 & 2,27 & chr19 \\
\hline PRSS16 & 10279 & protease, serine, 16 (thymus) & 6,65 & chr6 \\
\hline PRSS8 & 5652 & protease, serine, 8 (prostasin) & 5,16 & chr16 \\
\hline PRTG & 283659 & Protogenin homolog (Gallus gallus) & 9,91 & chr15 \\
\hline PRUNE & 58497 & prune homolog (Drosophila) & 2,65 & chr1 \\
\hline PSARL & 55486 & presenilin associated, rhomboid-like & 2,34 & chr6 \\
\hline PSEN2 & 5664 & presenilin 2 (Alzheimer disease 4) & 2,29 & chr1 \\
\hline PSF1 & 9837 & DNA replication complex GINS protein PSF1 & 13,11 & chr20 \\
\hline PSIP1 & 11168 & PC4 and SFRS1 interacting protein 1 & 12,30 & chr9 \\
\hline PSMA2 & 5683 & proteasome (prosome, macropain) subunit, alpha type, 2 & 4,09 & $\mathrm{chr} 7$ \\
\hline PSMA3 & 5684 & proteasome (prosome, macropain) subunit, alpha type, 3 & 2,70 & chr14 \\
\hline PSMB1 & 5689 & proteasome (prosome, macropain) subunit, beta type, 1 & 2,00 & chr6 \\
\hline PSMB4 & 5692 & proteasome (prosome, macropain) subunit, beta type, 4 & 2,31 & chr1 \\
\hline PSMB6 & 5694 & proteasome (prosome, macropain) subunit, beta type, 6 & 2,11 & chr17 \\
\hline PSMC4 & 5704 & proteasome (prosome, macropain) 26S subunit, ATPase, 4 & 2,02 & chr19 \\
\hline PSMD11 & 5717 & proteasome (prosome, macropain) 26S subunit, non-ATPase, 11 & 3,40 & chr17 \\
\hline PSMD3 & 5709 & proteasome (prosome, macropain) 26S subunit, non-ATPase, 3 & 2,07 & $\operatorname{chr} 17$ \\
\hline PSME4 & 23198 & Proteasome (prosome, macropain) activator subunit 4 & 2,83 & chr2 \\
\hline PSPC1 & 55269 & Paraspeckle component 1 & 2,35 & chr13 \\
\hline PSPC1 & $374491 / / / 55269$ & paraspeckle component 1 /// TPTE and PTEN homologous inositol lipid phospha & 3,73 & $\operatorname{chr} 13$ \\
\hline PSRC1 & 84722 & proline/serine-rich coiled-coil 1 & 6,19 & chr1 \\
\hline PTBP1 & 5725 & polypyrimidine tract binding protein 1 & 3,72 & chr19 \\
\hline PTBP2 & 58155 & Polypyrimidine tract binding protein 2 & 4,28 & chr1 \\
\hline PTCD1 & 26024 & pentatricopeptide repeat domain 1 & 2,88 & $\mathrm{chr} 7$ \\
\hline PTCH & 5727 & patched homolog (Drosophila) & 13,40 & chr9 \\
\hline PTCHD1 & 139411 & patched domain containing 1 & 2,43 & $\operatorname{chr} x$ \\
\hline PTER & 9317 & phosphotriesterase related & 2,80 & chr10 \\
\hline PTGES3 & 10728 & prostaglandin E synthase 3 (cytosolic) & 3,00 & chr2 \\
\hline PTMA & 5757 & prothymosin, alpha (gene sequence 28 ) & 4,00 & chr2 \\
\hline PTMA & $441454 / / / 4414$ & prothymosin, alpha (gene sequence 28 ) /// similar to prothymosin alpha /// hypot & 2,40 & chr3 \\
\hline PTOV1 & 53635 & prostate tumor overexpressed gene 1 & 2,03 & chr19 \\
\hline PTPMT1 & 114971 & protein tyrosine phosphatase, mitochondrial 1 & 2,52 & chr11 \\
\hline PTPN2 & 5771 & protein tyrosine phosphatase, non-receptor type 2 & 5,52 & chr13 \\
\hline PTPN4 & 5775 & protein tyrosine phosphatase, non-receptor type 4 (megakaryocyte) & 5,52 & chr2 \\
\hline PTPN5 & 84867 & protein tyrosine phosphatase, non-receptor type 5 (striatum-enriched) & 5,93 & chr11 \\
\hline PTPN6 & 5777 & protein tyrosine phosphatase, non-receptor type 6 & 4,52 & chr12 \\
\hline PTPRD & 5789 & Protein tyrosine phosphatase, receptor type, D & 15,44 & chr9 \\
\hline PTPRG & 5793 & Protein tyrosine phosphatase, receptor type, G & 2,86 & chr3 \\
\hline
\end{tabular}




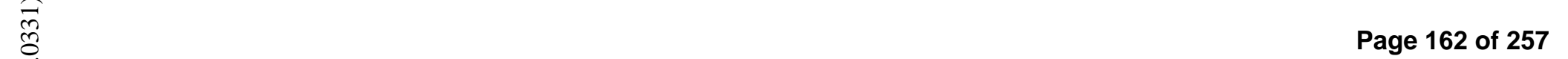

\begin{tabular}{|c|c|c|c|c|}
\hline PTPRS & 5802 & Protein tyrosine phosphatase, receptor type, $S$ & 2,66 & chr19 \\
\hline PTPRZ1 & 5803 & protein tyrosine phosphatase, receptor-type, Z polypeptide 1 & 176,52 & chr7 \\
\hline PTTG1 & 9232 & pituitary tumor-transforming 1 & 4,18 & chr5 \\
\hline PUM2 & 23369 & Pumilio homolog 2 (Drosophila) & 2,88 & chr2 \\
\hline PUNC & 9543 & putative neuronal cell adhesion molecule & 9,73 & chr15 \\
\hline PUS1 & 80324 & pseudouridylate synthase 1 & 5,16 & chr12 \\
\hline PUS3 & 83480 & pseudouridylate synthase $3 / / /$ pseudouridylate synthase 3 & 2,33 & $\operatorname{chr} 11$ \\
\hline PWP2H & 5822 & PWP2 periodic tryptophan protein homolog (yeast) & 5,31 & chr21 \\
\hline PX19 & 27166 & px19-like protein & 2,81 & chr1 \\
\hline PYCARD & 29108 & PYD and CARD domain containing & 5,75 & chr16 \\
\hline PYCR2 & 29920 & pyrroline-5-carboxylate reductase family, member 2 & 3,58 & chr1 \\
\hline QPRT & 23475 & quinolinate phosphoribosyltransferase (nicotinate-nucleotide pyrophosphorylase & 3,98 & chr16 \\
\hline QRSL1 & 55278 & glutaminyl-tRNA synthase (glutamine-hydrolyzing)-like 1 & 3,82 & chr6 \\
\hline QTRT1 & 81890 & queuine tRNA-ribosyltransferase 1 (tRNA-guanine transglycosylase) /// queuine & 2,77 & $\operatorname{chr} 19$ \\
\hline QTRTD1 & 79691 & queuine tRNA-ribosyltransferase domain containing 1 & 5,44 & chr3 \\
\hline RAB11FIP4 & 84440 & RAB11 family interacting protein 4 (class II) & 7,60 & chr17 \\
\hline RAB12 & 201475 & RAB12, member RAS oncogene family & 6,19 & chr18 \\
\hline RAB15 & 376267 & RAB15, member RAS onocogene family & 7,10 & chr14 \\
\hline RAB20 & 55647 & RAB20, member RAS oncogene family & 7,07 & chr13 \\
\hline RAB25 & 57111 & RAB25, member RAS oncogene family & 6,71 & chr1 \\
\hline RAB38 & 23682 & RAB38, member RAS oncogene family & 11,31 & chr11 \\
\hline RAB39B & 116442 & RAB39B, member RAS oncogene family & 5,31 & $\operatorname{chr} X$ \\
\hline RAB3D & 9545 & RAB3D, member RAS oncogene family & 4,14 & chr19 \\
\hline RAB3IP & 117177 & RAB3A interacting protein (rabin3) & 4,41 & chr12 \\
\hline RAB6B & 51560 & RAB6B, member RAS oncogene family & 2,41 & chr3 \\
\hline RAB9A & 9367 & RAB9A, member RAS oncogene family & 2,28 & $\operatorname{chr} X$ \\
\hline RABGAP1L & 9910 & RAB GTPase activating protein 1 -like & 11,26 & chr1 \\
\hline RABL4 & 11020 & RAB, member of RAS oncogene family-like 4 & 2,51 & chr22 \\
\hline RACGAP1 & 29127 & Rac GTPase activating protein 1 & 2,02 & chr12 \\
\hline RAD17 & 5884 & RAD17 homolog (S. pombe) & 2,44 & chr5 \\
\hline RAD23B & 5887 & RAD23 homolog B (S. cerevisiae) & 2,03 & chr9 \\
\hline RAD50 & 10111 & RAD50 homolog (S. cerevisiae) & 3,34 & chr5 \\
\hline RAD51 & 5888 & RAD51 homolog (RecA homolog, E. coli) (S. cerevisiae) & 7,17 & chr15 \\
\hline RAD51AP1 & 10635 & RAD51 associated protein 1 & 9,26 & chr12 \\
\hline RAD54B & 25788 & RAD54 homolog B (S. cerevisiae) & 3,48 & chr8 \\
\hline RAD54L & 8438 & RAD54-like (S. cerevisiae) & 5,69 & chr1 \\
\hline RAE1 & 8480 & RAE1 RNA export 1 homolog (S. pombe) & 2,45 & chr20 \\
\hline RAF1 & 5894 & V-raf-1 murine leukemia viral oncogene homolog 1 & 2,51 & chr3 \\
\hline RALGPS1 & 9649 & Ral GEF with $\mathrm{PH}$ domain and $\mathrm{SH} 3$ binding motif 1 & 3,24 & chr9 \\
\hline RAN & 5901 & RAN, member RAS oncogene family & 2,42 & chr6 \\
\hline RANBP1 & 5902 & RAN binding protein 1 & 4,44 & chr22 \\
\hline RANBP2 & 5903 & RAN binding protein 2 & 3,58 & chr2 \\
\hline RANBP5 & 3843 & RAN binding protein 5 & 2,48 & chr13 \\
\hline RANGAP1 & 5905 & Ran GTPase activating protein 1 & 2,86 & chr22 \\
\hline RANGNRF & 29098 & RAN guanine nucleotide release factor & 2,81 & chr17 \\
\hline RAPGEF5 & 9771 & Rap guanine nucleotide exchange factor (GEF) 5 & 5,10 & $\operatorname{chr} 7$ \\
\hline RARRES2 & 5919 & retinoic acid receptor responder (tazarotene induced) 2 & 76,23 & chr7 \\
\hline RASEF & 158158 & RAS and EF-hand domain containing & 13,45 & chr9 \\
\hline RASGEF1A & 221002 & RasGEF domain family, member $1 \mathrm{~A}$ & 22,42 & chr10 \\
\hline RASGEF1B & 153020 & RasGEF domain family, member $1 \mathrm{~B}$ & 3,18 & chr4 \\
\hline RASGRP2 & 10235 & RAS guanyl releasing protein 2 (calcium and DAG-regulated) & 2,89 & chr11 \\
\hline RASIP1 & 54922 & Ras interacting protein 1 & 2,03 & chr19 \\
\hline RASL11B & 65997 & RAS-like, family 11 , member B & 28,53 & chr4 \\
\hline RASL12 & 51285 & RAS-like, family 12 & 2,14 & chr15 \\
\hline RASSF2 & 9770 & Ras association (RalGDS/AF-6) domain family 2 & 2,22 & chr20 \\
\hline RB1CC1 & 9821 & RB1-inducible coiled-coil 1 & 2,90 & chr8 \\
\hline RBBP4 & 5928 & retinoblastoma binding protein 4 & 3,31 & chr1 \\
\hline RBBP6 & 5930 & retinoblastoma binding protein 6 & 3,00 & chr16 \\
\hline RBBP7 & 5931 & Retinoblastoma binding protein 7 & 6,06 & $\operatorname{chrX}$ \\
\hline RBBP8 & 5932 & retinoblastoma binding protein 8 & 4,19 & chr18 \\
\hline RBM10 & 8241 & RNA binding motif protein 10 & 3,51 & $\operatorname{chr} X$ \\
\hline RBM12 & 10137 & RNA binding motif protein 12 & 3,28 & chr20 \\
\hline RBM12B & 389677 & RNA binding motif protein $12 \mathrm{~B}$ & 3,78 & chr8 \\
\hline RBM13 & 84549 & RNA binding motif protein $13 / / /$ RNA binding motif protein 13 & 2,75 & chr8 \\
\hline RBM14 & 10432 & RNA binding motif protein 14 & 4,85 & chr11 \\
\hline RBM15 & 64783 & RNA binding motif protein 15 & 2,87 & chr1 \\
\hline RBM15B & 29890 & RNA binding motif protein $15 \mathrm{~B}$ & 2,72 & chr3 \\
\hline
\end{tabular}




\section{Pagge 163 of 257}

\begin{tabular}{|c|c|c|c|c|}
\hline RBM17 & 84991 & RNA binding motif protein 17 & 2,98 & chr10 \\
\hline RBM19 & 9904 & RNA binding motif protein 19 & 2,45 & chr12 \\
\hline RBM25 & 58517 & RNA binding motif protein 25 & 3,43 & chr14 \\
\hline RBM27 & 54439 & RNA binding motif protein 27 & 2,61 & chr5 \\
\hline RBM28 & 55131 & RNA binding motif protein 28 & 2,43 & $\mathrm{chr} 7$ \\
\hline RBM35A & 54845 & RNA binding motif protein $35 \mathrm{~A}$ & 119,95 & chr8 \\
\hline RBM35B & 80004 & RNA binding motif protein $35 \mathrm{~B}$ & 9,27 & $\operatorname{chr} 16$ \\
\hline RBM4 & 5936 & RNA binding motif protein 4 & 2,16 & chr11 \\
\hline RBM6 & 10180 & RNA binding motif protein 6 & 3,27 & chr3 \\
\hline RBM8A & 9939 & RNA binding motif protein $8 \mathrm{~A}$ & 3,52 & chr1 \\
\hline RBMX & 27316 & RNA binding motif protein, $X$-linked & 4,34 & chr9 \\
\hline RBMXL1 & 494115 & RNA binding motif protein, $X$-linked-like 1 & 2,39 & chr1 \\
\hline RBP1 & 5947 & retinol binding protein 1 , cellular & 4,50 & chr3 \\
\hline RBP7 & 116362 & retinol binding protein 7 , cellular & 2,91 & chr1 \\
\hline RBPMS & 11030 & RNA binding protein with multiple splicing & 5,28 & chr8 \\
\hline RBPMS2 & 348093 & RNA binding protein with multiple splicing 2 & 85,61 & $\operatorname{chr} 15$ \\
\hline RBPSUH & 3516 & recombining binding protein suppressor of hairless (Drosophila) & 3,12 & $\mathrm{chr} 4$ \\
\hline $\mathrm{RC} 74$ & 55756 & related to CPSF subunits $74 \mathrm{kDa}$ & 3,24 & chr8 \\
\hline RCBTB2 & 1102 & regulator of chromosome condensation (RCC1) and $\mathrm{BTB}(\mathrm{POZ})$ domain contain & 2,16 & $\operatorname{chr} 13$ \\
\hline RCC1 & 1104 & regulator of chromosome condensation 1 & 7,95 & chr1 \\
\hline RCC2 & 55920 & regulator of chromosome condensation 2 & 5,22 & chr11 \\
\hline $\mathrm{RCHY} 1$ & 25898 & ring finger and $\mathrm{CHY}$ zinc finger domain containing 1 & 3,38 & chr4 \\
\hline RCL1 & 10171 & RNA terminal phosphate cyclase-like 1 & 2,62 & chr9 \\
\hline RCOR2 & 283248 & REST corepressor 2 & 6,56 & chr11 \\
\hline RDH13 & 112724 & retinol dehydrogenase 13 (all-trans and 9-cis) & 3,30 & $\operatorname{chr} 19$ \\
\hline REC8L1 & 9985 & REC8-like 1 (yeast) & 9,34 & chr14 \\
\hline RECQL4 & 9401 & RecQ protein-like 4 & 8,26 & chr8 \\
\hline REPS1 & 85021 & RALBP1 associated Eps domain containing 1 /// RALBP1 associated Eps doma & 2,78 & chr6 \\
\hline RERE & 473 & Arginine-glutamic acid dipeptide (RE) repeats & 2,68 & chr1 \\
\hline RET & 5979 & Ret proto-oncogene (multiple endocrine neoplasia and medullary thyroid carcino & 3,04 & chr10 \\
\hline REXO4 & 57109 & REX4, RNA exonuclease 4 homolog (S. cerevisiae) & 2,52 & chr9 \\
\hline RFC2 & 5982 & replication factor C (activator 1) 2, 40kDa & 4,41 & $\mathrm{chr} 7$ \\
\hline RFC3 & 5983 & Replication factor C (activator 1) 3, 38kDa & 5,20 & $\operatorname{chr} 13$ \\
\hline RFC4 & 5984 & replication factor C (activator 1) 4, 37kDa & 7,31 & chr3 \\
\hline RFC5 & 5985 & replication factor C (activator 1) $5,36.5 \mathrm{kDa}$ & 4,76 & chr12 \\
\hline RFP2 & 10206 & ret finger protein 2 & 2,82 & $\operatorname{chr} 13$ \\
\hline RFT1 & 91869 & RFT1 homolog (S. cerevisiae) & 2,74 & chr3 \\
\hline RFWD3 & 55159 & ring finger and WD repeat domain 3 & 4,61 & chr16 \\
\hline RFX3 & 5991 & Regulatory factor X, 3 (influences HLA class II expression) & 2,83 & chr9 \\
\hline RFXAP & 5994 & regulatory factor $\mathrm{X}$-associated protein & 2,30 & chr13 \\
\hline RFXDC2 & 64864 & regulatory factor $\mathrm{X}$ domain containing 2 & 2,26 & chr15 \\
\hline RG9MTD1 & 54931 & RNA (guanine-9-) methyltransferase domain containing 1 & 3,76 & chr3 \\
\hline RGL3 & 57139 & ral guanine nucleotide dissociation stimulator-like 3 & 2,13 & chr19 \\
\hline RGS17 & 26575 & regulator of G-protein signalling 17 & 3,30 & chr6 \\
\hline RHBDL4 & 162494 & rhomboid, veinlet-like 4 (Drosophila) & 2,24 & chr17 \\
\hline RHEB & 6009 & Ras homolog enriched in brain & 2,07 & chr7 \\
\hline RHOT2 & 89941 & ras homolog gene family, member T2 & 3,02 & chr16 \\
\hline RHPN2 & 85415 & rhophilin, Rho GTPase binding protein 2 & 6,35 & chr16 \\
\hline RICS & 9743 & Rho GTPase-activating protein & 3,57 & chr11 \\
\hline RIF1 & 55183 & RAP1 interacting factor homolog (yeast) & 4,62 & chr2 \\
\hline RIMS2 & 9699 & Regulating synaptic membrane exocytosis 2 & 6,68 & chr8 \\
\hline RIMS3 & 9783 & regulating synaptic membrane exocytosis 3 & 9,28 & chr1 \\
\hline RIOK1 & 83732 & RIO kinase 1 (yeast) /// RIO kinase 1 (yeast) & 2,42 & chr6 \\
\hline RIP & 84268 & RPA interacting protein & 2,17 & chr17 \\
\hline RIPK4 & 54101 & receptor-interacting serine-threonine kinase 4 & 2,90 & chr21 \\
\hline RLF & 6018 & rearranged L-myc fusion & 2,42 & chr1 \\
\hline RMST & 196475 & Rhabdomyosarcoma 2 associated transcript (non-coding RNA) & 6,98 & chr12 \\
\hline RNASEH2A & 10535 & ribonuclease $\mathrm{H} 2$, large subunit & 9,22 & $\operatorname{chr} 19$ \\
\hline RNASEN & 29102 & ribonuclease III, nuclear & 2,40 & chr5 \\
\hline RND1 & 27289 & Rho family GTPase 1 & 2,96 & chr12 \\
\hline RND2 & 8153 & Rho family GTPase 2 & 8,85 & chr17 \\
\hline RNF10 & 9921 & Ring finger protein 10 & 5,28 & chr12 \\
\hline RNF125 & 54941 & ring finger protein 125 & 3,99 & chr18 \\
\hline RNF130 & 55819 & Ring finger protein 130 & 3,52 & chr5 \\
\hline RNF138 & 51444 & ring finger protein 138 & 5,66 & chr18 \\
\hline RNF175 & 285533 & ring finger protein 175 & 7,07 & chr4 \\
\hline RNF184 & 55167 & ring finger protein 184 & 2,83 & chr3 \\
\hline
\end{tabular}




\begin{tabular}{|c|c|c|c|c|}
\hline RNF26 & 79102 & ring finger protein 26 & 3,54 & chr11 \\
\hline RNF38 & 152006 & ring finger protein 38 & 2,88 & chr9 \\
\hline RNF44 & 22838 & ring finger protein 44 & 6,28 & chr5 \\
\hline RNF5 & 6048 & ring finger protein 5 & 2,82 & chr6 \\
\hline RNF8 & 9025 & ring finger protein 8 & 3,38 & chr6 \\
\hline RNMTL1 & 55178 & RNA methyltransferase like 1 & 2,41 & chr17 \\
\hline RNPC2 & 9584 & RNA-binding region (RNP1, RRM) containing 2 & 2,00 & chr20 \\
\hline RNPS1 & 10921 & RNA binding protein $\mathrm{S} 1$, serine-rich domain & 2,81 & chr4 \\
\hline RNU22 & 9304 & RNA, U22 small nucleolar & 4,21 & chr11 \\
\hline RNU3IP2 & 9136 & RNA, U3 small nucleolar interacting protein 2 & 4,43 & chr3 \\
\hline RNU47 & 26802 & RNA, U47 small nuclear & 2,22 & chr1 \\
\hline ROR1 & 4919 & receptor tyrosine kinase-like orphan receptor 1 & 7,53 & chr1 \\
\hline RORA & 6095 & RAR-related orphan receptor A & 2,80 & chr15 \\
\hline RP1-112K5.2 & 90121 & hypothetical protein DT1P1A10 & 2,20 & $\operatorname{chr} X$ \\
\hline RP11-311P8. & 139596 & hypothetical protein MGC23937 similar to CG4798 & 2,41 & $\operatorname{chr} \mathrm{X}$ \\
\hline RP11-50D16. & 387921 & Similar to RIKEN cDNA $8030451 \mathrm{~K} 01$ & 2,07 & chr13 \\
\hline RPA1 & 6117 & replication protein $\mathrm{A} 1,70 \mathrm{kDa}$ & 2,84 & chr17 \\
\hline RPA2 & 6118 & replication protein $\mathrm{A} 2,32 \mathrm{kDa}$ & 4,11 & chr1 \\
\hline RPA3 & 6119 & replication protein $\mathrm{A} 3,14 \mathrm{kDa}$ & 6,57 & chr7 \\
\hline RPAP1 & 26015 & RNA polymerase II associated protein 1 & 2,62 & chr15 \\
\hline RPL18A & $390354 / / / 6142$ & ribosomal protein L18a /// similar to ribosomal protein L18a; $60 \mathrm{~S}$ ribosomal prote & 2,20 & chr1 \\
\hline RPL39L & 116832 & ribosomal protein L39-like & 3,02 & chr3 \\
\hline RPP30 & 10556 & ribonuclease P/MRP 30kDa subunit & 3,40 & chr10 \\
\hline RPP38 & 10557 & ribonuclease P/MRP 38kDa subunit & 2,18 & $\operatorname{chr} 10$ \\
\hline RPP40 & 10799 & ribonuclease $\mathrm{P} 40 \mathrm{kDa}$ subunit & 2,42 & chr6 \\
\hline RPRM & 56475 & reprimo, TP53 dependant G2 arrest mediator candidate & 5,85 & chr2 \\
\hline RPS21 & 6227 & ribosomal protein S21 & 2,23 & chr20 \\
\hline RPS24 & 6229 & Ribosomal protein S24 & 2,43 & chr10 \\
\hline RPS6 & 6194 & Ribosomal protein S6 & 5,12 & chr9 \\
\hline RPS6KA1 & 6195 & ribosomal protein $\mathrm{S} 6$ kinase, 90kDa, polypeptide 1 & 5,70 & chr1 \\
\hline RPS6KA5 & 9252 & ribosomal protein S6 kinase, 90kDa, polypeptide 5 & 2,03 & chr14 \\
\hline RPS7 & 6201 & ribosomal protein S7 /// ribosomal protein S7 & 2,01 & chr2 \\
\hline RPUSD2 & 27079 & RNA pseudouridylate synthase domain containing 2 & 3,38 & chr15 \\
\hline RPUSD3 & 285367 & RNA pseudouridylate synthase domain containing 3 & 2,49 & chr3 \\
\hline RPUSD4 & 84881 & RNA pseudouridylate synthase domain containing 4 & 3,89 & chr11 \\
\hline RQCD1 & 9125 & RCD1 required for cell differentiation 1 homolog (S. pombe) & 3,02 & chr2 \\
\hline RRAGD & 58528 & Ras-related GTP binding D & 12,61 & chr6 \\
\hline RREB1 & 6239 & Ras responsive element binding protein 1 & 2,08 & chr6 \\
\hline RRM1 & 6240 & ribonucleotide reductase M1 polypeptide & 2,75 & chr11 \\
\hline RRM2 & 6241 & ribonucleotide reductase M2 polypeptide & 7,19 & chr2 \\
\hline RRS1 & 23212 & RRS1 ribosome biogenesis regulator homolog (S. cerevisiae) & 5,48 & chr8 \\
\hline RSBN1L & 222194 & Round spermatid basic protein 1 -like & 2,14 & chr7 \\
\hline RSC1A1 & 6248 & regulatory solute carrier protein, family 1 , member 1 & 3,74 & chr1 \\
\hline RSRC1 & 51319 & arginine/serine-rich coiled-coil 1 & 2,44 & chr3 \\
\hline RTKN & 6242 & rhotekin & 2,79 & chr2 \\
\hline RTN1 & 6252 & reticulon 1 & 3,45 & chr14 \\
\hline RTTN & 25914 & rotatin & 2,22 & chr18 \\
\hline RUNX1T1 & 862 & Runt-related transcription factor 1 ; translocated to, 1 (cyclin D-related) & 4,66 & chr8 \\
\hline RUVBL1 & 8607 & RuvB-like 1 (E. coli) & 3,17 & chr3 \\
\hline RUVBL2 & 10856 & RuvB-like 2 (E. coli) & 2,17 & chr19 \\
\hline RYBP & 23429 & RING1 and YY1 binding protein & 5,13 & chr3 \\
\hline RYR1 & 6261 & Ryanodine receptor 1 (skeletal) & 2,89 & chr14 \\
\hline S100PBPR & 64766 & S100P binding protein Riken & 3,01 & chr1 \\
\hline SAC3D1 & 29901 & SAC3 domain containing 1 & 2,22 & chr11 \\
\hline SAE1 & 10055 & SUMO-1 activating enzyme subunit 1 & 2,16 & chr11 \\
\hline SAFB & 6294 & scaffold attachment factor B & 3,67 & $\operatorname{chr} 19$ \\
\hline SALL1 & 6299 & sal-like 1 (Drosophila) & 5,39 & chr16 \\
\hline SALL2 & 6297 & sal-like 2 (Drosophila) & 16,27 & chr14 \\
\hline SALL4 & 57167 & sal-like 4 (Drosophila) & 162,24 & chr20 \\
\hline SAMD6 & 203286 & sterile alpha motif domain containing 6 & 3,85 & chr9 \\
\hline SAMHD1 & 25939 & SAM domain and HD domain 1 & 7,17 & chr20 \\
\hline SANG & 149775 & GNAS1 antisense & 3,77 & chr20 \\
\hline SAP130 & 79595 & mSin3A-associated protein 130 & 2,53 & chr2 \\
\hline SAP18 & 10284 & sin3-associated polypeptide, $18 \mathrm{kDa}$ & 2,63 & $\operatorname{chr} 13$ \\
\hline SAP30 & 8819 & sin3-associated polypeptide, $30 \mathrm{kDa}$ & 2,66 & chr4 \\
\hline SAPS3 & 55291 & SAPS domain family, member 3 & 3,88 & chr11 \\
\hline SARS2 & 54938 & seryl-tRNA synthetase 2 & 3,45 & chr19 \\
\hline
\end{tabular}




\section{Pagge 165 of 257}

\begin{tabular}{|c|c|c|c|c|}
\hline SART3 & 9733 & squamous cell carcinoma antigen recognised by T cells $3 / / /$ squamous cell carc & 4,60 & chr12 \\
\hline SASS6 & 163786 & spindle assembly 6 homolog (C. elegans) & 2,46 & chr1 \\
\hline SATB1 & 6304 & special AT-rich sequence binding protein 1 (binds to nuclear matrix/scaffold-ass & 3,11 & chr3 \\
\hline SAV1 & 60485 & salvador homolog 1 (Drosophila) & 2,46 & chr14 \\
\hline SBK1 & 388228 & SH3-binding domain kinase 1 & 28,31 & chr16 \\
\hline SBNO1 & 55206 & Sno, strawberry notch homolog 1 (Drosophila) & 2,08 & chr12 \\
\hline SC5DL & 6309 & sterol-C5-desaturase (ERG3 delta-5-desaturase homolog, fungal)-like & 2,49 & chr11 \\
\hline SCAMP5 & 192683 & secretory carrier membrane protein 5 & 3,83 & chr15 \\
\hline SCAND1 & 51282 & SCAN domain containing 1 & 2,49 & chr20 \\
\hline SCAP & 22937 & SREBP cleavage-activating protein & 4,16 & chr3 \\
\hline $\mathrm{SCD}$ & 6319 & stearoyl-CoA desaturase (delta-9-desaturase) /// stearoyl-CoA desaturase (delta & 4,64 & chr10 \\
\hline SCG3 & 29106 & secretogranin III & 21,28 & chr15 \\
\hline SCGB3A2 & 117156 & secretoglobin, family $3 \mathrm{~A}$, member 2 & 16,94 & chr5 \\
\hline SCLY & 51540 & selenocysteine lyase & 3,60 & chr2 \\
\hline SCNN1A & 6337 & sodium channel, nonvoltage-gated 1 alpha & 63,47 & chr12 \\
\hline SCNN1G & 6340 & sodium channel, nonvoltage-gated 1 , gamma & 2,17 & chr16 \\
\hline sco1 & 6341 & SCO cytochrome oxidase deficient homolog 1 (yeast) & 2,67 & chr17 \\
\hline SDAD1 1 & 55153 & SDA1 domain containing 1 & 2,35 & chr4 \\
\hline SDCCAG10 & 10283 & serologically defined colon cancer antigen 10 & 2,88 & chr5 \\
\hline SDCCAG3 /// & 10807 /// 388478 & serologically defined colon cancer antigen $3 / / /$ serologically defined colon cance & 2,55 & chr9 \\
\hline SDHC & 6391 & succinate dehydrogenase complex, subunit $\mathrm{C}$, integral membrane protein, $15 \mathrm{kD}$ & 2,05 & chr1 \\
\hline SEC11L1 & 23478 & SEC11-like 1 (S. cerevisiae) & 2,40 & chr8 \\
\hline SEC11L3 & 90701 & SEC11-like 3 (S. cerevisiae) & 2,96 & $\operatorname{chr} 18$ \\
\hline SEC14L5 & 9717 & SEC14-like 5 (S. cerevisiae) & 2,13 & chr16 \\
\hline SEC22L3 & 9117 & SEC22 vesicle trafficking protein-like 3 (S. cerevisiae) /// SEC22 vesicle traffickir & 3,01 & chr3 \\
\hline SEC5L1 & 55770 & SEC5-like 1 (S. cerevisiae) & 2,21 & chr6 \\
\hline SEC61A2 & 55176 & Sec61 alpha 2 subunit (S. cerevisiae) & 3,11 & chr10 \\
\hline SECISBP2 & 79048 & SECIS binding protein 2 & 5,82 & chr9 \\
\hline SECP43 & 54952 & tRNA selenocysteine associated protein & 2,70 & chr1 \\
\hline SEH1L & 81929 & SEH1-like (S. cerevisiae) & 2,41 & chr18 \\
\hline SELI & 85465 & selenoprotein I & 2,75 & chr2 \\
\hline SEMA3F & 6405 & sema domain, immunoglobulin domain (lg), short basic domain, secreted, (sem & 8,60 & chr3 \\
\hline SEMA4C & 54910 & sema domain, immunoglobulin domain $(\mathrm{lg})$, transmembrane domain $(\mathrm{TM})$ and $\mathrm{s}$ & 4,77 & chr2 \\
\hline SEMA4D & 10507 & sema domain, immunoglobulin domain $(\mathrm{lg})$, transmembrane domain $(\mathrm{TM})$ and $\mathrm{s}$ & 4,61 & chr9 \\
\hline SEMA5B & 54437 & sema domain, seven thrombospondin repeats (type 1 and type 1 -like), transmer & 3,24 & chr3 \\
\hline SEMA6A & 57556 & sema domain, transmembrane domain (TM), and cytoplasmic domain, (semaph & 114,93 & chr5 \\
\hline SENP2 & 59343 & SUMO1/sentrin/SMT3 specific peptidase 2 & 2,33 & chr3 \\
\hline SENP3 & 26168 & SUMO1/sentrin/SMT3 specific peptidase 3 & 2,38 & chr17 \\
\hline SENP6 & 26054 & SUMO1/sentrin specific peptidase 6 & 3,15 & chr6 \\
\hline SENP8 & 123228 & SUMO/sentrin specific peptidase family member 8 & 2,52 & chr15 \\
\hline SEPHS1 & 22929 & Selenophosphate synthetase 1 & 27,72 & chr2 \\
\hline SERBP1 & 26135 & SERPINE1 mRNA binding protein 1 & 4,31 & chr1 \\
\hline SERF1A & 8293 & small EDRK-rich factor $1 \mathrm{~A}$ (telomeric) & 3,05 & chr5 \\
\hline SERF1A & 56617 /// 8293 & small EDRK-rich factor $1 \mathrm{~A}$ (telomeric) /// small EDRK-rich factor $1 \mathrm{~B}$ (centromeric & 6,49 & chr5 \\
\hline SERF2 & $10169 / / / 25764$ & small EDRK-rich factor 2 /// Huntingtin interacting protein $\mathrm{K}$ & 2,61 & chr15 \\
\hline SERPINB6 & 5269 & serpin peptidase inhibitor, clade B (ovalbumin), member 6 & 13,45 & chr1 \\
\hline SERPINB9 & 5272 & serpin peptidase inhibitor, clade B (ovalbumin), member 9 & 7,56 & chr6 \\
\hline SERPINI1 & 5274 & serpin peptidase inhibitor, clade I (neuroserpin), member 1 & 4,85 & chr3 \\
\hline SET & 6418 & SET translocation (myeloid leukemia-associated) & 4,73 & chr1 \\
\hline SET & $389168 / / / 6418$ & SET translocation (myeloid leukemia-associated) /// similar to SET protein (Phos & 4,83 & $\operatorname{chrX}$ \\
\hline SETDB1 & 9869 & SET domain, bifurcated 1 & 2,45 & chr1 \\
\hline SF1 & 7536 & splicing factor 1 & 3,06 & chr11 \\
\hline SF3A1 & 10291 & splicing factor $3 \mathrm{a}$, subunit $1,120 \mathrm{kDa}$ & 2,02 & chr22 \\
\hline SF3A2 & 8175 & splicing factor $3 \mathrm{a}$, subunit $2,66 \mathrm{kDa}$ & 3,18 & chr19 \\
\hline SF3A3 & 10946 & splicing factor $3 \mathrm{a}$, subunit $3,60 \mathrm{kDa}$ & 2,47 & chr1 \\
\hline SF3B1 & 23451 & splicing factor $3 \mathrm{~b}$, subunit $1,155 \mathrm{kDa}$ & 3,54 & chr2 \\
\hline SF3B3 & 23450 & splicing factor $3 \mathrm{~b}$, subunit $3,130 \mathrm{kDa}$ & 2,34 & chr16 \\
\hline SFMBT1 & 51460 & Scm-like with four mbt domains 1 & 5,51 & chr3 \\
\hline SFMBT2 & 57713 & Scm-like with four mbt domains 2 & 2,37 & chr10 \\
\hline SFN & 2810 & stratifin & 6,90 & chr1 \\
\hline SFPQ & 6421 & Splicing factor proline/glutamine-rich (polypyrimidine tract binding protein associa & 3,61 & chr1 \\
\hline SFRP2 & 6423 & secreted frizzled-related protein 2 & 113,98 & chr4 \\
\hline SFRS1 & 6426 & Splicing factor, arginine/serine-rich 1 (splicing factor 2, alternate splicing factor) & 4,65 & chr17 \\
\hline SFRS10 & 6434 & splicing factor, arginine/serine-rich 10 (transformer 2 homolog, Drosophila) & 3,15 & chr3 \\
\hline SFRS12 & 140890 & Splicing factor, arginine/serine-rich 12 & 2,11 & chr5 \\
\hline SFRS15 & 57466 & splicing factor, arginine/serine-rich 15 & 2,82 & chr21 \\
\hline SFRS2 & 6427 & splicing factor, arginine/serine-rich 2 & 3,19 & chr17 \\
\hline
\end{tabular}




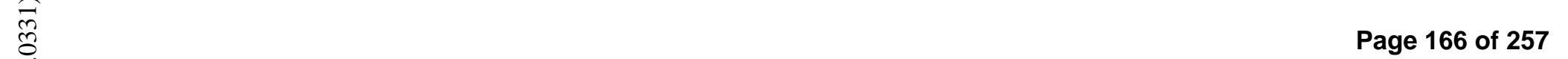

\begin{tabular}{|c|c|c|c|c|}
\hline SFRS3 & 6428 & splicing factor, arginine/serine-rich 3 & 3,82 & chr6 \\
\hline SFRS4 & 6429 & Splicing factor, arginine/serine-rich 4 & 2,19 & chr1 \\
\hline SFRS5 & 6430 & splicing factor, arginine/serine-rich 5 & 2,09 & chr14 \\
\hline SFRS7 & 6432 & splicing factor, arginine/serine-rich $7,35 \mathrm{kDa}$ & 6,09 & chr2 \\
\hline SFT2D1 & 113402 & SFT2 domain containing 1 & 2,23 & chr6 \\
\hline SFXN2 & 118980 & sideroflexin 2 & 3,66 & chr10 \\
\hline SFXN5 & 94097 & sideroflexin 5 & 2,09 & chr2 \\
\hline SGEF & 26084 & Src homology 3 domain-containing guanine nucleotide exchange factor & 6,00 & chr3 \\
\hline SGNE1 & 6447 & secretory granule, neuroendocrine protein 1 (7B2 protein) & 2,70 & chr15 \\
\hline SGOL2 & 151246 & shugoshin-like 2 (S. pombe) & 3,78 & chr2 \\
\hline SGTA & 6449 & small glutamine-rich tetratricopeptide repeat (TPR)-containing, alpha & 2,22 & chr19 \\
\hline SH3GL2 & 6456 & SH3-domain GRB2-like 2 & 4,09 & chr9 \\
\hline SH3GL3 & 6457 & SH3-domain GRB2-like 3 & 3,72 & chr15 \\
\hline SHANK2 & 22941 & $\mathrm{SH} 3$ and multiple ankyrin repeat domains 2 & 8,11 & chr11 \\
\hline SHMT1 & 6470 & serine hydroxymethyltransferase 1 (soluble) & 3,69 & chr17 \\
\hline SHPRH & 257218 & SNF2 histone linker PHD RING helicase & 3,64 & chr6 \\
\hline SIAH1 & 6477 & seven in absentia homolog 1 (Drosophila) & 2,52 & chr16 \\
\hline SIAHBP1 & 22827 & fuse-binding protein-interacting repressor & 2,00 & chr8 \\
\hline SIGIRR & 59307 & single immunoglobulin and toll-interleukin 1 receptor (TIR) domain & 3,44 & chr11 \\
\hline SIL & 6491 & TAL1 (SCL) interrupting locus & 4,52 & chr1 \\
\hline SILV & 6490 & silver homolog (mouse) & 30,56 & chr12 \\
\hline SIN3A & 25942 & SIN3 homolog A, transcription regulator (yeast) & 3,51 & chr15 \\
\hline SIP1 & 8487 & survival of motor neuron protein interacting protein 1 & 2,35 & chr14 \\
\hline SIPA1L2 & 57568 & signal-induced proliferation-associated 1 like 2 & 3,70 & chr1 \\
\hline SIRT1 & 23411 & sirtuin (silent mating type information regulation 2 homolog) 1 (S. cerevisiae) & 6,15 & chr10 \\
\hline SIRT5 & 23408 & sirtuin (silent mating type information regulation 2 homolog) 5 (S. cerevisiae) & 2,69 & chr6 \\
\hline SITPEC & 51295 & signaling intermediate in Toll pathway, evolutionarily conserved & 2,73 & chr19 \\
\hline SIVA & 10572 & CD27-binding (Siva) protein & 3,49 & chr14 \\
\hline SKB1 & 10419 & SKB1 homolog (S. pombe) & 2,09 & chr14 \\
\hline SKIL & 6498 & SKI-like & 2,26 & chr3 \\
\hline SKIV2L2 & 23517 & superkiller viralicidic activity 2-like 2 (S. cerevisiae) & 3,94 & chr5 \\
\hline SKP2 & 6502 & S-phase kinase-associated protein 2 (p45) & 8,02 & chr5 \\
\hline SLC11A2 & 4891 & solute carrier family 11 (proton-coupled divalent metal ion transporters), membe & 4,97 & chr12 \\
\hline SLC12A9 & 56996 & solute carrier family 12 (potassium/chloride transporters), member 9 & 2,21 & chr7 \\
\hline SLC15A4 & 121260 & solute carrier family 15 , member 4 & 2,21 & chr12 \\
\hline SLC16A1 & 6566 & solute carrier family 16 (monocarboxylic acid transporters), member 1 & 7,32 & chr1 \\
\hline SLC16A10 & 117247 & solute carrier family 16 (monocarboxylic acid transporters), member 10 & 20,68 & chr6 \\
\hline SLC16A4 & 9122 & Solute carrier family 16 (monocarboxylic acid transporters), member 4 & 6,68 & chr1 \\
\hline SLC16A9 & 220963 & solute carrier family 16 (monocarboxylic acid transporters), member 9 & 21,54 & chr10 \\
\hline SLC19A1 & 6573 & solute carrier family 19 (folate transporter), member 1 & 2,27 & chr21 \\
\hline SLC19A2 & 10560 & solute carrier family 19 (thiamine transporter), member 2 & 4,38 & chr1 \\
\hline SLC1A2 & 6506 & solute carrier family 1 (glial high affinity glutamate transporter), member 2 & 2,30 & chr11 \\
\hline SLC1A6 & 6511 & solute carrier family 1 (high affinity aspartate/glutamate transporter), member 6 & 30,29 & chr19 \\
\hline SLC24A3 & 57419 & solute carrier family 24 (sodium/potassium/calcium exchanger), member 3 & 2,69 & chr20 \\
\hline SLC25A13 & 10165 & solute carrier family 25 , member 13 (citrin) & 5,65 & chr7 \\
\hline SLC25A15 & 10166 & solute carrier family 25 (mitochondrial carrier; ornithine transporter) member 15 & 3,06 & chr13 \\
\hline SLC25A19 & 60386 & solute carrier family 25 (mitochondrial deoxynucleotide carrier), member 19 & 5,05 & chr17 \\
\hline SLC25A21 & 89874 & solute carrier family 25 (mitochondrial oxodicarboxylate carrier), member 21 & 3,13 & chr14 \\
\hline SLC25A26 & 115286 & solute carrier family 25, member 26 & 2,67 & chr3 \\
\hline SLC25A29 & 123096 & solute carrier family 25, member 29 & 3,93 & chr14 \\
\hline SLC25A37 & 51312 & solute carrier family 25, member 37 & 2,15 & chr8 \\
\hline SLC25A4 & 291 & solute carrier family 25 (mitochondrial carrier; adenine nucleotide translocator), n & 3,21 & chr4 \\
\hline SLC25A5 & 292 & solute carrier family 25 (mitochondrial carrier; adenine nucleotide translocator), $n$ & 2,35 & chr7 \\
\hline SLC26A6 & 65010 & solute carrier family 26 , member 6 & 2,77 & chr3 \\
\hline SLC27A2 & 11001 & solute carrier family 27 (fatty acid transporter), member 2 & 5,76 & chr15 \\
\hline SLC27A3 & 11000 & solute carrier family 27 (fatty acid transporter), member 3 & 10,12 & chr1 \\
\hline SLC27A5 & 10998 & solute carrier family 27 (fatty acid transporter), member 5 & 2,54 & chr19 \\
\hline SLC29A1 & 2030 & solute carrier family 29 (nucleoside transporters), member 1 & 7,02 & chr6 \\
\hline SLC29A2 & 3177 & solute carrier family 29 (nucleoside transporters), member 2 & 2,26 & chr11 \\
\hline SLC29A3 & 55315 & solute carrier family 29 (nucleoside transporters), member 3 & 2,37 & chr10 \\
\hline SLC2A3 & 6515 & solute carrier family 2 (facilitated glucose transporter), member 3 & 10,38 & chr12 \\
\hline SLC2A3 & $144195 / / / 6515$ & solute carrier family 2 (facilitated glucose transporter), member 3 /// solute carrie & 7,96 & chr12 \\
\hline SLC35B4 & 84912 & solute carrier family 35, member B4 & 2,14 & chr7 \\
\hline SLC35F1 & 222553 & solute carrier family 35 , member F1 & 9,40 & chr6 \\
\hline SLC37A1 & 54020 & solute carrier family 37 (glycerol-3-phosphate transporter), member 1 & 2,87 & chr21 \\
\hline SLC37A4 & 2542 & solute carrier family 37 (glycerol-6-phosphate transporter), member 4 & 2,39 & chr11 \\
\hline SLC38A1 & 81539 & Solute carrier family 38 , member 1 & 2,97 & chr12 \\
\hline
\end{tabular}




\begin{tabular}{|c|c|c|c|c|}
\hline SLC38A5 & 92745 & solute carrier family 38, member 5 & 3,25 & $\operatorname{chr} X$ \\
\hline SLC39A1 & 27173 & solute carrier family 39 (zinc transporter), member 1 & 3,20 & chr1 \\
\hline SLC39A10 & 57181 & Solute carrier family 39 (zinc transporter), member 10 & 5,79 & chr2 \\
\hline SLC39A8 & 64116 & solute carrier family 39 (zinc transporter), member 8 & 4,51 & chr4 \\
\hline SLC43A1 & 8501 & solute carrier family 43 , member 1 & 2,71 & chr11 \\
\hline SLC43A3 & 29015 & solute carrier family 43 , member 3 & 2,62 & chr11 \\
\hline SLC44A1 & 23446 & solute carrier family 44 , member 1 & 6,79 & chr9 \\
\hline SLC45A4 & 57210 & solute carrier family 45 , member 4 & 2,86 & chr8 \\
\hline SLC4A11 & 83959 & solute carrier family 4 , sodium bicarbonate transporter-like, member 11 & 12,98 & chr20 \\
\hline SLC5A10 & 125206 & Solute carrier family 5 (sodium/glucose cotransporter), member 10 & 2,37 & chr17 \\
\hline SLC5A6 & 8884 & solute carrier family 5 (sodium-dependent vitamin transporter), member 6 & 10,17 & chr2 \\
\hline SLC6A6 & 6533 & solute carrier family 6 (neurotransmitter transporter, taurine), member 6 & 2,22 & chr3 \\
\hline SLC6A8 & 6535 & solute carrier family 6 (neurotransmitter transporter, creatine), member 8 & 5,42 & $\operatorname{chr} X$ \\
\hline SLC6A8 & $386757 / / / 6535$ & solute carrier family 6 (neurotransmitter transporter, creatine), member $8 / / /$ simil & 3,89 & chr16 \\
\hline SLC7A3 & 84889 & solute carrier family 7 (cationic amino acid transporter, $y+$ system), member 3 & 215,51 & $\operatorname{chr} \mathrm{X}$ \\
\hline SLC7A8 & 23428 & Solute carrier family 7 (cationic amino acid transporter, $y+$ system), member 8 & 22,38 & chr14 \\
\hline SLC9A3R1 & 9368 & solute carrier family 9 (sodium/hydrogen exchanger), member 3 regulator 1 & 3,15 & chr17 \\
\hline SLCO3A1 & 28232 & solute carrier organic anion transporter family, member $3 \mathrm{~A} 1$ & 4,24 & chr15 \\
\hline SLCO4A1 & 28231 & solute carrier organic anion transporter family, member $4 \mathrm{~A} 1$ & 3,58 & chr20 \\
\hline SLCO4C1 & 353189 & solute carrier organic anion transporter family, member 4C1 & 7,24 & chr5 \\
\hline SLD5 & 84296 & SLD5 homolog /// SLD5 homolog & 4,37 & chr8 \\
\hline SLITRK5 & 26050 & SLIT and NTRK-like family, member 5 & 2,12 & chr13 \\
\hline SMA4 & 11039 & SMA4 & 4,70 & chr5 \\
\hline SMARCA3 & 6596 & SWI/SNF related, matrix associated, actin dependent regulator of chromatin, suf & 2,14 & chr3 \\
\hline SMARCA4 & 6597 & SWI/SNF related, matrix associated, actin dependent regulator of chromatin, sul & 4,67 & chr19 \\
\hline SMARCA5 & 8467 & SWI/SNF related, matrix associated, actin dependent regulator of chromatin, sul & 3,07 & chr4 \\
\hline SMARCAD1 & 56916 & SWI/SNF-related, matrix-associated actin-dependent regulator of chromatin, sul & 2,20 & $\operatorname{chr} 4$ \\
\hline SMARCB1 & 6598 & SWI/SNF related, matrix associated, actin dependent regulator of chromatin, sul & 2,30 & chr22 \\
\hline SMARCC1 & 6599 & SWI/SNF related, matrix associated, actin dependent regulator of chromatin, sul & 7,65 & chr3 \\
\hline SMARCD2 & 6603 & SWI/SNF related, matrix associated, actin dependent regulator of chromatin, suf & 3,03 & chr17 \\
\hline SMARCE1 & 6605 & SWI/SNF related, matrix associated, actin dependent regulator of chromatin, sul & 2,85 & chr17 \\
\hline SMC1L1 & 8243 & SMC1 structural maintenance of chromosomes 1 -like 1 (yeast) & 2,25 & $\operatorname{chr} \mathrm{X}$ \\
\hline SMC2L1 & 10592 & SMC2 structural maintenance of chromosomes 2 -like 1 (yeast) & 5,26 & chr9 \\
\hline SMC4L1 & 10051 & SMC4 structural maintenance of chromosomes 4 -like 1 (yeast) & 2,41 & chr3 \\
\hline SMC6L1 & 79677 & SMC6 structural maintenance of chromosomes 6-like 1 (yeast) & 2,45 & chr2 \\
\hline SMG1 & $40345 / / / 440354$ & PI-3-kinase-related kinase SMG-1 /// KIAA0220-like protein /// hypothetical prote & 2,20 & chr16 \\
\hline SMN1 & $6606 / / / 6607$ & survival of motor neuron 1 , telomeric /// survival of motor neuron 2 , centromeric & 2,51 & chr5 \\
\hline SMPDL3B & 27293 & sphingomyelin phosphodiesterase, acid-like 3B & 7,74 & chr1 \\
\hline SMU1 & 55234 & Smu-1 suppressor of mec-8 and unc-52 homolog (C. elegans) & 2,44 & chr9 \\
\hline SMUG1 & 23583 & Single-strand-selective monofunctional uracil-DNA glycosylase 1 & 2,10 & chr12 \\
\hline SMYD4 & 114826 & SET and MYND domain containing 4 & 5,93 & chr17 \\
\hline SNCA & 6622 & synuclein, alpha (non A4 component of amyloid precursor) /// synuclein, alpha (n) & 3,47 & chr4 \\
\hline SND1 & 27044 & Staphylococcal nuclease domain containing $1 / / /$ Staphylococcal nuclease doma & 2,58 & (vide) \\
\hline SNN & 8303 & stannin & 5,18 & chr16 \\
\hline SNRPA1 & 6627 & Small nuclear ribonucleoprotein polypeptide $\mathrm{A}^{\prime}$ & 4,71 & chr15 \\
\hline SNRPB & 6628 & small nuclear ribonucleoprotein polypeptides B and B1 & 2,07 & chr20 \\
\hline SNRPC & 6631 & small nuclear ribonucleoprotein polypeptide C & 2,44 & chr5 \\
\hline SNRPD1 & 6632 & small nuclear ribonucleoprotein $\mathrm{D} 1$ polypeptide $16 \mathrm{kDa}$ & 3,87 & chr18 \\
\hline SNRPD2 & 6633 & small nuclear ribonucleoprotein D2 polypeptide $16.5 \mathrm{kDa}$ & 2,00 & chr19 \\
\hline SNRPD3 & 6634 & small nuclear ribonucleoprotein D3 polypeptide $18 \mathrm{kDa}$ & 3,83 & chr22 \\
\hline SNRPE & 6635 & small nuclear ribonucleoprotein polypeptide $\mathrm{E}$ & 2,46 & chr1 \\
\hline SNRPN & 6638 & Small nuclear ribonucleoprotein polypeptide $\mathrm{N}$ & 25,36 & chr15 \\
\hline SNRPN & $6638 / / / 8926$ & small nuclear ribonucleoprotein polypeptide $\mathrm{N}$ /// SNRPN upstream reading fram & 4,75 & chr15 \\
\hline SNX10 & 29887 & sorting nexin 10 & 9,38 & chr7 \\
\hline SNX5 & 27131 & sorting nexin 5 & 3,27 & chr20 \\
\hline socs1 & 8651 & suppressor of cytokine signaling 1 & 2,57 & chr16 \\
\hline socs2 & 8835 & suppressor of cytokine signaling 2 & 3,62 & chr12 \\
\hline socs7 & 30837 & Suppressor of cytokine signaling 7 & 2,92 & chr17 \\
\hline SORBS1 & 10580 & sorbin and $\mathrm{SH} 3$ domain containing 1 & 13,18 & chr10 \\
\hline SORD & 6652 & sorbitol dehydrogenase & 2,55 & chr15 \\
\hline SORL1 & 6653 & sortilin-related receptor, $L(D L R$ class) A repeats-containing & 39,53 & $\operatorname{chr11}$ \\
\hline sox11 & 6664 & SRY (sex determining region Y)-box 11 & 3,41 & chr2 \\
\hline sox13 & 9580 & SRY (sex determining region Y)-box 13 & 2,12 & chr1 \\
\hline sox15 & 6665 & SRY (sex determining region Y)-box 15 & 2,72 & chr17 \\
\hline sox2 & 6657 & SRY (sex determining region Y)-box 2 & 240,38 & chr3 \\
\hline sox3 & 6658 & SRY (sex determining region Y)-box 3 & 5,34 & $\operatorname{chr} \mathrm{X}$ \\
\hline sox4 & 6659 & SRY (sex determining region Y)-box 4 & 4,26 & chr6 \\
\hline
\end{tabular}




\begin{tabular}{|c|c|c|c|c|c|}
\hline$\infty$ & Table S5: Ge & downregu & din MPC compared to hES (Fold Change $>2 ; \alpha<0.05$ ) & & \\
\hline & sox7 & 83595 & SRY (sex determining region Y)-box 7 & 2,20 & chr8 \\
\hline : & sox8 & 30812 & SRY (sex determining region Y)-box 8 & 4,23 & chr16 \\
\hline & SP1 & 6667 & Sp1 transcription factor & 2,33 & chr12 \\
\hline E. & SP3 & 6670 & Sp3 transcription factor & 2,93 & chr2 \\
\hline 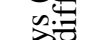 & SP4 & 6671 & Sp4 transcription factor & 7,37 & chr7 \\
\hline$>$ & SP8 & 221833 & Sp8 transcription factor & 3,32 & chr7 \\
\hline$\Xi$ & SPAG5 & 10615 & sperm associated antigen 5 & 8,31 & chr17 \\
\hline 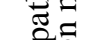 & SPAG7 & 9552 & sperm associated antigen $7 / / /$ sperm associated antigen 7 & 2,03 & chr17 \\
\hline 0 & SPATA13 & 221178 & spermatogenesis associated 13 & 3,38 & chr13 \\
\hline के & SPATA2 & 9825 & spermatogenesis associated 2 & 2,66 & chr20 \\
\hline $\mathrm{z}$ & SPATA5L1 & 79029 & spermatogenesis associated 5 -like 1 & 2,23 & chr15 \\
\hline 00 & SPATA6 & 54558 & Spermatogenesis associated 6 & 3,58 & chr1 \\
\hline$\sqrt{2}$ & SPBC24 & 147841 & spindle pole body component 24 homolog (S. cerevisiae) & 3,53 & (vide) \\
\hline ف를 & SPBC25 & 57405 & spindle pole body component 25 homolog (S. cerevisiae) & 11,32 & chr2 \\
\hline 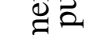 & SPEN & 23013 & spen homolog, transcriptional regulator (Drosophila) & 2,03 & chr1 \\
\hline हีत & SPHK2 & 56848 & sphingosine kinase 2 & 3,44 & chr19 \\
\hline 으ㅇㅛㅛ & SPIB & 6689 & Spi-B transcription factor (Spi-1/PU.1 related) /// Spi-B transcription factor (Spi-1) & 2,92 & chr19 \\
\hline 20 & SPINT1 & 6692 & serine peptidase inhibitor, Kunitz type 1 & 7,99 & chr15 \\
\hline 酌 & SPINT2 & 10653 & serine peptidase inhibitor, Kunitz type, 2 & 21,93 & chr19 \\
\hline$\stackrel{\dot{\Xi}}{>}$ & SPON1 & 10418 & spondin 1 , extracellular matrix protein & 2,51 & chr11 \\
\hline$\stackrel{\bar{g}}{\varrho}$ & SPP1 & 6696 & secreted phosphoprotein 1 (osteopontin, bone sialoprotein I, early T-lymphocyte & 5,65 & chr4 \\
\hline 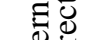 & SPRY1 & 10252 & sprouty homolog 1, antagonist of FGF signaling (Drosophila) & 10,38 & chr4 \\
\hline$\overline{0}$ & SPRY4 & 81848 & Sprouty homolog 4 (Drosophila) & 2,48 & chr11 \\
\hline s. & SPSB2 & 84727 & spIA/ryanodine receptor domain and SOCS box containing 2 & 2,42 & chr12 \\
\hline 눙 & SPSB4 & 92369 & spIA/ryanodine receptor domain and SOCS box containing 4 & 3,46 & chr3 \\
\hline$>$ & SQLE & 6713 & squalene epoxidase & 2,55 & chr8 \\
\hline$=0$ & SR140 & 23350 & U2-associated SR140 protein & 6,93 & chr3 \\
\hline 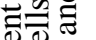 & SREBF1 & 6720 & Sterol regulatory element binding transcription factor 1 & 2,48 & chr17 \\
\hline 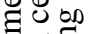 & SRPK1 & 6732 & SFRS protein kinase 1 & 2,25 & chr6 \\
\hline$\Xi$ & SRRM2 & 23524 & serine/arginine repetitive matrix 2 & 3,63 & chr16 \\
\hline ¿ & SRY & 6736 & sex determining region $\mathrm{Y}$ & 2,88 & $\operatorname{chrY}$ \\
\hline 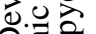 & SS18L2 & 51188 & synovial sarcoma translocation gene on chromosome 18 -like 2 & 3,87 & chr3 \\
\hline 8 & SSB & 6741 & Sjogren syndrome antigen B (autoantigen La) & 4,23 & chr2 \\
\hline Ėo & SSBP1 & 6742 & single-stranded DNA binding protein 1 & 2,73 & chr7 \\
\hline 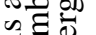 & SSBP2 & 23635 & Single-stranded DNA binding protein 2 & 2,52 & chr5 \\
\hline$\overline{0} \frac{0}{0}$ & SSBP3 & 23648 & single stranded DNA binding protein 3 & 3,10 & chr7 \\
\hline$\Xi$ & SSRP1 & 6749 & structure specific recognition protein 1 & 3,53 & chr11 \\
\hline E요 & ST14 & 6768 & suppression of tumorigenicity 14 (colon carcinoma, matriptase, epithin) & 3,11 & chr11 \\
\hline ¿ & ST3GAL6 & 10402 & ST3 beta-galactoside alpha-2,3-sialyltransferase 6 & 2,11 & chr3 \\
\hline 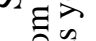 & ST6GAL1 & 6480 & ST6 beta-galactosamide alpha-2,6-sialyltranferase 1 & 12,34 & chr3 \\
\hline$\stackrel{\Xi}{\Xi}$ & ST6GALNAC & 256435 & ST6 (alpha-N-acetyl-neuraminyl-2,3-beta-galactosyl-1,3)-N-acetylgalactosaminid & 6,05 & chr1 \\
\hline DE & ST8SIA3 & 51046 & ST8 alpha-N-acetyl-neuraminide alpha-2,8-sialyltransferase 3 & 2,13 & chr18 \\
\hline & STAG2 & 10735 & stromal antigen 2 & 3,36 & $\operatorname{chrX}$ \\
\hline$\tilde{\tilde{E}}$ & STAP2 & 55620 & signal-transducing adaptor protein-2 & 2,29 & chr19 \\
\hline & STARD7 & 56910 & START domain containing 7 & 2,65 & chr2 \\
\hline ప.ల్ & STAT5B & 6777 & signal transducer and activator of transcription 5B & 2,30 & chr17 \\
\hline 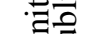 & STC1 & 6781 & Stanniocalcin 1 & 4,83 & chr8 \\
\hline ع & STIP1 & 10963 & stress-induced-phosphoprotein 1 (Hsp70/Hsp90-organizing protein) & 2,45 & chr11 \\
\hline : & STK11IP & 114790 & serine/threonine kinase 11 interacting protein & 2,03 & chr2 \\
\hline & STK24 & 8428 & serine/threonine kinase 24 (STE20 homolog, yeast) & 2,55 & chr13 \\
\hline בై & STK33 & 65975 & serine/threonine kinase 33 & 2,79 & chr11 \\
\hline కై & STK35 & 140901 & serine/threonine kinase 35 & 3,26 & chr20 \\
\hline ¿ृ & STK6 & 6790 & serine/threonine kinase 6 & 6,21 & chr20 \\
\hline & STMN3 & 50861 & stathmin-like 3 & 2,58 & chr20 \\
\hline ฮ & STN2 & 85439 & stonin 2 & 6,44 & chr14 \\
\hline$=0$ & STOML2 & 30968 & stomatin (EPB72)-like 2 & 3,17 & chr9 \\
\hline$\equiv$ & STOX2 & 56977 & storkhead box 2 & 10,44 & chr4 \\
\hline$=0$ & STRA13 & 201254 & stimulated by retinoic acid 13 homolog (mouse) & 2,68 & chr17 \\
\hline$\exists$ & STRBP & 55342 & Spermatid perinuclear RNA binding protein & 33,04 & chr9 \\
\hline$\overline{0}=\pi$ & STRN & 6801 & Striatin, calmodulin binding protein & 3,33 & chr2 \\
\hline 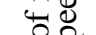 & STX3A & 6809 & syntaxin $3 \mathrm{~A}$ & 5,38 & chr11 \\
\hline & STX6 & 10228 & syntaxin 6 & 2,64 & chr1 \\
\hline$\Xi \nsubseteq$ & SUB1 & 10923 & SUB1 homolog (S. cerevisiae) & 2,50 & chr5 \\
\hline & SUMO1 & 7341 & SMT3 suppressor of mif two 3 homolog 1 (yeast) & 2,13 & chr1 \\
\hline בี & SUMO2 & 6613 & SMT3 suppressor of mif two 3 homolog 2 (yeast) & 2,25 & chr5 \\
\hline $\bar{\pi} \underline{0}$ & SUPT16H & 11198 & suppressor of Ty 16 homolog (S. cerevisiae) & 4,09 & chr12 \\
\hline 0 & |SUPT3H & 8464 & suppressor of Ty 3 homolog (S. cerevisiae) & 2,73 & chr6 \\
\hline
\end{tabular}




\section{Pagge 169 of 257}

\begin{tabular}{|c|c|c|c|c|}
\hline SURF2 & 6835 & surfeit 2 & 2,32 & chr9 \\
\hline SURF5 & 6837 & surfeit 5 & 5,46 & chr9 \\
\hline SURF6 & 6838 & surfeit 6 & 2,61 & chr9 \\
\hline SUV39H1 & 6839 & suppressor of variegation 3-9 homolog 1 (Drosophila) & 2,36 & $\operatorname{chr} \mathrm{X}$ \\
\hline SUV39H2 & 79723 & suppressor of variegation 3-9 homolog 2 (Drosophila) & 7,01 & chr10 \\
\hline SUV420H1 & 51111 & Suppressor of variegation 4-20 homolog 1 (Drosophila) & 4,63 & chr11 \\
\hline SUZ12 & 23512 & suppressor of zeste 12 homolog (Drosophila) & 2,72 & chr17 \\
\hline SYMPK & 8189 & symplekin & 3,56 & chr19 \\
\hline SYN2 & 6854 & synapsin II & 2,09 & chr3 \\
\hline SYNC1 & 81493 & Syncoilin, intermediate filament 1 & 4,58 & chr1 \\
\hline SYNCRIP & 10492 & synaptotagmin binding, cytoplasmic RNA interacting protein & 2,44 & chr20 \\
\hline SYNE2 & 23224 & spectrin repeat containing, nuclear envelope 2 & 15,87 & chr14 \\
\hline SYNGR3 & 9143 & synaptogyrin 3 & 3,49 & chr16 \\
\hline SYT1 & 6857 & synaptotagmin I & 5,88 & chr12 \\
\hline SYT13 & 57586 & synaptotagmin XIII & 19,22 & chr11 \\
\hline SYT17 & 51760 & Synaptotagmin XVII & 3,45 & chr16 \\
\hline SYT4 & 6860 & synaptotagmin IV & 4,69 & chr18 \\
\hline SYT6 & 148281 & synaptotagmin VI & 17,41 & chr1 \\
\hline TAC1 & 6863 & tachykinin, precursor 1 (substance $\mathrm{K}$, substance $\mathrm{P}$, neurokinin 1 , neurokinin $2, \mathrm{n}$ & 2,80 & chr7 \\
\hline TACC2 & 10579 & transforming, acidic coiled-coil containing protein 2 & 2,65 & chr10 \\
\hline TACC3 & 10460 & transforming, acidic coiled-coil containing protein 3 & 11,97 & chr4 \\
\hline TACSTD1 & 4072 & tumor-associated calcium signal transducer 1 & 373,33 & chr2 \\
\hline TACSTD2 & 4070 & tumor-associated calcium signal transducer 2 & 2,30 & chr1 \\
\hline TADA1L & 117143 & transcriptional adaptor 1 (HFI1 homolog, yeast)-like & 3,14 & chr1 \\
\hline TAF11 & 6882 & TAF11 RNA polymerase II, TATA box binding protein (TBP)-associated factor, 2 & 2,23 & chr6 \\
\hline TAF15 & 8148 & TAF15 RNA polymerase II, TATA box binding protein (TBP)-associated factor, 6 & 3,92 & chr17 \\
\hline TAF1A & 9015 & TATA box binding protein (TBP)-associated factor, RNA polymerase I, A, 48kDa & 3,11 & chr1 \\
\hline TAF1C & 9013 & TATA box binding protein (TBP)-associated factor, RNA polymerase I, C, 110kD & 4,04 & chr16 \\
\hline TAF4 & 6874 & TAF4 RNA polymerase II, TATA box binding protein (TBP)-associated factor, 13 & 4,19 & chr20 \\
\hline TAF4B & 6875 & TAF4b RNA polymerase II, TATA box binding protein (TBP)-associated factor, 1 & 9,58 & chr18 \\
\hline TAF5 & 6877 & TAF5 RNA polymerase II, TATA box binding protein (TBP)-associated factor, 10 & 6,35 & chr10 \\
\hline |TAF5L & 27097 & TAF5-like RNA polymerase II, p300/CBP-associated factor (PCAF)-associated f & 2,99 & chr1 \\
\hline TAF9L & 51616 & TAF9-like RNA polymerase II, TATA box binding protein (TBP)-associated factor & 2,35 & chrX_random \\
\hline TALDO1 & 6888 & transaldolase 1 & 2,07 & chr11 \\
\hline TAOK1 & 57551 & TAO kinase 1 & 2,30 & chr17 \\
\hline TARBP2 & 6895 & TAR (HIV) RNA binding protein 2 & 2,88 & chr12 \\
\hline TARDBP & 23435 & TAR DNA binding protein /// TAR DNA binding protein & 2,33 & chr1 \\
\hline TARS & 6897 & threonyl-tRNA synthetase & 2,80 & chr5 \\
\hline TARSL1 & 80222 & threonyl-tRNA synthetase-like 1 & 2,68 & chr1 \\
\hline TATDN1 & 83940 & TatD DNase domain containing 1 & 2,05 & chr10 \\
\hline TBC1D1 & 23216 & TBC1 (tre-2/USP6, BUB2, cdc16) domain family, member 1 & 2,85 & chr4 \\
\hline TBC1D14 & 57533 & TBC1 domain family, member 14 & 2,27 & chr4 \\
\hline TBC1D16 & 125058 & TBC1 domain family, member 16 & 3,69 & chr17 \\
\hline TBC1D4 & 9882 & TBC1 domain family, member 4 & 2,03 & chr13 \\
\hline TBC1D7 & 51256 & TBC1 domain family, member 7 & 2,14 & chr6 \\
\hline TBC1D8 & 11138 & TBC1 domain family, member 8 (with GRAM domain) & 5,48 & chr2 \\
\hline TBCD & 6904 & tubulin-specific chaperone $\mathrm{d} / / /$ tubulin-specific chaperone $\mathrm{d}$ & 3,29 & chr17 \\
\hline TBCE & 6905 & tubulin-specific chaperone e & 2,10 & chr1 \\
\hline TBL1Y & 90665 & Transducin (beta)-like 1Y-linked & 9,51 & $\operatorname{chrY}$ \\
\hline TBL3 & 10607 & transducin (beta)-like 3 & 2,52 & chr16 \\
\hline TBP & 6908 & TATA box binding protein & 2,40 & chr6 \\
\hline TBPIP & 29893 & TBP-1 interacting protein & 2,63 & chr17 \\
\hline TBRG4 & 9238 & transforming growth factor beta regulator 4 & 3,66 & chr7 \\
\hline TCEA1 & 6917 & transcription elongation factor A (SII), 1 & 2,51 & chr3 \\
\hline TCEAL2 & 140597 & transcription elongation factor A (SII)-like 2 & 31,45 & $\operatorname{chr} \mathrm{X}$ \\
\hline TCERG1L & 256536 & transcription elongation regulator 1 -like & 2,45 & chr10 \\
\hline TCF12 & 6938 & Transcription factor 12 (HTF4, helix-loop-helix transcription factors 4) & 2,38 & chr15 \\
\hline TCF15 & 6939 & transcription factor 15 (basic helix-loop-helix) & 2,53 & chr20 \\
\hline TCF20 & 6942 & transcription factor 20 (AR1) & 2,89 & chr22 \\
\hline TCF3 & 6929 & transcription factor 3 (E2A immunoglobulin enhancer binding factors E12/E47) & 3,27 & chr19 \\
\hline TCF7L1 & 83439 & transcription factor 7-like 1 (T-cell specific, HMG-box) /// transcription factor 7-likg & 4,74 & chr2 \\
\hline TCF7L2 & 6934 & Transcription factor 7-like 2 (T-cell specific, HMG-box) & 3,86 & chr10 \\
\hline TCFL5 & 10732 & Transcription factor-like 5 (basic helix-loop-helix) & 4,08 & chr20 \\
\hline TCOF1 & 6949 & Treacher Collins-Franceschetti syndrome 1 & 6,25 & chr5 \\
\hline TCP1 & 6950 & t-complex 1 & 2,13 & chr7 \\
\hline TDGF1 & 6997 & teratocarcinoma-derived growth factor 1 & 648,52 & chr3 \\
\hline TDP1 & 55775 & |tyrosyl-DNA phosphodiesterase 1 & 2,54 & chr14 \\
\hline
\end{tabular}




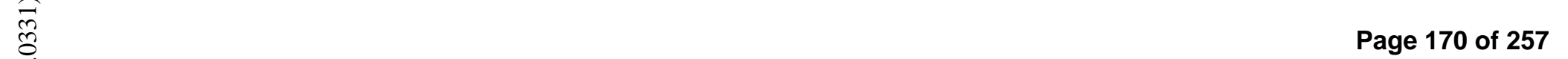

\begin{tabular}{|c|c|c|c|c|}
\hline TDRKH & 11022 & tudor and $\mathrm{KH}$ domain containing & 4,46 & chr1 \\
\hline TEAD2 & 8463 & TEA domain family member 2 & 3,30 & chr19 \\
\hline TEAD4 & 7004 & TEA domain family member 4 & 4,21 & chr12 \\
\hline TERF1 & 7013 & telomeric repeat binding factor (NIMA-interacting) 1 & 42,46 & chr8 \\
\hline TERF2 & 7014 & telomeric repeat binding factor 2 & 2,22 & chr16 \\
\hline TERT & 7015 & telomerase reverse transcriptase & 2,08 & chr5 \\
\hline TESK2 & 10420 & testis-specific kinase 2 & 4,25 & chr1 \\
\hline TEX10 & 54881 & Testis expressed sequence 10 & 3,46 & chr9 \\
\hline TEX15 & 56154 & testis expressed sequence 15 & 5,03 & chr8 \\
\hline TEX9 & 374618 & testis expressed sequence 9 & 2,42 & chr15 \\
\hline TFAM & 7019 & Transcription factor A, mitochondrial & 5,25 & chr10 \\
\hline TFAP2C & 7022 & transcription factor AP-2 gamma (activating enhancer binding protein 2 gamma) & 3,67 & chr20 \\
\hline TFB1M & 51106 & transcription factor B1, mitochondrial & 2,87 & chr6 \\
\hline TFCP2 & 7024 & |transcription factor CP2 & 2,71 & chr12 \\
\hline TFDP2 & 7029 & Transcription factor Dp-2 (E2F dimerization partner 2) & 9,05 & chr3 \\
\hline TGFBR1 & 7046 & Transforming growth factor, beta receptor I (activin A receptor type II-like kinase & 2,40 & chr9 \\
\hline TGFBR3 & 7049 & transforming growth factor, beta receptor III (betaglycan, $300 \mathrm{kDa}$ ) & 2,04 & chr1 \\
\hline TGIF & 7050 & TGFB-induced factor (TALE family homeobox) & 4,53 & chr18 \\
\hline TGIF2 & 60436 & TGFB-induced factor 2 (TALE family homeobox) & 5,27 & chr20 \\
\hline THADA & 63892 & thyroid adenoma associated & 2,39 & chr2 \\
\hline THAP11 & 57215 & THAP domain containing 11 & 4,09 & chr16 \\
\hline THAP4 & 51078 & THAP domain containing 4 & 2,29 & chr2_random \\
\hline THAP9 & 79725 & THAP domain containing 9 & 12,16 & chr4 \\
\hline THBS4 & 7060 & thrombospondin 4 & 10,39 & chr5 \\
\hline THEM2 & 55856 & thioesterase superfamily member 2 & 3,46 & chr6 \\
\hline THNSL1 & 79896 & threonine synthase-like 1 (bacterial) & 4,16 & chr10 \\
\hline THOC1 & 9984 & THO complex 1 & 2,82 & chr18 \\
\hline THOC2 & 57187 & THO complex 2 & 2,11 & $\operatorname{chrX}$ \\
\hline THOC3 & 84321 & THO complex 3 & 3,33 & chr5 \\
\hline THOC4 & 10189 & THO complex 4 & 2,90 & chr17_random \\
\hline THRAP1 & 9969 & Thyroid hormone receptor associated protein 1 & 2,96 & chr17 \\
\hline THRAP2 & 23389 & Thyroid hormone receptor associated protein 2 & 2,94 & chr12 \\
\hline THRAP3 & 9967 & thyroid hormone receptor associated protein 3 & 2,17 & chr1 \\
\hline THRAP6 & 90390 & thyroid hormone receptor associated protein 6 & 4,11 & chr8 \\
\hline THRB & 7068 & thyroid hormone receptor, beta (erythroblastic leukemia viral (v-erb-a) oncogene & 4,24 & chr3 \\
\hline THUMPD2 & 80745 & THUMP domain containing 2 & 2,30 & chr2 \\
\hline THUMPD3 & 25917 & THUMP domain containing 3 & 2,97 & chr3 \\
\hline THY28 & 29087 & thymocyte protein thy 28 & 6,98 & chr11 \\
\hline TIA1 & 7072 & TIA1 cytotoxic granule-associated RNA binding protein & 2,51 & chr2 \\
\hline TIAM1 & 7074 & T-cell lymphoma invasion and metastasis 1 & 2,46 & chr21 \\
\hline TIAM2 & 26230 & T-cell lymphoma invasion and metastasis 2 & 3,03 & chr6 \\
\hline TIGA1 & 114915 & TIGA1 & 2,55 & chr5 \\
\hline |TIGD7 & 91151 & tigger transposable element derived $7 / / /$ tigger transposable element derived 7 & 3,07 & chr16 \\
\hline TIMELESS & 8914 & timeless homolog (Drosophila) & 12,87 & chr12 \\
\hline TIMM10 & 26519 & translocase of inner mitochondrial membrane 10 homolog (yeast) & 2,77 & chr11 \\
\hline TIMM13 & 26517 & translocase of inner mitochondrial membrane 13 homolog (yeast) & 3,79 & chr19 \\
\hline TIMM22 & 29928 & translocase of inner mitochondrial membrane 22 homolog (yeast) & 2,43 & chr17 \\
\hline TIMM44 & 10469 & translocase of inner mitochondrial membrane 44 homolog (yeast) & 2,57 & chr19 \\
\hline TIMM50 & 92609 & translocase of inner mitochondrial membrane 50 homolog (yeast) & 2,77 & chr19 \\
\hline TIMM8A & 1678 & translocase of inner mitochondrial membrane 8 homolog $\mathrm{A}$ (yeast) & 3,45 & chr2 \\
\hline TIMM8B & 26521 & translocase of inner mitochondrial membrane 8 homolog $B$ (yeast) & 2,06 & chr11 \\
\hline TIMP4 & 7079 & TIMP metallopeptidase inhibitor 4 & 2,93 & chr3 \\
\hline TJP2 & 9414 & tight junction protein 2 (zona occludens 2) & 5,26 & chr9 \\
\hline TJP3 & 27134 & tight junction protein 3 (zona occludens 3 ) & 4,91 & chr19 \\
\hline TKT & 7086 & transketolase (Wernicke-Korsakoff syndrome) & 6,38 & chr3 \\
\hline TLE1 & 7088 & transducin-like enhancer of split 1 (E(sp1) homolog, Drosophila) & 6,48 & chr9 \\
\hline TLE1 & $389863 / / / 7088$ & transducin-like enhancer of split 1 (E(sp1) homolog, Drosophila) /// hypothetical & 2,37 & $\operatorname{chrX}$ \\
\hline TLE2 & 7089 & transducin-like enhancer of split 2 (E(sp1) homolog, Drosophila) & 3,89 & chr19 \\
\hline TLE3 & 7090 & transducin-like enhancer of split 3 (E(sp1) homolog, Drosophila) & 2,32 & chr15 \\
\hline TLK1 & 9874 & tousled-like kinase 1 & 2,14 & chr2 \\
\hline TM7SF2 & 7108 & transmembrane 7 superfamily member 2 & 7,70 & chr11 \\
\hline TMCC1 & 23023 & transmembrane and coiled-coil domain family 1 & 2,61 & chr3 \\
\hline TMEFF1 & 8577 & transmembrane protein with EGF-like and two follistatin-like domains 1 & 6,99 & chr9 \\
\hline TMEM11 & 8834 & transmembrane protein 11 & 2,20 & chr17 \\
\hline TMEM118 & 84900 & Transmembrane protein 118 & 5,24 & chr12 \\
\hline TMEM28 & 27112 & transmembrane protein 28 & 2,49 & $\operatorname{chrX}$ \\
\hline TMEM30B & 161291 & transmembrane protein $30 \mathrm{~B}$ & 8,60 & chr14 \\
\hline
\end{tabular}




\begin{tabular}{|c|c|c|c|c|}
\hline TMEM33 & 55161 & Transmembrane protein 33 & 3,05 & chr4 \\
\hline TMEM37 & 140738 & transmembrane protein 37 & 4,91 & chr2 \\
\hline TMEM4 & 10330 & transmembrane protein 4 & 2,75 & chr12 \\
\hline TMEM48 & 55706 & transmembrane protein 48 & 6,28 & chr1 \\
\hline TMEM53 & 79639 & transmembrane protein 53 & 2,80 & chr1 \\
\hline TMEM63A & 9725 & transmembrane protein $63 \mathrm{~A}$ & 2,11 & chr1 \\
\hline TMEM64 & 169200 & transmembrane protein 64 & 2,39 & chr8 \\
\hline TMEM68 & 137695 & transmembrane protein 68 & 3,70 & chr8 \\
\hline TMEM70 & 54968 & transmembrane protein 70 & 2,20 & chr8 \\
\hline TMPO & 7112 & Thymopoietin & 4,75 & chr12 \\
\hline TMPRSS2 & 7113 & transmembrane protease, serine 2 & 4,23 & chr21 \\
\hline TMSL8 & 11013 & thymosin-like 8 & 6,34 & $\operatorname{chr} X$ \\
\hline TNFSF11 & 8600 & tumor necrosis factor (ligand) superfamily, member 11 & 5,07 & $\operatorname{chr} 13$ \\
\hline TNNI3 & 7137 & troponin I type 3 (cardiac) & 5,38 & $\operatorname{chr} 19$ \\
\hline TNNT1 & 7138 & troponin T type 1 (skeletal, slow) & 9,44 & chr19 \\
\hline TNPO3 & 23534 & transportin 3 & 3,24 & $\operatorname{chr} 7$ \\
\hline TNRC6A & 27327 & trinucleotide repeat containing $6 \mathrm{~A}$ & 4,04 & chr16 \\
\hline TNRC6B & 23112 & Trinucleotide repeat containing 6B & 2,91 & chr22 \\
\hline TNRC6C & 57690 & trinucleotide repeat containing $6 \mathrm{C}$ & 2,44 & chr17 \\
\hline TNRC9 & 27324 & trinucleotide repeat containing 9 & 58,38 & chr16 \\
\hline TOE1 & 114034 & target of EGR1, member 1 (nuclear) & 3,83 & chr1 \\
\hline TOM1L1 & 10040 & target of myb1-like 1 (chicken) & 2,90 & chr17 \\
\hline TOMM22 & 56993 & translocase of outer mitochondrial membrane 22 homolog (yeast) & 2,36 & chr22 \\
\hline TOMM40 & 10452 & translocase of outer mitochondrial membrane 40 homolog (yeast) & 2,43 & chr14 \\
\hline TOMM40L & 84134 & translocase of outer mitochondrial membrane 40 homolog-like (yeast) & 2,05 & chr1 \\
\hline TOP1MT & 116447 & topoisomerase (DNA) I, mitochondrial & 5,91 & chr8 \\
\hline TOP2A & 7153 & topoisomerase (DNA) II alpha $170 \mathrm{kDa}$ & 4,98 & chr17 \\
\hline TOPЗА & 7156 & topoisomerase (DNA) III alpha & 2,43 & chr17 \\
\hline TOPBP1 & 11073 & topoisomerase (DNA) II binding protein 1 & 2,02 & chr3 \\
\hline TOPORS & 10210 & topoisomerase I binding, arginine/serine-rich & 2,13 & chr9 \\
\hline TOR3A & 64222 & torsin family 3 , member A & 2,97 & chr1 \\
\hline TOX & 9760 & Full-length cDNA clone CSODM012YE14 of Fetal liver of Homo sapiens (human & 2,05 & chr8 \\
\hline TP53RK & 112858 & TP53 regulating kinase & 2,31 & chr20 \\
\hline TPD52 & 7163 & tumor protein D52 & 34,89 & chr8 \\
\hline TPK1 & 27010 & thiamin pyrophosphokinase 1 & 2,05 & $\operatorname{chr} 7$ \\
\hline TPP2 & 7174 & tripeptidyl peptidase II & 2,82 & chr13 \\
\hline TPR & 7175 & translocated promoter region (to activated MET oncogene) & 3,12 & chr1 \\
\hline TPRT & 23590 & trans-prenyltransferase & 2,67 & chr10 \\
\hline TPST2 & 8459 & tyrosylprotein sulfotransferase 2 & 3,67 & chr22 \\
\hline TPX2 & 22974 & TPX2, microtubule-associated, homolog (Xenopus laevis) & 6,28 & chr20 \\
\hline TRA16 & 126382 & TR4 orphan receptor associated protein TRA16 & 3,03 & chr19 \\
\hline TRA2A & 29896 & Transformer-2 alpha & 2,33 & $\operatorname{chr} 7$ \\
\hline TRAF2 & 7186 & TNF receptor-associated factor 2 & 2,37 & chr9 \\
\hline TRAF4 & 9618 & TNF receptor-associated factor 4 & 3,00 & chr17 \\
\hline TRAP1 & 10131 & TNF receptor-associated protein 1 & 3,01 & chr16 \\
\hline TRAPPC6A & 79090 & trafficking protein particle complex $6 \mathrm{~A}$ & 3,18 & chr19 \\
\hline TREX1 & 11277 & three prime repair exonuclease 1 & 4,60 & chr3 \\
\hline TREX2 & $11219 / / / 55559$ & three prime repair exonuclease $2 / / / 26 \mathrm{~S}$ proteasome-associated $\mathrm{UCH}$ interactin & 2,02 & $\operatorname{chr} \mathrm{X}$ \\
\hline TRFP & 9477 & Trf (TATA binding protein-related factor)-proximal homolog (Drosophila) & 2,69 & chr6 \\
\hline TRIB1 & 10221 & tribbles homolog 1 (Drosophila) & 4,16 & chr8 \\
\hline TRIB2 & 28951 & tribbles homolog 2 (Drosophila) & 3,03 & chr2 \\
\hline TRIM14 & 9830 & |tripartite motif-containing 14 & 3,11 & chr9 \\
\hline TRIM2 & 23321 & tripartite motif-containing 2 & 2,32 & chr4 \\
\hline TRIM24 & 8805 & tripartite motif-containing 24 & 5,73 & chr7 \\
\hline TRIM28 & 10155 & tripartite motif-containing 28 & 2,52 & chr19 \\
\hline TRIM33 & 51592 & tripartite motif-containing 33 & 3,57 & chr1 \\
\hline TRIM36 & 55521 & tripartite motif-containing 36 & 7,96 & chr5 \\
\hline TRIM37 & 4591 & tripartite motif-containing 37 & 3,21 & chr17 \\
\hline TRIM39 & 56658 & tripartite motif-containing 39 & 2,00 & chr6 \\
\hline TRIM45 & 80263 & Tripartite motif-containing 45 & 5,32 & chr1 \\
\hline TRIM59 & 286827 & tripartite motif-containing 59 & 4,13 & chr3 \\
\hline TRIM6 & 117854 & tripartite motif-containing 6 & 5,51 & chr11 \\
\hline TRIP & 10293 & TRAF interacting protein & 2,23 & chr3 \\
\hline TRIP10 & 9322 & thyroid hormone receptor interactor 10 & 2,40 & chr19 \\
\hline TRIP12 & 9320 & Thyroid hormone receptor interactor 12 & 3,99 & (vide) \\
\hline TRIP13 & 9319 & thyroid hormone receptor interactor 13 & 4,64 & chr5 \\
\hline TRIT1 & 54802 & tRNA isopentenyltransferase 1 & 2,91 & chr1 \\
\hline
\end{tabular}




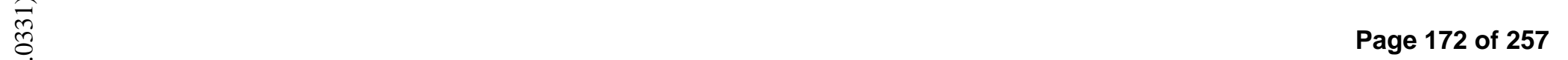

\begin{tabular}{|c|c|c|c|c|}
\hline TRMT1 & 55621 & TRM1 tRNA mothyltrancforace 1 homolog (s corevician) & & \\
\hline TRMT12 & 55039 & tRNA methyltranferase 12 homolog (S. cerevisiae) & 240 & chr8 \\
\hline TRMT5 & 57570 & TRM5 tRNA methyltransferase 5 homolog (S. cerevisiae) & 2,44 & chr14 \\
\hline TRUB2 & 26995 & TruB pseudouridine (psi) synthase homolog 2 (E. coli) & 2,45 & chr9 \\
\hline TSC22D1 & 8848 & TSC22 domain family, member 1 & 2,88 & chr13 \\
\hline TSEN2 & 80746 & tRNA splicing endonuclease 2 homolog (SEN2, S. cerevisiae) & 2,69 & chr3 \\
\hline TSEN54 & 283989 & tRNA splicing endonuclease 54 homolog (SEN54, S. cerevisiae) & 4,46 & chr17 \\
\hline TSGA10 & 80705 & Testis specific, 10 & 3,53 & chr2 \\
\hline TSN & 7247 & translin & 2,33 & chr2 \\
\hline TSPAN12 & 23554 & tetraspanin 12 & 3,63 & $\mathrm{chr} 7$ \\
\hline TSPAN18 & 90139 & tetraspanin 18 & 2,29 & chr11 \\
\hline TSPAN2 & 10100 & tetraspanin 2 & 7,19 & chr1 \\
\hline TSPAN33 & 340348 & tetraspanin 33 & 3,98 & chr7 \\
\hline TSPAN6 & 7105 & tetraspanin 6 & 2,31 & $\operatorname{chrX}$ \\
\hline TTC19 & 54902 & Tetratricopeptide repeat domain 19 & 5,51 & chr17 \\
\hline TTC9 & 23508 & tetratricopeptide repeat domain 9 & 4,33 & chr14 \\
\hline TTF1 & 7270 & transcription termination factor, RNA polymerase I & 3,18 & chr9 \\
\hline TTF2 & 8458 & transcription termination factor, RNA polymerase II & 3,11 & chr1 \\
\hline TTK & 7272 & TTK protein kinase & 14,89 & chr6 \\
\hline TTLL4 & 9654 & tubulin tyrosine ligase-like family, member 4 & 2,91 & chr2 \\
\hline TUBA1 & 7277 & tubulin, alpha 1 (testis specific) & 2,51 & chr2 \\
\hline TUBB2 & $347733 / / / / 7280$ & tubulin, beta 2 /// tubulin, beta polypeptide paralog & 3,72 & chr6 \\
\hline TUBB3 & 10381 & tubulin, beta 3 & 2,02 & chr16 \\
\hline TUBB-PARAL & 347733 & tubulin, beta polypeptide paralog & 5,07 & chr6 \\
\hline TUBD1 & 51174 & tubulin, delta 1 & 4,26 & chr17 \\
\hline TUBG1 & 7283 & tubulin, gamma 1 & 2,58 & $\mathrm{chr} 7$ \\
\hline TUBGCP3 & 10426 & tubulin, gamma complex associated protein 3 & 2,12 & chr13 \\
\hline TUFM & 7284 & Tu translation elongation factor, mitochondrial & 2,78 & chr16 \\
\hline TXNDC & 81542 & thioredoxin domain containing /// thioredoxin domain containing & 3,19 & chr14 \\
\hline TXNL2 & 10539 & thioredoxin-like 2 & 2,39 & chr6 \\
\hline TYMS & 7298 & thymidylate synthetase & 2,78 & chr18 \\
\hline TYRO3 & 7301 & TYRO3 protein tyrosine kinase & 5,79 & chr15 \\
\hline U1SNRNPBP & 11066 & U11/U12 snRNP 35K & 2,87 & chr12 \\
\hline U2AF1 & 7307 & U2(RNU2) small nuclear RNA auxiliary factor 1 & 3,61 & chr15 \\
\hline U2AF2 & 11338 & U2 (RNU2) small nuclear RNA auxiliary factor 2 & 4,34 & chr19 \\
\hline UBA2 & 10054 & SUMO-1 activating enzyme subunit 2 & 2,40 & chr19 \\
\hline UBADC1 & 10422 & ubiquitin associated domain containing 1 & 2,70 & chr9 \\
\hline UBAP2 & 55833 & ubiquitin associated protein 2 & 2,88 & chr9 \\
\hline UBE2C & 11065 & ubiquitin-conjugating enzyme E2C & 10,33 & chr20 \\
\hline UBE2D2 & 7322 & Ubiquitin-conjugating enzyme E2D 2 (UBC4/5 homolog, yeast) & 2,60 & chr5 \\
\hline UBE2E2 & 7325 & Ubiquitin-conjugating enzyme E2E 2 (UBC4/5 homolog, yeast) & 2,09 & chr3 \\
\hline UBE2G1 & 7326 & ubiquitin-conjugating enzyme E2G 1 (UBC7 homolog, yeast) & 3,08 & chr17 \\
\hline UBE2I & 7329 & Ubiquitin-conjugating enzyme E2I (UBC9 homolog, yeast) & 2,80 & chr16 \\
\hline UBE2L6 & 9246 & ubiquitin-conjugating enzyme E2L 6 & 3,28 & chr11 \\
\hline UBE2N & 7334 & ubiquitin-conjugating enzyme E2N (UBC13 homolog, yeast) & 2,01 & chr12 \\
\hline UBE2S & 27338 & ubiquitin-conjugating enzyme E2S & 4,83 & chr17 \\
\hline UBE2T & 29089 & ubiquitin-conjugating enzyme E2T (putative) & 6,45 & chr1 \\
\hline UBE3B & 89910 & ubiquitin protein ligase E3B & 2,10 & chr12 \\
\hline UBE3C & 9690 & Ubiquitin protein ligase E3C & 3,73 & $\mathrm{chr} 7$ \\
\hline UBE4B & 10277 & ubiquitination factor E4B (UFD2 homolog, yeast) & 3,97 & chr1 \\
\hline UBTF & 7343 & upstream binding transcription factor, RNA polymerase I & 2,04 & chr17 \\
\hline UCK1 & 83549 & uridine-cytidine kinase 1 & 2,11 & $\operatorname{chr} 9$ \\
\hline UCK2 & 7371 & |uridine-cytidine kinase 2 & 2,10 & chr1 \\
\hline UCKL1 & 54963 & |uridine-cytidine kinase 1 -like 1 & 3,58 & chr20 \\
\hline UGCGL2 & 55757 & UDP-glucose ceramide glucosyltransferase-like 2 & 2,24 & $\operatorname{chr} 13$ \\
\hline UGP2 & 7360 & UDP-glucose pyrophosphorylase 2 & 3,37 & chr2 \\
\hline UGT8 & 7368 & UDP glycosyltransferase 8 (UDP-galactose ceramide galactosyltransferase) & 19,31 & chr4 \\
\hline UIP1 & 55559 & $26 \mathrm{~S}$ proteasome-associated $\mathrm{UCH}$ interacting protein 1 & 3,20 & $\operatorname{chrX}$ \\
\hline UMPS & 7372 & uridine monophosphate synthetase (orotate phosphoribosyl transferase and oro & 3,38 & chr3 \\
\hline UNC13B & 10497 & unc-13 homolog B (C. elegans) & 3,48 & chr9 \\
\hline UNC5D & 137970 & Unc-5 homolog D (C. elegans) & 31,19 & chr8 \\
\hline UNG & 7374 & uracil-DNA glycosylase & 13,88 & chr12 \\
\hline UNQ501 & 374882 & MBC3205 & 3,68 & chr19 \\
\hline UPF3B & 65109 & UPF3 regulator of nonsense transcripts homolog $B$ (yeast) & 4,20 & $\operatorname{chrX}$ \\
\hline UPP1 & 7378 & uridine phosphorylase 1 & 4,10 & $\mathrm{chr} 7$ \\
\hline UQCR & 10975 & ubiquinol-cytochrome $\mathrm{c}$ reductase, $6.4 \mathrm{kDa}$ subunit & 2,70 & chr19 \\
\hline UQCRFS1 & 7386 & ubiquinol-cytochrome $\mathrm{c}$ reductase, Rieske iron-sulfur polypeptide 1 & 2,00 & chr22 \\
\hline
\end{tabular}




\section{Pagge 173 of 257}

\begin{tabular}{|c|c|c|c|c|}
\hline UQCRH & 7388 & ubiquinol-cytochrome $\mathrm{c}$ reductase hinge protein & 2,64 & chr1 \\
\hline USP1 & 7398 & ubiquitin specific peptidase 1 & 3,68 & chr1 \\
\hline USP10 & 9100 & ubiquitin specific peptidase 10 & 2,45 & chr14 \\
\hline USP13 & 8975 & Ubiquitin specific peptidase 13 (isopeptidase T-3) & 3,14 & chr5 \\
\hline USP25 & 29761 & ubiquitin specific peptidase 25 & 3,14 & chr21 \\
\hline USP28 & 57646 & ubiquitin specific peptidase 28 & 8,39 & chr11 \\
\hline USP31 & 57478 & ubiquitin specific peptidase 31 & 5,43 & chr16 \\
\hline USP32 & 84669 & |ubiquitin specific peptidase 32 & 2,30 & chr17 \\
\hline USP34 & 9736 & Ubiquitin specific peptidase 34 & 2,22 & chr2 \\
\hline USP37 & 57695 & ubiquitin specific peptidase 37 & 3,68 & chr2 \\
\hline USP39 & 10713 & ubiquitin specific peptidase 39 & 2,33 & chr2 \\
\hline USP44 & 84101 & ubiquitin specific peptidase 44 & 50,78 & chr12 \\
\hline USP45 & 85015 & Ubiquitin specific peptidase 45 & 2,02 & chr6 \\
\hline USP46 & 64854 & ubiquitin specific peptidase 46 & 2,54 & chr4 \\
\hline |USP51 & 158880 & |ubiquitin specific peptidase 51 & 4,06 & $\operatorname{chrX}$ \\
\hline USP52 & 9924 & ubiquitin specific peptidase 52 & 2,70 & chr12 \\
\hline USP54 & 159195 & ubiquitin specific peptidase 54 & 6,98 & chr10 \\
\hline USP6NL & 9712 & USP6 N-terminal like & 2,33 & chr10 \\
\hline USP7 & 7874 & Unknown protein & 2,94 & chr16 \\
\hline USP9X & 8239 & ubiquitin specific peptidase 9, X-linked (fat facets-like, Drosophila) & 3,60 & $\operatorname{chrX}$ \\
\hline UST & 10090 & uronyl-2-sulfotransferase & 3,03 & chr6 \\
\hline UTX & 7403 & ubiquitously transcribed tetratricopeptide repeat, $\mathrm{X}$ chromosome & 2,99 & $\operatorname{chrX}$ \\
\hline UXS1 & 80146 & UDP-glucuronate decarboxylase 1 & 2,12 & chr2 \\
\hline VAMP8 & 8673 & vesicle-associated membrane protein 8 (endobrevin) & 15,21 & chr2 \\
\hline VANGL2 & 57216 & vang-like 2 (van gogh, Drosophila) & 8,83 & chr1 \\
\hline VARSL & 57176 & valyl-tRNA synthetase like & 2,55 & chr6 \\
\hline VAV3 & 10451 & vav 3 oncogene & 8,21 & chr1 \\
\hline VDP & 8615 & Vesicle docking protein $\mathrm{p} 115$ & 2,61 & chr4 \\
\hline VIL2 & 7430 & villin 2 (ezrin) & 6,34 & chr6 \\
\hline VprBP & 9730 & Vpr-binding protein & 2,21 & chr3 \\
\hline VPS4A & 27183 & Vacuolar protein sorting 4A (yeast) & 2,32 & chr16 \\
\hline VRK1 & 7443 & vaccinia related kinase 1 & 9,09 & chr14 \\
\hline VRK2 & 7444 & vaccinia related kinase 2 & 2,01 & chr2 \\
\hline VSNL1 & 7447 & visinin-like 1 & 17,97 & chr2 \\
\hline WASL & 8976 & Wiskott-Aldrich syndrome-like & 3,39 & chr7 \\
\hline WBSCR16 & 81554 & Williams-Beuren syndrome chromosome region $16 / / /$ Williams-Beuren syndrom & 2,06 & chr7 \\
\hline WBSCR17 & 64409 & Williams-Beuren syndrome chromosome region 17 & 6,65 & chr7 \\
\hline WBSCR20C & 260294 & Williams Beuren syndrome chromosome region $20 \mathrm{C}$ & 4,69 & chr7 \\
\hline WDHD1 & 11169 & WD repeat and HMG-box DNA binding protein 1 & 10,80 & chr14 \\
\hline WDR12 & 55759 & WD repeat domain 12 & 4,46 & chr2 \\
\hline WDR18 & 57418 & WD repeat domain 18 & 3,47 & chr19 \\
\hline WDR23 & 80344 & WD repeat domain 23 & 2,99 & chr14 \\
\hline WDR27 & 253769 & CDNA FLJ46815 fis, clone TRACH3036897 /// WD repeat domain 27 & 2,95 & chr6 \\
\hline WDR3 & 10885 & WD repeat domain 3 & 2,13 & chr1 \\
\hline WDR33 & 55339 & WD repeat domain 33 & 3,29 & chr2 \\
\hline WDR34 & 89891 & WD repeat domain 34 & 2,77 & chr9 \\
\hline WDR35 & 57539 & WD repeat domain 35 & 3,92 & chr2 \\
\hline WDR40B & 139170 & WD repeat domain $40 \mathrm{~B}$ & 3,67 & $\operatorname{chrX}$ \\
\hline WDR42A & 50717 & WD repeat domain $42 \mathrm{~A}$ & 5,14 & chr1 \\
\hline WDR43 & 23160 & WD repeat domain 43 & 2,25 & chr2 \\
\hline WDR46 & 9277 & WD repeat domain 46 & 2,28 & chr6 \\
\hline WDR48 & 57599 & WD repeat domain 48 & 2,32 & chr3 \\
\hline WDR5 & 11091 & WD repeat domain 5 & 2,88 & chr9 \\
\hline WDR50 & 51096 & WD repeat domain 50 & 3,12 & chr18 \\
\hline WDR51A & 25886 & WD repeat domain $51 \mathrm{~A}$ & 6,29 & chr3 \\
\hline WDR54 & 84058 & WD repeat domain 54 & 4,21 & chr2 \\
\hline WDR57 & 9410 & WD repeat domain 57 (U5 snRNP specific) & 3,25 & chr1 \\
\hline WDR58 & 79228 & WD repeat domain 58 & 2,71 & chr16 \\
\hline WDR59 & 79726 & WD repeat domain 59 & 3,14 & chr16 \\
\hline WDR6 & 11180 & WD repeat domain 6 & 2,10 & chr3 \\
\hline WDR67 & 93594 & WD repeat domain 67 & 3,94 & chr8 \\
\hline WDR70 & 55100 & WD repeat domain 70 & 2,45 & chr5 \\
\hline WDR72 & 256764 & WD repeat domain 72 & 9,40 & chr15 \\
\hline WDR74 & 54663 & WD repeat domain 74 /// WD repeat domain 74 & 4,27 & chr11 \\
\hline WDR75 & 84128 & WD repeat domain 75 & 3,33 & chr2 \\
\hline WDR76 & 79968 & WD repeat domain 76 & 2,15 & chr15 \\
\hline WDR77 & 79084 & WD repeat domain 77 & 3,33 & chr1 \\
\hline
\end{tabular}




\begin{tabular}{|c|c|c|c|c|}
\hline WDR79 & 55135 & WD repeat domain 79 & 4,97 & chr17 \\
\hline WDSUB1 & 151525 & WD repeat, SAM and U-box domain containing 1 & 2,10 & chr2 \\
\hline WEE1 & 7465 & WEE1 homolog (S. pombe) & 2,37 & chr11 \\
\hline WFDC2 & 10406 & WAP four-disulfide core domain 2 & 4,23 & chr20 \\
\hline WHSC1 & 7468 & Wolf-Hirschhorn syndrome candidate 1 & 2,16 & chr4 \\
\hline WHSC1L1 & 54904 & Wolf-Hirschhorn syndrome candidate 1 -like 1 & 2,17 & chr8 \\
\hline WHSC2 & 7469 & Wolf-Hirschhorn syndrome candidate 2 & 2,29 & chr4 \\
\hline WIBG & 84305 & within bgen homolog (Drosophila) /// within bgen homolog (Drosophila) & 3,52 & chr12 \\
\hline WIF1 & 11197 & WNT inhibitory factor 1 & 17,67 & chr12 \\
\hline WNK3 & 65267 & WNK lysine deficient protein kinase 3 & 13,44 & $\operatorname{chr} X$ \\
\hline WRNIP1 & 56897 & Werner helicase interacting protein 1 & 2,07 & chr6 \\
\hline WTAP & 9589 & Wilms tumor 1 associated protein & 3,56 & chr6 \\
\hline WWP1 & 11059 & WW domain containing E3 ubiquitin protein ligase 1 & 2,16 & chr3 \\
\hline XKR4 & 114786 & X Kell blood group precursor-related family, member 4 & 2,36 & chr8 \\
\hline XPO4 & 64328 & exportin 4 & 2,28 & chr13 \\
\hline XPO5 & 57510 & exportin 5 & 3,38 & chr6 \\
\hline XPO7 & 23039 & exportin 7 & 2,18 & chr8 \\
\hline XPR1 & 9213 & Xenotropic and polytropic retrovirus receptor & 2,62 & chr1 \\
\hline XRCC1 & 7515 & X-ray repair complementing defective repair in Chinese hamster cells 1 & 3,36 & chr19 \\
\hline XRCC2 & 7516 & X-ray repair complementing defective repair in Chinese hamster cells 2 & 2,25 & chr7 \\
\hline XRCC5 & 7520 & X-ray repair complementing defective repair in Chinese hamster cells 5 (double- & 3,18 & chr2 \\
\hline XRCC6 & 2547 & X-ray repair complementing defective repair in Chinese hamster cells 6 (Ku auto & 2,23 & chr22 \\
\hline XRN2 & 22803 & 5'-3' exoribonuclease 2 & 2,82 & chr20 \\
\hline ХTРЗТРА & 79077 & XTP3-transactivated protein A & 3,37 & chr16 \\
\hline YARS & 8565 & tyrosyl-tRNA synthetase & 3,24 & chr1 \\
\hline YBX1 & 4904 & Y box binding protein 1 & 2,97 & chr1 \\
\hline YEATS4 & 8089 & YEATS domain containing 4 & 4,67 & chr12 \\
\hline YME1L1 & 10730 & YME1-like 1 (S. cerevisiae) & 3,17 & chr10 \\
\hline YPEL1 & 29799 & yippee-like 1 (Drosophila) & 2,30 & chr22 \\
\hline YT521 & 91746 & splicing factor YT521-B & 2,89 & chr4 \\
\hline YTHDC2 & 64848 & YTH domain containing 2 & 2,71 & chr5 \\
\hline YTHDF2 & 51441 & YTH domain family, member 2 & 2,47 & chr1 \\
\hline YWHAE & 7531 & tyrosine 3-monooxygenase/tryptophan 5-monooxygenase activation protein, eps & 3,15 & chr7 \\
\hline YY1 & 7528 & YY1 transcription factor & 2,03 & chr14 \\
\hline ZA20D3 & 54469 & Zinc finger, A20 domain containing 3 & 4,01 & chr15 \\
\hline ZADH1 & 145482 & zinc binding alcohol dehydrogenase, domain containing 1 & 2,99 & chr14 \\
\hline ZBED1 & 9189 & zinc finger, BED-type containing 1 & 2,29 & chrY \\
\hline ZBTB24 & 9841 & zinc finger and BTB domain containing 24 & 4,28 & chr6 \\
\hline ZВТВ3 & 79842 & zinc finger and BTB domain containing 3 & 5,64 & chr11 \\
\hline ZBTB8OS & 339487 & zinc finger and BTB domain containing 8 opposite strand & 2,52 & chr1 \\
\hline ZBTB9 & 221504 & zinc finger and BTB domain containing 9 & 3,10 & chr6 \\
\hline $\mathrm{ZC} 3 \mathrm{H} 10$ & 84872 & zinc finger $\mathrm{CCCH}$-type containing 10 & 2,74 & chr12 \\
\hline ZC3HAV1 & 56829 & zinc finger $\mathrm{CCCH}$-type, antiviral 1 & 3,05 & chr7 \\
\hline $\mathrm{ZC} 3 \mathrm{HC} 1$ & 51530 & zinc finger, C3HC-type containing 1 & 2,40 & chr7 \\
\hline $\mathrm{ZCCHC} 10$ & 54819 & zinc finger, $\mathrm{CCHC}$ domain containing 10 & 2,09 & chr5 \\
\hline $\mathrm{ZCCHC} 11$ & 23318 & Zinc finger, $\mathrm{CCHC}$ domain containing 11 & 2,69 & chr1 \\
\hline $\mathrm{ZCCHC} 14$ & 23174 & zinc finger, $\mathrm{CCHC}$ domain containing 14 & 2,06 & chr16 \\
\hline $\mathrm{ZCCHC} 3$ & 85364 & zinc finger, $\mathrm{CCHC}$ domain containing 3 & 2,58 & chr20 \\
\hline $\mathrm{ZCCHC7}$ & 84186 & Zinc finger, $\mathrm{CCHC}$ domain containing 7 & 3,72 & chr9 \\
\hline ZD52F10 & 93099 & dermokine & 28,48 & chr19 \\
\hline ZDHHC11 & 79844 & zinc finger, DHHC-type containing 11 & 5,57 & chr5 \\
\hline ZDHHC15 & 158866 & zinc finger, DHHC-type containing 15 & 3,54 & $\operatorname{chrX}$ \\
\hline ZDHHC22 & 283576 & zinc finger, DHHC-type containing 22 & 2,85 & chr14 \\
\hline ZDHHC23 & 254887 & zinc finger, DHHC-type containing 23 & 10,00 & chr3 \\
\hline $\mathrm{ZF}$ & 58487 & HCF-binding transcription factor Zhangfei & 2,01 & chr11 \\
\hline ZFAND3 & 60685 & Zinc finger, AN1-type domain 3 & 2,19 & chr6 \\
\hline ZFP161 & 7541 & zinc finger protein 161 homolog (mouse) & 2,07 & chr18 \\
\hline ZFP36L2 & 678 & zinc finger protein $36, \mathrm{C} 3 \mathrm{H}$ type-like 2 & 2,90 & chr2 \\
\hline ZFP42 & 132625 & zinc finger protein 42 & 107,59 & chr4 \\
\hline ZFP62 & 92379 & zinc finger protein 62 homolog (mouse) & 3,93 & chr5_random \\
\hline ZFPL & 162967 & zinc finger protein like & 3,52 & chr19 \\
\hline ZGPAT & 84619 & zinc finger, $\mathrm{CCCH}$-type with $\mathrm{G}$ patch domain & 2,35 & chr20 \\
\hline ZIC2 & 7546 & Zic family member 2 (odd-paired homolog, Drosophila) & 65,62 & chr13 \\
\hline ZIC3 & 7547 & Zic family member 3 heterotaxy 1 (odd-paired homolog, Drosophila) & 68,18 & $\operatorname{chrX}$ \\
\hline ZIK1 & 284307 & zinc finger protein interacting with $\mathrm{K}$ protein 1 & 9,74 & chr19 \\
\hline ZMAT4 & 79698 & zinc finger, matrin type 4 & 4,76 & chr8 \\
\hline ZMYM1 & 79830 & zinc finger, MYM-type 1 & 2,37 & chr1 \\
\hline
\end{tabular}




\section{Pagge 175 of 257}

0

zinc finger, MYND-type containing 19

ZNF10

\begin{tabular}{c|l}
116225 & zinc finger, MYND \\
7556 & zinc finger protein 10
\end{tabular}

ZNF114

zinc finger protein 114

ZNF11B

7558

zinc finger protein 11B

ZNF124

7678

7690

zinc finger protein 124 (HZF-16)

ZNF134

ZNF137

ZNF138

ZNF14

ZNF140

ZNF141

ZNF142

ZNF143

ZNF165

ZNF167

ZNF180

ZNF195

ZNF198

ZNF20

ZNF200

ZNF202

ZNF204

ZNF206

ZNF207

ZNF21

ZNF215

ZNF217

ZNF219

ZNF22

ZNF226

ZNF227

ZNF232

ZNF239

ZNF253

ZNF256

ZNF26

ZNF261

ZNF262

ZNF267

ZNF273

ZNF278

ZNF281

ZNF285

ZNF286

ZNF292

ZNF3

ZNF30

ZNF300

ZNF302

ZNF311

ZNF313

ZNF323

ZNF326

ZNF331

ZNF33A

ZNF342

ZNF35

ZNF367

ZNF38

ZNF394

ZNF395

ZNF397

7693

Zinc finger protein 131 (clone $\mathrm{pHZ}-10$ )

zinc finger protein 137 (clone $\mathrm{pHZ}-30$ )

7697 zinc finger protein 138

7561 zinc finger protein 14 (KOX 6)

7699 Zinc finger protein 140 (clone pHZ-39)

$7700 \quad$ Zinc finger protein 141 (clone pHZ-44)

7701 zinc finger protein 142 (clone pHZ-49)

7702 zinc finger protein 143 (clone pHZ-1)

7718 zinc finger protein 165

55888 zinc finger protein 167

7733 zinc finger protein 180 (HHZ168)

7748 zinc finger protein 195

$7750 \quad$ Zinc finger protein 198

7568 zinc finger protein 20 (KOX 13)

7752 zinc finger protein 200

7753 zinc finger protein 202

7754 zinc finger protein 204

84891 zinc finger protein 206

7756 zinc finger protein 207

7569 zinc finger protein 21 (KOX 14)

7762 zinc finger protein 215

7764 zinc finger protein 217

51222 zinc finger protein 219

7570 zinc finger protein 22 (KOX 15)

7769 zinc finger protein 226

7770 zinc finger protein 227

7775 zinc finger protein 232

8187 zinc finger protein 239

114977 /// 56242 zinc finger protein 253 /// hypothetical protein BC014148

10172 zinc finger protein 256

$7574 \quad$ zinc finger protein 26 (KOX 20)

9203 zinc finger protein 261

9202 zinc finger protein 262

10308 zinc finger protein 267

10793 zinc finger protein 273

23598 zinc finger protein 278

23528 zinc finger protein 281

26974 zinc finger protein 285

57335 zinc finger protein 286

23036 zinc finger protein 292

7551 zinc finger protein 3 (A8-51)

90075 zinc finger protein 30 (KOX 28)

91975 zinc finger protein 300

55900 zinc finger protein 302

282890 zinc finger protein 311

55905 zinc finger protein 313

64288 zinc finger protein 323

284695 zinc finger protein 326

55422 zinc finger protein 331

7581 zinc finger protein $33 \mathrm{~A}$

162979 zinc finger protein 342

7584 zinc finger protein 35 (clone HF.10)

195828 zinc finger protein 367

7589 zinc finger protein 38

84124 zinc finger protein 394

55893 zinc finger protein 395

84307 zinc finger protein 397

$57541 \quad$ zinc finger protein 398

ZNF398

55786

zinc finger protein 398
zinc finger protein 415

55659 zinc finger protein 416

\begin{tabular}{|c|c|}
\hline 3,47 & chr9 \\
\hline 3,69 & chr12 \\
\hline 3,86 & chr19 \\
\hline 6,82 & chr19 \\
\hline 3,51 & chr10 \\
\hline 5,80 & chr1 \\
\hline 3,80 & chr5 \\
\hline 3,58 & chr19 \\
\hline 3,44 & chr19 \\
\hline 12,07 & chr7 \\
\hline 2,80 & chr19 \\
\hline 2,32 & chr12 \\
\hline 2,58 & chr4 \\
\hline 2,18 & chr2 \\
\hline 3,04 & chr11 \\
\hline 12,07 & chr6 \\
\hline 2,52 & chr3 \\
\hline 3,07 & chr19 \\
\hline 5,65 & chr11 \\
\hline 8,00 & chr13 \\
\hline 3,14 & chr19 \\
\hline 2,09 & chr16 \\
\hline 4,95 & chr11 \\
\hline 13,28 & chr6 \\
\hline 12,42 & chr16 \\
\hline 2,96 & chr17 \\
\hline 2,01 & $\operatorname{chrX}$ \\
\hline 2,32 & chr11 \\
\hline 2,15 & chr20 \\
\hline 3,06 & chr14 \\
\hline 2,79 & chr10 \\
\hline 2,26 & chr19 \\
\hline 3,47 & chr19 \\
\hline 6,79 & chr17 \\
\hline 3,74 & chr10 \\
\hline 5,46 & chr19 \\
\hline 7,30 & chr19 \\
\hline 2,45 & chr12 \\
\hline 3,17 & $\operatorname{chrX}$ \\
\hline 2,03 & chr1 \\
\hline 2,28 & chr16 \\
\hline 9,27 & chr7 \\
\hline 5,75 & chr22 \\
\hline 2,83 & chr1 \\
\hline 2,46 & chr2 \\
\hline 4,17 & chr17 \\
\hline 2,54 & chr6 \\
\hline 2,18 & chr7 \\
\hline 3,02 & chr19 \\
\hline 5,54 & chr5 \\
\hline 2,77 & chr19 \\
\hline 2,77 & chr6 \\
\hline 2,77 & chr20 \\
\hline 2,19 & chr6 \\
\hline 3,59 & chr1 \\
\hline 11,91 & chr19 \\
\hline 3,75 & chr10 \\
\hline 2,28 & chr19 \\
\hline 2,57 & chr3 \\
\hline 4,92 & chr9 \\
\hline 4,30 & chr7 \\
\hline 2,54 & chr7 \\
\hline 2,17 & chr8 \\
\hline 6,58 & chr18 \\
\hline 9,43 & chr7 \\
\hline 3,06 & chr19 \\
\hline 2,05 & chr19 \\
\hline
\end{tabular}




\begin{tabular}{|c|c|c|c|c|}
\hline ZNF418 & 147686 & zinc finger protein 418 & 4,65 & (vide) \\
\hline ZNF420 & 147923 & zinc finger protein 420 & 2,45 & $\operatorname{chr} 19$ \\
\hline ZNF423 & 23090 & zinc finger protein 423 & 9,65 & $\operatorname{chr} 16$ \\
\hline ZNF43 & 7594 & zinc finger protein 43 (HTF6) & 4,61 & chr19 \\
\hline ZNF430 & 80264 & zinc finger protein 430 & 2,45 & chr19 \\
\hline ZNF435 & 80345 & zinc finger protein 435 & 4,22 & chr6 \\
\hline ZNF44 & 51710 & Zinc finger protein 44 (KOX 7) & 2,11 & $\operatorname{chr} 19$ \\
\hline ZNF443 & 10224 & zinc finger protein 443 & 3,93 & chr19 \\
\hline ZNF447 & 65982 & zinc finger protein 447 & 2,54 & chr19 \\
\hline ZNF462 & 58499 & zinc finger protein 462 & 7,48 & chr9 \\
\hline ZNF483 & 158399 & zinc finger protein 483 & 2,10 & chr9 \\
\hline ZNF488 & 118738 & zinc finger protein 488 & 2,05 & chr10 \\
\hline ZNF493 & 284443 & zinc finger protein 493 & 2,41 & chr19 \\
\hline ZNF496 & 84838 & zinc finger protein 496 & 3,03 & chr1 \\
\hline ZNF505 & 81931 & zinc finger protein $505 / / /$ zinc finger protein 505 & 16,37 & chr19 \\
\hline ZNF507 & 22847 & zinc finger protein 507 & 2,64 & chr19 \\
\hline ZNF511 & 118472 & zinc finger protein 511 & 3,81 & chr10 \\
\hline ZNF518 & 9849 & Zinc finger protein 518 & 6,44 & (vide) \\
\hline ZNF519 & 162655 & zinc finger protein 519 & 8,99 & chr18 \\
\hline ZNF529 & 57711 & zinc finger protein 529 & 2,22 & chr19 \\
\hline ZNF532 & 55205 & zinc finger protein 532 & 2,61 & chr18 \\
\hline ZNF544 & 27300 & Zinc finger protein 544 & 5,14 & chr19 \\
\hline ZNF545 & 284406 & zinc finger protein 545 & 2,72 & chr19 \\
\hline ZNF551 & 90233 & zinc finger protein 551 /// zinc finger protein 551 & 8,74 & chr19 \\
\hline ZNF553 & 197407 & zinc finger protein 553 & 2,52 & chr16 \\
\hline ZNF559 & 84527 & zinc finger protein 559 /// zinc finger protein 559 & 7,44 & chr19 \\
\hline ZNF560 & 147741 & zinc finger protein 560 & 2,40 & $\operatorname{chr} 19$ \\
\hline ZNF561 & 93134 & zinc finger protein 561 & 2,17 & chr5 \\
\hline ZNF562 & 54811 & zinc finger protein 562 & 2,60 & $\operatorname{chr} 19$ \\
\hline ZNF567 & 163081 & zinc finger protein 567 & 3,70 & chr19 \\
\hline ZNF573 & 126231 & zinc finger protein 573 & 2,62 & chr19 \\
\hline ZNF574 & 64763 & zinc finger protein 574 & 2,36 & chr19 \\
\hline ZNF586 & 54807 & zinc finger protein 586 & 4,70 & chr19 \\
\hline ZNF587 & 84914 & Zinc finger protein 587 & 2,11 & chr19 \\
\hline ZNF588 & 51427 & zinc finger protein 588 & 15,58 & $\operatorname{chr} 7$ \\
\hline ZNF589 & 51385 & zinc finger protein 589 & 16,80 & chr3 \\
\hline ZNF6 & 7552 & zinc finger protein 6 (CMPX1) & 2,31 & $\operatorname{chrX}$ \\
\hline ZNF600 & 162966 & zinc finger protein 600 & 2,66 & chr19 \\
\hline ZNF605 & 90462 & zinc finger protein 605 & 2,60 & chr12 \\
\hline ZNF606 & 80095 & zinc finger protein 606 & 2,32 & chr19 \\
\hline ZNF607 & 84775 & zinc finger protein 607 & 4,26 & chr19 \\
\hline ZNF608 & 57507 & zinc finger protein 608 & 3,16 & chr5 \\
\hline ZNF610 & 162963 & zinc finger protein 610 & 2,50 & chr19 \\
\hline ZNF614 & 80110 & zinc finger protein 614 & 4,51 & chr19 \\
\hline ZNF616 & 90317 & zinc finger protein 616 & 6,45 & chr19 \\
\hline ZNF638 & 27332 & zinc finger protein 638 & 3,74 & chr2 \\
\hline ZNF639 & 51193 & zinc finger protein 639 & 2,08 & chr3 \\
\hline ZNF643 & 65243 & zinc finger protein 643 & 3,02 & chr1 \\
\hline ZNF644 & 84146 & zinc finger protein 644 & 2,37 & chr1 \\
\hline ZNF649 & 65251 & zinc finger protein 649 & 9,20 & chr19 \\
\hline ZNF652 & 22834 & Zinc finger protein 652 & 5,10 & chr17 \\
\hline ZNF667 & 63934 & Zinc finger protein 667 & 4,41 & chr19 \\
\hline ZNF669 & 79862 & zinc finger protein 669 & 2,01 & chr1 \\
\hline ZNF670 & 93474 & zinc finger protein 670 & 4,26 & chr1 \\
\hline ZNF675 & 171392 & zinc finger protein 675 & 2,48 & chr19 \\
\hline ZNF677 & 342926 & Zinc finger protein 677 & 4,29 & $\operatorname{chr} 19$ \\
\hline ZNF678 & 339500 & hypothetical protein MGC15634 & 8,91 & chr1 \\
\hline ZNF680 & 340252 & zinc finger protein 680 & 8,15 & $\mathrm{chr} 7$ \\
\hline ZNF682 & 91120 & zinc finger protein 682 & 7,89 & chr19 \\
\hline ZNF689 & 115509 & zinc finger protein 689 & 3,65 & chr16 \\
\hline ZNF690 & 146050 & zinc finger protein 690 & 2,46 & chr15 \\
\hline ZNF691 & 51058 & zinc finger protein 691 & 3,92 & chr1 \\
\hline ZNF692 & 55657 & zinc finger protein 692 & 2,78 & chr1 \\
\hline ZNF694 & 342357 & zinc finger protein 694 & 2,16 & chr16 \\
\hline ZNF700 & 90592 & zinc finger protein 700 & 2,58 & chr19 \\
\hline ZNF708 & 7562 & Zinc finger protein 708 (KOX8) & 4,08 & chr19 \\
\hline ZNF71 & 58491 & zinc finger protein 71 (Cos26) & 2,03 & $\operatorname{chr} 19$ \\
\hline
\end{tabular}




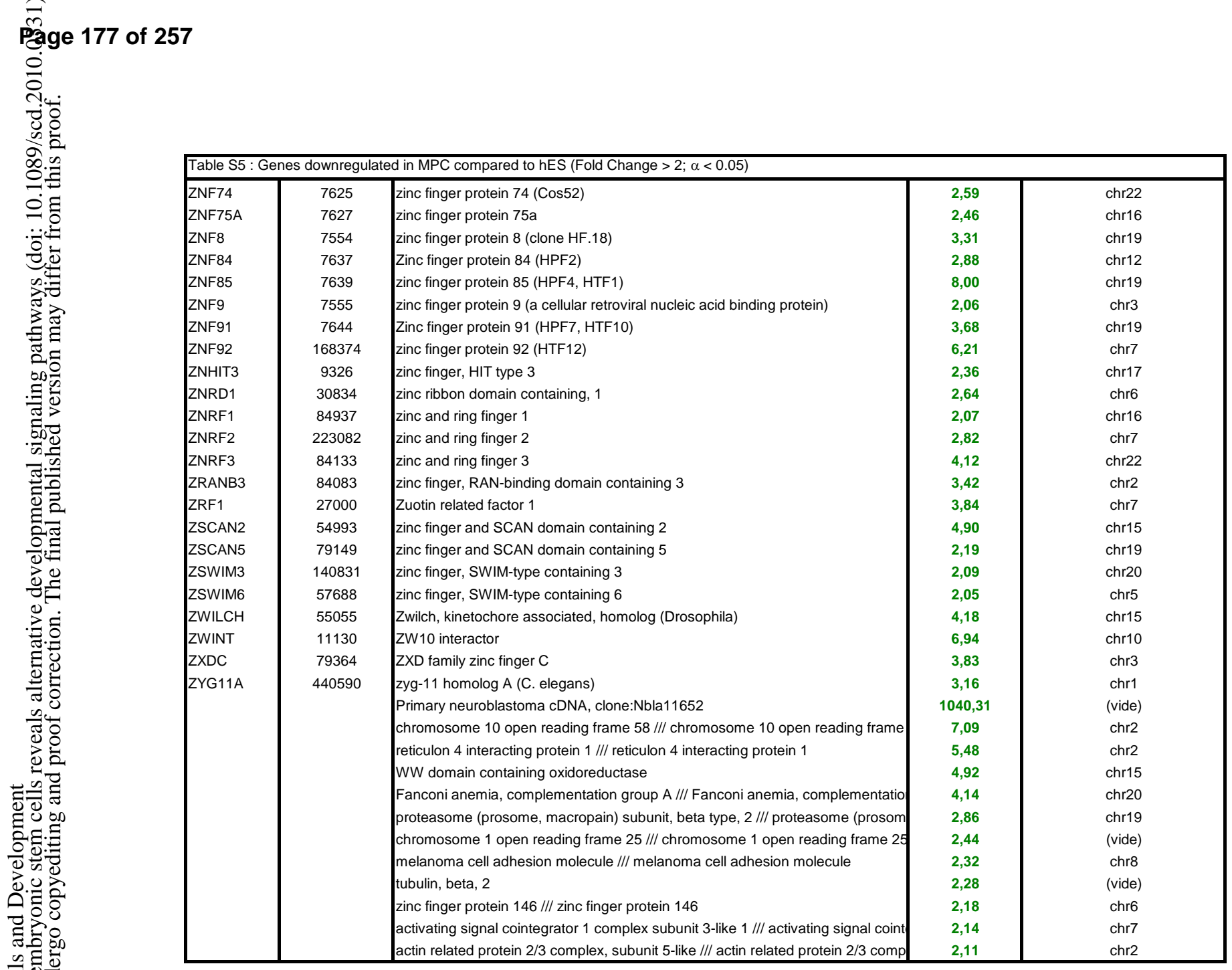




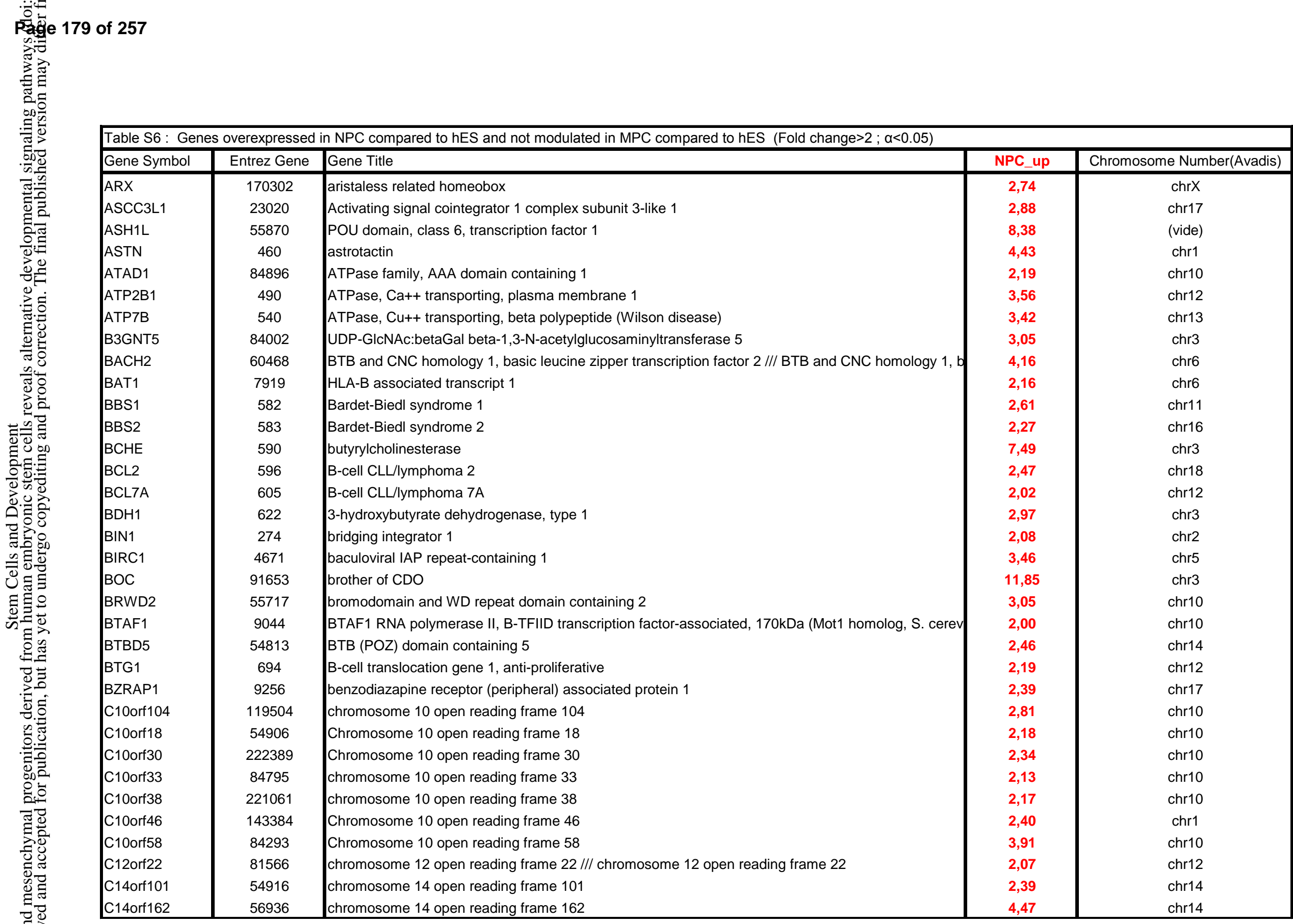




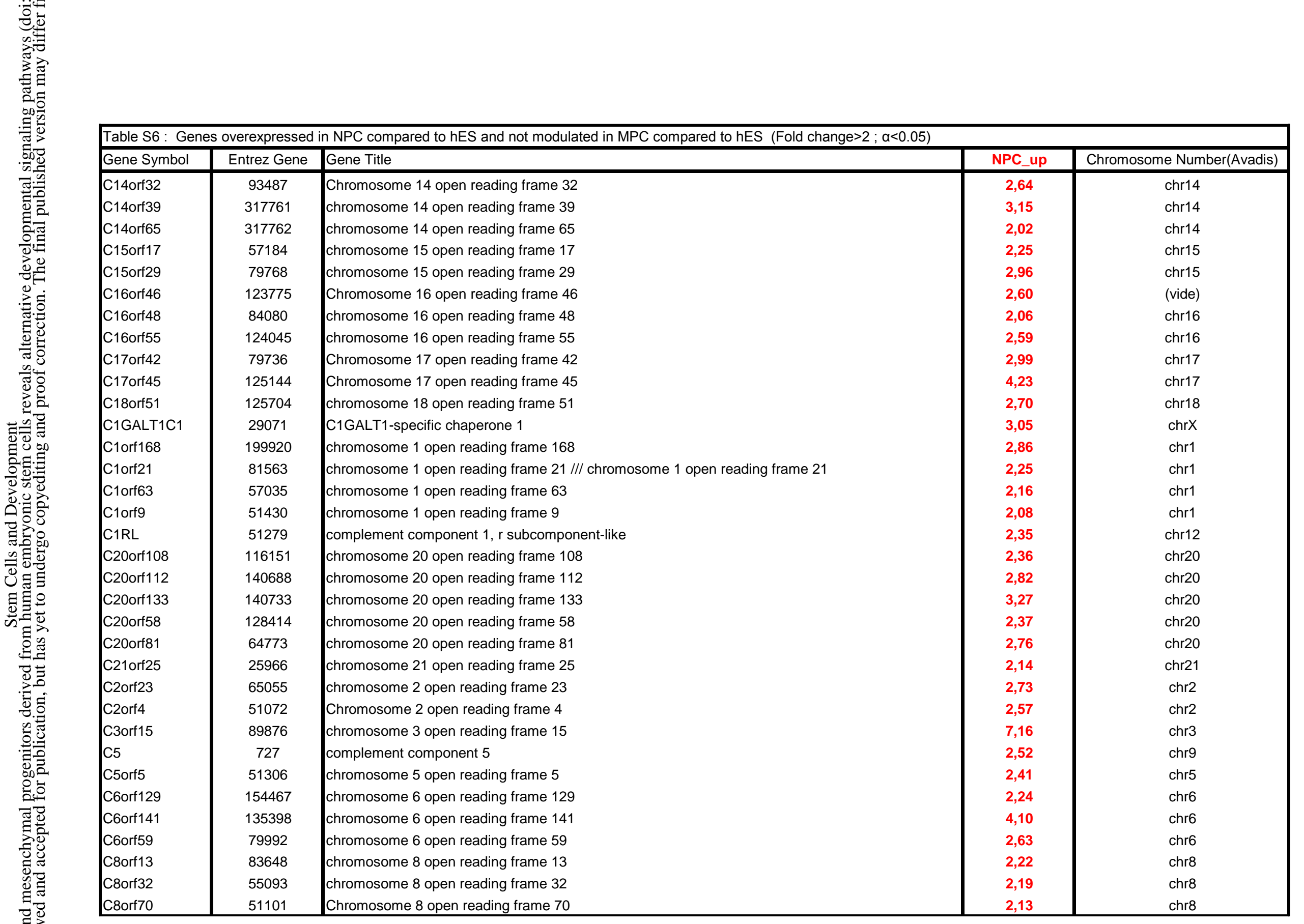




\section{Page 181 of 257}

\begin{tabular}{|c|c|c|c|c|}
\hline Gene Symbol & Entrez Gene & Gene Title & NPC_up & Chromosome Number(Avadis) \\
\hline C8orf72 & 90362 & chromosome 8 open reading frame 72 & 5,84 & chr8 \\
\hline C9orf28 & 89853 & chromosome 9 open reading frame 28 & 2,56 & chr9 \\
\hline C9orf5 & 23731 & chromosome 9 open reading frame 5 & 2,17 & chr9 \\
\hline C9orf7 & 11094 & chromosome 9 open reading frame 7 & 2,46 & chr9 \\
\hline CABLES2 & 81928 & Cdk5 and Abl enzyme substrate 2 & 2,03 & chr20 \\
\hline CACNA1A & 773 & calcium channel, voltage-dependent, $\mathrm{P} / \mathrm{Q}$ type, alpha $1 \mathrm{~A}$ subunit & 3,30 & chr19 \\
\hline CALM2 & 805 & Calmodulin 2 (phosphorylase kinase, delta) & 2,30 & chr2 \\
\hline CAND1 & 55832 & cullin-associated and neddylation-dissociated 1 & 2,44 & chr12 \\
\hline CAP350 & 9857 & centrosome-associated protein 350 & 2,11 & chr1 \\
\hline CAPN1 & 823 & calpain 1, (mu/l) large subunit & 3,99 & chr11 \\
\hline CAPN6 & 827 & calpain 6 & 6,34 & $\operatorname{chr} \mathrm{X}$ \\
\hline CARD8 & 22900 & caspase recruitment domain family, member 8 & 2,80 & chr19 \\
\hline CASKIN1 & 57524 & CASK interacting protein 1 & 2,06 & chr16 \\
\hline CCDC3 & 83643 & coiled-coil domain containing 3 & 3,17 & chr10 \\
\hline CCDC52 & 152185 & Coiled-coil domain containing 52 & 2,39 & chr3 \\
\hline CCNL2 & 81669 & cyclin L2 & 2,64 & chr1_random \\
\hline ССт6В & 10693 & chaperonin containing TCP1, subunit 6B (zeta 2) & 2,07 & chr17 \\
\hline CDC42BPB & 9578 & CDC42 binding protein kinase beta (DMPK-like) & 2,84 & chr14 \\
\hline CDK5RAP3 & 80279 & CDK5 regulatory subunit associated protein 3 & 2,15 & chr17 \\
\hline CDKL3 & 51265 & cyclin-dependent kinase-like 3 & 2,12 & chr5 \\
\hline CDV1 & 28981 & carnitine deficiency-associated, expressed in ventricle 1 & 3,30 & chr12 \\
\hline CEP1 & 11064 & centrosomal protein 1 & 2,32 & chr9 \\
\hline Cep164 & 22897 & |KIAA1052 protein & 2,54 & chr11 \\
\hline CHD9 & 80205 & Chromodomain helicase DNA binding protein 9 & 3,66 & chr16 \\
\hline $\mathrm{CHN} 2$ & 1124 & Chimerin (chimaerin) 2 & 3,09 & chr7 \\
\hline CHST5 & 23563 & carbohydrate (N-acetylglucosamine 6-O) sulfotransferase 5 & 2,87 & chr16 \\
\hline CHST5 & $23563 / / / 84836$ & carbohydrate (N-acetylglucosamine 6-O) sulfotransferase 5 /// hypothetical protein MGC15429 & 2,15 & chr16 \\
\hline CKLFSF3 & 123920 & chemokine-like factor superfamily 3 & 2,39 & chr16 \\
\hline CLK1 & 1195 & CDC-like kinase 1 & 2,62 & chr2 \\
\hline CMKOR1 & 57007 & chemokine orphan receptor 1 & 4,25 & chr2 \\
\hline CNTN2 & 6900 & contactin 2 (axonal) & 3,66 & chr1 \\
\hline CNTN4 & 152330 & contactin 4 & 4,47 & chr3 \\
\hline COL22A1 & 169044 & collagen, type XXII, alpha 1 & 2,71 & chr8 \\
\hline COL27A1 & 85301 & Collagen, type XXVII, alpha 1 & 2,69 & chr9 \\
\hline
\end{tabular}




\section{Page 183 of 257}

\begin{tabular}{|c|c|c|c|c|}
\hline Gene Symbol & Entrez Gene & Gene Title & NPC_up & Chromosome Number(Avadis) \\
\hline DKFZp313A2432 & 258010 & hypothetical protein DKFZp313A2432 & 2,35 & chr11 \\
\hline DKFZP434H011 & 83538 & hypothetical protein DKFZp434H0115 & 2,36 & chr17 \\
\hline DKFZp451M211 & 285023 & Hypothetical protein DKFZp451M2119 & 3,08 & chr2 \\
\hline DKFZP566N034 & 81615 & hypothetical protein DKFZp566N034 & 2,62 & chr2 \\
\hline DKFZP761N091 & 57183 & hypothetical protein DKFZp761N09121 & 10,12 & (vide) \\
\hline DKFZp76102018 & 92293 & hypothetical protein DKFZp76102018 & 4,07 & chr12 \\
\hline DKFZp762A217 & 160335 & hypothetical protein DKFZp762A217 & 3,19 & chr12 \\
\hline DLK1 & 8788 & delta-like 1 homolog (Drosophila) & 39,62 & chr14 \\
\hline DMD & 1756 & dystrophin (muscular dystrophy, Duchenne and Becker types) & 3,45 & $\operatorname{chrX}$ \\
\hline DMRT3 & 58524 & doublesex and mab-3 related transcription factor 3 & 3,27 & chr9 \\
\hline DMXL2 & 23312 & Dmx-like 2 & 5,22 & chr15 \\
\hline DNAJC12 & 56521 & DnaJ (Hsp40) homolog, subfamily C, member 12 & 3,13 & chr10 \\
\hline DNALI1 & 7802 & dynein, axonemal, light intermediate polypeptide 1 & 4,36 & chr1 \\
\hline DNM1 & 1759 & dynamin 1 & 4,23 & chr9 \\
\hline DOCK7 & 85440 & Dedicator of cytokinesis 7 & 2,75 & chr1 \\
\hline DPY19L2 & 283417 & dpy-19-like 2 (C. elegans) & 2,11 & chr7 \\
\hline DPYSL5 & 56896 & dihydropyrimidinase-like 5 & 3,84 & chr2 \\
\hline DREV1 & 51108 & DORA reverse strand protein 1 & 2,32 & chr16 \\
\hline DSCR6 & 53820 & Down syndrome critical region gene 6 & 2,65 & chr21 \\
\hline DST & 667 & dystonin & 4,69 & chr6 \\
\hline DUSP4 & 1846 & dual specificity phosphatase 4 & 7,87 & chr8 \\
\hline ECHDC1 & 55862 & Enoyl Coenzyme A hydratase domain containing 1 & 3,52 & chr6 \\
\hline EFHD1 & 80303 & EF-hand domain family, member D1 & 3,22 & chr2 \\
\hline EFNA1 & 1942 & ephrin-A1 & 2,08 & chr1 \\
\hline EFNB2 & 1948 & ephrin-B2 & 5,61 & chr13 \\
\hline EFS & 10278 & embryonal Fyn-associated substrate & 3,80 & chr14 \\
\hline EIF2C3 & 192669 & eukaryotic translation initiation factor $2 \mathrm{C}, 3$ & 2,38 & chr1 \\
\hline EIF2C4 & 192670 & Eukaryotic translation initiation factor 2C, 4 & 2,87 & chr1 \\
\hline ELAC1 & 55520 & elaC homolog 1 (E. coli) & 2,01 & chr18 \\
\hline ELAVL4 & 1996 & ELAV (embryonic lethal, abnormal vision, Drosophila)-like 4 (Hu antigen D) & 4,59 & chr1 \\
\hline ELOVL5 & 60481 & ELOVL family member 5 , elongation of long chain fatty acids (FEN1/Elo2, SUR4/Elo3-like, yeast) & 2,05 & chr6 \\
\hline EMILIN2 & 84034 & elastin microfibril interfacer 2 /// elastin microfibril interfacer 2 & 2,47 & chr18 \\
\hline EML1 & 2009 & echinoderm microtubule associated protein like 1 & 2,27 & chr14 \\
\hline EMP2 & 2013 & epithelial membrane protein 2 & 3,91 & chr16 \\
\hline
\end{tabular}




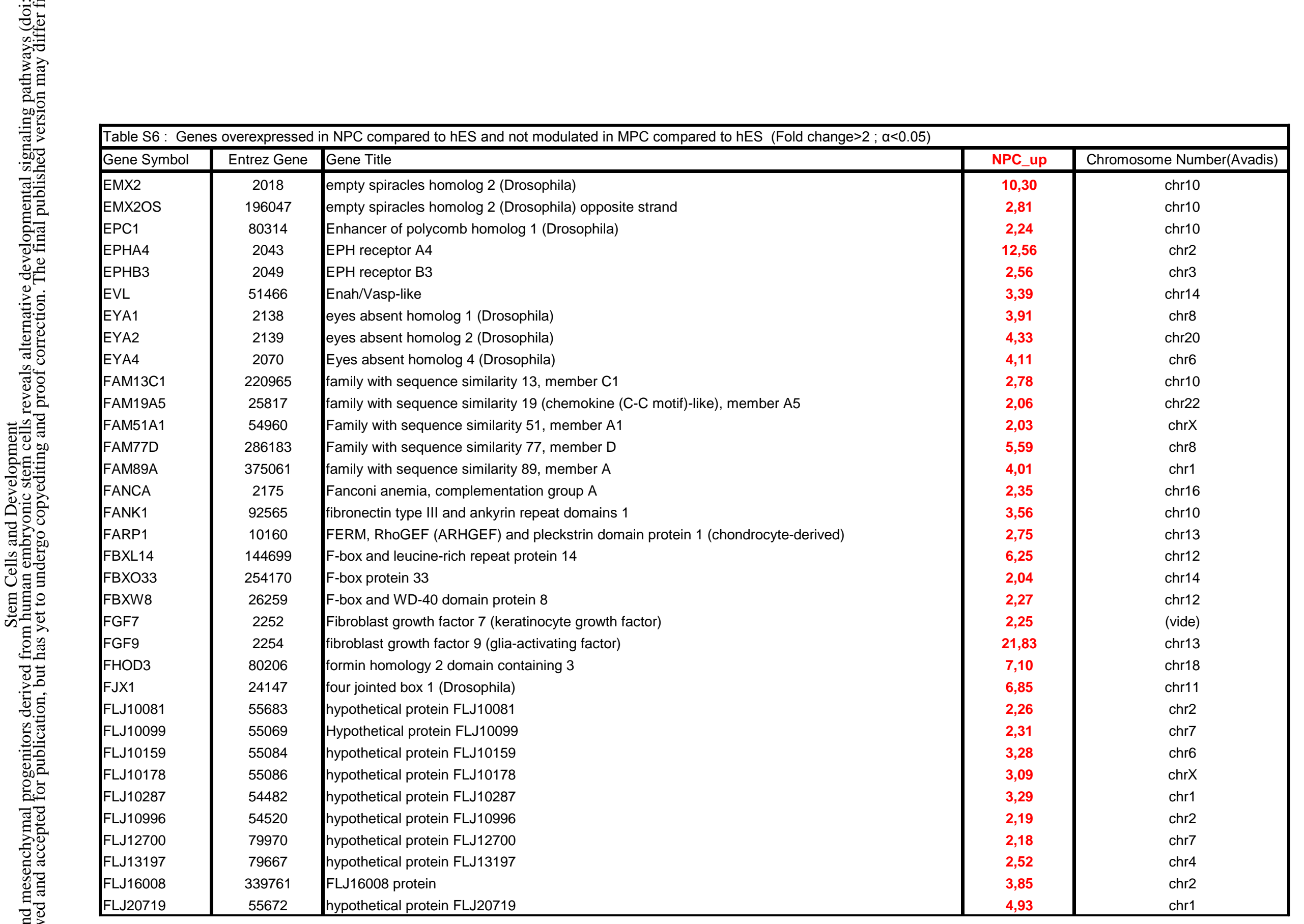




\section{Page 185 of 257}

\begin{tabular}{|c|c|c|c|c|}
\hline Gene Symbol & Entrez Gene & Gene Title & NPC_up & Chromosome Number(Avadis) \\
\hline FLJ21125 & 79680 & hypothetical protein FLJ21125 & 2,04 & chr22 \\
\hline FLJ21616 & 79618 & Hypothetical protein FLJ21616 & 2,28 & chr8 \\
\hline FLJ23191 & 79625 & hypothetical protein FLJ23191 & 4,87 & chr4 \\
\hline FLJ25476 & 149076 & FLJ25476 protein & 2,24 & chr1 \\
\hline FLJ25694 & 283492 & hypothetical protein FLJ25694 & 5,59 & chr13 \\
\hline FLJ25715 & 284241 & Hypothetical protein FLJ25715 & 2,12 & $\operatorname{chr} 18$ \\
\hline FLJ30092 & 196515 & AF-1 specific protein phosphatase /// AF-1 specific protein phosphatase & 2,01 & chr12 \\
\hline FLJ30901 & 150378 & hypothetical protein FLJ30901 & 7,03 & chr22 \\
\hline FLJ31438 & 130162 & hypothetical protein FLJ31438 & 2,95 & chr2 \\
\hline FLJ31818 & 154743 & hypothetical protein FLJ31818 & 2,18 & $\mathrm{chr} 7$ \\
\hline FLJ31951 & 153830 & Hypothetical protein FLJ31951 & 3,92 & chr5 \\
\hline FLJ34443 & 285464 & hypothetical protein FLJ34443 & 2,90 & chr4 \\
\hline FLJ36166 & 349152 & Hypothetical protein FLJ36166 & 2,36 & chr7 \\
\hline FLJ37440 & 129804 & hypothetical protein FLJ37440 & 2,05 & chr2 \\
\hline FLJ38379 & 285097 & hypothetical protein FLJ38379 & 2,87 & chr2 \\
\hline FLJ39155 & 133584 & hypothetical protein FLJ39155 & 3,06 & chr5 \\
\hline FLJ39653 & 202020 & hypothetical protein FLJ39653 & 2,30 & chr4 \\
\hline FLJ42393 & 401105 & FLJ42393 protein & 2,27 & chr3 \\
\hline FLJ42957 & 400077 & FLJ42957 protein & 2,28 & chr12 \\
\hline FLJ44216 & 375484 & FLJ44216 protein & 2,75 & chr5 \\
\hline FLJ45187 & 387640 & FLJ45187 protein & 13,87 & chr10 \\
\hline FLJ90757 & 440465 & FLJ90757 protein & 4,70 & chr17 \\
\hline FLRT2 & 23768 & fibronectin leucine rich transmembrane protein 2 & 6,72 & chr14 \\
\hline FLRT3 & 23767 & fibronectin leucine rich transmembrane protein 3 & 20,56 & chr20 \\
\hline FNBP1 & 23048 & Formin binding protein 1 & 3,16 & chr9 \\
\hline FNBP4 & 23360 & formin binding protein 4 & 2,24 & chr11 \\
\hline FNDC5 & 252995 & fibronectin type III domain containing 5 & 3,30 & chr1 \\
\hline FOXG1B & 2290 & forkhead box G1B & 14,70 & chr14 \\
\hline FOXO3A & 2309 & forkhead box О3A & 2,14 & chr6 \\
\hline FRMD4A & $1810 / / / 55691$ & FERM domain containing 4A /// Down-regulator of transcription 1, TBP-binding (negative cofactor 2) & 6,08 & chr1 \\
\hline FRMD4B & 23150 & FERM domain containing 4B & 2,36 & chr3 \\
\hline FRZB & 2487 & frizzled-related protein & 13,30 & chr2 \\
\hline FSD1CL & 405752 & GTPase activating Rap/RanGAP domain-like 1 & 3,17 & chr9 \\
\hline FSD1L & 405752 & FSD1-like & 2,01 & chr9 \\
\hline
\end{tabular}




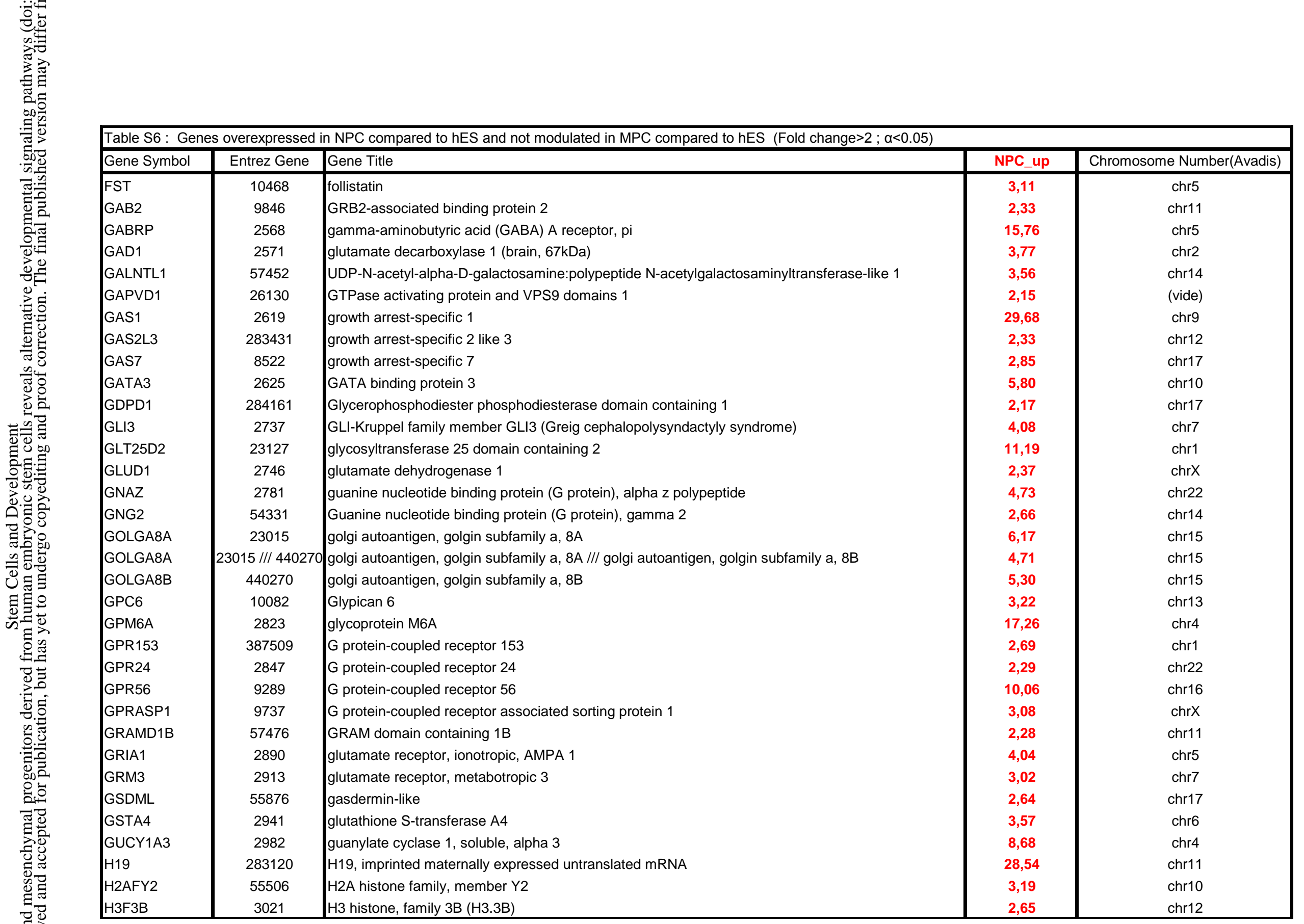




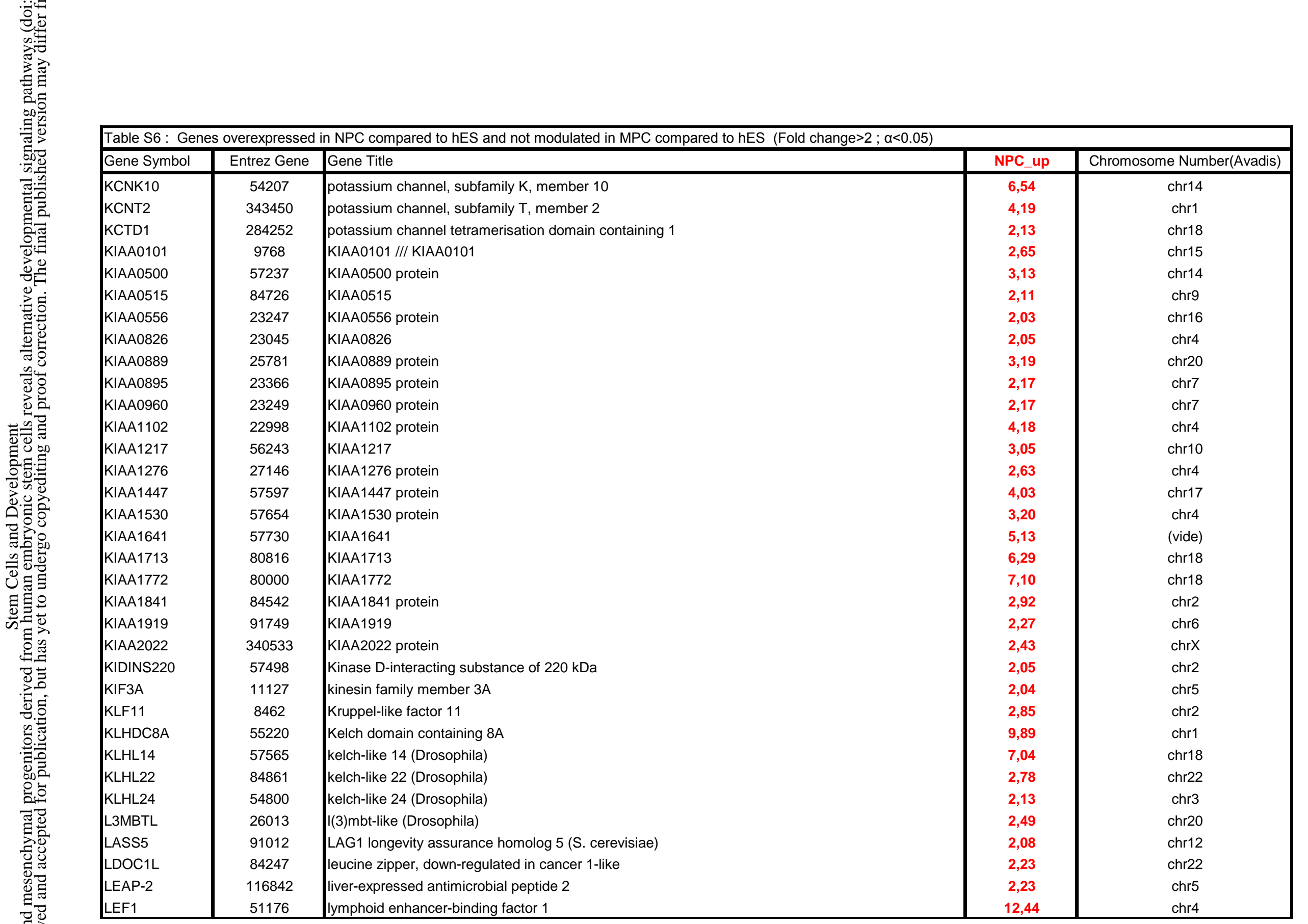




\section{Page 189 of 257}

\begin{tabular}{|c|c|c|c|c|}
\hline Gene Symbol & Entrez Gene & Gene Title & NPC_up & Chromosome Number(Avadis) \\
\hline LEMD1 & 93273 & LEM domain containing 1 & 3,95 & chr1 \\
\hline LFNG & 3955 & lunatic fringe homolog (Drosophila) & 2,74 & chr7 \\
\hline LGI1 & 9211 & leucine-rich, glioma inactivated 1 & 8,42 & chr10 \\
\hline LGR5 & 8549 & leucine-rich repeat-containing $\mathrm{G}$ protein-coupled receptor 5 & 6,45 & chr12 \\
\hline LHX2 & 9355 & LIM homeobox 2 & 135,71 & chr9 \\
\hline LHX9 & 56956 & LIM homeobox 9 & 3,81 & chr1 \\
\hline LIX1 & 167410 & Lix1 homolog (mouse) & 24,29 & chr5 \\
\hline LKAP & 9665 & limkain b1 & 2,03 & $\operatorname{chr} 16$ \\
\hline LMO2 & 4005 & LIM domain only 2 (rhombotin-like 1) & 2,35 & chr11 \\
\hline LMO3 & 55885 & LIM domain only 3 (rhombotin-like 2) & 26,62 & chr12 \\
\hline LOC112476 & 112476 & similar to lymphocyte antigen 6 complex, locus G5B; G5b protein; open reading frame 31 & 4,16 & chr16 \\
\hline LOC113386 & 113386 & similar to envelope protein & 2,25 & chr19 \\
\hline LOC144997 & 144997 & hypothetical protein LOC144997 & 7,95 & chr13 \\
\hline LOC147670 & 147670 & hypothetical protein LOC147670 & 2,25 & (vide) \\
\hline LOC150759 & 150759 & hypothetical protein LOC150759 & 4,35 & chr2 \\
\hline LOC153682 & 153682 & Hypothetical protein LOC153682 & 2,28 & chr5 \\
\hline LOC158563 & 158563 & hypothetical protein LOC158563 & 3,15 & $\operatorname{chr} \mathrm{X}$ \\
\hline LOC220930 & 220930 & hypothetical protein LOC220930 & 3,37 & chr10 \\
\hline LOC221362 & 221362 & hypothetical protein LOC221362 & 2,68 & chr6 \\
\hline LOC221981 & 221981 & hypothetical protein LOC221981 & 2,99 & chr7 \\
\hline LOC255326 & 255326 & hypothetical protein LOC255326 & 2,64 & chr10 \\
\hline LOC284244 & 284244 & hypothetical protein LOC284244 & 2,93 & $\operatorname{chr} 18$ \\
\hline LOC284262 & 284262 & hypothetical protein LOC284262 & 3,78 & chr18 \\
\hline LOC284356 & 284356 & hypothetical protein LOC284356 & 3,97 & chr19 \\
\hline LOC284409 & 284409 & hypothetical LOC284409 & 2,65 & $\operatorname{chr} 19$ \\
\hline LOC285535 & 285535 & hypothetical protein LOC285535 & 3,18 & chr4 \\
\hline LOC285989 & 285989 & hypothetical protein LOC285989 & 2,26 & chr7 \\
\hline LOC286052 & 286052 & hypothetical protein LOC286052 & 2,19 & chr8 \\
\hline LOC286334 & 286334 & Hypothetical protein LOC286334 & 2,61 & chr9 \\
\hline LOC286382 & 286382 & hypothetical protein LOC286382 & 2,95 & chr9 \\
\hline LOC338758 & 338758 & hypothetical protein LOC338758 & 3,78 & chr12 \\
\hline LOC339025 & 339025 & Hypothetical LOC339025 & 2,12 & chr15 \\
\hline LOC339287 & 339287 & hypothetical protein LOC339287 & 2,25 & chr17 \\
\hline LOC340281 & 340281 & hypothetical protein LOC340281 & 2,55 & chr7 \\
\hline
\end{tabular}




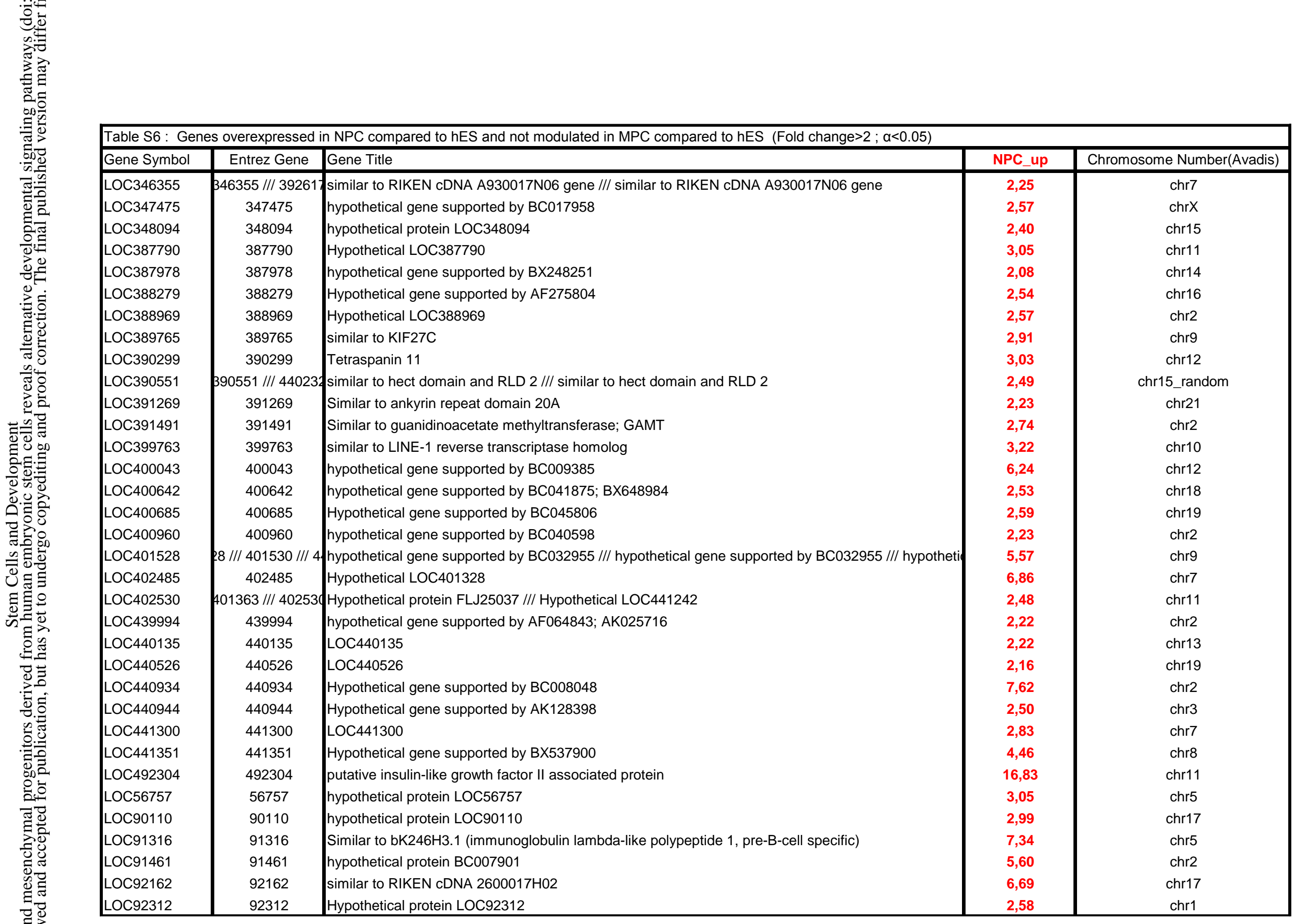




\begin{tabular}{|c|c|c|c|c|c|}
\hline. & Gene Symbol & Entrez Gene & Gene Title & NPC up & Chromosome Number(Avadis) \\
\hline$\Xi \stackrel{6}{=}$ & LOC92691 & 92691 & hypothetical protein BC008604 & 7,23 & chr2 \\
\hline$\underline{\varrho}$ & LPL & 4023 & lipoprotein lipase & 3,81 & chr8 \\
\hline 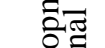 & LRCH2 & 57631 & leucine-rich repeats and calponin homology $(\mathrm{CH})$ domain containing 2 & 2,07 & $\operatorname{chrX}$ \\
\hline Die & LRCH3 & 84859 & leucine-rich repeats and calponin homology $(\mathrm{CH})$ domain containing 3 & 3,56 & chr3 \\
\hline 造 & LRP2 & 4036 & Low density lipoprotein-related protein 2 & 21,79 & chr2 \\
\hline 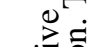 & LRP4 & 4038 & low density lipoprotein receptor-related protein 4 & 3,17 & chr11 \\
\hline$E$ & LRRC3B & 116135 & leucine rich repeat containing $3 \mathrm{~B}$ & 2,35 & chr3 \\
\hline 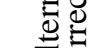 & LRRC49 & 54839 & leucine rich repeat containing 49 & 2,20 & chr15 \\
\hline 50 & LRRC4C & 57689 & leucine rich repeat containing $4 \mathrm{C}$ & 3,58 & chr11 \\
\hline 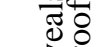 & |LRRN3 & 54674 & leucine rich repeat neuronal 3 & 6,49 & chr7 \\
\hline (1) & LSAMP & 4045 & limbic system-associated membrane protein & 2,27 & chr3 \\
\hline$\Xi$ & LSM8 & 51691 & LSM8 homolog, U6 small nuclear RNA associated (S. cerevisiae) & 2,69 & chr7 \\
\hline so & LUM & 4060 & lumican & 16,09 & chr12 \\
\hline$\Xi$ & LZIC & 84328 & Leucine zipper and CTNNBIP1 domain containing & 2,15 & chr1 \\
\hline & MAB21L1 & 4081 & mab-21-like 1 (C. elegans) & 5,33 & chr13 \\
\hline 8े & MAF & 4094 & v-maf musculoaponeurotic fibrosarcoma oncogene homolog (avian) & 6,36 & chr16 \\
\hline 58 & MAGI3 & 260425 & membrane associated guanylate kinase, $\mathrm{WW}$ and PDZ domain containing 3 & 2,96 & chr1 \\
\hline \% & MAML3 & 55534 & Mastermind-like 3 (Drosophila) & 2,68 & chr4 \\
\hline 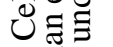 & MAN2A2 & 4122 & mannosidase, alpha, class 2A, member 2 & 2,72 & chr15 \\
\hline 9 & MAP2 & 4133 & Microtubule-associated protein 2 & 25,86 & chr2 \\
\hline & MAP2K1IP1 & 8649 & Mitogen-activated protein kinase kinase 1 interacting protein 1 & 2,40 & chr4 \\
\hline$\tilde{z}$ & MAP6 & 4135 & microtubule-associated protein 6 & 4,48 & chr11 \\
\hline$E$ & MAPK10 & 5602 & mitogen-activated protein kinase 10 & 9,24 & chr4 \\
\hline & MARCH6 & 10299 & membrane-associated ring finger (C3HC4) 6 & 2,16 & chr5 \\
\hline & MASP2 & 10747 & Mannan-binding lectin serine peptidase 2 & 6,08 & chr1 \\
\hline ల్ & MCART6 & 401612 & Mitochondrial carrier triple repeat 6 & 3,04 & $\operatorname{chrX}$ \\
\hline $\bar{z}$ & MCF2L & 23263 & MCF.2 cell line derived transforming sequence-like & 3,95 & chr13 \\
\hline & ME3 & 10873 & malic enzyme 3, NADP(+)-dependent, mitochondrial & 8,66 & chr11 \\
\hline$=$ & MECP2 & 4204 & methyl $\mathrm{CpG}$ binding protein 2 (Rett syndrome) & 2,09 & $\operatorname{chrX}$ \\
\hline$\stackrel{0}{\pi}$ & MEIS1 & 4211 & Meis1, myeloid ecotropic viral integration site 1 homolog (mouse) & 8,07 & chr2 \\
\hline 0 & MGAT3 & 4248 & Mannosyl (beta-1,4-)-glycoprotein beta-1,4-N-acetylglucosaminyltransferase & 2,44 & chr22 \\
\hline 㞼 & MGC12760 & 84809 & hypothetical protein MGC12760 /// hypothetical protein MGC12760 & 3,31 & chr1 \\
\hline & MGC13057 & 84281 & Hypothetical protein MGC13057 & 2,58 & chr2 \\
\hline & MGC15407 & 112942 & Similar to RIKEN cDNA 4931428D14 gene & 2,54 & chr2 \\
\hline
\end{tabular}




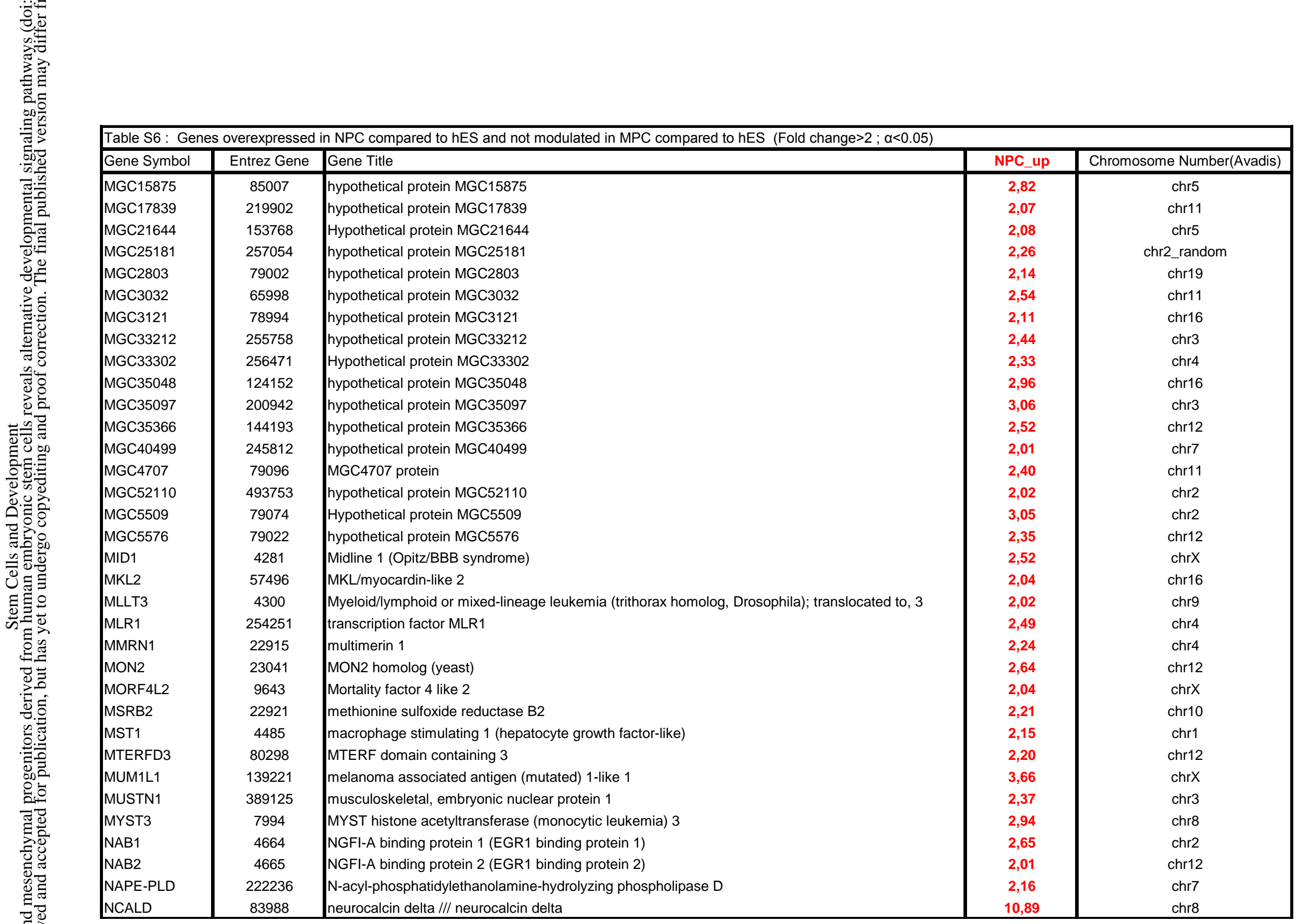




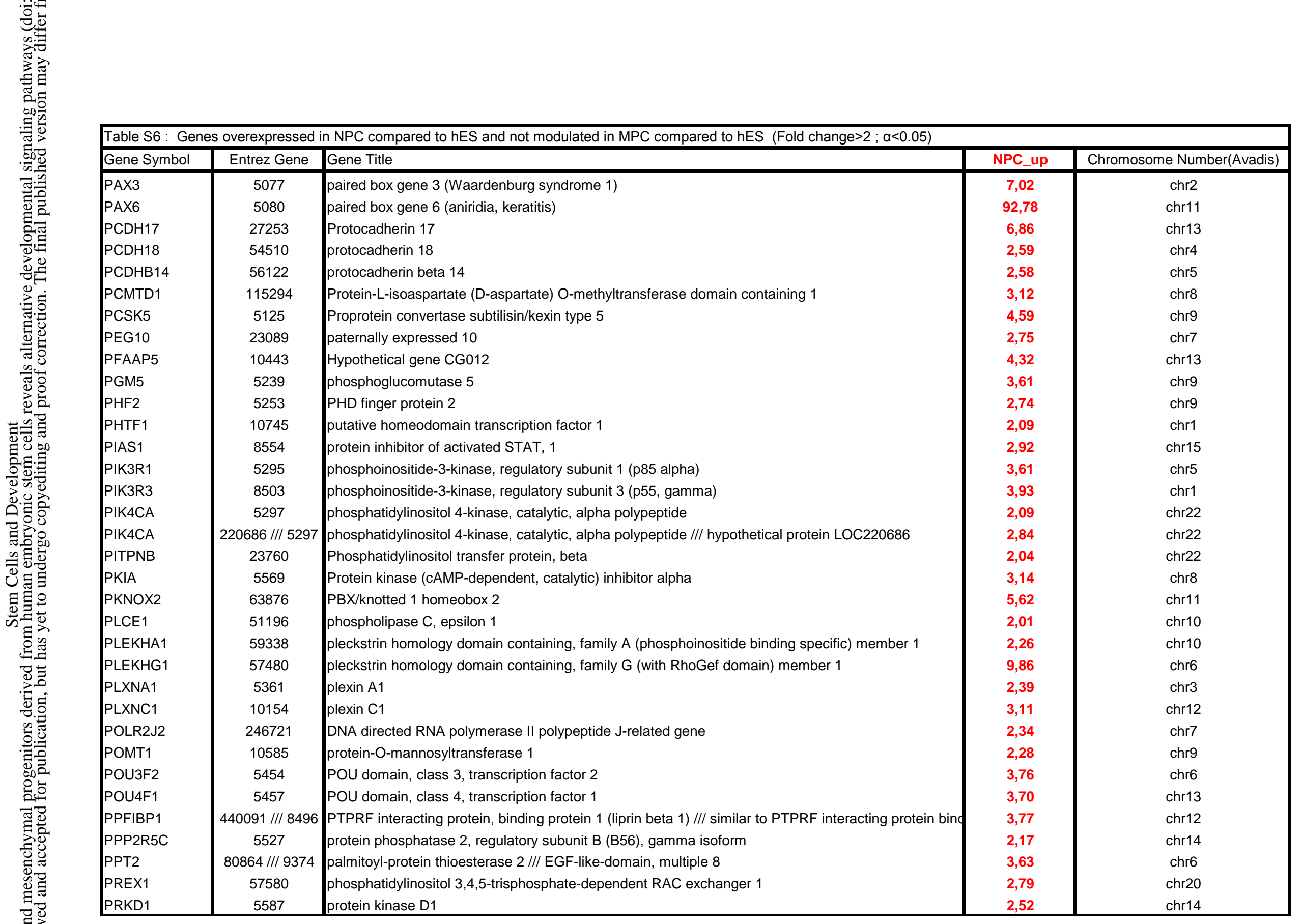




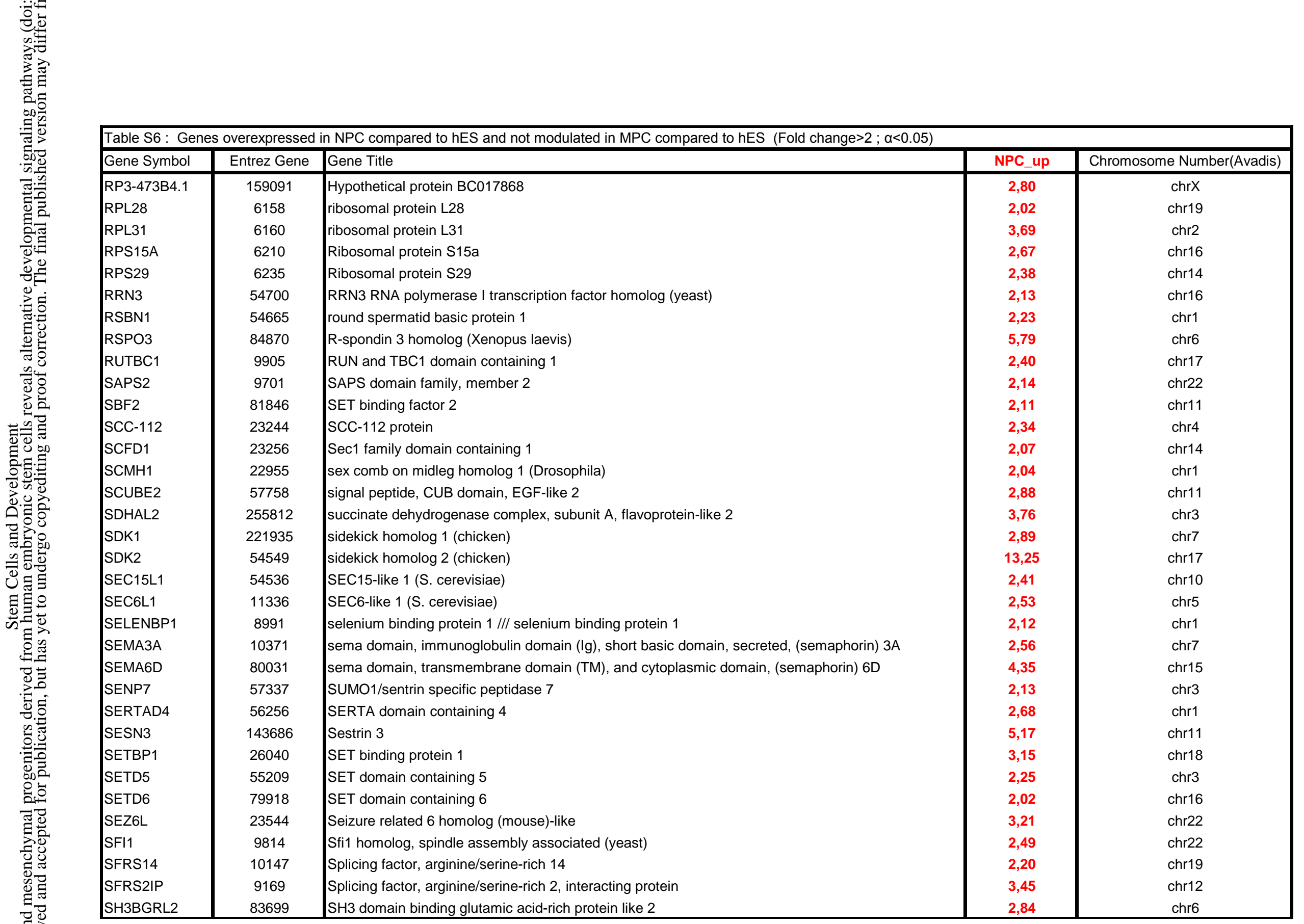




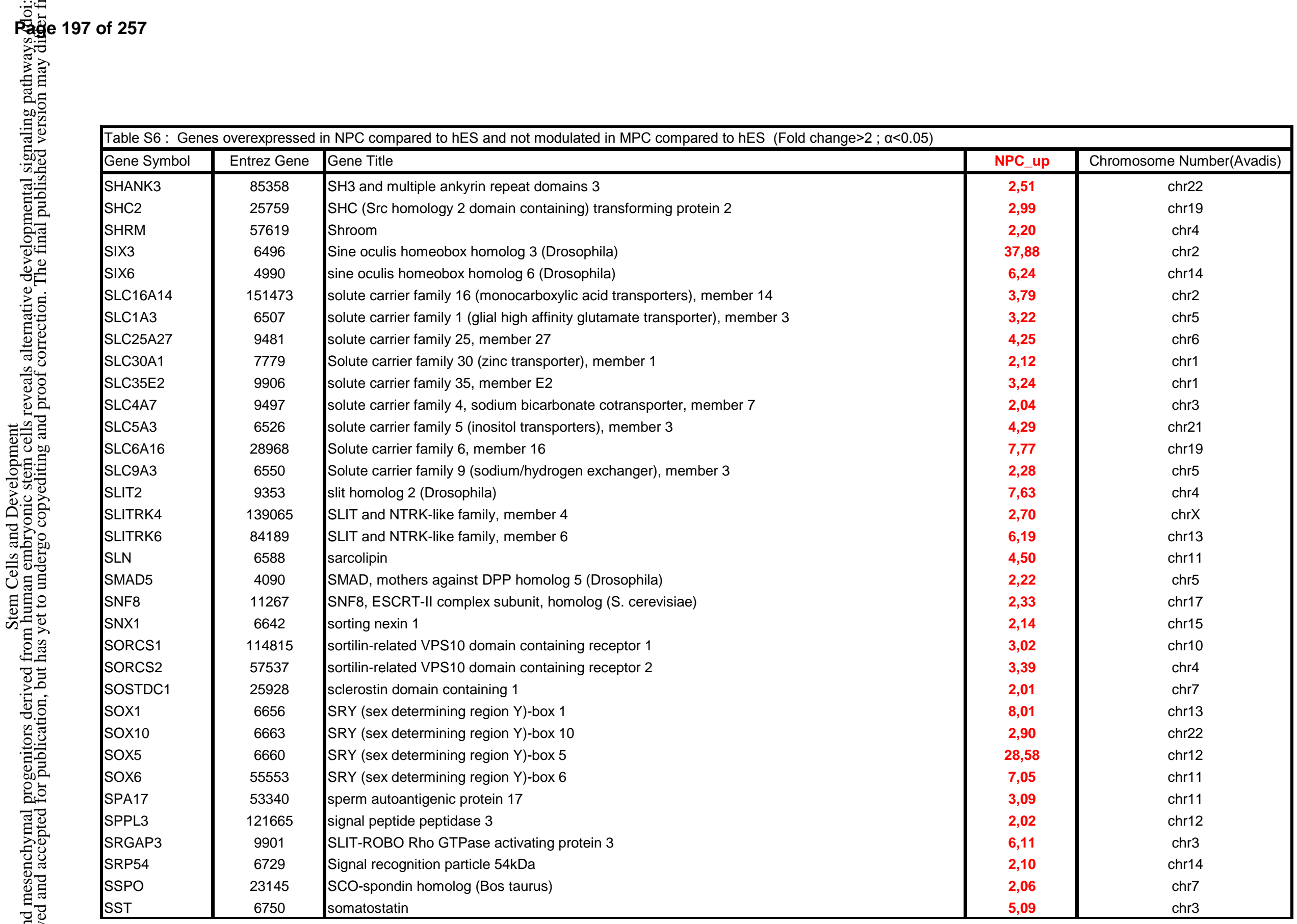




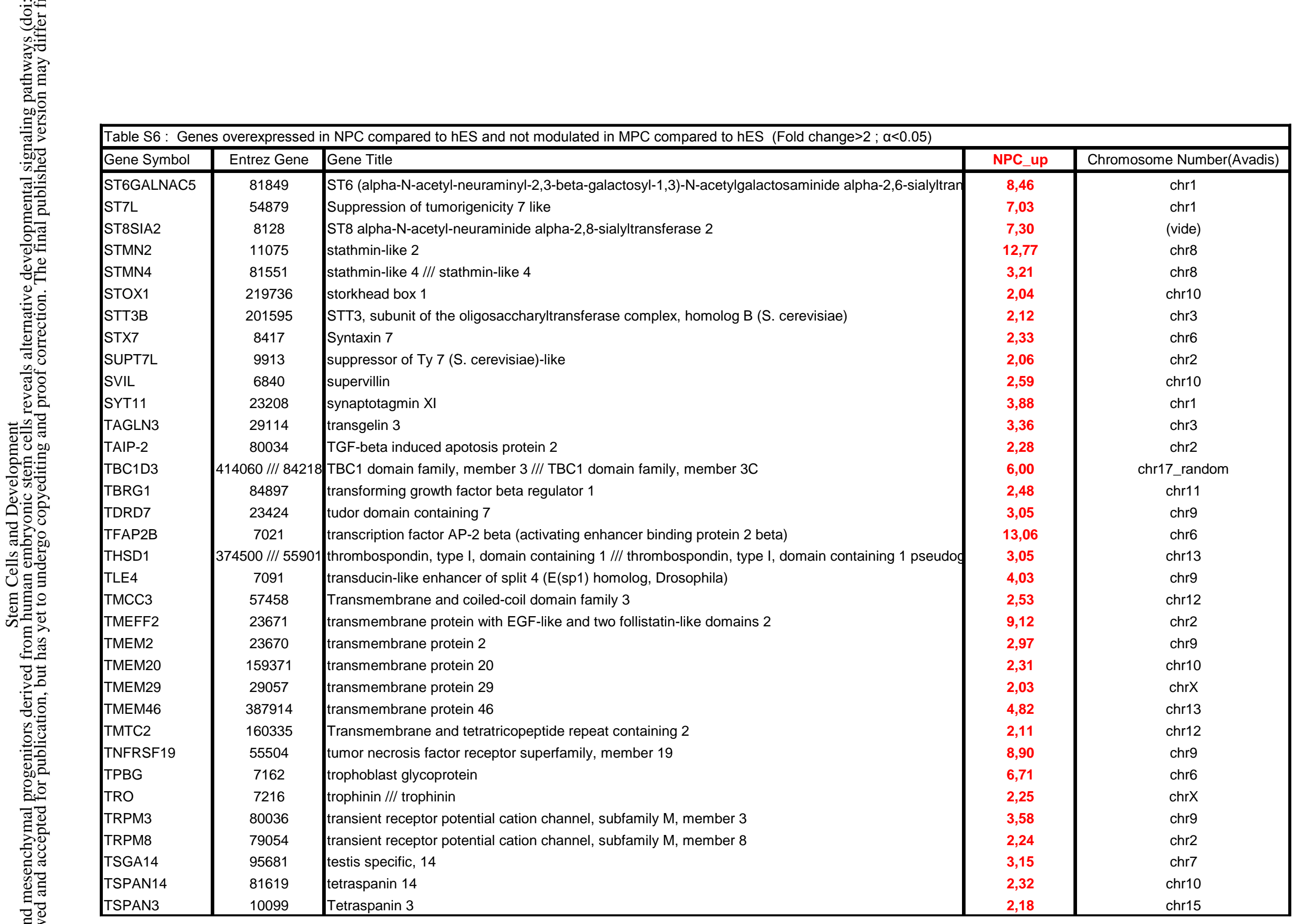




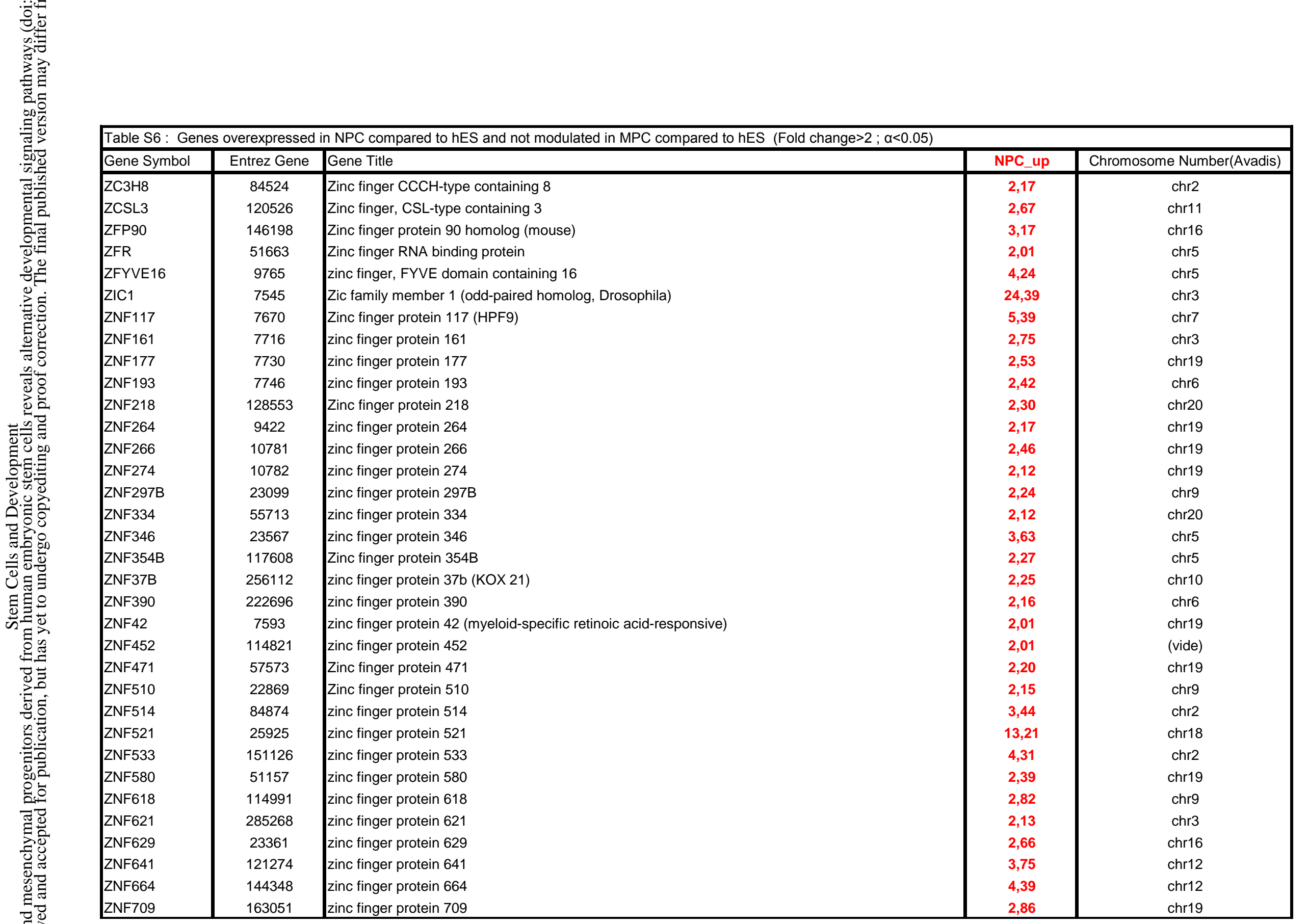




\section{Page 201 of 257}

\begin{tabular}{|l|c|l|c|c|}
\hline \multicolumn{2}{|l|}{ Table S6: Genes overexpressed in NPC compared to hES and not modulated in MPC compared to hES (Fold change>2; $\alpha<0.05)$} & NPC_up & Chromosome Number(Avadis) \\
\hline Gene Symbol & Entrez Gene & Gene Title & 3,79 & \\
ZNF83 & 55769 & Zinc finger protein 83 (HPF1) & 4,39 & chr19 \\
& & myosin, light polypeptide kinase /// myosin, light polypeptide kinase & 2,25 & chr6 \\
& & secreted protein, acidic, cysteine-rich (osteonectin) /// secreted protein, acidic, cysteine-rich (osteone & 2 \\
\hline
\end{tabular}




\section{Pag 203 of 257}

\begin{tabular}{|c|c|c|c|c|c|}
\hline Gene Symbol & Entrez Gene & Gene Title & NPC_up & MPC_down & Chromosome Number(Avadis) \\
\hline CALML4 & 91860 & calmodulin-like 4 & 2,34 & 2,05 & chr15 \\
\hline CAMTA1 & 23261 & calmodulin binding transcription activator 1 & 3,03 & 2,05 & chr1 \\
\hline CAST1 & 26059 & CAZ-associated structural protein & 2,17 & 3,19 & chr3 \\
\hline CBR4 & 84869 & carbonic reductase 4 & 2,28 & 4,76 & chr4 \\
\hline CBX5 & 23468 & Chromobox homolog 5 (HP1 alpha homolog, Drosophila) & 2,23 & 2,76 & chr12 \\
\hline CCAR1 & 55749 & Cell division cycle and apoptosis regulator 1 & 2,07 & 3,12 & chr10 \\
\hline CCDC14 & 64770 & coiled-coil domain containing 14 & 2,04 & 2,16 & chr3 \\
\hline CDKN1C & 1028 & Cyclin-dependent kinase inhibitor 1C (p57, Kip2) & 3,14 & 2,83 & chr11 \\
\hline CDON & 50937 & Cdon homolog (mouse) & 3,91 & 6,95 & chr11 \\
\hline CENTG2 & 116987 & Centaurin, gamma 2 & 2,01 & 2,77 & chr2 \\
\hline Cep152 & 22995 & KIAA0912 protein & 2,03 & 3,56 & chr15 \\
\hline CEP68 & 23177 & Centrosomal protein $68 \mathrm{kDa}$ & 2,54 & 2,49 & chr2 \\
\hline CHD1L & 9557 & Chromodomain helicase DNA binding protein 1 -like & 2,83 & 3,07 & chr1 \\
\hline CHD7 & 55636 & chromodomain helicase DNA binding protein 7 & 5,92 & 5,99 & chr8 \\
\hline CHKB /// CPT1B & 1120 & choline kinase beta /// carnitine palmitoyltransferase $1 \mathrm{~B}$ (muscle) & 3,33 & 2,45 & chr22 \\
\hline CLCN4 & 1183 & Chloride channel 4 & 2,43 & 2,66 & $\operatorname{chrX}$ \\
\hline CLGN & 1047 & calmegin & 6,29 & 3,12 & chr4 \\
\hline CNNM3 & 26505 & Cyclin M3 & 2,43 & 4,25 & chr2 \\
\hline CNOT7 & 29883 & CCR4-NOT transcription complex, subunit 7 & 2,28 & 2,44 & chr1 \\
\hline COL9A1 & 1297 & collagen, type IX, alpha 1 & 3,10 & 4,24 & chr6 \\
\hline COPG2 & 26958 & Coatomer protein complex, subunit gamma 2 & 6,24 & 2,62 & chr7 \\
\hline CPXM & 56265 & carboxypeptidase X (M14 family) & 2,40 & 4,77 & chr20 \\
\hline CRLF3 & 51379 & Cytokine receptor-like factor 3 & 5,71 & 2,78 & chr17 \\
\hline CTNNA2 & 1496 & catenin (cadherin-associated protein), alpha 2 & 7,17 & 3,17 & chr2 \\
\hline CTTNBP2 & 83992 & Cortactin binding protein 2 & 2,30 & 3,52 & chr7 \\
\hline CUGBP2 & 10659 & CUG triplet repeat, RNA binding protein 2 & 3,44 & 7,20 & chr10 \\
\hline CUL1 & 8454 & Cullin 1 & 2,13 & 5,56 & chr7 \\
\hline CUL3 & 8452 & Cullin 3 & 3,04 & 2,10 & chr2 \\
\hline CXCR4 & 7852 & chemokine (C-X-C motif) receptor 4 & 4,95 & 13,89 & chr2 \\
\hline DCX & 1641 & doublecortex; lissencephaly, X-linked (doublecortin) & 3,86 & 2,51 & $\operatorname{chrX}$ \\
\hline DDX17 & 10521 & DEAD (Asp-Glu-Ala-Asp) box polypeptide 17 & 2,13 & 3,06 & chr22 \\
\hline DKFZp434P055 & 91531 & hypothetical protein DKFZp434P055 & 2,42 & 4,60 & chr2 \\
\hline DKFZP761M151 & 54492 & hypothetical protein DKFZP761M1511 & 2,22 & 2,83 & chr5 \\
\hline DLL1 & 28514 & delta-like 1 (Drosophila) & 24,34 & 2,48 & chr6 \\
\hline
\end{tabular}




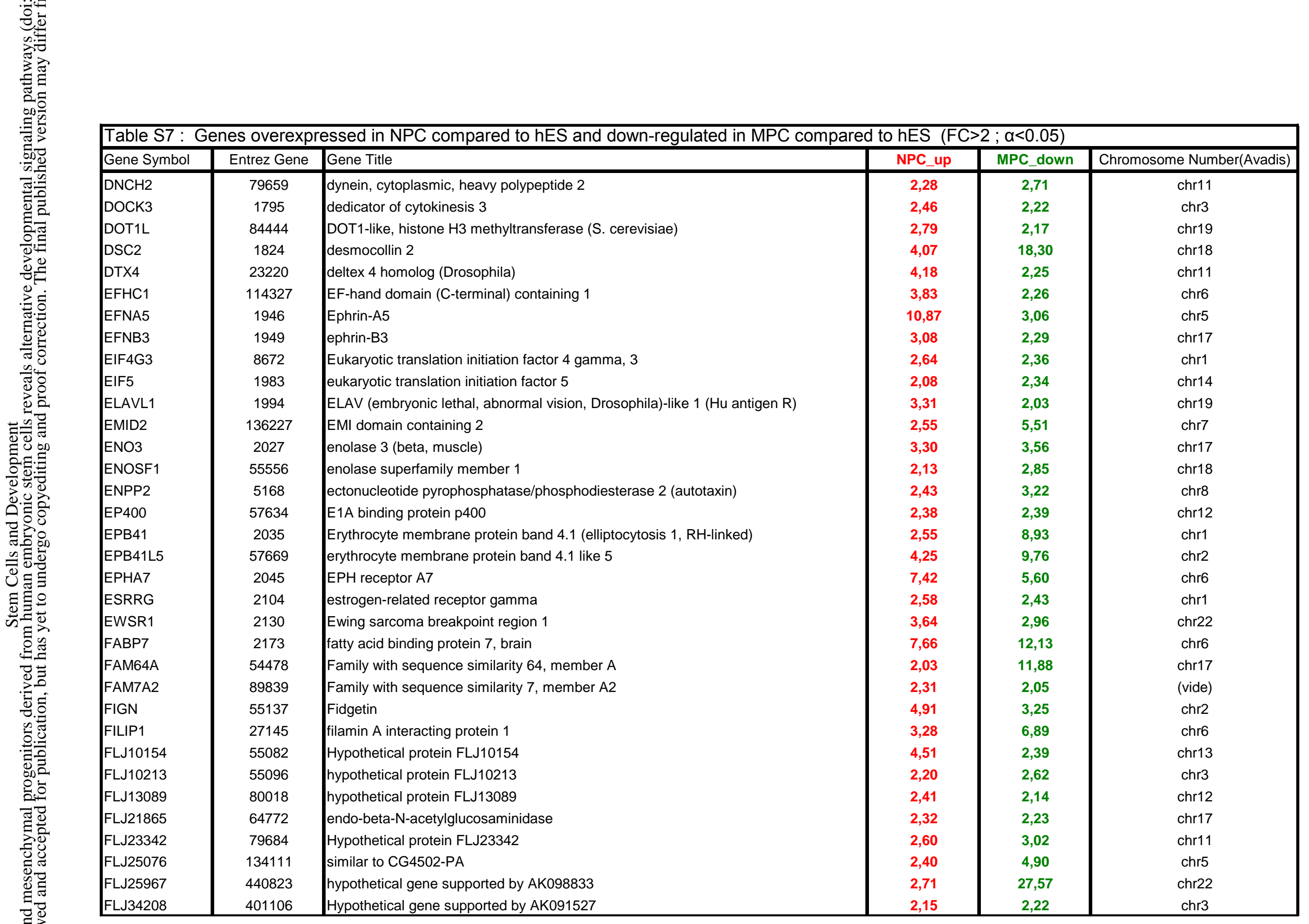




\section{Page 205 of 257}

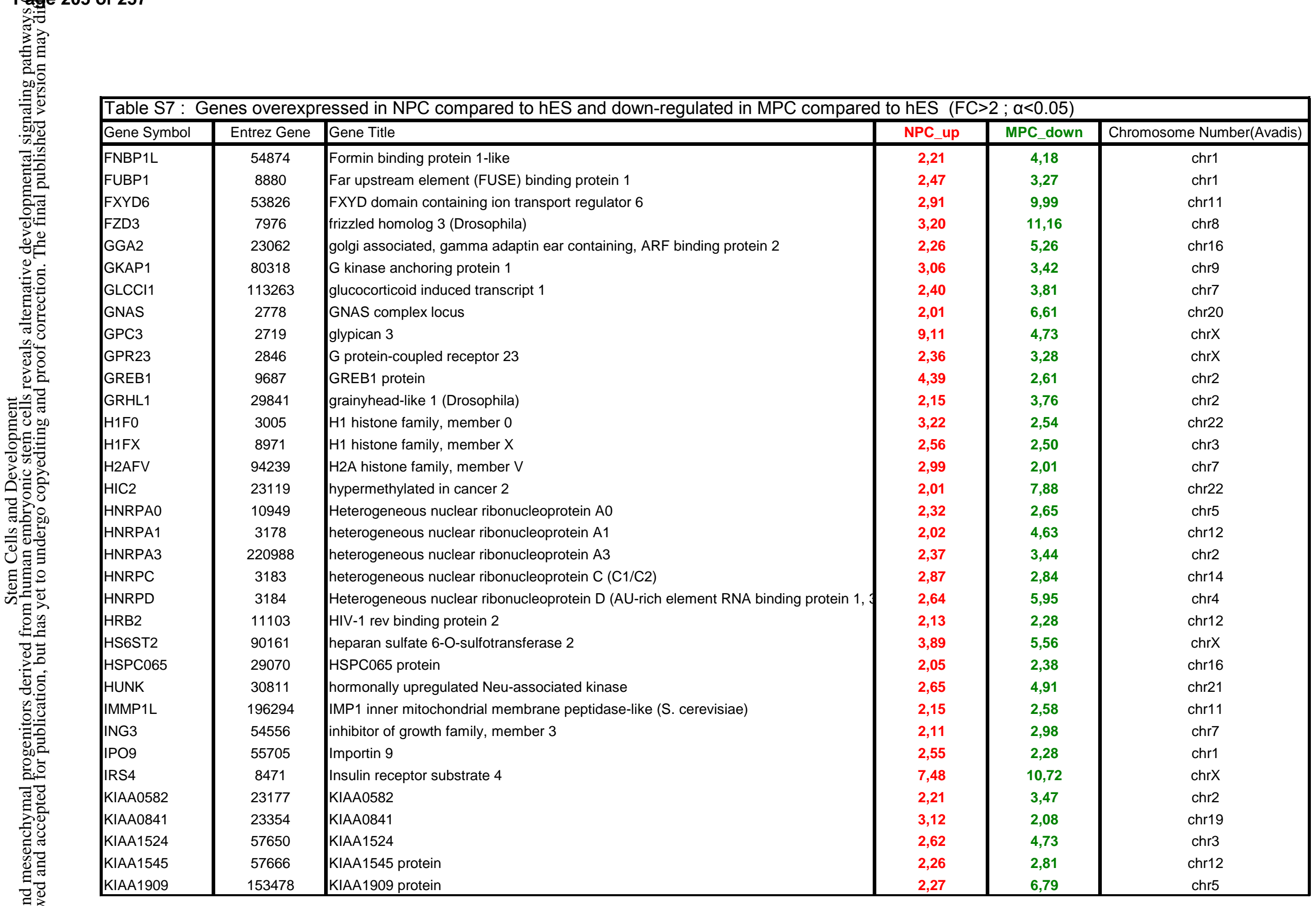




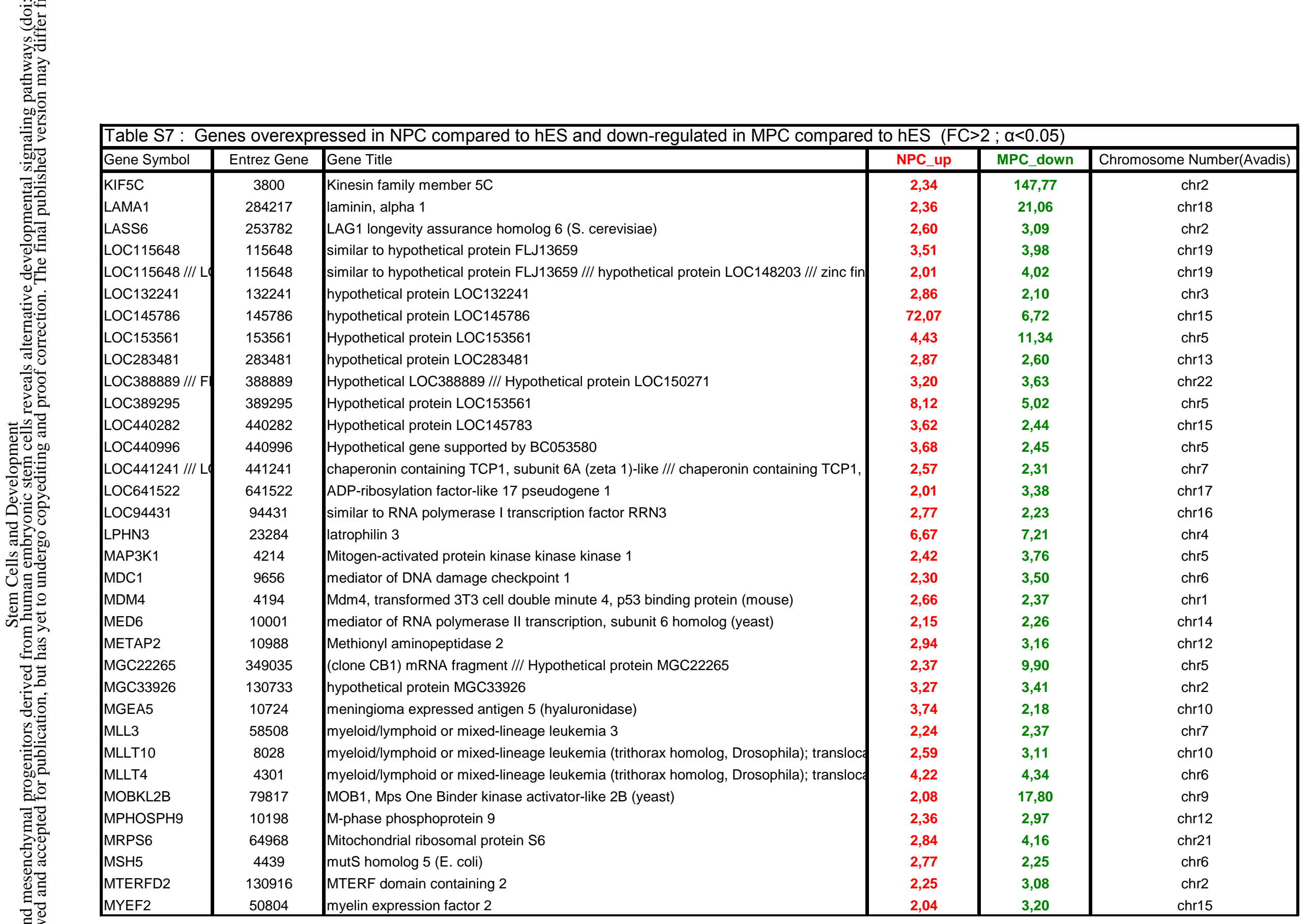




\begin{tabular}{|c|c|c|c|c|c|c|}
\hline .000 & Gene Symbol & Entrez Gene & Gene Title & NPC_up & MPC_down & $\begin{array}{l}\text { Chromosome Number(Avadis) } \\
\text { (a) }\end{array}$ \\
\hline $8=$ & NAALAD2 & 10003 & N-acetylated alpha-linked acidic dipeptidase 2 & 2,66 & 2,87 & chr11 \\
\hline $\bar{z}$ & NAG6 & 64753 & hypothetical protein DKFZp434G156 & 2,52 & 3,25 & $\operatorname{chr} 7$ \\
\hline 2त्] & NASP & 4678 & Nuclear autoantigenic sperm protein (histone-binding) & 2,41 & 33,83 & chr1 \\
\hline 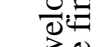 & NBLA04196 & 64921 & Putative protein product of Nbla04196 & 2,86 & 2,95 & $\mathrm{chr} 7$ \\
\hline 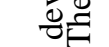 & NCOA5 & 57727 & Nuclear receptor coactivator 5 & 2,49 & 2,49 & chr20 \\
\hline & NEBL & 10529 & nebulette & 4,19 & 8,36 & chr10 \\
\hline & NELL2 & 4753 & NEL-like 2 (chicken) /// NEL-like 2 (chicken) & 3,91 & 69,31 & chr12 \\
\hline & NEO1 & 4756 & neogenin homolog 1 (chicken) & 2,57 & 2,53 & chr15 \\
\hline $\bar{\partial}$ & NOL7 & 51406 & Nucleolar protein 7, 27kDa & 2,36 & 2,15 & chr6 \\
\hline 0 & NOVA1 & 4857 & neuro-oncological ventral antigen 1 & 2,89 & 2,65 & chr14 \\
\hline$\vec{a}$ & NR2C2 & 7182 & Nuclear receptor subfamily 2, group C, member 2 & 2,19 & 2,25 & chr3 \\
\hline $\bar{E}$ & NSUN6 & 221078 & NOL1/NOP2/Sun domain family, member 6 & 6,89 & 2,16 & chr10 \\
\hline o & NUDT5 & 11164 & Nudix (nucleoside diphosphate linked moiety X)-type motif 5 & 2,43 & 2,44 & chr10 \\
\hline$\Xi$ & NUPL1 & 9818 & Nucleoporin like 1 & 2,59 & 2,48 & chr13 \\
\hline & отх2 & 5015 & orthodenticle homolog 2 (Drosophila) & 2,24 & 109,06 & chr14 \\
\hline 0 & ovos2 & 144203 & ovostatin 2 & 2,70 & 14,87 & chr12 \\
\hline 50 & PABPN1 & 8106 & poly(A) binding protein, nuclear 1 & 4,03 & 3,57 & chr14 \\
\hline $\bar{\omega}$ & PAN3 & 255967 & PABP1-dependent poly A-specific ribonuclease subunit PAN3 & 2,12 & 4,92 & chr13 \\
\hline 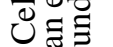 & PAQR8 & 85315 & progestin and adipoQ receptor family member VIII & 2,76 & 4,28 & chr6 \\
\hline & PCBP2 & 5094 & Poly $(\mathrm{rC})$ binding protein 2 & 2,28 & 2,19 & chr12 \\
\hline & PCGF3 & 10336 & Polycomb group ring finger 3 & 2,57 & 2,49 & chr4 \\
\hline$\Xi$ & РCM1 & 5108 & Pericentriolar material 1 & 2,91 & 2,32 & chr8 \\
\hline & PDE7A & 5150 & phosphodiesterase 7A & 2,00 & 7,01 & chr8 \\
\hline & PGAP1 & 80055 & GPI deacylase & 6,04 & 3,02 & chr2 \\
\hline & PHF10 & 55274 & PHD finger protein 10 & 2,31 & 2,83 & chr6 \\
\hline & PHF21B & 112885 & PHD finger protein 21B & 2,15 & 8,54 & chr22 \\
\hline 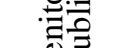 & PIK3С2A & 5286 & Phosphoinositide-3-kinase, class 2, alpha polypeptide & 2,80 & 2,33 & chr11 \\
\hline & PILRB & 29990 & paired immunoglobin-like type 2 receptor beta & 2,31 & 3,19 & chr7 \\
\hline & PKP4 & 8502 & Plakophilin 4 & 2,36 & 2,54 & chr2 \\
\hline & PLCL3 & 23007 & phospholipase C-like 3 & 2,01 & 8,50 & chr3 \\
\hline & PLEKHA5 & 54477 & Pleckstrin homology domain containing, family A member 5 & 2,21 & 8,88 & chr12 \\
\hline & PMS2L1 /// PMS & 5379 & postmeiotic segregation increased 2-like 1 /// postmeiotic segregation increased 2-like & 2,13 & 2,16 & chr7 \\
\hline & PPM1L & 151742 & Protein phosphatase 1 (formerly 2C)-like & 2,71 & 3,64 & chr3 \\
\hline & PPOX & 5498 & protoporphyrinogen oxidase & 3,33 & 2,60 & chr1 \\
\hline
\end{tabular}




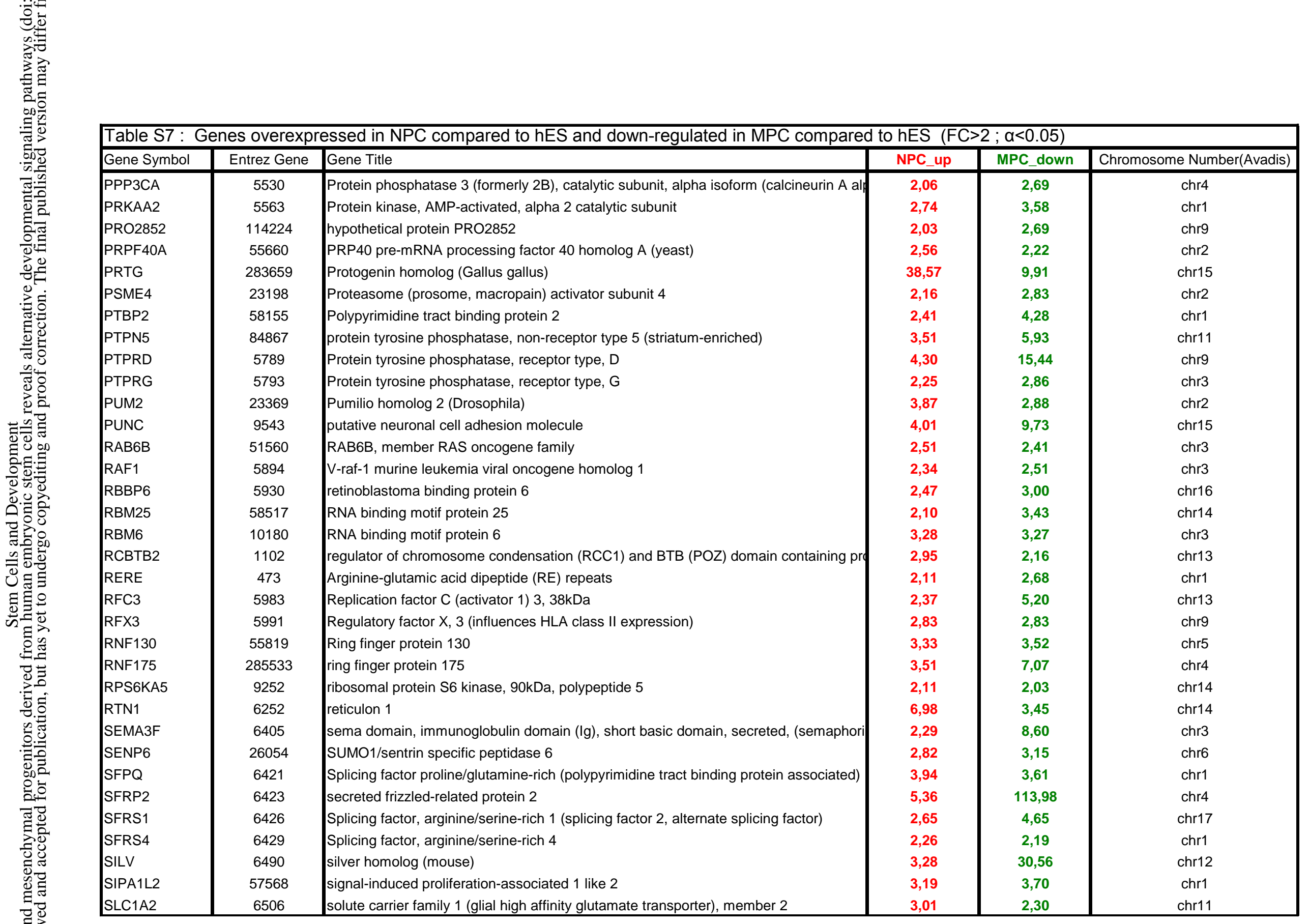




\begin{tabular}{|c|c|c|c|c|c|c|}
\hline 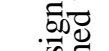 & Gene Symbol & Entrez Gene & Gene Title & NPC up & MPC down & Chromosome Number(Avadis) \\
\hline & SLC25A37 & 51312 & solute carrier family 25, member 37 & 3,10 & 2,15 & chr8 \\
\hline $\bar{z}$ & SLC35F1 & 222553 & solute carrier family 35 , member $\mathrm{F} 1$ & 2,10 & 9,40 & chr6 \\
\hline हैं & SLITRK5 & 26050 & SLIT and NTRK-like family, member 5 & 3,43 & 2,12 & chr13 \\
\hline $0 ; \equiv$ & SMARCC1 & 6599 & SWI/SNF related, matrix associated, actin dependent regulator of chromatin, subfamil & 3,05 & 7,65 & chr3 \\
\hline \& & SMARCE1 & 6605 & SWI/SNF related, matrix associated, actin dependent regulator of chromatin, subfamil & 2,05 & 2,85 & chr17 \\
\hline & SMU1 & 55234 & Smu-1 suppressor of mec-8 and unc-52 homolog (C. elegans) & 2,41 & 2,44 & chr9 \\
\hline & SNCA & 6622 & synuclein, alpha (non A4 component of amyloid precursor) /// synuclein, alpha (non A & 2,70 & 3,47 & chr4 \\
\hline & SNRPA1 & 6627 & Small nuclear ribonucleoprotein polypeptide A' & 2,47 & 4,71 & chr15 \\
\hline & Sox11 & 6664 & SRY (sex determining region Y)-box 11 & 8,85 & 3,41 & chr2 \\
\hline & sox3 & 6658 & SRY (sex determining region Y)-box 3 & 3,38 & 5,34 & $\operatorname{chr} \mathrm{X}$ \\
\hline ة & SP8 & 221833 & Sp8 transcription factor & 16,95 & 3,32 & chr7 \\
\hline$\Xi$ & SPON1 & 10418 & spondin 1 , extracellular matrix protein & 9,34 & 2,51 & chr11 \\
\hline$\underbrace{\infty}_{E}$ & SPSB4 & 92369 & splA/ryanodine receptor domain and SOCS box containing 4 & 2,16 & 3,46 & chr3 \\
\hline$=$ & SREBF1 & 6720 & Sterol regulatory element binding transcription factor 1 & 2,06 & 2,48 & chr17 \\
\hline & SSBP2 & 23635 & Single-stranded DNA binding protein 2 & 7,89 & 2,52 & chr5 \\
\hline ¿ & SUV420H1 & 51111 & Suppressor of variegation 4-20 homolog 1 (Drosophila) & 2,41 & 4,63 & chr11 \\
\hline 50 & SYT17 & 51760 & Synaptotagmin XVII & 3,76 & 3,45 & chr16 \\
\hline 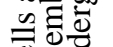 & TAF15 & 8148 & TAF15 RNA polymerase II, TATA box binding protein (TBP)-associated factor, 68kDa & 2,84 & 3,92 & chr17 \\
\hline$\Xi$ & TARDBP & 23435 & TAR DNA binding protein /// TAR DNA binding protein & 2,19 & 2,33 & chr1 \\
\hline & TBC1D8 & 11138 & TBC1 domain family, member 8 (with GRAM domain) & 2,20 & 5,48 & chr2 \\
\hline & TCF12 & 6938 & Transcription factor 12 (HTF4, helix-loop-helix transcription factors 4) & 2,55 & 2,38 & chr15 \\
\hline$\approx$ & TEX10 & 54881 & Testis expressed sequence 10 & 2,17 & 3,46 & chr9 \\
\hline & TFDP2 & 7029 & Transcription factor Dp-2 (E2F dimerization partner 2) & 2,21 & 9,05 & chr3 \\
\hline & TGFBR3 & 7049 & transforming growth factor, beta receptor III (betaglycan, $300 \mathrm{kDa}$ ) & 5,34 & 2,04 & chr1 \\
\hline & TIA1 & 7072 & TIA1 cytotoxic granule-associated RNA binding protein & 2,69 & 2,51 & chr2 \\
\hline & TIAM1 & 7074 & T-cell lymphoma invasion and metastasis 1 & 3,51 & 2,46 & chr21 \\
\hline & TIGA1 & 114915 & TIGA1 & 2,74 & 2,55 & chr5 \\
\hline & TMCC1 & 23023 & transmembrane and coiled-coil domain family 1 & 2,08 & 2,61 & chr3 \\
\hline & TMEM118 & 84900 & Transmembrane protein 118 & 3,17 & 5,24 & chr12 \\
\hline & TMSL8 & 11013 & thymosin-like 8 & 2,67 & 6,34 & $\operatorname{chr} x$ \\
\hline & TRA2A & 29896 & Transformer-2 alpha & 4,88 & 2,33 & chr7 \\
\hline & TRIB2 & 28951 & tribbles homolog 2 (Drosophila) & 2,63 & 3,03 & chr2 \\
\hline & TRIM45 & 80263 & Tripartite motif-containing 45 & 2,69 & 5,32 & chr1 \\
\hline & TSPAN18 & 90139 & tetraspanin 18 & 3,72 & 2,29 & chr11 \\
\hline
\end{tabular}




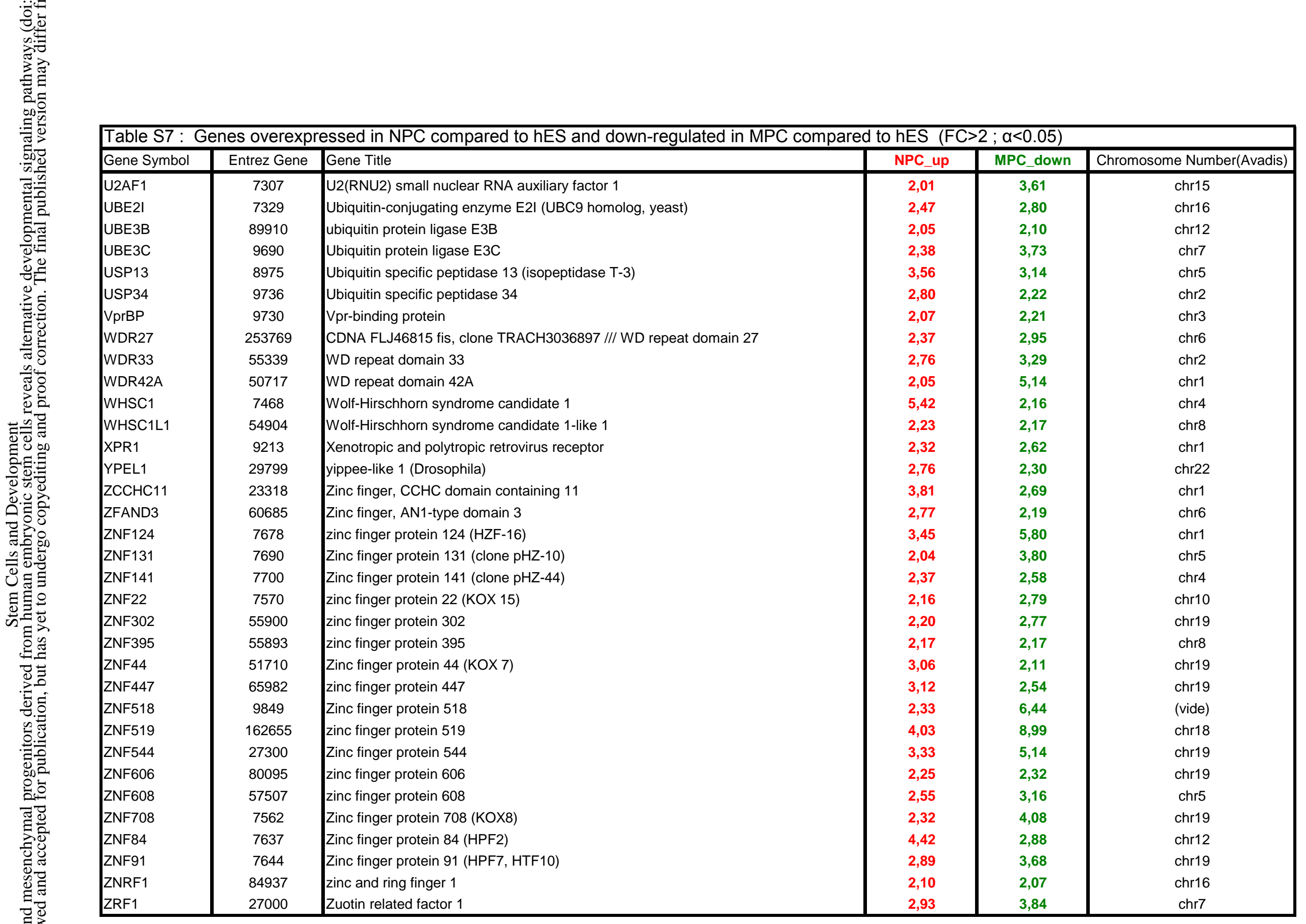




\section{Page 211 of 257}

\begin{tabular}{|c|c|c|c|c|}
\hline Gene Symbol & Entrez Gene & Gene Title & MPC_up & Chromosome Number(Avadis) \\
\hline sept-06 & $23157 / / / 84656$ & septin 6 /// cytokine-like nuclear factor n-pac & 2,66 & $\operatorname{chrX}$ \\
\hline 39326 & 989 & Septin 7 & 2,10 & chr7 \\
\hline 40787 & 55752 & Septin 11 & 73,88 & chr4 \\
\hline AADACL1 & 57552 & arylacetamide deacetylase-like 1 & 4,26 & chr3 \\
\hline ABHD2 & 11057 & abhydrolase domain containing 2 & 2,32 & chr15 \\
\hline ABI1 & 10006 & abl-interactor 1 & 2,24 & chr10 \\
\hline ABI3BP & 25890 & ABI gene family, member 3 (NESH) binding protein & 5,82 & chr3 \\
\hline ABLIM3 & 22885 & actin binding LIM protein family, member 3 & 2,00 & chr5 \\
\hline ACBD3 & 64746 & acyl-Coenzyme A binding domain containing 3 & 2,33 & chr1 \\
\hline ACP2 & 53 & acid phosphatase 2, lysosomal & 2,32 & chr11 \\
\hline ACSL4 & 2182 & acyl-CoA synthetase long-chain family member 4 & 3,23 & $\operatorname{chr} X$ \\
\hline ACSS2 & 55902 & acyl-CoA synthetase short-chain family member 2 & 2,59 & chr20 \\
\hline ACTG2 & 72 & actin, gamma 2, smooth muscle, enteric & 92,67 & chr2 \\
\hline ACTN1 & 87 & actinin, alpha 1 & 5,49 & chr14 \\
\hline ACTN4 & 81 & actinin, alpha 4 & 3,50 & chr19 \\
\hline ACTR10 & 55860 & actin-related protein 10 homolog (S. cerevisiae) & 2,52 & chr14 \\
\hline ACTR1A & 10121 & ARP1 actin-related protein 1 homolog A, centractin alpha (yeast) & 2,60 & chr10 \\
\hline ACTR2 & 10097 & ARP2 actin-related protein 2 homolog (yeast) & 4,01 & chr2 \\
\hline ACTR3 & 10096 & ARP3 actin-related protein 3 homolog (yeast) & 3,56 & chr2 \\
\hline ACVR1 & 90 & activin A receptor, type I & 2,11 & chr2 \\
\hline ADA & 100 & adenosine deaminase & 2,90 & chr20 \\
\hline ADAM10 & 102 & ADAM metallopeptidase domain 10 & 2,14 & chr15 \\
\hline ADAM9 & 8754 & ADAM metallopeptidase domain 9 (meltrin gamma) & 18,84 & chr8 \\
\hline ADAMTS5 & 11096 & ADAM metallopeptidase with thrombospondin type 1 motif, 5 (aggrecanase-2) & 9,73 & (vide) \\
\hline ADCY9 & 115 & adenylate cyclase 9 & 2,59 & chr16 \\
\hline ADD1 & 118 & adducin 1 (alpha) & 6,50 & chr4 \\
\hline ADK & 132 & adenosine kinase & 4,23 & chr10 \\
\hline AEBP1 & 165 & AE binding protein 1 & 3,30 & chr7 \\
\hline AFAP & 60312 & actin filament associated protein & 6,64 & chr4 \\
\hline AGA & 175 & aspartylglucosaminidase & 3,03 & chr4 \\
\hline AGTR1 & 185 & angiotensin II receptor, type 1 & 6,52 & chr3 \\
\hline AHR & 196 & aryl hydrocarbon receptor & 8,35 & chr7 \\
\hline AK1 & 203 & adenylate kinase 1 & 5,98 & chr9 \\
\hline AK5 & 26289 & adenylate kinase 5 & 4,34 & chr1 \\
\hline
\end{tabular}




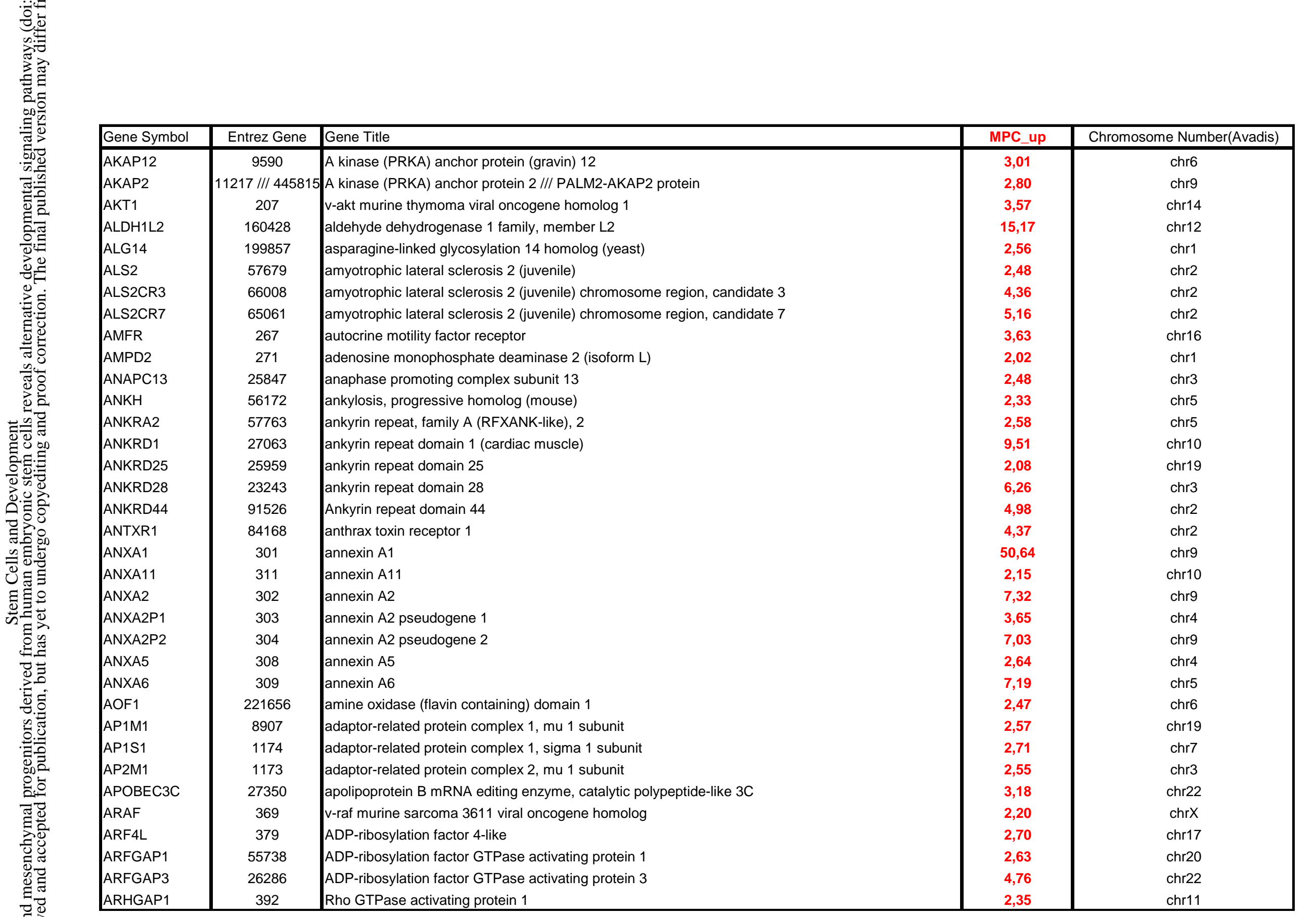




\begin{tabular}{|c|c|c|c|c|c|}
\hline 可 & Gene Symbol & Entrez Gene & Gene Title & MPC_up & Chromosome Number(Avadis) \\
\hline$\pi$ & ARHGAP18 & 93663 & Rho GTPase activating protein 18 & 4,52 & chr6 \\
\hline$\Xi$ & ARHGAP23 & 57636 & Rho GTPase activating protein 23 & 4,45 & chr17 \\
\hline 醇 & ARHGDIA & 396 & Rho GDP dissociation inhibitor (GDI) alpha /// Rho GDP dissociation inhibitor (GDI) alpha & 3,69 & chr17_random \\
\hline อีำ & ARHGEF12 & 23365 & Rho guanine nucleotide exchange factor (GEF) 12 & 3,21 & chr11 \\
\hline Q & ARL1 & 400 & ADP-ribosylation factor-like 1 & 2,71 & chr12 \\
\hline 8 & ARL2BP & 23568 & ADP-ribosylation factor-like 2 binding protein & 3,01 & chr16 \\
\hline 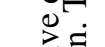 & ARPC1B & 10095 & actin related protein $2 / 3$ complex, subunit $1 \mathrm{~B}, 41 \mathrm{kDa}$ & 2,24 & $\operatorname{chr} 7$ \\
\hline 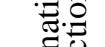 & ARPC2 & 10109 & actin related protein $2 / 3$ complex, subunit $2,34 \mathrm{kDa}$ & 2,13 & chr2 \\
\hline 象 & ARPC5 & 10092 & actin related protein $2 / 3$ complex, subunit $5,16 \mathrm{kDa}$ & 3,48 & chr1 \\
\hline$\pi 0$ & ARSB & 411 & arylsulfatase B & 3,20 & chr5 \\
\hline (1) & ARSJ & 79642 & arylsulfatase $\mathrm{J}$ & 70,99 & chr4 \\
\hline . & ARTS-1 & 51752 & type 1 tumor necrosis factor receptor shedding aminopeptidase regulator & 3,05 & chr5 \\
\hline$\exists=$ & ASAH1 & 427 & N-acylsphingosine amidohydrolase (acid ceramidase) 1 & 2,03 & chr8 \\
\hline$\underbrace{\infty}_{E}$ & ASAM & 79827 & Adipocyte-specific adhesion molecule & 5,27 & chr11 \\
\hline$\Xi$ & ASB8 & 140461 & ankyrin repeat and SOCS box-containing 8 & 4,22 & chr12 \\
\hline & ATG4A & 115201 & ATG4 autophagy related 4 homolog A (S. cerevisiae) & 3,34 & $\operatorname{chr} \mathrm{x}$ \\
\hline 3 & ATG7 & 10533 & ATG7 autophagy related 7 homolog (S. cerevisiae) & 2,99 & chr3 \\
\hline ? & ATM & 472 & ataxia telangiectasia mutated (includes complementation groups $\mathrm{A}, \mathrm{C}$ and $\mathrm{D}$ ) & 2,49 & chr11 \\
\hline 西 & ATP10A & 57194 & ATPase, Class V, type 10A & 2,95 & chr15 \\
\hline$\Xi$ & ATP11B & 23200 & ATPase, Class VI, type 11B & 4,34 & chr3 \\
\hline & ATP13A3 & 79572 & ATPase type 13 A3 & 4,08 & chr3 \\
\hline & ATP2A2 & 488 & ATPase, Ca++ transporting, cardiac muscle, slow twitch 2 & 2,04 & chr12 \\
\hline $8 \pi$ & |ATP5E & 514 & ATP synthase, $\mathrm{H}+$ transporting, mitochondrial $\mathrm{F} 1$ complex, epsilon subunit & 2,17 & chr20 \\
\hline & ATP6AP2 & 10159 & ATPase, $\mathrm{H}+$ transporting, lysosomal accessory protein 2 & 2,17 & $\operatorname{chr} \mathrm{x}$ \\
\hline & ATP6V0D1 & 9114 & ATPase, $\mathrm{H}+$ transporting, lysosomal $38 \mathrm{kDa}$, V0 subunit d isoform 1 & 2,09 & chr16 \\
\hline & ATP6VOE & 8992 & ATPase, $\mathrm{H}+$ transporting, lysosomal 9kDa, V0 subunit e & 3,70 & chr5 \\
\hline हैं & ATP8B1 & 5205 & ATPase, Class I, type 8B, member 1 & 24,44 & chr18 \\
\hline ב⿱艹 & ATXN1 & 6310 & $\operatorname{ataxin} 1$ & 10,66 & chr6 \\
\hline & AVO3 & 253260 & TORC2-specific protein AVO3 & 2,26 & chr5 \\
\hline 然 & AZI2 & 64343 & 5-azacytidine induced 2 & 3,19 & chr3 \\
\hline (8) & B2M & 567 & beta-2-microglobulin & 7,86 & chr15 \\
\hline$\bigotimes_{0}^{2}$ & B4GALT1 & 2683 & UDP-Gal:betaGlcNAc beta 1,4- galactosyltransferase, polypeptide 1 & 2,38 & chr9 \\
\hline & bA16L21.2.1 & 548645 & DnaJ-like protein & 2,04 & chr9 \\
\hline & BACE2 & 25825 & beta-site APP-cleaving enzyme 2 & 4,07 & chr21 \\
\hline & $\mathrm{BACH} 1$ & 571 & BTB and CNC homology 1 , basic leucine zipper transcription factor 1 & 4,61 & chr21 \\
\hline
\end{tabular}




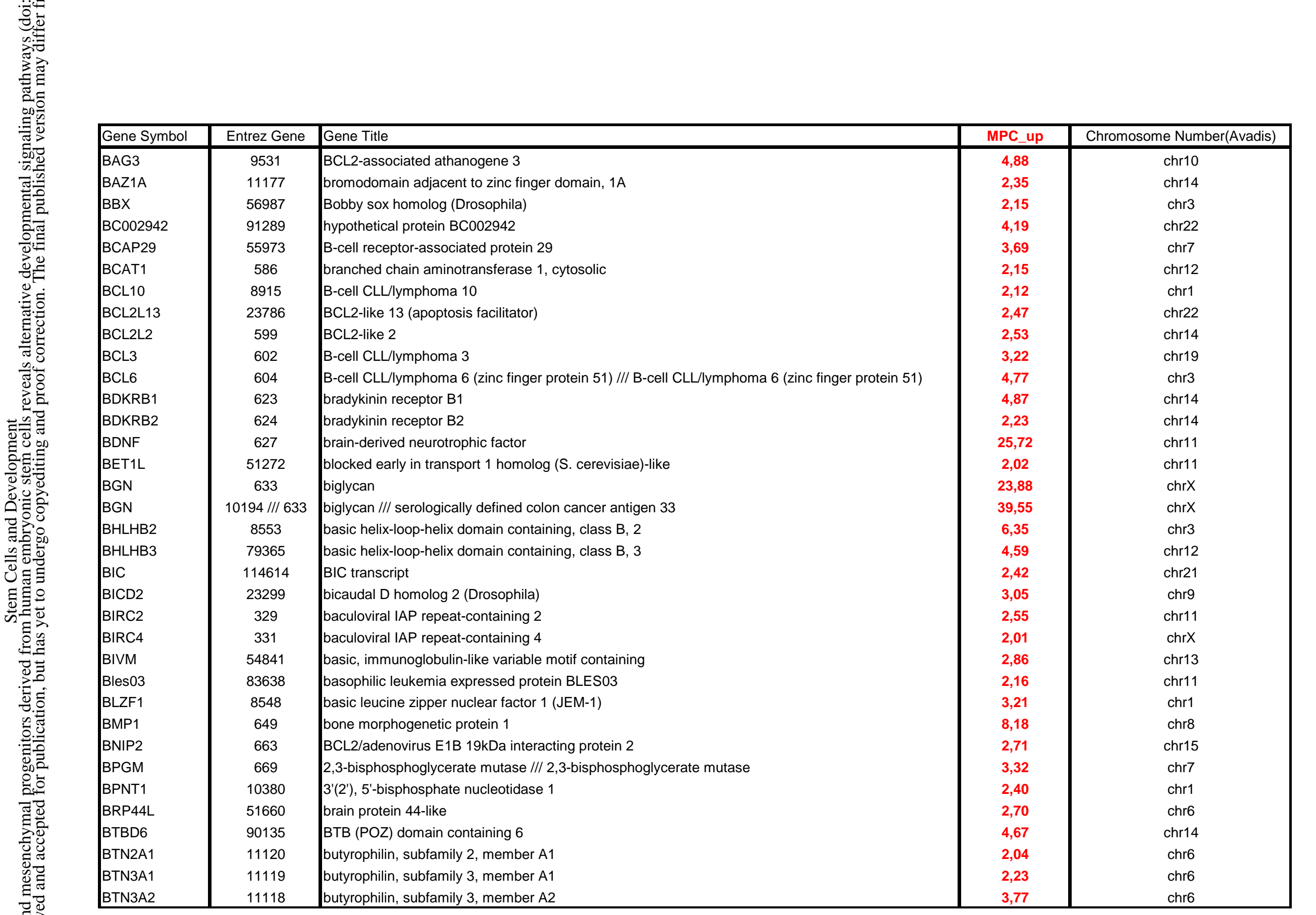




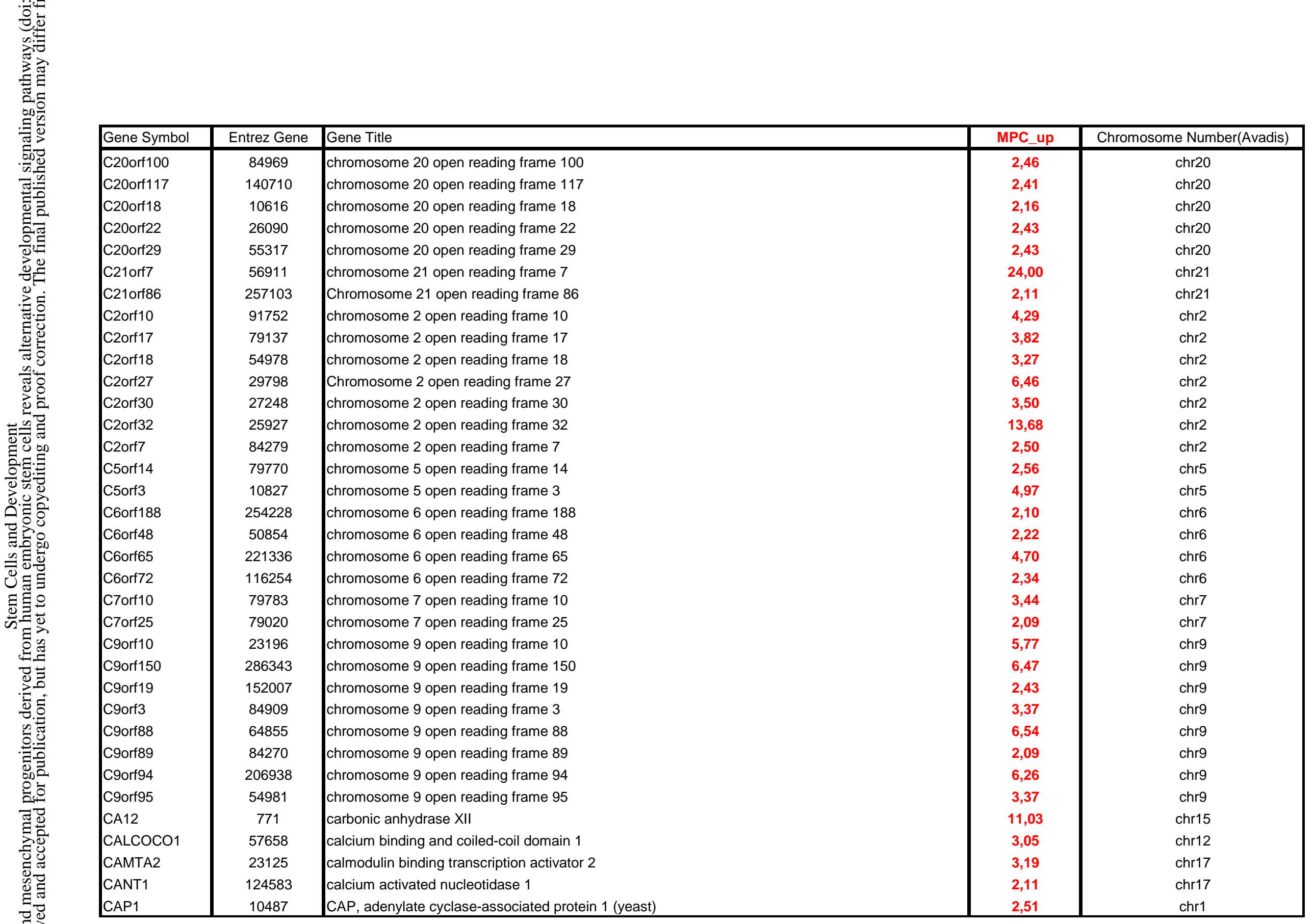




\begin{tabular}{|c|c|c|c|c|c|}
\hline 可 & Gene Symbol & Entrez Gene & Gene Title & MPC_up & Chromosome Number(Avadis) \\
\hline 50] & CAPN7 & 23473 & calpain 7 & 3,02 & chr3 \\
\hline Iే & CAPNS1 & 826 & calpain, small subunit $1 / / /$ calpain, small subunit 1 & 3,94 & chr19 \\
\hline 演 & CASC4 & 113201 & cancer susceptibility candidate 4 & 3,50 & chr15 \\
\hline हैं & CASP4 & 837 & caspase 4 , apoptosis-related cysteine peptidase & 3,52 & chr11 \\
\hline QE & CASP7 & 840 & caspase 7 , apoptosis-related cysteine peptidase & 3,07 & chr10 \\
\hline 远 & CASP8 & 841 & caspase 8 , apoptosis-related cysteine peptidase & 4,36 & chr2 \\
\hline$D$ & CAV1 & 857 & caveolin 1 , caveolae protein, $22 \mathrm{kDa}$ & 27,31 & chr7 \\
\hline 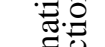 & CAV2 & 858 & caveolin 2 & 46,79 & chr7 \\
\hline 过 & CBX4 & 8535 & chromobox homolog 4 (Pc class homolog, Drosophila) & 6,13 & chr17 \\
\hline न & CBX6 & 23466 & Chromobox homolog 6 & 3,65 & chr22 \\
\hline 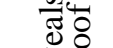 & CCBE1 & 147372 & collagen and calcium binding EGF domains 1 & 2,79 & chr18 \\
\hline . & CCDC6 & 8030 & coiled-coil domain containing 6 & 2,23 & chr10 \\
\hline$\exists=$ & CCDC75 & 253635 & Coiled-coil domain containing 75 & 2,67 & chr2 \\
\hline so & CCND1 & 595 & cyclin D1 & 6,46 & chr11 \\
\hline 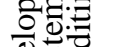 & CCND3 & 896 & cyclin D3 & 2,20 & chr6 \\
\hline$\sum_{2}^{D}$ & CCPG1 & 9236 & cell cycle progression 1 & 9,01 & chr15 \\
\hline o. & CD109 & 135228 & CD109 antigen (Gov platelet alloantigens) & 10,06 & chr6 \\
\hline 60 & CD151 & 977 & CD151 antigen & 6,14 & chr11 \\
\hline E & CD248 & 57124 & CD248 antigen, endosialin & 12,50 & chr11 \\
\hline$\Xi$ & CD274 & 29126 & CD274 antigen & 40,81 & chr9 \\
\hline \& & CD44 & 960 & CD44 antigen (homing function and Indian blood group system) & 111,41 & chr11 \\
\hline & CD58 & 965 & CD58 antigen, (Iymphocyte function-associated antigen 3) & 2,04 & chr1 \\
\hline $8 \pi$ & CD59 & 966 & CD59 antigen p18-20 (antigen identified by monoclonal antibodies 16.3A5, EJ16, EJ30, EL32 and G3 & 15,10 & chr11 \\
\hline & CDA08 & 81533 & T-cell immunomodulatory protein & 3,36 & chr16 \\
\hline & CDC27 & 996 & Cell division cycle 27 & 2,65 & chr17 \\
\hline & CDC42 & 998 & cell division cycle 42 (GTP binding protein, $25 \mathrm{kDa}$ ) & 2,39 & chr1 \\
\hline : & CDC42BPA & 8476 & CDC42 binding protein kinase alpha (DMPK-like) & 2,33 & chr1 \\
\hline$\frac{2}{3}$ & CDC42EP5 & 148170 & CDC42 effector protein (Rho GTPase binding) 5 & 26,82 & chr19 \\
\hline & CDGAP & 57514 & Cdc42 GTPase-activating protein & 2,48 & chr3 \\
\hline \pm & $\mathrm{CDH} 13$ & 1012 & cadherin $13, \mathrm{H}$-cadherin (heart) & 14,00 & chr16 \\
\hline (8) & CDKN1A & 1026 & cyclin-dependent kinase inhibitor 1A (p21, Cip1) & 55,11 & chr6 \\
\hline ठ্రి & CDKN2A & 1029 & cyclin-dependent kinase inhibitor 2A (melanoma, p16, inhibits CDK4) & 22,27 & chr9 \\
\hline & CDKN2B & 1030 & cyclin-dependent kinase inhibitor 2B ( $\mathrm{p} 15$, inhibits CDK4) & 45,10 & chr9 \\
\hline & CDKN2C & 1031 & cyclin-dependent kinase inhibitor $2 \mathrm{C}(\mathrm{p} 18$, inhibits CDK4) & 6,71 & chr1 \\
\hline & CEBPD & 1052 & CCAAT/enhancer binding protein (C/EBP), delta & 5,63 & chr8 \\
\hline
\end{tabular}




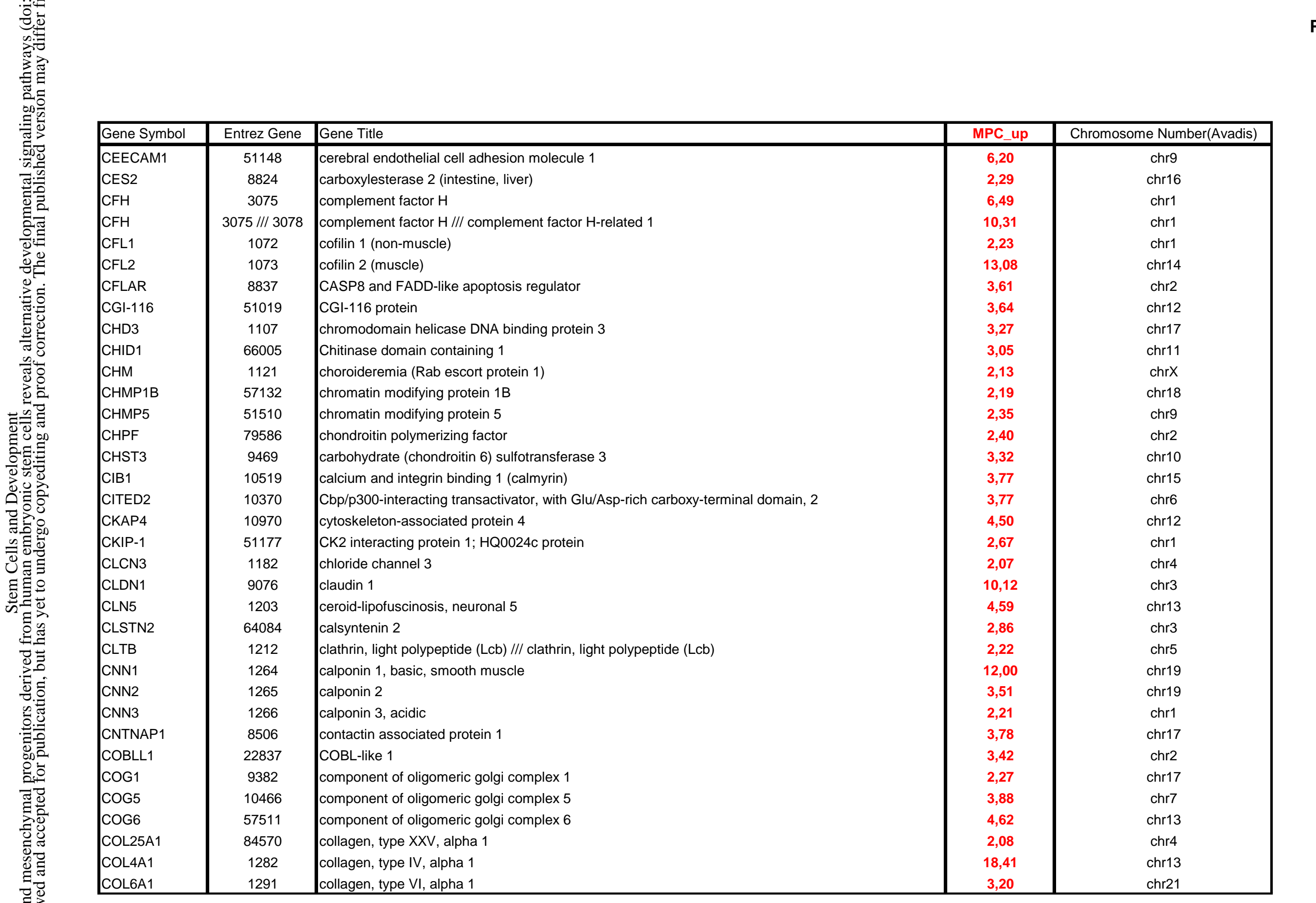




\begin{tabular}{|c|c|c|c|c|c|}
\hline : & Gene Symbol & Entrez Gene & Gene Title & MPC_up & Chromosome Number(Avadis \\
\hline 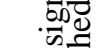 & COL6A2 & 1292 & collagen, type $\mathrm{VI}$, alpha 2 & 51,34 & chr21 \\
\hline$\underline{\underline{n}}$ & COL8A1 & 1295 & Collagen, type VIII, alpha 1 & 291,36 & chr3 \\
\hline $\bar{E}$ & COMMD8 & 54951 & COMM domain containing 8 & 3,62 & chr4 \\
\hline हैं & СОМт & 1312 & catechol-O-methyltransferase & 5,97 & chr22 \\
\hline QE & COPA & 1314 & coatomer protein complex, subunit alpha & 2,51 & chr1 \\
\hline 远 & COPB & 1315 & coatomer protein complex, subunit beta & 3,08 & chr11 \\
\hline 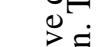 & COPB2 & 9276 & coatomer protein complex, subunit beta 2 (beta prime) & 2,27 & chr3 \\
\hline$=$ & COPE & 11316 & coatomer protein complex, subunit epsilon & 2,19 & chr19 \\
\hline 幽 & COPG & 22820 & coatomer protein complex, subunit gamma & 2,74 & $\operatorname{chr} 3$ \\
\hline$\pi \overline{0}$ & COPZ1 & 22818 & coatomer protein complex, subunit zeta 1 & 2,20 & chr12 \\
\hline 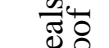 & COPZ2 & 51226 & coatomer protein complex, subunit zeta 2 & 20,86 & chr17 \\
\hline ة & COTL1 & 23406 & coactosin-like 1 (Dictyostelium) & 2,66 & chr16 \\
\hline$\Rightarrow \overline{=}$ & CPA4 & 51200 & carboxypeptidase A4 & 9,29 & chr7 \\
\hline D. & CPEB2 & 132864 & cytoplasmic polyadenylation element binding protein 2 & 8,67 & chr4 \\
\hline$\exists$ & CPEB4 & 80315 & cytoplasmic polyadenylation element binding protein 4 & 2,96 & chr5 \\
\hline & CRAT & 1384 & carnitine acetyltransferase & 3,90 & chr9 \\
\hline : & CREB3 & 10488 & CAMP responsive element binding protein 3 & 4,52 & chr9 \\
\hline 要 & CREB3L1 & 90993 & cAMP responsive element binding protein 3 -like 1 & 13,03 & chr11 \\
\hline 西 & CREB3L2 & 64764 & cAMP responsive element binding protein 3 -like 2 & 3,27 & $\operatorname{chr} 7$ \\
\hline$\Xi$ & CRI1 & 23741 & CREBBP/EP300 inhibitor $1 / / /$ CREBBP/EP300 inhibitor 1 & 2,73 & chr15 \\
\hline 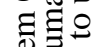 & CRIM1 & 51232 & cysteine rich transmembrane BMP regulator 1 (chordin-like) & 22,12 & chr2 \\
\hline & CRYL1 & 51084 & crystallin, lambda 1 & 2,12 & chr13 \\
\hline 2 & CSGICA-T & 54480 & chondroitin sulfate glucuronyltransferase & 7,26 & $\operatorname{chr} 7$ \\
\hline & CSNK1D & 1453 & casein kinase 1 , delta & 2,07 & chr17 \\
\hline & CSNK1G1 & 53944 & casein kinase 1 , gamma 1 & 2,41 & chr15 \\
\hline & CSPG4 & 1464 & Chondroitin sulfate proteoglycan 4 (melanoma-associated) & 13,86 & chr15 \\
\hline 毛 & CSRP1 & 1465 & cysteine and glycine-rich protein 1 & 14,42 & chr1 \\
\hline تح & css3 & 337876 & chondroitin sulfate synthase 3 & 24,41 & chr5 \\
\hline & CST3 & 1471 & cystatin C (amyloid angiopathy and cerebral hemorrhage) & 6,45 & chr20 \\
\hline 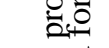 & CTBS & 1486 & chitobiase, di-N-acetyl- & 10,27 & chr1 \\
\hline (ב) & CTGF & 1490 & connective tissue growth factor & 20,66 & chr6 \\
\hline 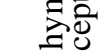 & CTSB & 1508 & cathepsin B & 17,12 & chr8 \\
\hline & CUEDC2 & 79004 & CUE domain containing 2 & 2,04 & chr10 \\
\hline$\Xi$ & CUL4B & 8450 & cullin 4B & 2,80 & chr10 \\
\hline & CUL5 & 8065 & cullin 5 & 2,16 & chr11 \\
\hline
\end{tabular}




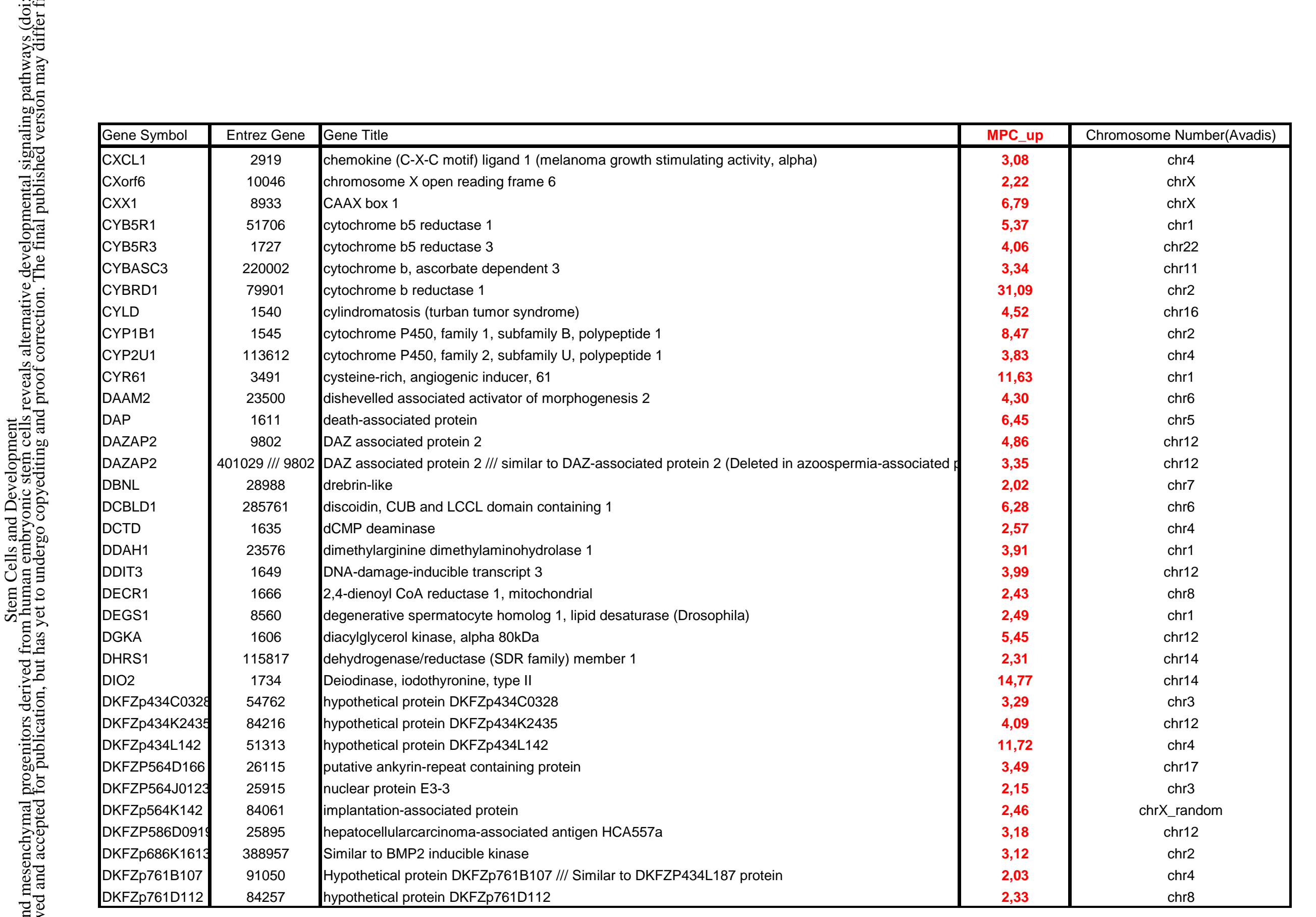




\section{Page 221 of 257}

\begin{tabular}{|c|c|c|c|c|}
\hline Gene Symbol & Entrez Gene & Gene Title & MPC_up & Chromosome Number(Avadis) \\
\hline DKK3 & 27122 & dickkopf homolog 3 (Xenopus laevis) & 19,69 & chr11 \\
\hline DLG1 & 1739 & discs, large homolog 1 (Drosophila) & 3,05 & chr3 \\
\hline DMN & 23336 & desmuslin & 7,70 & chr15 \\
\hline DNAJB12 & 54788 & DnaJ (Hsp40) homolog, subfamily B, member 12 & 2,60 & chr10 \\
\hline DNAJB14 & 79982 & DnaJ (Hsp40) homolog, subfamily B, member 14 & 2,74 & chr4 \\
\hline DNAJB4 & 11080 & DnaJ (Hsp40) homolog, subfamily B, member 4 & 7,82 & chr1 \\
\hline DNAJB9 & 4189 & DnaJ (Hsp40) homolog, subfamily B, member 9 & 3,73 & chr7 \\
\hline DNAJC13 & 23317 & DnaJ (Hsp40) homolog, subfamily C, member 13 & 2,71 & chr3 \\
\hline DNAJC3 & 5611 & Hypothetical protein LOC144871 & 4,16 & chr13 \\
\hline DNAPTP6 & 26010 & DNA polymerase-transactivated protein 6 & 2,23 & chr2 \\
\hline DNASE1L1 & 1774 & deoxyribonuclease I-like 1 & 7,70 & $\operatorname{chr} X$ \\
\hline DNCLI2 & 1783 & dynein, cytoplasmic, light intermediate polypeptide 2 & 2,08 & chr16 \\
\hline DOCK10 & 55619 & dedicator of cytokinesis 10 & 9,82 & chr2 \\
\hline DOCK2 & 1794 & Dedicator of cytokinesis 2 & 16,79 & chr5 \\
\hline DPF3 & 8110 & D4, zinc and double PHD fingers, family 3 & 6,47 & chr14 \\
\hline DPP8 & 54878 & Dipeptidyl-peptidase 8 & 2,27 & chr15 \\
\hline DPY19L4 & 286148 & dpy-19-like 4 (C. elegans) & 2,64 & chr8 \\
\hline DRAP1 & 10589 & DR1-associated protein 1 (negative cofactor 2 alpha) & 2,70 & chr11 \\
\hline DSP & 1832 & desmoplakin & 3,83 & chr6 \\
\hline DUSP1 & 1843 & dual specificity phosphatase 1 & 9,65 & chr5 \\
\hline DUSP10 & 11221 & dual specificity phosphatase 10 & 3,51 & chr1 \\
\hline DUSP14 & 11072 & dual specificity phosphatase 14 & 2,33 & chr17 \\
\hline DUSP18 & 150290 & dual specificity phosphatase 18 & 2,04 & chr22 \\
\hline DYM & 54808 & Dymeclin & 2,10 & chr18 \\
\hline DYRK4 & 8798 & dual-specificity tyrosine-(Y)-phosphorylation regulated kinase 4 & 3,97 & chr12 \\
\hline DYSF & 8291 & dysferlin, limb girdle muscular dystrophy 2B (autosomal recessive) & 2,48 & chr2 \\
\hline EBI2 & 1880 & Epstein-Barr virus induced gene 2 (Iymphocyte-specific G protein-coupled receptor) & 2,06 & chr13 \\
\hline ECE1 & 1889 & Endothelin converting enzyme 1 & 2,38 & chr1 \\
\hline ECM1 & 1893 & extracellular matrix protein 1 & 8,48 & chr1 \\
\hline ECM2 & 1842 & extracellular matrix protein 2 , female organ and adipocyte specific & 2,46 & chr9 \\
\hline EDEM1 & 9695 & ER degradation enhancer, mannosidase alpha-like 1 & 4,99 & chr3 \\
\hline EDIL3 & 10085 & EGF-like repeats and discoidin I-like domains 3 & 6,80 & chr5 \\
\hline EDN1 & 1906 & endothelin 1 & 3,78 & chr6 \\
\hline EEA1 & 8411 & early endosome antigen $1,162 \mathrm{kD}$ & 2,68 & chr12 \\
\hline EEF1D & 1936 & Eukaryotic translation elongation factor 1 delta (guanine nucleotide exchange protein) & 2,69 & chr8 \\
\hline
\end{tabular}




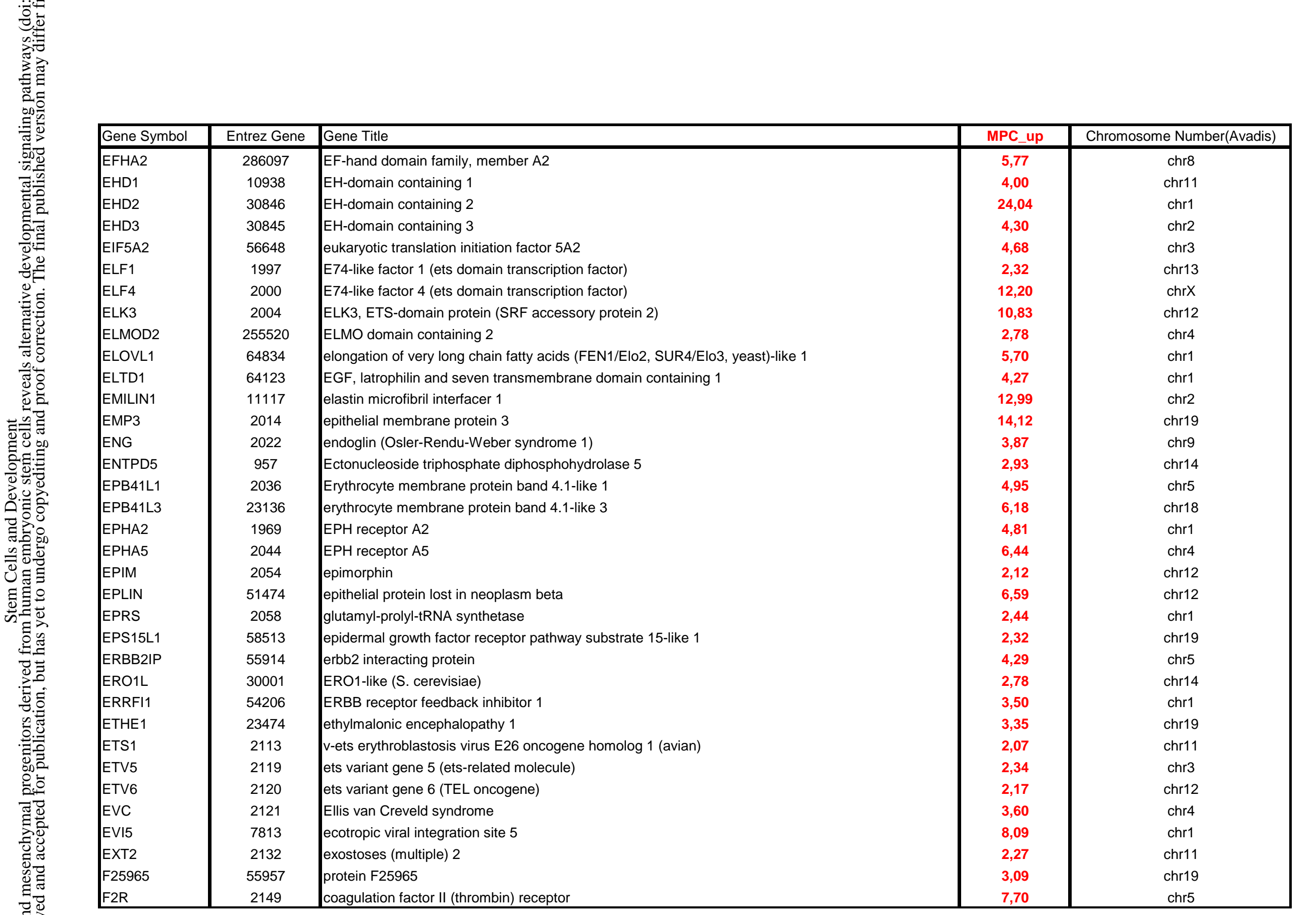




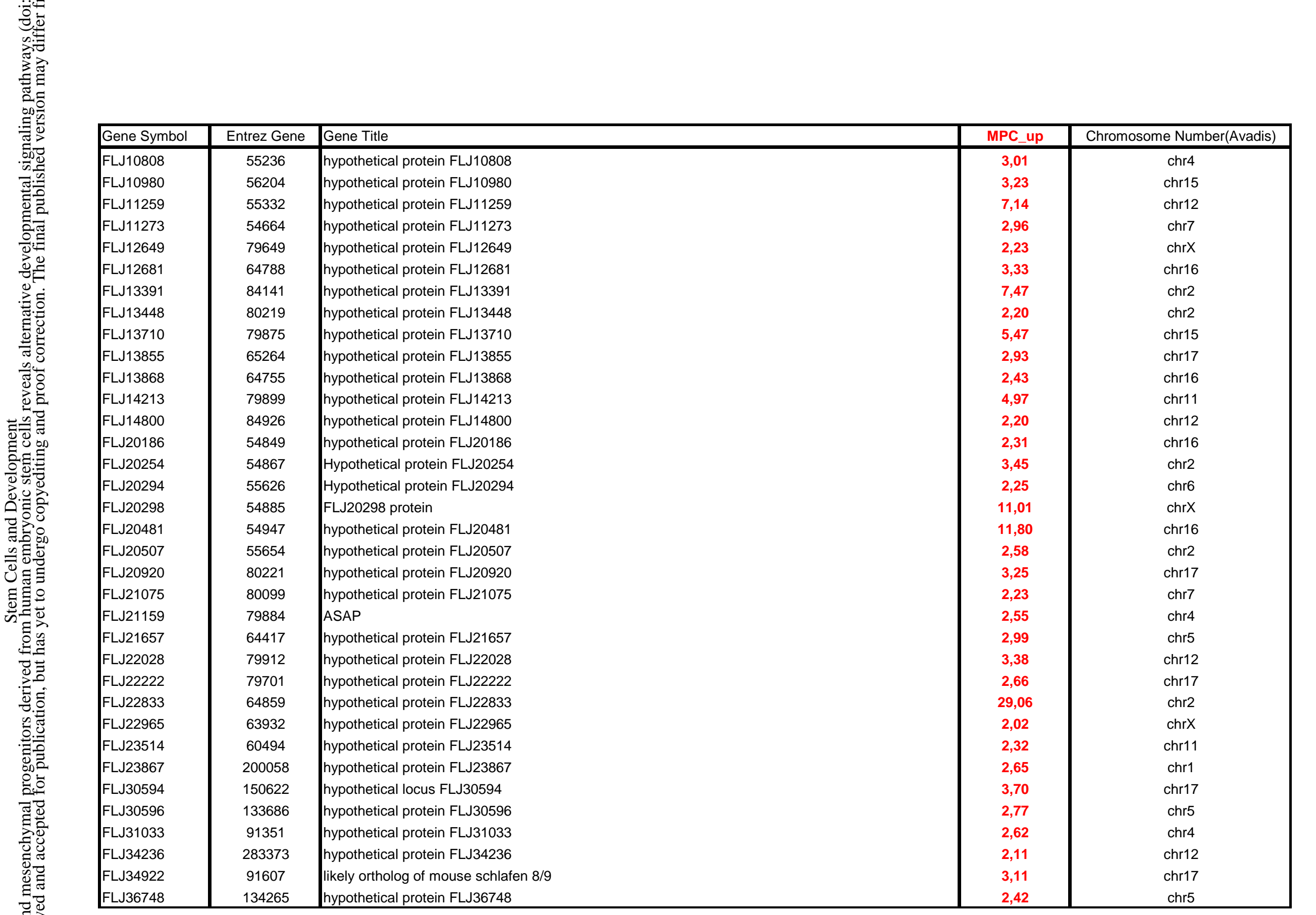




\section{Page 225 of 257}

\begin{tabular}{|c|c|c|c|c|}
\hline Gene Symbol & Entrez Gene & Gene Title & MPC_up & Chromosome Number(Avadis) \\
\hline FLJ38101 & 255919 & hypothetical protein FLJ38101 & 2,28 & chr16 \\
\hline FLJ38725 & 144811 & hypothetical protein FLJ38725 & 7,54 & chr13 \\
\hline FLJ39370 & 132720 & hypothetical protein FLJ39370 & 6,14 & chr4 \\
\hline FLJ39441 & 144108 & hypothetical protein FLJ39441 & 2,18 & chr11 \\
\hline FLJ43339 & 388115 & FLJ43339 protein & 4,21 & chr15 \\
\hline FLJ44635 & 392490 & TPT1-like protein & 2,26 & $\operatorname{chr} X$ \\
\hline FLJ90166 & 164284 & hypothetical protein FLJ90166 & 7,80 & chr20 \\
\hline FLNA & 2316 & filamin A, alpha (actin binding protein 280) & 6,93 & $\operatorname{chr} X$ \\
\hline FLNC & 2318 & filamin C, gamma (actin binding protein 280) & 9,72 & chr7 \\
\hline FLYWCH1 & 84256 & FLYWCH-type zinc finger 1 & 3,14 & chr16 \\
\hline FMN2 & 56776 & formin 2 & 6,91 & chr1 \\
\hline FN1 & 2335 & fibronectin 1 & 178,10 & chr2 \\
\hline FNDC3B & 64778 & fibronectin type III domain containing $3 \mathrm{~B}$ & 7,30 & chr3 \\
\hline FOSL1 & 8061 & FOS-like antigen 1 & 3,19 & chr11 \\
\hline FOXD1 & 2297 & forkhead box D1 & 25,91 & chr5 \\
\hline FOXF1 & 2294 & forkhead box F1 & 8,38 & chr16 \\
\hline FOXF2 & 2295 & forkhead box F2 & 3,12 & chr6 \\
\hline FOXJ2 & 55810 & forkhead box J2 & 2,42 & chr12 \\
\hline FOXL1 & 2300 & Forkhead box L1 & 6,23 & chr16 \\
\hline FREQ & 23413 & Frequenin homolog (Drosophila) & 2,16 & chr9 \\
\hline FRS2 & 10818 & Fibroblast growth factor receptor substrate 2 & 2,35 & chr12 \\
\hline FSTL1 & 11167 & follistatin-like 1 & 4,78 & chr3 \\
\hline FSTL3 & 10272 & follistatin-like 3 (secreted glycoprotein) & 6,37 & chr19 \\
\hline FTH1 & 2495 & ferritin, heavy polypeptide 1 & 2,38 & chr11 \\
\hline FTL & 2512 & Ferritin, light polypeptide & 2,50 & $\operatorname{chr} X$ \\
\hline FUT6 & 2528 & Fucosyltransferase 6 (alpha $(1,3)$ fucosyltransferase) & 2,79 & chr16 \\
\hline FVT1 & 2531 & Follicular Iymphoma variant translocation 1 & 3,31 & chr18 \\
\hline FYCO1 & 79443 & FYVE and coiled-coil domain containing 1 & 3,80 & chr3 \\
\hline FZD6 & 8323 & frizzled homolog 6 (Drosophila) & 5,16 & chr8 \\
\hline GABARAP & 11337 & GABA(A) receptor-associated protein & 2,04 & chr17 \\
\hline GABRB1 & 2560 & gamma-aminobutyric acid (GABA) A receptor, beta 1 & 2,41 & chr4 \\
\hline GADD45A & 1647 & growth arrest and DNA-damage-inducible, alpha & 11,34 & chr1 \\
\hline GADD45B & 4616 & growth arrest and DNA-damage-inducible, beta & 13,14 & chr19 \\
\hline GALNACT-2 & 55454 & chondroitin sulfate GalNAcT-2 & 6,52 & chr10 \\
\hline GALNT2 & 2590 & UDP-N-acetyl-alpha-D-galactosamine:polypeptide N-acetylgalactosaminyltransferase 2 (GalNAC-T2) & 6,45 & chr1 \\
\hline
\end{tabular}




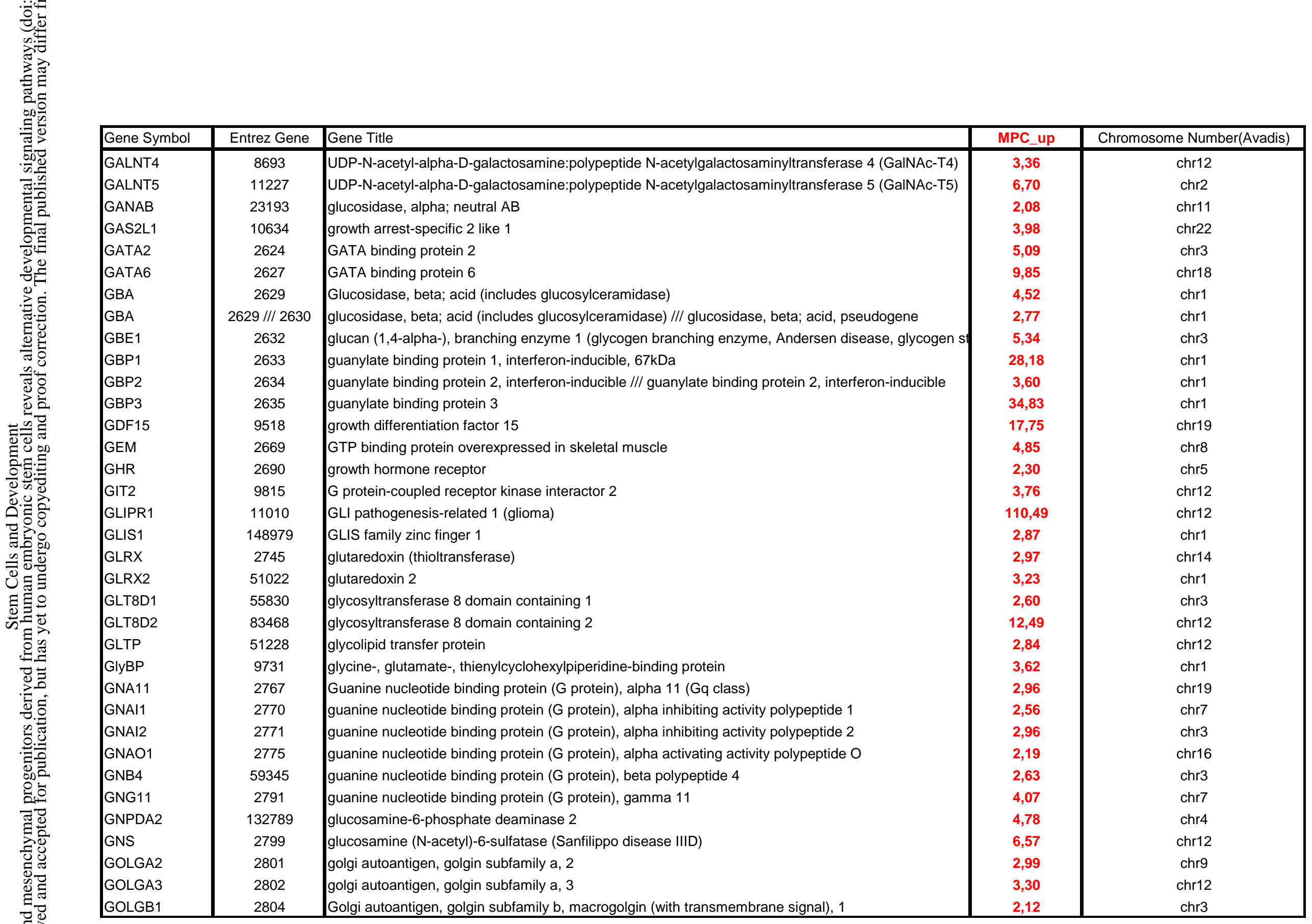




\begin{tabular}{|c|c|c|c|c|c|}
\hline 可 & Gene Symbol & Entrez Gene & Gene Title & MPC_up & Chromosome Number(Avadis) \\
\hline . & GOLPH4 & 27333 & golgi phosphoprotein 4 & 3,03 & chr3 \\
\hline$\Xi$ & GOLT1B & 51026 & golgi transport 1 homolog B (S. cerevisiae) & 7,34 & chr12 \\
\hline Ẽ & GOSR1 & 9527 & golgi SNAP receptor complex member 1 & 2,31 & chr17 \\
\hline ํㅐ을 & GPR124 & 25960 & G protein-coupled receptor 124 & 6,41 & chr8 \\
\hline$\stackrel{0}{0} \Xi$ & GPR126 & 57211 & G protein-coupled receptor 126 & 3,17 & chr6 \\
\hline 远 & GPR155 & 151556 & G protein-coupled receptor 155 & 5,24 & chr2 \\
\hline$D$ & GPRC5A & 9052 & G protein-coupled receptor, family C, group 5 , member A & 17,86 & chr12 \\
\hline$=0$ & GRN & 2896 & granulin & 2,32 & chr17 \\
\hline 要 & GSN & 2934 & gelsolin (amyloidosis, Finnish type) & 5,06 & chr9 \\
\hline $\begin{array}{lll}\pi \\
0\end{array}$ & GSTK1 & 373156 & glutathione S-transferase kappa 1 & 2,08 & chr7 \\
\hline 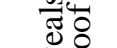 & GSTM2 & 2946 & glutathione S-transferase M2 (muscle) & 2,11 & chr1 \\
\hline ة & GTF2H1 & 2965 & general transcription factor IIH, polypeptide $1,62 \mathrm{kDa}$ & 2,29 & chr11 \\
\hline$\exists \Xi$ & GTPBP5 & 26164 & GTP binding protein 5 (putative) & 2,86 & chr20 \\
\hline S. & GUK1 & 2987 & guanylate kinase 1 /// guanylate kinase 1 & 2,41 & chr1 \\
\hline$E$ & H6PD & 9563 & hexose-6-phosphate dehydrogenase (glucose 1-dehydrogenase) & 4,63 & chr1 \\
\hline & HBEGF & 1839 & heparin-binding EGF-like growth factor & 11,83 & chr5 \\
\hline 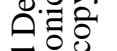 & HBP1 & 26959 & HMG-box transcription factor 1 & 2,71 & chr7 \\
\hline 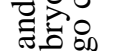 & HCFC2 & 29915 & host cell factor C2 & 3,62 & chr12 \\
\hline$E$ & HDLBP & 3069 & high density lipoprotein binding protein (vigilin) & 3,06 & chr2 \\
\hline$\Xi$ & HECTD2 & 143279 & HECT domain containing 2 & 2,37 & chr10 \\
\hline 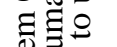 & HERC4 & 26091 & hect domain and RLD 4 & 2,76 & chr10 \\
\hline & HERPUD1 & 9709 & homocysteine-inducible, endoplasmic reticulum stress-inducible, ubiquitin-like domain member 1 & 9,52 & chr16 \\
\hline D̊ & HIF1A & 3091 & hypoxia-inducible factor 1 , alpha subunit (basic helix-loop-helix transcription factor) & 2,04 & chr14 \\
\hline & HIST1H2BC & 8347 & histone $1, \mathrm{H} 2 \mathrm{bc}$ & 2,42 & chr6 \\
\hline & $\mathrm{HIST} 1 \mathrm{H} 4 \mathrm{H}$ & 8365 & histone $1, \mathrm{H} 4 \mathrm{~h}$ & 2,57 & chr6 \\
\hline & HIVEP3 & 59269 & Human immunodeficiency virus type I enhancer binding protein 3 & 2,40 & chr1 \\
\hline 蔍 & HLA-B & 3106 & major histocompatibility complex, class I, B & 2,92 & chr6 \\
\hline$\underline{E}$ & HLA-C & 3107 & major histocompatibility complex, class I, C & 2,61 & chr6 \\
\hline & HLA-E & 3133 & major histocompatibility complex, class I, E & 5,94 & chr6 \\
\hline 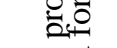 & HLX1 & 3142 & H2.0-like homeo box 1 (Drosophila) & 2,35 & chr1 \\
\hline 8 & HNRPLL & 92906 & heterogeneous nuclear ribonucleoprotein L-like & 2,32 & chr2 \\
\hline$\overbrace{0}^{2}$ & HNRPUL2 & 221092 & heterogeneous nuclear ribonucleoprotein U-like 2 & 2,01 & chr11 \\
\hline ta & HOM-TES-103 & 25900 & HOM-TES-103 tumor antigen-like & 2,52 & chr12 \\
\hline$=$ & HOOK3 & 84376 & Hook homolog 3 (Drosophila) & 2,95 & chr8 \\
\hline & HOXB7 & 3217 & homeo box B7 & 2,76 & chr17 \\
\hline
\end{tabular}




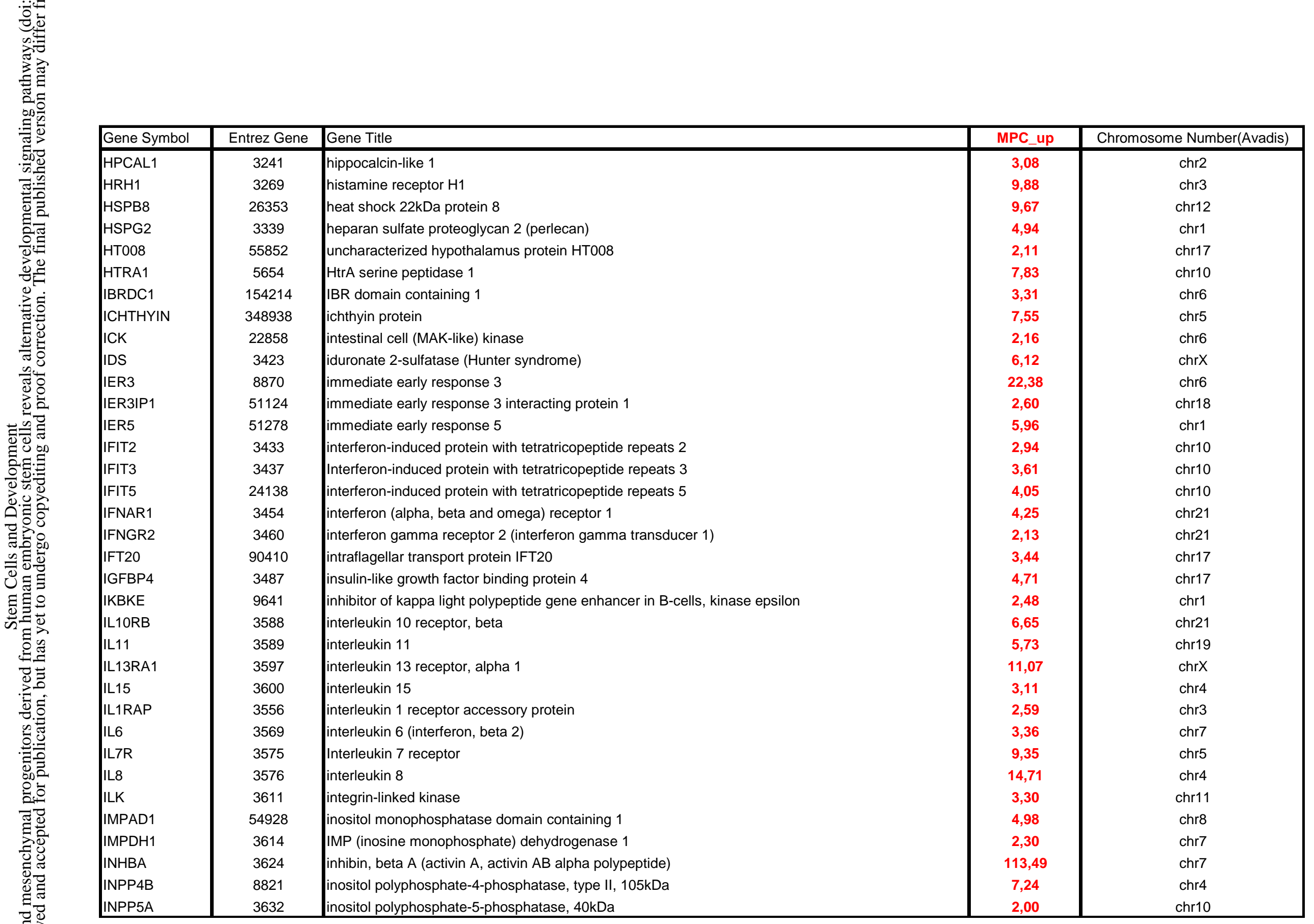




\begin{tabular}{|c|c|c|c|c|c|}
\hline$\Xi$ & Gene Symbol & Entrez Gene & Gene Title & MPC_up & Chromosome Number(Avadis) \\
\hline 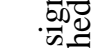 & IQWD1 & 55827 & IQ motif and WD repeats 1 & 3,48 & chr1 \\
\hline$\Xi \bar{\Xi}=$ & |IRAK2 & 3656 & interleukin-1 receptor-associated kinase 2 & 4,82 & chr3 \\
\hline $\bar{\Xi} \bar{z}$ & IRF2BP2 & 359948 & linterferon regulatory factor 2 binding protein 2 & 3,69 & chr1 \\
\hline อีำ & ISGF3G & 10379 & Interferon-stimulated transcription factor 3 , gamma $48 \mathrm{kDa}$ & 2,21 & chr14 \\
\hline Q & |TGA11 & 22801 & |integrin, alpha 11 & 12,78 & chr15 \\
\hline 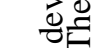 & ITGA2 & 3673 & Integrin, alpha 2 (CD49B, alpha 2 subunit of VLA-2 receptor) & 8,92 & chr5 \\
\hline 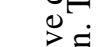 & ITGA3 & 3675 & integrin, alpha 3 (antigen CD49C, alpha 3 subunit of VLA-3 receptor) & 6,45 & chr17 \\
\hline 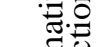 & ITGA5 & 3678 & Iintegrin, alpha 5 (fibronectin receptor, alpha polypeptide) & 14,20 & chr12 \\
\hline & |TGB1 & 3688 & Integrin, beta 1 (fibronectin receptor, beta polypeptide, antigen CD29 includes MDF2, MSK12) & 8,89 & chr10 \\
\hline$\pi \delta$ & |TGB1BP1 & 9270 & integrin beta 1 binding protein 1 & 3,04 & chr2 \\
\hline 당 & ITGB3 & 3690 & integrin, beta 3 (platelet glycoprotein IIla, antigen CD61) & 3,21 & chr17 \\
\hline ية & ITM2B & 9445 & integral membrane protein $2 \mathrm{~B}$ & 2,02 & chr13 \\
\hline$=\overline{0}$ & ITPR2 & 3709 & Family with sequence similarity 20 , member $\mathrm{C}$ & 2,21 & chr12 \\
\hline 6 & JAK1 & $3716 / / / 391045$ & Janus kinase 1 (a protein tyrosine kinase) /// similar to Solute carrier family 2 , facilitated glucose trans & 9,31 & chr1 \\
\hline$\Xi$ & JAK2 & 3717 & Janus kinase 2 (a protein tyrosine kinase) & 2,41 & chr9 \\
\hline & JRKL & 8690 & jerky homolog-like (mouse) & 2,56 & chr11 \\
\hline 5 & JuN & 3725 & V-jun sarcoma virus 17 oncogene homolog (avian) & 5,16 & chr1 \\
\hline E 60 & JUND & 3727 & jun D proto-oncogene & 2,01 & chr19 \\
\hline 西 & |KATNAL1 & 84056 & katanin p60 subunit A-like 1 & 3,79 & chr13 \\
\hline$\Xi$ & |KCNG1 & 3755 & potassium voltage-gated channel, subfamily G, member 1 & 3,92 & chr20 \\
\hline & KCNMA1 & 3778 & potassium large conductance calcium-activated channel, subfamily M, alpha member 1 & 18,21 & chr10 \\
\hline & KCTD10 & 83892 & potassium channel tetramerisation domain containing 10 & 3,83 & chr12 \\
\hline $8 \pi$ & KCTD11 & 147040 & potassium channel tetramerisation domain containing 11 & 2,09 & chr17 \\
\hline & |KCTD18 & 130535 & potassium channel tetramerisation domain containing 18 & 4,78 & chr2 \\
\hline & KCTD9 & 54793 & potassium channel tetramerisation domain containing 9 & 2,44 & chr9 \\
\hline & KDELR2 & 11014 & KDEL (Lys-Asp-Glu-Leu) endoplasmic reticulum protein retention receptor 2 & 4,73 & $\operatorname{chr} 7$ \\
\hline 㟒苞 & KDELR3 & 11015 & KDEL (Lys-Asp-Glu-Leu) endoplasmic reticulum protein retention receptor 3 & 43,78 & chr22 \\
\hline 눌 & KIAA0063 & 9929 & KIAA0063 gene product & 2,75 & chr22 \\
\hline & KIAA0090 & 23065 & KIAA0090 & 2,14 & chr1 \\
\hline \pm & KIAA0143 & 23167 & KIAA0143 protein & 2,03 & chr8 \\
\hline 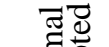 & KIAA0256 & 9728 & |KIAA0256 gene product & 2,42 & chr15 \\
\hline త్రి & |KIAA0268 & $7 / / / / 375056 / / / 4$ & C219-reactive peptide /// AAAP6077 /// similar to C219-reactive peptide & 2,32 & chr1_random \\
\hline 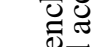 & |KIAA0310 & 9919 & |KIAA0310 & 2,07 & chr9 \\
\hline 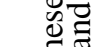 & |KIAA0372 & 9652 & |KIAA0372 & 4,17 & chr5 \\
\hline & KIAA0427 & 9811 & KIAA0427 & 4,02 & chr18 \\
\hline
\end{tabular}




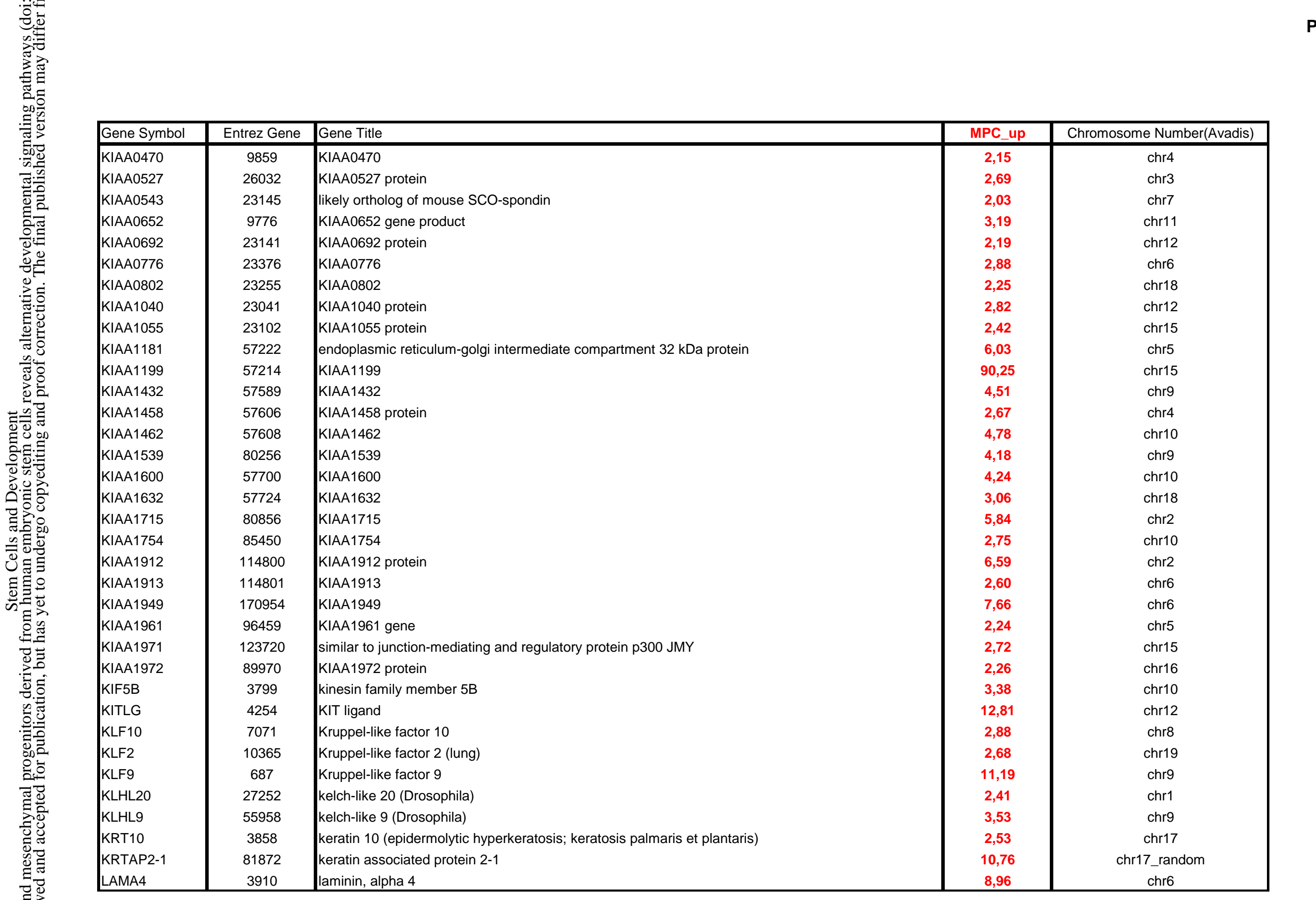




\begin{tabular}{|c|c|c|c|c|c|}
\hline 灵 & Gene Symbol & Entrez Gene & Gene Title & MPC_up & Chromosome Number(Avadis) \\
\hline 50] & LAMB1 & 3912 & laminin, beta 1 & 3,05 & chr7 \\
\hline$\Xi \overline{0}$ & LAMC1 & 3915 & laminin, gamma 1 (formerly LAMB2) & 4,84 & chr1 \\
\hline 婹 & LARP6 & 55323 & La ribonucleoprotein domain family, member 6 & 7,55 & chr15 \\
\hline हैं & |LASP1 & 3927 & LIM and SH3 protein 1 & 3,61 & chr17 \\
\hline QE & LATS2 & 26524 & LATS, large tumor suppressor, homolog 2 (Drosophila) & 2,71 & chr13 \\
\hline 远 & LDB3 & 11155 & LIM domain binding 3 & 14,73 & chr10 \\
\hline$D$ & LENG4 & 79143 & leukocyte receptor cluster (LRC) member 4 & 2,11 & chr19 \\
\hline$=$ & |LEPR & $3953 / / / 54741$ & leptin receptor /// leptin receptor overlapping transcript & 3,49 & chr1 \\
\hline Ẽ & LEPRE1 & 64175 & leucine proline-enriched proteoglycan (leprecan) 1 & 4,05 & chr1 \\
\hline 藏 & |LEPREL2 & 10536 & leprecan-like 2 & 2,51 & chr12 \\
\hline एँ & LGALS3 & $3958 / / / 81625$ & lectin, galactoside-binding, soluble, 3 (galectin 3) /// galectin-3 internal gene & 10,80 & chr14 \\
\hline 象 & LGALS3BP & 3959 & lectin, galactoside-binding, soluble, 3 binding protein & 7,85 & chr17 \\
\hline$\Rightarrow \overline{=}$ & LGMN & 5641 & legumain & 2,55 & chr13 \\
\hline 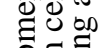 & LHFP & 10186 & lipoma HMGIC fusion partner & 8,04 & chr13 \\
\hline$\Xi$ & LHX8 & 431707 & LIM homeobox 8 & 5,89 & chr1 \\
\hline & LIF & 3976 & leukemia inhibitory factor (cholinergic differentiation factor) & 8,10 & chr22 \\
\hline : & |LIG4 & 3981 & ligase IV, DNA, ATP-dependent & 3,28 & chr13 \\
\hline 耐品 & LIMS1 & 3987 & LIM and senescent cell antigen-like domains 1 & 3,84 & chr2 \\
\hline 西 & LIMS3 & 96626 & LIM and senescent cell antigen-like domains 3 & 7,42 & chr2 \\
\hline$\overline{\tilde{U}} \Xi \bar{\Xi}$ & |LMAN1 & 3998 & lectin, mannose-binding, 1 & 3,18 & chr18 \\
\hline 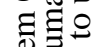 & LMBRD2 & 92255 & LMBR1 domain containing 2 & 3,11 & chr5 \\
\hline & |LMCD1 & 29995 & LIM and cysteine-rich domains 1 & 11,48 & chr3 \\
\hline O & LMNA & 4000 & $\operatorname{lamin} A / C$ & 11,58 & chr1 \\
\hline & LMO7 & 4008 & LIM domain 7 & 22,68 & chr13 \\
\hline & |LMOD1 & 25802 & leiomodin 1 (smooth muscle) & 9,05 & chr1 \\
\hline & LOC126917 & 126917 & hypothetical protein LOC126917 & 3,01 & chr1 \\
\hline 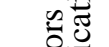 & LOC133308 & 133308 & hypothetical protein BC009732 & 2,30 & chr4 \\
\hline 늘 & LOC134147 & 134147 & similar to mouse 2310016A09Rik gene & 2,24 & chr5 \\
\hline & LOC143903 & 143903 & layilin & 19,22 & chr11 \\
\hline 每 & LOC144363 & 144363 & hypothetical protein LOC144363 & 3,55 & chr12 \\
\hline 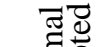 & LOC144871 & 144871 & Hypothetical protein LOC144871 & 3,58 & chr13 \\
\hline ఖ్రి & LOC149478 & 149478 & Hypothetical protein LOC149478 & 2,32 & chr1 \\
\hline 5 & LOC162073 & 162073 & Hypothetical protein LOC162073 & 10,19 & chr16 \\
\hline 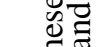 & LOC168850 & 168850 & hypothetical protein LOC168850 & 3,81 & $\operatorname{chr} 7$ \\
\hline & LOC196463 & 196463 & Hypothetical protein LOC196463 & 2,79 & chr12 \\
\hline
\end{tabular}




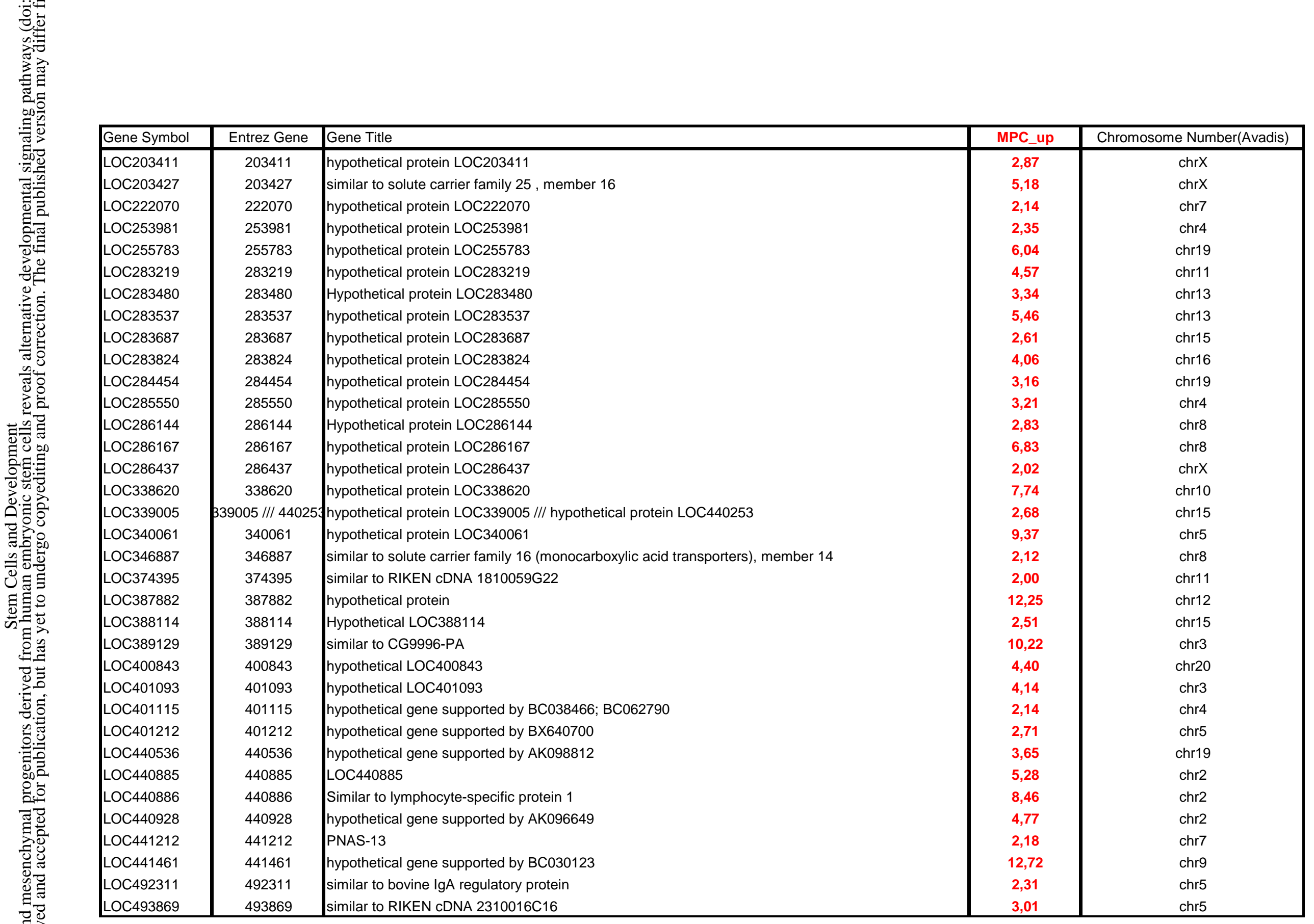




\begin{tabular}{|c|c|c|c|c|c|}
\hline 毒 & Gene Symbol & Entrez Gene & Gene Title & MPC_up & Chromosome Number(Avadis) \\
\hline 50] & LOC51315 & 51315 & hypothetical protein LOC51315 & 3,50 & chr2 \\
\hline 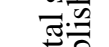 & LOC51334 & 51334 & mesenchymal stem cell protein DSC54 & 14,29 & chr5 \\
\hline $\bar{\Xi} \bar{\Xi}$ & LOC554202 & 554202 & hypothetical LOC554202 & 2,23 & chr9 \\
\hline हैं & LOC92689 & 92689 & hypothetical protein BC001096 & 39,06 & chr4 \\
\hline QE & LOC93349 & 93349 & hypothetical protein BC004921 & 9,80 & chr2 \\
\hline 远 & LOC96610 & 96610 & Hypothetical protein similar to KIAA0187 gene product & 3,16 & chr9 \\
\hline 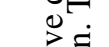 & LOX & 4015 & lysyl oxidase & 388,97 & chr5 \\
\hline$=$ & |LOXL4 & 84171 & lysyl oxidase-like 4 & 2,54 & chr10 \\
\hline 递 & LPP & 4026 & LIM domain containing preferred translocation partner in lipoma & 4,68 & chr3 \\
\hline$\pi \overline{0}$ & LRAP & 64167 & Leukocyte-derived arginine aminopeptidase & 4,38 & chr5 \\
\hline 8 & LRIG3 & 121227 & leucine-rich repeats and immunoglobulin-like domains 3 & 3,72 & chr12 \\
\hline 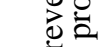 & LRP1 & 4035 & low density lipoprotein-related protein 1 (alpha-2-macroglobulin receptor) & 4,26 & chr12 \\
\hline$\Rightarrow \overline{=}$ & LRP10 & 26020 & low density lipoprotein receptor-related protein 10 & 15,41 & chr14 \\
\hline 6 & LRP11 & 84918 & low density lipoprotein receptor-related protein 11 & 3,70 & chr6 \\
\hline$\Xi \Xi$ & LRRC15 & 131578 & leucine rich repeat containing 15 & 2,69 & chr3 \\
\hline & LRRC35 & 219899 & Leucine rich repeat containing 35 & 2,02 & chr11 \\
\hline zे & LRRC41 & 10489 & leucine rich repeat containing 41 & 2,31 & chr6 \\
\hline : & LRRC8C & 84230 & leucine rich repeat containing 8 family, member $\mathrm{C}$ & 3,01 & chr1 \\
\hline 西 & LRRFIP1 & 9208 & leucine rich repeat (in FLII) interacting protein 1 & 2,48 & chr2 \\
\hline$\Xi$ & LTB4DH & 22949 & leukotriene B4 12-hydroxydehydrogenase & 2,20 & chr9 \\
\hline 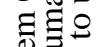 & LTBP2 & 4053 & latent transforming growth factor beta binding protein 2 & 13,08 & chr14 \\
\hline & LTBP3 & 4054 & latent transforming growth factor beta binding protein 3 & 9,77 & chr11 \\
\hline 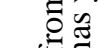 & LXN & 56925 & latexin & 4,64 & chr3 \\
\hline & LY96 & 23643 & lymphocyte antigen 96 & 3,86 & chr8 \\
\hline & LYPD1 & 116372 & LYG/PLAUR domain containing 1 & 7,27 & chr2 \\
\hline & LYPLA3 & 23659 & lysophospholipase 3 (lysosomal phospholipase A2) & 2,15 & chr16 \\
\hline 㟒苞 & LYSMD3 & 116068 & LysM, putative peptidoglycan-binding, domain containing 3 & 2,57 & chr5 \\
\hline 눌 & LYST & 1130 & lysosomal trafficking regulator & 6,29 & chr1 \\
\hline & LZTR2 & 89866 & leucine zipper transcription regulator 2 & 3,05 & chr1 \\
\hline 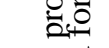 & |LZTS1 & 11178 & leucine zipper, putative tumor suppressor 1 & 3,77 & chr8 \\
\hline 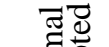 & M6PRBP1 & 10226 & mannose-6-phosphate receptor binding protein 1 & 3,61 & chr19 \\
\hline $\mathrm{C}_{0}^{2}$ & MAFF & 23764 & $\mathrm{v}$-maf musculoaponeurotic fibrosarcoma oncogene homolog $\mathrm{F}$ (avian) & 13,02 & chr22 \\
\hline : & MAGED1 & 9500 & melanoma antigen family D, 1 & 2,62 & $\operatorname{chr} \mathrm{X}$ \\
\hline$=$ & MAGED2 & 10916 & melanoma antigen family D, 2 & 2,62 & $\operatorname{chr} \mathrm{X}$ \\
\hline & MAN1B1 & 11253 & mannosidase, alpha, class 1B, member 1 & 2,03 & chr9 \\
\hline
\end{tabular}




\begin{tabular}{|c|c|c|c|c|c|}
\hline$\overline{7}$ & Gene Symbol & Entrez Gene & Gene Title & MPC_up & Chromosome Number(Avadis) \\
\hline $\bar{n}$ & MGC3123 & 79089 & hypothetical protein MGC3123 & 2,23 & chr17 \\
\hline $\bar{J} \bar{g}$ & MGC34646 & 157807 & Hypothetical protein MGC34646 & 10,17 & chr8 \\
\hline $\bar{E}$ & MGC34830 & 120196 & hypothetical protein MGC34830 & 4,48 & chr11 \\
\hline 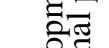 & MGC4677 & 112597 & hypothetical protein MGC4677 & 34,47 & chr2 \\
\hline QE & MGC5370 & 84825 & hypothetical protein MGC5370 & 3,31 & chr12 \\
\hline \pm & MGC5508 & 79073 & hypothetical protein MGC5508 & 2,29 & chr11 \\
\hline 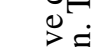 & MGC5618 & 79099 & hypothetical protein MGC5618 & 2,25 & chr6 \\
\hline$=$ & MGLL & 11343 & monoglyceride lipase /// monoglyceride lipase & 10,84 & chr3 \\
\hline 幽 & MICA & $4276 / / / 4277$ & MHC class I polypeptide-related sequence A /// MHC class I polypeptide-related sequence B & 8,37 & chr6 \\
\hline ते & MICA & 4276 & MHC class I polypeptide-related sequence A & 7,74 & chr6 \\
\hline (1) & MICAL2 & 9645 & microtubule associated monoxygenase, calponin and LIM domain containing 2 & 127,34 & chr11 \\
\hline 常 & MINA & 84864 & MYC induced nuclear antigen & 2,01 & chr3 \\
\hline$=\overline{0}$ & MIRN21 & 406991 & microRNA 21 & 7,85 & chr9 \\
\hline 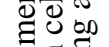 & MKRN2 & 23609 & makorin, ring finger protein, 2 & 2,93 & chr3 \\
\hline ఏి & MLLT11 & 10962 & myeloid/lymphoid or mixed-lineage leukemia (trithorax homolog, Drosophila); translocated to, $11 / / / \mathrm{my}$ & 2,34 & chr1 \\
\hline & MLPH & 79083 & melanophilin & 8,12 & chr2 \\
\hline 0 & MLXIP & 22877 & MLX interacting protein & 2,75 & chr12 \\
\hline : & MME & 4311 & membrane metallo-endopeptidase (neutral endopeptidase, enkephalinase, CALLA, CD10) & 7,11 & chr3 \\
\hline 西 & MMP1 & 4312 & matrix metallopeptidase 1 (interstitial collagenase) & 114,26 & chr11 \\
\hline 拝 & MMP14 & 4323 & matrix metallopeptidase 14 (membrane-inserted) & 2,53 & chr14 \\
\hline & MMP16 & 4325 & matrix metallopeptidase 16 (membrane-inserted) & 3,45 & chr8 \\
\hline & MMP19 & 4327 & matrix metallopeptidase 19 & 2,74 & chr12 \\
\hline O & MMP2 & 4313 & matrix metallopeptidase 2 (gelatinase A, 72kDa gelatinase, $72 \mathrm{kDa}$ type IV collagenase) & 20,82 & chr16 \\
\hline & MOBKL2A & 126308 & MOB1, Mps One Binder kinase activator-like 2A (yeast) & 6,42 & chr19 \\
\hline & MOcs2 & 4338 & molybdenum cofactor synthesis 2 & 2,85 & chr5 \\
\hline & MONDOA & 22877 & MondoA & 2,18 & chr12 \\
\hline t艹 & MOSPD1 & 56180 & motile sperm domain containing 1 & 2,48 & $\operatorname{chr} \mathrm{X}$ \\
\hline & MOSPD2 & 158747 & motile sperm domain containing 2 & 2,23 & $\operatorname{chrX}$ \\
\hline & MRAS & 22808 & muscle RAS oncogene homolog & 5,80 & chr3 \\
\hline \pm & MRCL3 & 10627 & myosin regulatory light chain MRCL3 & 2,21 & chr18 \\
\hline (ב) & MRCL3 & $103910 / / / 10627$ & myosin regulatory light chain MRCL3 /// myosin regulatory light chain MRLC2 & 2,50 & chr18 \\
\hline : & MRLC2 & 103910 & myosin regulatory light chain MRLC2 & 2,26 & chr18 \\
\hline & MRVI1 & 10335 & Murine retrovirus integration site 1 homolog & 3,75 & chr11 \\
\hline 8 & MSN & 4478 & moesin & 4,80 & chr5 \\
\hline & MTCBP-1 & 55256 & membrane-type 1 matrix metalloproteinase cytoplasmic tail binding protein-1 & 2,21 & chr2 \\
\hline
\end{tabular}




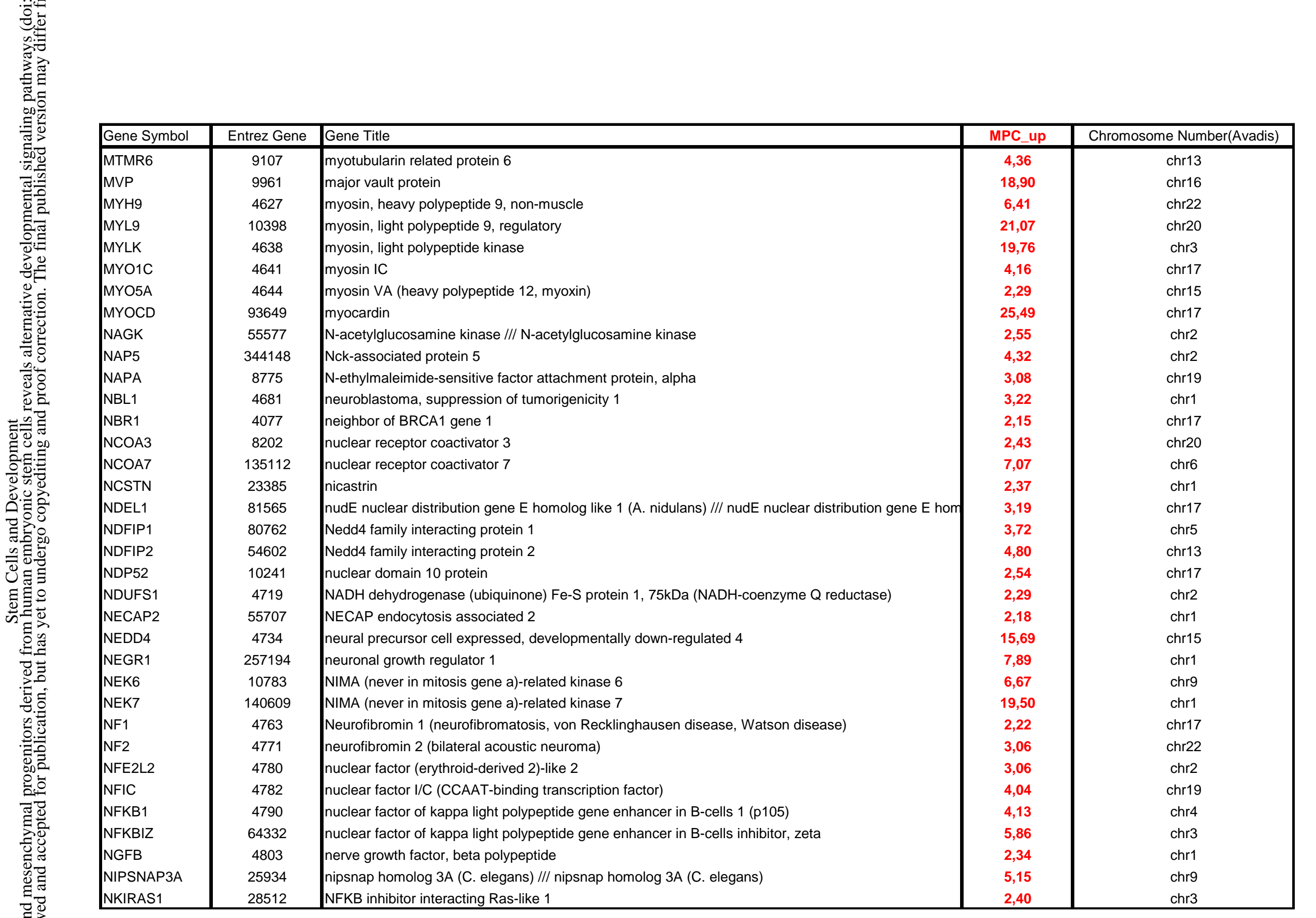




\begin{tabular}{|c|c|c|c|c|c|}
\hline : & Gene Symbol & Entrez Gene & Gene Title & MPC_up & Chromosome Number(Avadis) \\
\hline 50] & NKIRAS2 & 28511 & NFKB inhibitor interacting Ras-like 2 & 2,35 & chr17 \\
\hline$\Xi \overline{0}$ & NKX3-1 & 4824 & NK3 transcription factor related, locus 1 (Drosophila) & 2,35 & chr8 \\
\hline बे & NNMT & 4837 & nicotinamide N-methyltransferase & 261,83 & chr11 \\
\hline อีำ & NNT & 23530 & nicotinamide nucleotide transhydrogenase & 3,92 & chr5 \\
\hline Q & NOD27 & 84166 & nucleotide-binding oligomerization domains 27 & 2,94 & chr16 \\
\hline 远 & NOL3 & 8996 & nucleolar protein 3 (apoptosis repressor with CARD domain) & 2,25 & chr16 \\
\hline$D$ & NPAL3 & 57185 & NIPA-like domain containing 3 & 9,52 & chr1 \\
\hline 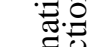 & NPAS2 & 4862 & neuronal PAS domain protein 2 & 3,09 & chr2 \\
\hline 过 & NQO1 & 1728 & $\mathrm{NAD}(\mathrm{P}) \mathrm{H}$ dehydrogenase, quinone 1 & 6,69 & chr16 \\
\hline 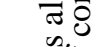 & NR3C1 & 2908 & nuclear receptor subfamily 3, group C, member 1 (glucocorticoid receptor) /// nuclear receptor subfam & 7,55 & chr5 \\
\hline ¿艹 & NRXN3 & 9369 & neurexin 3 & 3,03 & chr14 \\
\hline 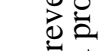 & NS3TP2 & 65983 & HCV NS3-transactivated protein 2 & 2,89 & chr5 \\
\hline$\exists=$ & NS5ATP13TP2 & 220323 & NS5ATP13TP2 protein & 5,13 & chr11 \\
\hline Bo & NSF & 4905 & N-ethylmaleimide-sensitive factor & 2,57 & chr17 \\
\hline$\exists$ & NT5E & 4907 & 5'-nucleotidase, ecto (CD73) & 83,19 & chr6 \\
\hline$\sum_{2}^{D}$ & NTN4 & 59277 & netrin 4 & 68,83 & chr12 \\
\hline o. & NUCB1 & 4924 & nucleobindin 1 & 2,67 & chr19 \\
\hline 6 & NUCB2 & 4925 & nucleobindin 2 & 4,76 & chr11 \\
\hline E & NUDT4 & 11163 & Nudix (nucleoside diphosphate linked moiety X)-type motif 4 pseudogene 2 & 4,85 & chr5 \\
\hline$\Xi$ & NUMB & 8650 & numb homolog (Drosophila) & 2,52 & chr14 \\
\hline \& & ОАСТ2 & 129642 & O-acyltransferase (membrane bound) domain containing 2 & 2,58 & chr2 \\
\hline & OCRL & 4952 & oculocerebrorenal syndrome of Lowe & 2,34 & $\operatorname{chrX}$ \\
\hline Dृ & OGFRL1 & 79627 & opioid growth factor receptor-like 1 & 2,63 & chr6 \\
\hline & ORMDL1 & 94101 & ORM1-like 1 (S. cerevisiae) & 2,79 & chr2 \\
\hline & os9 & 10956 & amplified in osteosarcoma & 4,49 & chr12 \\
\hline & OSAP & 84709 & ovary-specific acidic protein & 11,35 & chr4 \\
\hline 气ूة & OSMR & 9180 & Oncostatin M receptor & 49,48 & chr5 \\
\hline 눌 & OSTF1 & 26578 & osteoclast stimulating factor 1 & 2,21 & chr9 \\
\hline & OXTR & 5021 & oxytocin receptor & 94,66 & chr3 \\
\hline 年 & P4HA1 & 5033 & procollagen-proline, 2-oxoglutarate 4-dioxygenase (proline 4-hydroxylase), alpha polypeptide I & 5,06 & chr10 \\
\hline (8) & P4HA2 & 8974 & procollagen-proline, 2-oxoglutarate 4-dioxygenase (proline 4-hydroxylase), alpha polypeptide II & 21,34 & chr5 \\
\hline : & P4HA3 & 283208 & procollagen-proline, 2-oxoglutarate 4-dioxygenase (proline 4-hydroxylase), alpha polypeptide III & 3,18 & chr11 \\
\hline 둠주 & P4HB & 5034 & procollagen-proline, 2-oxoglutarate 4-dioxygenase (proline 4-hydroxylase), beta polypeptide (protein di & 3,93 & chr17 \\
\hline 5 & PACS1 & 55690 & phosphofurin acidic cluster sorting protein 1 & 2,20 & chr11 \\
\hline & PAEP & 5047 & progestagen-associated endometrial protein (placental protein 14, pregnancy-associated endometrial & 3,72 & chr19 \\
\hline
\end{tabular}




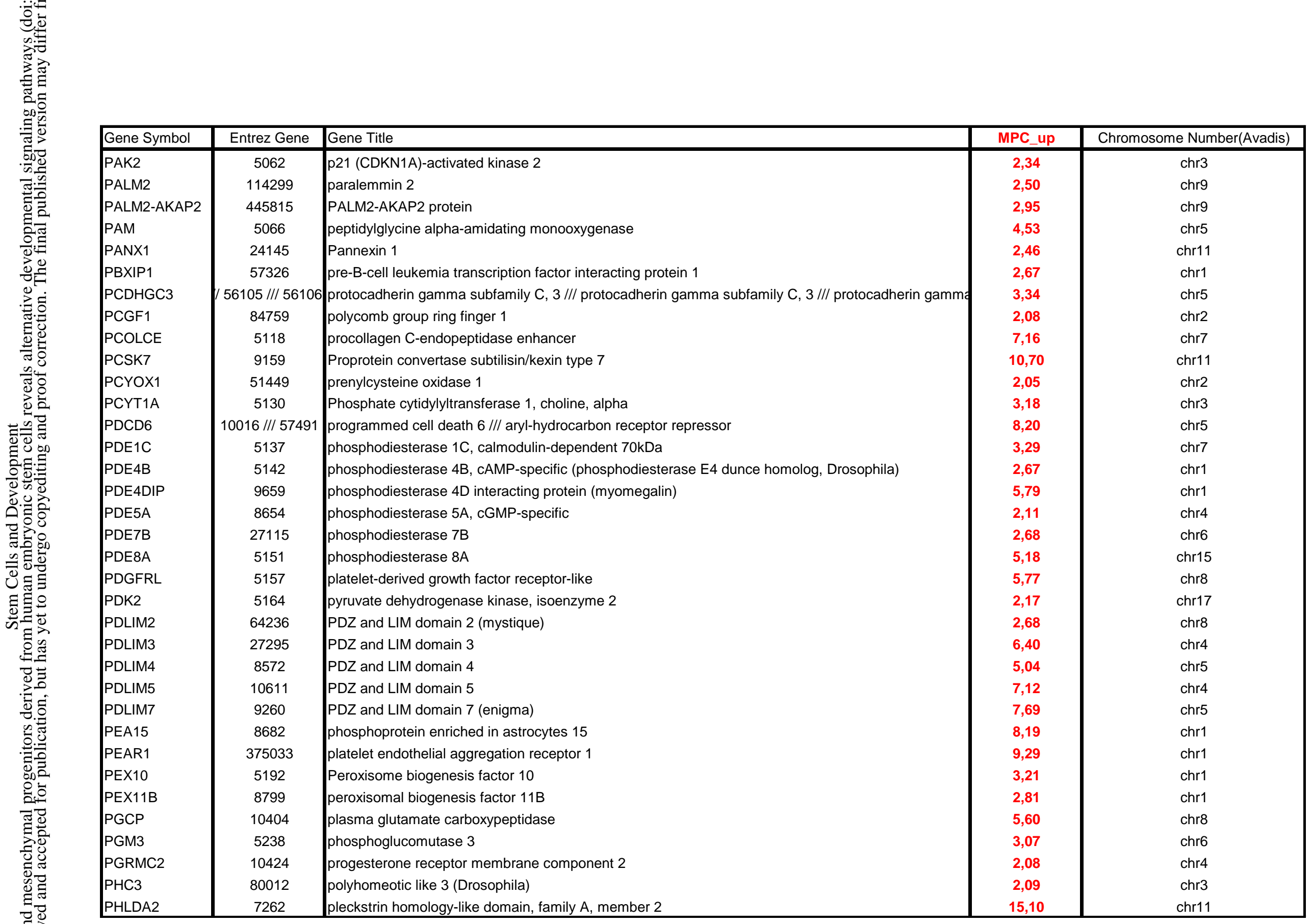




\begin{tabular}{|c|c|c|c|c|c|}
\hline 可 & Gene Symbol & Entrez Gene & Gene Title & MPC_up & Chromosome Number(Avadis) \\
\hline 50] & PHLDA3 & 23612 & pleckstrin homology-like domain, family A, member 3 & 4,83 & chr1 \\
\hline 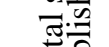 & PHLDB2 & 90102 & pleckstrin homology-like domain, family B, member 2 & 14,96 & chr3 \\
\hline बे & PHTF2 & 57157 & putative homeodomain transcription factor 2 & 2,69 & chr7 \\
\hline हैं & PICALM & 8301 & phosphatidylinositol binding clathrin assembly protein & 4,52 & chr11 \\
\hline QE & PIGX & 54965 & phosphatidylinositol glycan, class $\mathrm{X}$ & 2,28 & chr3 \\
\hline \pm & PIK4CB & 5298 & phosphatidylinositol 4-kinase, catalytic, beta polypeptide & 3,53 & chr1 \\
\hline$\therefore$ & PIP5K1C & 23396 & phosphatidylinositol-4-phosphate 5-kinase, type I, gamma & 2,21 & chr19 \\
\hline$=$ & PITRM1 & 10531 & pitrilysin metallopeptidase 1 & 2,67 & chr10 \\
\hline 过 & PJA2 & 9867 & praja 2, RING-H2 motif containing & 3,02 & chr5 \\
\hline$\pi \overline{0}$ & PKIG & 11142 & protein kinase (cAMP-dependent, catalytic) inhibitor gamma & 2,08 & chr20 \\
\hline (1) & PLA2G4A & 5321 & phospholipase A2, group IVA (cytosolic, calcium-dependent) & 3,38 & chr1 \\
\hline 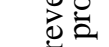 & PLAU & 5328 & plasminogen activator, urokinase & 16,09 & chr10 \\
\hline$\Rightarrow \overline{=}$ & PLAUR & 5329 & plasminogen activator, urokinase receptor & 18,67 & chr19 \\
\hline 3 & PLD1 & 5337 & phospholipase D1, phophatidylcholine-specific & 4,36 & chr3 \\
\hline$\exists$ & PLD3 & 23646 & phospholipase D family, member 3 & 3,91 & chr19 \\
\hline & PLDN & 26258 & pallidin homolog (mouse) & 2,39 & chr15 \\
\hline 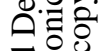 & PLEC1 & 5339 & plectin 1 , intermediate filament binding protein $500 \mathrm{kDa}$ & 2,66 & chr8 \\
\hline 0 & PLEKHA2 & 59339 & pleckstrin homology domain containing, family A (phosphoinositide binding specific) member 2 & 18,71 & chr8 \\
\hline 西 & PLEKHA3 & 65977 & pleckstrin homology domain containing, family A (phosphoinositide binding specific) member 3 & 2,58 & chr2 \\
\hline$\Xi$ & PLEKHC1 & 10979 & pleckstrin homology domain containing, family C (with FERM domain) member 1 & 2,27 & chr14 \\
\hline & PLK2 & 10769 & polo-like kinase 2 (Drosophila) & 18,51 & chr5 \\
\hline & PLK3 & 1263 & polo-like kinase 3 (Drosophila) & 2,02 & chr1 \\
\hline ప్రై & PLOD1 & 5351 & procollagen-lysine 1, 2-oxoglutarate 5-dioxygenase 1 & 9,76 & chr1 \\
\hline & |PLOD2 & 5352 & procollagen-lysine, 2-oxoglutarate 5-dioxygenase 2 & 10,14 & chr3 \\
\hline & PLOD3 & 8985 & procollagen-lysine, 2-oxoglutarate 5-dioxygenase 3 & 3,27 & chr7 \\
\hline & PLP2 & 5355 & proteolipid protein 2 (colonic epithelium-enriched) & 20,06 & $\operatorname{chr} \mathrm{x}$ \\
\hline 㟒苞 & PLSCR3 & $254863 / / / 57048$ & phospholipid scramblase 3 /// hypothetical protein MGC40107 & 3,10 & chr17 \\
\hline 눌 & PLSCR4 & 57088 & phospholipid scramblase 4 & 2,86 & chr3 \\
\hline & PLXNA3 & 55558 & plexin A3 & 2,83 & $\operatorname{chr} \mathrm{X}$ \\
\hline$=$ & PLXND1 & 23129 & plexin D1 & 3,22 & chr3 \\
\hline 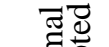 & PME-1 & 51400 & protein phosphatase methylesterase-1 & 3,23 & chr11 \\
\hline ఖ్రి & PMM1 & 5372 & phosphomannomutase 1 & 3,75 & chr22 \\
\hline$\pi$ & POLH & 5429 & Polymerase (DNA directed), eta & 3,42 & chr6 \\
\hline$=$ & POLK & 51426 & polymerase (DNA directed) kappa & 3,54 & chr5 \\
\hline & POLR3GL & 84265 & polymerase (RNA) III (DNA directed) polypeptide G (32kD) like & 6,75 & chr1 \\
\hline
\end{tabular}




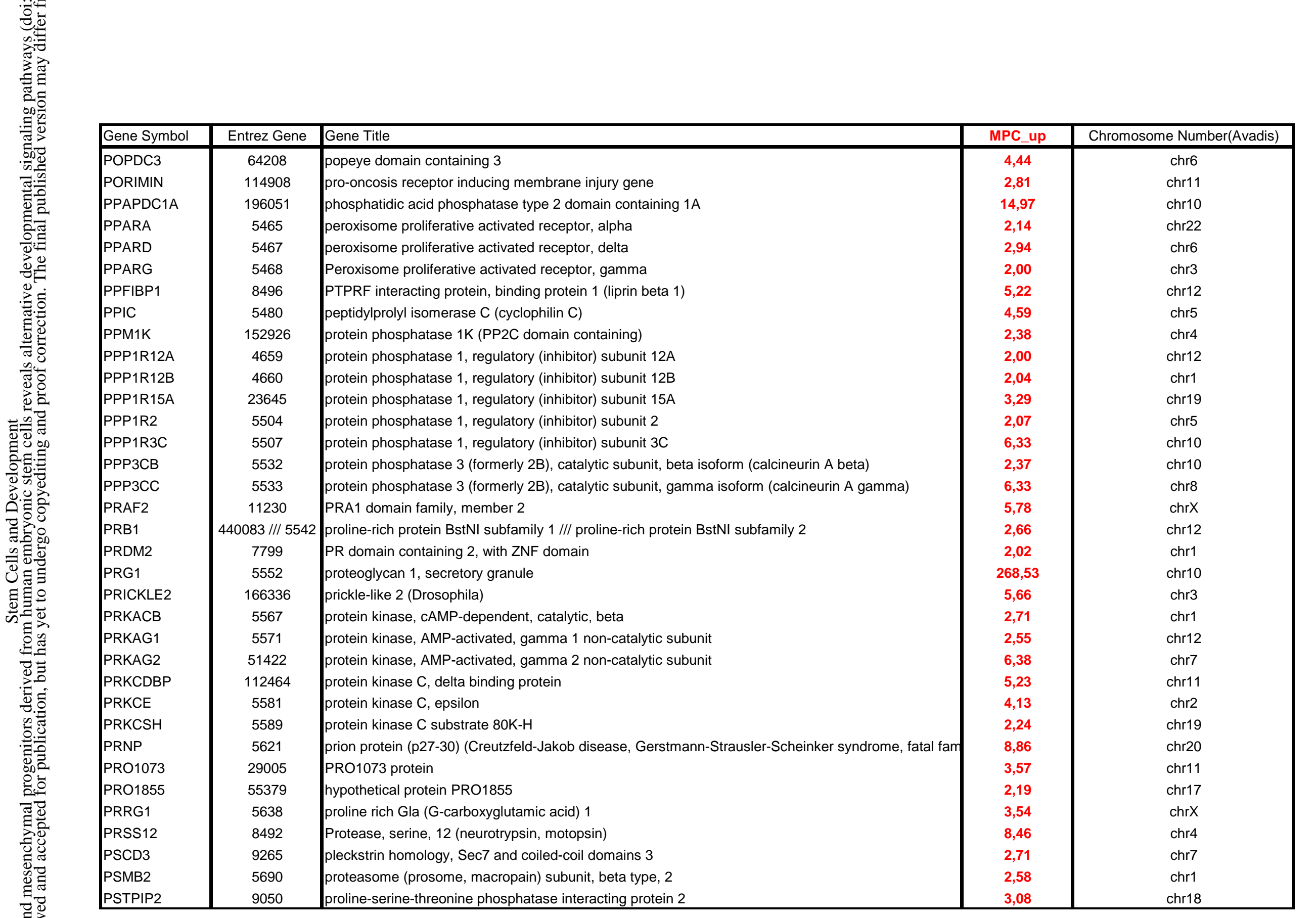




\begin{tabular}{|c|c|c|c|c|c|}
\hline 可 & Gene Symbol & Entrez Gene & Gene Title & MPC_up & Chromosome Number(Avadis) \\
\hline$\infty$ & PTGER2 & 5732 & prostaglandin E receptor 2 (subtype EP2), 53kDa & 2,68 & chr14 \\
\hline 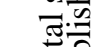 & PTGER4 & 5734 & prostaglandin E receptor 4 (subtype EP4) & 4,84 & chr5 \\
\hline बे & PTGFR & 5737 & prostaglandin $\mathrm{F}$ receptor $(\mathrm{FP})$ & 2,67 & chr1 \\
\hline हैं & PTGFRN & 5738 & prostaglandin F2 receptor negative regulator & 3,47 & chr1 \\
\hline 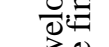 & PTGS2 & 5743 & prostaglandin-endoperoxide synthase 2 (prostaglandin $\mathrm{G} / \mathrm{H}$ synthase and cyclooxygenase) & 8,89 & chr1 \\
\hline 远 & PTHLH & 5744 & parathyroid hormone-like hormone /// parathyroid hormone-like hormone & 8,74 & chr12 \\
\hline$D$ & PTK9 & 5756 & PTK9 protein tyrosine kinase 9 & 4,53 & chr12 \\
\hline$=$ & PTP4A1 & 7803 & protein tyrosine phosphatase type IVA, member 1 & 3,48 & chr6 \\
\hline 可 & PTP4A2 & 8073 & protein tyrosine phosphatase type IVA, member 2 & 2,64 & chr1 \\
\hline 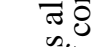 & PTPLA & 9200 & protein tyrosine phosphatase-like (proline instead of catalytic arginine), member a & 2,53 & chr10 \\
\hline 8 & PTPN11 & 5781 & protein tyrosine phosphatase, non-receptor type 11 (Noonan syndrome 1) & 3,00 & chr12 \\
\hline 年 & PTPN9 & 5780 & protein tyrosine phosphatase, non-receptor type 9 & 2,21 & chr15 \\
\hline$=\overline{0}$ & PTPNS1 & 140885 & protein tyrosine phosphatase, non-receptor type substrate 1 & 2,90 & chr20 \\
\hline 6 & PTRF & 284119 & polymerase I and transcript release factor & 22,23 & chr17 \\
\hline$\Xi \Xi$ & PTX1 & 51290 & PTX1 protein & 2,21 & chr12 \\
\hline & PVR & 5817 & poliovirus receptor & 3,52 & chr19 \\
\hline zे & PXDN & 7837 & peroxidasin homolog (Drosophila) & 3,26 & chr2 \\
\hline 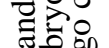 & PXK & 54899 & PX domain containing serine/threonine kinase & 5,67 & chr3 \\
\hline 西 & PXN & 5829 & paxillin & 2,21 & chr12 \\
\hline$\Xi$ & PYCR1 & 5831 & pyrroline-5-carboxylate reductase 1 & 2,10 & chr17 \\
\hline & PYGB & 5834 & phosphorylase, glycogen; brain & 3,06 & chr20 \\
\hline & QIL1 & 125988 & QIL1 protein & 2,87 & chr19 \\
\hline ప్ర & QSCN6 & 5768 & quiescin Q6 & 5,39 & chr1 \\
\hline & RAB11FIP2 & 22841 & RAB11 family interacting protein 2 (class I) & 2,07 & chr10 \\
\hline & RAB11FIP5 & 26056 & RAB11 family interacting protein 5 (class I) & 4,01 & chr2 \\
\hline & RAB18 & 22931 & RAB18, member RAS oncogene family & 2,36 & chr10 \\
\hline 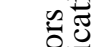 & RAB2 & 5862 & RAB2, member RAS oncogene family & 2,97 & chr8 \\
\hline 늘 & RAB23 & 51715 & RAB23, member RAS oncogene family & 4,32 & chr6 \\
\hline & RAB27A & 5873 & RAB27A, member RAS oncogene family & 4,20 & chr15 \\
\hline بح & RAB32 & 10981 & RAB32, member RAS oncogene family & 15,55 & chr6 \\
\hline 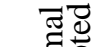 & RAB33A & 9363 & RAB33A, member RAS oncogene family & 2,53 & $\operatorname{chrX}$ \\
\hline త్రి & RAB3GAP1 & 22930 & RAB3 GTPase activating protein subunit 1 (catalytic) & 2,33 & chr2 \\
\hline & RAB6A & $5870 / / / 84084$ & RAB6A, member RAS oncogene family /// RAB6C, member RAS oncogene family & 2,01 & chr2 \\
\hline$=$ & RAB6IP2 & 23085 & RAB6 interacting protein 2 & 2,73 & chr12 \\
\hline 7 & RAB9B & 51209 & RAB9B, member RAS oncogene family & 2,03 & $\operatorname{chr} X$ \\
\hline
\end{tabular}




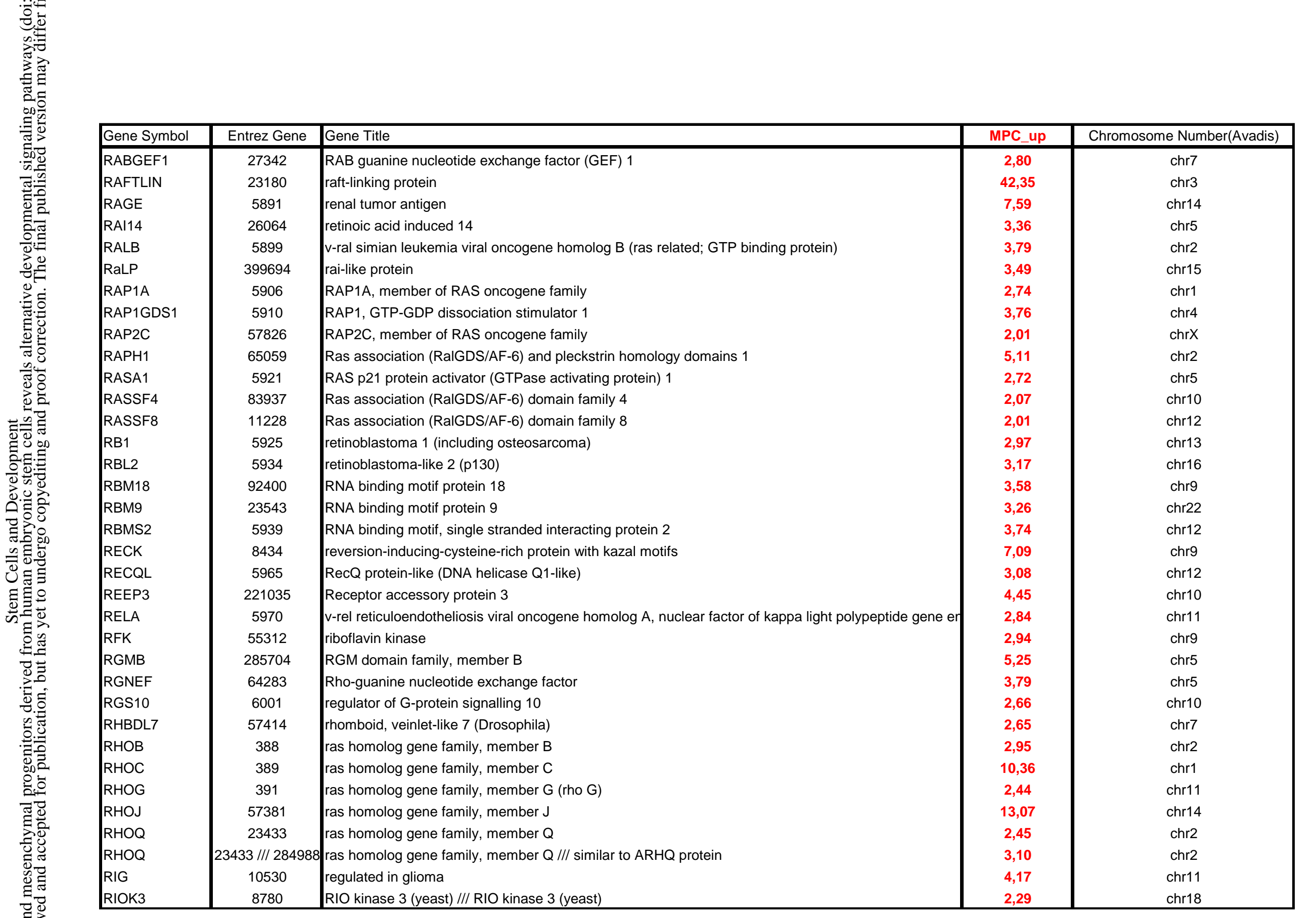




\section{Page 243 of 257}

\begin{tabular}{|c|c|c|c|c|}
\hline Gene Symbol & Entrez Gene & Gene Title & MPC_up & Chromosome Number(Avadis) \\
\hline RIPK1 & 8737 & receptor (TNFRSF)-interacting serine-threonine kinase 1 & 3,05 & chr6 \\
\hline RNASE4 & 6038 & ribonuclease, RNase A family, 4 & 10,14 & chr14 \\
\hline RND3 & 390 & Rho family GTPase 3 & 7,69 & chr2 \\
\hline RNF11 & 26994 & ring finger protein 11 & 2,28 & chr1 \\
\hline RNF14 & 9604 & ring finger protein 14 & 3,12 & chr5 \\
\hline RNF150 & 57484 & ring finger protein 150 & 2,03 & chr4 \\
\hline RNF185 & 91445 & ring finger protein 185 & 2,60 & chr22 \\
\hline RNF6 & 6049 & ring finger protein (С3H2C3 type) 6 & 3,18 & chr13 \\
\hline RNH1 & 6050 & ribonuclease/angiogenin inhibitor 1 & 3,39 & chr11 \\
\hline RP11-378J18.4 & 375056 & C219-reactive peptide & 2,95 & chr1 \\
\hline RP2 & 6102 & retinitis pigmentosa 2 (X-linked recessive) & 3,03 & $\operatorname{chrX}$ \\
\hline RPL23AP7 & 118433 & ribosomal protein L23a pseudogene 7 & 3,44 & chr1 \\
\hline RPS6KA2 & 6196 & ribosomal protein $\mathrm{S} 6$ kinase, 90kDa, polypeptide 2 & 4,08 & chr6 \\
\hline RRAD & 6236 & Ras-related associated with diabetes & 2,65 & chr16 \\
\hline RRAGB & 10325 & Ras-related GTP binding B & 2,23 & $\operatorname{chr} X$ \\
\hline RRAS & 6237 & related RAS viral (r-ras) oncogene homolog & 16,59 & chr19 \\
\hline RSN & 6249 & restin (Reed-Steinberg cell-expressed intermediate filament-associated protein) & 5,89 & chr12 \\
\hline RTN4 & 57142 & reticulon 4 & 2,24 & chr2 \\
\hline RUNX2 & 860 & runt-related transcription factor 2 & 10,68 & chr6 \\
\hline RUSC2 & 9853 & $\mathrm{RUN}$ and $\mathrm{SH} 3$ domain containing 2 & 3,56 & chr9 \\
\hline RXRB & 6257 & retinoid $\mathrm{X}$ receptor, beta & 2,65 & chr6 \\
\hline S100A11 & 6282 & S100 calcium binding protein A11 (calgizzarin) & 11,04 & chr1 \\
\hline S100A13 & 6284 & S100 calcium binding protein $\mathrm{A} 13$ & 2,75 & chr1 \\
\hline S100A16 & 140576 & S100 calcium binding protein A16 & 14,87 & chr1 \\
\hline SACS & 26278 & spastic ataxia of Charlevoix-Saguenay (sacsin) & 2,33 & chr13 \\
\hline SAMD4 & 23034 & sterile alpha motif domain containing 4 & 5,95 & chr14 \\
\hline SAMD9 & 54809 & sterile alpha motif domain containing 9 & 6,51 & chr7 \\
\hline SAR1B & 51128 & SAR1 gene homolog B (S. cerevisiae) & 3,44 & chr5 \\
\hline SAT & 6303 & Spermidine/spermine $\mathrm{N} 1$-acetyltransferase & 6,26 & $\operatorname{chr} X$ \\
\hline SATB2 & 23314 & SATB family member 2 & 2,94 & chr2 \\
\hline SATL1 & 340562 & Spermidine/spermine N1-acetyl transferase-like 1 & 4,19 & $\operatorname{chr} X$ \\
\hline SBDS & 51119 & Shwachman-Bodian-Diamond syndrome & 3,63 & chr7 \\
\hline SBDS /// SBDSP & $155370 / / / 51119$ & Shwachman-Bodian-Diamond syndrome /// Shwachman-Bodian-Diamond syndrome pseudogene & 3,67 & $\operatorname{chr} 7$ \\
\hline SC65 & 10609 & synaptonemal complex protein SC65 & 5,32 & chr17 \\
\hline SCAP2 & 8935 & src family associated phosphoprotein 2 & 2,24 & chr7 \\
\hline
\end{tabular}




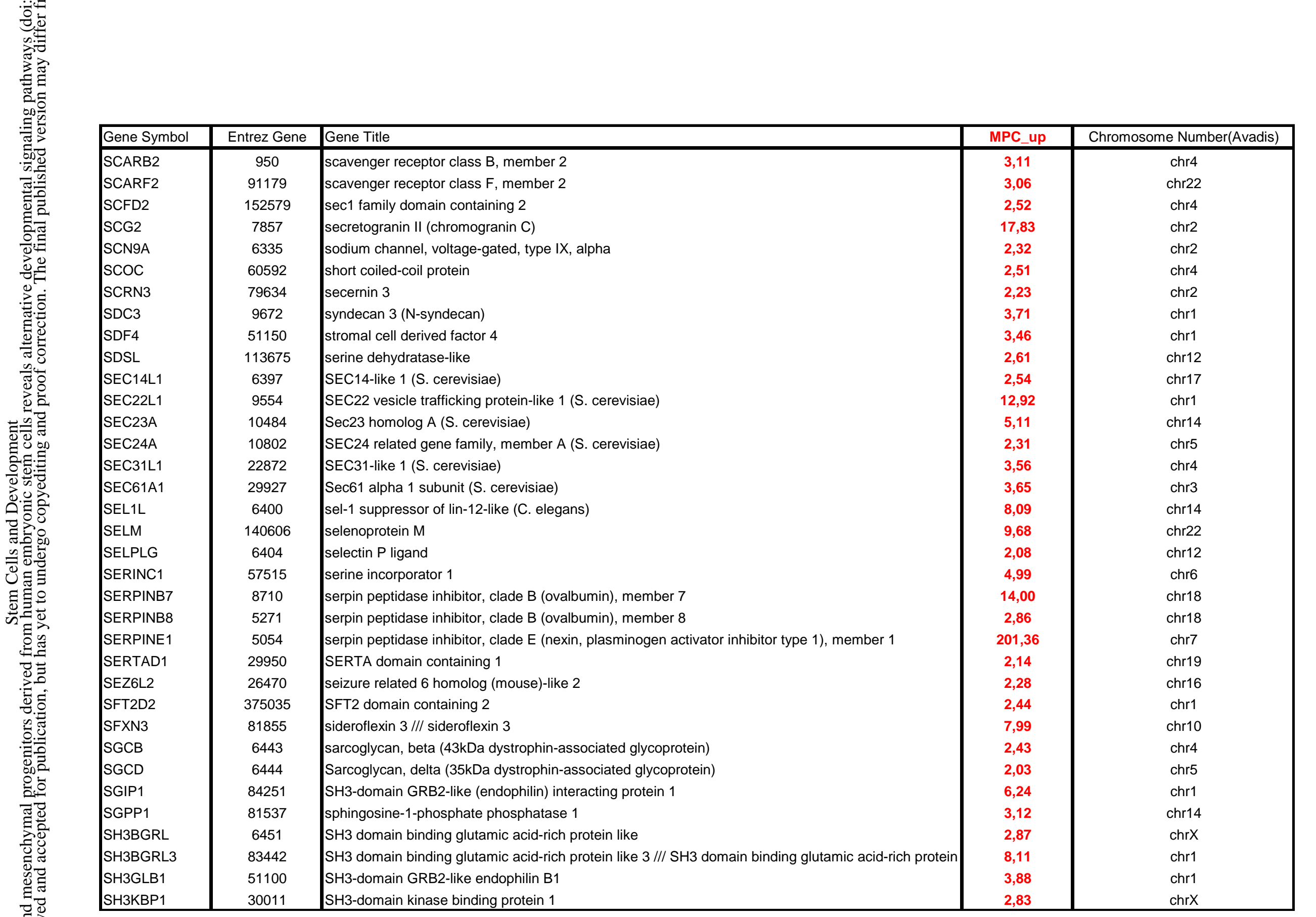




\begin{tabular}{|c|c|c|c|c|c|}
\hline$\pi$ & Gene Symbol & Entrez Gene & Gene Title & MPC_up & Chromosome Number(Avadis) \\
\hline क & SH3MD2 & 57630 & SH3 multiple domains 2 & 5,41 & chr4 \\
\hline క్రా:- & SH3MD4 & 344558 & SH3 multiple domains 4 & 5,15 & chr2 \\
\hline$\overline{\mathrm{d}} \overline{\mathrm{z}}$ & SH3RF2 & 153769 & SH3 domain containing ring finger 2 & 3,56 & chr5 \\
\hline ลีำ & SHB & 6461 & Src homology 2 domain containing adaptor protein B & 2,10 & chr9 \\
\hline อ & SHC1 & 6464 & SHC (Src homology 2 domain containing) transforming protein 1 & 2,21 & chr1 \\
\hline 8 & SIL1 & 64374 & SIL1 homolog, endoplasmic reticulum chaperone (S. cerevisiae) & 7,07 & chr5 \\
\hline$\stackrel{2}{2}$ & SIRT2 & 22933 & sirtuin (silent mating type information regulation 2 homolog) 2 (S. cerevisiae) & 2,87 & chr19 \\
\hline$=$ & SIX1 & 6495 & Sine oculis homeobox homolog 1 (Drosophila) & 112,74 & chr14 \\
\hline 8 & SLC10A3 & 8273 & solute carrier family 10 (sodium/bile acid cotransporter family), member 3 & 2,72 & $\operatorname{chr} \mathrm{x}$ \\
\hline $\bar{\pi}$ & SLC12A2 & 6558 & solute carrier family 12 (sodium/potassium/chloride transporters), member 2 & 2,07 & chr5 \\
\hline$=0$ & SLC12A4 & 6560 & solute carrier family 12 (potassium/chloride transporters), member 4 & 2,84 & chr16 \\
\hline ة & SLC16A7 & 9194 & solute carrier family 16 (monocarboxylic acid transporters), member 7 & 4,54 & chr12 \\
\hline$\exists \Xi$ & SLC17A5 & 26503 & solute carrier family 17 (anion/sugar transporter), member 5 & 7,10 & chr6 \\
\hline 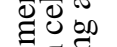 & SLC20A2 & 6575 & solute carrier family 20 (phosphate transporter), member 2 & 2,33 & chr8 \\
\hline$\Xi$ & SLC22A18 & 5002 & solute carrier family 22 (organic cation transporter), member 18 & 4,89 & chr11 \\
\hline & SLC22A4 & 6583 & solute carrier family 22 (organic cation transporter), member 4 & 2,24 & chr5 \\
\hline 2 & SLC25A20 & 788 & solute carrier family 25 (carnitine/acylcarnitine translocase), member 20 & 2,63 & chr3 \\
\hline 品 & SLC25A24 & 29957 & solute carrier family 25 (mitochondrial carrier; phosphate carrier), member 24 & 2,07 & chr1 \\
\hline 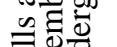 & SLC25A32 & 81034 & solute carrier family 25 , member 32 /// solute carrier family 25 , member 32 & 2,69 & chr8 \\
\hline$\Xi$ & SLC26A5 & 375611 & Solute carrier family 26 , member 5 (prestin) & 2,72 & chr7 \\
\hline & SLC2A10 & 81031 & solute carrier family 2 (facilitated glucose transporter), member $10 / / /$ solute carrier family 2 (facilitated & 5,13 & chr20 \\
\hline & SLC30A5 & 64924 & solute carrier family 30 (zinc transporter), member 5 & 2,22 & chr5 \\
\hline R & SLC30A7 & 148867 & solute carrier family 30 (zinc transporter), member 7 & 4,96 & chr1 \\
\hline & SLC31A2 & 1318 & solute carrier family 31 (copper transporters), member 2 & 3,06 & chr9 \\
\hline & SLC35A3 & 23443 & solute carrier family 35 (UDP-N-acetylglucosamine (UDP-GlcNAc) transporter), member A3 & 2,31 & chr1 \\
\hline छี & SLC35B2 & 347734 & solute carrier family 35, member B2 & 2,47 & chr6 \\
\hline 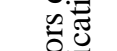 & SLC35B3 & 51000 & solute carrier family 35, member B3 & 4,68 & chr6 \\
\hline & SLC35C1 & 55343 & solute carrier family 35, member $\mathrm{C} 1$ & 2,03 & chr11 \\
\hline & SLC35E1 & 79939 & solute carrier family 35, member E1 & 3,25 & chr19 \\
\hline & SLC35F5 & 80255 & solute carrier family 35, member F5 & 4,00 & chr2 \\
\hline 递 & SLC39A13 & 91252 & solute carrier family 39 (zinc transporter), member 13 & 2,82 & chr11 \\
\hline$\overbrace{8}^{8}$ & SLC39A6 & 25800 & solute carrier family 39 (zinc transporter), member 6 & 2,72 & chr18 \\
\hline & SLC41A2 & 84102 & solute carrier family 41 , member 2 & 2,05 & chr12 \\
\hline & SLC41A3 & 54946 & solute carrier family 41 , member 3 & 2,02 & chr3 \\
\hline & SLC4A4 & 8671 & solute carrier family 4 , sodium bicarbonate cotransporter, member 4 & 5,47 & chr4 \\
\hline
\end{tabular}




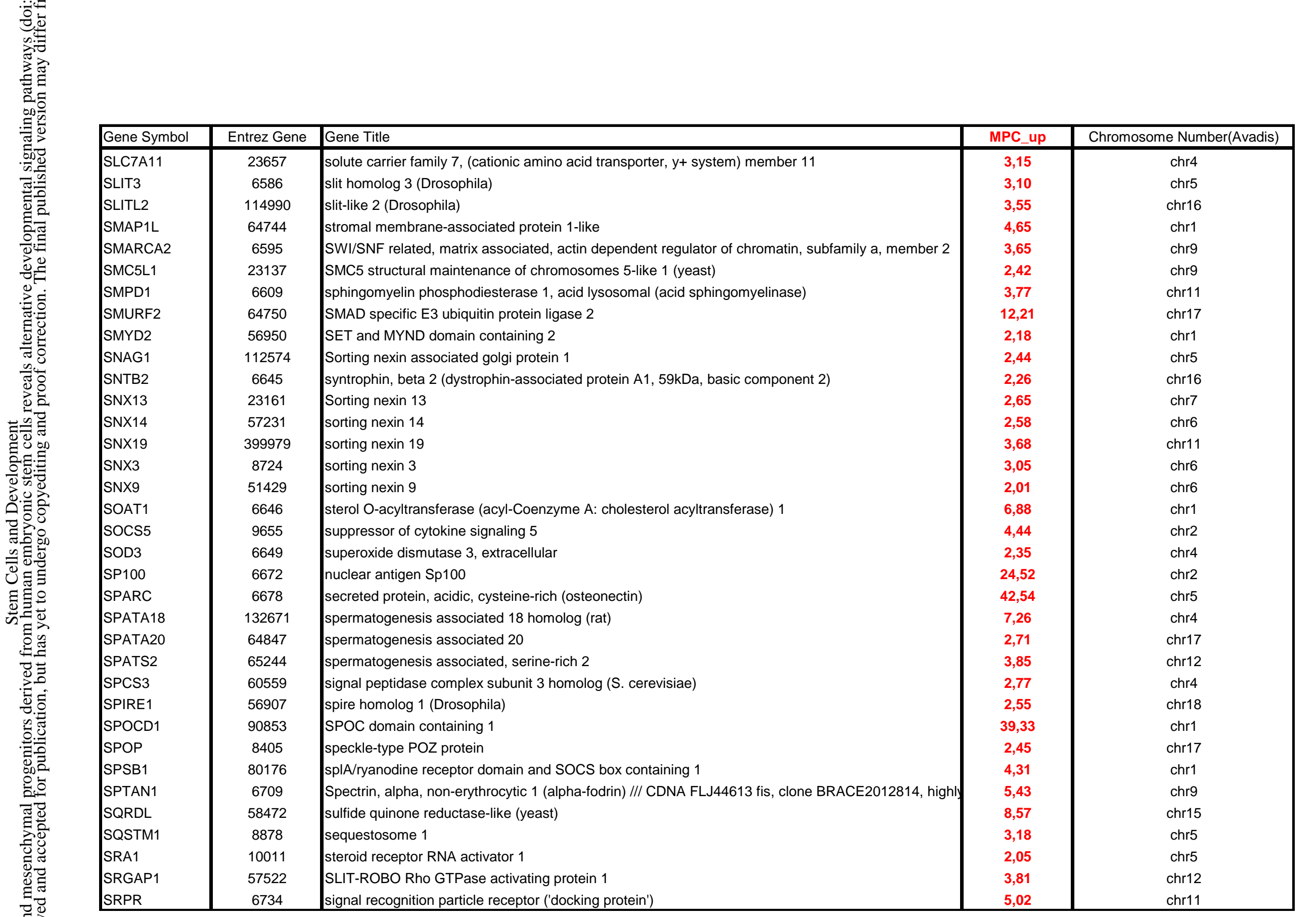




\begin{tabular}{|c|c|c|c|c|c|}
\hline 毒 & Gene Symbol & Entrez Gene & Gene Title & MPC_up & Chromosome Number(Avadis) \\
\hline .50 & SRPX & 8406 & sushi-repeat-containing protein, $X$-linked & 7,97 & $\operatorname{chrX}$ \\
\hline 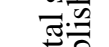 & SRPX2 & 27286 & sushi-repeat-containing protein, X-linked 2 & 18,18 & $\operatorname{chr} \mathrm{x}$ \\
\hline 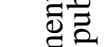 & SRXN1 & 140809 & sulfiredoxin 1 homolog (S. cerevisiae) & 2,09 & chr20 \\
\hline हैं & SSPN & 8082 & sarcospan (Kras oncogene-associated gene) & 16,19 & chr12 \\
\hline QE & SSR2 & 6746 & signal sequence receptor, beta (translocon-associated protein beta) & 2,83 & chr1 \\
\hline 远 & ST3GAL1 & 6482 & ST3 beta-galactoside alpha-2,3-sialyltransferase 1 & 5,49 & chr8 \\
\hline$D$ & ST7 & 7982 & suppression of tumorigenicity 7 & 2,02 & chr7 \\
\hline 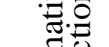 & STAM2 & 10254 & signal transducing adaptor molecule (SH3 domain and ITAM motif) 2 & 3,97 & chr2 \\
\hline 焉 & STARD13 & 90627 & START domain containing 13 & 7,46 & chr13 \\
\hline 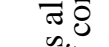 & STAT1 & 6772 & signal transducer and activator of transcription $1,91 \mathrm{kDa}$ & 2,12 & (vide) \\
\hline "艹 & STAT2 & 6773 & signal transducer and activator of transcription 2, $113 \mathrm{kDa}$ & 2,11 & chr12 \\
\hline ب & STAT6 & 6778 & signal transducer and activator of transcription 6 , interleukin- 4 induced & 2,49 & chr12 \\
\hline$\Rightarrow \overline{=}$ & STCH & 6782 & stress 70 protein chaperone, microsome-associated, $60 \mathrm{kDa}$ & 2,70 & chr21 \\
\hline 30 & STK10 & 6793 & serine/threonine kinase 10 & 2,21 & chr5 \\
\hline$\Xi$ & STK17B & 9262 & Basic leucine zipper and W2 domains 1 & 13,41 & chr2 \\
\hline$\sum_{2}^{D}$ & STK32B & 55351 & serine/threonine kinase $32 \mathrm{~B}$ & 4,59 & chr4 \\
\hline : & STOML1 & 9399 & stomatin (EPB72)-like 1 & 3,40 & chr15 \\
\hline : & STS & 412 & steroid sulfatase (microsomal), arylsulfatase C, isozyme S & 2,37 & $\operatorname{chr} X$ \\
\hline 西 & STS-1 & 84959 & Cbl-interacting protein Sts-1 & 13,09 & chr11 \\
\hline$\Xi$ & STX5A & 6811 & syntaxin $5 \mathrm{~A}$ & 2,81 & chr11 \\
\hline 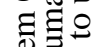 & STXBP1 & 6812 & syntaxin binding protein 1 & 2,79 & chr9 \\
\hline & SUMF1 & 285362 & sulfatase modifying factor 1 & 2,32 & chr3 \\
\hline है & SUPT6H & 6830 & suppressor of Ty 6 homolog (S. cerevisiae) & 2,27 & chr17 \\
\hline & SUSD1 & 64420 & sushi domain containing 1 & 2,64 & chr9 \\
\hline & SYBL1 & 6845 & synaptobrevin-like 1 & 2,90 & $\operatorname{chr} \mathrm{X}$ \\
\hline & SYDE1 & 85360 & synapse defective 1, Rho GTPase, homolog 1 (C. elegans) & 3,99 & chr19 \\
\hline 毛 & SYNJ1 & 8867 & synaptojanin 1 & 2,29 & chr21 \\
\hline 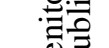 & SYNJ2 & 8871 & synaptojanin 2 & 7,52 & chr6 \\
\hline 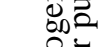 & SYNPO & 11346 & synaptopodin & 25,03 & chr5 \\
\hline 年 & SYNPO2 & 171024 & synaptopodin 2 & 32,72 & chr4 \\
\hline 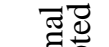 & SYTL4 & 94121 & Synaptotagmin-like 4 (granuphilin-a) & 3,38 & $\operatorname{chr} \mathrm{X}$ \\
\hline త్రి & |TACC1 & 6867 & transforming, acidic coiled-coil containing protein 1 & 8,32 & chr8 \\
\hline$\tilde{\pi}$ & TAF13 & 6884 & TAF13 RNA polymerase II, TATA box binding protein (TBP)-associated factor, 18kDa & 2,18 & chr1 \\
\hline$=$ & TAGLN & 6876 & transgelin & 25,80 & chr11 \\
\hline 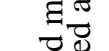 & TAGLN2 & 8407 & transgelin 2 & 6,46 & chr1 \\
\hline
\end{tabular}




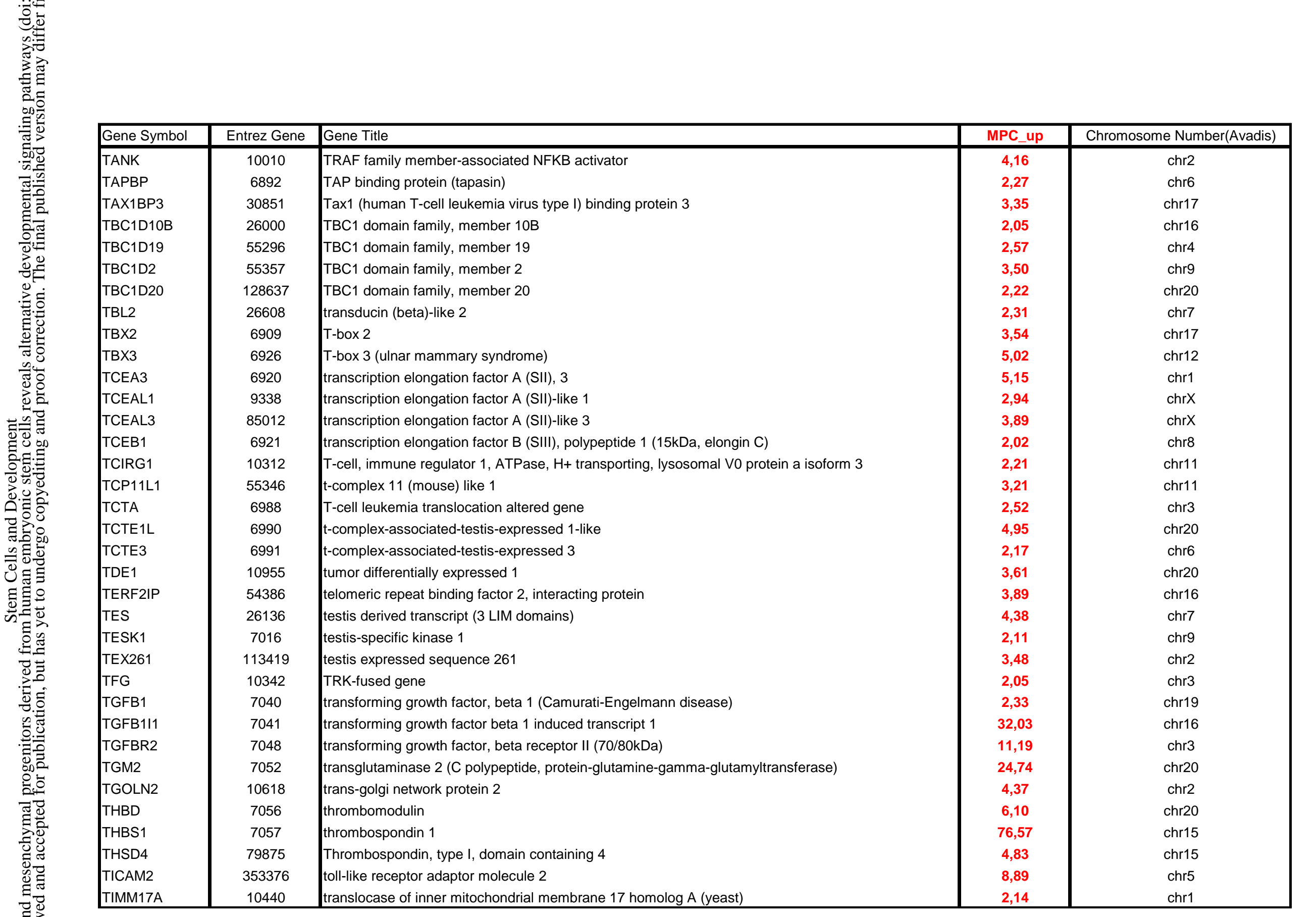




\begin{tabular}{|c|c|c|c|c|c|}
\hline 可 & Gene Symbol & Entrez Gene & Gene Title & MPC_up & Chromosome Number(Avadis) \\
\hline 50] & TIMP1 & 7076 & TIMP metallopeptidase inhibitor 1 & 6,85 & $\operatorname{chrX}$ \\
\hline$\Xi$ & TIPARP & 25976 & TCDD-inducible poly(ADP-ribose) polymerase & 10,37 & chr3 \\
\hline 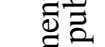 & TLOC1 & 7095 & translocation protein 1 & 3,35 & chr3 \\
\hline हैं & TLR4 & 7099 & toll-like receptor 4 /// toll-like receptor 4 & 4,58 & chr9 \\
\hline QE & TM4SF1 & 4071 & transmembrane $4 \mathrm{~L}$ six family member 1 & 48,84 & chr3 \\
\hline 远 & TM7SF1 & 7107 & transmembrane 7 superfamily member 1 (upregulated in kidney) & 2,27 & chr1 \\
\hline$D$ & TM9SF1 & 10548 & transmembrane 9 superfamily member 1 & 2,73 & chr14 \\
\hline $\bar{J}$ & TMBIM1 & 64114 & transmembrane BAX inhibitor motif containing 1 & 2,78 & chr2 \\
\hline E & TMCO1 & 54499 & transmembrane and coiled-coil domains 1 & 2,37 & chr1 \\
\hline न & TMCO3 & 55002 & transmembrane and coiled-coil domains 3 & 4,78 & chr13 \\
\hline (1) & TMED3 & 23423 & transmembrane emp24 protein transport domain containing 3 & 2,28 & chr15 \\
\hline 至 & TMED7 & 51014 & transmembrane emp24 protein transport domain containing 7 & 2,44 & chr5 \\
\hline$\exists=$ & TMEM14A & 28978 & transmembrane protein $14 \mathrm{~A}$ & 2,43 & chr6 \\
\hline Sol & TMEM16D & 121601 & transmembrane protein 16D & 2,46 & chr12 \\
\hline$\because \Xi$ & TMEM16F & 196527 & transmembrane protein $16 \mathrm{~F}$ & 2,61 & chr12 \\
\hline$\sum_{2}^{D}$ & TMEM17 & 200728 & transmembrane protein 17 & 2,04 & chr2 \\
\hline o. & TMEM30A & 55754 & transmembrane protein $30 \mathrm{~A}$ & 7,85 & chr6 \\
\hline 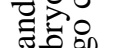 & TMEM43 & 79188 & transmembrane protein 43 & 2,80 & chr3 \\
\hline E & TMEM45A & 55076 & transmembrane protein $45 \mathrm{~A}$ & 2,67 & chr3 \\
\hline$\Xi$ & TMEM47 & 83604 & transmembrane protein 47 & 2,45 & $\operatorname{chr} X$ \\
\hline \& & TMEM49 & 81671 & transmembrane protein 49 & 4,57 & chr17 \\
\hline & TMEM55A & 55529 & transmembrane protein $55 \mathrm{~A}$ & 2,21 & chr8 \\
\hline పี & TMEM65 & 157378 & transmembrane protein 65 & 3,26 & chr8 \\
\hline & TMEM87B & 84910 & Transmembrane protein $87 \mathrm{~B}$ & 4,46 & chr2 \\
\hline & TMEM9B & 56674 & TMEM9 domain family, member $B$ & 2,23 & chr11 \\
\hline & TMF1 & 7110 & TATA element modulatory factor 1 & 2,82 & chr3 \\
\hline 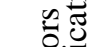 & TMOD3 & 29766 & tropomodulin 3 (ubiquitous) & 3,17 & chr15 \\
\hline$\frac{7}{3}$ & TMSB10 & 9168 & thymosin, beta 10 & 2,57 & chr2 \\
\hline & TNFAIP6 & 7130 & tumor necrosis factor, alpha-induced protein 6 & 2,49 & chr2 \\
\hline \pm & TNFRSF10B & 8795 & tumor necrosis factor receptor superfamily, member $10 \mathrm{~b}$ & 4,42 & chr8 \\
\hline 焉 & TNFRSF10D & 8793 & tumor necrosis factor receptor superfamily, member $10 \mathrm{~d}$, decoy with truncated death domain & 17,91 & chr8 \\
\hline : & TNFRSF1A & 7132 & tumor necrosis factor receptor superfamily, member $1 \mathrm{~A}$ & 6,21 & chr12 \\
\hline & TNFSF4 & 7292 & tumor necrosis factor (ligand) superfamily, member 4 (tax-transcriptionally activated glycoprotein 1, 34 & 8,61 & chr1 \\
\hline & TNIP1 & 10318 & TNFAIP3 interacting protein 1 & 2,96 & chr5 \\
\hline & TNS3 & 64759 & Tensin 3 & 4,74 & chr7 \\
\hline
\end{tabular}




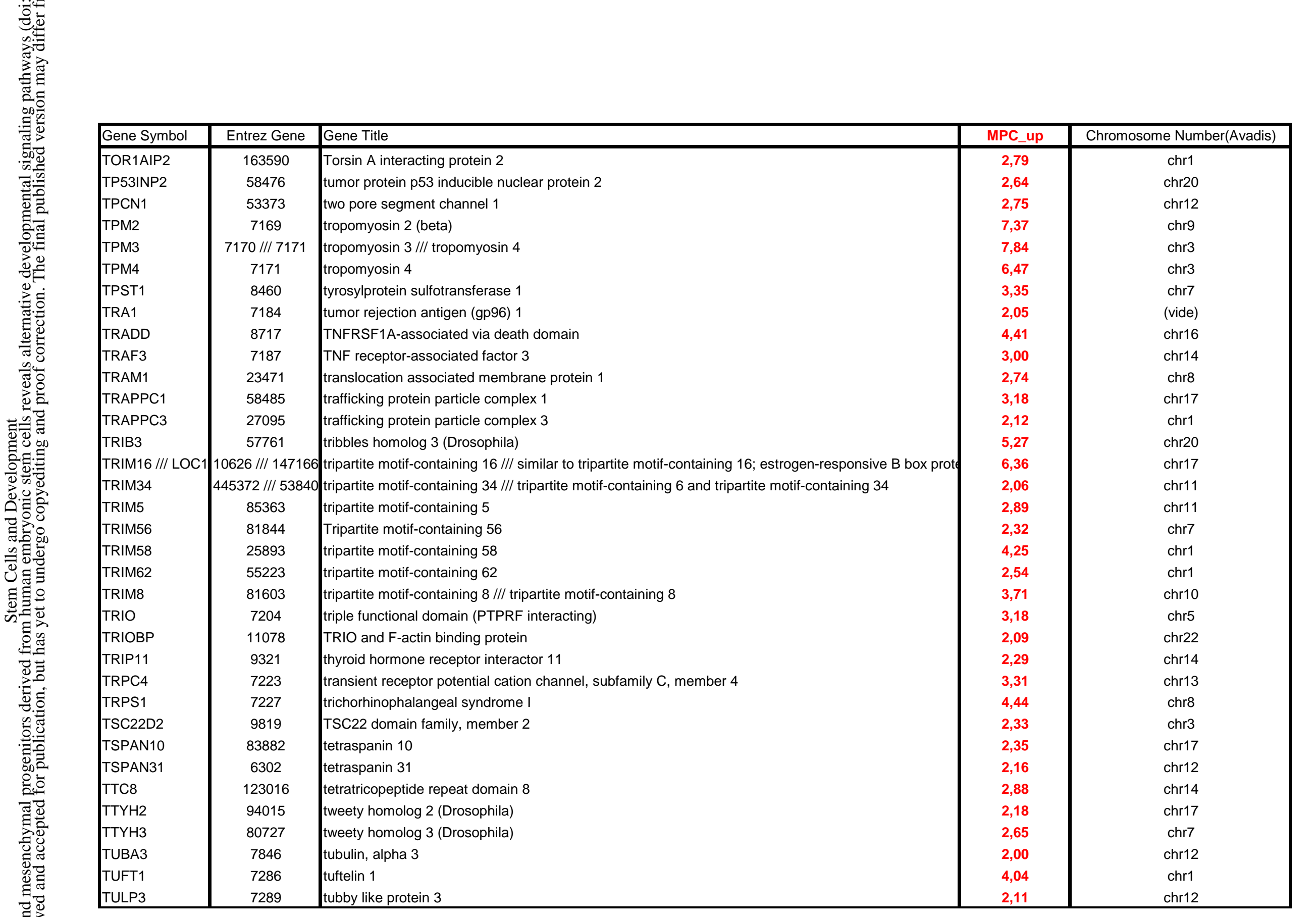




\begin{tabular}{|c|c|c|c|c|c|}
\hline 可 & Gene Symbol & Entrez Gene & Gene Title & MPC_up & Chromosome Number(Avadis) \\
\hline 50 & TWIST1 & 7291 & twist homolog 1 (acrocephalosyndactyly 3; Saethre-Chotzen syndrome) (Drosophila) & 10,84 & chr7 \\
\hline$\Xi \overline{0}$ & TWSG1 & 57045 & twisted gastrulation homolog 1 (Drosophila) & 3,64 & chr18 \\
\hline 醇 & TXNDC10 & 54495 & thioredoxin domain containing 10 & 3,71 & chr18 \\
\hline हैं & TXNDC11 & 51061 & thioredoxin domain containing 11 & 3,94 & chr16 \\
\hline 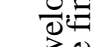 & TXNDC13 & 56255 & thioredoxin domain containing 13 & 2,65 & chr20 \\
\hline 远 & TXNRD1 & 7296 & thioredoxin reductase 1 & 3,06 & chr12 \\
\hline$D$ & TXNRD3 & 114112 & thioredoxin reductase 3 & 2,06 & chr3 \\
\hline $\bar{J}$ & UACA & 55075 & uveal autoantigen with coiled-coil domains and ankyrin repeats & 5,87 & chr15 \\
\hline 远 & UAP1 & 6675 & UDP-N-acteylglucosamine pyrophosphorylase 1 & 5,83 & chr1 \\
\hline$\pi \overline{0}$ & |UBE2B & 7320 & ubiquitin-conjugating enzyme E2B (RAD6 homolog) /// ubiquitin-conjugating enzyme E2B (RAD6 hom & 2,59 & chr5 \\
\hline एँ. & UBE2Q2 & 92912 & ubiquitin-conjugating enzyme E2Q (putative) 2 & 2,76 & chr15 \\
\hline 象 & JUBE2W & 55284 & ubiquitin-conjugating enzyme E2W (putative) & 2,32 & chr8 \\
\hline$=\overline{0}$ & UBL3 & 5412 & ubiquitin-like 3 & 2,60 & chr13 \\
\hline 30 & UBXD1 & 80700 & UBX domain containing 1 & 3,08 & chr19 \\
\hline$\Xi \Xi$ & JEV3 & 55293 & Ubiquitin-conjugating enzyme E2-like & 8,22 & chr11 \\
\hline$\sum_{2}^{D}$ & UFM1 & 51569 & ubiquitin-fold modifier 1 & 3,62 & chr13 \\
\hline 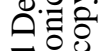 & UGCG & 7357 & UDP-glucose ceramide glucosyltransferase & 4,24 & chr9 \\
\hline 路品 & UGCGL1 & 56886 & UDP-glucose ceramide glucosyltransferase-like 1 & 2,14 & chr2 \\
\hline 西 & |UHMK1 & 127933 & U2AF homology motif (UHM) kinase 1 & 6,04 & chr1 \\
\hline$\Xi$ & |ULBP2 & 80328 & UL16 binding protein 2 & 24,08 & chr6 \\
\hline & |UNQ1912 & 345757 & HGS_RE408 & 5,13 & chr5 \\
\hline & URB & 151887 & steroid sensitive gene 1 & 147,43 & chr3 \\
\hline ప్ర & UROS & 7390 & uroporphyrinogen III synthase (congenital erythropoietic porphyria) & 2,02 & chr10 \\
\hline & |USP15 & 9958 & ubiquitin specific peptidase 15 & 2,63 & chr12 \\
\hline & |USP38 & 84640 & ubiquitin specific peptidase 38 & 2,19 & chr4 \\
\hline & USP40 & 55230 & ubiquitin specific peptidase 40 & 2,38 & chr2 \\
\hline t. & VAMP3 & 9341 & vesicle-associated membrane protein 3 (cellubrevin) /// vesicle-associated membrane protein 3 (cellub & 3,14 & chr1 \\
\hline 눌 & VCPIP1 & 80124 & Valosin containing protein (p97)/p47 complex interacting protein 1 & 2,83 & chr8 \\
\hline & VDR & 7421 & vitamin D (1,25- dihydroxyvitamin D3) receptor & 3,81 & chr12 \\
\hline 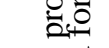 & VEGF & 7422 & vascular endothelial growth factor & 3,89 & chr6 \\
\hline 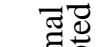 & VEPH1 & 79674 & ventricular zone expressed PH domain homolog 1 (zebrafish) & 16,65 & chr3 \\
\hline 8 & VGCNL1 & 259232 & Voltage gated channel like 1 & 6,11 & chr13 \\
\hline z & VGL-3 & 389136 & vestigial-like 3 & 26,25 & chr3 \\
\hline$=$ & VIM & 7431 & vimentin & 4,65 & chr10 \\
\hline & WARS & 7453 & tryptophanyl-tRNA synthetase & 2,30 & chr14 \\
\hline
\end{tabular}




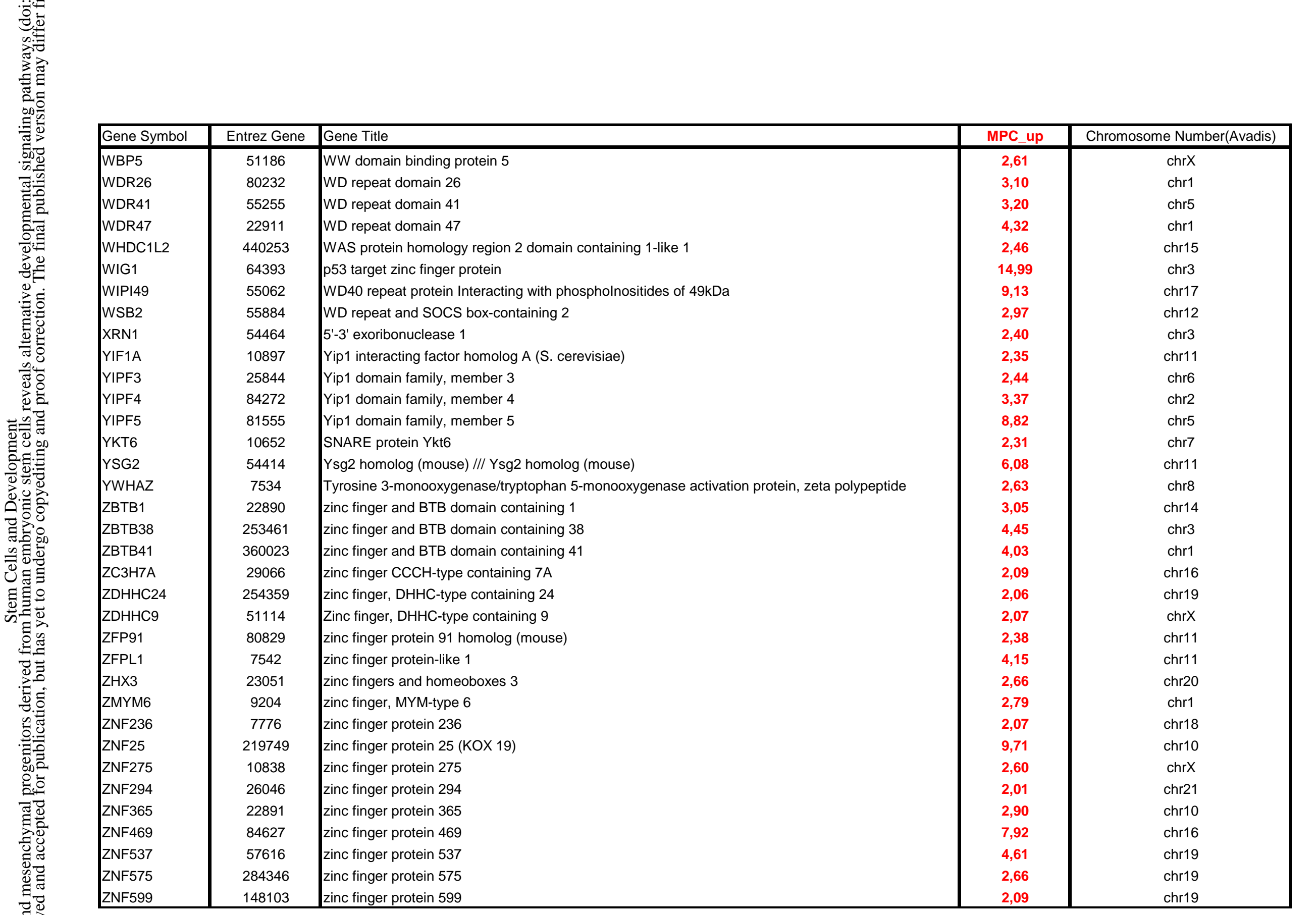




\section{Page 253 of 257}

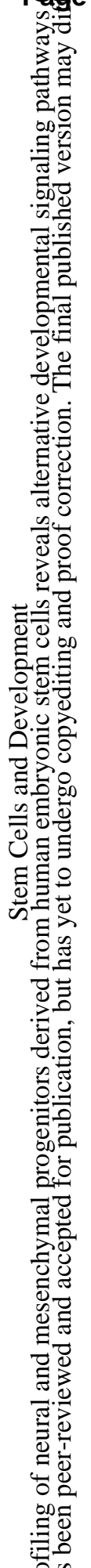

\begin{tabular}{|c|c|c|c|c|}
\hline Gene Symbol & Entrez Gene & Gene Title & MPC_up & Chromosome Number(Avadis) \\
\hline ZNF650 & 130507 & zinc finger protein 650 & 2,13 & chr2 \\
\hline ZNF654 & 55279 & Zinc finger protein 654 & 2,78 & chr3 \\
\hline ZNHIT1 & 10467 & zinc finger, HIT type 1 & 2,48 & chr7 \\
\hline & & (vide) & 66,75 & chr17 \\
\hline & & wingless-type MMTV integration site family, member 5A /// wingless-type MMTV integration site family & 37,13 & chr2 \\
\hline & & syncoilin, intermediate filament $1 / / /$ syncoilin, intermediate filament 1 & 9,57 & chr3 \\
\hline & & protein kinase C, alpha & 6,99 & chr5 \\
\hline & & Fucosyltransferase 1 (galactoside 2-alpha-L-fucosyltransferase, $\mathrm{H}$ blood group) & 4,06 & chrY \\
\hline & & sterol carrier protein 2 /// sterol carrier protein 2 & 2,44 & (vide) \\
\hline & & transmembrane protein with EGF-like and two follistatin-like dor & 2,30 & chr4 \\
\hline
\end{tabular}




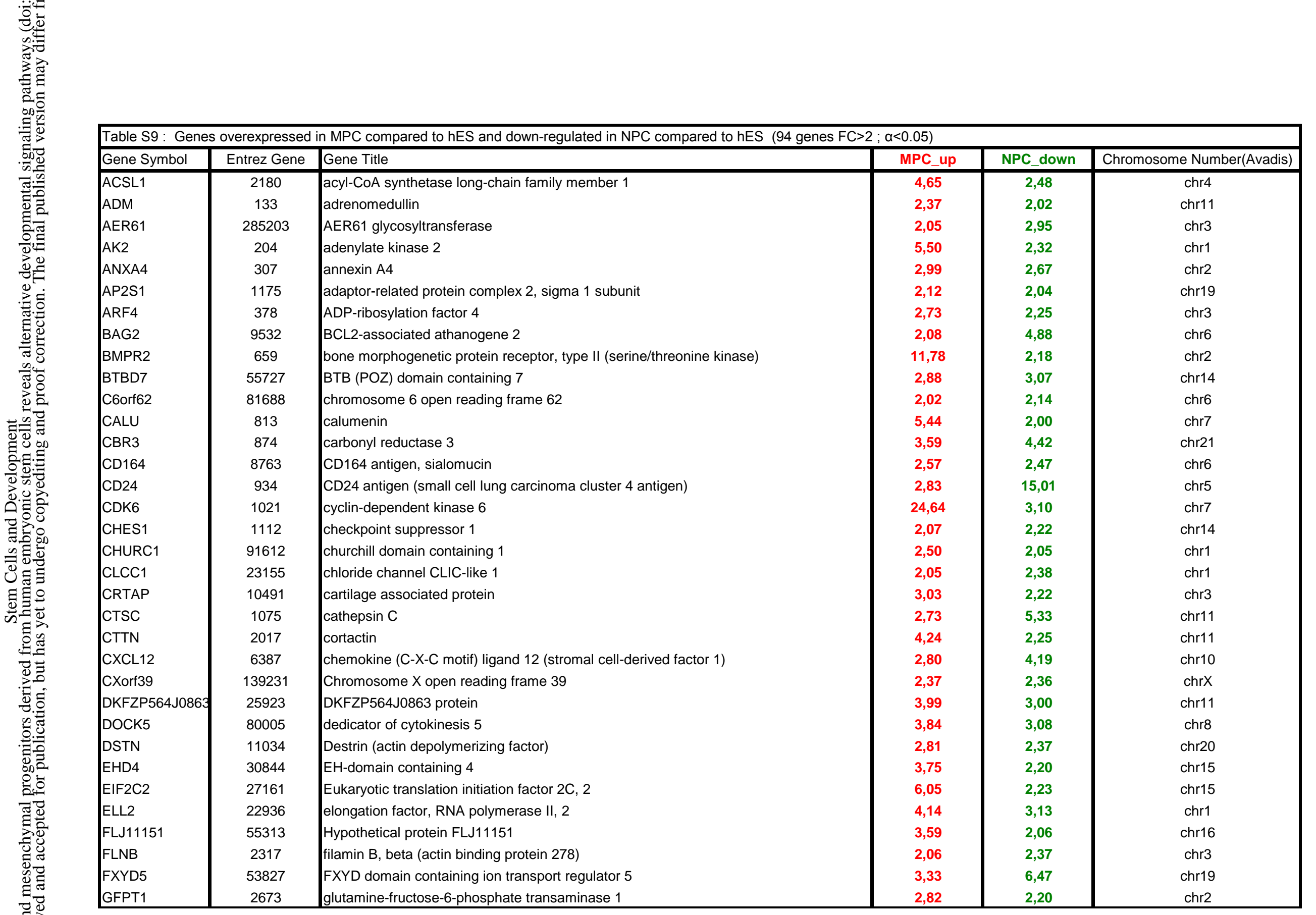




\begin{tabular}{|c|c|c|c|c|c|c|}
\hline & & & |Gene IItle & & & \\
\hline & GLS & 2744 & glutaminase & 17,00 & 2,14 & chr2 \\
\hline $\bar{D} \bar{z}$ & GNB1 & 2782 & guanine nucleotide binding protein ( $G$ protein), beta polypeptide 1 & 3,97 & 2,06 & chr1 \\
\hline 言苛 & HSPB1 & 3315 & heat shock $27 \mathrm{kDa}$ protein 1 & 11,96 & 2,93 & $\operatorname{chr} 7$ \\
\hline$\Xi$ & IGFBP6 & 3489 & insulin-like growth factor binding protein 6 & 7,75 & 2,36 & chr12 \\
\hline 刍 & ITCH & 83737 & litchy homolog E3 ubiquitin protein ligase (mouse) & 2,85 & 2,04 & chr20 \\
\hline 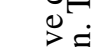 & JAK1 & 3716 & Janus kinase 1 (a protein tyrosine kinase) & 5,81 & 2,20 & chr1 \\
\hline$=$ & JAZF1 & 221895 & juxtaposed with another zinc finger gene 1 & 2,18 & 4,31 & $\operatorname{chr} 7$ \\
\hline 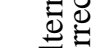 & KIAA1404 & 57169 & KIAA1404 protein & 2,00 & 2,16 & chr20 \\
\hline 䓽 & KLF7 & 8609 & Kruppel-like factor 7 (ubiquitous) & 3,30 & 2,26 & chr2 \\
\hline 5 & LACTB & 114294 & lactamase, beta & 2,37 & 2,62 & chr15 \\
\hline$\vec{a}$ & LAMP2 & 3920 & lysosomal-associated membrane protein 2 & 6,02 & 2,16 & $\operatorname{chr} \mathrm{x}$ \\
\hline$\vec{a}=\vec{\sigma}$ & LGALS1 & 3956 & lectin, galactoside-binding, soluble, 1 (galectin 1) & 52,69 & 13,73 & chr22 \\
\hline$\underbrace{\infty}_{\infty}$ & LOC151194 & 151194 & similar to hepatocellular carcinoma-associated antigen HCA557b & 2,09 & 2,47 & chr2 \\
\hline$\Xi$ & LOC201895 & 201895 & hypothetical protein LOC201895 & 4,58 & 2,28 & chr4 \\
\hline$\underset{D}{0}$ & LOC441762 & 441762 & Similar to CG7467-PA /// Similar to CG7467-PA & 2,16 & 2,64 & chr16 \\
\hline$\frac{2}{0}$ & MAP1LC3B & 81631 & microtubule-associated protein 1 light chain 3 beta & 4,53 & 3,04 & chr12 \\
\hline 㱐品 & MAP3K5 & 4217 & mitogen-activated protein kinase kinase kinase 5 & 2,25 & 2,98 & chr6 \\
\hline 焉 & MAP4 & 4134 & microtubule-associated protein 4 & 5,40 & 2,64 & chr3 \\
\hline$\Xi$ & MAPK1 & 5594 & mitogen-activated protein kinase 1 & 2,26 & 2,51 & chr22 \\
\hline 0 & MAPKAP1 & 79109 & mitogen-activated protein kinase associated protein 1 & 2,72 & 2,96 & chr9 \\
\hline & ME1 & 4199 & Malic enzyme 1, NADP(+)-dependent, cytosolic & 12,94 & 2,20 & chr6 \\
\hline$\pi$ & MEF2A & 4205 & MADS box transcription enhancer factor 2, polypeptide A (myocyte enhancer factor 2A) & 2,45 & 2,22 & chr15 \\
\hline & MGC11324 & 84803 & hypothetical protein MGC11324 /// hypothetical protein MGC11324 & 2,89 & 2,30 & chr4 \\
\hline & MT2A & 4502 & metallothionein $2 \mathrm{~A}$ & 3,32 & 5,41 & $\operatorname{chr} 16$ \\
\hline छَّ. & NCBP2 & 22916 & Nuclear cap binding protein subunit 2, 20kDa & 2,14 & 2,33 & chr3 \\
\hline ฮ艹 & PAPSS2 & 9060 & 3'-phosphoadenosine 5'-phosphosulfate synthase 2 & 6,50 & 4,71 & chr10 \\
\hline$\frac{7}{7}$ & PHLDA1 & 22822 & Pleckstrin homology-like domain, family A, member 1 & 3,23 & 3,12 & chr12 \\
\hline & PINK1 & 65018 & PTEN induced putative kinase 1 & 2,72 & 2,16 & chr1 \\
\hline & PKIB & 5570 & protein kinase (cAMP-dependent, catalytic) inhibitor beta & 2,33 & 2,69 & $\operatorname{chr} 6$ \\
\hline 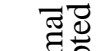 & PPGB & 5476 & protective protein for beta-galactosidase (galactosialidosis) & 2,61 & 2,34 & chr20 \\
\hline & PRKAA1 & 5562 & protein kinase, AMP-activated, alpha 1 catalytic subunit & 3,78 & 2,02 & chr5 \\
\hline & PTEN & 5728 & Phosphatase and tensin homolog (mutated in multiple advanced cancers 1 ) & 2,70 & 2,12 & chr10 \\
\hline & RAB3B & 5865 & RAB3B, member RAS oncogene family & 3,19 & 2,14 & chr1 \\
\hline & RCN3 & 57333 & reticulocalbin 3, EF-hand calcium binding domain & 3,50 & 2,75 & chr19 \\
\hline
\end{tabular}




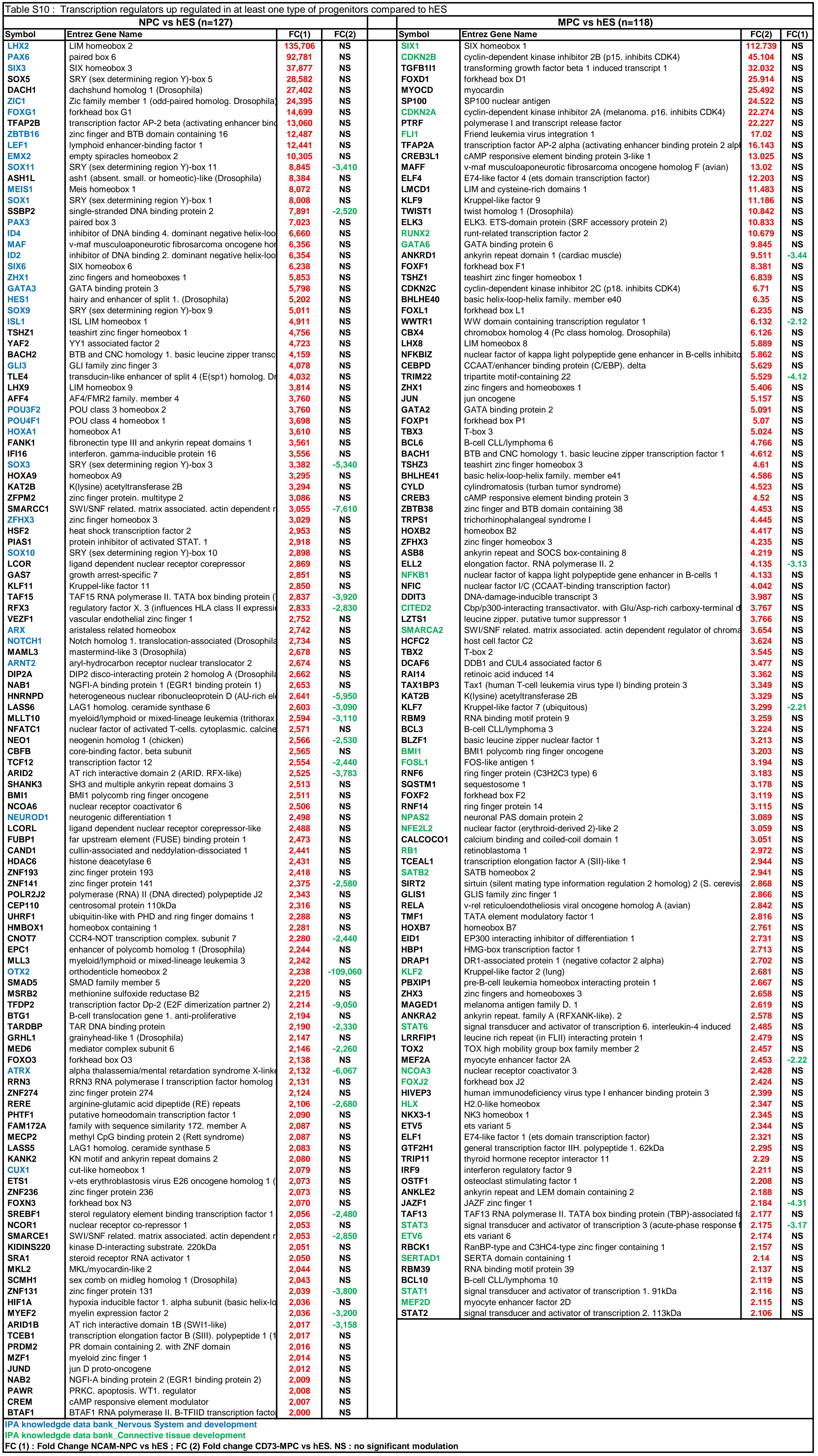

